Western Kentucky University

TopSCHOLAR®

Masters Theses \& Specialist Projects

Graduate School

Spring 2020

\title{
Load Balancing of Financial Data Using Machine Learning and Cloud Analytics
}

Dimple Jaiswal

Western Kentucky University, dimple.jaiswal230@topper.wku.edu

Follow this and additional works at: https://digitalcommons.wku.edu/theses

Part of the Computer and Systems Architecture Commons, and the Data Storage Systems Commons

\section{Recommended Citation}

Jaiswal, Dimple, "Load Balancing of Financial Data Using Machine Learning and Cloud Analytics" (2020).

Masters Theses \& Specialist Projects. Paper 3215.

https://digitalcommons.wku.edu/theses/3215

This Thesis is brought to you for free and open access by TopSCHOLARß. It has been accepted for inclusion in Masters Theses \& Specialist Projects by an authorized administrator of TopSCHOLARß. For more information, please contact topscholar@wku.edu. 
LOAD BALANCING OF FINANCIAL DATA USING MACHINE LEARNING AND CLOUD ANALYTICS

\author{
A Thesis \\ Presented to \\ The Faculty of the School of Engineering and Applied Sciences \\ Western Kentucky University \\ Bowling Green, Kentucky
}

In Partial Fulfillment

Of the Requirements for the Degree

Master of Science

By

Dimple Jaiswal

May 2020 
LOAD BALANCING OF FINANCIAL DATA USING MACHINE LEARNING AND CLOUD ANALYTICS

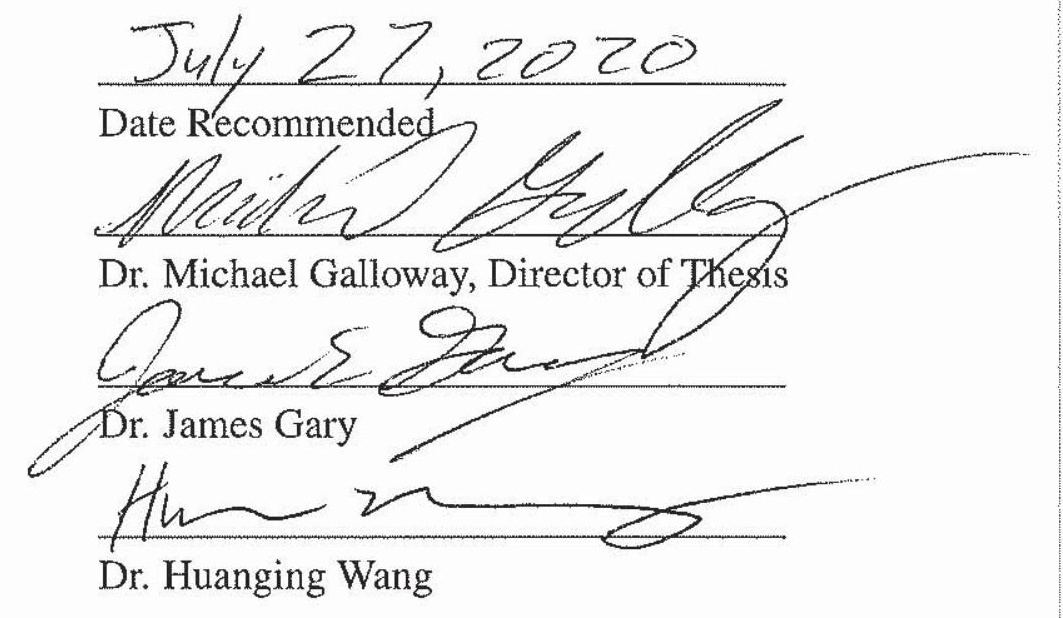

Ranjit T. Koodali Digitally signed by Ranjit T. Koodali

Associa e rovos for esearch and $\mathrm{rad}$ a e Ed ca ion 
To my grandparents, mother, father, brother and friends.

$\&$

To the faculty of the Computer Science Department at Western Kentucky University. 


\section{ACKNOWLEDGMENTS}

I would first especially like to thank my professor and my advisor, Dr. Galloway, for giving me an opportunity to work in the cloud lab and for introducing me to gain practical knowledge in the cloud computing field. I really appreciate his support and contribution for my work. Dr. Galloway not only helped in my research but also guided me throughout my masters. Dr. Galloway supports and welcomes every student's innovative ideas for technology. I'm very glad to see various research done by students in cloud lab. It motivated me and gave me a chance to think different. Dr. Galloway encouraged me to participate in various conferences and supported me in every aspect he could. His goal-oriented approach is the best take away from my master's journey. Moreover, I'm very thankful for his time and flexibility he has given me for my work.

I would also like to thank my professors, Dr. Wang, and Dr. Gary, for their teachings and guidance in my academic journey. Dr. Wang is the sweetest professor I met at WKU. She is very generous and caring. She makes everything so simple, concise and achievable for students. I really had a great time taking her class. Thank you ma'am for your support and help.

I would like to thank and appreciate Dr. Gary for making my master's journey memorable. I'm surely going to miss your class, sir. Your hilarious jokes and your class's interactive environment made attending your class worth it. Thank you sir for creating such a beautiful memory at WKU.

I express my gratitude towards to all the other faculty members of Computer Sci- 
ence Department and SEAS for giving me a chance to work as a graduate assistant and helping me to grow and learn more, every day. I also want to thank the graduate school and Ogden College for all the financial support they have given me. It gave me a chance to explore more in my field. Thank you for providing all the resources and support at every step.

Last but not least, I would like to thank my parents for believing in me and giving me an opportunity to travel so far to grow in my career. I owe every bit of my success to you and to your teachings. I wouldn't have been here without your support. Thank you god for sending so many beautiful souls in my life. Thank you! 


\section{CONTENTS}

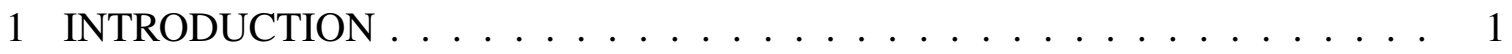

1.1 History and Analysis of Existing Problems . . . . . . . . . . . 1

1.2 Brief Discussion on Financial Data and Various Job Requests . . . . . . . 2

1.2.1 Data Preprocessing ................. 2

1.2.2 Commonly Known User Requests on Financial Web Application . . 2

1.2.3 Analysis of Execution Time of Different Job Requests . . . . . . 5

1.3 Proposed Solution . . . . . . . . . . . . . . . . 6

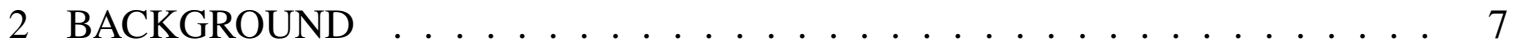

2.1 Cloud Computing . . . . . . . . . . . . . . . . . 7

2.1.1 Several Characteristics of Cloud Computing . . . . . . . . . . 7

2.1.2 Services Provided by Cloud Computing . . . . . . . . . . . . 8

2.2 Load Balancing . . . . . . . . . . . . . . . . 9

2.2 .1 Introduction . . . . . . . . . . . . . . . 9

2.3 Machine Learning . . . . . . . . . . . . . . . . . 13

2.3.1 Classification Technique ................ 15 
2.4 Cloud Analytics . . . . . . . . . . . . . . . . . . . . . . . 18

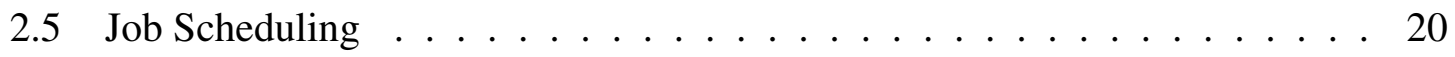

2.5 .1 Genetic Algorithm . . . . . . . . . . . . . . 20

2.5.2 Imperialist Competitive Algorithm . . . . . . . . . . . . 23

2.6 Comparison of AWS and IBM Clouds $\ldots \ldots \ldots \ldots$

2.7 Amazon Web Services _ . . . . . . . . . . . . . . . . 26

2.7.1 AWS Tools used for Implementation . . . . . . . . . . . . . 28

3 HARDWARE AND SOFTWARE ARCHITECTURE . . . . . . . . . . . 30

3.1 Hardware Architecture . . . . . . . . . . . . . . . . . . . . . 31

3.2 Software Architecture . . . . . . . . . . . . . . 32

3.2.1 Front-end Architecture . . . . . . . . . . . . . . . . . 33

3.2.2 Back-end Architecture . . . . . . . . . . . . . 33

4 DESIGN AND IMPLEMENTATION . . . . . . . . . . . . . . . . . 35

$4.1 \quad$ Stock Data Preprocessing . . . . . . . . . . . . . . . 36

4.2 Web Application Development . . . . . . . . . . . . . . . . 37

4.2.1 Implementation of Different Types of Job Requests . . . . . . . 37

4.3 Training Set Creation using Locust $\ldots \ldots \ldots \ldots$

4.3.1 Load Testing Performed at Every Node . . . . . . . . . . 40

4.3.2 Analysis of Data Generated at Nodes by Locust Testing _. . . . . 40

$4.4 \quad$ Load Balancing $\ldots \ldots \ldots \ldots \ldots \ldots$

4.4 .1 Types of Load Balancing . . . . . . . . . . . . . . . . 45

$4.4 .2 \quad \log$ Analysis $\ldots \ldots \ldots \ldots \ldots$ 
5 COMPARATIVE STUDY AND CONCLUSION . . . . . . . . . . 55

5.1 Comparative Study . . . . . . . . . . . . . . . 55

5.2 Future work . . . . . . . . . . . . . . . . . 59

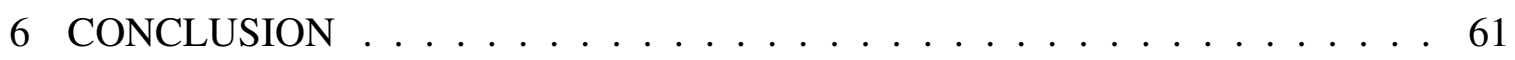

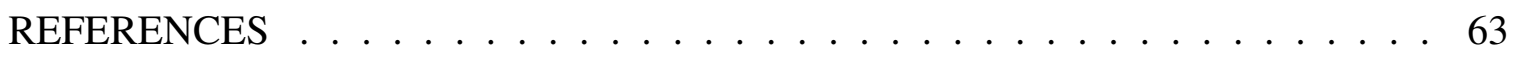

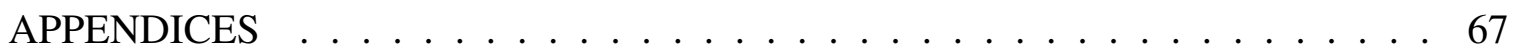

A APPENDIX A: SETTING UP THE ARCHITECTURE . . . . . . . . . . . . 68

A.1 Stock Data Pre-processing script . . . . . . . . . . . . . 68

A.2 Web Application Design . . . . . . . . . . . . . 73

A.2.1 Directory Tree Structure for Web Application . . . . . . . . . . . . 77

A.2.2 Files in project Finance . . . . . . . . . . . . . . 77

A.2.3 Files in finWeb app . . . . . . . . . . . . . 81

A.2.4 Execution . . . . . . . . . . . . . . . 197

A.2.5 Initial testing of jobs using ab-benchmark tool . . . . . . . . . 198

A.3 Comparison of appilcation hosted on IBM and AWS Cloud . . . . . . . 199

A.3.1 Web application on IBM Cloud . . . . . . . . . . . . . . 199

A.3.2 Web application on AWS Cloud . . . . . . . . . . . . . 200

A.3.3 Stress load testing using ab-benchmark tool . . . . . . . . . . 202

A.4 Generation of training dataset . . . . . . . . . . . 206

A.5 Load Balancing Component _ . . . . . . . . . . . . . . . . 212

A.5.1 Installation of NGINX and setup NGINX for cluster . . . . . . . 212

A.5.2 Types of Load Balancing . . . . . . . . . . . . . . . . 213 
A.6 Data Analysis . . . . . . . . . . . . . . . . . . . 217

A.6.1 Response time analysis for different types of job requests . . . . 217

A.6.2 Data analysis of data generated by Locust testing at all the nodes . 220

A.6.3 Data analysis of data generated by Locust testing using different types of load balancing approach . . . . . . . . . . . . 234

A.6.4 Analysis of logs generated by nginx . . . . . . . . . . . . . 250

A.6.5 Data analysis of data generated by Locust testing for web application hosted on AWS cloud . . . . . . . . . . . . . 266 


\section{LIST OF TABLES}

3.1 Harware Architecture of Master Node and Computing Nodes . . . . . . . . 31

A.1 Execution time for all the types of job requests using ab-benchmark tool . . 199

A.2 Shell script output for load testing . . . . . . . . . . . 206 


\section{LIST OF FIGURES}

1.1 Types of User Requests . . . . . . . . . . . . . . . . . . . . . . . 4

1.2 Total Time Taken for Execution of Individual Job . . . . . . . . . . . 6

2.1 Cloud Reference Architecture [Kumar and Goudar, 2012] . . . . . . . . . . 9

2.2 Round Robin Approach used to Balance Various Requests Acting as Load on Given Two Servers [Villanueva, 2015] . . . . . . . . . . . . . . . . 10

2.3 Weighted Round Robin Approach used to Balance Various Requests Acting as Load on Given Two servers [Villanueva, 2015] . . . . . . . . . . . . . . 11

2.4 Flow Diagram of the Behavioral Control Structure for Honey Bee Behavior Inspired Load Balancing of Tasks in Cloud Computing Inspired by Foraging Behavior of Real Honey Bees Adapted from Johnson and Nieh with Minor Changes. [Johnson and Nieh, 2010] . . . . . . . . . . . . . . . . . . 12

2.5 Channel Agent Searching for Virtual Machines [Vig, Kushwah, Tomar, and Kushwah, 2016] . . . . . . . . . . . . . . . 13

2.6 Learning = Representation + Evaluation + Optimization [Domingos, 2012] 14

2.7 Proposed Framework to Transform Decision Table or Decision Tree to Directly Generate Set of Rules [Shamim, Hussain, and Maqbool Uddin Shaikh, 2010] . . . . . . . . . . . . . . . . . . 16

2.8 Log Analytics on Cloud [Bhole, Adinarayana, and Shenoy, 2015] . . . . . 20

2.9 Flowchart for Genetic Algorithm [Rajput and Kushwah, 2016] . . . . . . . 22

2.10 Apache benchmarking results [Kaur, Raj, Yadav, and Choudhury, 2018] . . 24

2.11 Dbenchmark benchmarking results [Kaur et al., 2018] . . . . . . . . . . 24

2.12 RAMSpeed benchmarking results [Kaur et al., 2018] . . . . . . . . . 25

2.13 AWS vs. IBM Enterprise Scorecard [Kaur et al., 2018] . . . . . . . . . . . 25

2.14 Performance of Web Application hosted on AWS vs. IBM Cloud . . . . 26 
2.15 Cloud Service Provider Competitive Positioning [Reno and Group, Reno

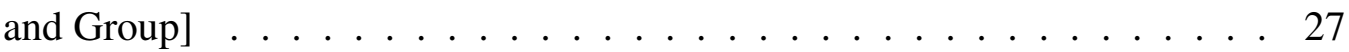

3.1 Load Balancing Diagram $[$ NGINX, 2020] . . . . . . . . . . . . 30

3.2 Cluster Architecture . . . . . . . . . . . . . . . . . . . . . . . 32

4.1 Design and Workflow of the Project $\ldots \ldots \ldots \ldots \ldots$

4.2 ETL- Extract, Transform and Load Process _ . . . . . . . . . . . . . 37

4.3 Example of Stock Data after Completion of Data Preprocessing . . . . . . . 37

4.4 Response Time Analysis for Different Types of Job Requests . . . . . . . . 39

4.5 Example of stats.csv file generated $\ldots \ldots \ldots \ldots \ldots$

4.6 Success Request Rate for All the Nodes _ . . . . . . . . . . . . . . . . 42

4.7 Failure Request Rate for All the Nodes _ . . . . . . . . . . . . . . . . 43

4.8 Average Response Time(in seconds) for All the Nodes . . . . . . . . . . . 44

4.9 Success Request Rate for Different Types of Load Balancers _ . . . . . . . 48

4.10 Failure Request Rate for Different Types of Load Balancers _ . . . . . . . 49

4.11 Average Response Time(in seconds) for Different Types of Load Balancers 50

4.12 Example of Error Log Generated by Nginx _ . . . . . . . . . . 51

4.13 Total Number of Requests made by Different Types of Load Balancing

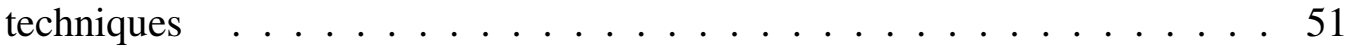

4.14 Total Number of Successful Requests for Every Node using Different Types of Load Balancing Techniques . . . . . . . . . . . . . . . . . . 52

4.15 Total Number of Requests Failed by Different Types of Load Balancing

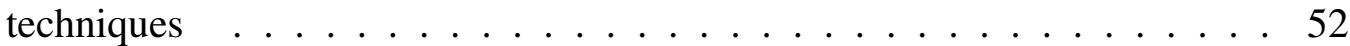

4.16 Total Number of Failure Requests for Every Node using Different Types of Load Balancing Techniques $\ldots \ldots \ldots \ldots$ 
5.1 Success Request Rate for Round Robin Load Balancing Method with AWS Load Balancer . . . . . . . . . . . . . . . . . . . 57

5.2 Failure Request Rate for Round Robin Load Balancing Method with AWS Load Balancer . . . . . . . . . . . . . . . . . . . 58

5.3 Average Response Time(in seconds) for Round Robin Load Balancing Method with AWS Load Balancer . . . . . . . . . . . . . . . . . . . 59

A.1 Work flow of methods called in Data preprocessing script $\ldots \ldots \ldots 73$

A.2 Directory Tree Structure for Web Application . . . . . . . . . . 77

A.3 Directory Tree Structure for templates folder $\ldots \ldots \ldots \ldots$

A.4 Dashboard of web application hosted on IBM Cloud _ . . . . . . . . 199

A.5 Deployement of web application on IBM Cloud- part $1 \ldots \ldots$. . . . . 200

A.6 Deployement of web application on IBM Cloud- part $2 \ldots \ldots \ldots$. . . . 200

A.7 Dashboard of web application hosted on AWS Cloud . . . . . . . . . 201

A.8 Dashboard for monitoring performance of web application $\ldots \ldots \ldots 201$

A.9 Deployement of web application on AWS Cloud _ . . . . . . . . . . 201

A.10 Directory Tree Structure for output generated by automateLocush.sh . . . 212

A.11 Example of directory containing output generated by automateLocush.sh $\quad 212$

A.12 Example of locust output on web interface $\ldots \ldots \ldots \ldots \ldots . \ldots 212$

A.13 Response time analysis for different types of job requests . . . . . . . 220

A.14 Success request rate for all the nodes $\ldots \ldots \ldots \ldots \ldots \ldots \ldots$

A.15 Failure request rate for all the nodes $\ldots \ldots \ldots \ldots \ldots \ldots$

A.16 Average response time(in seconds) for all the nodes . . . . . . . . . 234

A.17 Success request rate for different types of load balancers $\ldots \ldots \ldots . .248$

A.18 Failure request rate for different types of load balancers _ . . . . . . . 249 
A.19 Average response time(in seconds) for different types of load balancers . . 250

A.20 Total number of requests made by different types of load balancing tech-

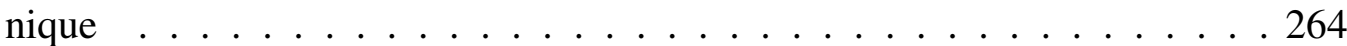

A.21 Total number of successful requests for every node using different types of load balancing techniques . . . . . . . . . . . . . . 265

A.22 Total number of requests failed by different types of load balancing tech-

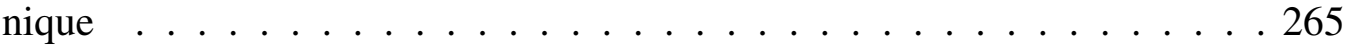

A.23 Total number of failure requests for every node using different types of load

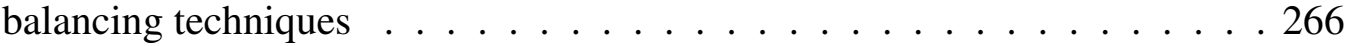

A.24 Success request rate for round robin load balancing method with AWS balancer . . . . . . . . . . . . . . . . . 275

A.25 Failure request rate for round robin load balancing method with AWS bal-

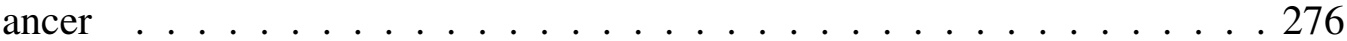

A.26 Average response time(in seconds) for round robin load balancing method with AWS balancer . . . . . . . . . . . . . . . . . . . 277 


\section{LIST OF CODES}

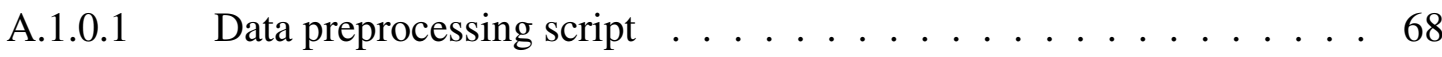

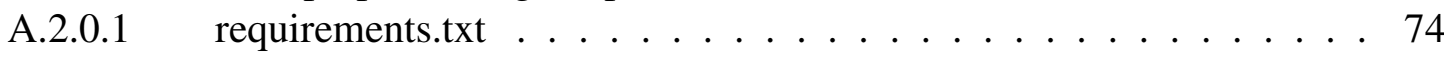

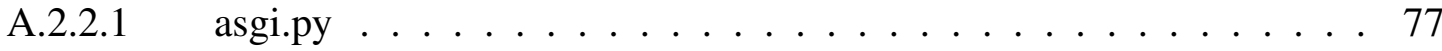

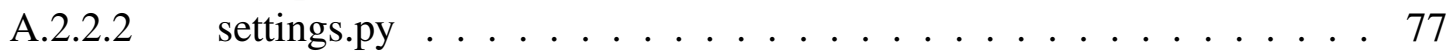

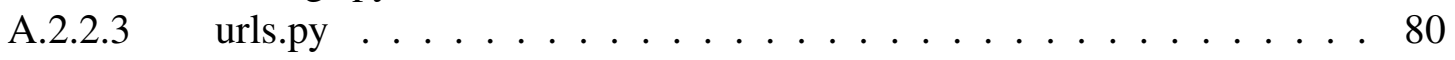

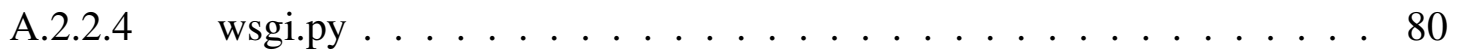

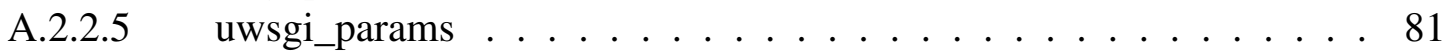

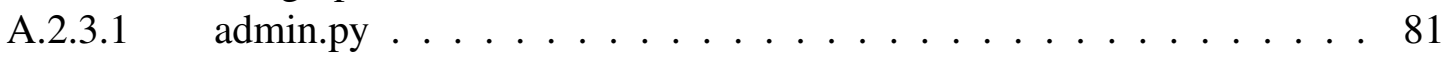

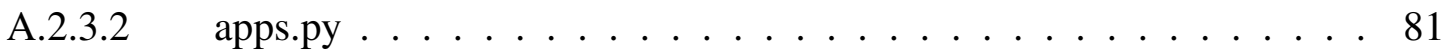

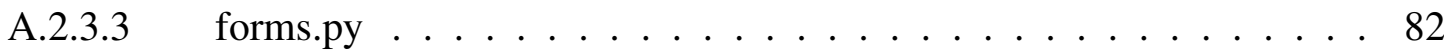

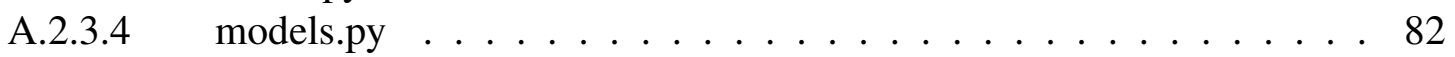

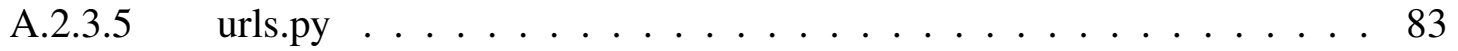

A.2.3.6 views.py .................. 83

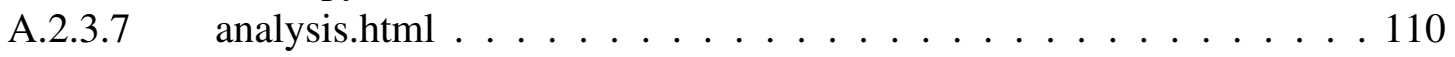

A.2.3.8 analysisResult.html . . . . . . . . . . . . . . 114

A.2.3.9 analysisResultLine.html . . . . . . . . . . . . . . . . 119

A.2.3.10 analysisResultTable.html . . . . . . . . . . . . . . 124

A.2.3.11 analysisResultCandlestick.html . . . . . . . . . . . 128

A.2.3.12 compare.html . . . . . . . . . . . . . 133

A.2.3.13 compareResult.html . . . . . . . . . . . . . . 137

A.2.3.14 compareCandleStick.html . . . . . . . . . . . . . . 142

A.2.3.15 compareResultLine.html . . . . . . . . . . . . . . . . 148

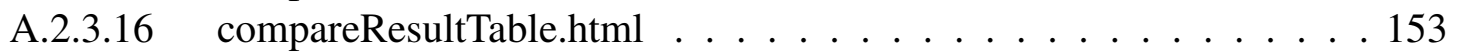

A.2.3.17 compareSameStock.html . . . . . . . . . . . . 158

A.2.3.18 measure.html . . . . . . . . . . . . 163

A.2.3.19 measureResults.html . . . . . . . . . . . . . 166

A.2.3.20 measureResultLine.html . . . . . . . . . . . . . . 170

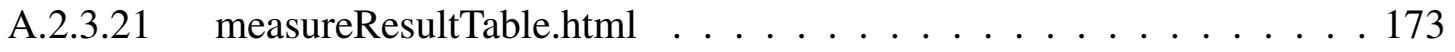

A.2.3.22 movers.html . . . . . . . . . . . . . . . 177

A.2.3.23 moversResult.html . . . . . . . . . . . . . . . 179

A.2.3.24 prediction.html . . . . . . . . . . . . . 182

A.2.3.25 predictionResult.html . . . . . . . . . . . . 185

A.2.3.26 predictionResultNoData.html . . . . . . . . . . . 188

A.2.3.27 signup.html . . . . . . . . . . . . . . . . 191

A.2.3.28 Loginpage.html . . . . . . . . . . . . . . . . 192

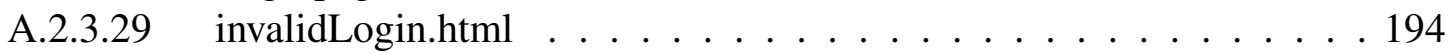

A.2.3.30 home.html . . . . . . . . . . . . . . 195

A.2.4.1 manage.py . . . . . . . . . . . . . . 197 
A.2.5.1 Execution time for all the types of job requests using ab-benchmark tool . . . . . . . . . . . . . . . . . . 198

A.3.3.1 Shell script for load testing . . . . . . . . . . . . 202

A.4.0.1 locustfile.py . . . . . . . . . . . . . 206

A.4.0.2 automateLocust.sh . . . . . . . . . . . . 211

A.5.1.1 Nginx Configuration file . . . . . . . . . . . . . 213

A.5.2.1 Round Robin load balancing . . . . . . . . . . . . . . . 213

A.5.2.2 Least connected load balancing . . . . . . . . . . . . . . . . . . . . . 214

A.5.2.3 Session persistence load balancing . . . . . . . . . . 215

A.5.2.4 Weighted load balancing . . . . . . . . . . . . 216

A.5.2.5 Locust stress testing for all types of load balancing approach . . . . . 217

A.6.1.1 Response time analysis for different types of job requests . . . . . 217

A.6.2.1 Data preprocessing of data generated by Locust testing for all the nodes . . . . . . . . . . . . . . . . . 220

A.6.2.2 Output generated for node 1 . . . . . . . . . . . . . 224

A.6.2.3 Output generated for node 2 . . . . . . . . . . . 225

A.6.2.4 Output generated for node $3 \ldots \ldots$. . . . . . . . . 226

A.6.2.5 Analysis of data generated by locust for all nodes . . . . . . . . . . 227

A.6.3.1 Data preprocessing of data generated by Locust testing using differ-

ent types of load balancers . . . . . . . . . . . . . . . . 234

A.6.3.2 Output generated using Round robin approach . . . . . . . . . . . . 239

A.6.3.3 Output generated using Least connection approach . . . . . . . . . . 240

A.6.3.4 Output generated using Session persistance approach . . . . . . . . . 241

A.6.3.5 Output generated using Weighted round robin approach . . . . . . . 242

A.6.3.6 Analysis of data generated by locust using different types of load balancing technique ... . . . . . . . . . . . 243

A.6.4.1 Analysis of logs generated by nginx using different types of load balancing technique ................ . . 250

A.6.5.1 Data preprocessing of data generated by Locust testing for application on AWS cloud . . . . . . . . . . . . . . . 266

A.6.5.2 Output generated for node 1 . . . . . . . . . . . . . 270 A.6.5.3 Analysis of data generated by Locust testing for application on AWS cloud with application using round robin approach on local cluster .271 


\section{LOAD BALANCING OF FINANCIAL DATA USING MACHINE LEARNING AND CLOUD ANALYTICS}

Dimple Jaiswal

May 2020

277 Pages

Directed by: Dr. Michael Galloway, Dr. Huanging Wang, Dr. James Gary

School of Engineering and Applied Sciences

Western Kentucky University

The rising use of technology for web applications, android applications, digital marketing, and e-application systems for financial investments benefits a large sector of stakeholders and common people. It allows investors to make an appropriate choice for investment and to increase their capital growth. This requires proper research of investment companies, their trends in price and analysis of historical and current information. In addition, prediction of prices makes the process of investment more comfortable and reliable for investors as shares are the most volatile type of investment. To offer this service to multiple users spread across the globe, there are certain vulnerable challenges for developers: specially to handle peak conditions and to channelize requests to servers in an optimal way. This demands high performance, cost-effective and sustainable approach to manage the concurrent user request on servers. It also needs to manage increasing utilization of computing resources to satisfy the increasing requirement.

The process of balancing simultaneous requests is highly complicated, non-trivial and critical at times, which forces to add a subsystem or an external service to handle requests and balance the resource utilization as per requirements. Load balancing is a method used to channelize requests across the servers in back-end. It helps in improving the performance of the system by optimizing the use of resources, maximizing the throughput and reducing the latency. The traditional method to load balancing is inadequate and inflexible 
to satisfy the growing demand. Increasing only hardware resources to balance the system is not an effective approach all the time. This needs an in-depth study of jobs to be performed and analysis of various approaches to organize sub-tasks to fulfill the objective.

In this project, four different type of techniques were used for balancing the user requests; they are: round robin, least connected, IP hash and weighted round robin methods. By analyzing the performance of all the computing nodes and by analyzing the logs generated using different load balancing approaches, it was found that weighted round robin resulted in better performance in all the factors: success request rate, failure request rate and average response time. The weight parameter for node 2 and node 3 was 2 and for node 1, it was 1 , as computing node 2 and node 3 have better performance than node 1 . 


\section{Chapter 1}

\section{INTRODUCTION}

\subsection{History and Analysis of Existing Problems}

Resource utilization and management has become a major concern with advent use of technology for development to improve functionality, flexibility, reliability and efficiency of existing systems. Cloud computing plays a major role in maximizing resource utilization without affecting services and features provided by the system. It provides essential services like high availability, high throughput, scalability and optimized approach in model design. As Wang [Wang, 2011] suggests that stock market data analysis is an important research domain of natural sciences, economics, and financial trading, and poor market economy will affect stock investments.

In [Hargreaves and Hao, 2012], Hargreaves and Hao discusses how stock data is dually concerned among financial companies and people. Many financial companies are discovering different ways to find productive information from large data sets of stock market and on the other hand, how private investors have keen interest in prediction of stock prices so that they result in profitable investments with minimum risk. Fonseka and Liyanage [Fonseka and Liyanage, 2008] focuses on how efficient market hypothesis is associated with the idea of "random walk", and discusses different ways in which an investor can reduce short term risks while investing in stock market using data mining. Since trading being a secondary source of income for massive number of people, it gives an exponential 
increase in the number of users accessing financial website during market hours. This generates heavy traffic on web applications, which affects the performance of the system and sometimes, results in adverse conditions like malfunctioning of server, computing node failure and at worst system failure. This needs strategic and optimized allocation of requests and hardware resources. Every user searches for various information- with different time frame and different types of view according to their requirement. The processing time of these requests varies based on their type, view and complexity. The requests are diversified on various attributes. Moreover, the stock market generates lots of data in short span. Capturing and processing stock data is the most difficult task as the data is generated very frequently, which results in lots of ambiguity and missing data. The most demanded request is for prediction of stock prices, which basically deals with data preprocessing and model design.

\subsection{Brief Discussion on Financial Data and Various Job Requests}

\subsubsection{Data Preprocessing}

Data preprocessing is done using Extract, Transform and Load(ETL) process. Data is extracted using 'yfinance' library. Data is available from the year 2015 and is updated everyday. Data preprocessing involves data extraction, data cleaning and loading the processed data in the storage node.

\subsubsection{Commonly Known User Requests on Financial Web Application}

There are many types of user requests are made to analyze the trends in the market. The most commonly found user requests are- 


\subsubsection{Prediction of Stock Prices}

Prediction of stock prices for a particular stock for a provided date is most highly demanded user request. Prediction of stock price is usually done using data mining or using machine learning algorithm, which involves mathematical computation and thereby, utilizes large amount of resources and results in high execution time. Here, Prophet is used for prediction purposes. It is an open-source software released by Facebook's Core Data Science team. It is a procedure used for forecasting time series data based on an additive model, where non-linear trends are fit with yearly, weekly, and daily seasonality, plus holiday effects. It is an accurate and fast, fully automatic, tunable forecast and available in R/Python language [Taylor and Letham, 2017]. It is based on concepts of joint probability approach as probablistic approach has better predictive results as compared to other techniques. Thus, it can be treated as complexed request and should be processed to a node, which has ample amount of resources.

\subsubsection{Display Historical Data}

Stock data visualization with different properties and representation is used to analyze stock data to obtain common trends in market. It can be visualized in various different types of representation methods. In the designed application, a line graph, a candlestick chart and a simple table is used to analyze the stock data. This type of request can be classified as moderate type of requests as they do not involve any computation operation.

\subsubsection{Display Market Movers}

To display market movers, i.e., top gainers and losers, can be treated as minor request as this request can be processed as a batch process and will result same for every user 
requesting in the same time frame. Users can analyze market movers for last day or for a week or for a month.

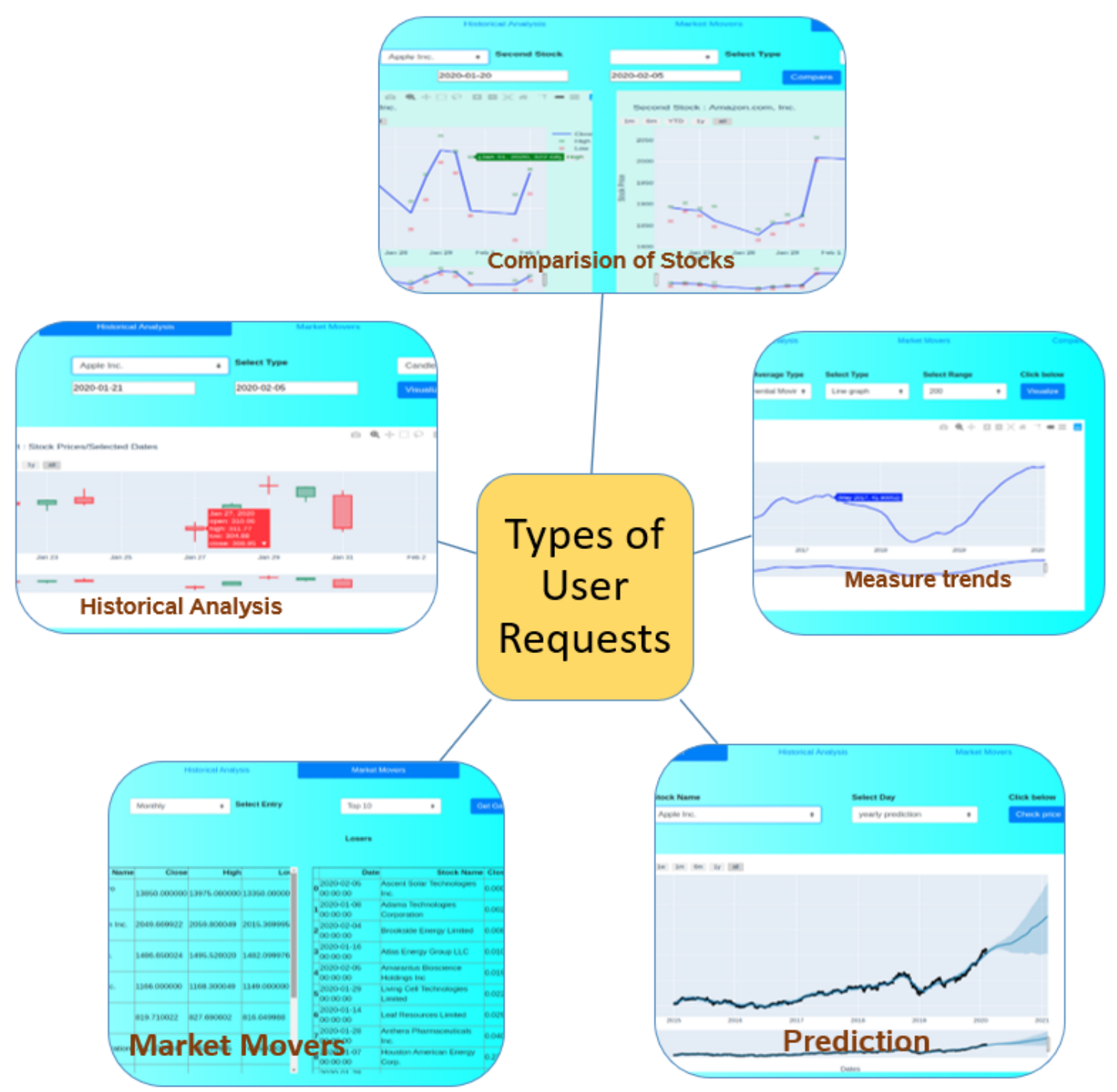

Figure 1.1: Types of User Requests

1.2.2.4 Measure Trends: Simple Moving Average(SMA) and Exponential Moving Aver$\operatorname{age}(E M A)$

SMA and EMA are technical indicators to make decisions to buy or sell an asset based on crossovers and divergences from the historical average. Computation of SMA 
and EMA with different timeframes and different representation methods can be classified as moderate or complicated requests based on the timeframe and type of representation selected by the user.

\subsubsection{Comparison of Two Stocks}

Sometimes, when situations are tough to make a decision for an investor and investor is not sure of choosing a specific stock for investment, comparing two different stock data helps investor to make a more valuable and logical choice for an investment. These requests can be categorized as moderate because it doesn't require any computation and data of selected stocks is just fetched and represented.

\subsubsection{Analysis of Execution Time of Different Job Requests}

The web application design commonly consist of 5 different types of job. On the basis of their complexity and time consumption, they are primarily classified as- low, moderate and highly complexed jobs. The given bar graph below provides the execution time taken for both the type of request- GET and POST request for every type of job without any additional parameter. More about analysis of execution time of each type of job request is

discussed briefly in Chapter 4. This testing is performed using ab - Apache HTTP server benchmarking tool. 


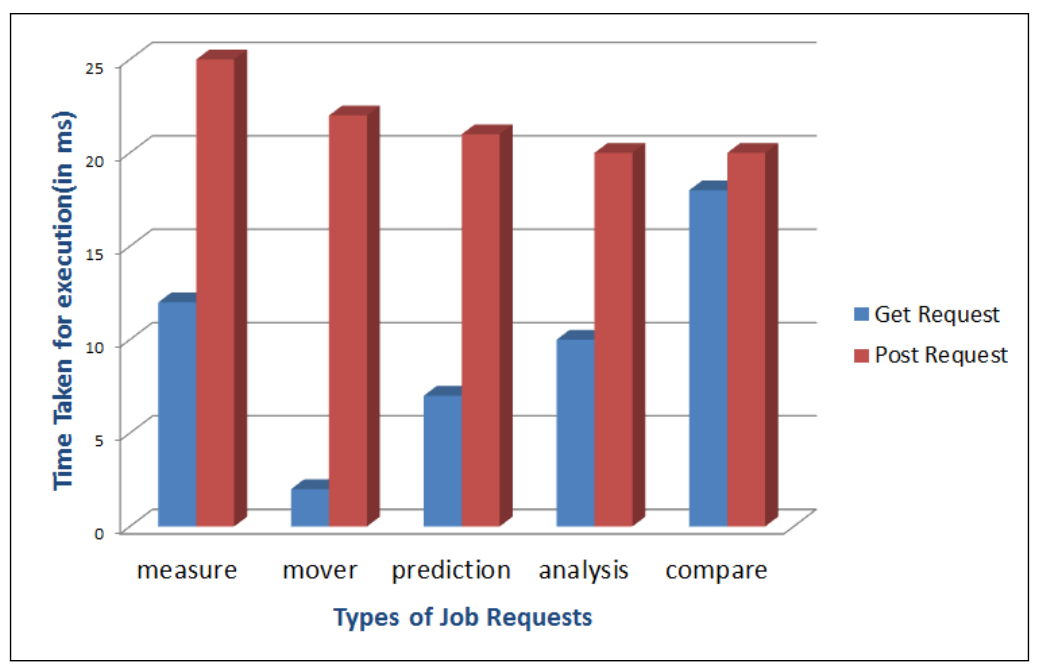

Figure 1.2: Total Time Taken for Execution of Individual Job

\subsection{Proposed Solution}

This work intends to focus on analysis of various strategic approaches to solve the complexity faced at the time of decision making to schedule jobs and to learn load balancing concepts so that deployment of application on any additional node, could be easily achieved in faster and in more secured way. Moreover, it aims on applying knowledge of Machine Learning for analyzing the training data and preparing a comparative study of deploying an application on public and private cloud. So, that the resultant study can be used as pre-requisites for future application deployment process with consideration of different performance parameters. The next chapter describes brief study of topics involved in research and development. The chapter 3 provides the detailed view of hardware and software architecture. In chapter 4, the design and work flow of project is discussed briefly. In last chapter, performance analysis is computed by comparing performance of web application deployed on local cloud with web application hosted on public cloud. It also talks about the results obtained in research and topics that can be implemented in future. 


\section{Chapter 2}

\section{BACKGROUND}

\subsection{Cloud Computing}

Due to arising information revolution, the need for hardware resources and its optimized utilization has exponentially increased. This demands excessive computation for analysis of data generated by machines to improve the quality, response time, reduce maintenance cost and mitigate problems in existing system. Cloud computing provides the most preeminent solution to these existing problems. It aims to reduce computing cost, increasing reliability and adding flexibility to scale up, down and across according to use. Essential part of this is cloud rely on virtualization and virtualization is a process for mapping of Information Technology resources to business needs[Foster, Zhao, Raicu, and Lu, 2008]. Thus, reducing the cost of hardware, improving computing power and storage capacity.

\subsubsection{Several Characteristics of Cloud Computing}

The common key factors which makes cloud computing most popular and sustainable field as described by [NOVKOVIC, 2017] are as follows:

1. Virtualization: cloud computing utilizes server and storage virtualization extensively to allocate/reallocate resources rapidly

2. Multi-tenancy and resource pooling: Resources can be pooled and shared among multiple users working on either same application or same physical infrastructure. 
3. Broad network access: Resources are available on network and can be accessed via web-browser or thin client by diverse customer platforms.

4. On-demand self-service: Resources are self-provisioned and available online.Thereby, do not require any human interaction from service provider.

5. Rapid elasticity and scalability: Resources can scale up or down, automatically.

\subsubsection{Services Provided by Cloud Computing}

On the basis of various features of Cloud Computing given above, services provided by Cloud Computing are broadly classified as -

\subsubsection{Infrastructure As a Service(Iaas)}

Compute, storage, networking, and other elements (security, tools) are provided by the IaaS provider via public Internet, VPN, or dedicated network connection. Users own and manage operating systems, applications, and information running on the infrastructure and pay by usage.

\subsubsection{Software As a Service(Saas)}

Software runs on computers owned and managed by the SaaS provider, versus installed and managed on user computers. The software is accessed over the public internet and generally offered on a monthly or yearly subscription.

\subsubsection{Platform As a Service(Paas)}

All software and hardware required to build and operate cloud-based applications are provided by the PaaS provider via public Internet, VPN, or dedicated network connection. Users pay by use of the platform and control how applications are utilized throughout their lifecycle. 


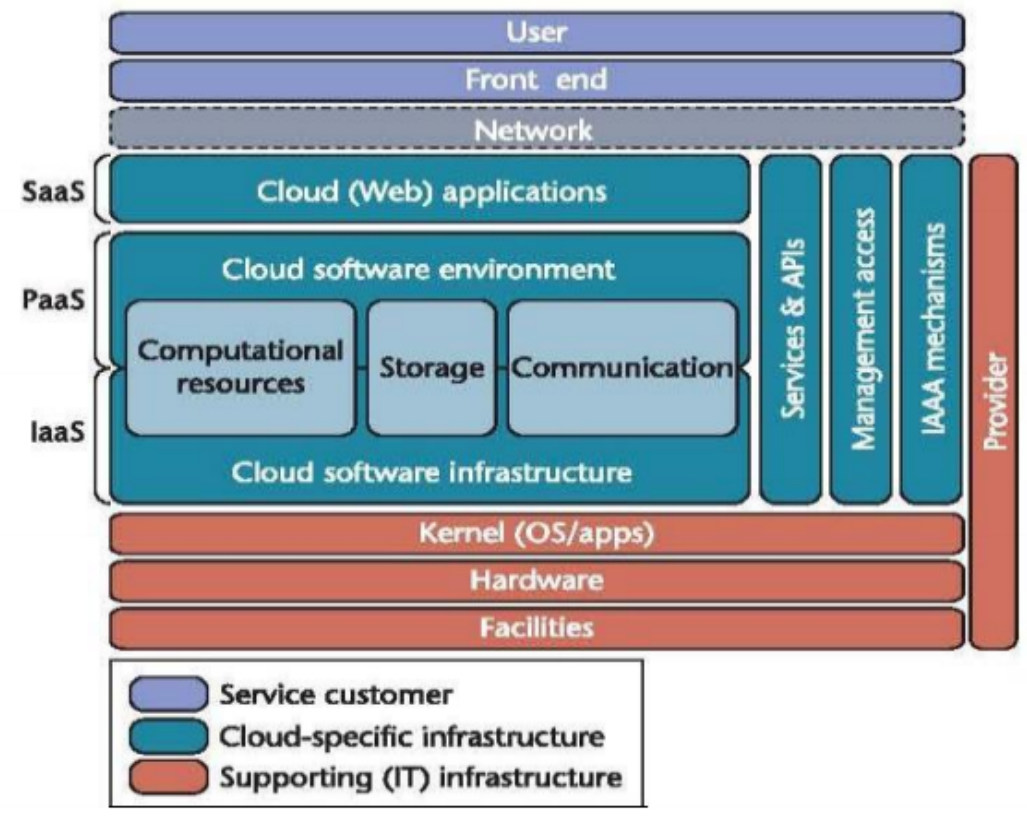

Figure 2.1: Cloud Reference Architecture [Kumar and Goudar, 2012]

Cloud computing also provides various other services, such as Cloud-Based Analyticsas-a-Service (CLAaaS), Database-as-a-Service (DBaaS/DaaS), Machine Learning-as-a-service (MLaas), Disaster recovery-as-a-service (DRaas) etc, which are dependent on the three basic services provided by cloud [Sharma, Chang, Tim, Wong, and Gadia, 2016]. Thus, it has numerous benefits like high availability, faster implementation and rapid scalability with low infrastructure and facility costs.

\subsection{Load Balancing}

\subsubsection{Introduction}

The traditional load balance technique uses master-slave architecture to balance the load, in which, slave-servers sends a heartbeat to master-node (load balancer) to indicate that it is up and alive every time and the master-node accordingly redistributes the load. The most common approach used by master node to distribute traffic is round robin method . 
Round robin scheduling approach is based on time sharing among jobs in equal slice / quantum and in FIFO(First In First Out) queue basis. The scheduling is done completely on the basis of job fairness, and jobs are independent from each other for their execution. However, it results in starvation or indefinite blocking for a job, which extremely varies in size and requirements due to unsatisfactory [Raj, Singh, and Bansal, 2013].

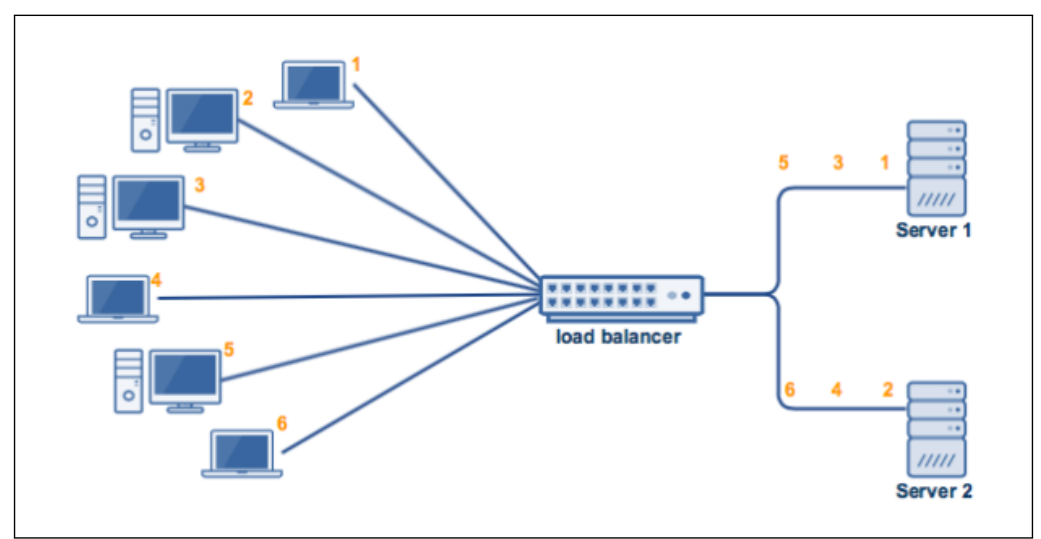

Figure 2.2: Round Robin Approach used to Balance Various Requests Acting as Load on Given Two Servers [Villanueva, 2015]

When heterogeneous servers are used based on different configurations in a system, using round robin approach for load balancing leads to over-loaded condition on less hardware configured servers and under-loaded condition for a highly configured server. This results in a highly imbalance system. Thus, a modified form of round robin approach is used, termed as Weighted Round Robin load balancing method. It distributes load on basis of the capacity of servers. 


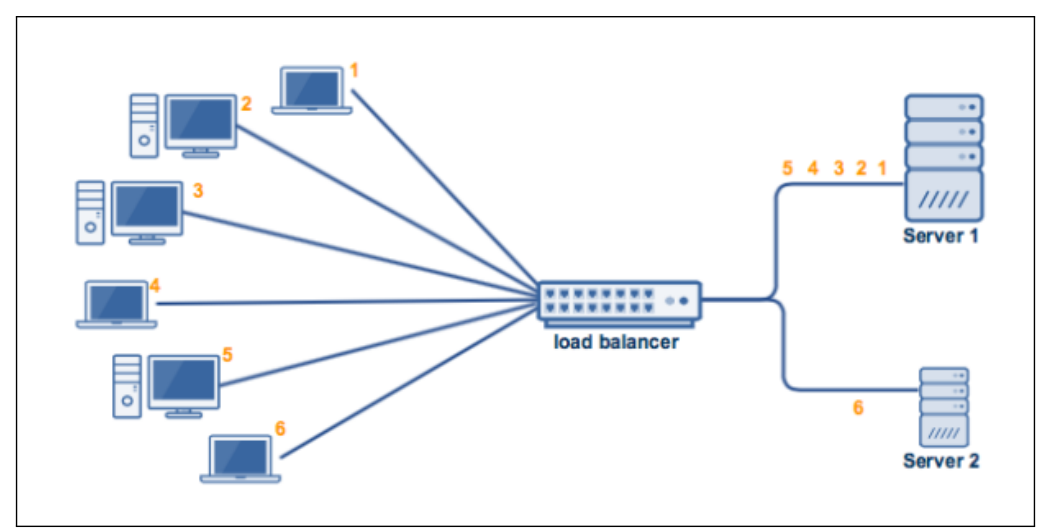

Figure 2.3: Weighted Round Robin Approach used to Balance Various Requests Acting as Load on Given Two servers [Villanueva, 2015]

In the master-slave architecture used in round robin and weighted round robin methods, if any slave-server node dies, then master node(load balancer) redistributes the load of that node to other working nodes. Thus, making interaction between master and slave nodes tightly coupled, time and energy are consumed. The most risky drawback of this method is that if master node dies, it lead to single point failure and all slave-servers becomes orphans; which is very critical and unpredictable. This usually happens in static load balancing. Thus, this needs a technique which is dynamic and fault-tolerant. To avoid such conditions, a queue is added between load balancer and slave-servers, which channelizes the communication. Thus, working are not completely dependant on master node and makes system loosely coupled and uses less energy and resources.

In Honey bee behavior based approach for dynamic load balancing, the capacity and load of all virtual machines are calculated and if load is greater than maximum capacity, then, the honey bee foraging behaviour is used for load balancing. This approach clusters virtual machines into low loaded(LVM), balanced loaded (BLM) and over loaded(OLM) VM groups. Then, Algorithm calculates supply and demand of all LVMs and OLMs, and 
sorts OVMs with descending order and LVMs with ascending order. Thus, it goes on optimizing the request iteratively in loop until all request are fulfilled and also the change machine state from OVM to BVM/LVM or LVM to OVM/BVM based on conditions dynamically[L.D. and Krishna, 2013]. Thereby, optimizing use of resources and improvising the system efficiency.

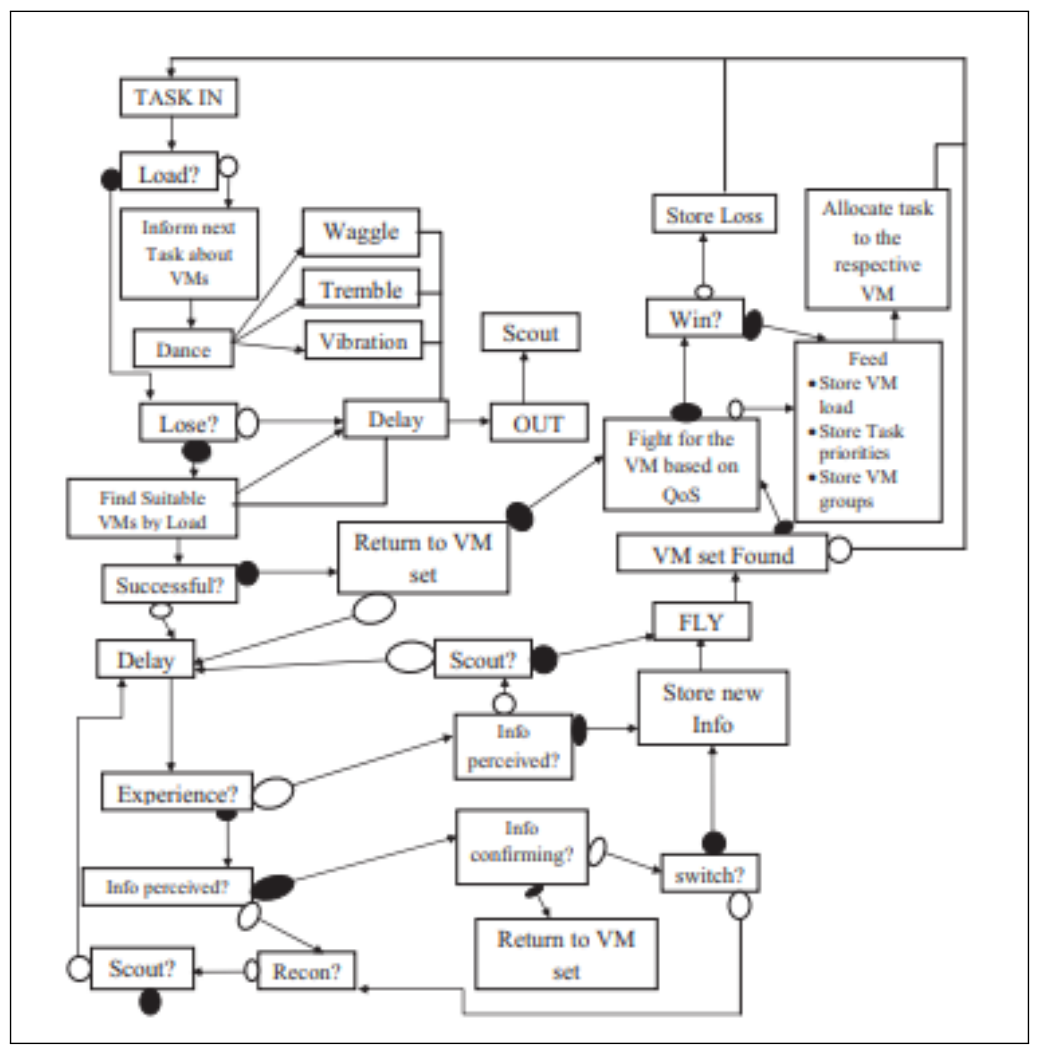

Figure 2.4: Flow Diagram of the Behavioral Control Structure for Honey Bee Behavior Inspired Load Balancing of Tasks in Cloud Computing Inspired by Foraging Behavior of Real Honey Bees Adapted from Johnson and Nieh with Minor Changes. [Johnson and Nieh, 2010]

In autonomous agent based shortest path load balancing method, the channel agent creates local agents and keeps information of each local agent in its table. On the basis of this information, channel agent distributes its neighbour information to its all local agent. All the local agents maintains information about its neighbour and whenever local agent do not have enough space to execute process, it broadcast its id, process id and required 
space to all its neighbour. If neighbour has enough space, then, they send true message. Otherwise, the neighbour forwards the request to its neighbour. If local agent gets same packets from different neighbour, then, it chooses shortest path neighbour to execute its process and if local agent receives multiple true messages, then, it makes decision based on shortest distance and more memory of neighbour. This dynamic approach reduces overall request time and simulation time for different cloudlet. [Vig et al., 2016]

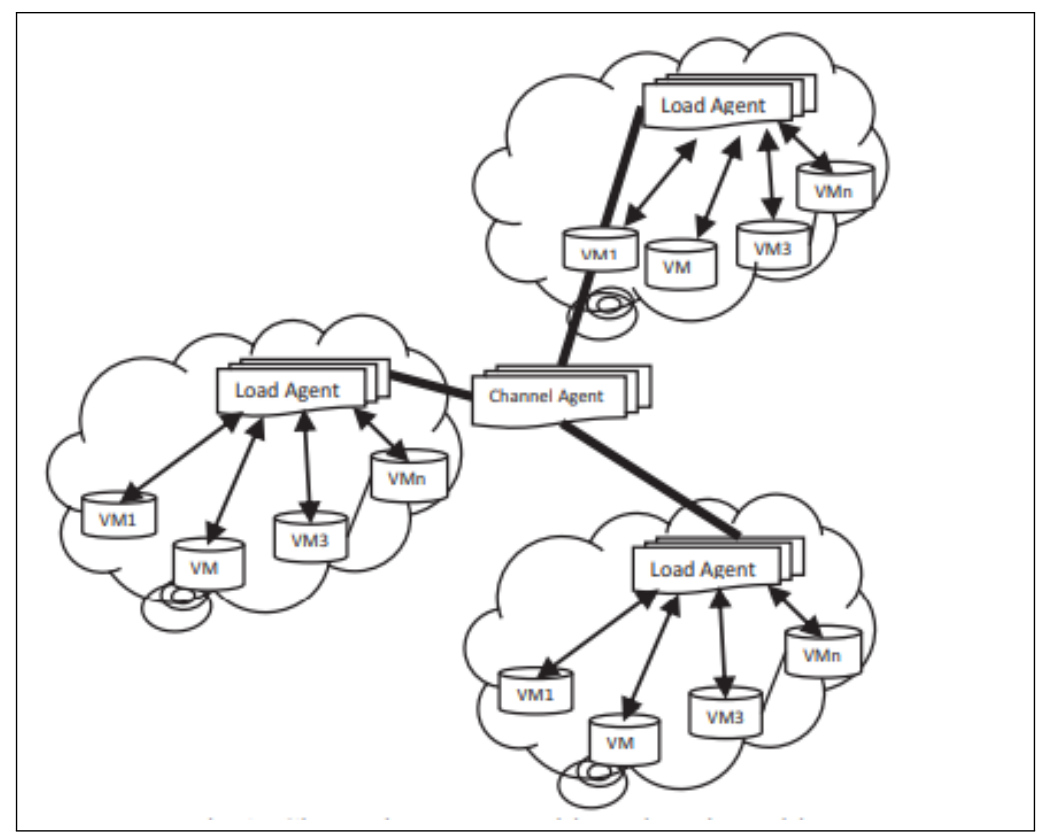

Figure 2.5: Channel Agent Searching for Virtual Machines [Vig et al., 2016]

\subsection{Machine Learning}

Machine learning is scientific study of algorithms and mathematical model to solve real-time problems. An algorithm can be made trained in three broadly classified ways supervised, unsupervised and reinforcement learning [Wikipedia contributors, 2019b]. It is kind of Artificial Intelligence technique, which mainly focuses to make system learn automatically without human intervention [Saravanan and Sujatha, 2018]. In supervised learning, the algorithm is trained and using training set and results are supervised on the 
basis of predicted outcomes. This approach can be used to develop load balancer for a specific applications, which has ample information about resource utilization by a specific job and wide range of data-set to train the algorithm. Supervised technique used with Dijkstra algorithm can be used to find and predict an optimized path for balancing load.

The most widely used technique to train algorithm is classification. In order to select the most suitable and optimized approach to solve the problem, three parameters can be used - representation, evaluation and optimization. These components makes the process of selection of algorithm much easier and simple [Domingos, 2012].

\begin{tabular}{|c|c|c|}
\hline Representation & Evaluation & Optimization \\
\hline Instances & Accuracy/Error rate & Combinatorial optimization \\
\hline K-nearest neighbor & Precision and recall & Greedy search \\
\hline Support vector machines & Squared error & Beam search \\
\hline Hyperplanes & Likelihood & Branch-and-bound \\
\hline Naive Bayes & Posterior probability & Continuous optimization \\
\hline Logistic regression & Information gain & Unconstrained \\
\hline Decision trees & $\mathrm{K}-\mathrm{L}$ divergence & Gradient descent \\
\hline Sets of rules & Cost/Utility & Conjugate gradient \\
\hline Propositional rules & Margin & Quasi-Newton methods \\
\hline Logic programs & & Constrained \\
\hline Neural networks & & Linear programming \\
\hline Graphical models & & Quadratic programming \\
\hline \multicolumn{3}{|l|}{ Bayesian networks } \\
\hline Conditional random fields & & \\
\hline
\end{tabular}

Figure 2.6: Learning = Representation + Evaluation + Optimization [Domingos, 2012]

In unsupervised learning approach, training data is unclassified and unlabelled, as used by [Tang, Liu, Wang, and Liu, 2010] for static load balancing algorithm in homogeneous multiprocessor system since it has no relation with current state of load and do not store load information. So, it has great blindness and low efficiency. The dynamic algorithm has load information of each node of current state. Hence, it has high performance and real-time applications.

Reinforcement technique interacts with the environment by responding with errors 
or rewards [Saravanan and Sujatha, 2018]. This can be used to improvise service provided by the developed load balancer to test efficiency and judge the results. However, this technique cannot be used as a direct method for load balancing.

\subsubsection{Classification Technique}

Classification is a supervised learning approach, in which data is categorized into classes. It can be either classified as binary classification or multi-class classification, depending on the training dataset used for training the algorithm. The type of classification technique to be used for training a model can be decided by visualizing the dataset and observing how the data points are outspreaded over the graph. As [Domingos, 2012] mentioned that classification is the most popular technique used for training algorithm, given below are the different types of classification methods commonly used-

\subsubsection{Logistic Regression}

Logistic Regression(LR) is a statistical model uses a logistic function to model a binary dependent variable [Wikipedia contributors, 2020c]. Basically, it is an approach used to find the best fitted relation between dependent and independent attributes for a given dataset. For example, in [Zhou and Yan, 2016] the author uses LR for software test management, to find the best fitting relationship between the test quality of software project and the test management data collected during test process. LR is most effective and highly recommended technique only when the data is to be classified or predicted as binary. Otherwise, accuracy is not guaranteed.

\subsubsection{Decision tree}

Decision tree approach is a predictive model that uses decision tree that undergoes from observations about an item (represented in the branches) to conclusions about the 
item's target value (represented in the leaves). On the basis of the predicted outcome, decision tree can be classified as Regression tree or Classification tree. When the prediction outcome is a real number, then, it is called as regression tree and when the prediction outcome is class (discrete), it is known as Classification tree [Wikipedia contributors, 2020a]. Decision trees are very informative as compare to any other supervised approach. Generating rules is the most part in decision tree and in [Shamim et al., 2010], the author proposed a framework to transform the knowledge presented in decision trees and decision tables into knowledge base, their aim is to automate the construction of rules from decision tree and decision table.

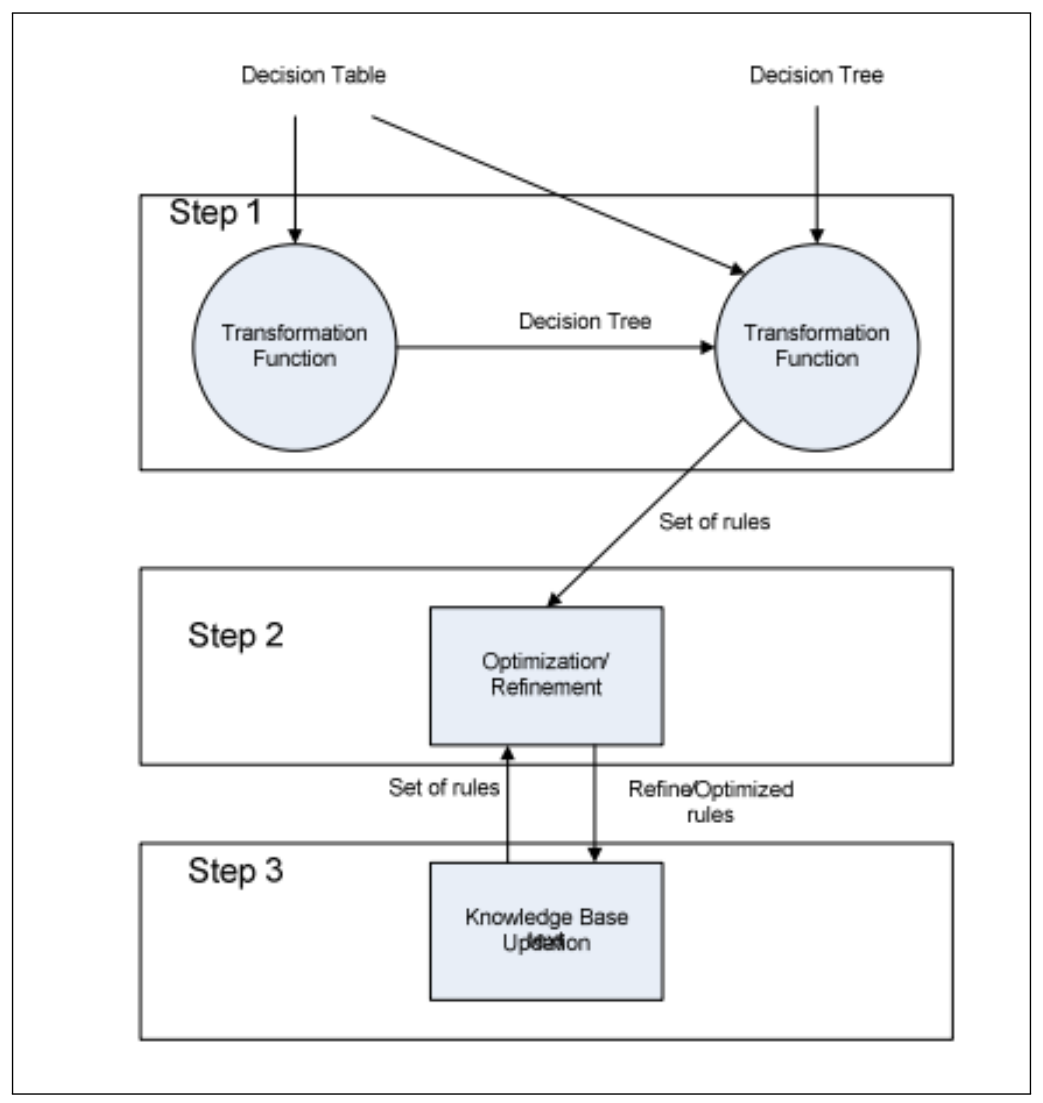

Figure 2.7: Proposed Framework to Transform Decision Table or Decision Tree to Directly Generate Set of Rules [Shamim et al., 2010] 


\subsubsection{Naive Bayes Classifier}

Naive Bayes classifiers are known as simple "probabilistic classifiers" and are based on applying Bayes' theorem with strong (naïve) independence assumptions between the features. It is typically based on conditional probability model and is one of the oldest method used for spam filtering. It is highly scalable [Wikipedia contributors, 2020d]. In [Solanki, Verma, and Kumar, 2015], a hybrid approach is used for spam filtering, the technique is based on local and global classifier, which results in improved classification accuracy. As it is based on Bayes' theorem and probabilistic approach has better predictive outcome, Naive Bayes classifier is effective in terms of accuracy.

\subsubsection{K-nearest neighbor Classifier}

K-nearest neighbor Classifier ( $\mathrm{k}-\mathrm{NN}$ ) is a type of instance-based learning, or lazy learning, in which the function is only approximated locally and all computation is deferred until the function evaluation. As classification is majorly depended upon the distance metrics, normalizing the training set brings a lot of improvement in accuracy [Wikipedia contributors, 2020b]. It is most popular non-parametric classification method and therefore, requires a huge amount of classification time to search $\mathrm{k}$ nearest neighbors of an unlabelled object point. Thus, in [Yu and yu, 2006], the author proposed an adaptive k-nearest neighbors classifier (AKNNAC) technique to improve the performance and efficiency. The algorithm involves two steps, first step is to find the $\mathrm{k}$ nearest neighbor of the object point and the second step is to label that object point. The algorithm results indicate that when $\mathrm{k}$ is settled, AKNNAC is the most better approach than any other algorithms, in terms of performance. 


\subsubsection{Support Vector Machine}

Support vector Machine (SVM) constructs a hyperplane or set of hyperplanes in a high or infinite-dimensional space, which can be used for classification, regression, or other tasks like outliers detection [Wikipedia contributors, 2020e]. SVMs are originally designed for binary classifications and for multi-classifications, the values are converted into binary. Thus, multi-classification results in large amount of unclassified regions and thus, in [Bo Liu, Zhi-Feng Hao, and Xiao-Wei Yang, 2005], the author suggests a novel method for multi-classification called as Nesting Support Vector Machine (NSVMS). NSVMS is basically designed to deal with unclassifiable regions in the One-against-One algorithm. The algorithm first constructs hyperplanes based on One-against-One approach. Secondly, it selects the data points in the middle unclassifiable region and lastly, it uses the data points in the unclassifiable region alone to construct hyperplanes with the same hyperparameters. The last two steps are called repetitively until there are no data points in middle of region or the region disappears. Thus, results shows that the training accuracy of NSVM is higher than those of One-Against-One and Fuzzy Least Square Support Vector Machine (FLS-SVM), and its generalization performance is also comparable with them.

\subsection{Cloud Analytics}

Cloud computing is mostly widely used for hosting applications and increasing demand to adopt cloud-based architecture urges for more agility and cost-effectiveness in system. The use of cloud delivery models as Analytics as a service simplifies obtaining useful information from an operational enterprise data center helping to cost and performance optimizations. This uses Big data analytics to extract actionable information from system 
management metrics data, which brings more agility, elasticity and scalability in system [Routray, 2015]. Thus, benefits process of load balancing either by making an appropriate decision for scaling the application or by using it for developing another independent similar system with different configurations.

In [Wu and Marotta, 2013], Wu and Marotta talks about developing a Framework for Assessing Cloud Trustworthiness (FACT) that treats cloud as a black box and assesses its trustworthiness from the trusted cloud client. This is implemented by designing set of diagnostic tests, the diagnostic tests for data objects stored in the cloud are based on a separate cryptographic hash-based check that verifies their data integrity. The diagnostic tests are based on the acceptance tests that are already generated during the application development and testing lifecycle to make sure that they test the functionality of the application in the cloud accurately. They are integrated with the local application client, to ensure that they run within a single process. The results of the diagnostic tests are evaluated at the client side and if a test fails, the framework attempts to redeploy the cloud application on alternative cloud resources and rerun the instrumented client. This process is conducted repetitively until all diagnostic test pass or until time constraints are exceeded. This provides high data security for large scale applications and helps developer to evaluate the cloud performance.

The proposed method of designing web robots for collecting data to analyze stock data automatically suggested in [Ching-Te Wang and Yung-Yu Lin, 2015], which can be used to gather data and necessary information to analyze cloud data to improve the performance.

[Bhole et al., 2015] describes use of logs generated for analysis and prediction. The 
individual events that occur in a sequence of logs are extracted and grouped together based on domain knowledge into patterns. These patterns are considered for detailed analysis, which can be also served as an input to generate the statistics of the overall behavior. The stages involved in analyzing the log files are - data extraction, data cleaning, pattern recognition and visualization. The results of analysis can be used evaluate performance issues and furthermore, helps in improving the overall performance of the applications.

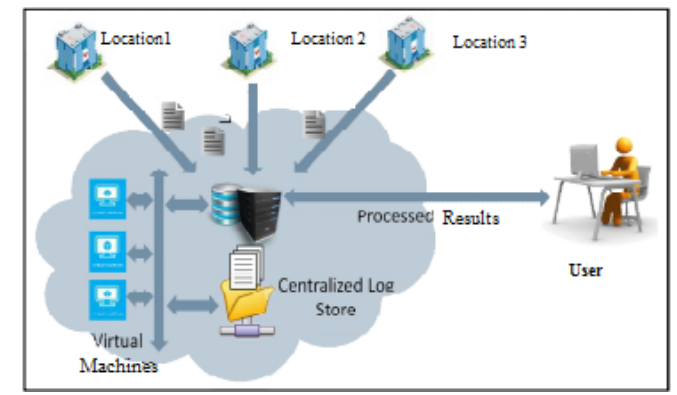

Figure 2.8: Log Analytics on Cloud [Bhole et al., 2015]

\subsection{Job Scheduling}

Job scheduling is a NP Complete problem, there is no specific methods to get solve these problems. It depends and varies according to different properties of a job or request. Usually, a single conventional queue is used for scheduling and it has several drawbacks like more waiting time for execution, enlarging size of queue due to delay and FIFO approach.Thus, a different technique to handle requests is needed such as multilevel queuing or divide and conquer approach.

\subsubsection{Genetic Algorithm}

The Genetic Algorithm (GA) is an optimization algorithm which uses the method of computerized search based on natural selection and genetics. Each solution to a problem is represented by chromosome, which consist set of elements called genes. The algorithm 
in beginning starts with random population of chromosomes, and quality of population is evaluated using an objective function, called as 'Fitness Function'. After initialization of population, with randomly generated solutions, new offspring chromosomes are generated by cross over or mutation. Again, the newly generated solutions are evaluated using fitness function to test with the previous one.If solution produced is optimal, then, that population is adopted. This process of generating offspring is repeated to obtain an optimal solution until best offspring are created. In Improved Load Balanced Min-Min (ILBMM) algorithm, the execution time of task on the virtual machine is calculated. This provides the minimum or maximum time of task on virtual machine (VM), which can be used in combination with Genetic Algorithm [Rajput and Kushwah, 2016]. 


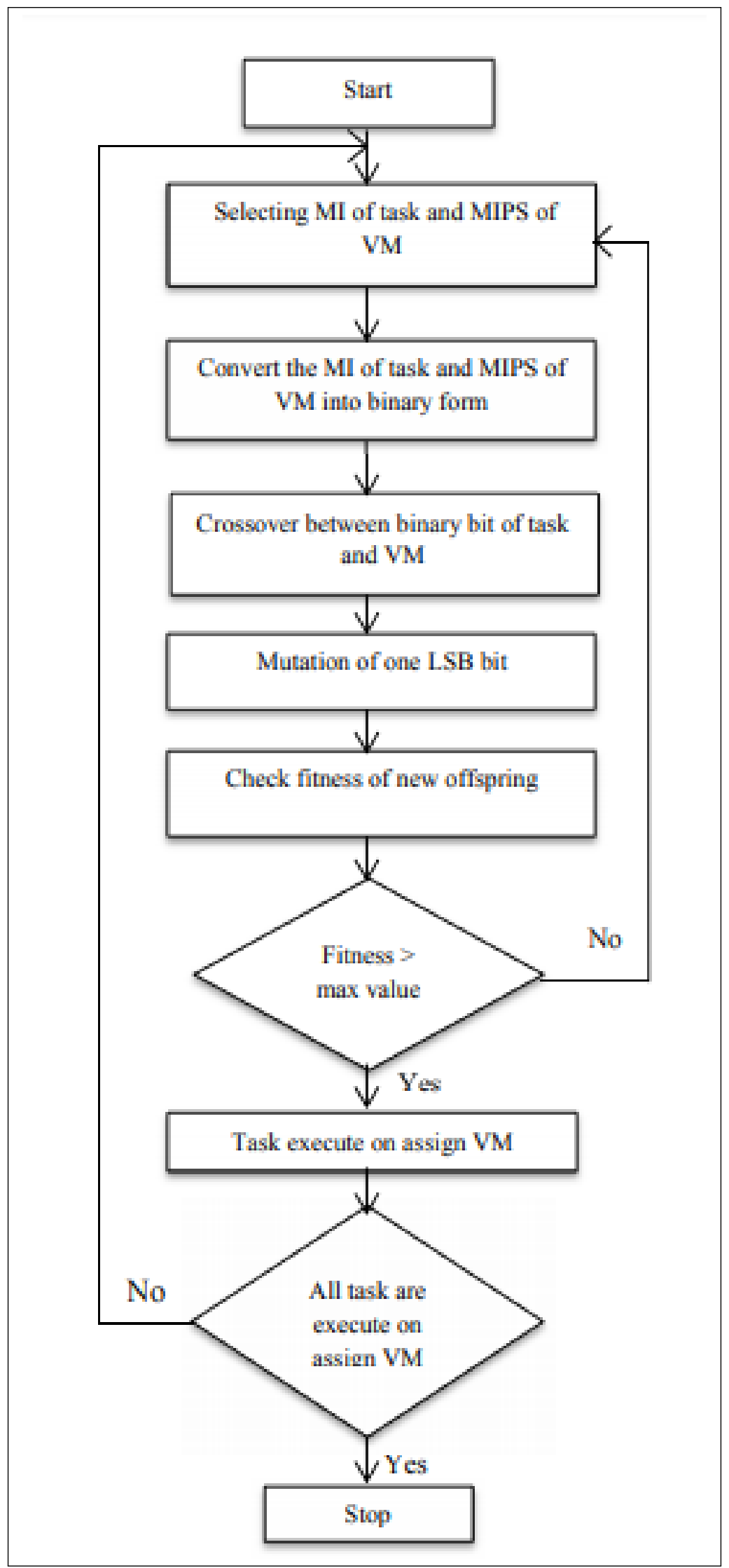

Figure 2.9: Flowchart for Genetic Algorithm [Rajput and Kushwah, 2016] 


\subsubsection{Imperialist Competitive Algorithm}

In Imperialist Competitive Algorithm, machine can simulate natural selection. The algorithm initializes population of countries and each country represents solution to a problem. The best countries are termed as Imperialists, based on a fitness function and rest of population become colonies to imperialists. The imperialists assimilate colony's characteristics based on optimization criteria to improve them. This builds a healthy competition among imperialist to become a powerful one and thus, weaker imperialists gets eliminated. The powerful country is considered as most optimized solution. This also helps in optimizing energy consumption with comparatively more reliable solution [Dutta and Aggarwal, 2017].

\subsection{Comparison of AWS and IBM Clouds}

In [Kaur et al., 2018], the author compares AWS and IBM cloud on the basis of performance and security measures, the testing of performance is done on AWS Elastic Compute Cloud[EC2] and Virtual server of IBM cloud using Phoronix Test Suite. The results in given below figures indicates that AWS services are easy to use on linux platform and offers more highlights in the linux Virtual machines. AWS has more adop-tion rate than any other cloud services available. It has better disk performance and RAM Speed than IBM. Also, AWS has an additional security feature of RSA security technique while IBM lacks behind in this aspect. 


\section{Apache Results}

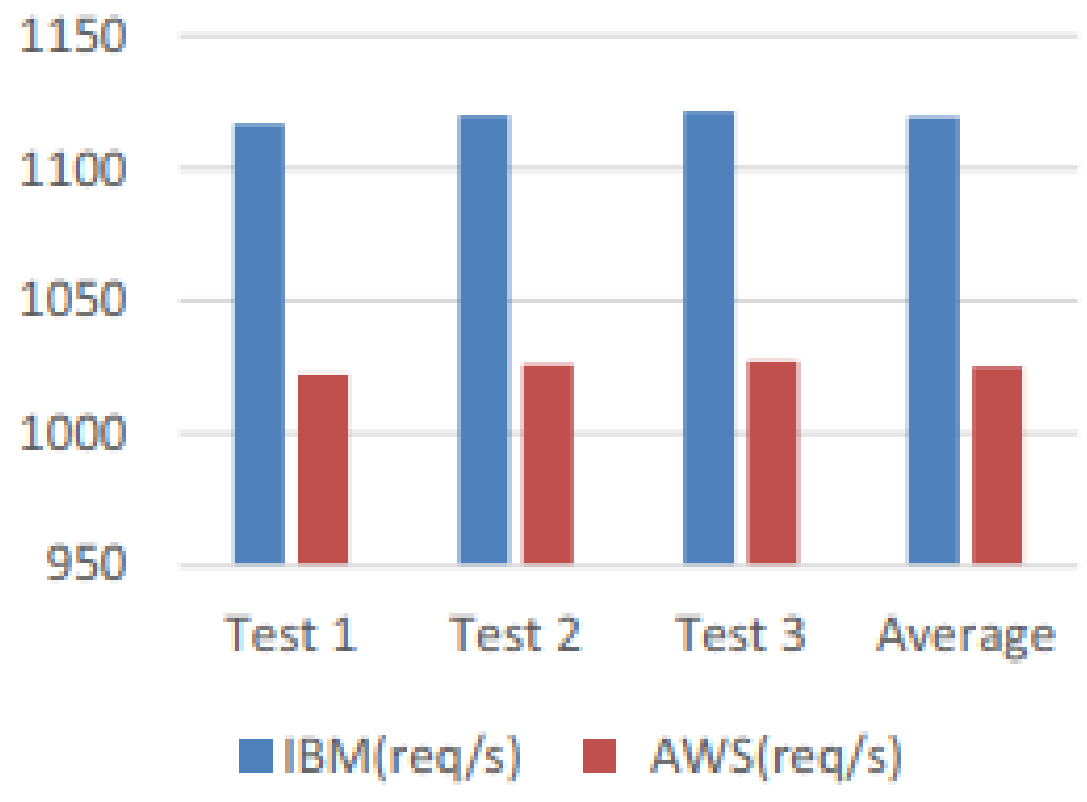

Figure 2.10: Apache benchmarking results [Kaur et al., 2018]

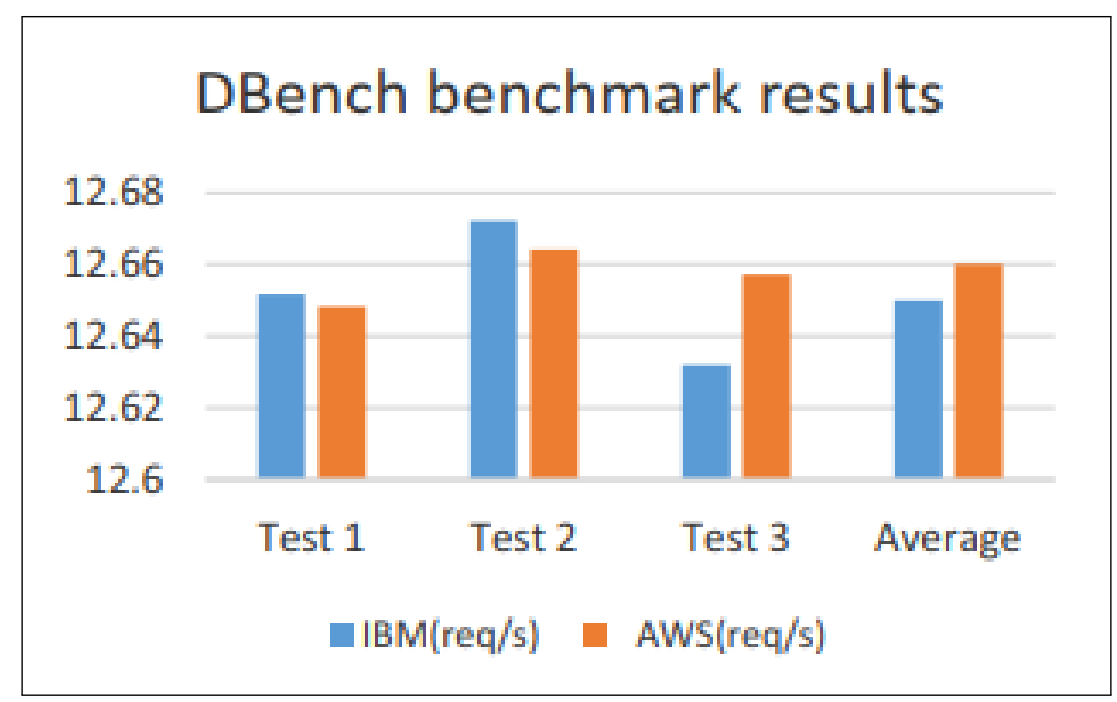

Figure 2.11: Dbenchmark benchmarking results [Kaur et al., 2018] 


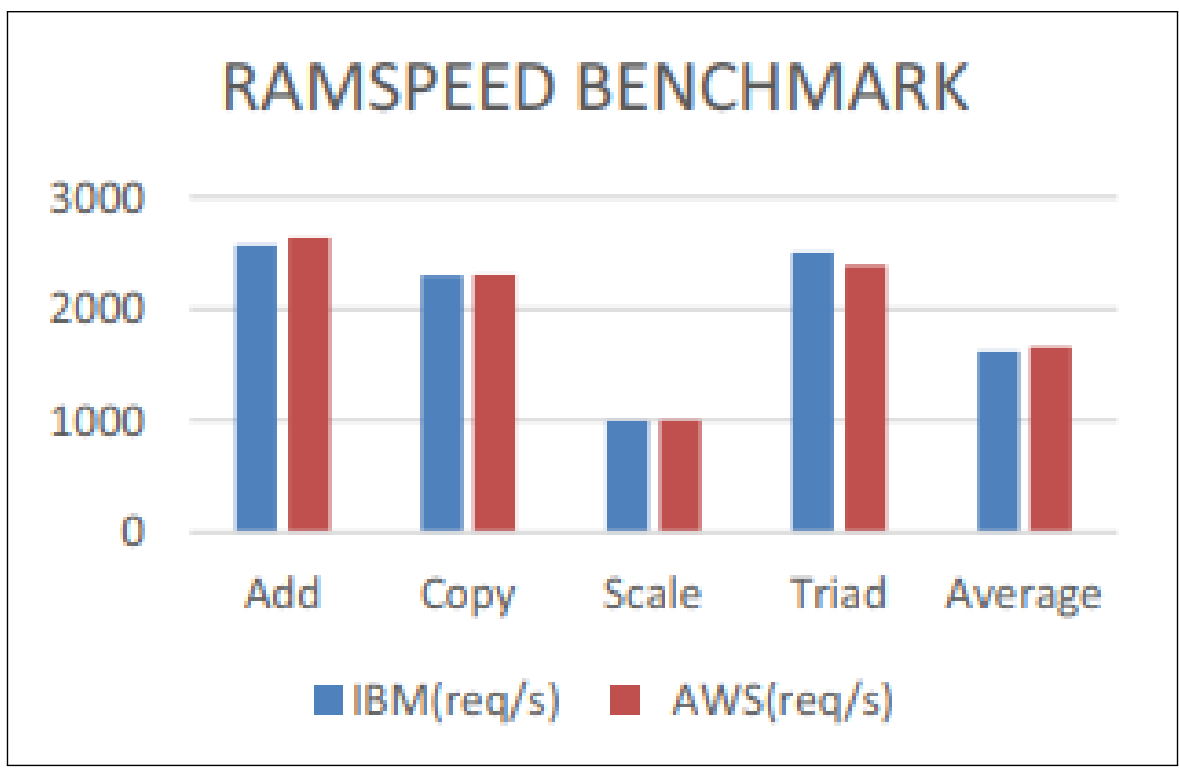

Figure 2.12: RAMSpeed benchmarking results [Kaur et al., 2018]

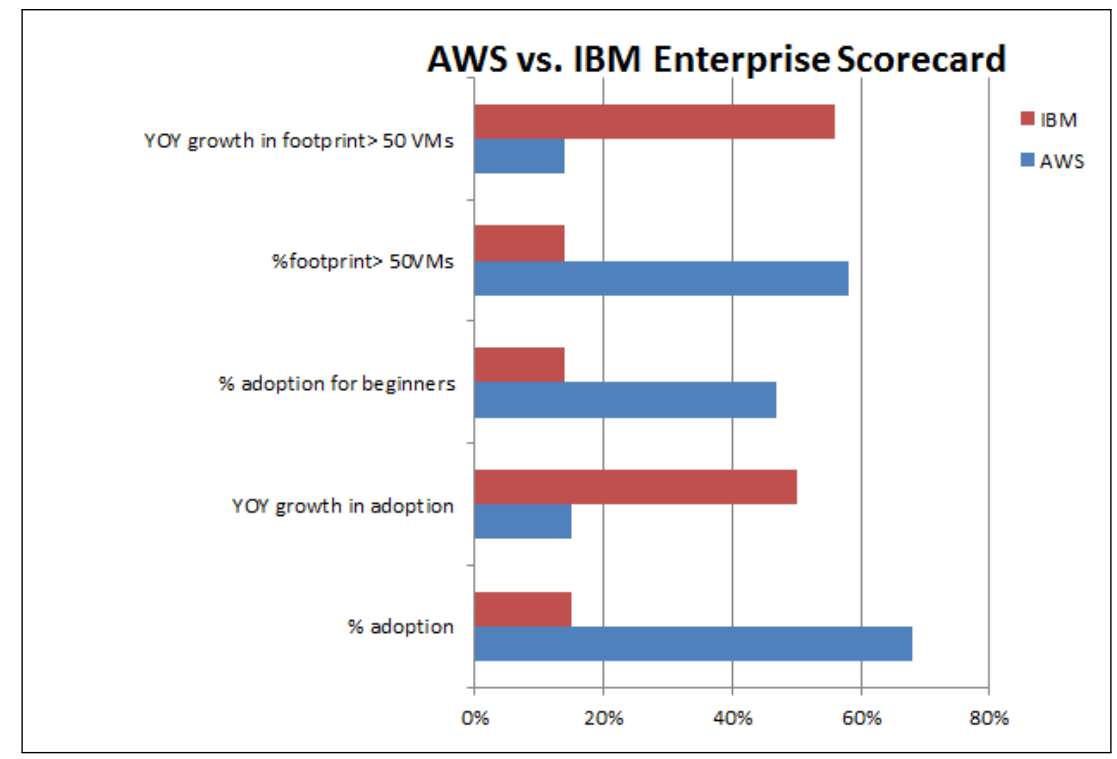

Figure 2.13: AWS vs. IBM Enterprise Scorecard [Kaur et al., 2018]

Similarly, here the financial web application was hosted on both the clouds. On IBM cloud, the application was hosted on Cloud Foundry app and on AWS, the application was hosted on AWS Elastic Beanstalk. The performance was analysed by conducting load testing with the help of ab - Apache HTTP server benchmarking tool and following results were obtained- 


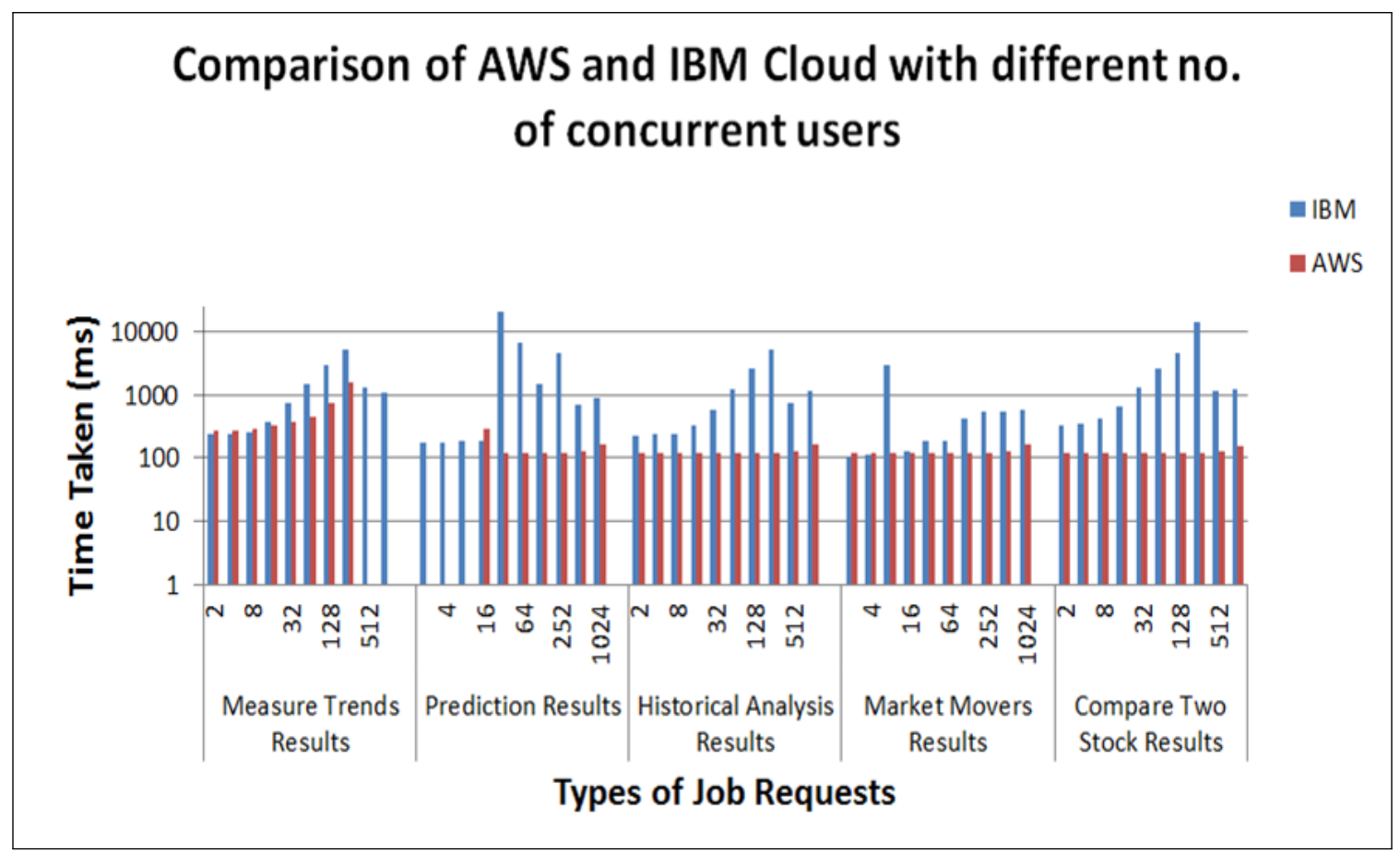

Figure 2.14: Performance of Web Application hosted on AWS vs. IBM Cloud

The above figure shows that the time taken for execution for all the types of requests for IBM cloud is comparatively more than AWS cloud and AWS cloud performance is better than IBM cloud. Moreover, AWS provides a lot of features to monitor the performance of the application as compare to IBM cloud. It is also observed that the application on IBM cloud automatically stops working within a range of 8 to 10 days and service needs to be restarted again. Thus, AWS cloud is considered for further performance analysis work.

\subsection{Amazon Web Services}

Amazon Web Services(AWS) is the first and most popular "pay-as-you-go" service that provides on-demand cloud computing platforms and APIs to wide variety of clients by Amazon.com. The services provided that are broadly classified on the basis of- computing, storage, networking, database, analytics, application services, deployment, management, mobile, developer tools, and tools for the Internet of Things [Sharma et al., 2016; Wikipedia 
contributors, 2019a]. AWS attracts the attention of scholars in the field of high performance computing because of its high bandwidth, low latency, high computing power and price characteristics [Li, Wang, and $\mathrm{Hu}, 2015]$.

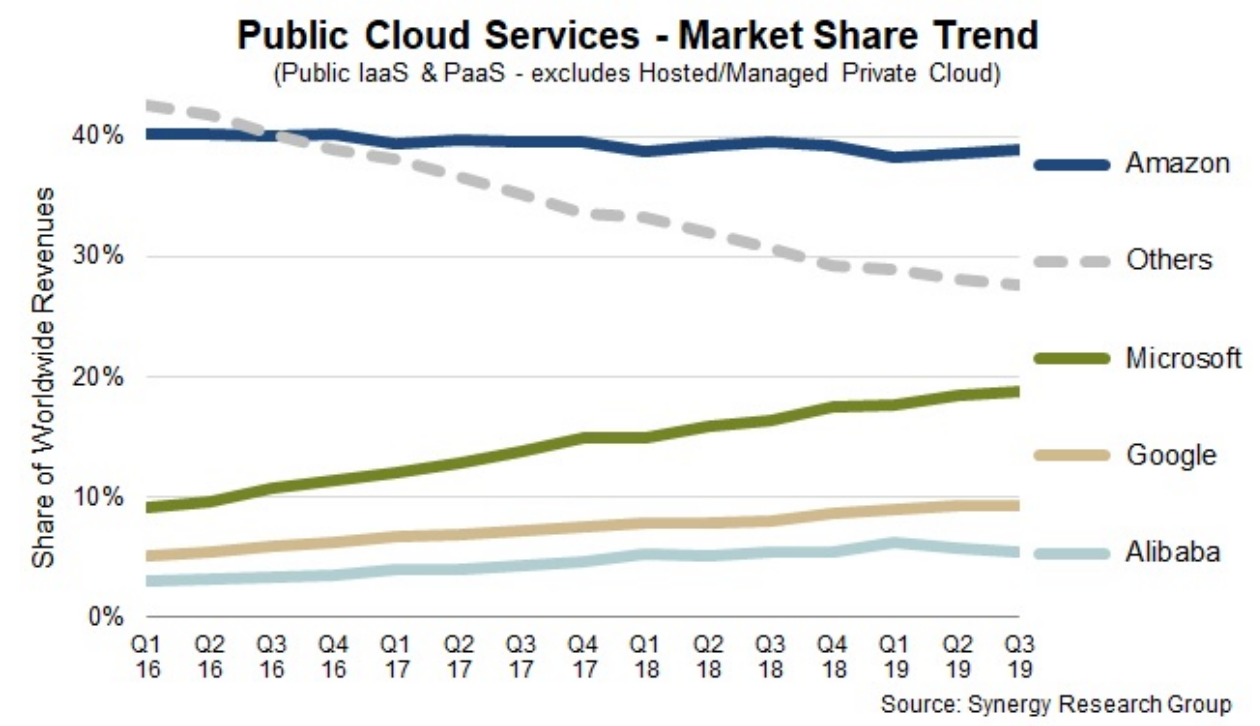

Figure 2.15: Cloud Service Provider Competitive Positioning [Reno and Group, Reno and Group]

Amazon Simple Storage Service (S3) is the storage service provided by Amazon, where customer data is organized by means of objects stored in buckets. A bucket is a logical unit of storage uniquely identified and belonging to one of the locations in which the provider has deployed its storage infrastructures [Persico, Montieri, and Pescapè, 2016]. Amazon Elastic Compute Cloud (Amazon EC2) is a web service that provides resizable computing capacity provided by Amazon [Li et al., 2015]. Similarly, there around 165 services and tools provided by Amazon [Amazon, Amazon] . 


\subsubsection{AWS Tools used for Implementation}

\subsubsection{Amazon Simple Storage Service (Amazon S3)}

Amazon Simple Storage Service (Amazon S3) is an object storage service that provides scalability, data availability, security, and performance. Different features provide various applications like -S3 Storage Class Analysis to analyze access patterns; S3 Lifecycle policies to transfer objects to lower-cost storage classes; S3 Cross-Region Replication to replicate data into other regions and many more. This provides a highly safe and secured environment to store data [Amazon, Amazon].

\subsubsection{Amazon Elastic Compute Cloud (Amazon EC2)}

Amazon Elastic Compute Cloud (Amazon EC2) is a web service that provides secure, resizable compute capacity required in the cloud. It is designed to make web-scale cloud computing easier for developers. Amazon EC2's web service interface is very developer friendly and simple, helps developers to obtain and configure capacity with minimal friction. It also provides developers to have a complete control of all the computing resources used by them to the run application on Amazon's proven computing environment [Amazon, Amazon].

\subsubsection{AWS Elastic Beanstalk}

AWS Elastic Beanstalk is an easy-to-use service for deploying and scaling web applications and services developed using various language and frameworks. In this project, the web application is developed with Python, using Django framework. The AWS Elastice Beanstalk is known as "Easy to begin, impossible to outgrow" service as just by uploading the code, it not only automatically handles the deployment, capacity provisioning, load 
balancing, auto-scaling and health monitoring of an application, but also, gives its users access to its underlying resources and full control over the AWS resources used in their application. Moreover, there is no additional charges for using Elastic Beanstalk; users only pay for AWS resources need to store and run the application. Amazon S3 bucket is used for storing the application and Amazon EC2 instance for running the web application. Thus, the costs of running a web application varies based on several factors such as the number of Amazon EC2 instances needed to handle the web application traffic, the bandwidth consumed by web application, and the database or storage used for application. The primary cost is typically based on Amazon EC2 instance(s) used and for the elastic load balancing that distributes traffic between the instances running the application [Amazon, Amazon]. 


\section{Chapter 3}

\section{HARDWARE AND SOFTWARE ARCHITECTURE}

A load balancer acts as the "traffic cop" sitting in front of all the servers and routes client requests across all the servers in different ways. The process of directing request is done in such a way that it maximizes the speed and capacity utilization without overloading a particular server. Thus, it ensures high availability and reliability by sending requests only to servers that are online and provides flexibility to add or remove servers according to the demand [NGINX, 2020]. Here, nginx is used as a HTTP load balancer to distribute traffic to various computing nodes. The complete load balancing system acts as a single system. The given diagram below shows how a load balancer handles the client requests. A similar approach is used for development of the project.

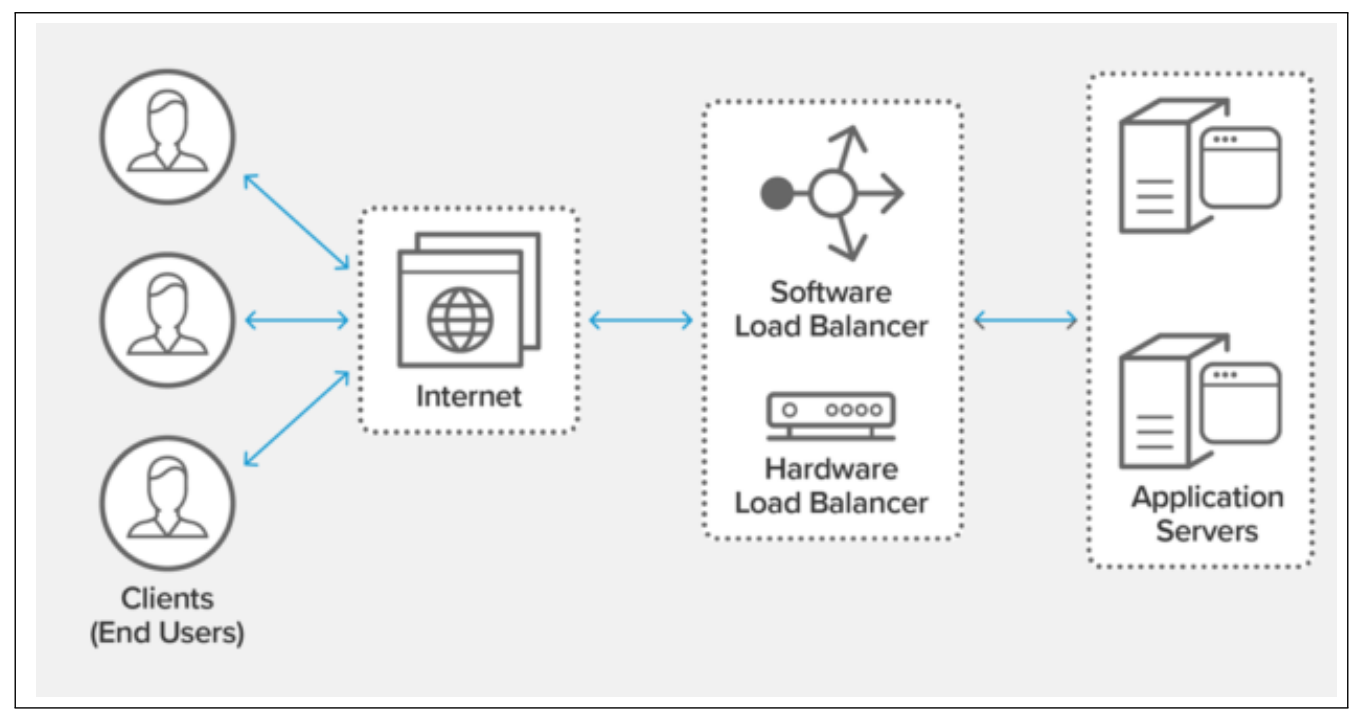

Figure 3.1: Load Balancing Diagram [NGINX, 2020] 


\subsection{Hardware Architecture}

There are two main components used for the development of the project: master node and computing nodes. Thus, the architecture used for directing requests is master/slave architecture. The cluster has one master node and three computing nodes. The user requests are directed to master node and these request are then internally executed on one of the three worker/computing nodes. The selection of computing node for execution of job is decided by the type of load balancing approach is used.

Nginx is used as a web server for balancing the load and is sitting on master node. Thus, the major task of load balancing is performed on master node. Master node also acts as a storage node. It stores the entire stock data and all log files generated by nginx. Therefore, it plays a crucial part in the project, and all the configuration settings to add or remove a computing node in cluster can be performed at master node. The job execution takes place at all the computing nodes. The given table below provides the information for master node and all the computing nodes.

\begin{tabular}{|c|c|c|c|c|}
\hline ID & $\begin{array}{l}\text { CPU } \\
\text { Cores }\end{array}$ & $\begin{array}{l}\text { RAM } \\
\text { Size }\end{array}$ & $\begin{array}{l}\text { Disk } \\
\text { Size }\end{array}$ & $\begin{array}{l}\text { IP } \\
\text { Address }\end{array}$ \\
\hline WS (Master Node) & 4 & 16 & 240GB & $\begin{array}{l}161.6 .5 .209 \\
192.168 .1 .167 \\
\text { (Local) }\end{array}$ \\
\hline NODE 1 & 4 & 8 & $131 \mathrm{~GB}$ & 192.168 .1 .240 \\
\hline NODE 2 & 4 & 8 & $233 \mathrm{~GB}$ & 192.168 .1 .158 \\
\hline NODE 3 & 4 & 32 & $163 \mathrm{~GB}$ & 192.168 .1 .243 \\
\hline
\end{tabular}

Table 3.1: Harware Architecture of Master Node and Computing Nodes 


\subsection{Software Architecture}

The software architecture comprises of two parts: front-end and back-end architecture. The front-end deals with web application design. All the users make request through web interface; these requests are executed on back-end servers and the response is directed back to client. The complete process involves interaction between the client and the server. Thus, communication between master and computing nodes needs to be very efficient so that the overall performance of the system is not affected. The architecture supports adding of more computing nodes to handle heavy traffic on application without making changes in the present system. Thus, the system is horizontally scalable. The below given diagram displays the interaction of end user with the load balancing system.

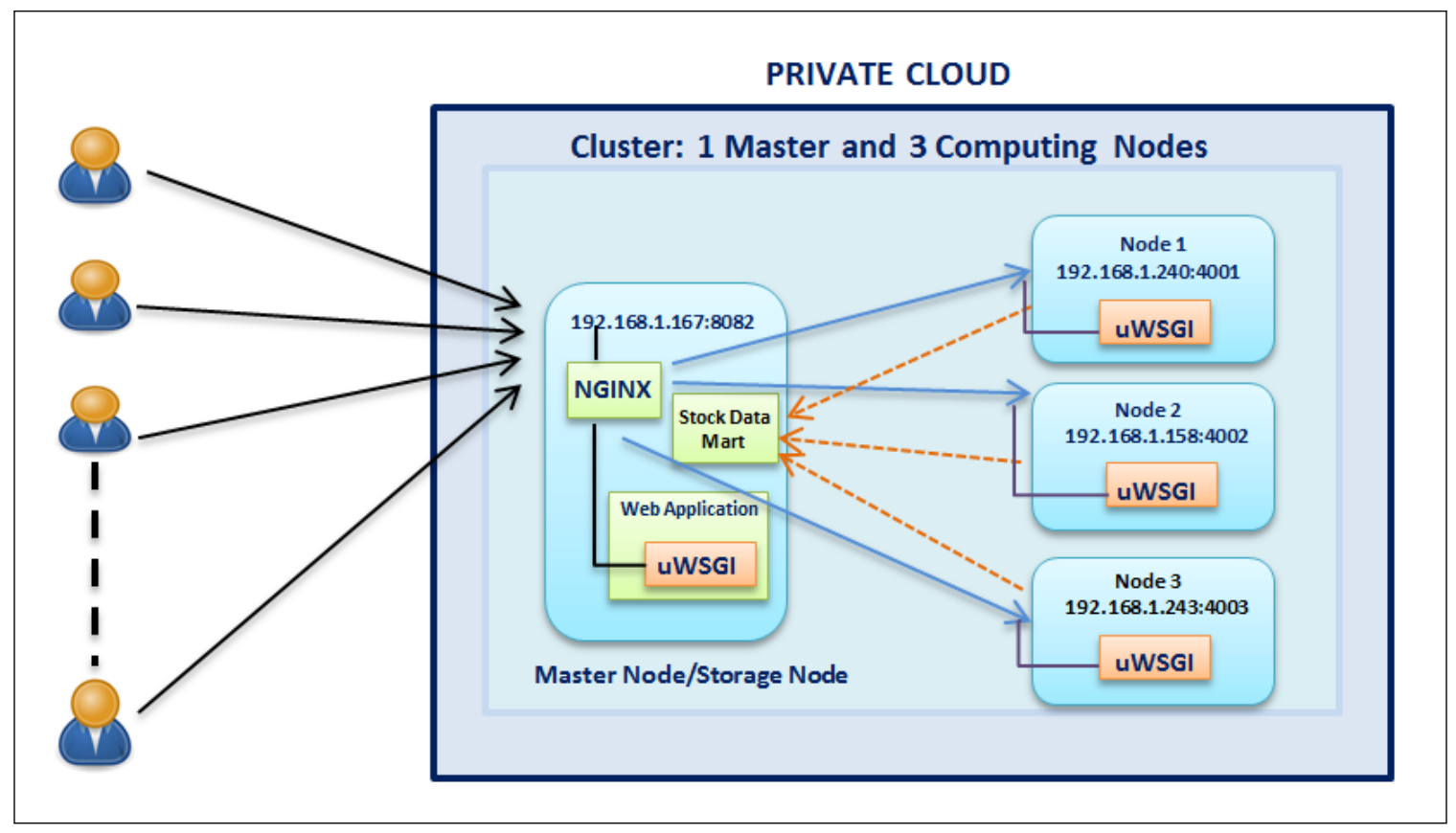

Figure 3.2: Cluster Architecture 


\subsubsection{Front-end Architecture}

The front-end architecture handles the client side of the application. It basically involves web application design. Here, django is used for web application development. Django is the most popular web server framework that follows the model-template-view (MVC) architectural pattern. It is developed in python and has several benefits. First, it provides functionality for user authentication, content administration, site maps and many more tasks. Second, it handles security attacks like SQL injection, cross-site scripting, cross-site request forgery and clickjacking. Third, it has an ability to scale quickly to handle heavy traffic on applications [Django, Django]. Bootstrap4 is used for front-end design.

\subsubsection{Back-end Architecture}

The back-end is completely implemented using shell script and python language. The implementation of data preprocesssing is done using python. Django is used for building and designing the web application. The application uses SQLite database for storing user information. It is the default database provided by django. The application is successfully deployed on each computing node and is ready to handle user request.

The user makes a request on IP address of master node(192.168.1.167) and at 8082 port, nginx HTTP server is listening for incoming connections. When a request is encountered by the nginx, nginx interacts with uWSGI server and then, schedules the request to one of the computing node for execution. The uWSGI server is used to deploy WSGI applications and is responsible for loading django application using the WSGI interface. It also helps in faster communication between users and application. 
Each node is listening at its port number and IP address. Here, node 1 is listening on address: 192.168 .1 .240 and on 4001 port number. Similarly, node 2 is listening on 192.168.1.158 IP address and 4002 port number, and node 3 is listening on 192.168.1.243 IP address and 4003 port number. The uWSGI at each node handles the execution of a job. All the computing nodes make an internal request to access stock data using 'paramiko' library. The response is directed back to the user after successful execution of job. The complete process takes place in back-end and logs are updated whenever a request is addressed. 


\section{Chapter 4}

\section{DESIGN AND IMPLEMENTATION}

The load balancing component acts as a single system but involves various functions to distribute the requests to application servers. These functions are pipelined in such a way that the designed system is more effective and efficient, in terms of performance. The design and workflow for the project is divided into several steps as shown in the below given diagram and each step is discussed in detail in this chapter.

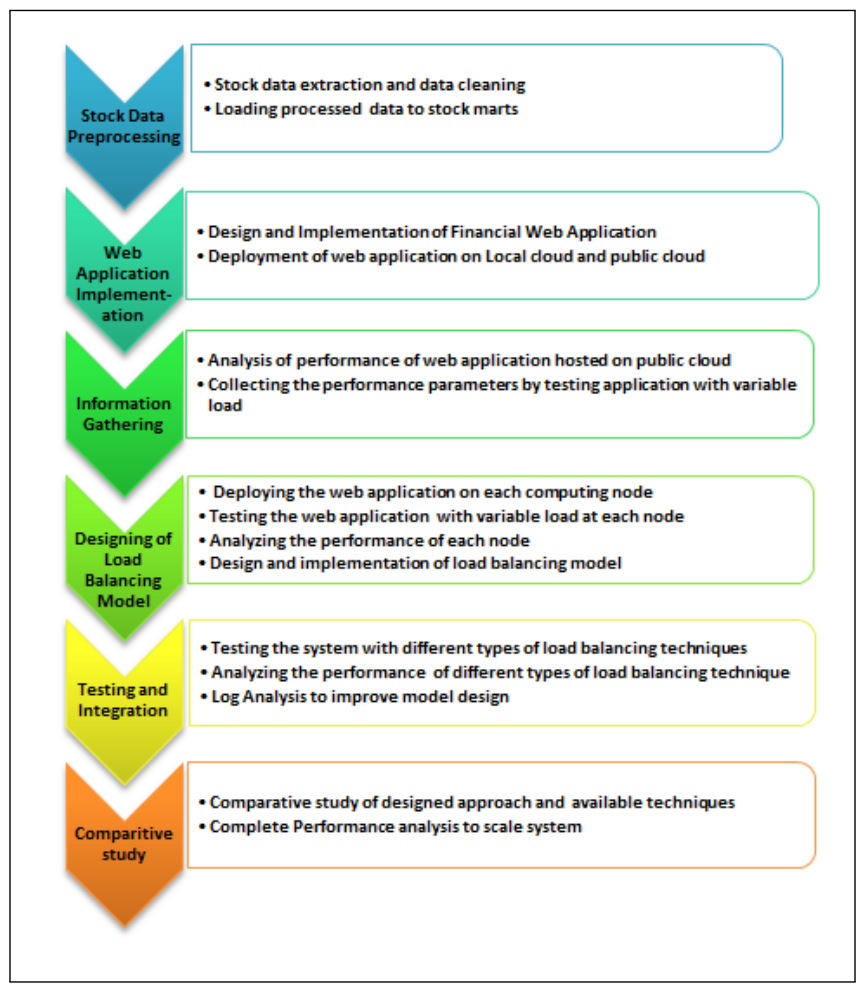

Figure 4.1: Design and Workflow of the Project 


\subsection{Stock Data Preprocessing}

The data preprocessing is implemented using Extract, Transfer and Load process; it is the traditional and most widely used technique for data preprocessing and data management. There are several financial data providers like Google Finance, Yahoo Finance, Alphavantage, Quandl and many more. The data is provided through APIs and this data can be used for generating technical indicators, which helps in designing trading strategies and monitoring the market price [Huang, 2020]. The data source used here is Yahoo Finance and data is extracted with the help of 'yfinance' python module.

The first step is data extraction. In this step, the stock price for the day is extracted. Each entry of data consist of 7 attributes- date, open, high, low, close, adj. close and volume. The next step is data cleaning and transformation, which mainly involves handling missing data and adding stock name to extracted data entry. In this step, missing data is replaced by computing the mean value of the missing attribute. After completion of this step, the data is set to load in stock data marts. This process is automated and scheduled job executes at 8pm, everyday. The complete process is performed only on master node and all other computing nodes request master node for the required data. The data preprocessing is implemented using pandas, numpy and scikit-learn libraries of python. 

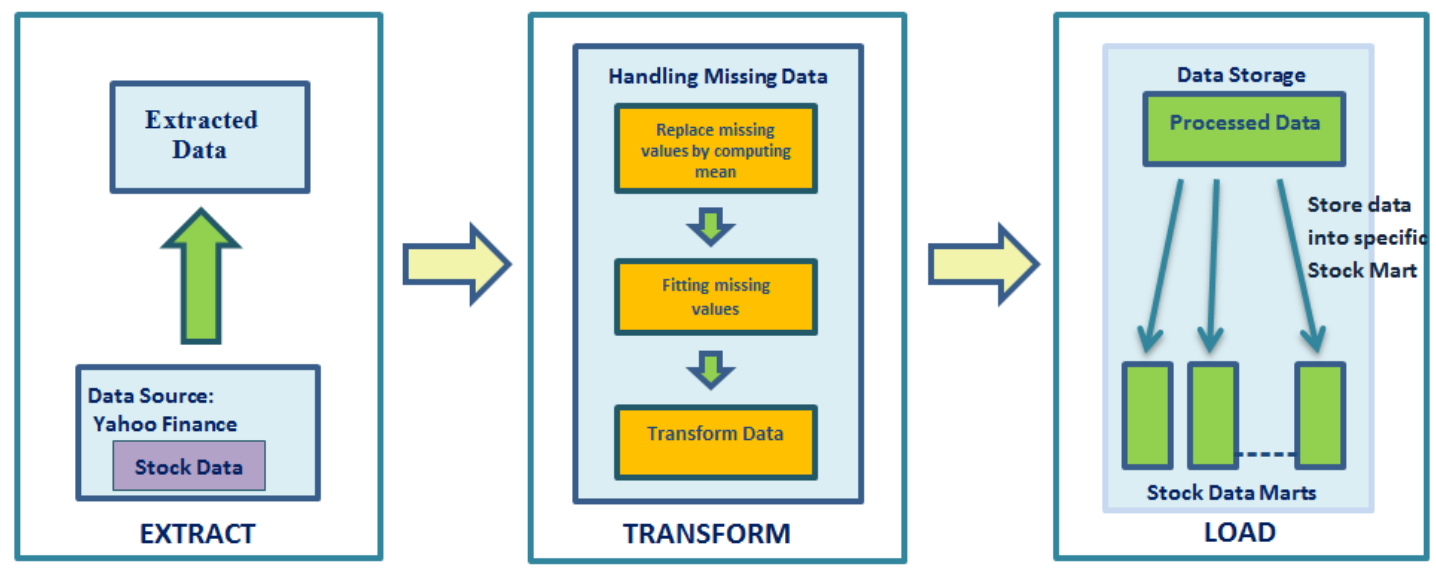

Figure 4.2: ETL- Extract, Transform and Load Process

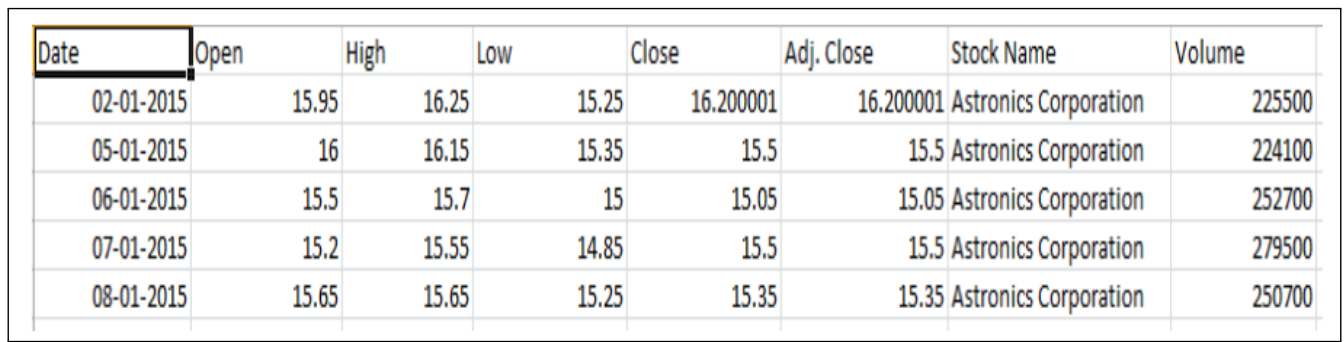

Figure 4.3: Example of Stock Data after Completion of Data Preprocessing

\subsection{Web Application Development}

The web application is developed by creating an isolated python virtual environment, which helps to keep dependencies required by the project separately and makes it easy to replicate on another node. The web application provides 5 types of function to users: prediction of stock price, historical analysis of stock data, top stock gainers and losers, comparison of two stocks and trend analysis. Analysis of each type of job request is discussed in below given section.

\subsubsection{Implementation of Different Types of Job Requests}

Each job is implemented and represented in a different way. All the jobs use: 'paramiko' library- to access stock data from master node, and 'pandas' and 'numpy' 
library- for performing operations. The following points provides the implementation of job requests:

1. Prediction of stock price is implemented using 'fbprophet' library and price can predicted either for next day, next week, next month or next year.

2. Historical analysis and comparison of stock data are implemented using 'mask' method of pandas, and are represented using 'plotly' library in three different forms: table, line graph and candlestick graph.

3. Market movers (top gainers and losers) are computed at master node and the result is read using paramiko. This reduces execution time as reading every stock data using paramiko consumes a lot of time.

4. Measure trends include computation of SMA and EMA for the provided range by user. The calculation of SMA and EMA to get trends in market is implemented using 'rolling' and 'ewn' function of pandas; the calculated values are represented in table and line graph.

To examine the performance of different jobs for all the computing nodes, one of locust test output is considered here. The total number of users are 32 with 8 concurrent users accessing at the same time. Following are the observations made:

1. For prediction requests, node 2 consumes large amount of time than node 3 and node 3 consumes more time than node 1.

2. For historical analysis requests, for table and candlestick representation, node 2 takes lot of time than node 3 and node 1 . For line representation, node 1 takes time than node 3 and node 2. 
3. For comparing two stocks, for all the type of representation, node 2 consumes more time than node 1 and node 3.

4. For market movers, node 1 and node 3 consumes more time than node 2 .

5. For measure trends, node 2 takes large amount of time than node 3 and node 1 .

Thus, it was found that for this test, the execution time for node 2 is comparatively higher than node 1 and the execution time for node 1 is more than node 3 for almost all types of jobs.

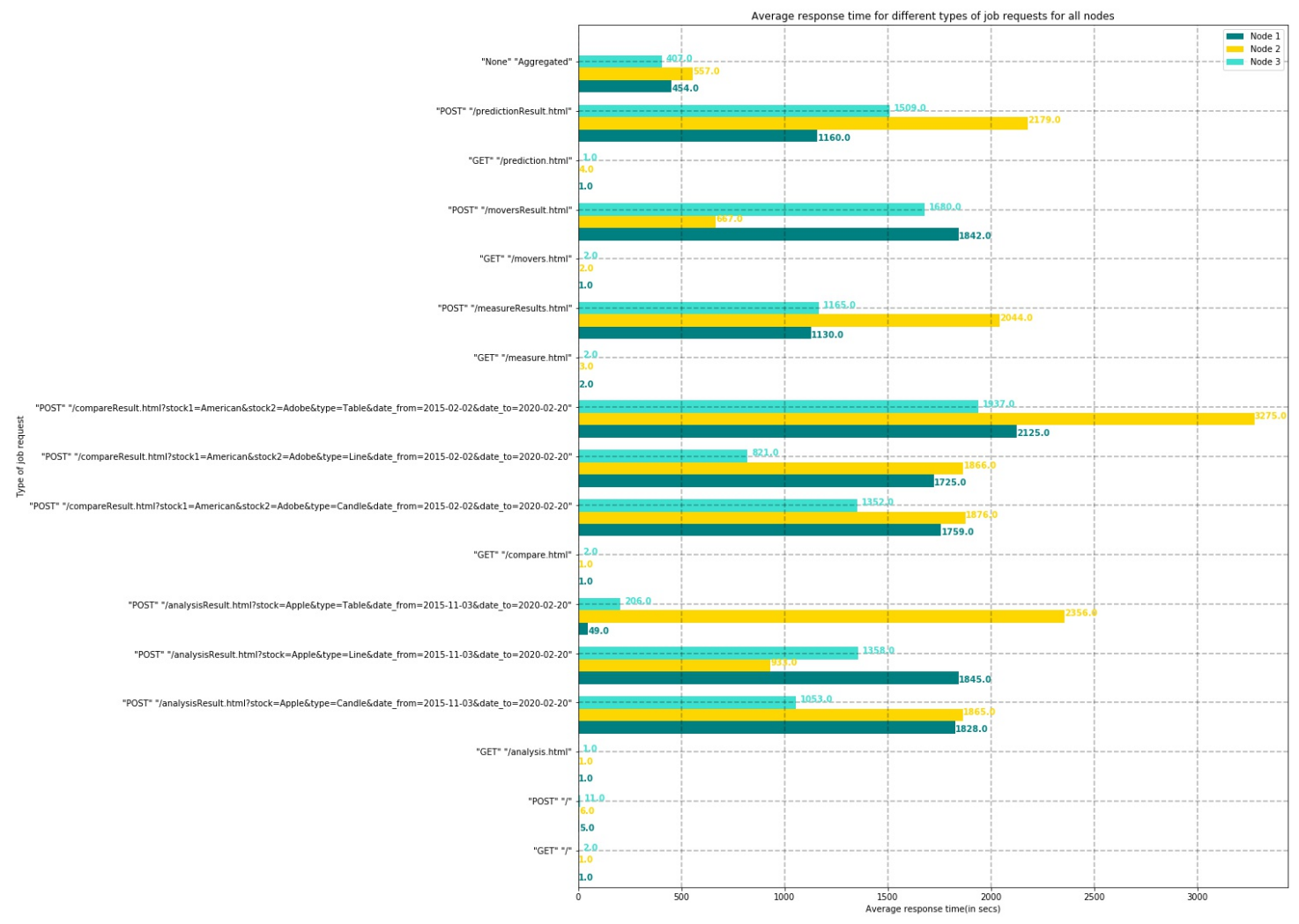

Figure 4.4: Response Time Analysis for Different Types of Job Requests 


\subsection{Training Set Creation using Locust}

\subsubsection{Load Testing Performed at Every Node}

To test the application for performance analysis at each node, stress testing is performed using locust. Locust is a load testing tool and helps in finding how many concurrent users can be handled by the system. It is event-based and thereby supports large number of concurrent users on a single machine. For every node, it generates 3 files: stats.csv, stats_history.csv and failures.csv files. The stats.csv provides the complete statistics about request with all the parameters. The stats_history.csv records request information with timestamp and failures.csv contains all the errors occured by a request. To compare the performance of nodes, stats.csv file is used for analysis.

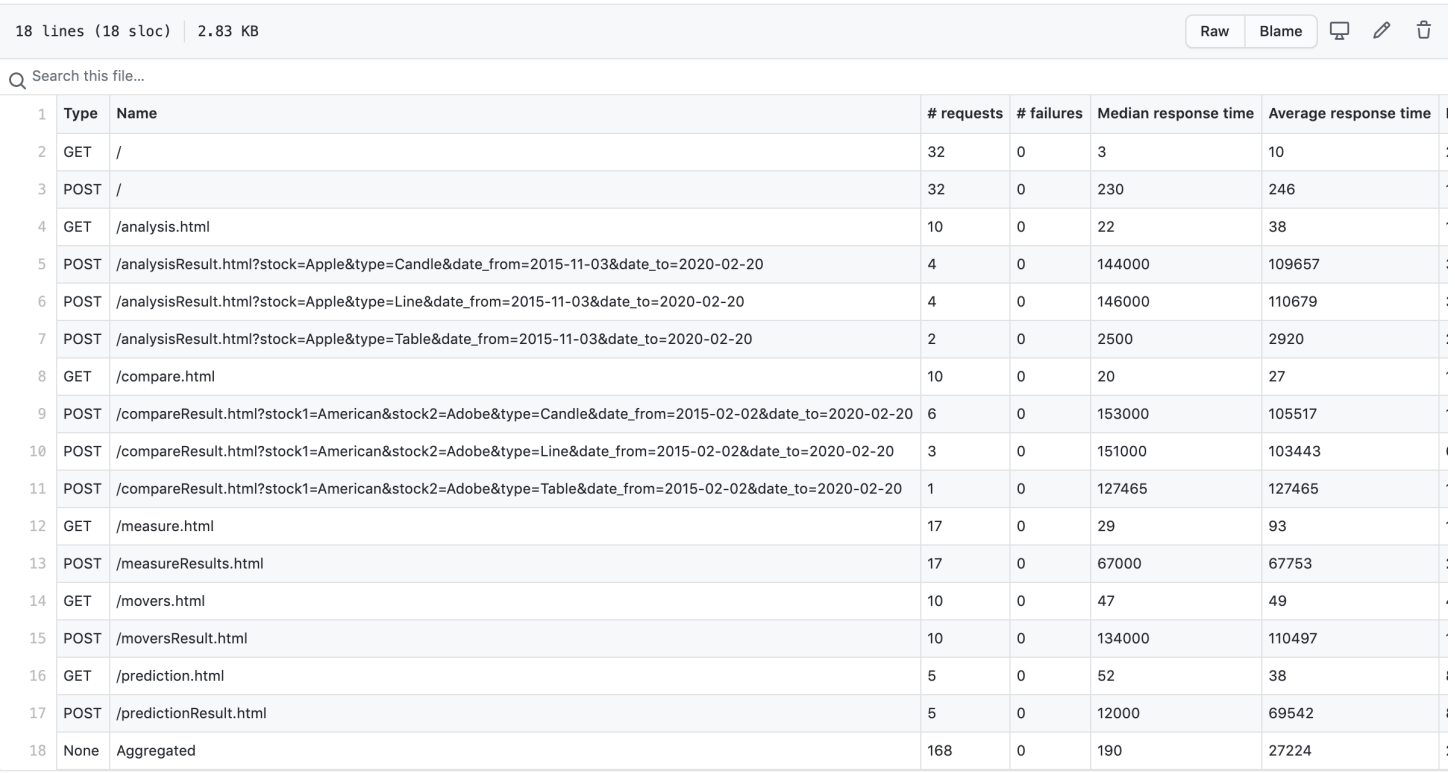

Figure 4.5: Example of stats.csv file generated

\subsubsection{Analysis of Data Generated at Nodes by Locust Testing}

The important attributes in 'stats.csv' file generated by the locust are: Type, Name, requests, failures, Median response time, Average response time, Min response time, Max 
response time, Average Content Size, Requests/s and Requests Failed/s. Out of all the mentioned attributes; requests, failures and Average response time are considered for performance analysis at node level. These 3 attributes are extracted for every type of load and following are the observations made:

1. The success rate for node 2 and node 3 is better than node 1 . It was obtained that all the three nodes were available till 256 total users. The success rate of node 2 was around $50 \%$ for total 256 users with 4 concurrent users; after that it was unavailable. The success rate for node 3 was around $30 \%$ for total 256 users with 4 concurrent users, it also became unavailable after this test. The success rate for node 1 was $50 \%$ for total 256 users with only 1 user accessing a time and it stopped working after that. Thus, node2 performance was better in general.

2. The failure rate for node 1 was comparatively more than node 2 and node 3 . Node 1 started failing with total 128 users with 128 concurrent users at failure rate around $38 \%$. Node 2 and node 3 started failing with total 256 users with 4 concurrent users with failure rate around $38 \%$ and $65 \%$, respectively. Node 2 has less failure requests comparatively.

3. The average response time for node 2 was relatively higher than node 1 and node 3 . The response time for node 2 was maximum for total 128 users with 8 concurrent users and was 1721 seconds. The response time for node 1 was maximum for total 64 users with 1 concurrent user and was 1397 seconds. The response time for node 3 was maximum for total 64 users with 4 concurrent users and was 1113 seconds. Thus, response time for node 3 is much better than node 1 and node 2 . 


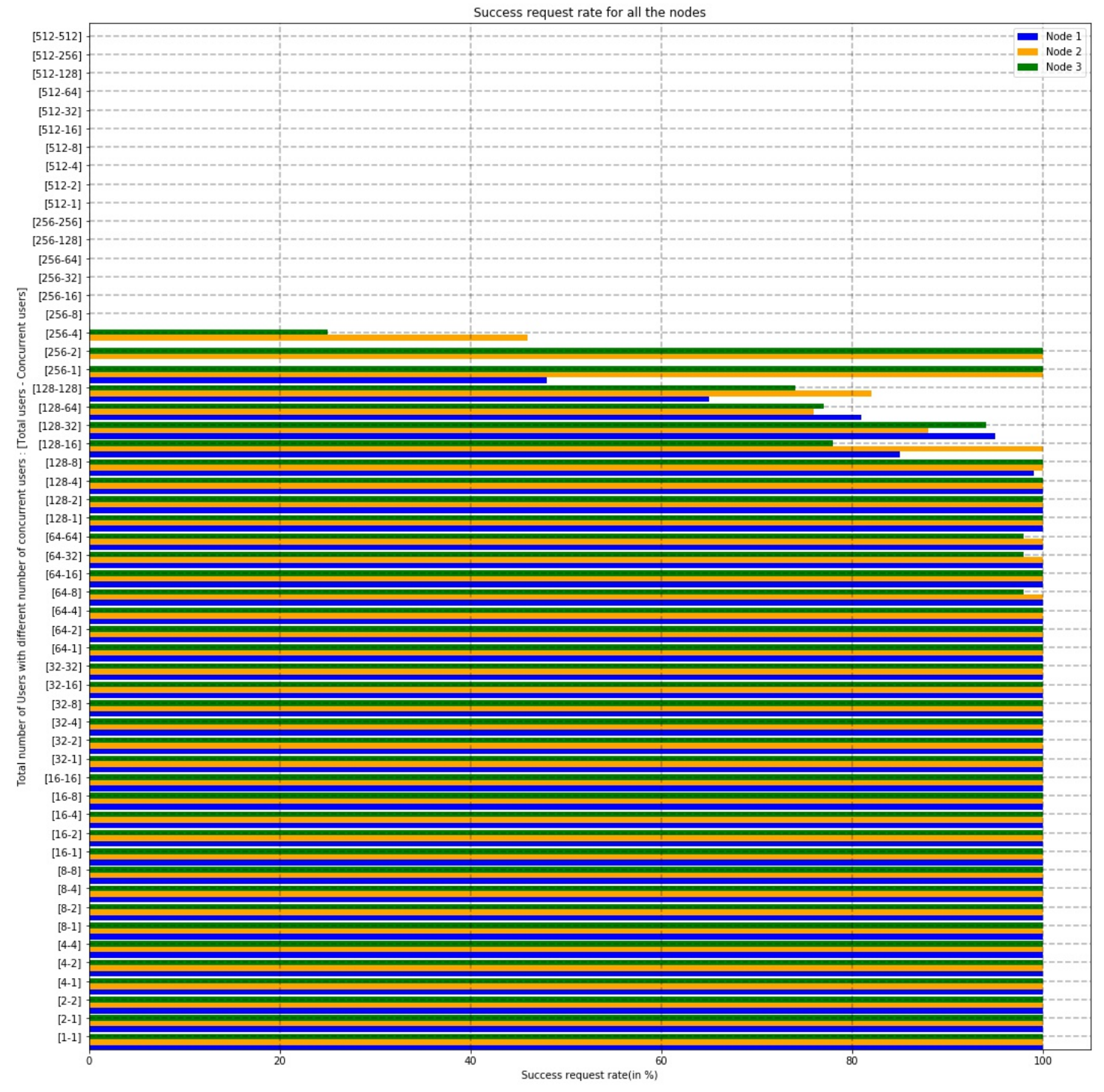

Figure 4.6: Success Request Rate for All the Nodes 


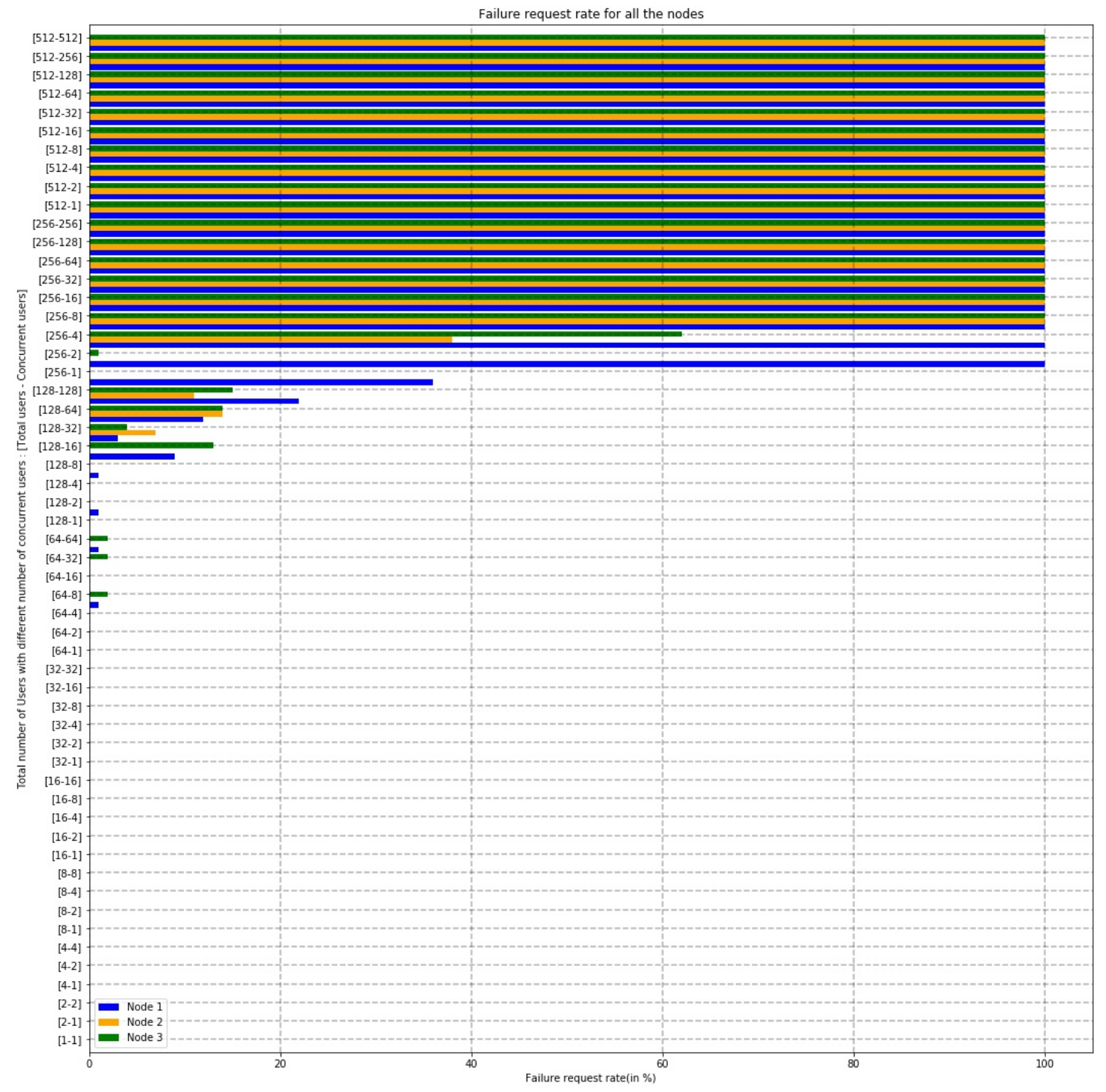

Figure 4.7: Failure Request Rate for All the Nodes 


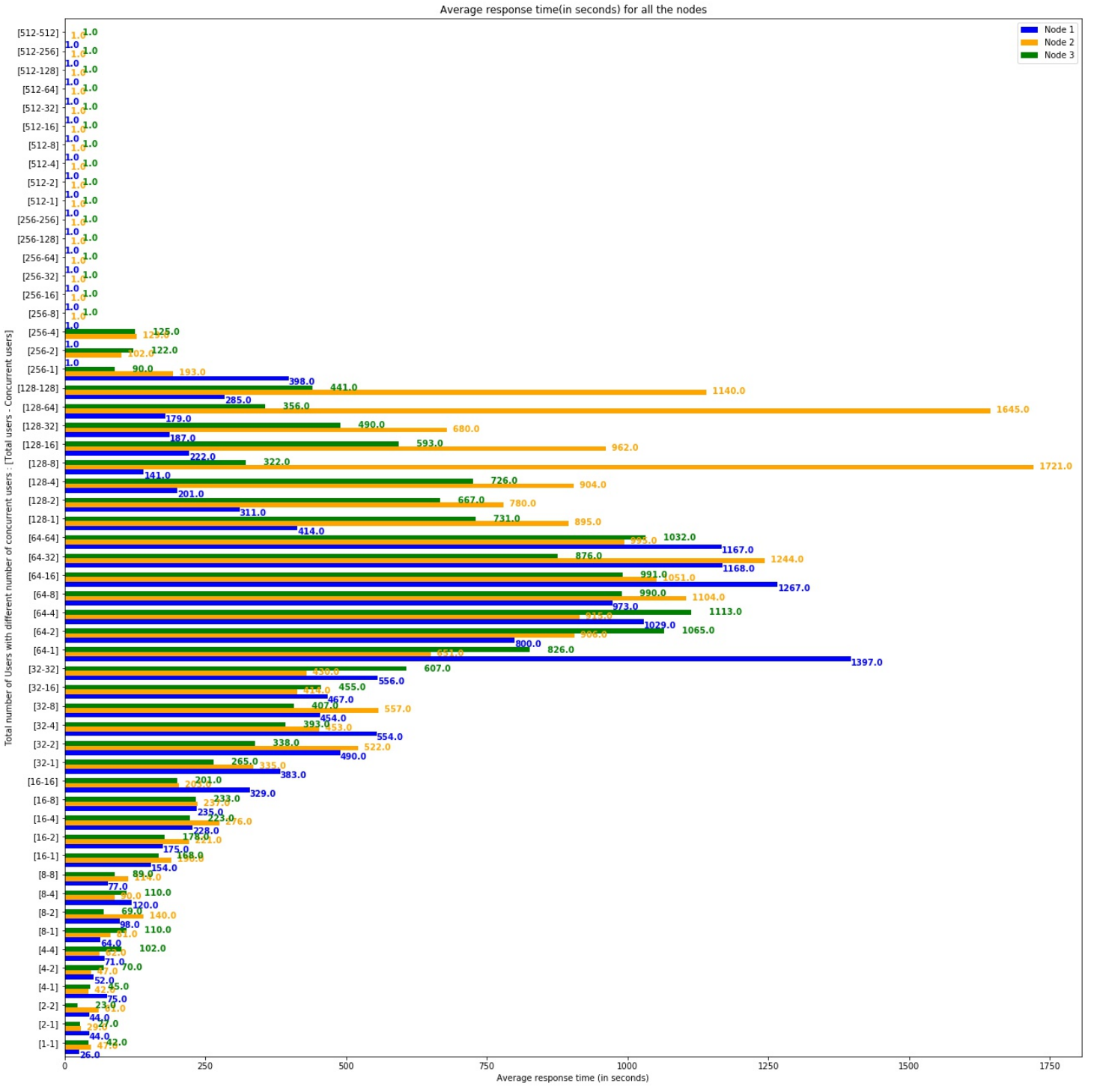

Figure 4.8: Average Response Time(in seconds) for All the Nodes

\subsection{Load Balancing}

To design load balancing system, a web server is required to handle the requests and in this project, nginx is used as a web server for HTTP load balancing purpose. The nginx uses uWSGI for loading django application. The 'ngx_http_uwsgi_module' module allows nginx to pass the request to a uwsgi server. Nginx provides four types of load 
balancing methods: round robin, least connection, iphash and weighted round robin. The below given section provides details for each type of technique.

\subsubsection{Types of Load Balancing}

\subsubsection{Round Robin Load Balancing Method}

In round robin approach, the requests are distributed across all the computing nodes sequentially. It is the default approach used for load balancing, if none of the load balancing method is specified. As we have seen in chapter 2 the round robin method is a static approach and doesn't utilize resources efficiently.

By performing testing using round robin approach in the developed system, it was found that the approach results in average performance and is not recommended for applying in the present developed system.

\subsubsection{Leasted Connection Load Balancing Method}

In situations, when some requests take a long time to respond, using least connection approach to balance the load will bring more stability and fairness in the system. In least connection approach, the new requests are distributed to servers, which are less burdened and will not overburden a busy application server. Thus, it builds a balanced environment and reduces the chances of performance and system issues. This approach can be used in nginx by adding 'least_conn' directive in server group configuration part of nginx configuration file.

\subsubsection{IP Hash Load Balancing Method}

In applications, when there is a need to direct some client to a specific server, nginx provides ip hash technique to attain the requirement. In other words, it makes client's session "sticky" or "persistent" to a particular application server. To attain this session per- 
sistence mechanism, ip hash method uses client's IP as a hashing key to choose application server from a group of application servers. It also ensures that the same client's request are directed to the same application server unless that application server is down or unavailable. This approach can be used in nginx by adding 'ip_hash' directive to server group configuration part of nginx configuration file.

\subsubsection{Weighted Round Robin Load Balancing Method}

Nginx provides an approach to assign weights to servers as per requirement of the application. This can be accomplished by using weighted round robin approach in nginx. The assigned weight in the weight parameter is considered for load balancing decision. By analyzing the performance of each node in the designed system, it was observed that the performance of node 2 and node 3 is similar and better than node 1 performance. Thus, weight parameter for node 2 and node 3 are same in the designed system. The assigned weight for node 2 and node 3 is 2 and for node 1 , the weight is 1 . Therefore, for every 5 new requests, the application will direct 2 requests to node 2 and node 3 , and one request to node 1 . The system was tested with the assigned weights and the performance of system was evaluated. It was observed that overall performance of the system was decent and recommendable for application, in consideration to all the factors like success rate, failure rate and average response time.

\subsubsection{Performance Analysis of Different Load Balancing Approaches}

1. It was observed that all the load balancing techniques worked fine for total 512 users with 512 concurrency rate but performance was varied from algorithm to algorithm. The success rate for IP hash technique was around 95\%, for weighted round robin, it was around $89 \%$, for least connected, it was around $62 \%$ and for round robin, it was 
$60 \%$. The IP hash and weighted round robin techniques have much better performance than least connected and round robin approach.

2. The failure rate for least connected method was comparatively high among all the other techniques for total 512 users with different concurrent users. The failure rate for least connected was maximum for total 512 users with 32 concurrent users and was $80 \%$. The failure rate for round robin was maximum for total 512 users with 128 concurrent users and was around 58\%. The failure rate for weighted round robin was maximum for total 512 users with 4 concurrent users and was around $63 \%$. The failure rate for IP hash was maximum for total 512 users with 32 concurrent users and was around $78 \%$. The overall failure rate for IP hash and weighted round robin, was very less often and less. However, the overall failure rate for least connected and round robin, was very often.

3. The average response time for IP hash technique was comparatively more than all other approaches. The average response time for IP hash was maximum for total 512 users with 128 concurrent users and was 1200 seconds. The average response time for least connected and round robin, was maximum for total 512 users with 128 concurrent users and was around 1100 seconds. The average response time for weighted round robin was maximum for total 512 users with 32 concurrent users and was around 1100 seconds.

Thus, it was observed that overall performance of least connected and round robin approach was similar and is not recommended for this application with the present architecture. The IP hash technique has lower failure rate and high success rate, but it directs re- 
quest to a particular server for a specific client, which overloads some servers. This creates imbalance in system and does not distribute requests evenly and moreover, it experiences high response time. Thus, it is not recommended for this application. The weighted round robin approach has better success rate, comparatively less error rate and average response time. Therefore, weighted round robin approach is suggested approach for load balancing this application.

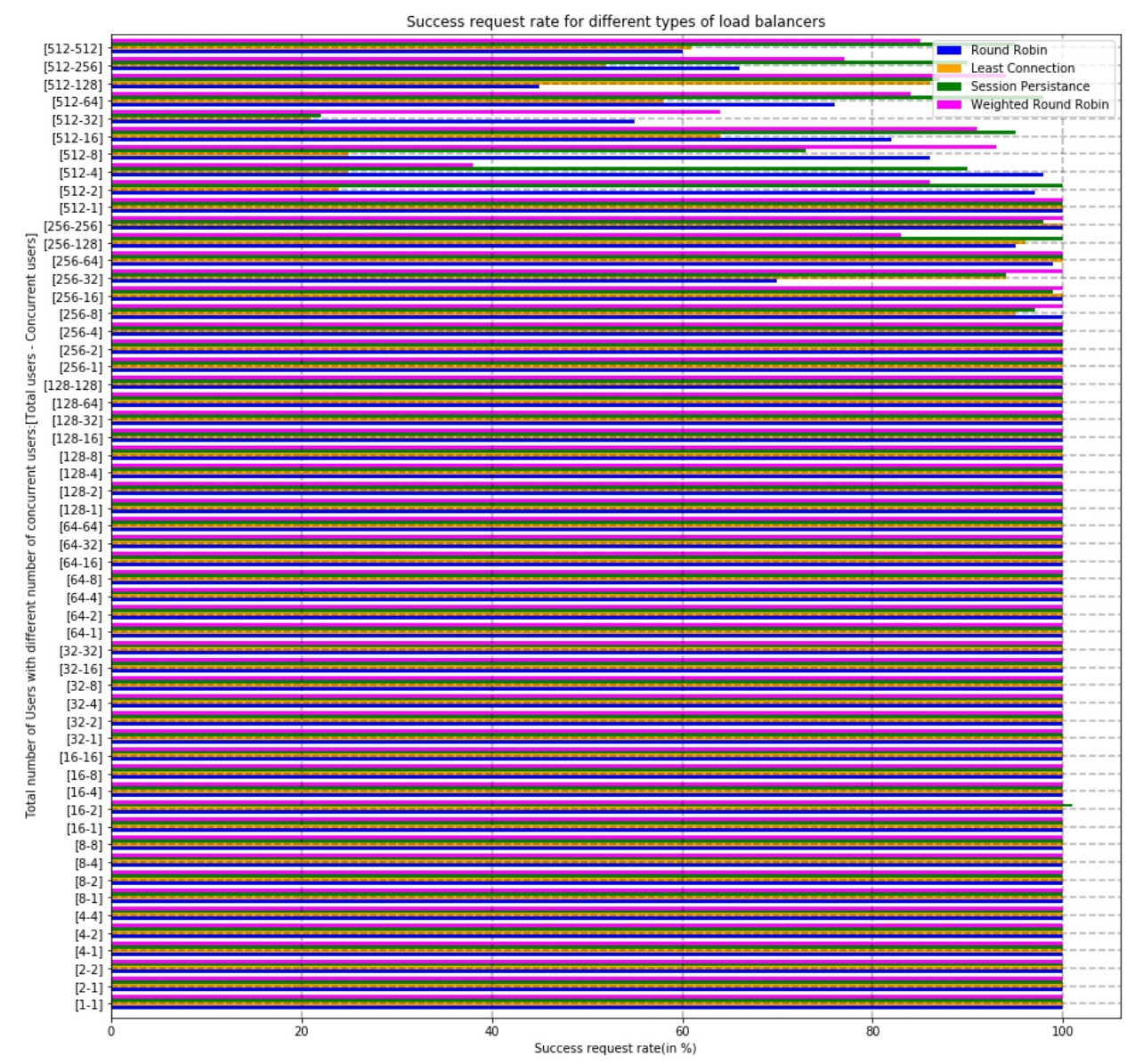

Figure 4.9: Success Request Rate for Different Types of Load Balancers 


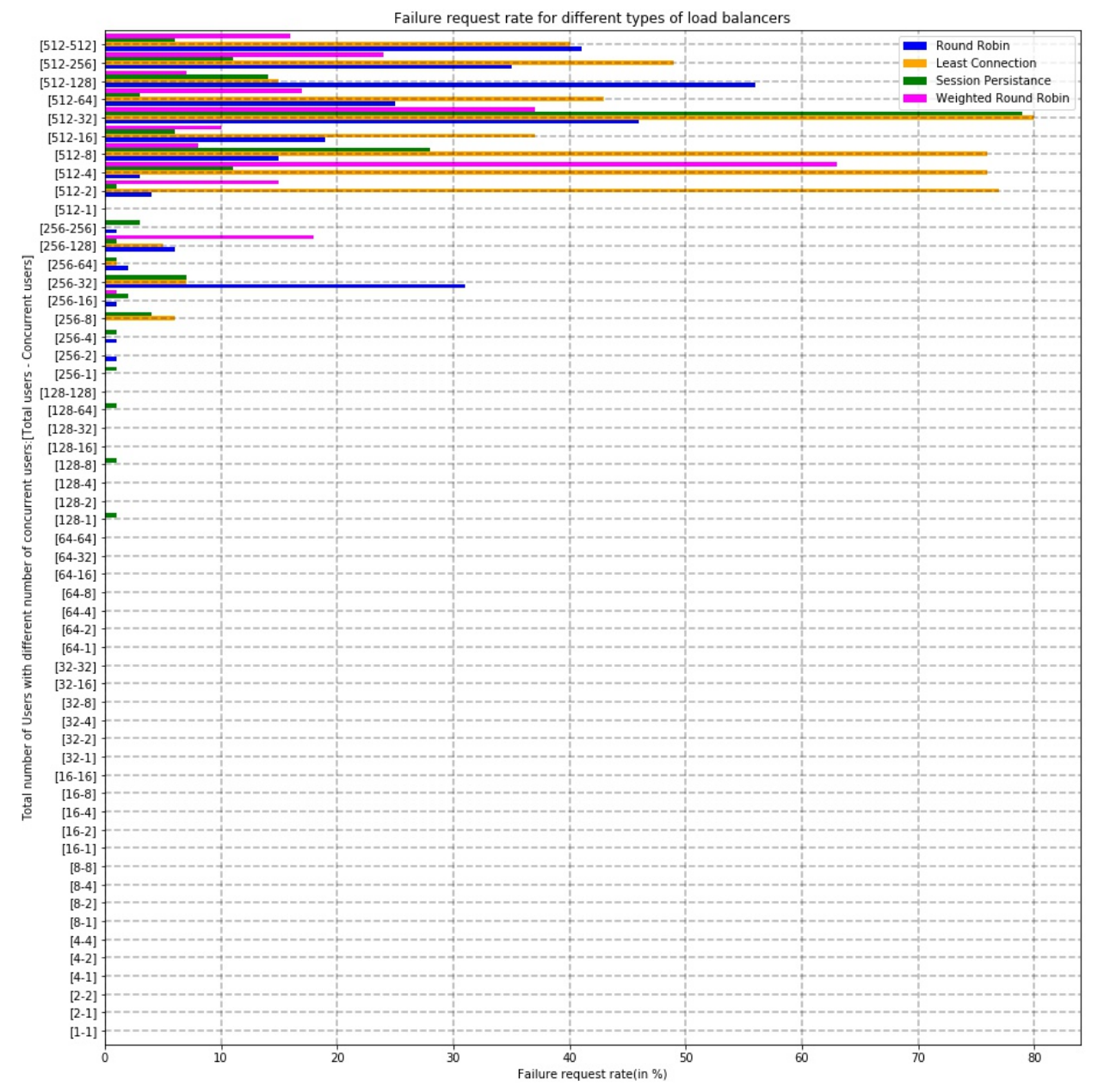

Figure 4.10: Failure Request Rate for Different Types of Load Balancers 


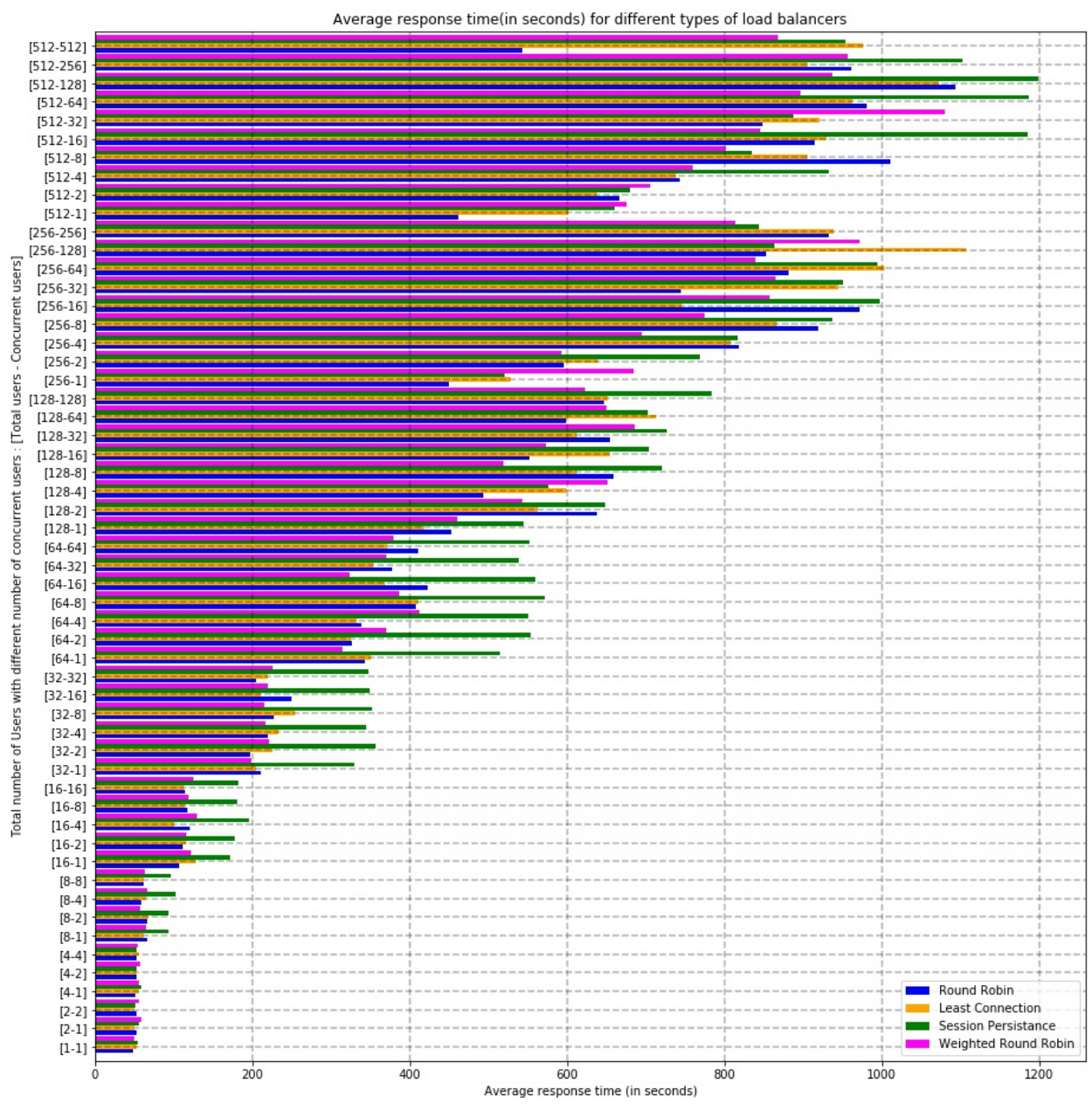

Figure 4.11: Average Response Time(in seconds) for Different Types of Load Balancers

\subsubsection{Log Analysis}

Nginx records its events in two types of logs: access log and error log. Access log provides all the information about the users accessing the web application. For example, it provides information like IP address of client, what type of request made, request time, response time and much more. Below given is the access log format: 
'\$upstream_addr - "\$http_x_forwarded_for" - \$remote_addr - \$remote_user [\$time_local] "\$request", '\$status \$body_bytes_sent, "\$http_referer" "\$http_user_agent" , \$request_time , Supstream_response_time'

The error log records all the issued encountered by nginx. It is used to debug issues in the system and can be used to monitor the performance of the system. Error logs do not have a specific format and the below given figure shows an example of error log generated.

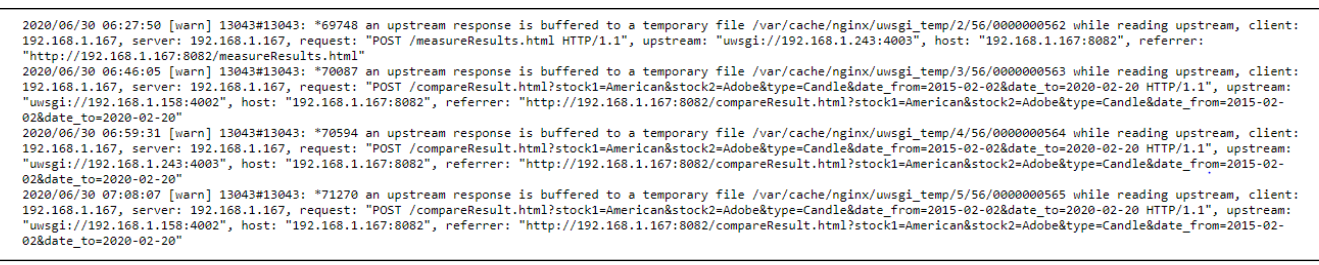

Figure 4.12: Example of Error Log Generated by Nginx

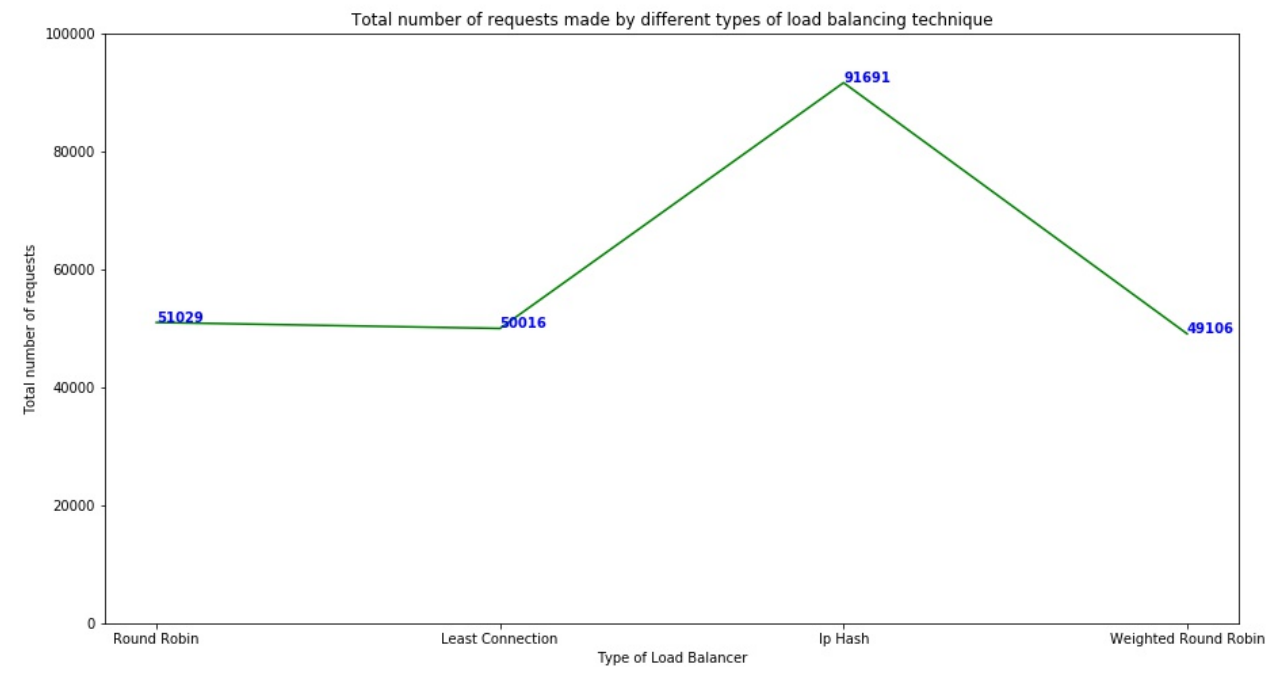

Figure 4.13: Total Number of Requests made by Different Types of Load Balancing techniques 


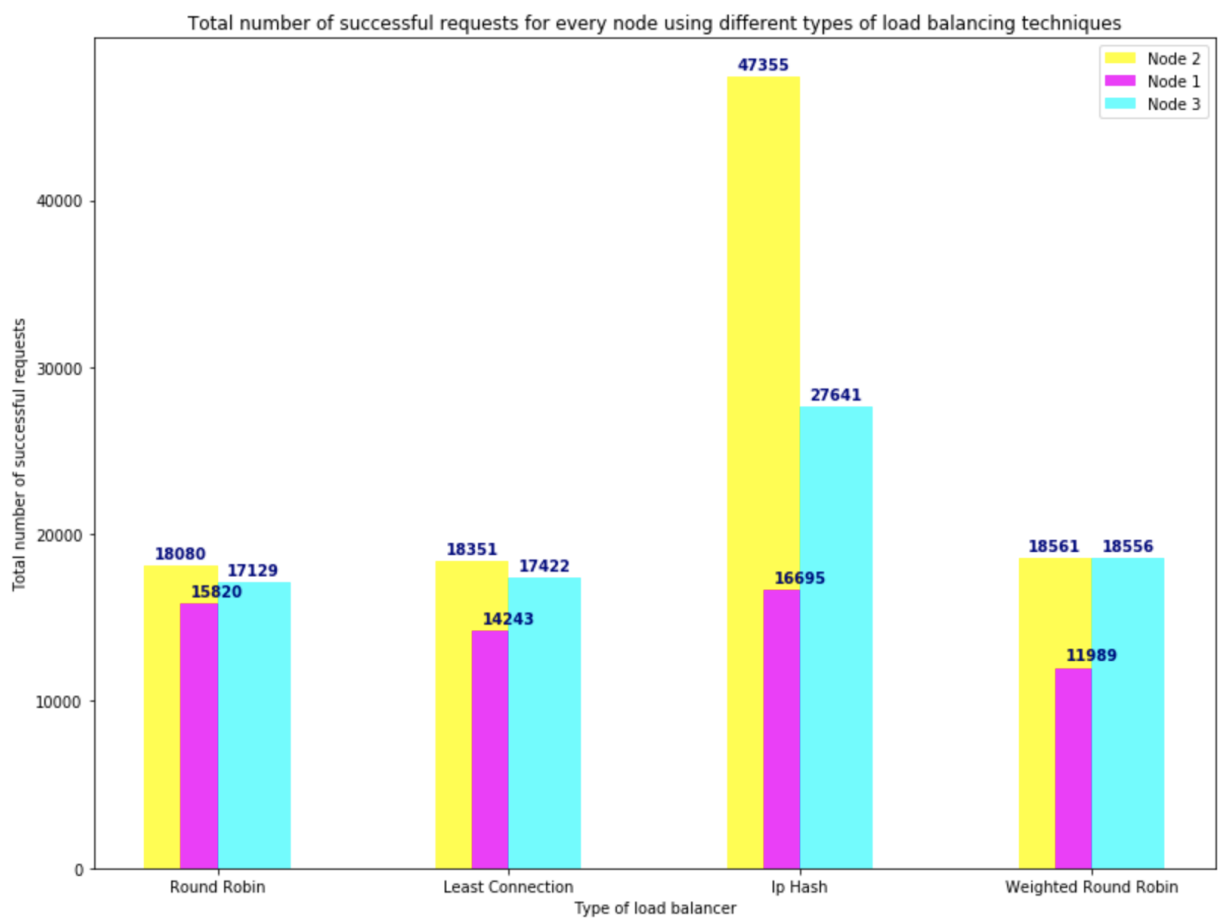

Figure 4.14: Total Number of Successful Requests for Every Node using Different Types of Load Balancing Techniques

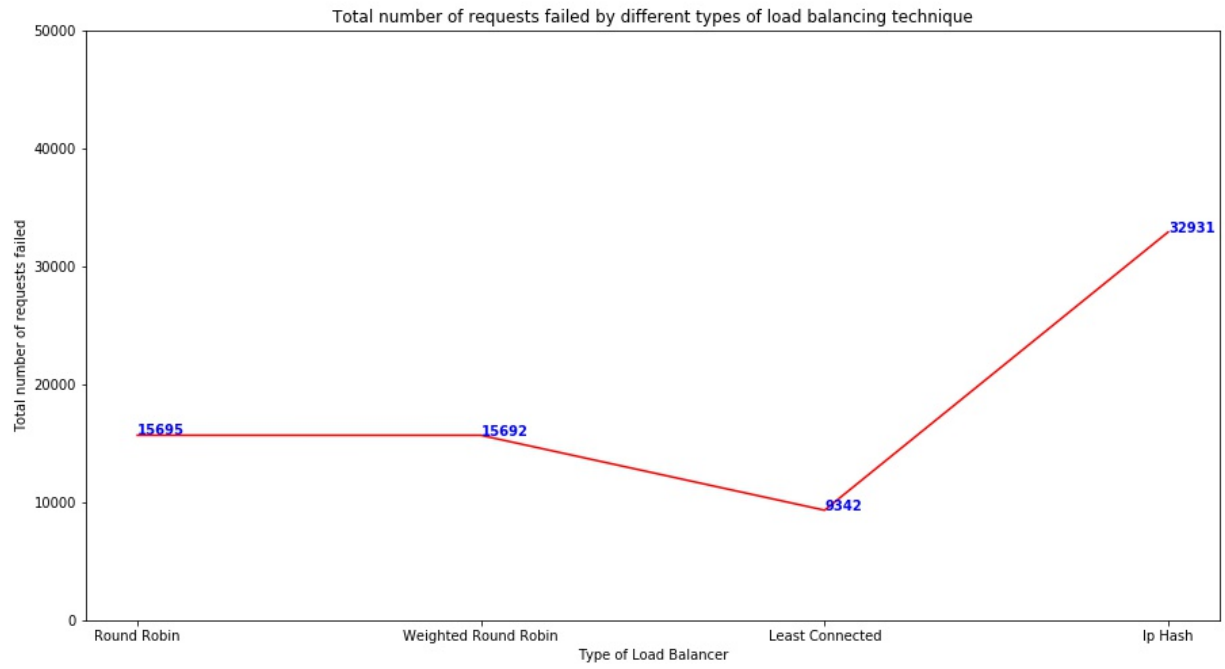

Figure 4.15: Total Number of Requests Failed by Different Types of Load Balancing techniques 


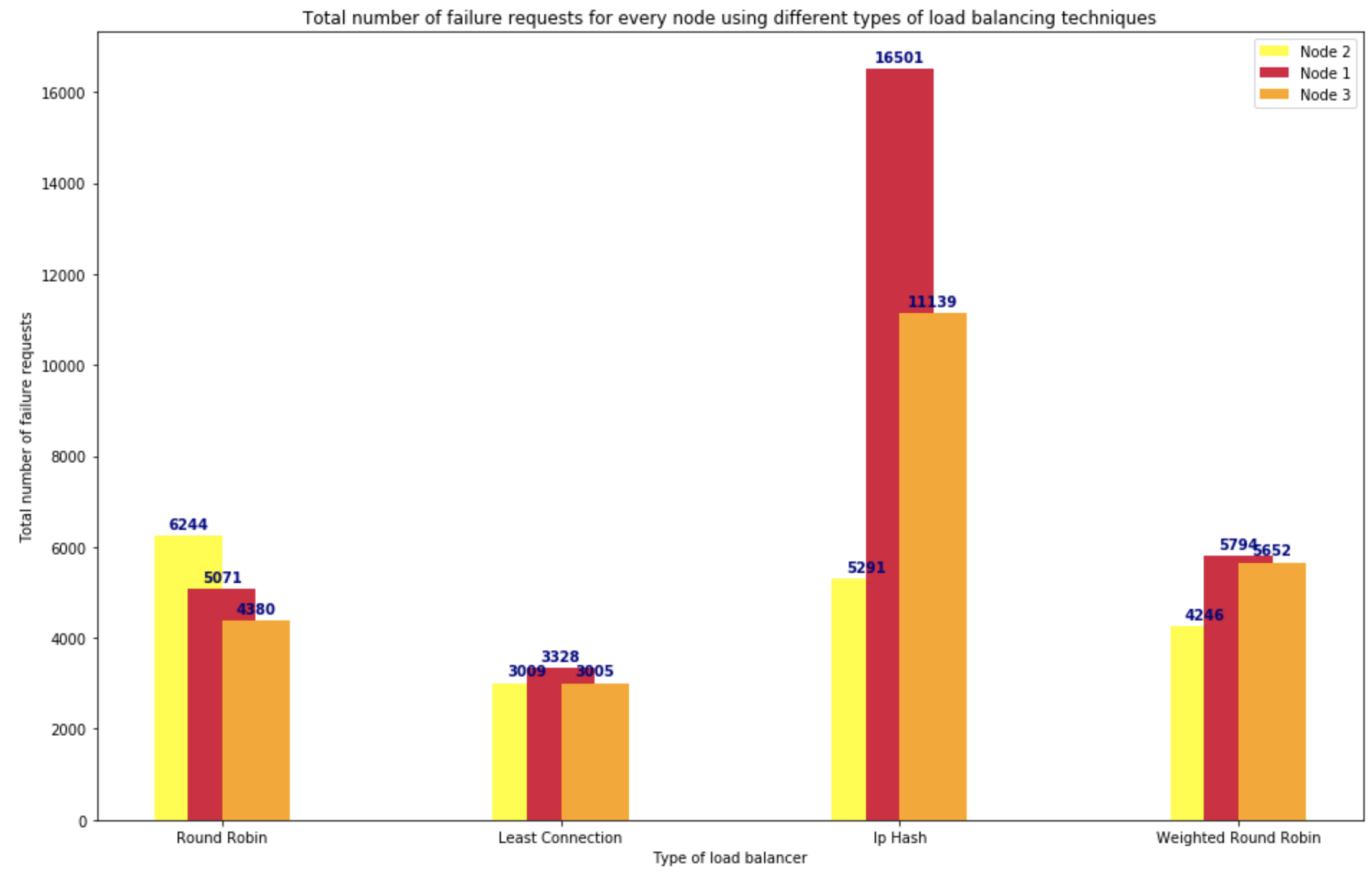

Figure 4.16: Total Number of Failure Requests for Every Node using Different Types of Load Balancing Techniques

Following are the observations made by analyzing both the logs:

1. It was observed that IP hash technique handles large number of requests as compared to other approaches. All other load balancing handles the same number of requests.

2. The number of successful requests and failure requests made by IP hash technique is comparatively higher than any other techniques.

3. The number of failed requests for least connected is comparatively less than all the other approaches. The number of failure requests made by round robin and weighted round robin is same.

4. For round robin, least connected and weighted round robin methods, node 2 and node 3 handles larger number of requests than node 1 . 
5. For IP hash technique, larger number of requests are handled by node 2 , than node 3 and then, node 1 .

Thus, it was observed by analyzing the logs that- node 2 and node 3 handles comparatively larger number of requests than node 1 . Therefore, descending order of performance of nodes can be represented as:

\author{
Node $2>$ Node $3>$ Node 1
}




\section{Chapter 5}

\section{COMPARATIVE STUDY AND CONCLUSION}

\subsection{Comparative Study}

In chapter 2, it was found that the application performance on AWS cloud was much better than the IBM cloud. In order, to conduct comparative study and analysis, the application on local cloud was compared with the application hosted on AWS cloud. To compare the load balancing module, designed system with round robin approach was selected to analyze the performance with AWS load balancer. After conducting stress testing of application on AWS cloud, it was observed that the performance of application on AWS cloud was not good enough to compare with other load balancing techniques than round robin. On AWS cloud, the application was deployed with the help of AWS Elastic Beanstalk service.

AWS free-tier account provides 750 hours of t2.micro instances for each month for a year and as studied in chapter 2 that the Elastic Beanstalk uses Amazon EC2 instance for running the web application. Since, t2.micro instances are low-cost and designed for serving general-purpose applications, it is not recommended to use for applications with heavy traffic. When undergoing heavy traffic, the load balancing and auto-scaling feature

of Elastic Beanstalk gets highly affected. As a result, EC2 instances are unavailable to serve the upcoming new requests. Thus, the application hosted on AWS cloud results in 
inefficient performance and upgrading instance type is the only solution to improve the performance of the application.

By performing stress testing using Locust on application on AWS cloud and application on local cluster using round robin approach, it was found that:

1. The request success rate for for AWS cloud was inconsistent and the application was unavailable for the most of the time. For round robin approach on local system, the success rate was $60 \%$ for total 512 users with 512 concurrent users. The application on AWS cloud was available for only few tests.

2. The request failure rate for application on AWS cloud was $100 \%$ for most of the tests and only for total 8 users with 1 concurrent user, it is $0 \%$. For local system, failure rate was around $58 \%$ for total 512 users with 128 concurrent users.

3. The average response time for application on AWS cloud was at maximum for a total of eight users with 1 concurrent user and was around 4900 seconds. For a local system, the average response time was maximum for total 512 users with 128 concurrent users and was around 1100 seconds.

Thus, application on AWS cloud is unavailable most of the time and response time is also very high. Thereby, results in poor performance. 


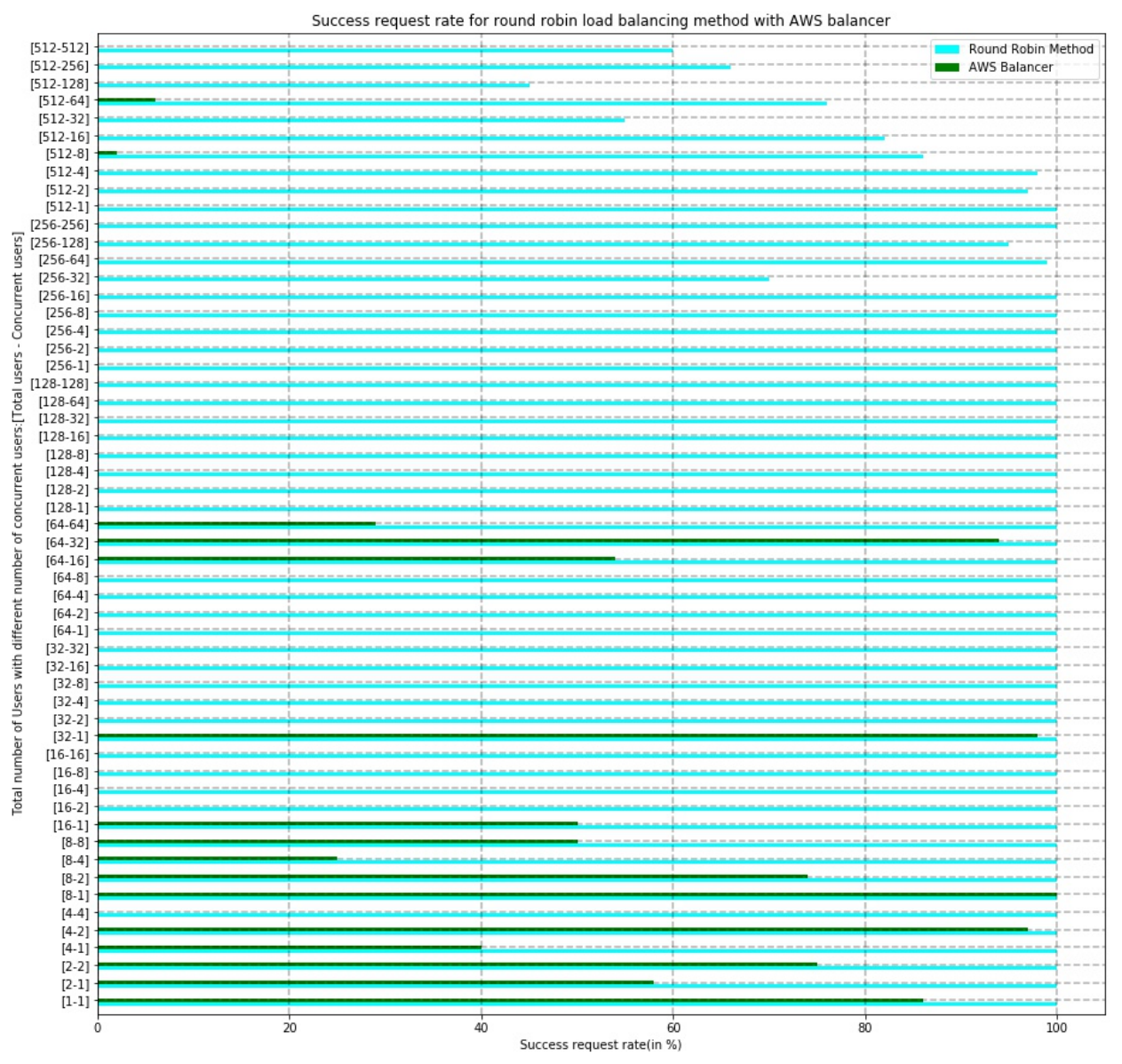

Figure 5.1: Success Request Rate for Round Robin Load Balancing Method with AWS Load Balancer 


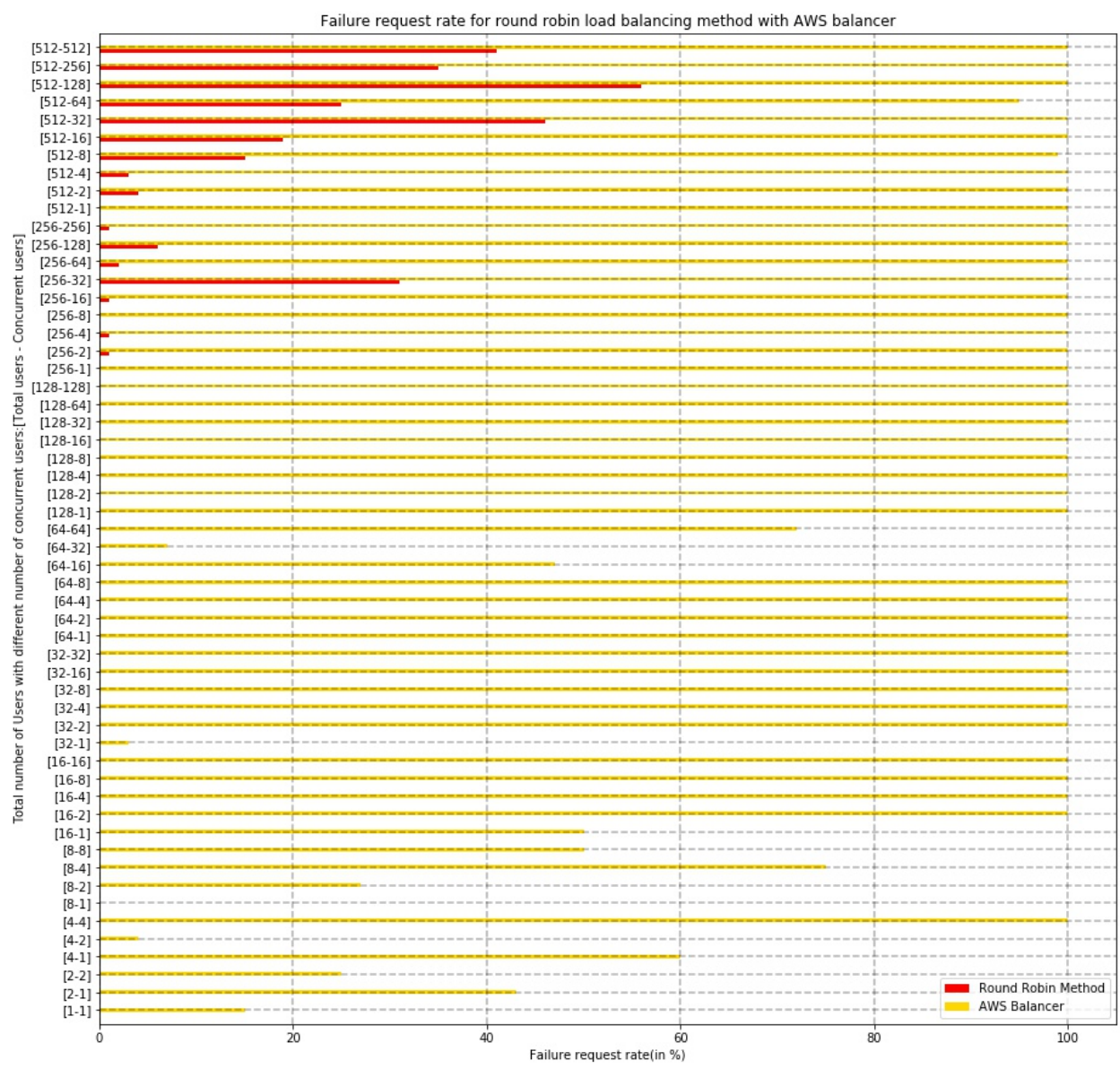

Figure 5.2: Failure Request Rate for Round Robin Load Balancing Method with AWS Load Balancer 


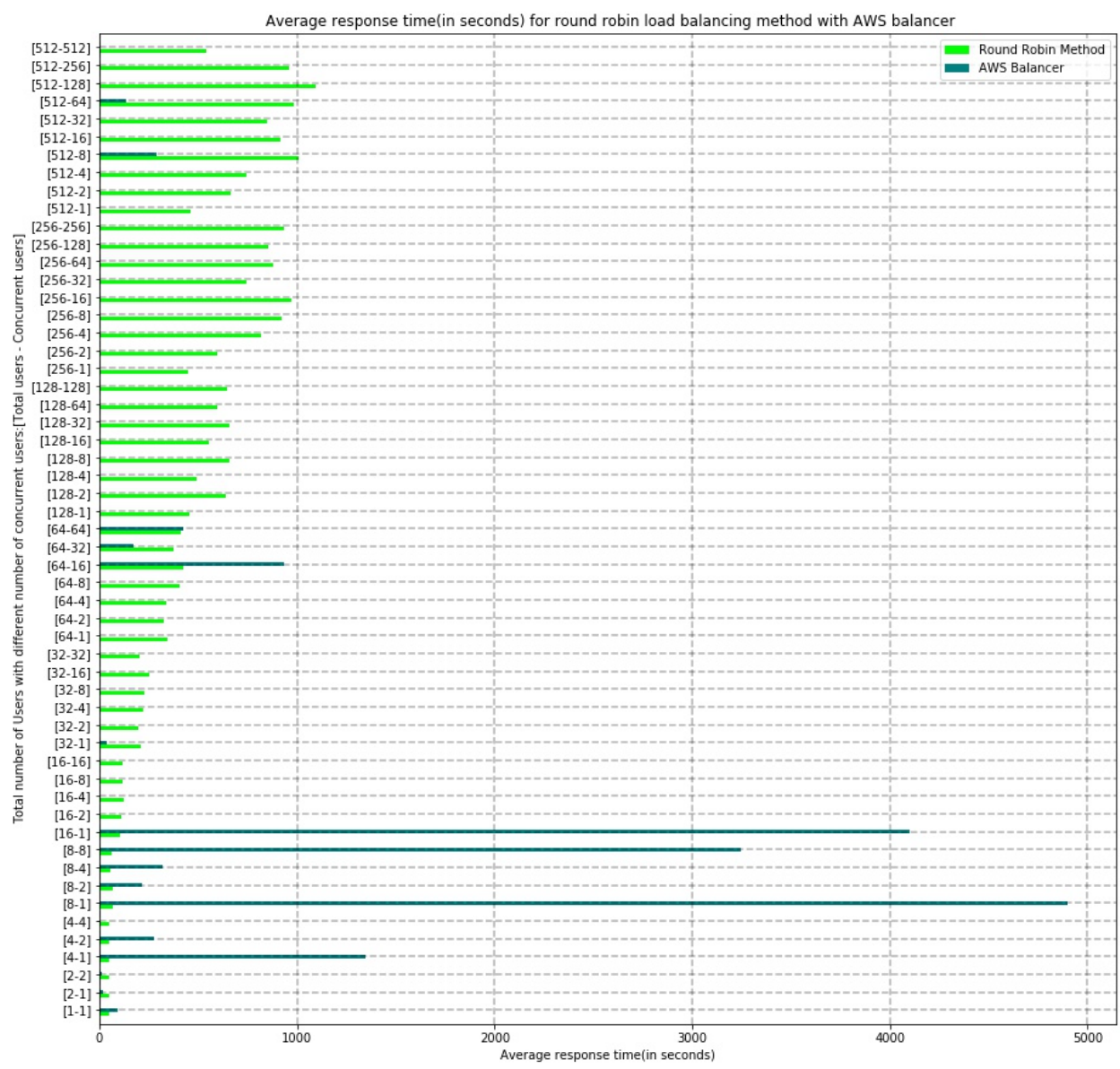

Figure 5.3: Average Response Time(in seconds) for Round Robin Load Balancing Method with AWS Load Balancer

\subsection{Future work}

In present designed system, the test duration for conducting stress testing was around 10 hours for performing testing on computing nodes at node level and for testing all the types of balancers, the test duration was around 5 hours, due to time constraints. Thus, by increasing testing time would be the next step, it will help for much better anal- 
ysis and will bring more accuracy in decision making of the system. Next step will be implementation of automated log analysis system for better and easy analysis purpose.

The designed application do not have any external security feature to handle security attacks like Distributed Denial of Service Attack (DDoS) attack, Cross-Site Scripting (XSS) attack etc. Thus, another step would be adding security mechanism to the existing system. Also, the present system can be scaled horizontally, without affecting the performance of existing system. However, the system is not vertically scalable and adding this feature will be next step for further implementation. 


\section{Chapter 6}

\section{CONCLUSION}

The main purpose of this project is to improve resource utilization and manage loads over system in an efficient way. The designed system helps user to analyze the stock data in different ways with less amount of time. The data is updated and processed daily, so user can analyze the stock price with updated data at the end of the day. The existing system has three computing nodes for processing the user requests, out of which, node 2 and node 3 have better performance in handling large number of requests than node 1 . The average response time for node 2 is comparatively higher than node 1 and the average response time

for node 1 is more than node 3 . Moreover, by performing log analysis, it was found that node 2 and node 3 are better than node 1 . Thus, overall performance of node 2 and node 3 is better than node 1 and more load can be distributed to these nodes. Also, by conducting the stress testing on the present system, it was observed that only hardware architecture cannot be used for making decision to balance the load, it also needs a complete analysis of jobs executed at each node. In addition, the designed system is horizontally scalable. Therefore, adding computing nodes will not affect the performance of the existing system. The developed application was tested with four types of load balancing approaches: Round Robin, Least Connected, IP Hash and Weighted Round Robin. Out of all, it was observed that the overall performance of weighted round robin was better in terms of: success request rate, failure request rate and average response time. For the present system, 
the weight parameter used for node 2 and node 3 is 2 , and for node 1 , it is 1 . The weight parameter was decided by looking at the performance of all the computing nodes used in the system. Also, here only one client was used to perform testing on the system. The type of client is also a contributing factor for selection of load balancing approach.

As client-base changes, it cannot be claimed that the same load balancing approach will be effective for all the situations. So, selection of type of load balancer depends on various factors like types of jobs, types of users, hardware architecture of the system and the performance of computing nodes used. The performance of designed system was also compared with application hosted on AWS cloud to analyze the performance of application on public cloud. It was observed that the application on AWS cloud has poor performance and was unavailable due to heavy traffic generated while stress testing. The performance of application on AWS cloud can be improved by upgrading the type of EC2 instance used. Also, AWS cloud provides services at cheap rates and the web interface provides a wide variety of factors for users to monitor the performance of the application . Thus, it is most widely used service. 


\section{REFERENCES}

Deploy django applications to the cloud.

Amazon. Amazon - amazon web services.

Baygan, M. and M. Baygan (2015, Nov). A new method for solving the open shop scheduling using imperialist competitive algorithm and tabu search with regard to maintenance of machine. In 2015 2nd International Conference on KnowledgeBased Engineering and Innovation (KBEI), pp. 972-977.

Bhole, A., B. Adinarayana, and S. Shenoy (2015, Oct). Log analytics on cloud using pattern recognition a practical perspective to cloud based approach. In 2015 International Conference on Green Computing and Internet of Things (ICGCIoT), pp. 699-703.

Bo Liu, Zhi-Feng Hao, and Xiao-Wei Yang (2005). Nesting support vector machinte for muti-classification [machinte read machine]. In 2005 International Conference on Machine Learning and Cybernetics, Volume 7, pp. 4220-4225 Vol. 7.

Ching-Te Wang and Yung-Yu Lin (2015, Aug). The prediction system for data analysis of stock market by using genetic algorithm. In 2015 12th International Conference on Fuzzy Systems and Knowledge Discovery (FSKD), pp. 1721-1725.

Django. Django overview.

Domingos, P. (2012, October). A few useful things to know about machine learning. Commun. ACM 55(10), 78-87.

Dutta, M. and N. Aggarwal (2017, Apr). A review of task scheduling based on metaheuristics approach in cloud computing.

Fonseka, C. and L. Liyanage (2008, Dec). A data mining algorithm to analyse stock market data using lagged correlation. In 2008 4th International Conference on Information and Automation for Sustainability, pp. 163-166.

Foster, I., Y. Zhao, I. Raicu, and S. Lu (2008, Nov). Cloud computing and grid computing 360-degree compared. In 2008 Grid Computing Environments Workshop, pp. $1-10$.

Hargreaves, C. and Y. Hao (2012, Sep.). Does the use of technical fundamental analy- 
sis improve stock choice? : A data mining approach applied to the australian stock market. In 2012 International Conference on Statistics in Science, Business and Engineering (ICSSBE), pp. 1-6.

Huang, S. (2020, Mar). Best 5 free stock market apis in 2020.

Johnson, B. and J. Nieh (2010, 9). Modeling the adaptive role of negative signaling in honey bee intraspecific competition. Journal of Insect Behavior 23(6), 459-471.

Kaur, A., G. Raj, S. Yadav, and T. Choudhury (2018, Dec). Performance evaluation of aws and ibm cloud platforms for security mechanism. In 2018 International Conference on Computational Techniques, Electronics and Mechanical Systems (CTEMS), pp. 516-520.

Kumar, S. and R. H. Goudar (2012). Cloud computing - research issues, challenges, architecture, platforms and applications: A survey.

L.D., D. B. and P. V. Krishna (2013). Honey bee behavior inspired load balancing of tasks in cloud computing environments. Applied Soft Computing 13(5), $2292-2303$.

Li, J., W. Wang, and X. Hu (2015, Aug). Parallel particle swarm optimization algorithm based on cuda in the aws cloud. In 2015 Ninth International Conference on Frontier of Computer Science and Technology, pp. 8-12.

NGINX (2020, May). What is load balancing? how load balancers work.

NOVKOVIC, G. N. (2017, Oct). Top 5 control engineering articles october 23-29: Modern hmi elements, father of the plc, cloud computing, more.

Persico, V., A. Montieri, and A. Pescapè (2016, Oct). On the network performance of amazon s3 cloud-storage service. In 2016 5th IEEE International Conference on Cloud Networking (Cloudnet), pp. 113-118.

Raj, G., D. Singh, and A. Bansal (2013, Sep.). Load balancing for resource provisioning using batch mode heuristic priority in round robin (pbrr) scheduling. In Confluence 2013: The Next Generation Information Technology Summit (4th International Conference), pp. 308-314.

Rajput, S. S. and V. S. Kushwah (2016, Dec). A genetic based improved load balanced min-min task scheduling algorithm for load balancing in cloud computing. In 2016 8th International Conference on Computational Intelligence and Communication Networks (CICN), pp. 677-681.

Reno and S. R. Group. Amazon, microsoft, google and alibaba strengthen their grip on the public cloud market. 
Routray, R. (2015, March). Cloud storage infrastructure optimization analytics. In 2015 IEEE International Conference on Cloud Engineering, pp. 92-92.

Saravanan, R. and P. Sujatha (2018, June). A state of art techniques on machine learning algorithms: A perspective of supervised learning approaches in data classification. In 2018 Second International Conference on Intelligent Computing and Control Systems (ICICCS), pp. 945-949.

Shamim, A., H. Hussain, and Maqbool Uddin Shaikh (2010). A framework for generation of rules from decision tree and decision table. In 2010 International Conference on Information and Emerging Technologies, pp. 1-6.

Sharma, S., V. Chang, U. Tim, J. Wong, and S. Gadia (2016, 03). Cloud-based emerging services systems. International Journal of Information Management.

Solanki, R. K., K. Verma, and R. Kumar (2015). Spam filtering using hybrid local-global naive bayes classifier. In 2015 International Conference on Advances in Computing, Communications and Informatics (ICACCI), pp. 829-833.

Tang, X., P. Liu, Z. Wang, and B. Liu (2010, July). A load balancing algorithm for homogeneous multiprocessor system. In 2010 International Conference on Machine Learning and Cybernetics, Volume 3, pp. 1186-1190.

Taylor, S. J. and B. Letham (2017, Sep). Forecasting at scale.

Vig, A., R. S. Kushwah, R. S. Tomar, and S. S. Kushwah (2016, Dec). Autonomous agent based shortest path load balancing in cloud. In 2016 8th International Conference on Computational Intelligence and Communication Networks (CICN), pp. 33-37.

Villanueva, J. C. $(2015,6)$. Comparing load balancing algorithms.

Wang, R. (2011, Dec). Stock selection based on data clustering method. In 2011 Seventh International Conference on Computational Intelligence and Security, pp. 15421545.

Wikipedia contributors (2019a). Amazon web services - Wikipedia, the free encyclopedia. [Online; accessed 21-June-2019].

Wikipedia contributors (2019b). Machine learning — Wikipedia, the free encyclopedia. [Online; accessed 21-June-2019].

Wikipedia contributors (2020a). Decision tree learning — Wikipedia, the free encyclopedia. [Online; accessed 14-July-2020]. 
Wikipedia contributors (2020b). K-nearest neighbors algorithm - Wikipedia, the free encyclopedia. [Online; accessed 14-July-2020].

Wikipedia contributors (2020c). Logistic regression - Wikipedia, the free encyclopedia. [Online; accessed 14-July-2020].

Wikipedia contributors (2020d). Naive bayes classifier - Wikipedia, the free encyclopedia. [Online; accessed 14-July-2020].

Wikipedia contributors (2020e). Support vector machine - Wikipedia, the free encyclopedia. [Online; accessed 14-July-2020].

Wu, C. and S. Marotta (2013, June). Framework for assessing cloud trustworthiness. In 2013 IEEE Sixth International Conference on Cloud Computing, pp. 956-957.

Yu, X. and X. yu (2006). The research on an adaptive k-nearest neighbors classifier. In 2006 5th IEEE International Conference on Cognitive Informatics, Volume 1, pp. 535-540.

Zhou, Y. and J. Yan (2016). A logistic regression based approach for software test management. In 2016 International Conference on Cyber-Enabled Distributed Computing and Knowledge Discovery (CyberC), pp. 268-271. 
Appendices 


\section{Appendix A}

\section{APPENDIX A: SETTING UP THE ARCHITECTURE}

1.Install Ubuntu 18.04.3 LTS

2. Set up hardware

3. Check CPU Cores, RAM and memory using -grep -c ^processor /proc/cpu

4. Install Anaconda3

5. Install Visual studio code

\section{A.1 Stock Data Pre-processing script}

The stock data entry is extracted, processed and stored every day using the following script-

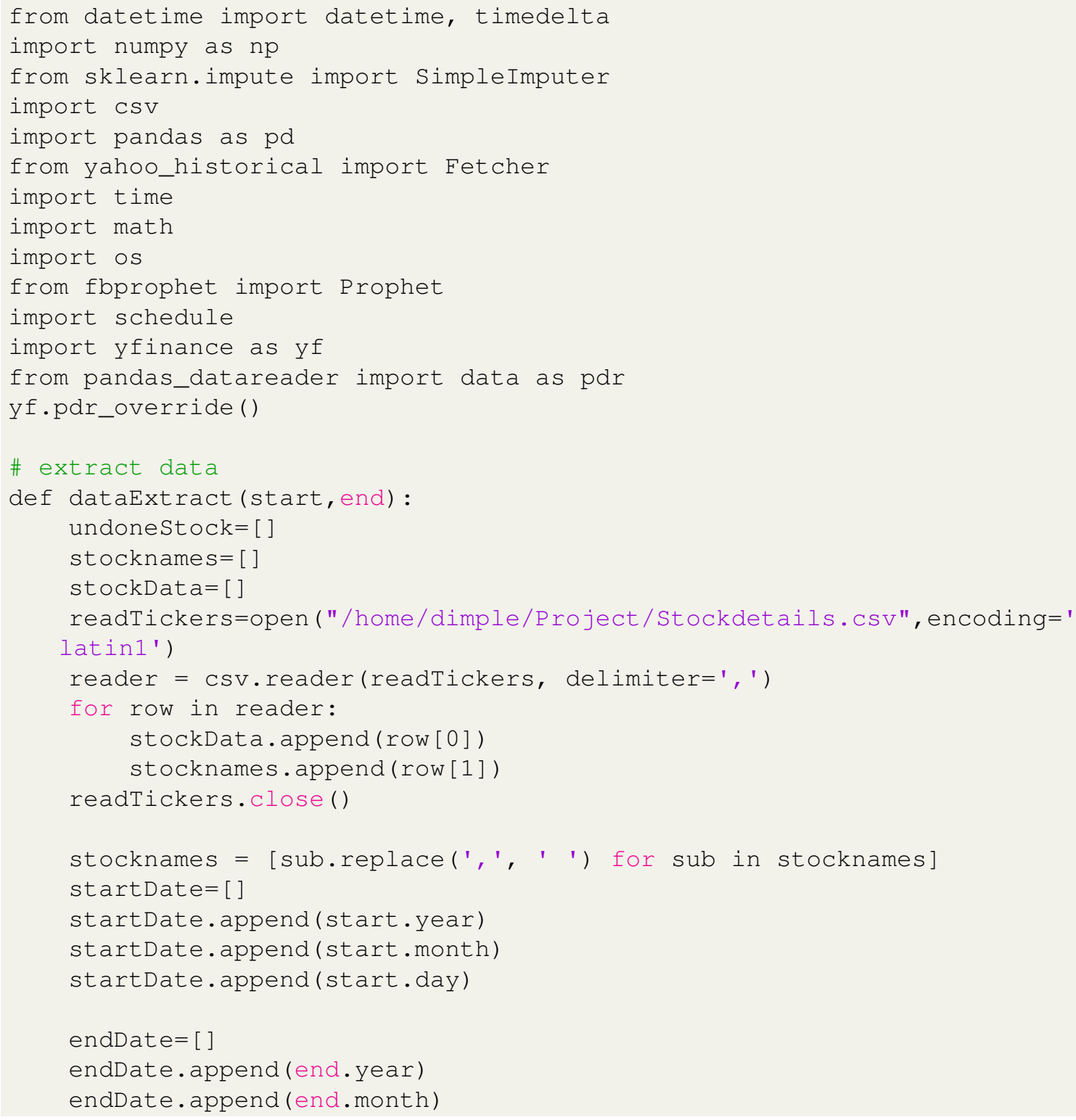




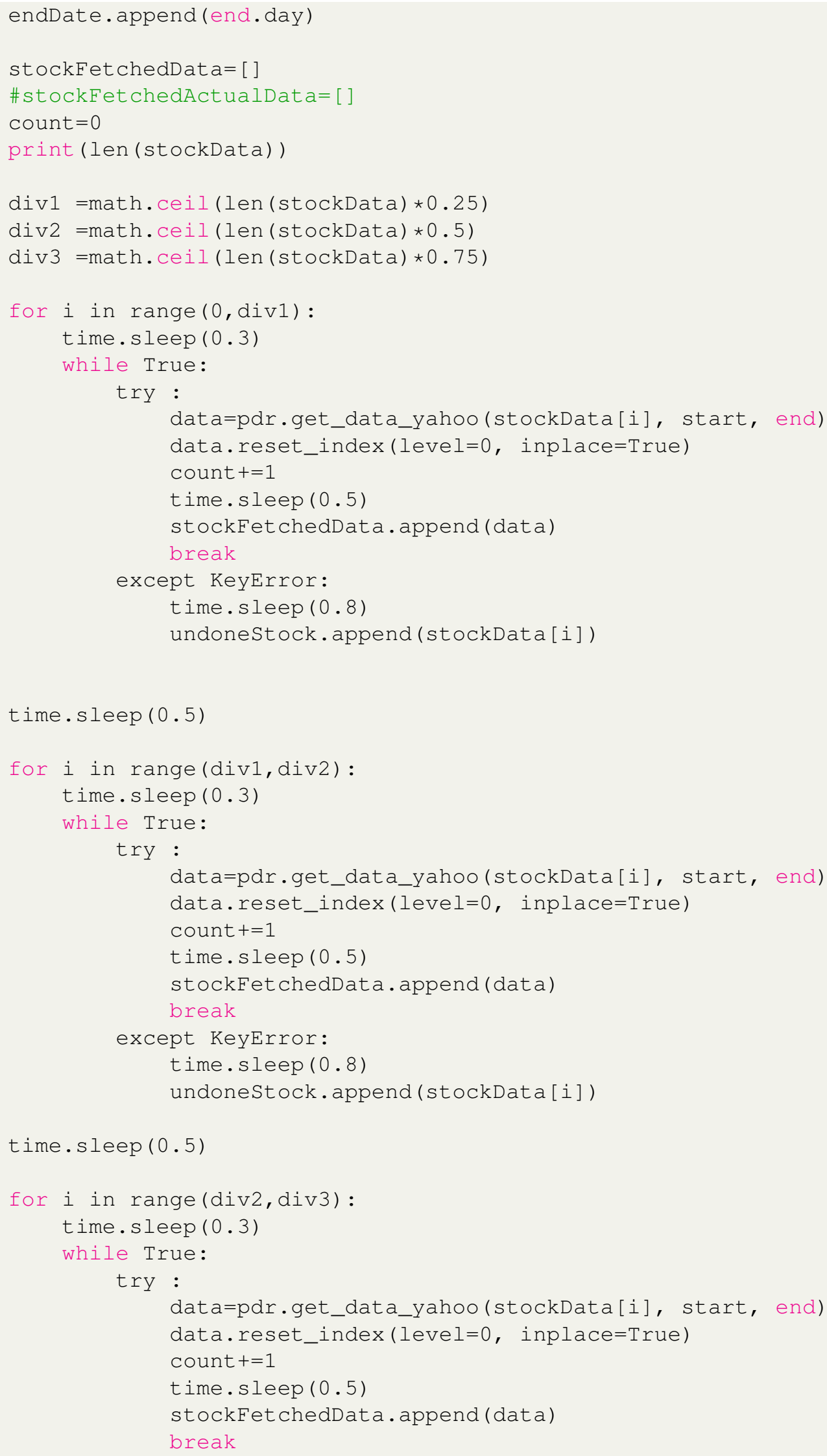




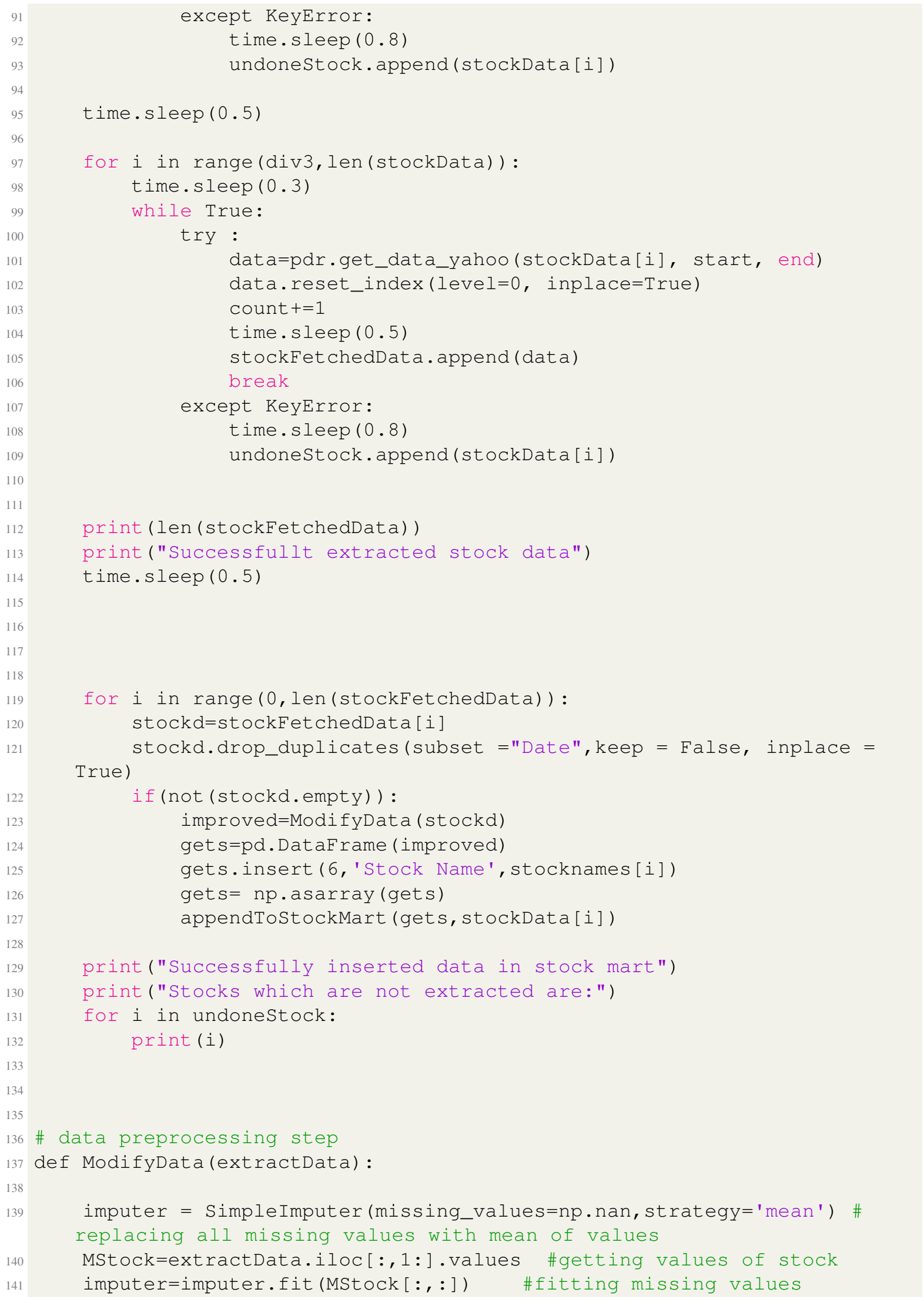




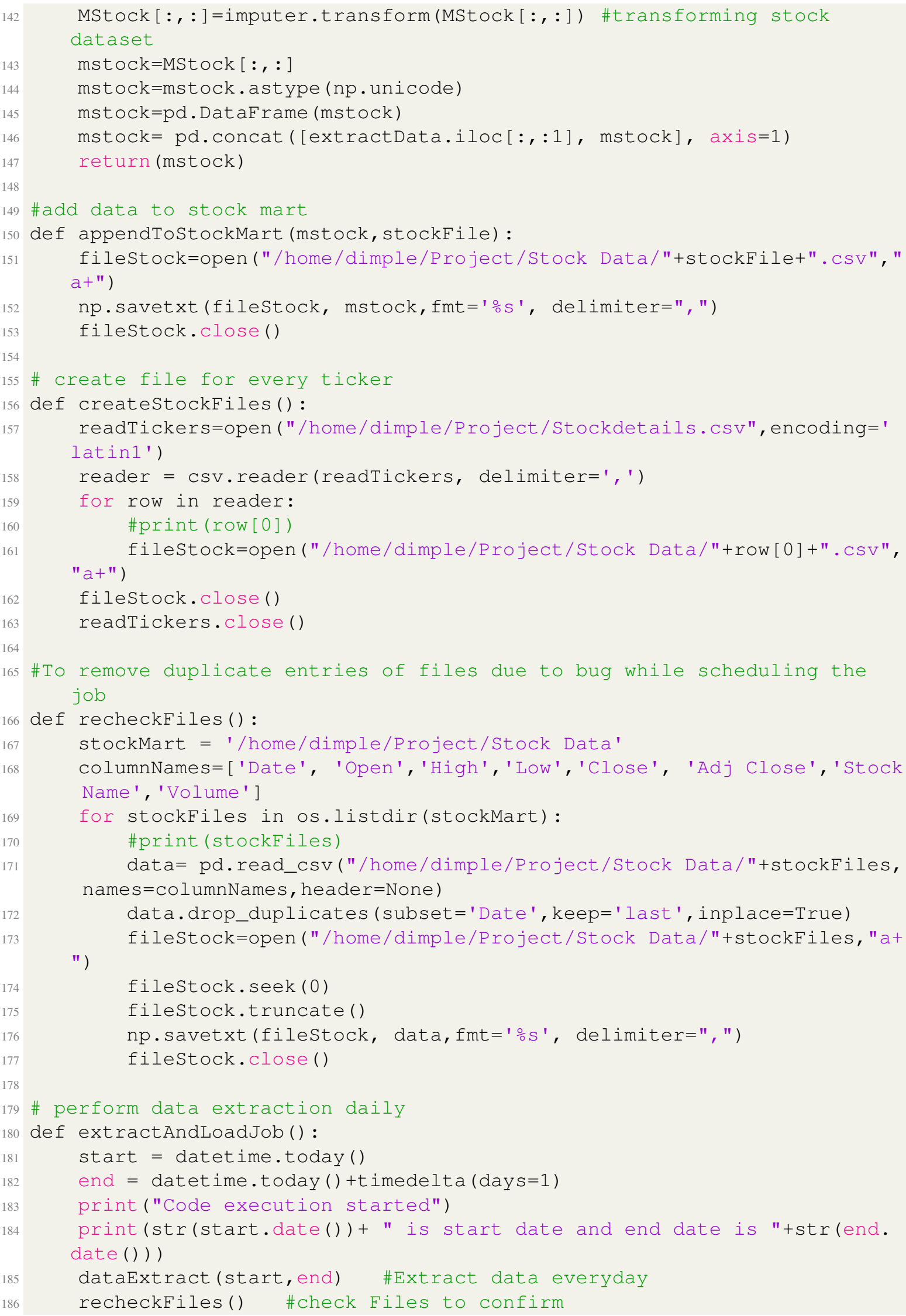




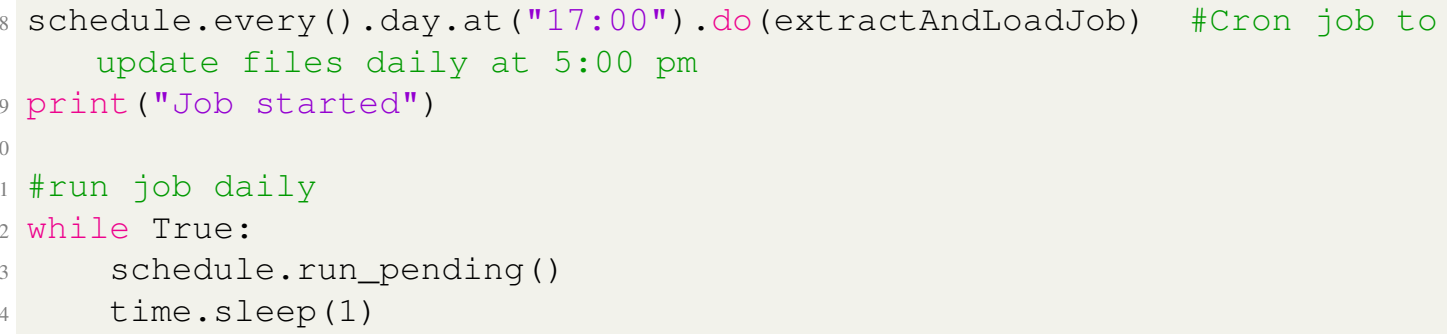

Code A.1.0.1: Data preprocessing script 


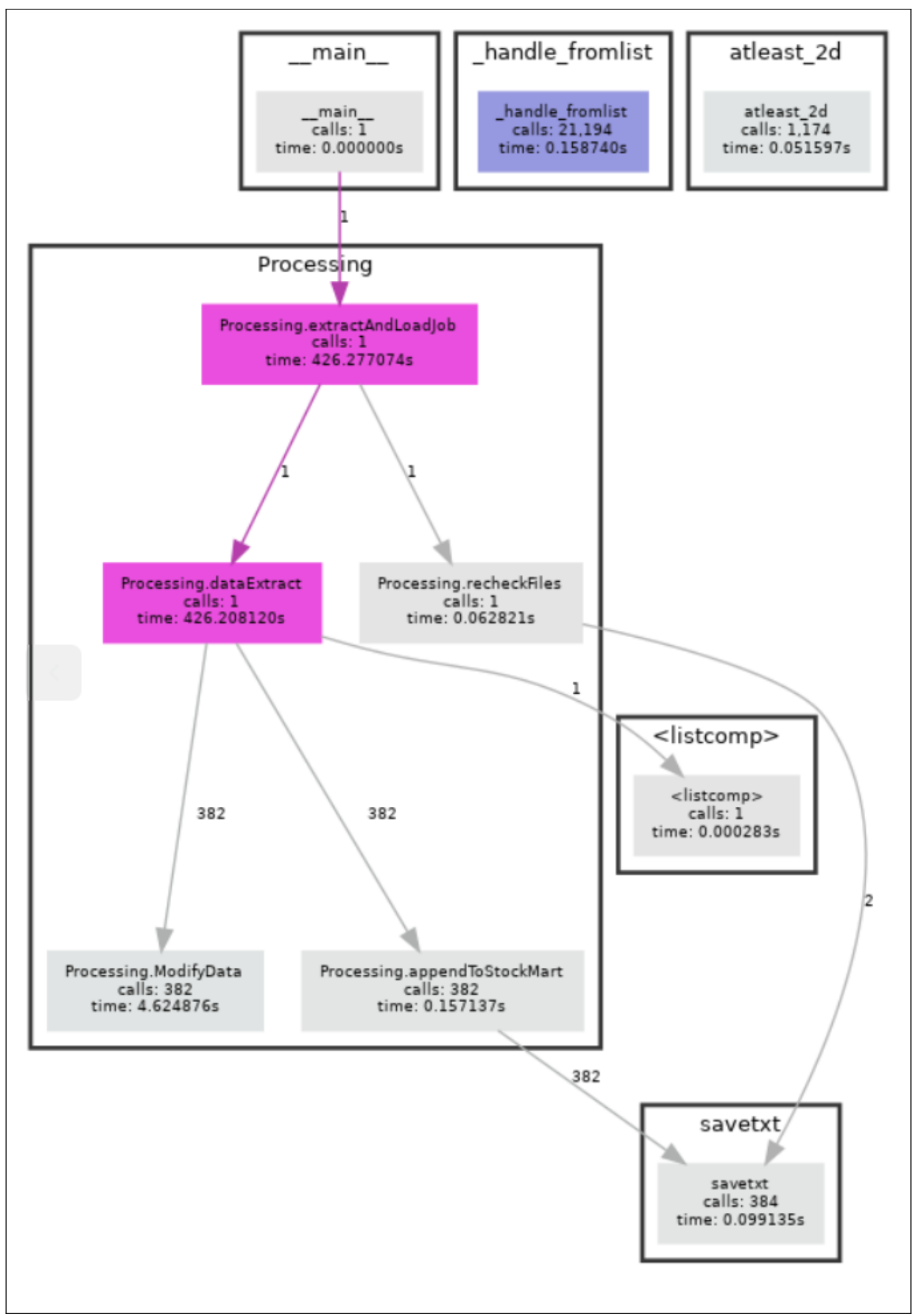

Figure A.1: Work flow of methods called in Data preprocessing script

\section{A.2 Web Application Design}

1. Create virtual environment using- python $-\mathrm{m}$ virtualenv - python=/usr/bin/python3.6 env

2. Install Django 3.0.2 using pip install Django==3.0.3 
3. Create project Finance using- django-admin startproject Finance

4. Create an app finWeb in project using- python manage.py startapp finWeb

5. Create a file urls.py in finWeb app

6. Add app finWeb in settings.py of Finance project by adding line 'finWeb.apps.FinwebConfig' in INSTALLED_APPS

7. Make migrations in database by given below commandsPython manage.py makemigrations finWeb python manage.py sqlmigrate finWeb 0001

8. Install all libraries required for development by executing- pip3 install -r requirements.txt

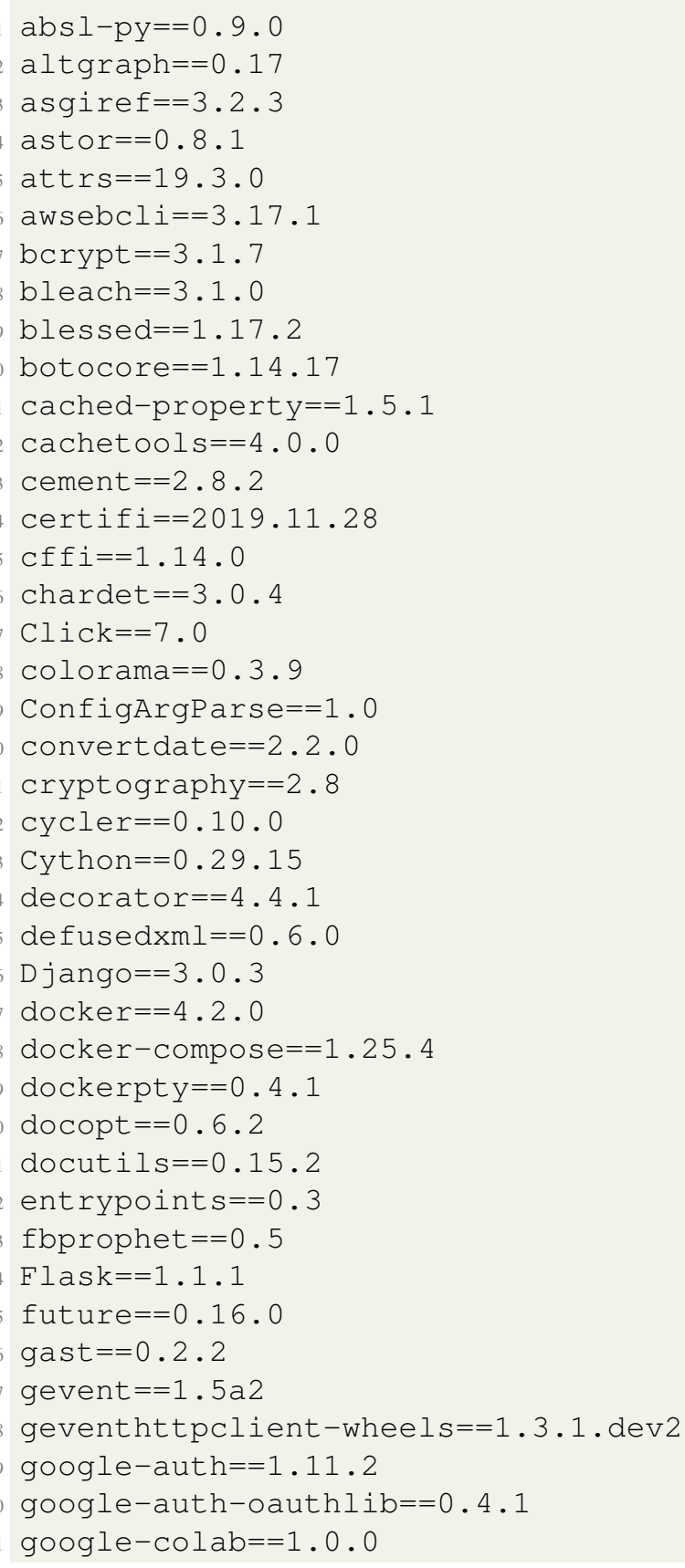




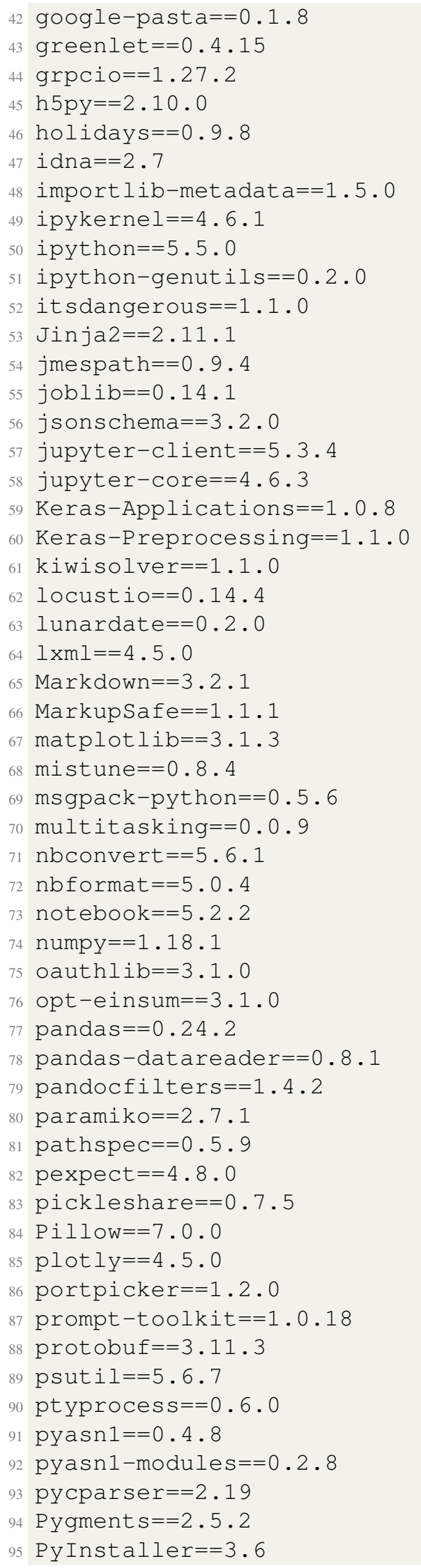




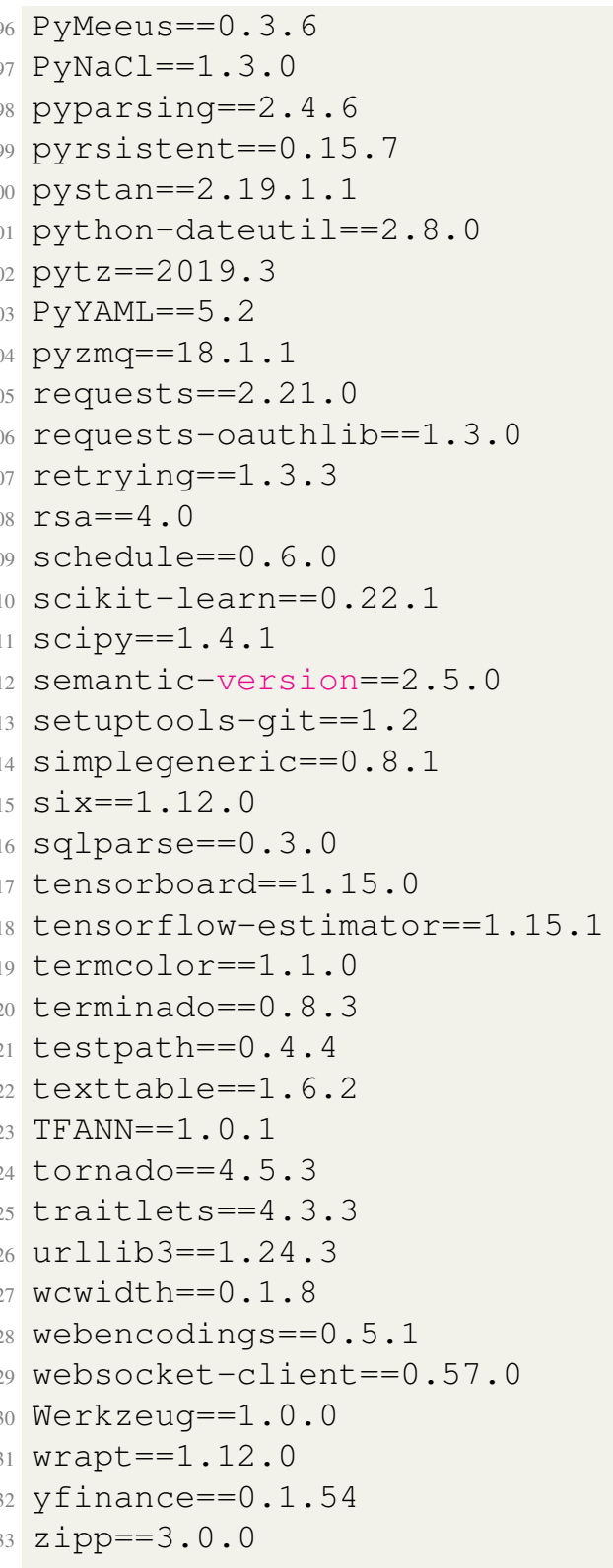

Code A.2.0.1: requirements.txt 


\section{A.2.1 Directory Tree Structure for Web Application}

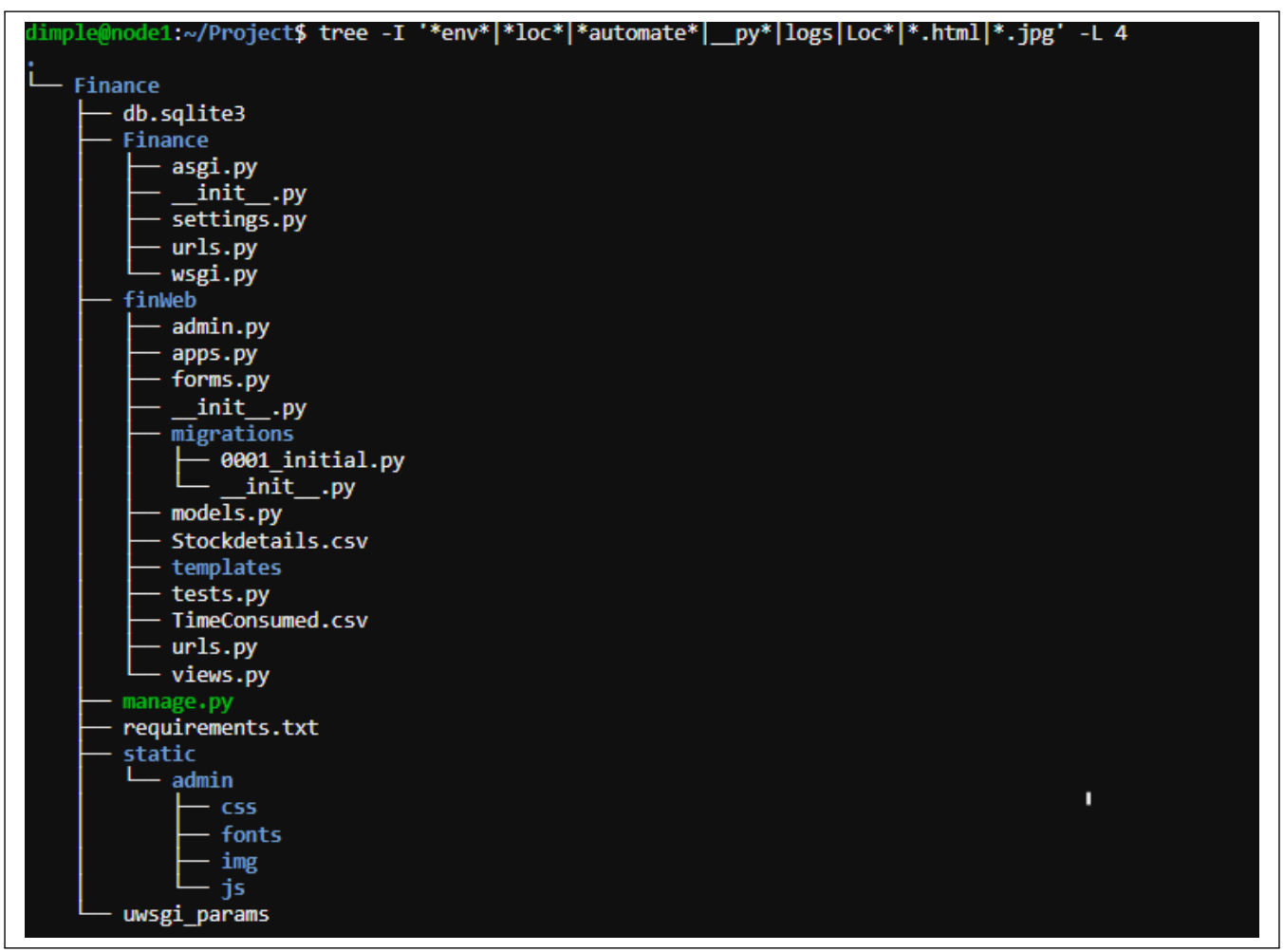

Figure A.2: Directory Tree Structure for Web Application

\section{A.2.2 Files in project Finance}

There are 5 files in Finance folder of project- asgi.py, settings.py, urls.py, wsgi.py and init.py, which is empty file.

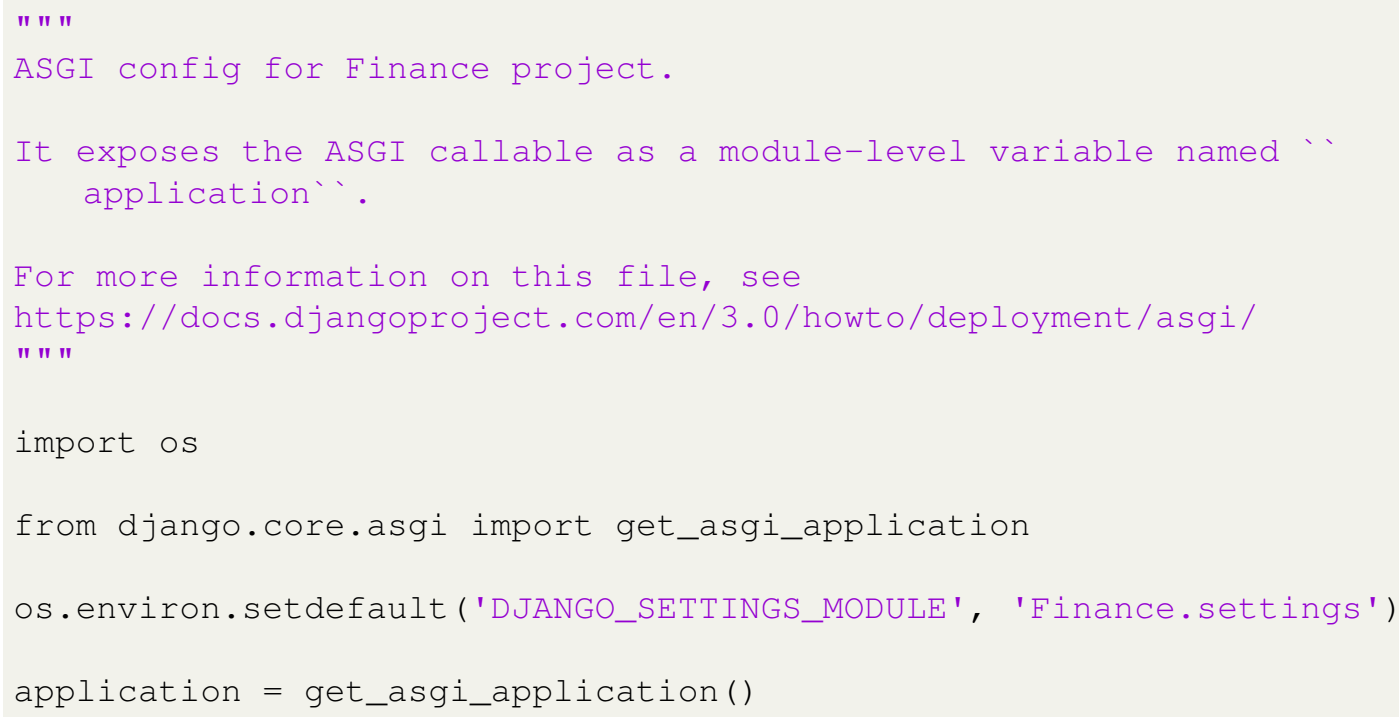

Code A.2.2.1: asgi.py 


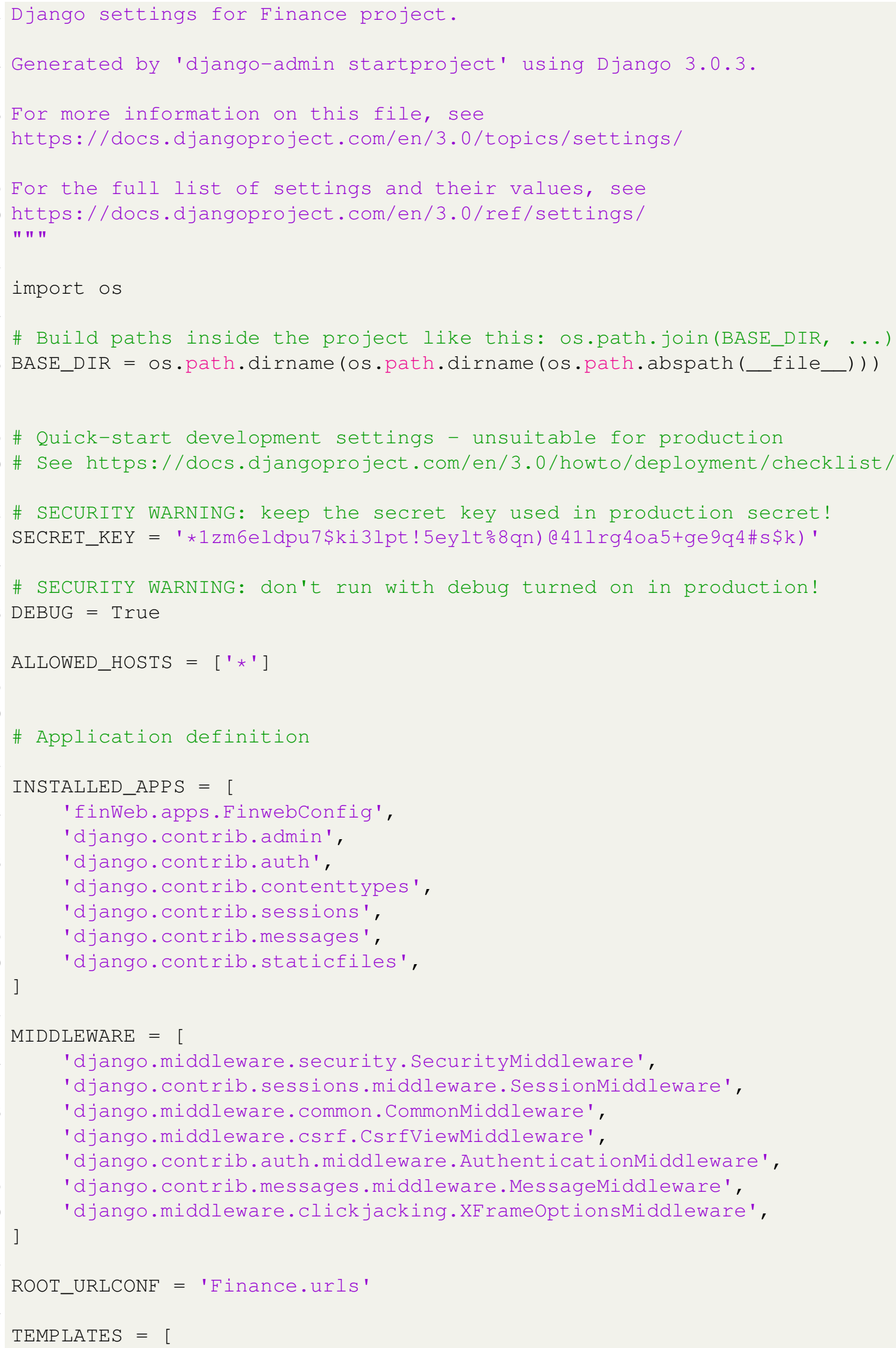




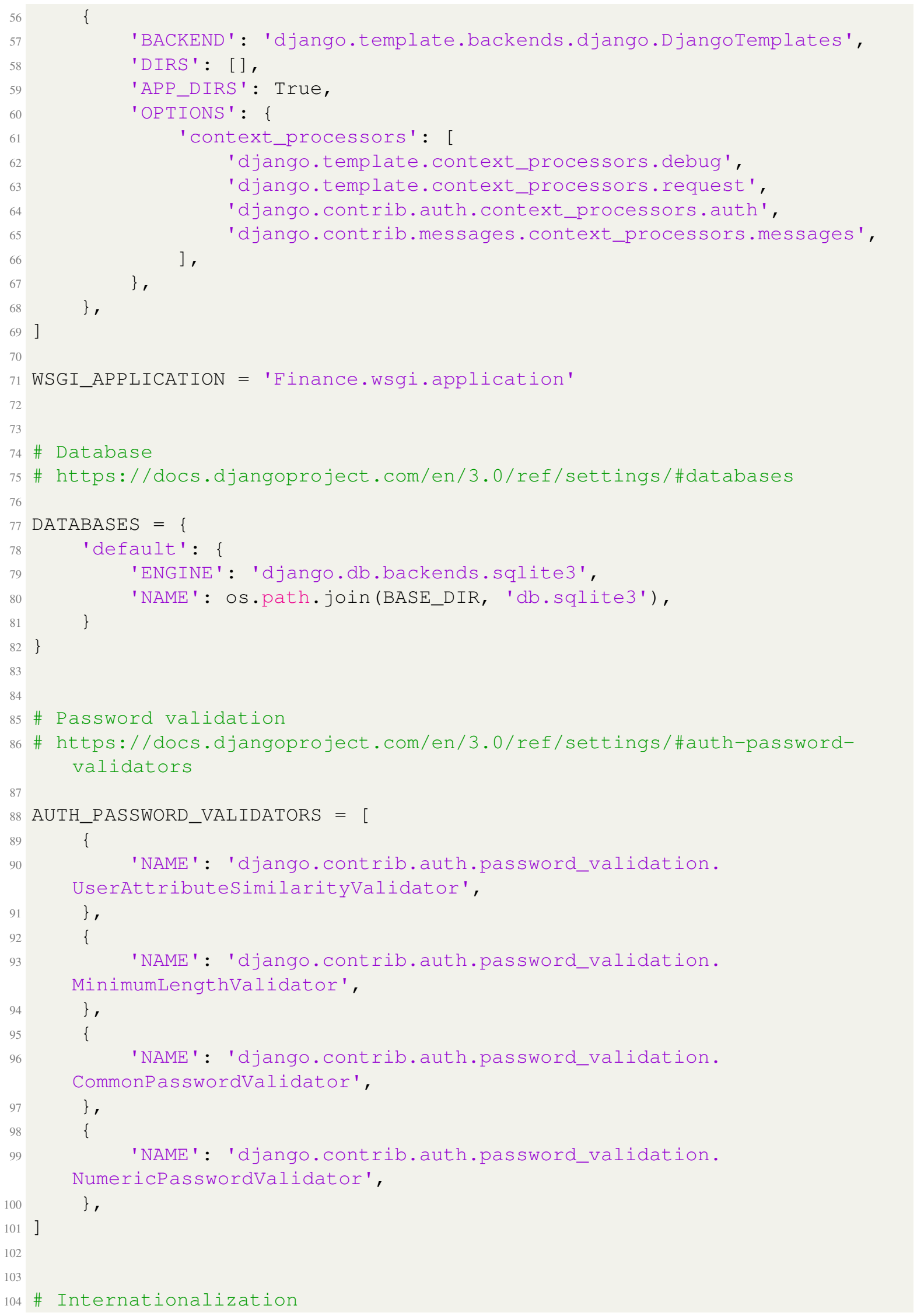




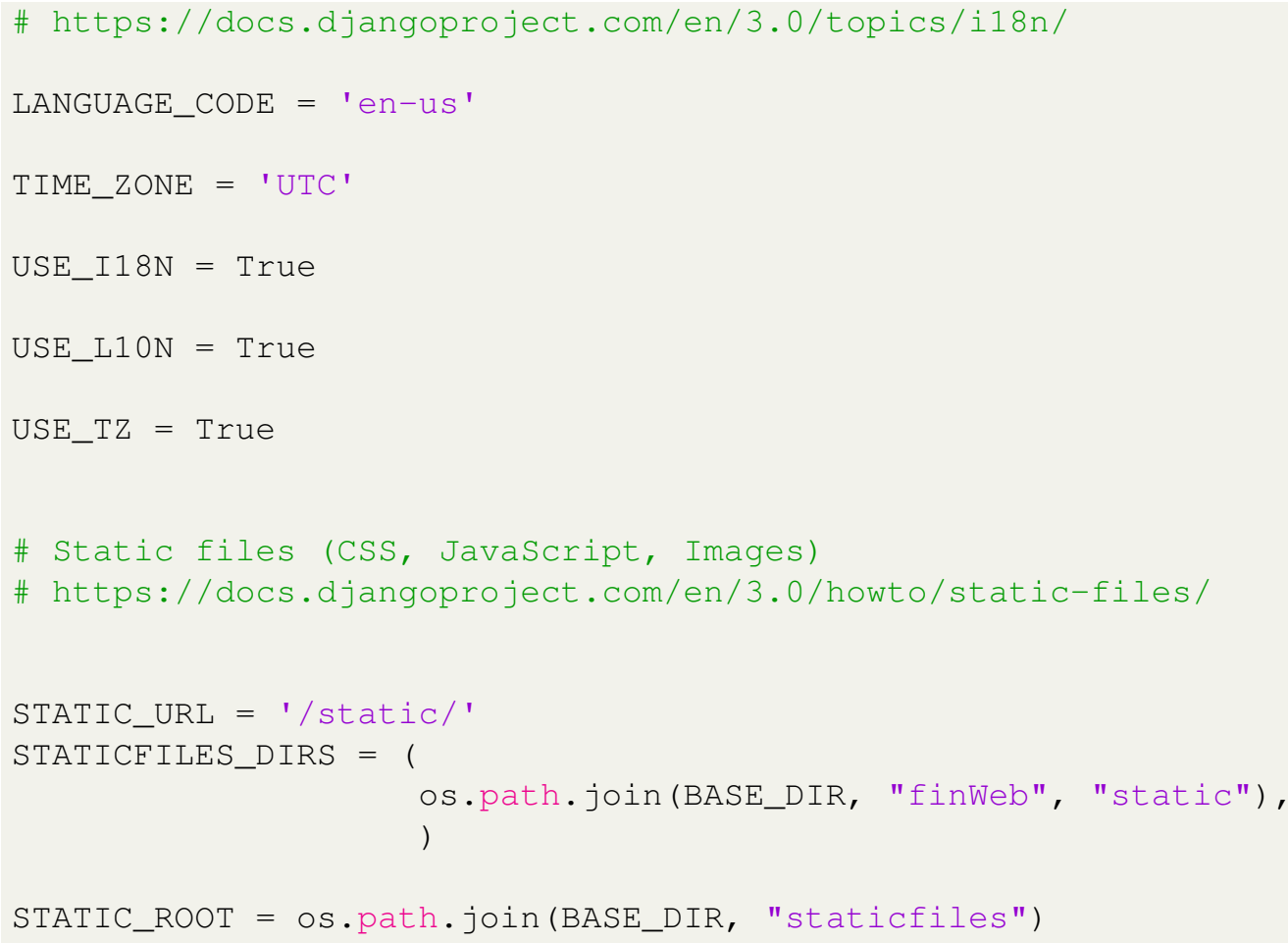

\section{Code A.2.2.2: settings.py}

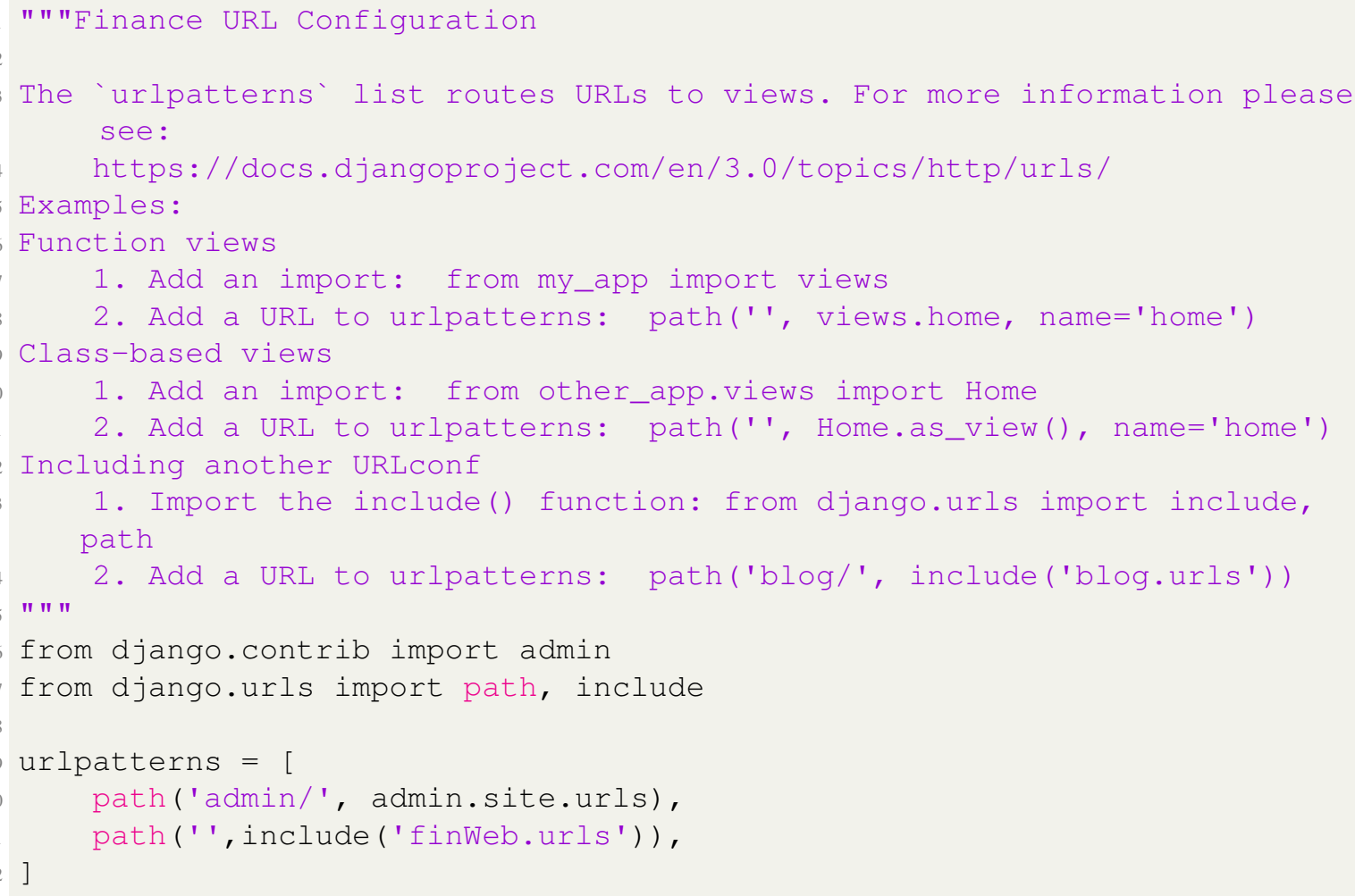

\section{Code A.2.2.3: urls.py}




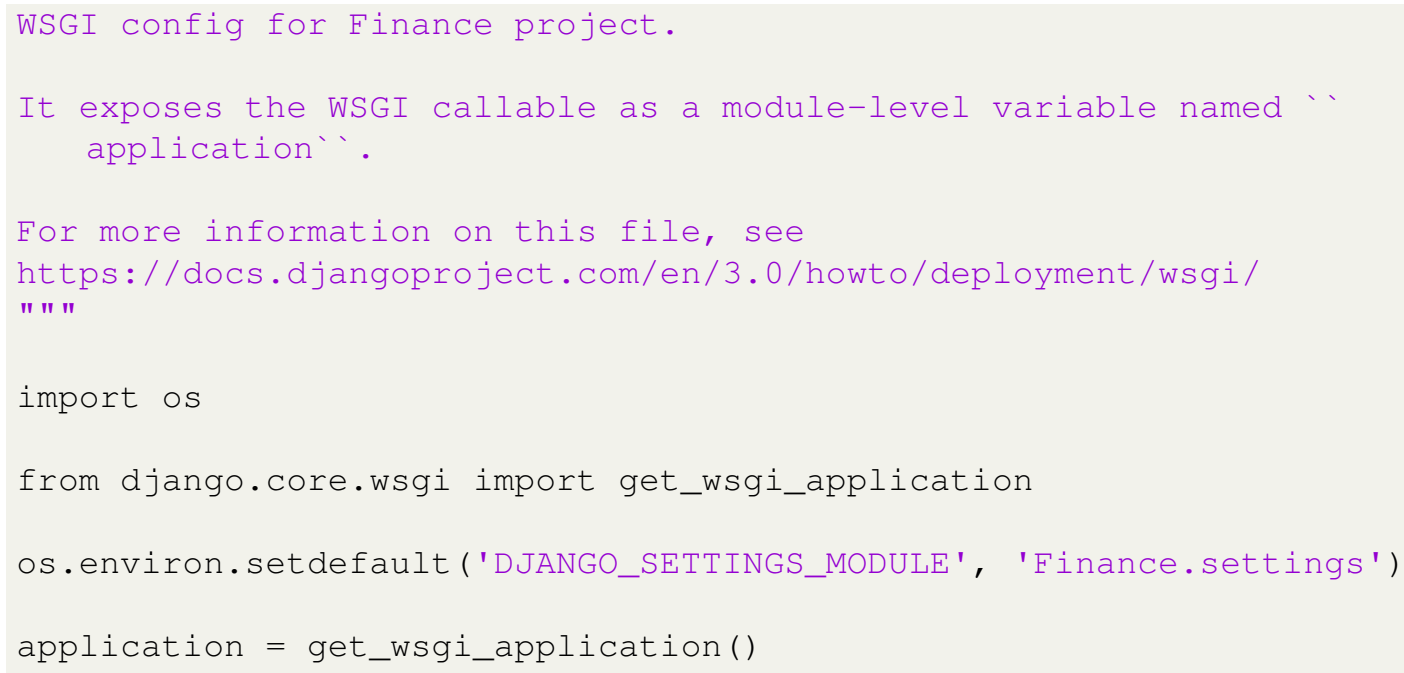

Code A.2.2.4: wsgi.py

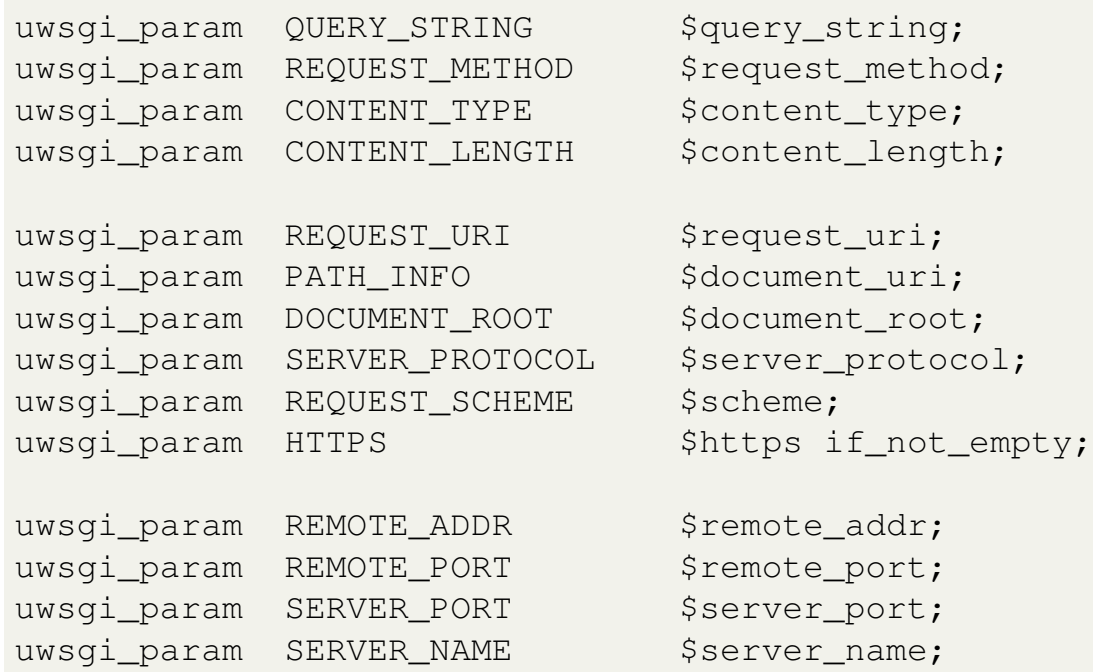

Code A.2.2.5: uwsgi_params

\section{A.2.3 Files in finWeb app}

The application consist of mainly six files - admin.py, apps.py, forms.py, models.py, urls.py, views.py and init.py, which is an empty file used to initialize attribute of class. All HTML pages are created in a separate folder called templates in Django.

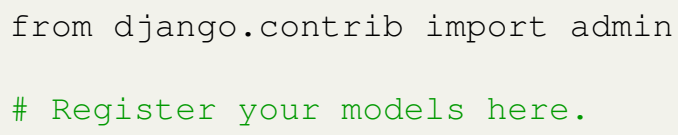

\section{Code A.2.3.1: admin.py}

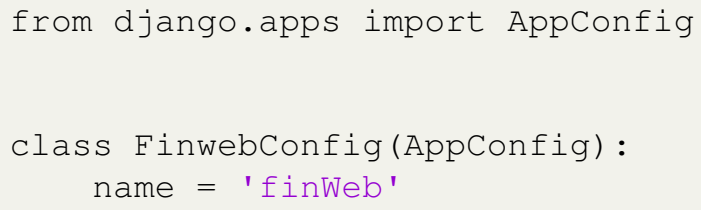




\section{Code A.2.3.2: apps.py}

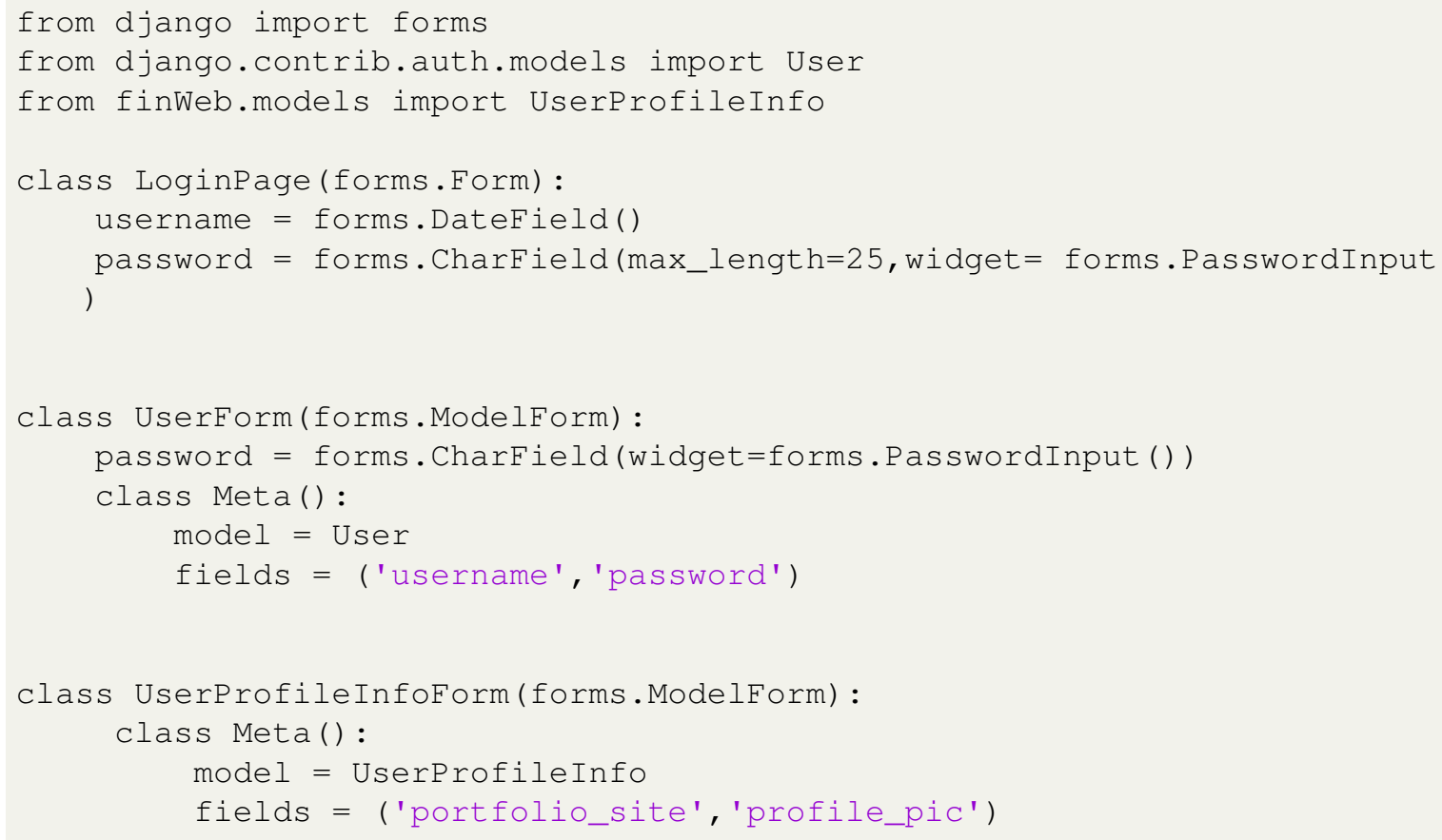

\section{Code A.2.3.3: forms.py}

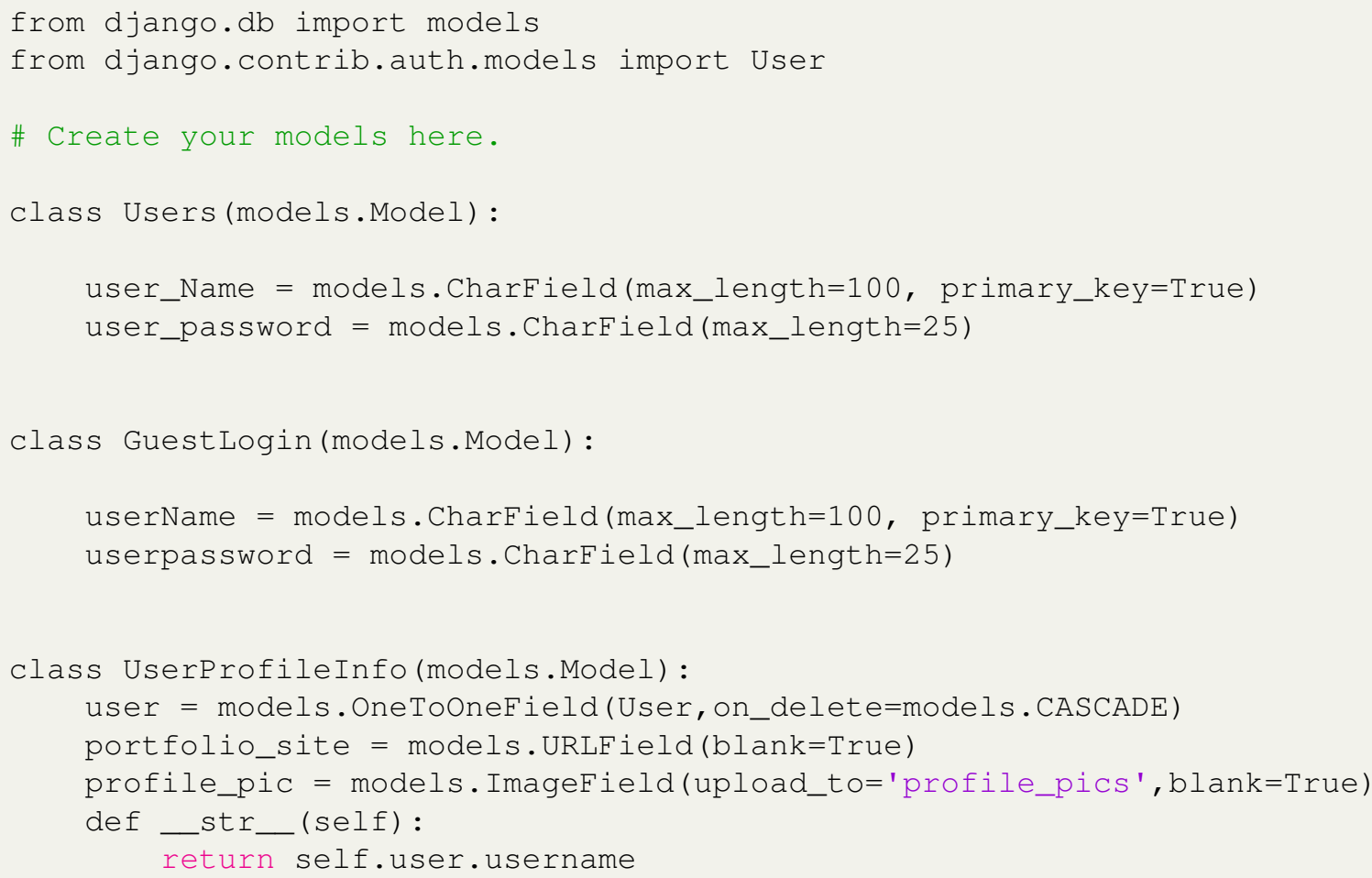

Code A.2.3.4: models.py 


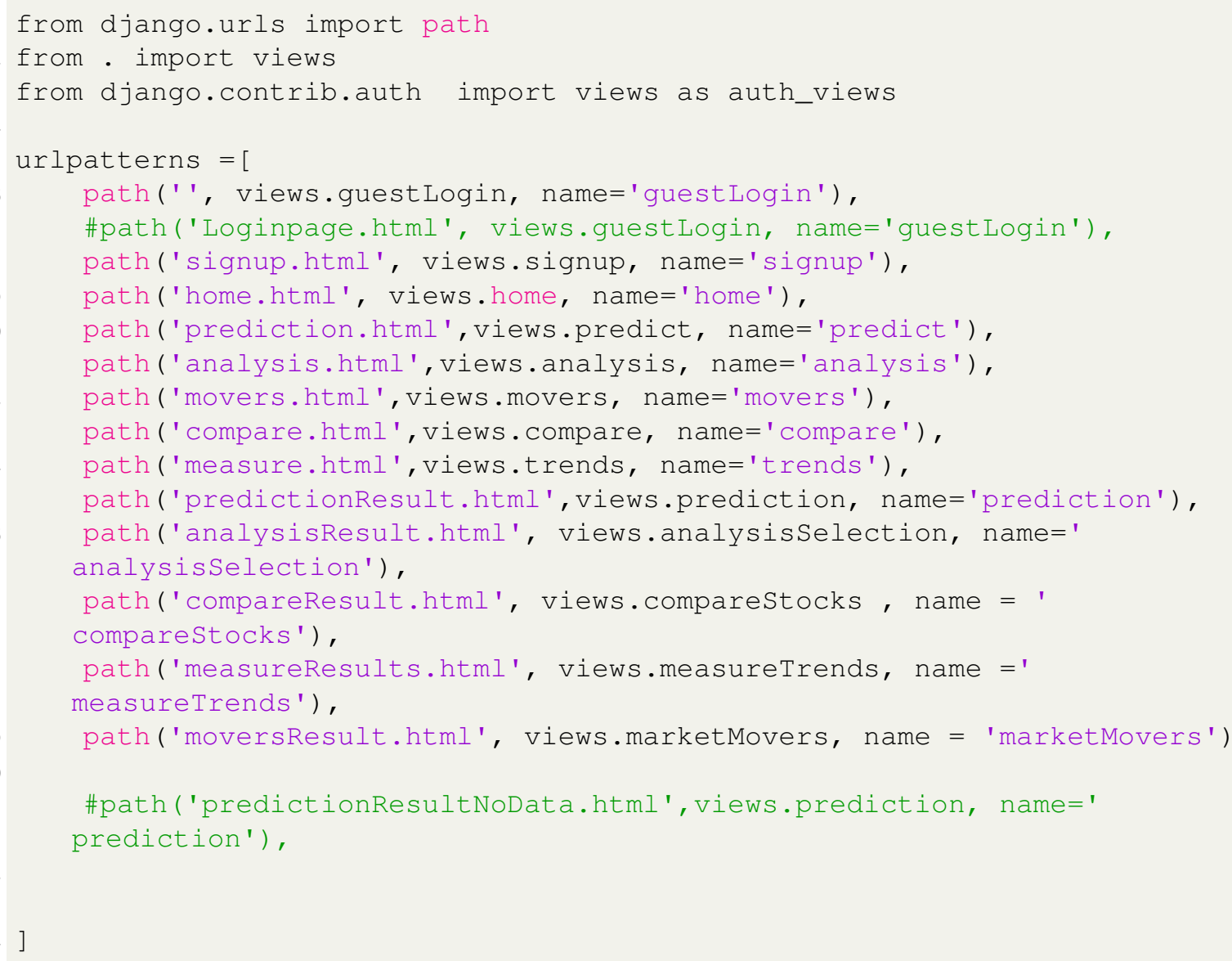

\section{Code A.2.3.5: urls.py}

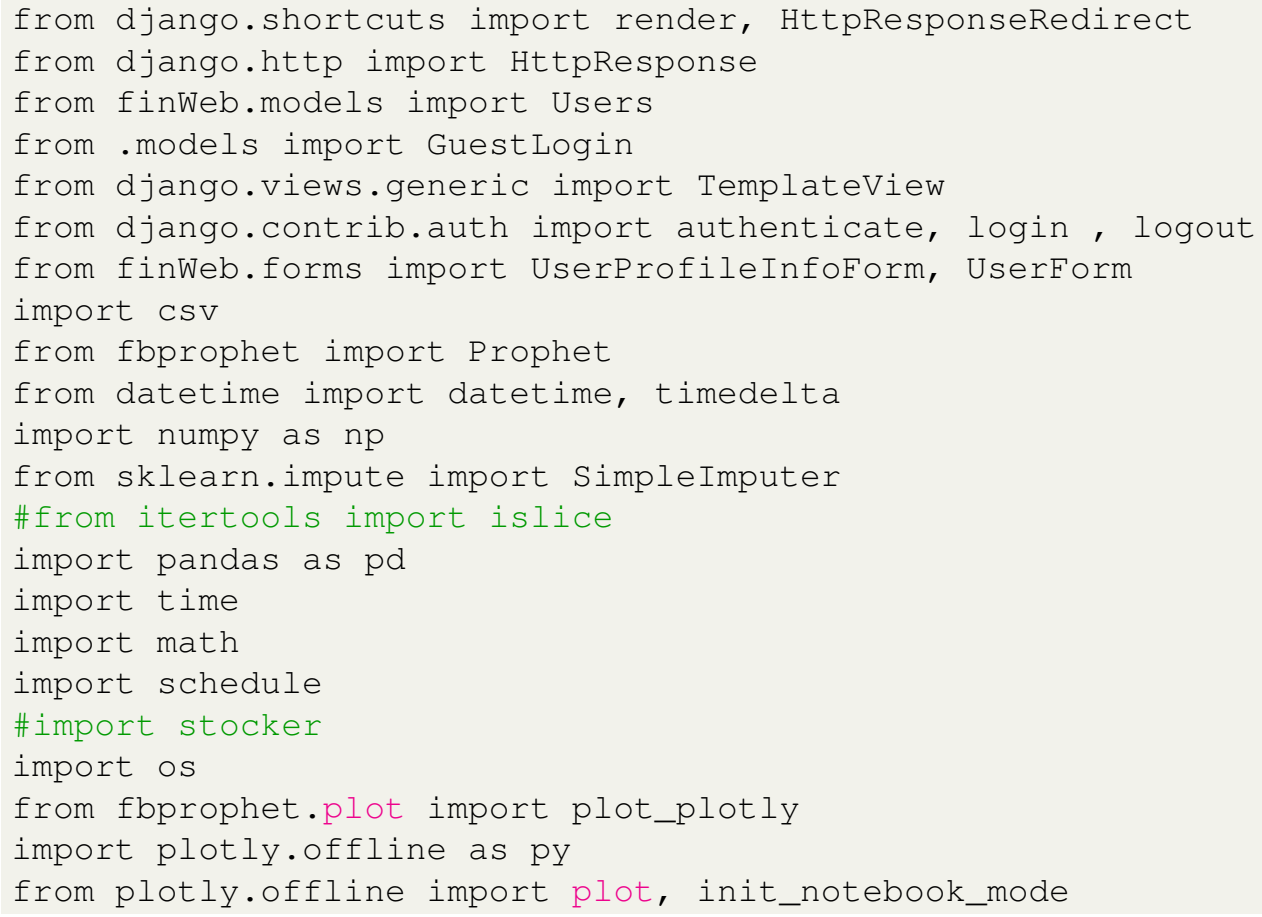




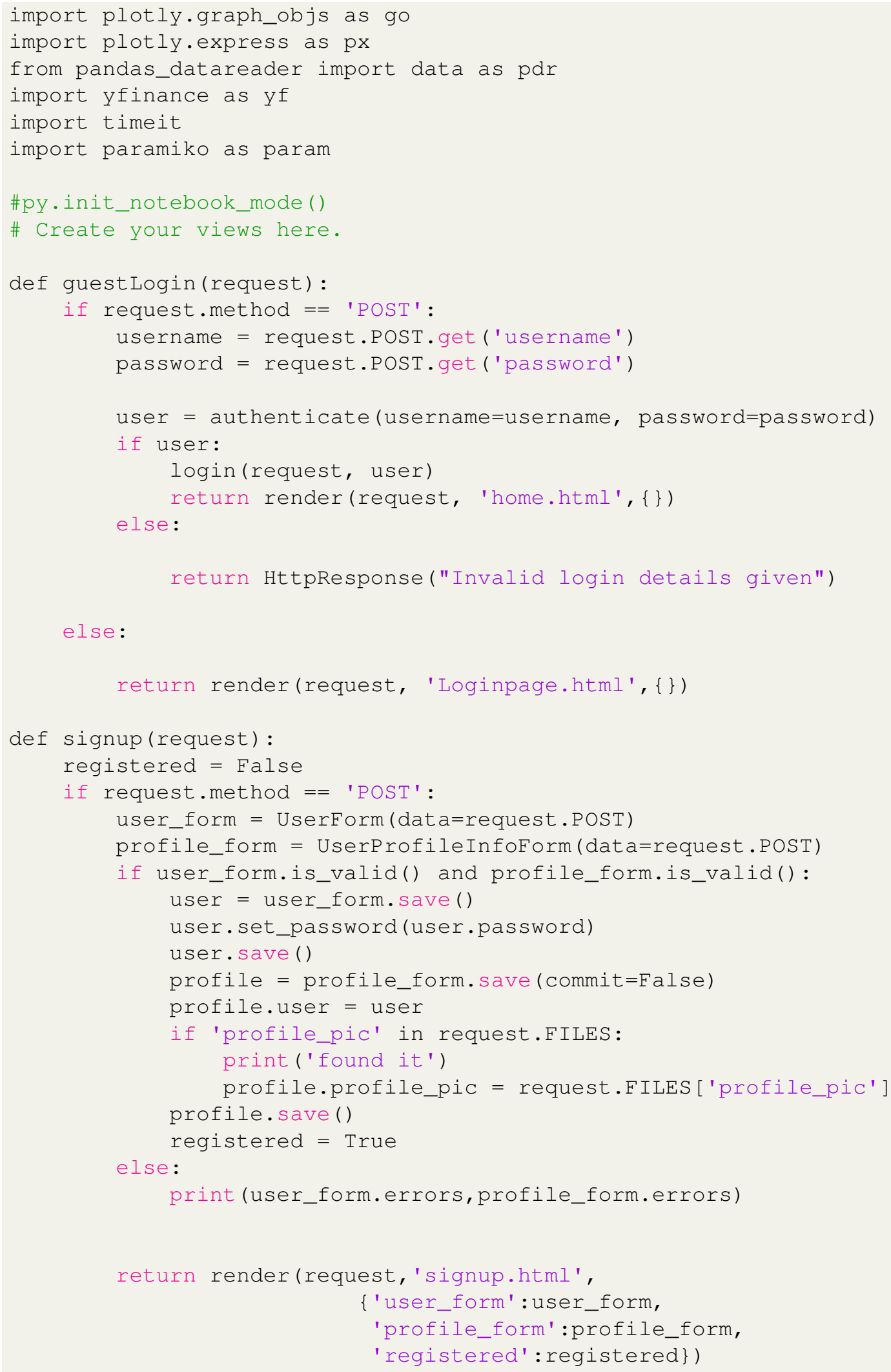




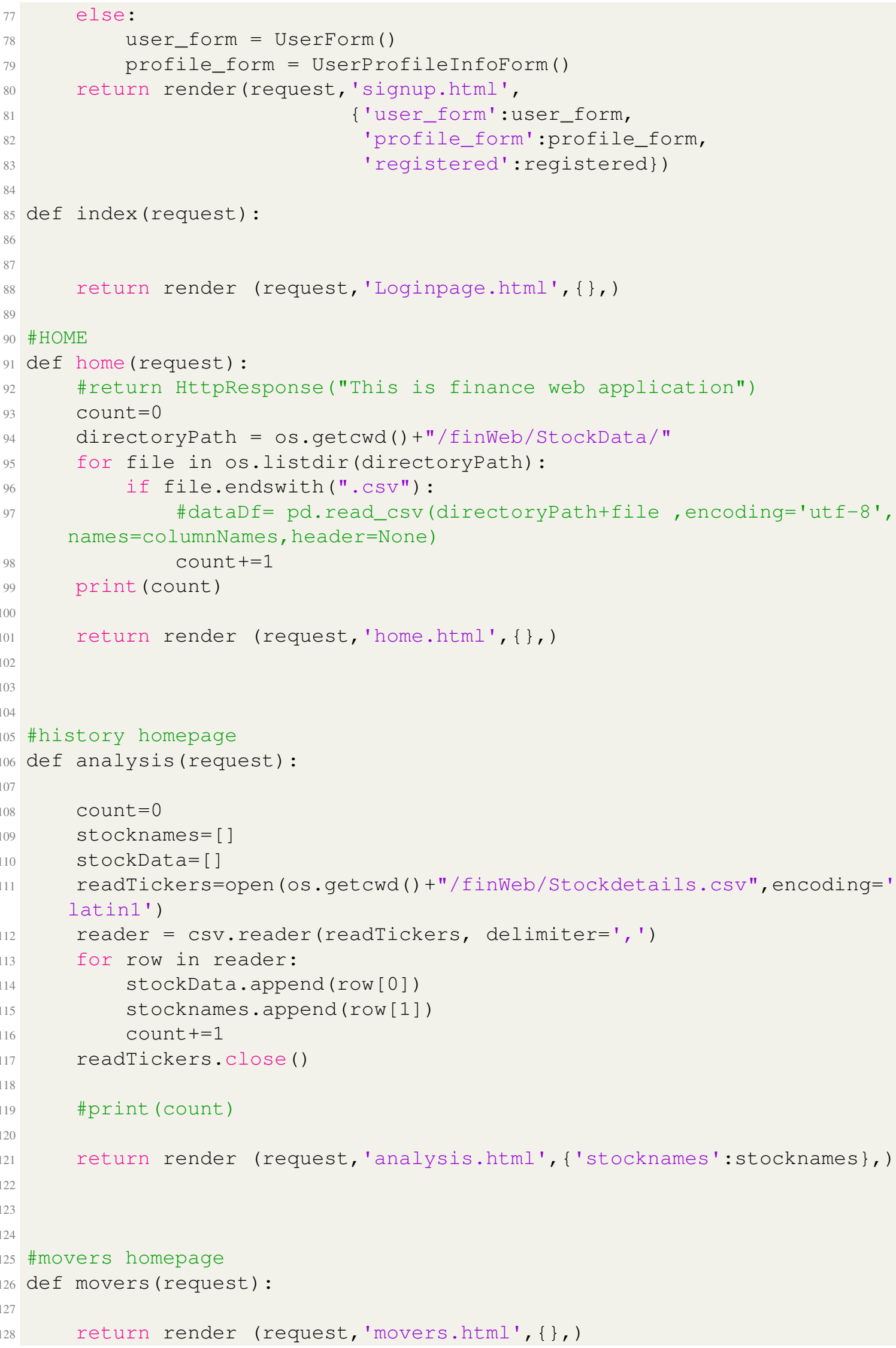




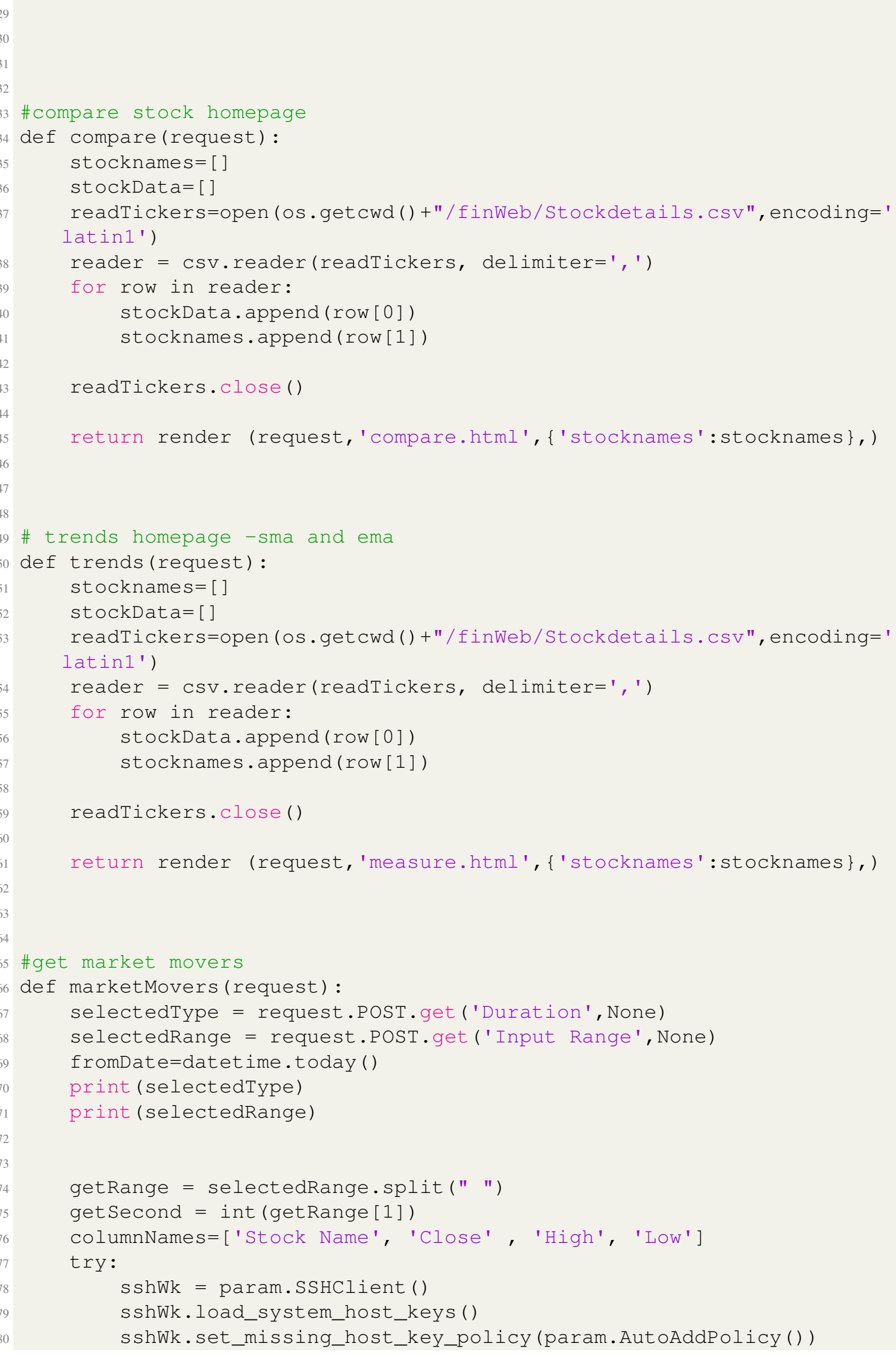




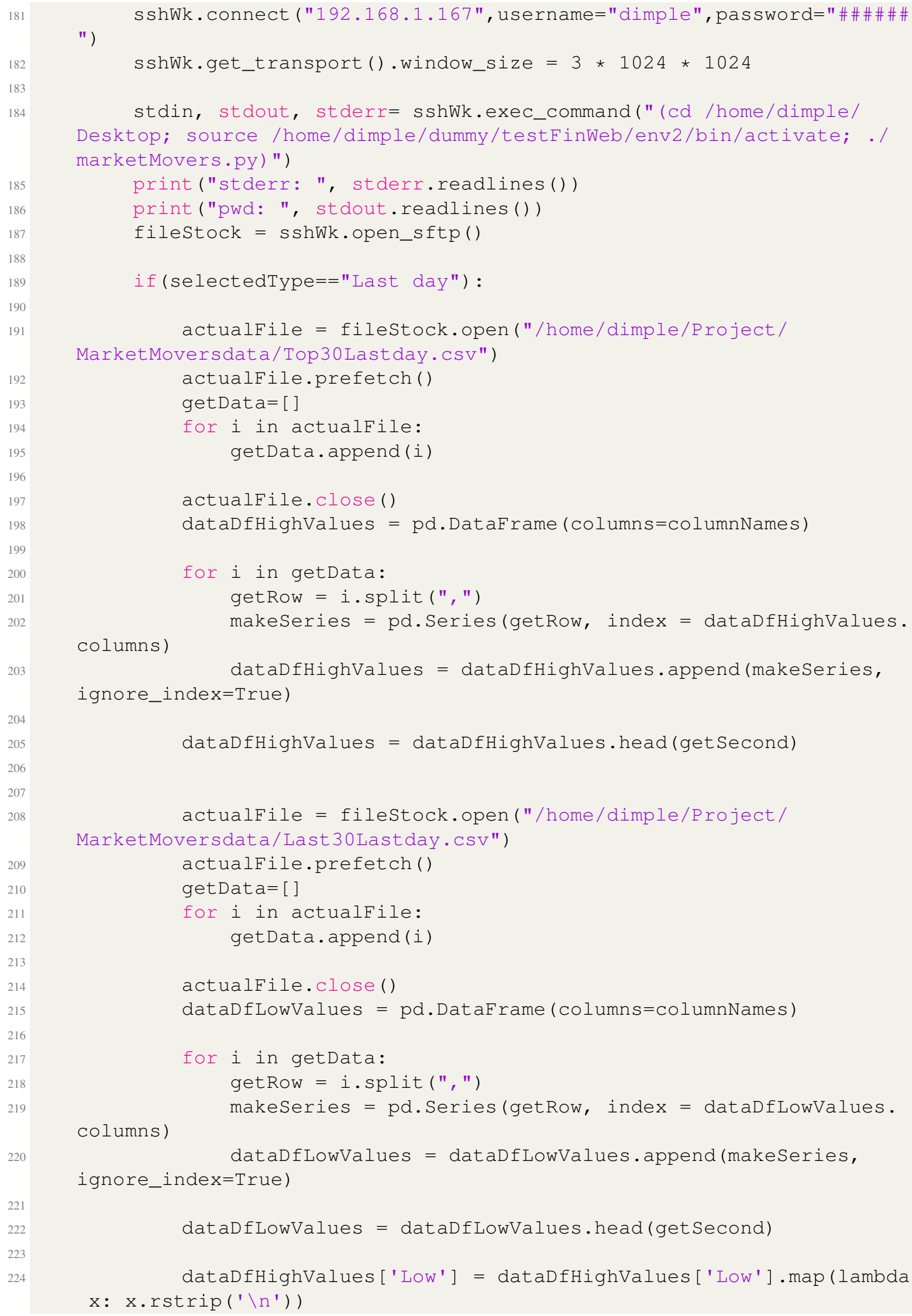




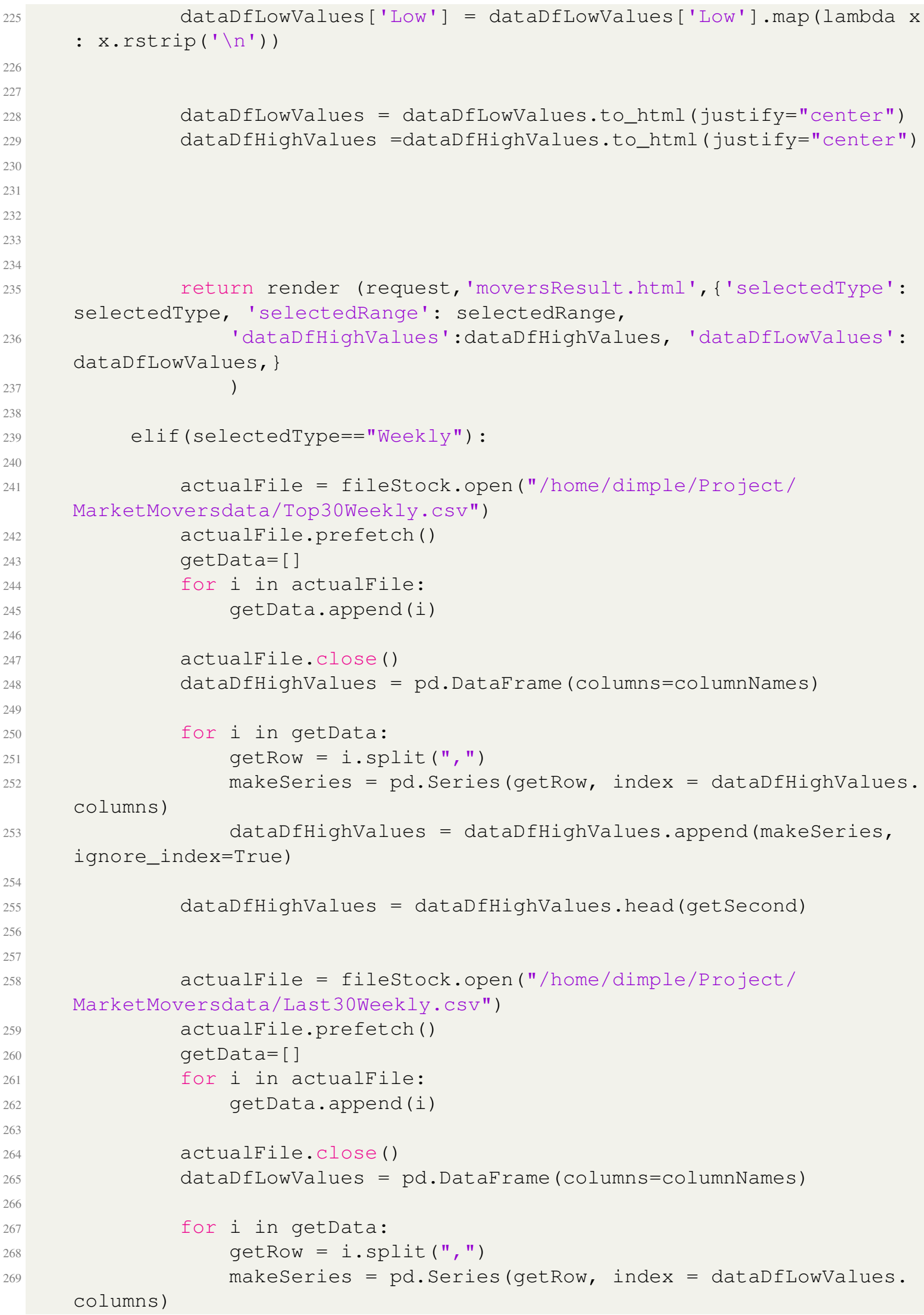




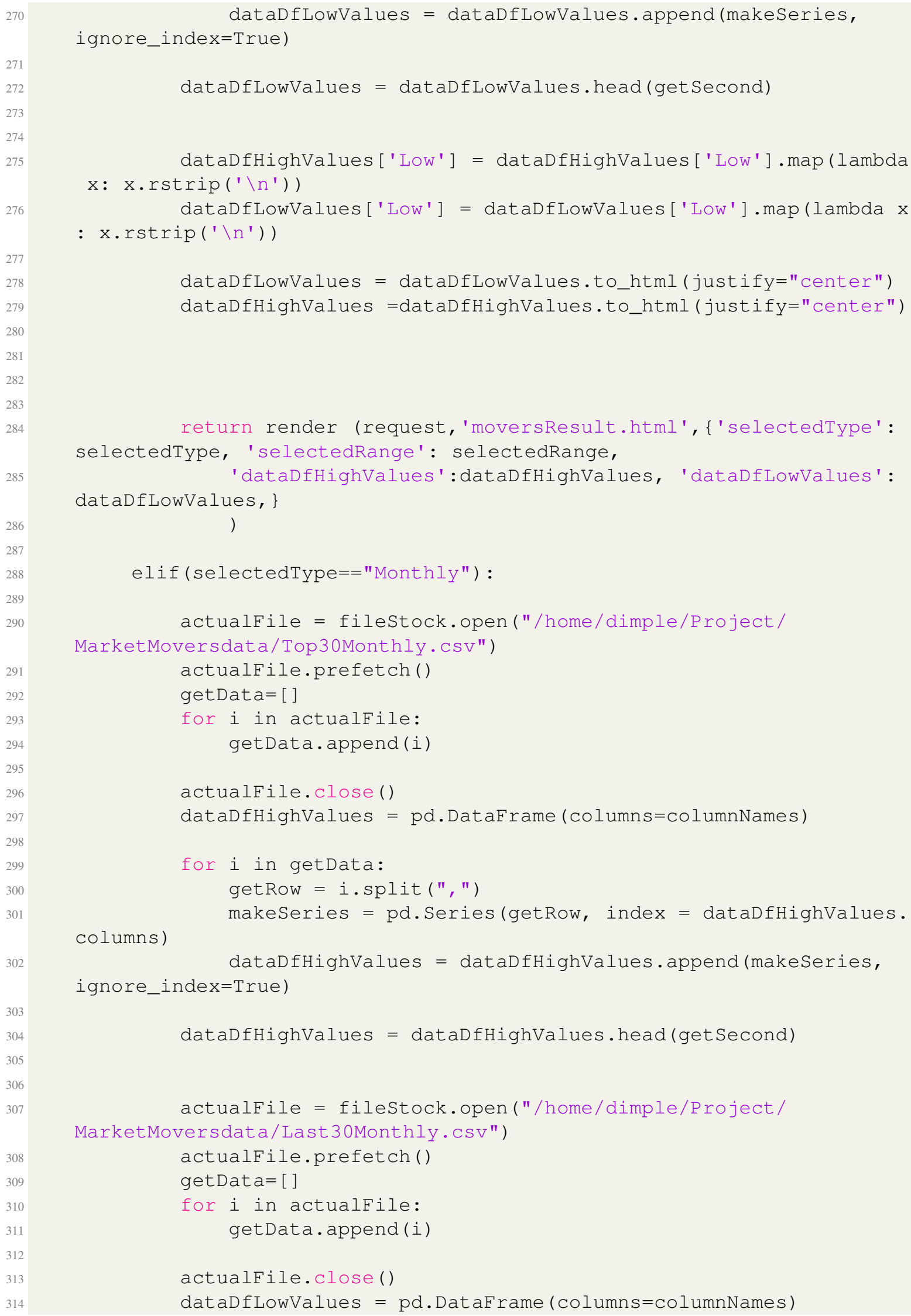




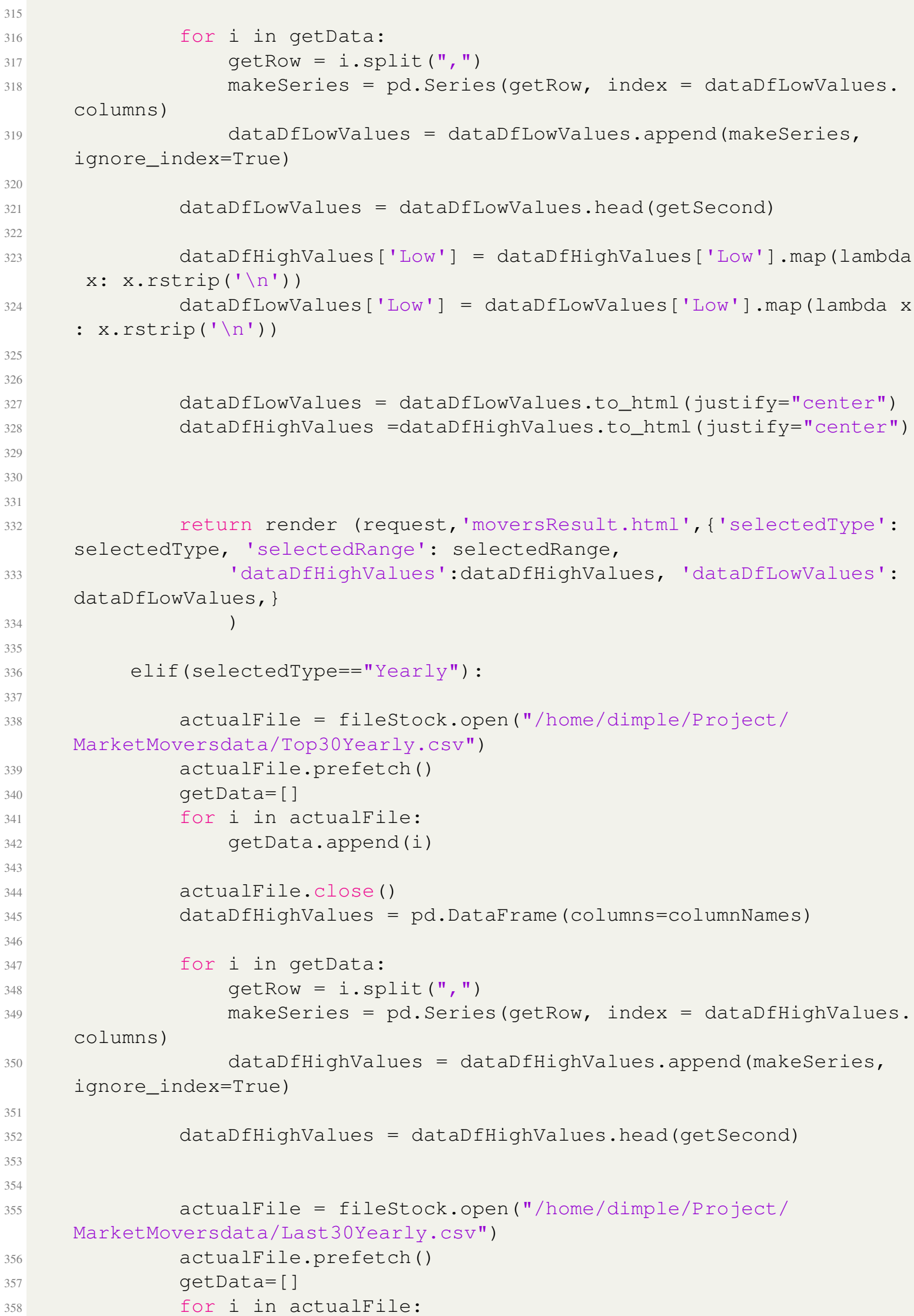

\section{for $i$ in getData:} getRow = i.split (",")

columns) makeSeries $=$ pd.Series (getRow, index = dataDfLowValues. ignore_index=True)

dataDfLowValues = dataDfLowValues.head(get.Second)

dataDfHighValues['Low'] = dataDfHighValues['Low'].map (lambda $x: x \cdot r s t r i p(' \backslash n '))$

dataDfLowValues ['Low'] = dataDfLowValues['Low'].map (lambda $x$ : $x$.rstrip (' $\left.\backslash \mathrm{n}^{\prime}\right)$ ) 


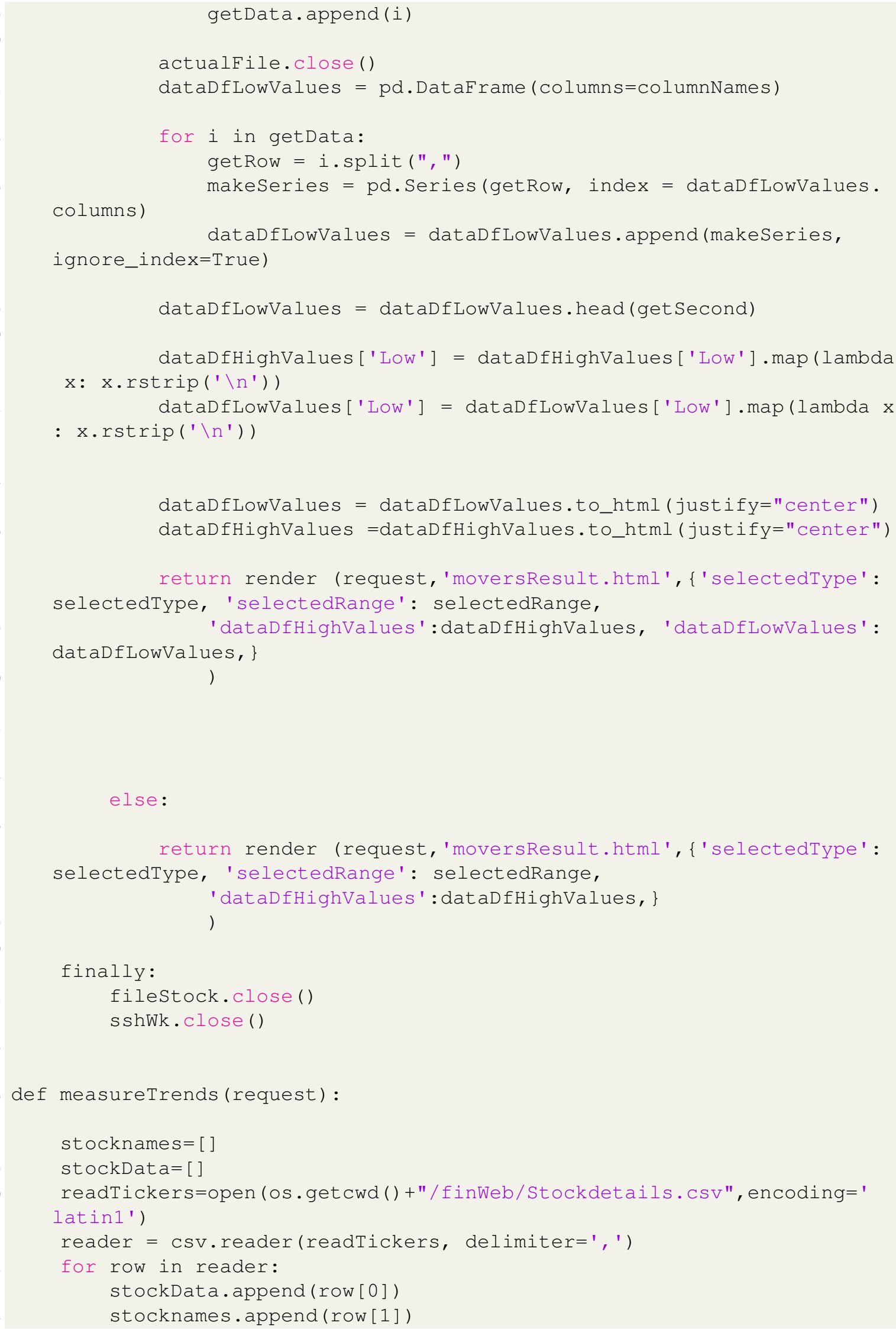




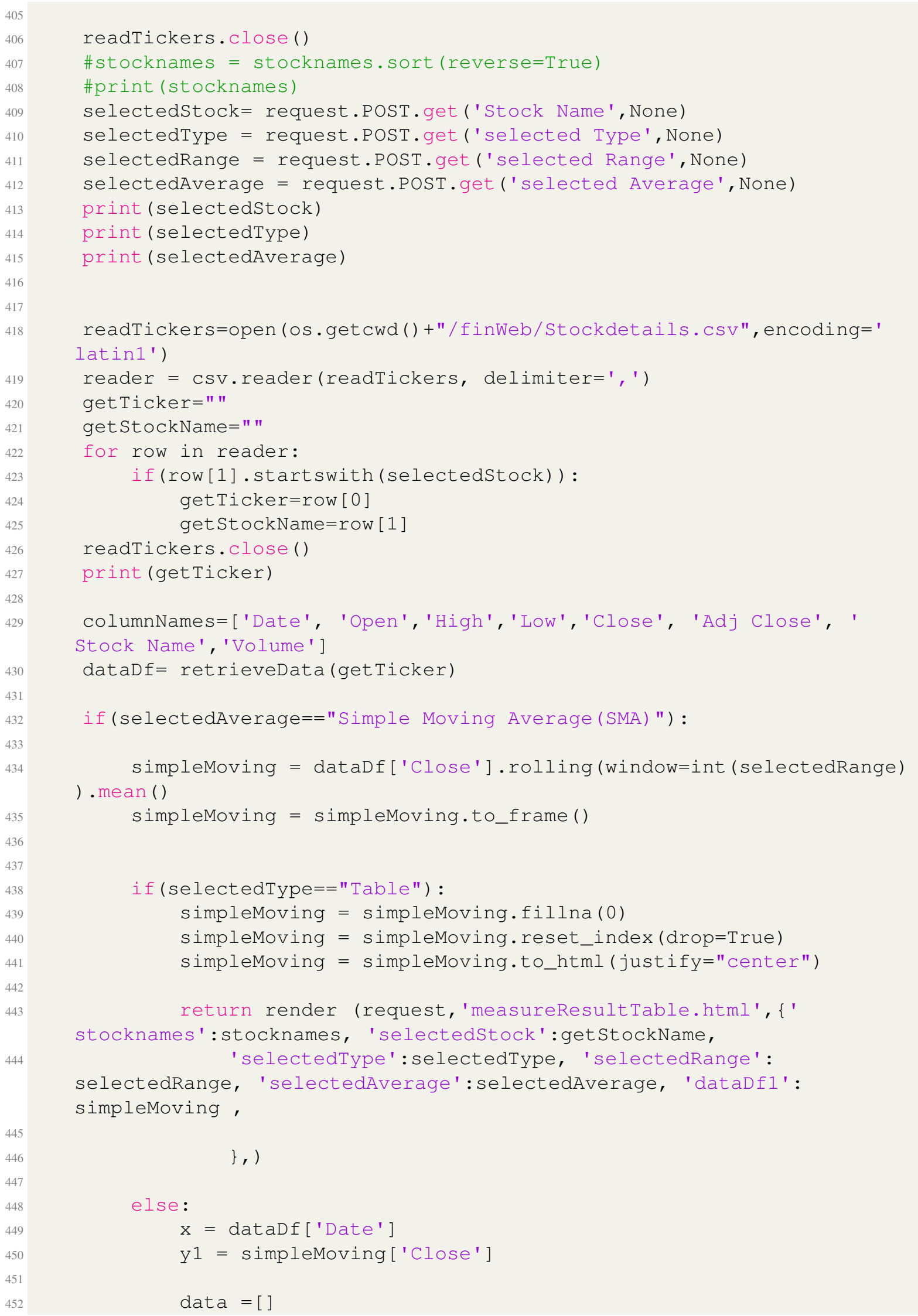




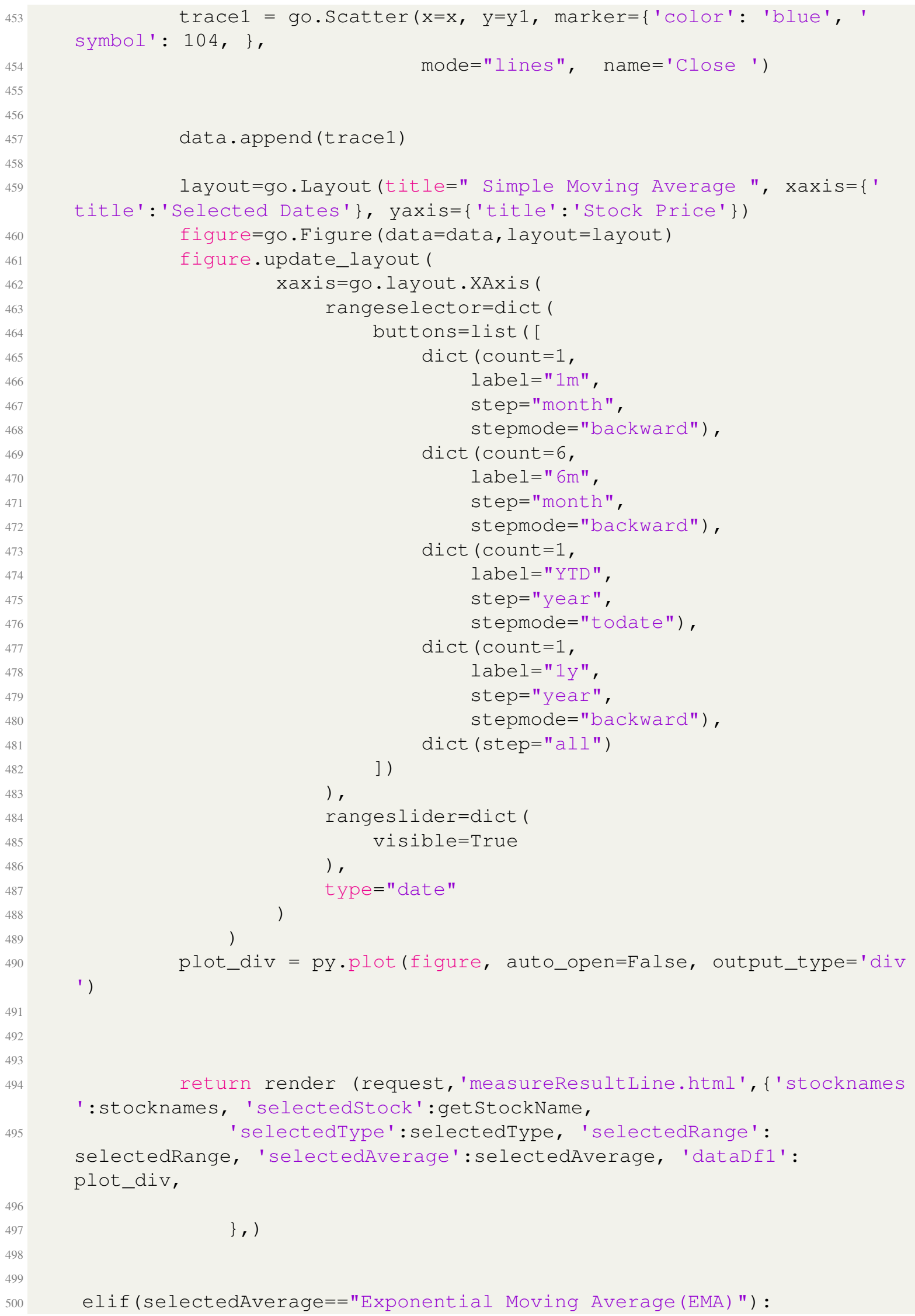




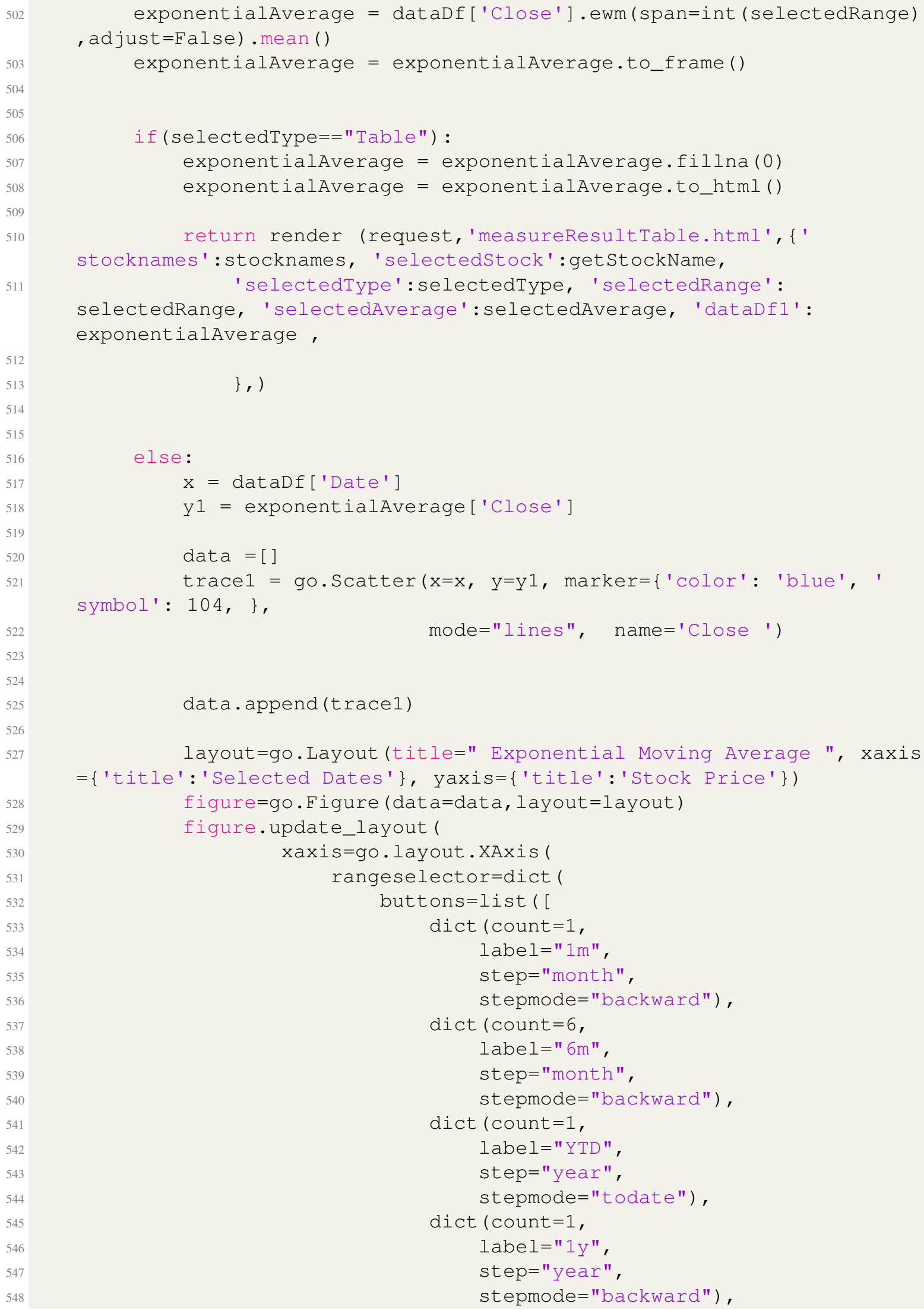




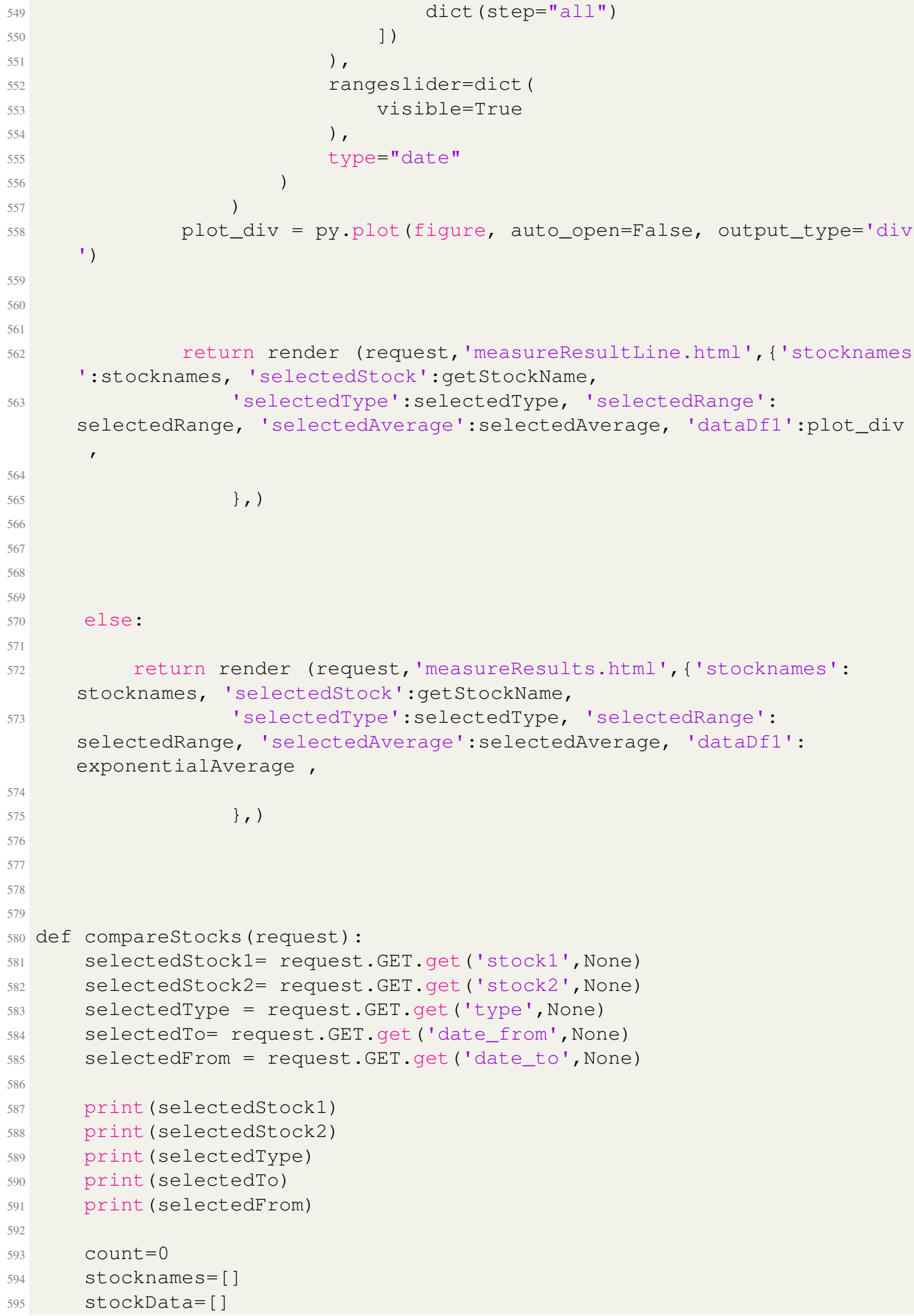




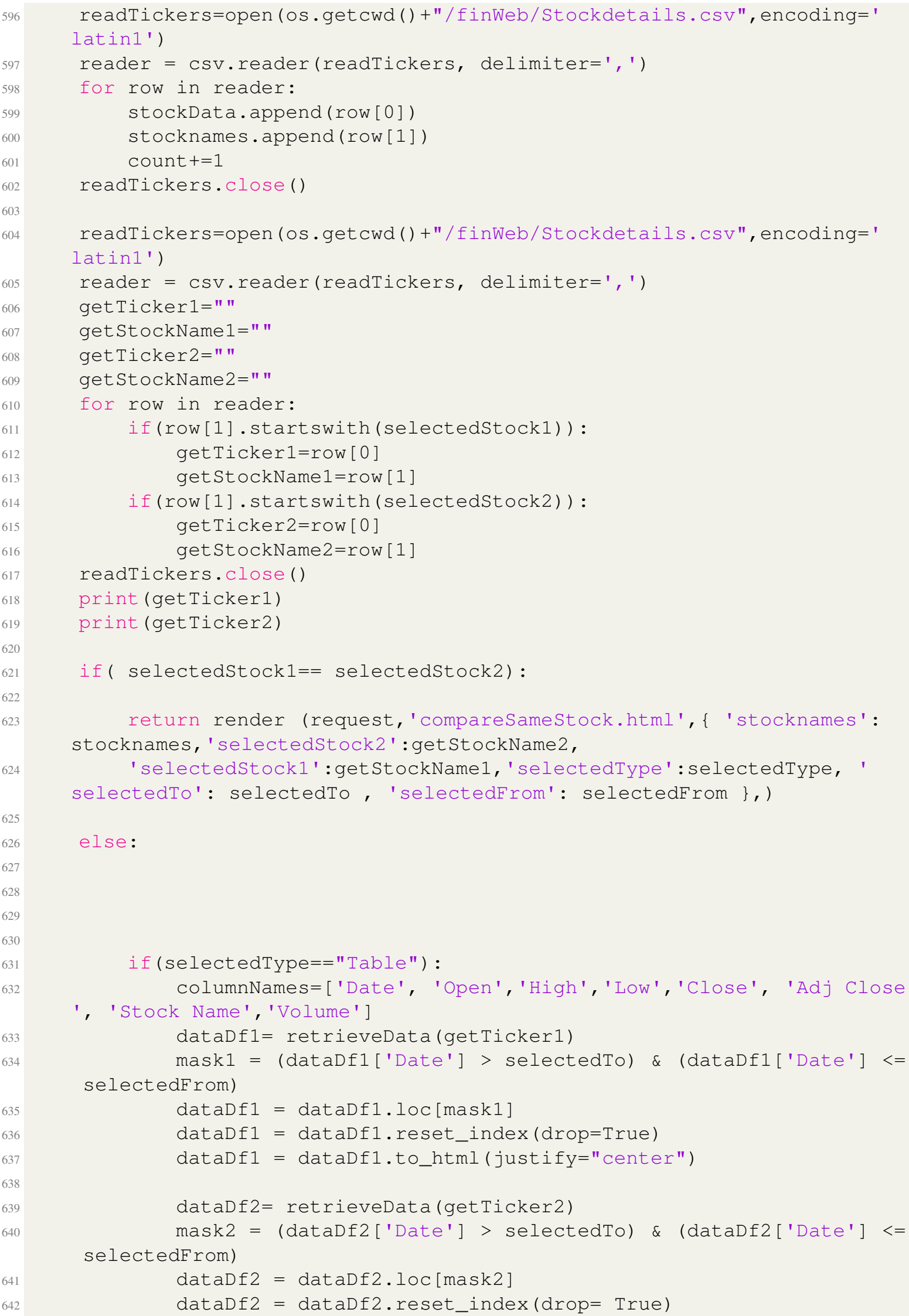




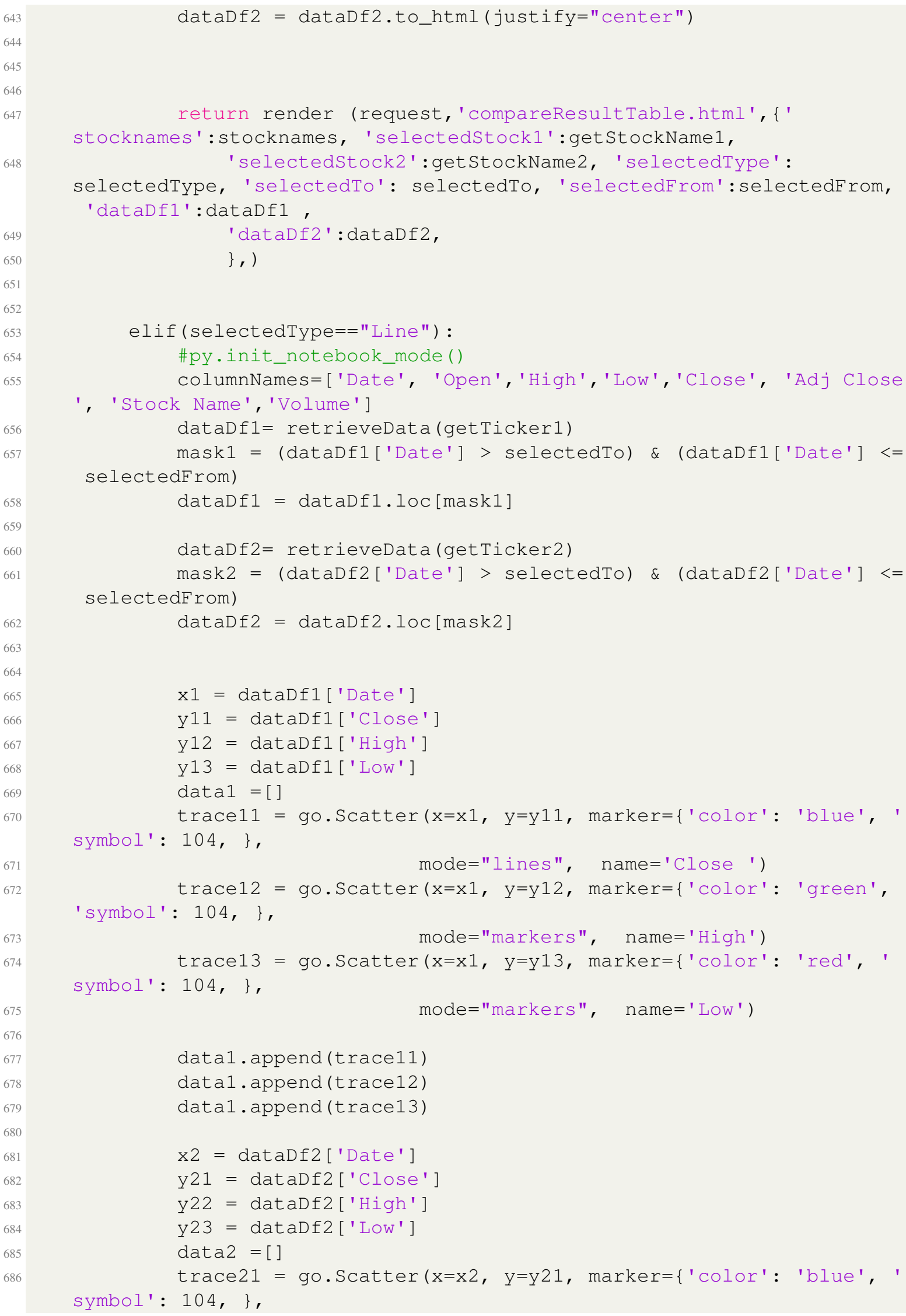




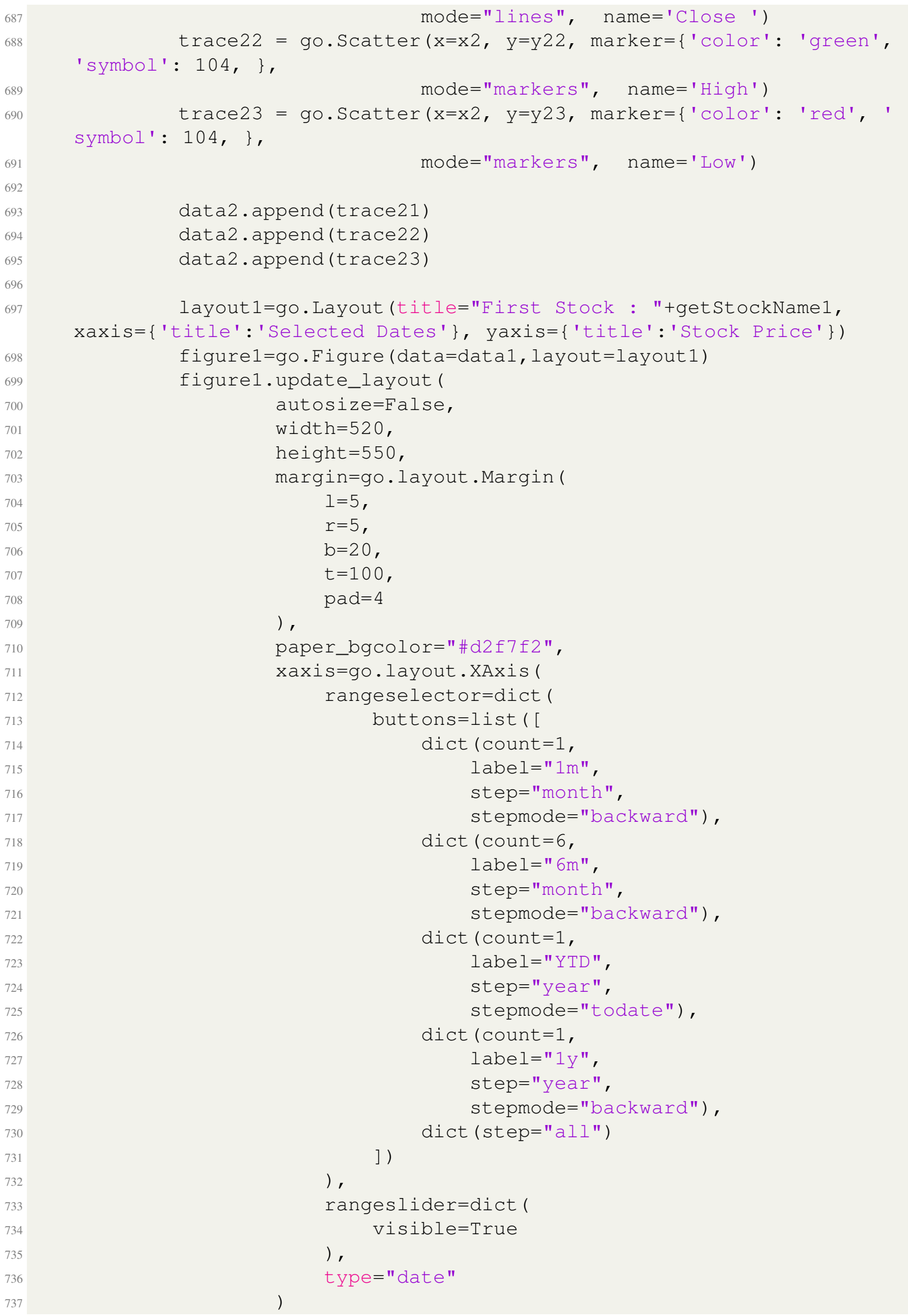




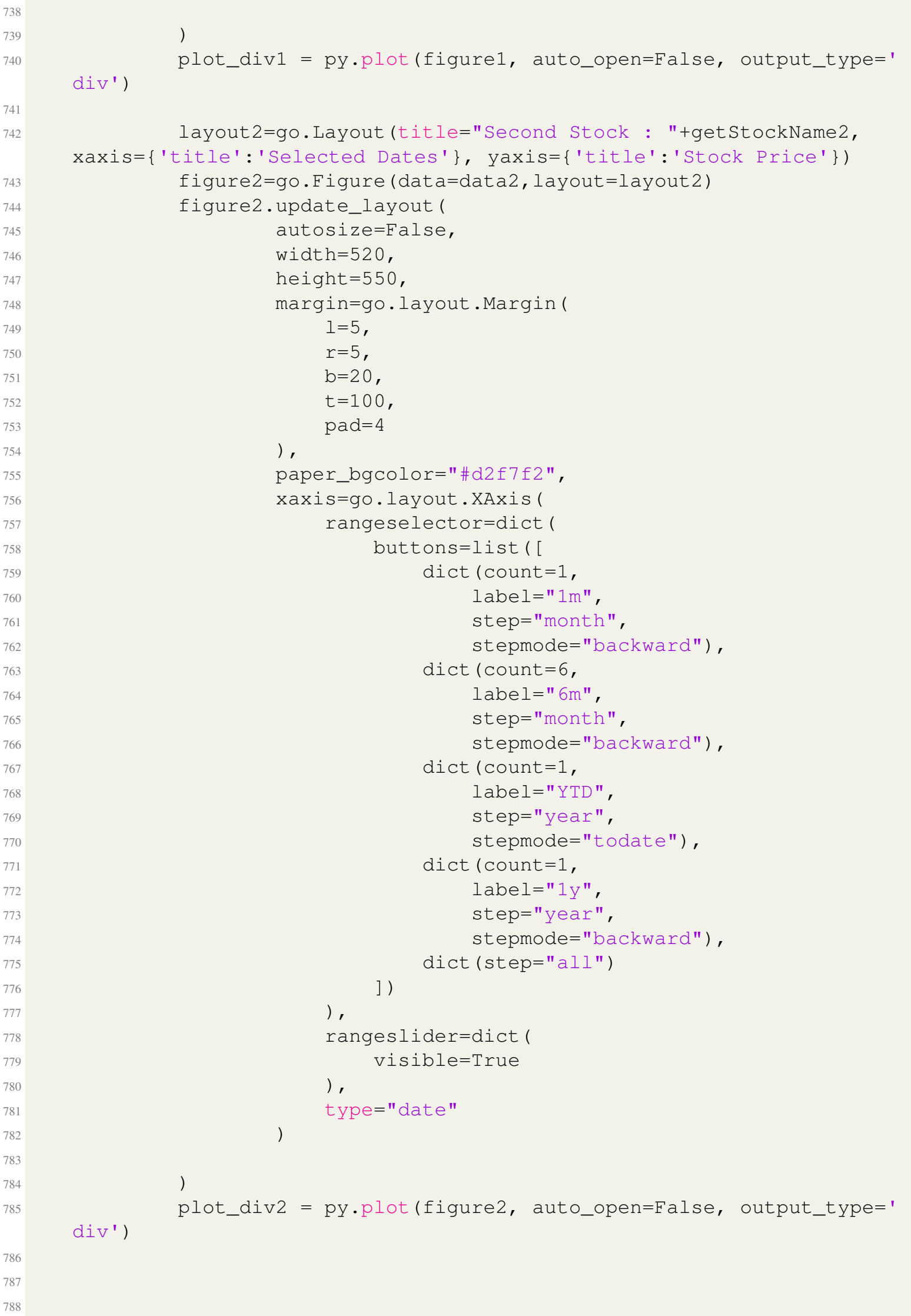




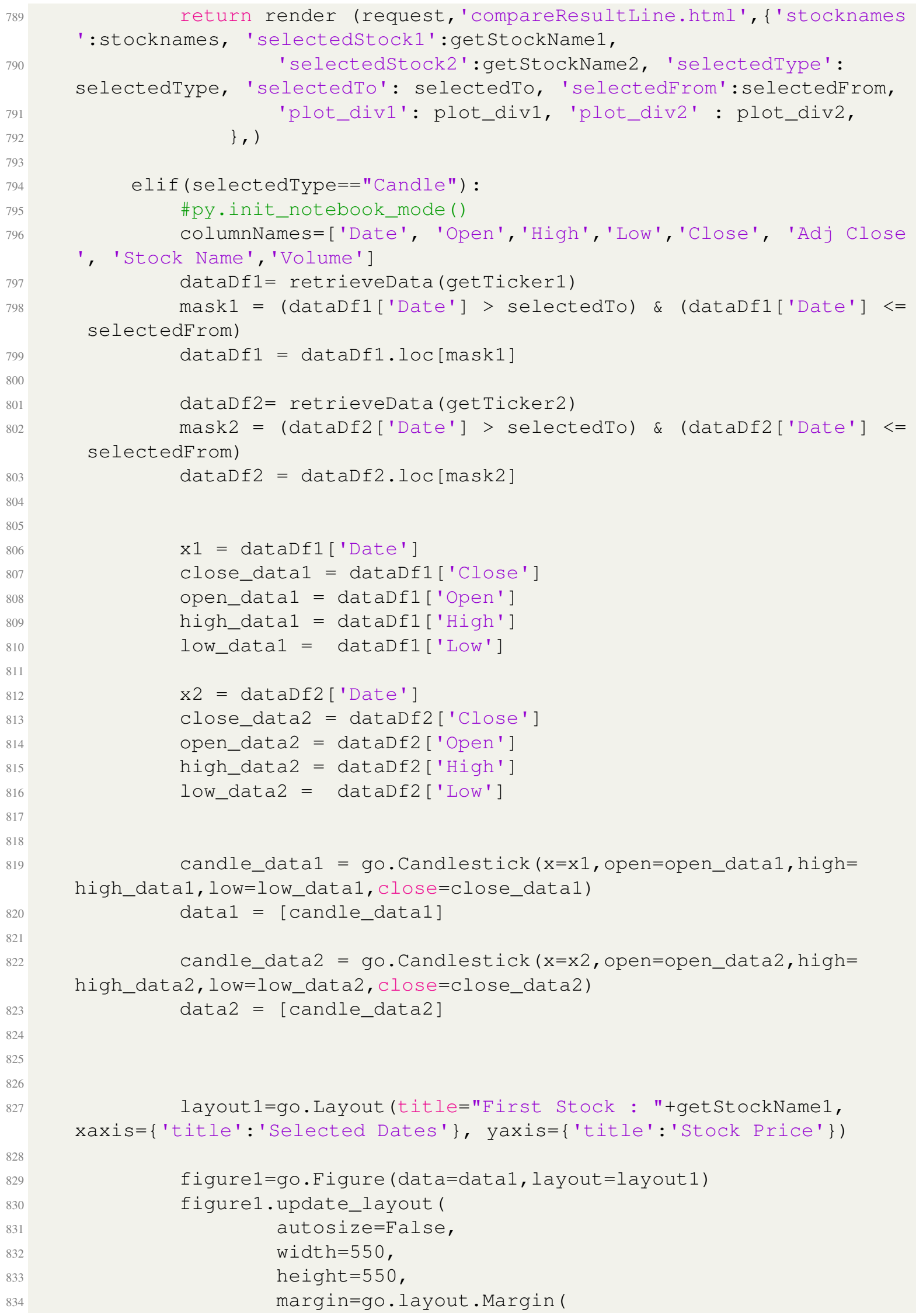




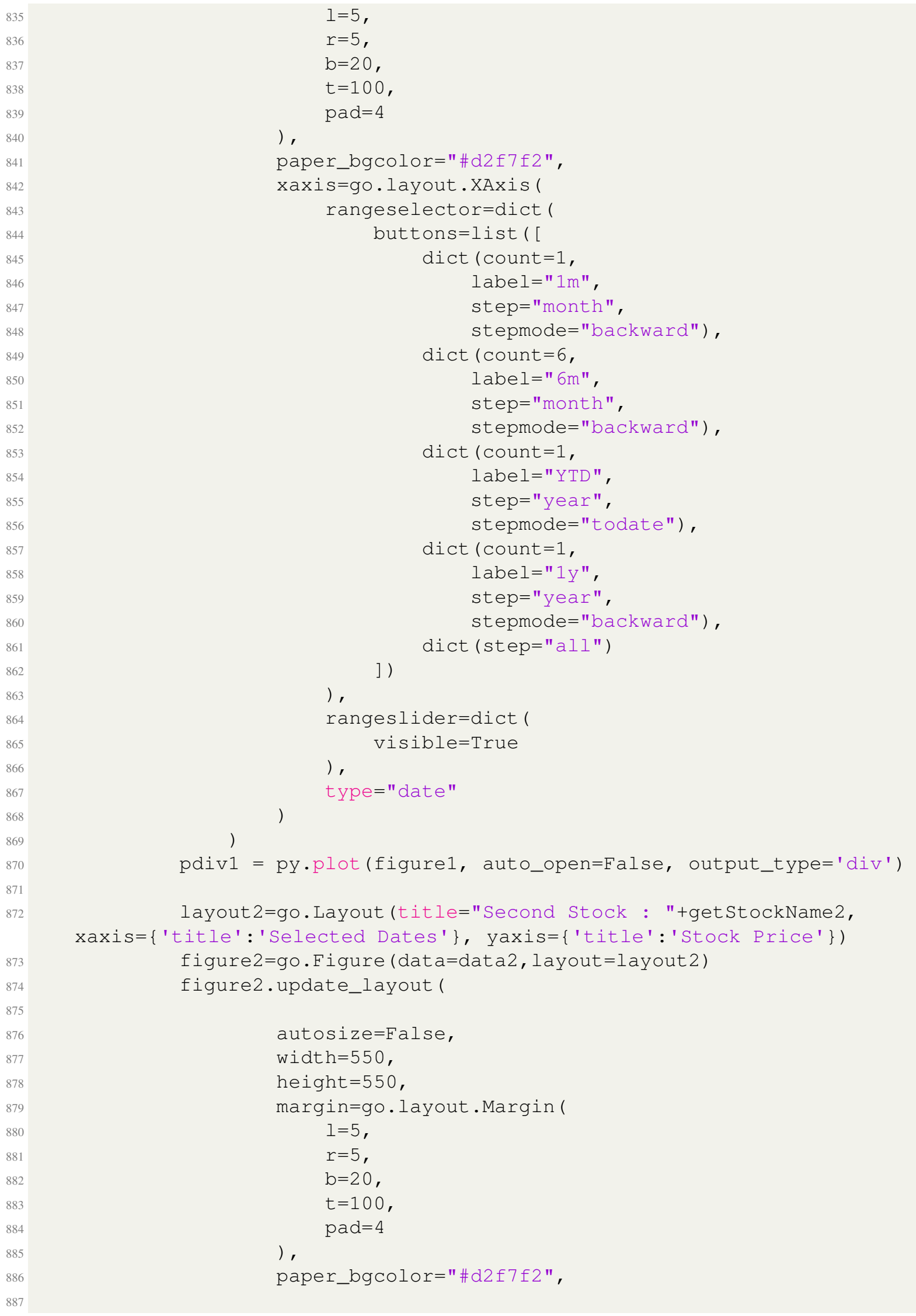




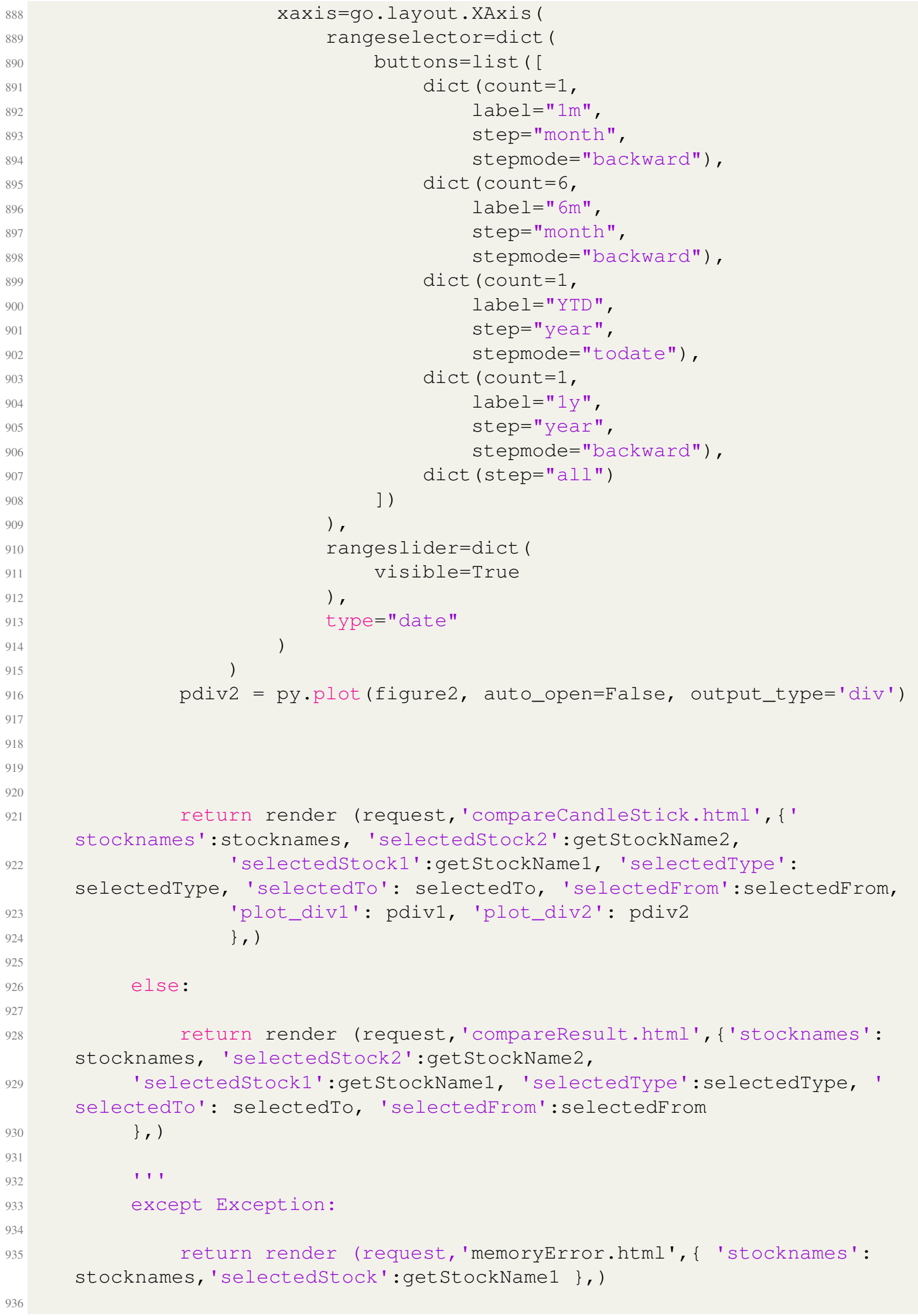




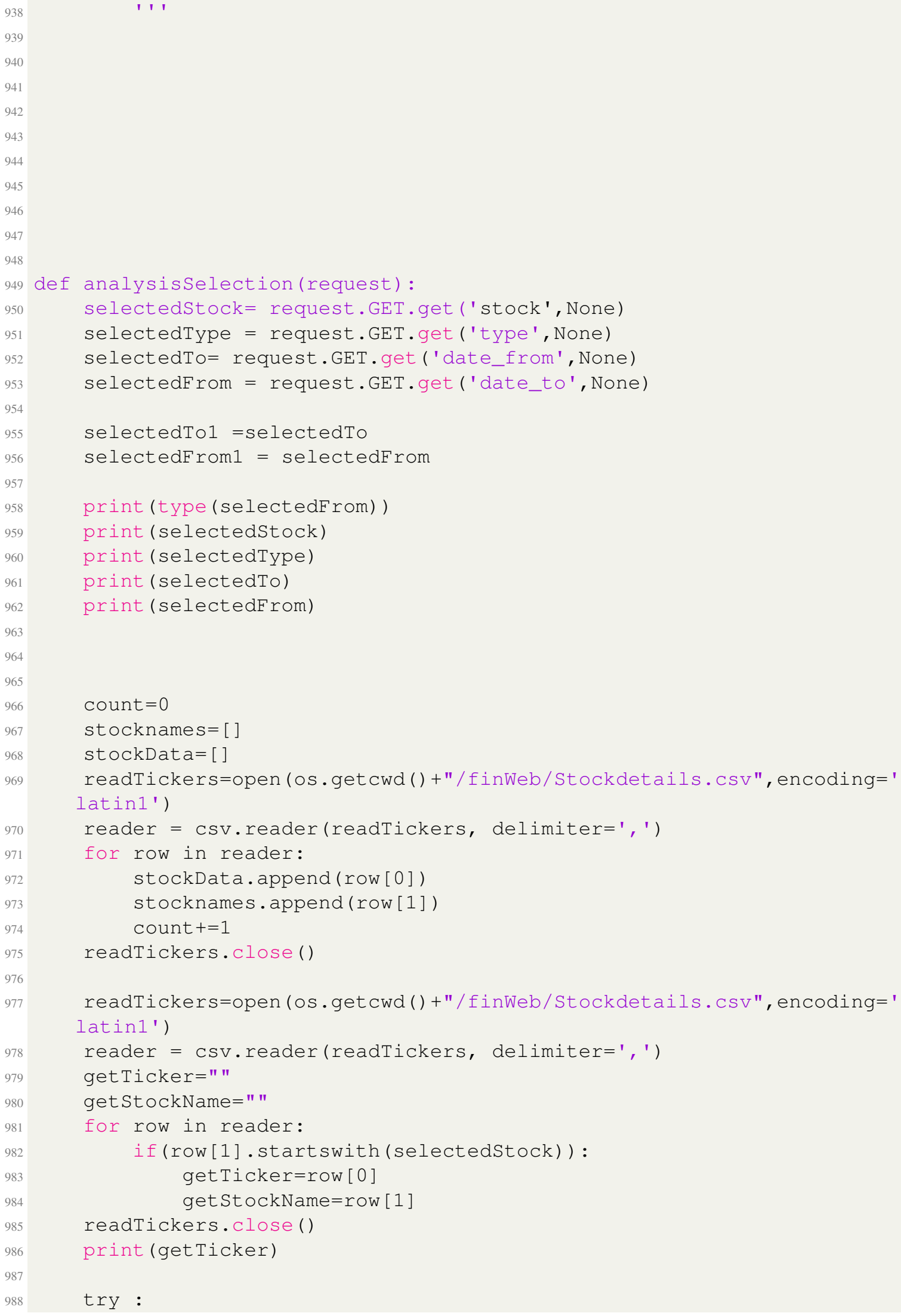




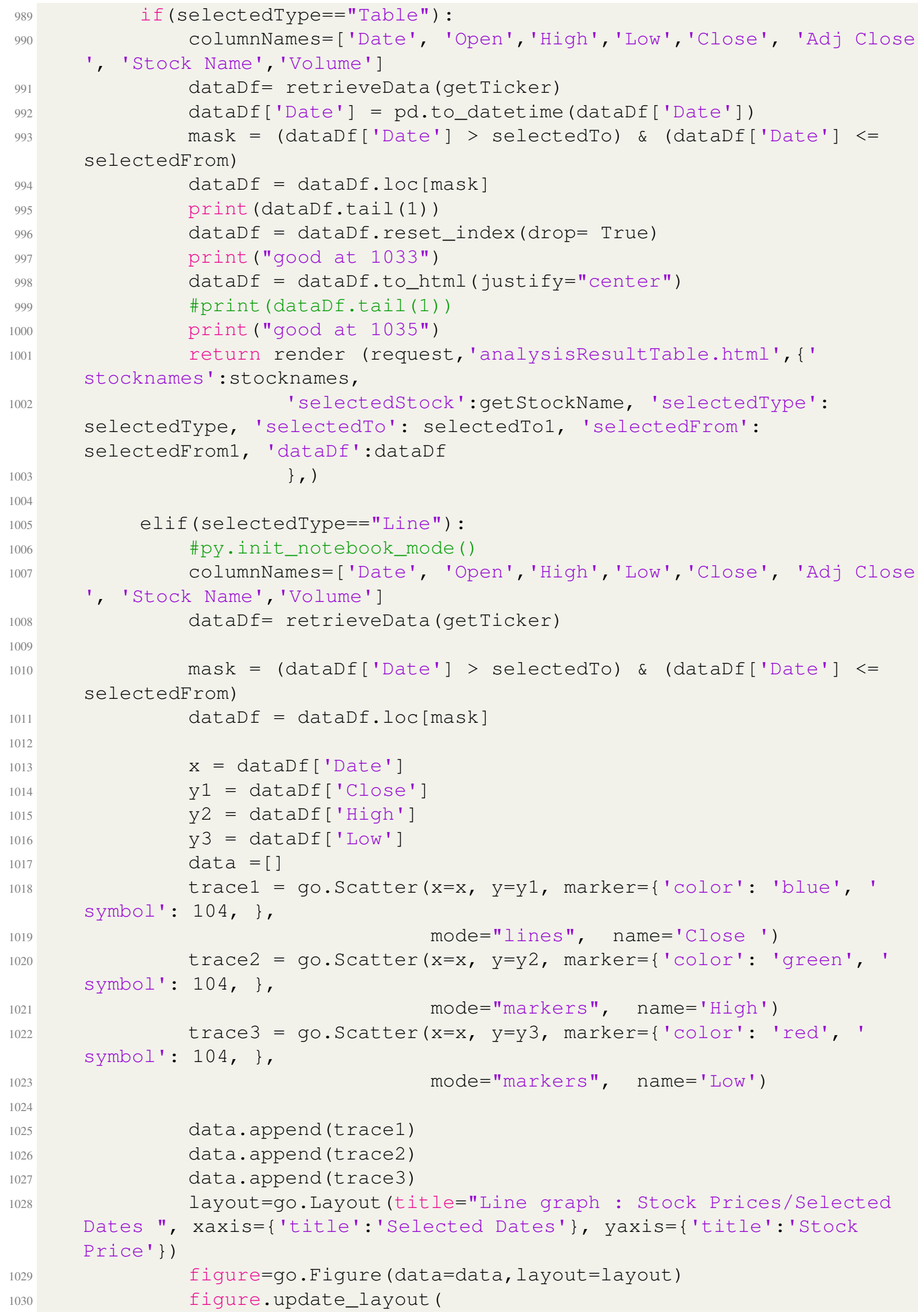




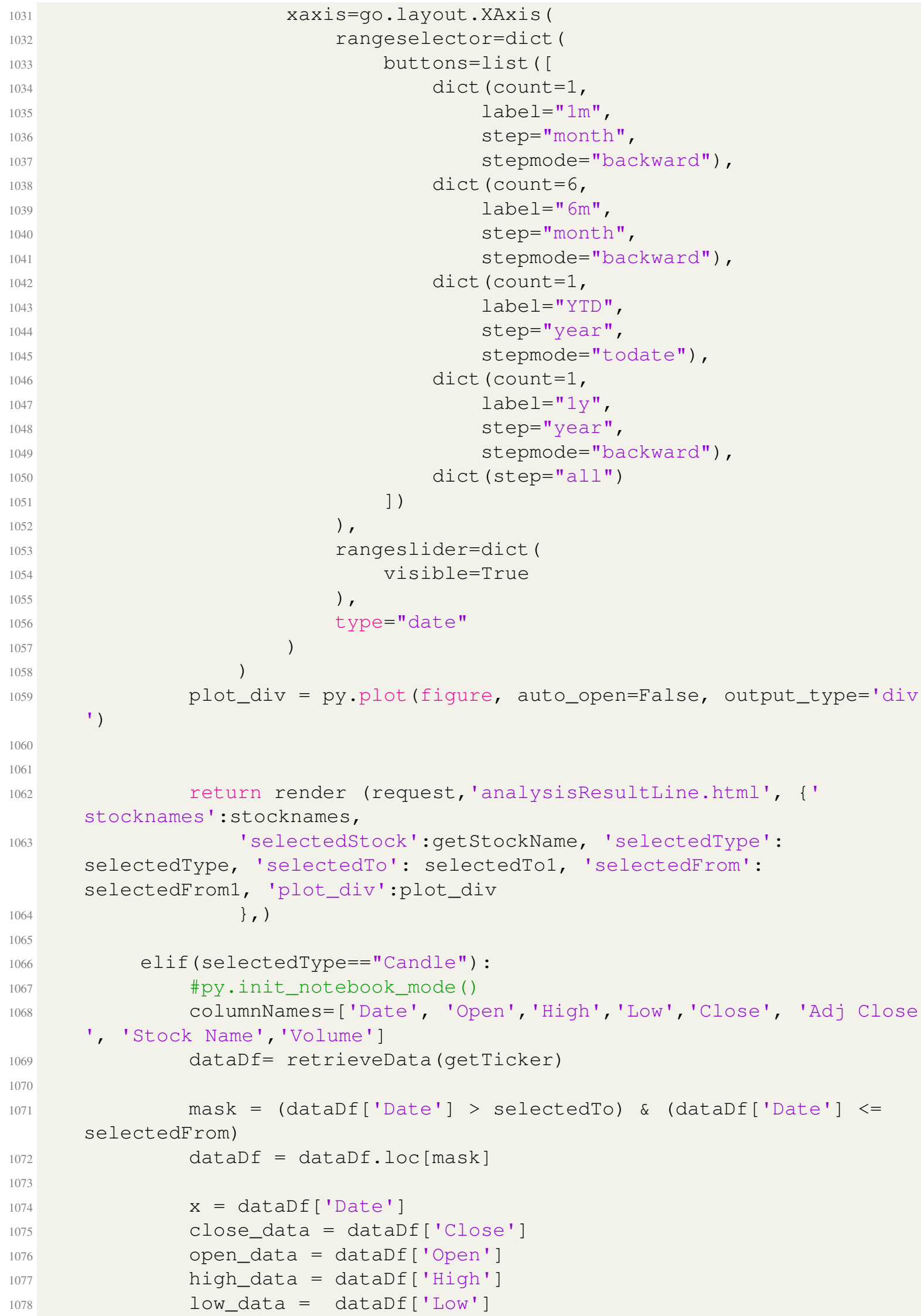

xaxis=go.layout. XAxis ( rangeselector=dict ( buttons=list ( [ dict ( count $=1$, label=" $1 \mathrm{~m} "$, step="month", stepmode="backward") , dict ( count $=6$, label $=" 6 \mathrm{~m} "$, step="month", stepmode="backward") , dict ( count $=1$, label="YTD", step="year", stepmode="todate"), dict ( count $=1$, label="1y", step="year", stepmode="backward") , $\operatorname{dict}($ step="all") ] ) 


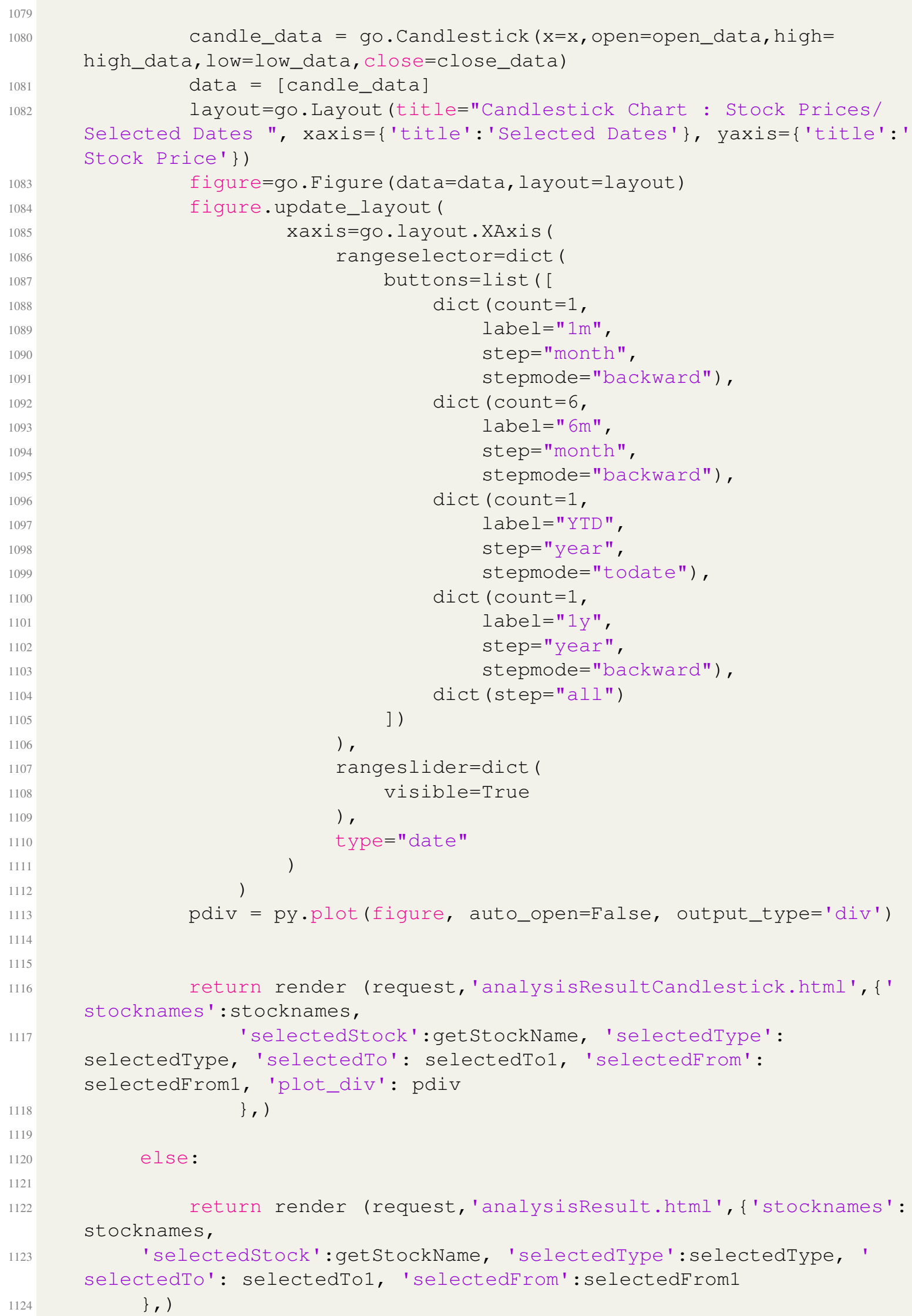




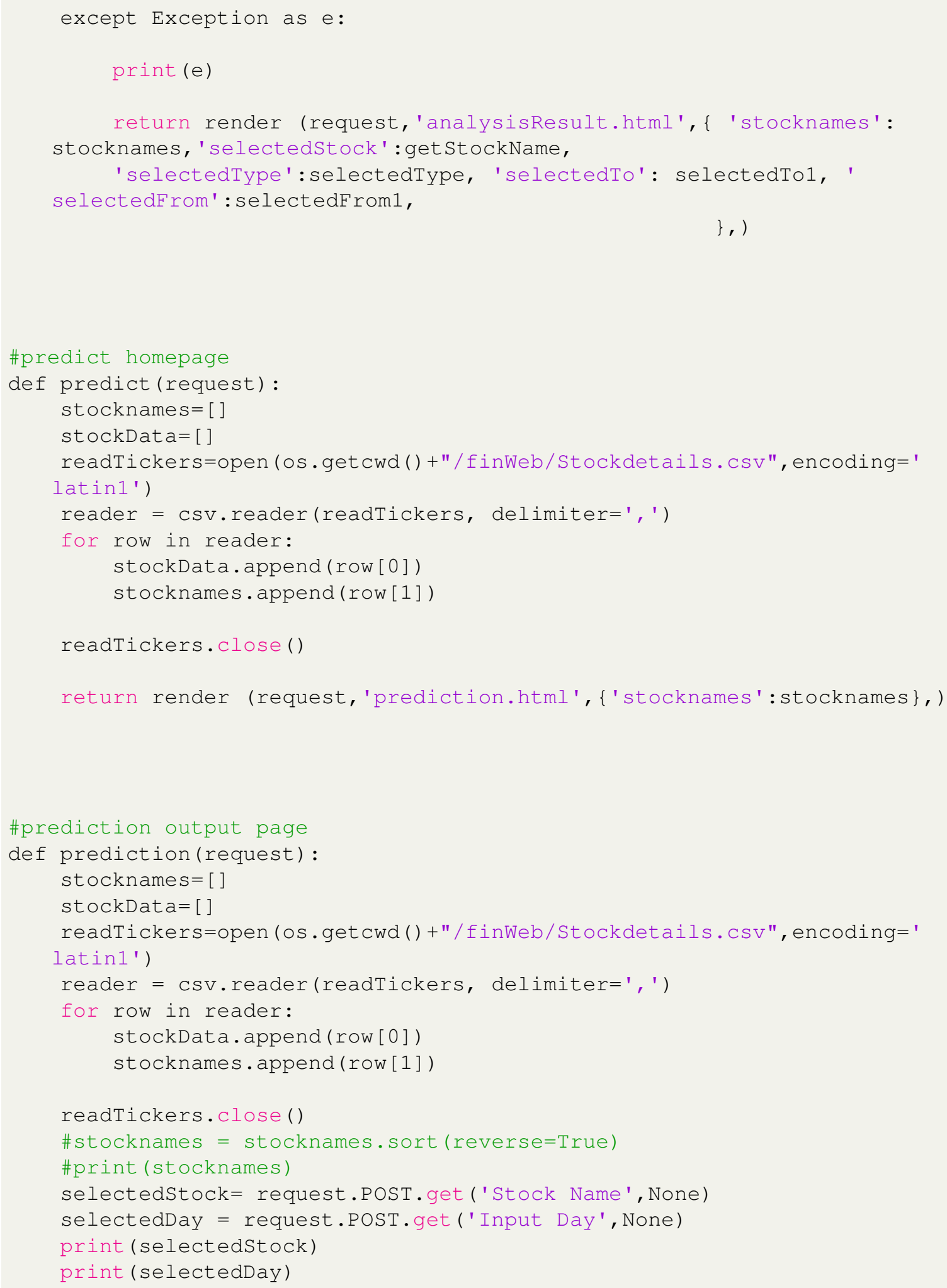




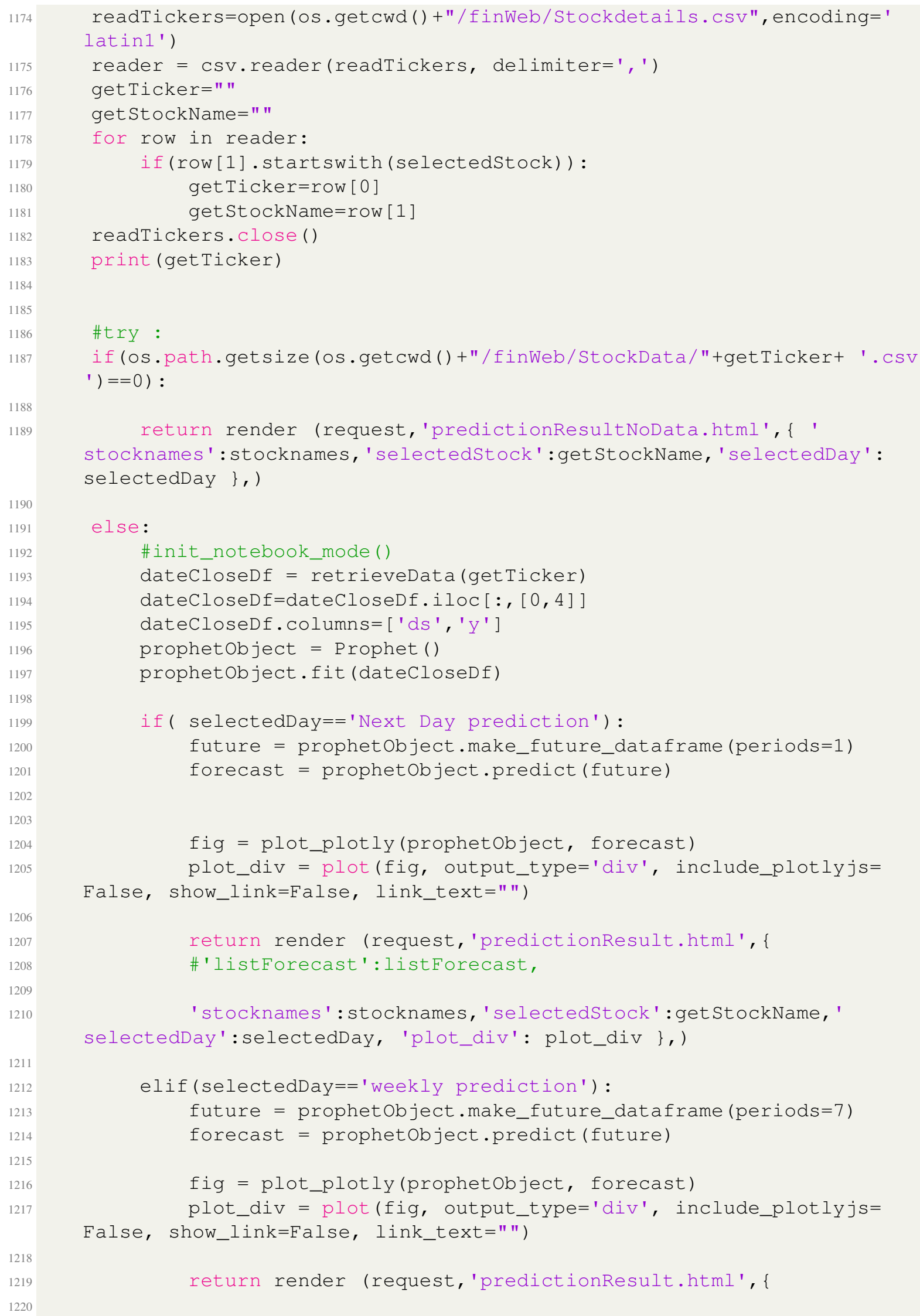
stocknames' : stocknames, 'selectedStock' : getStockName, 'selectedDay' : selectedDay \}, ) 


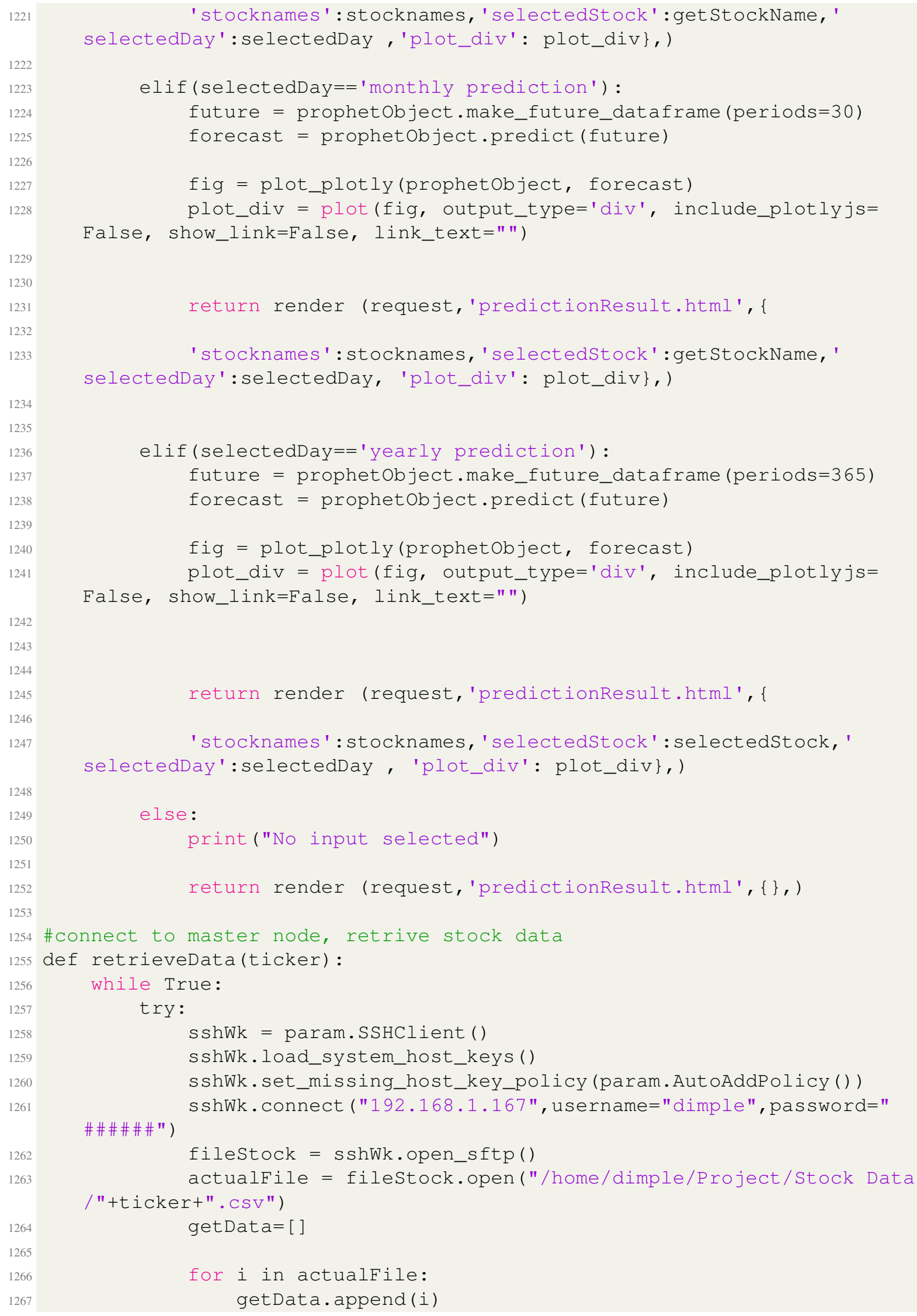




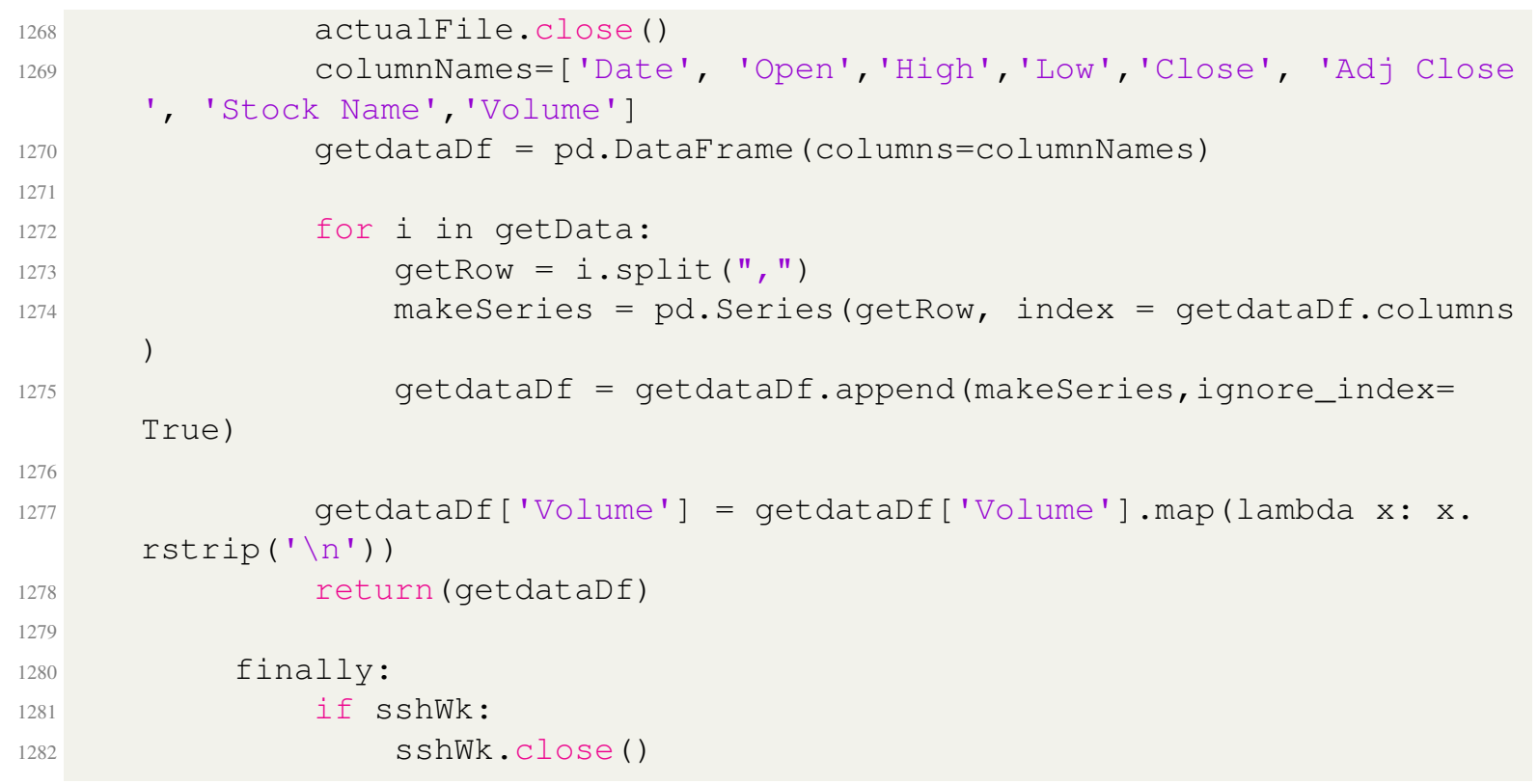

Code A.2.3.6: views.py

The templates folder contains of following files -

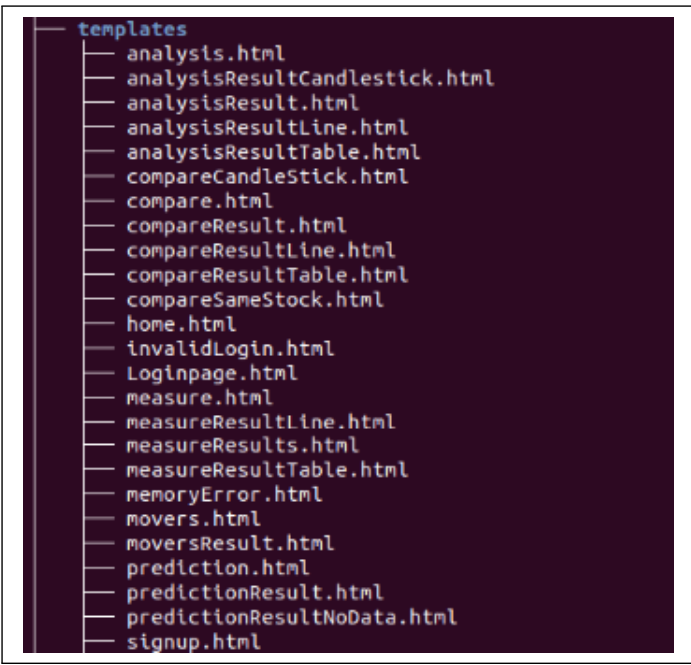

Figure A.3: Directory Tree Structure for templates folder

\section{For historical analysis}

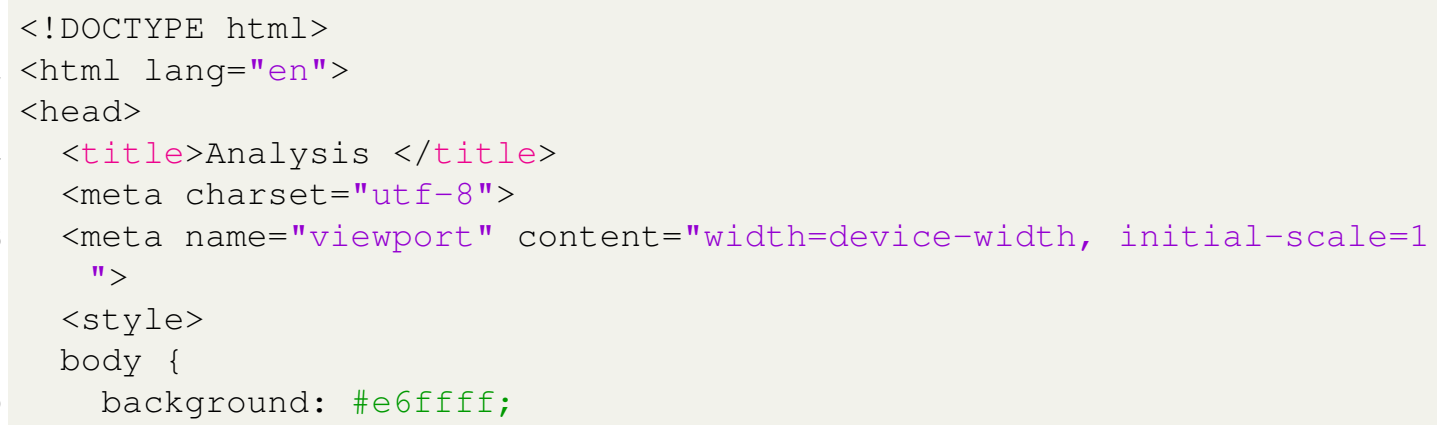




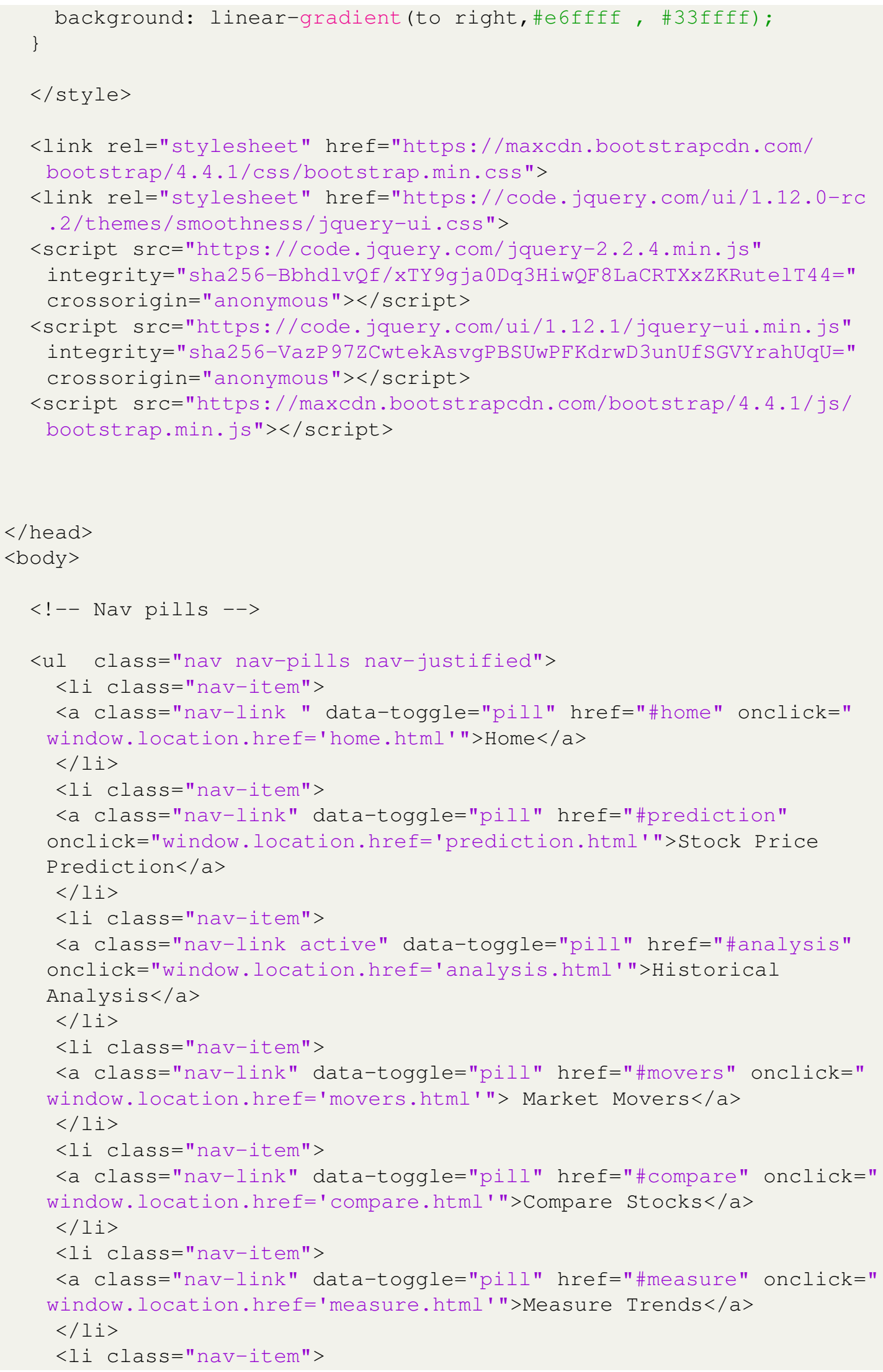




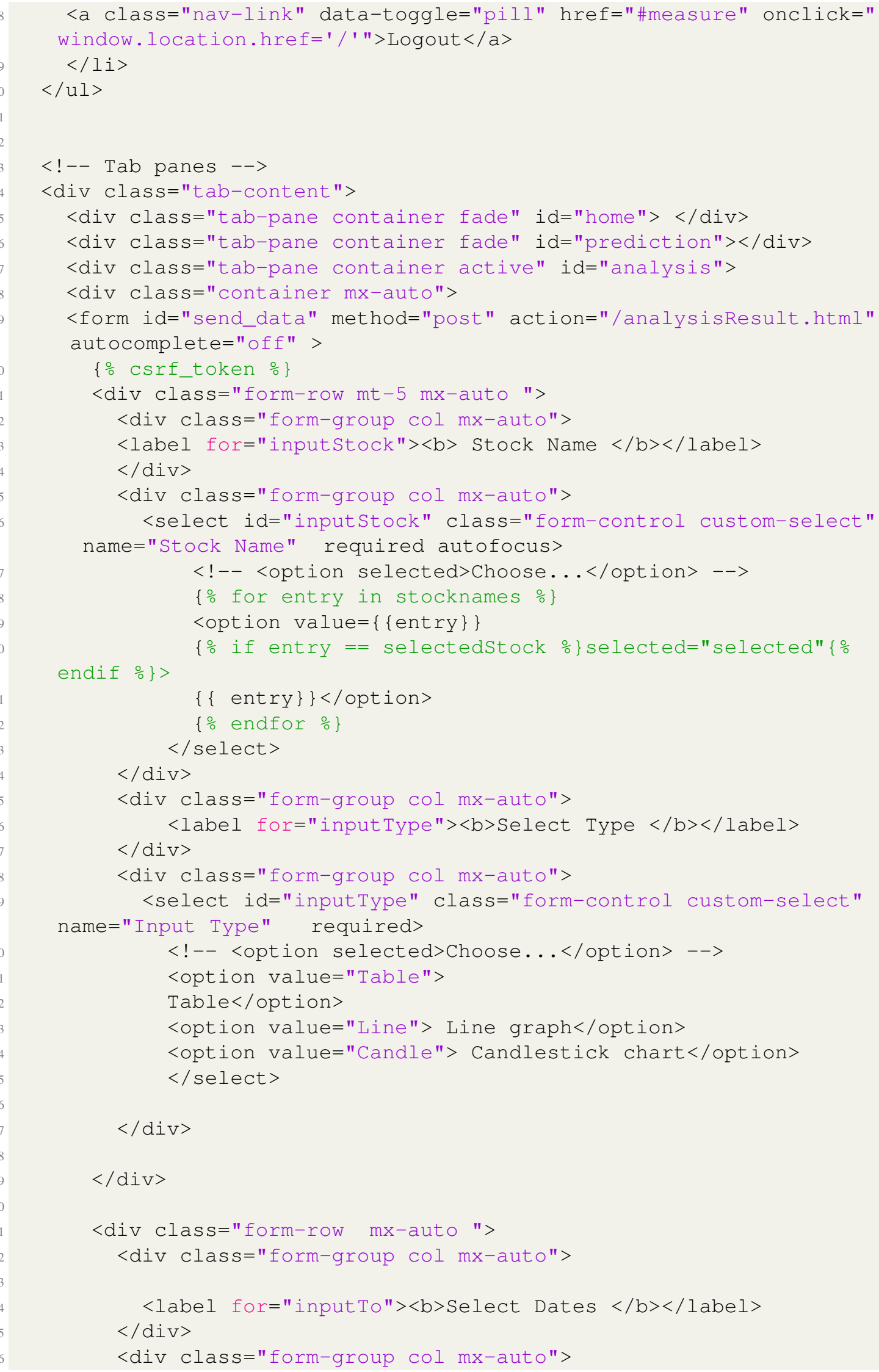




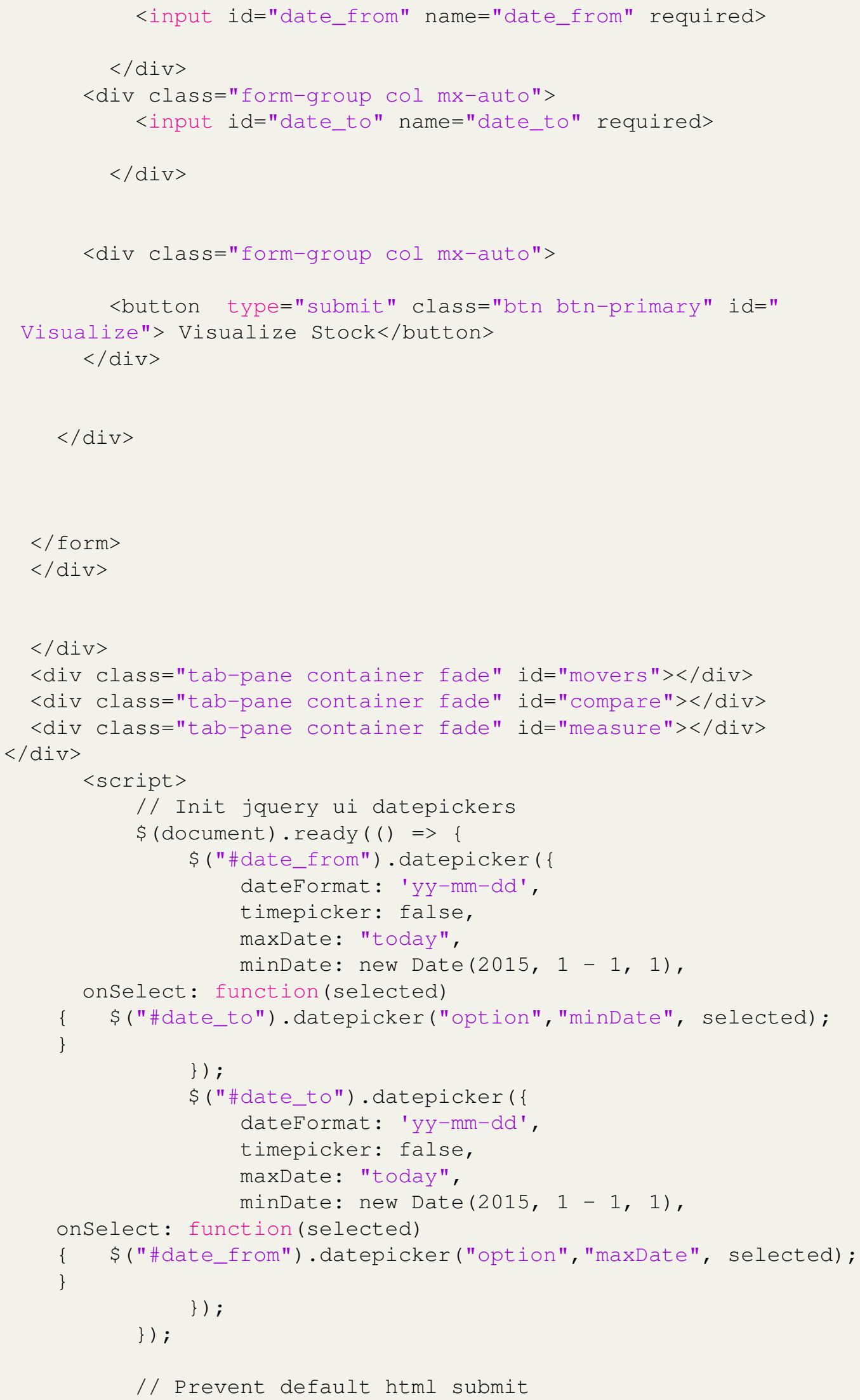




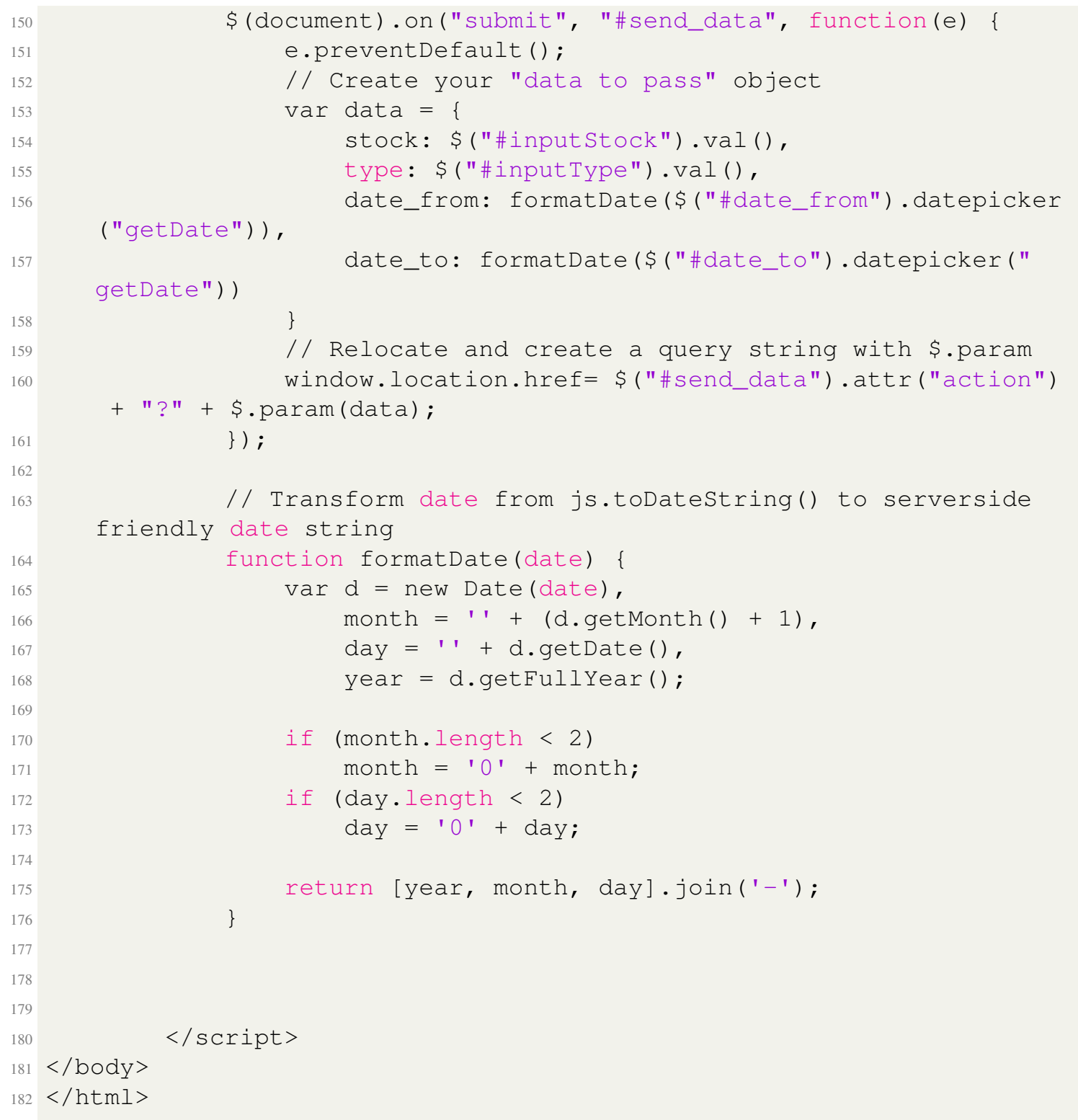

\section{Code A.2.3.7: analysis.html}

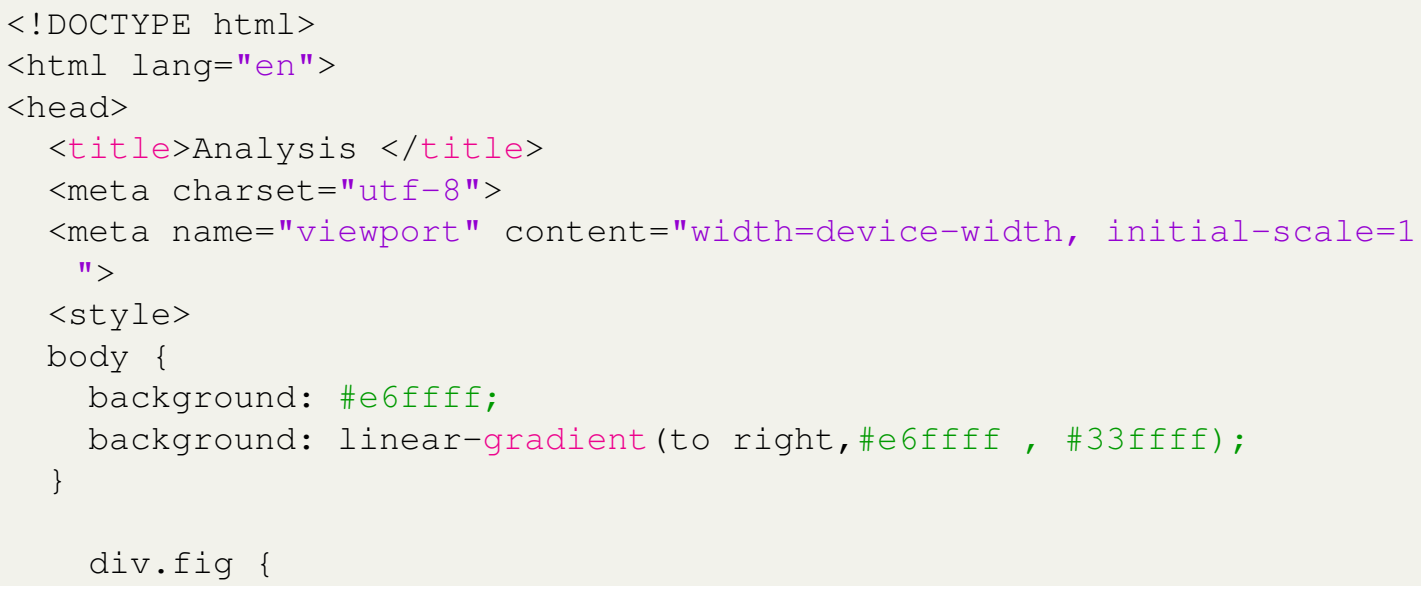




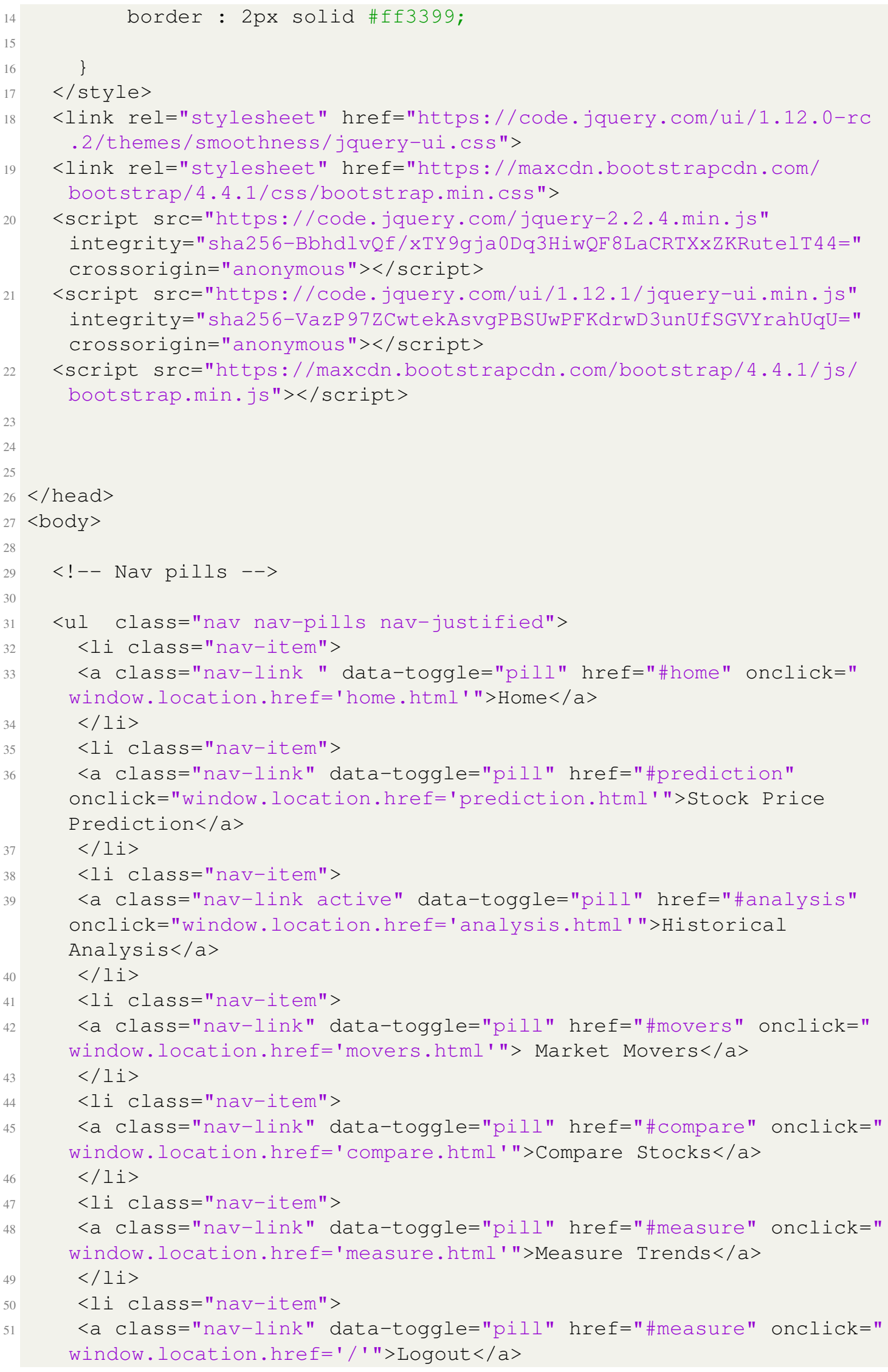




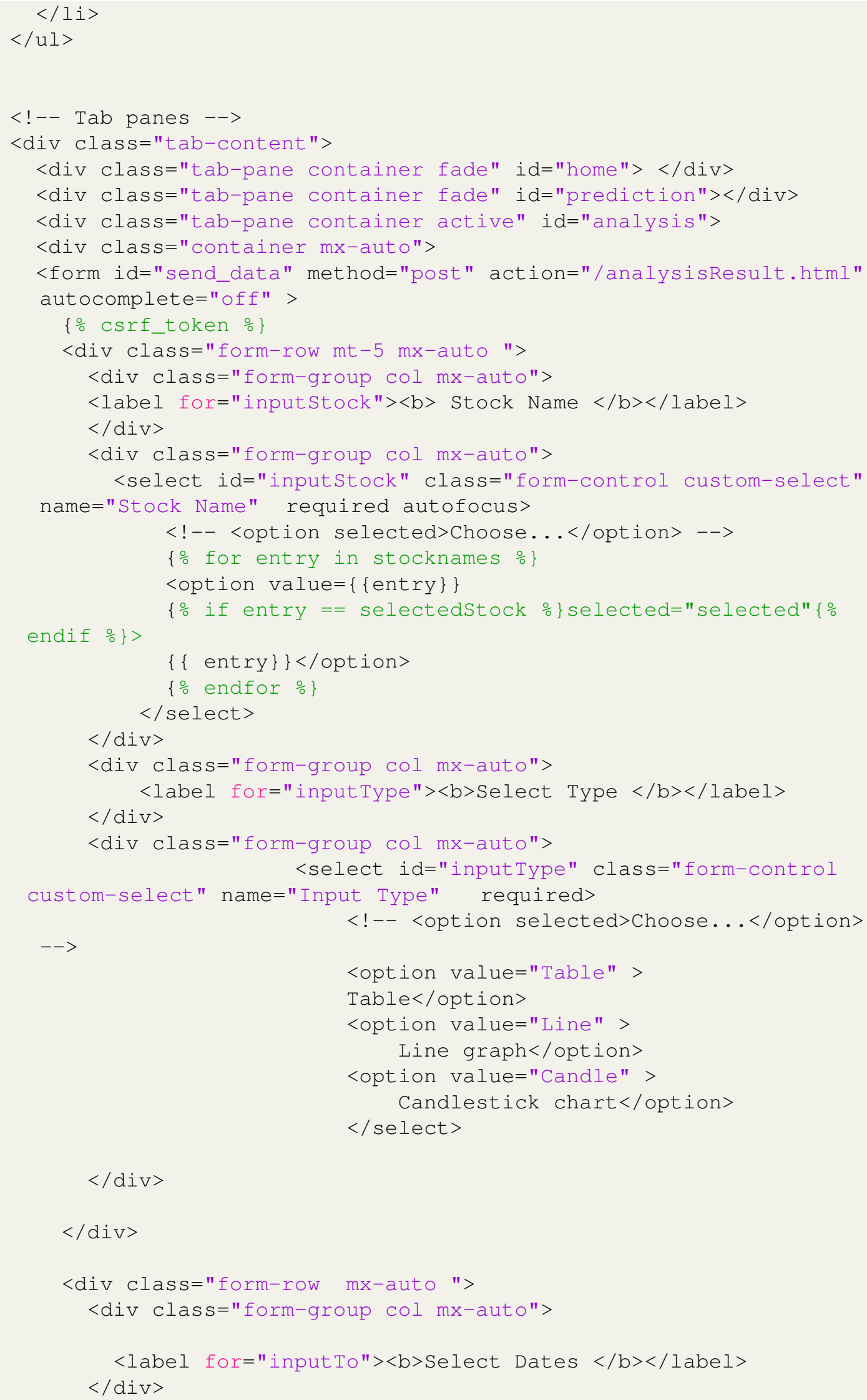




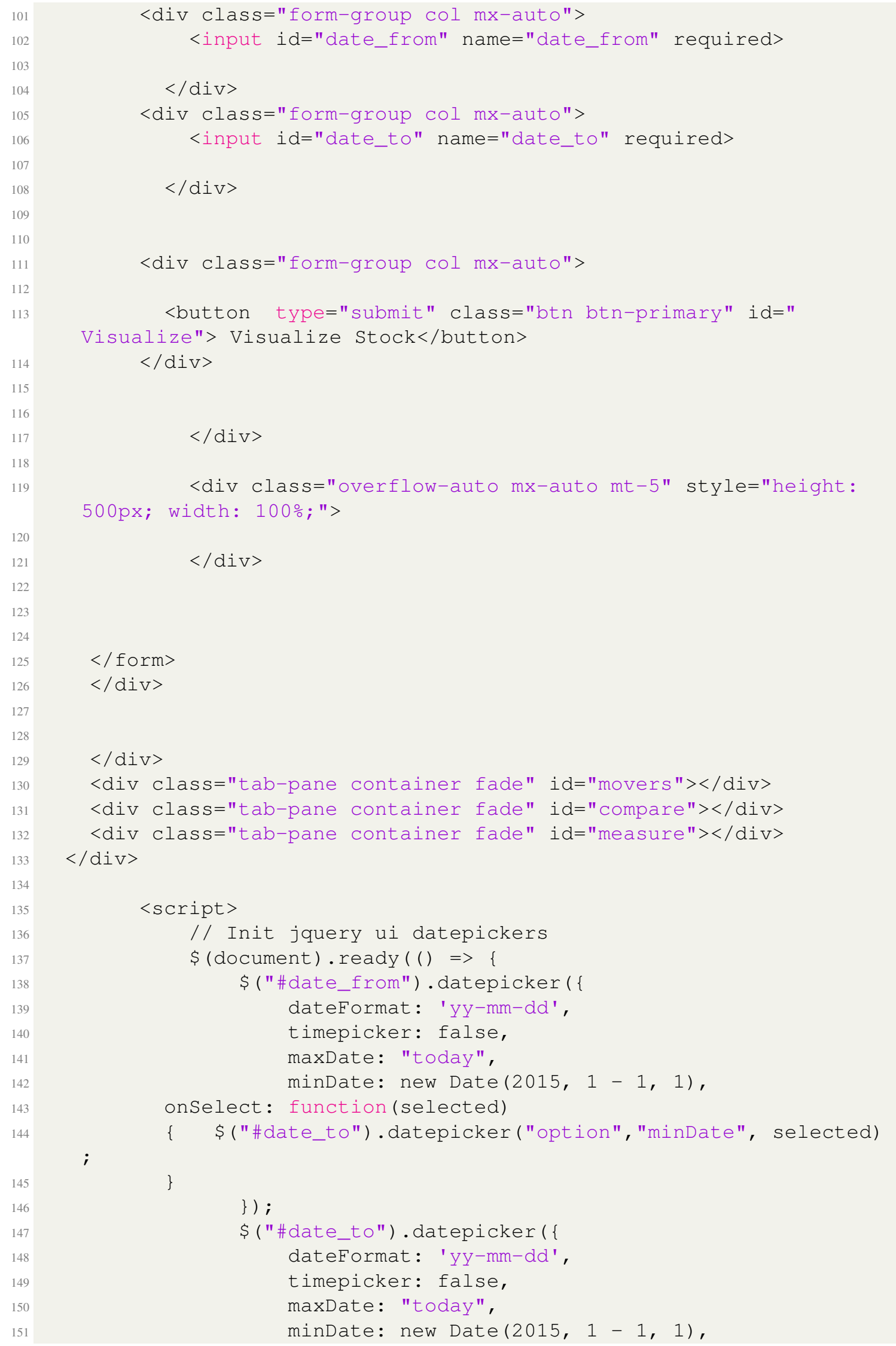




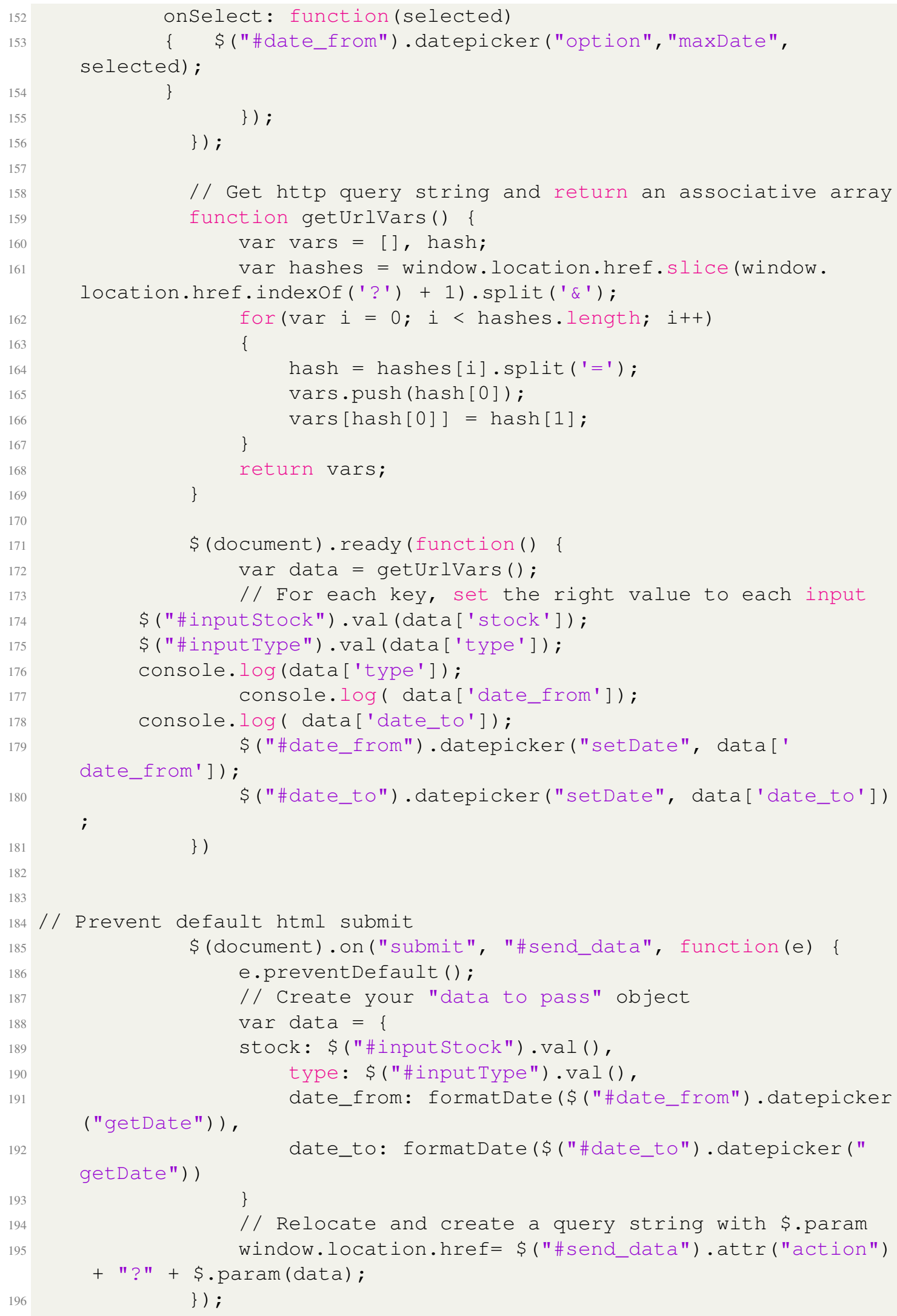




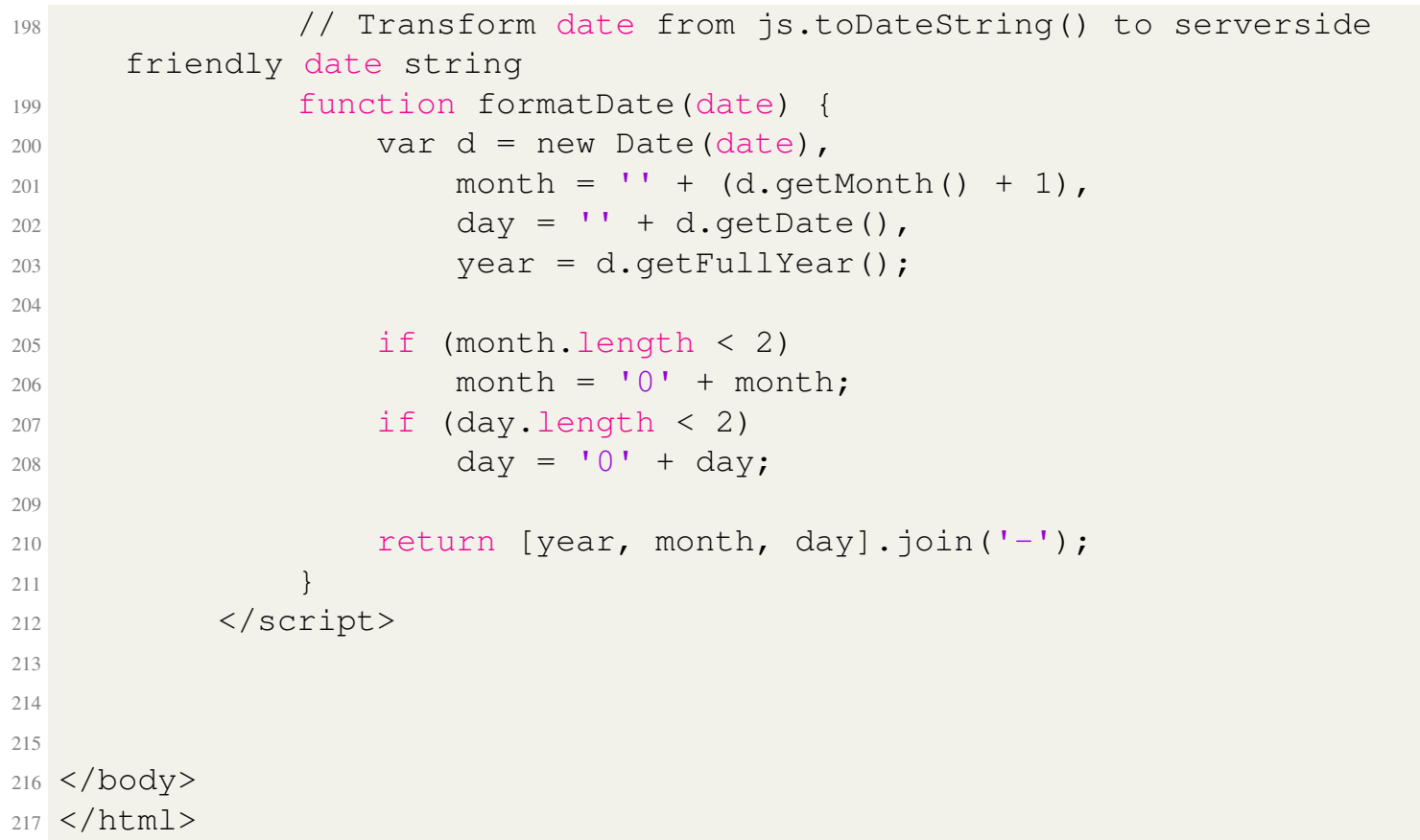

\section{Code A.2.3.8: analysisResult.html}

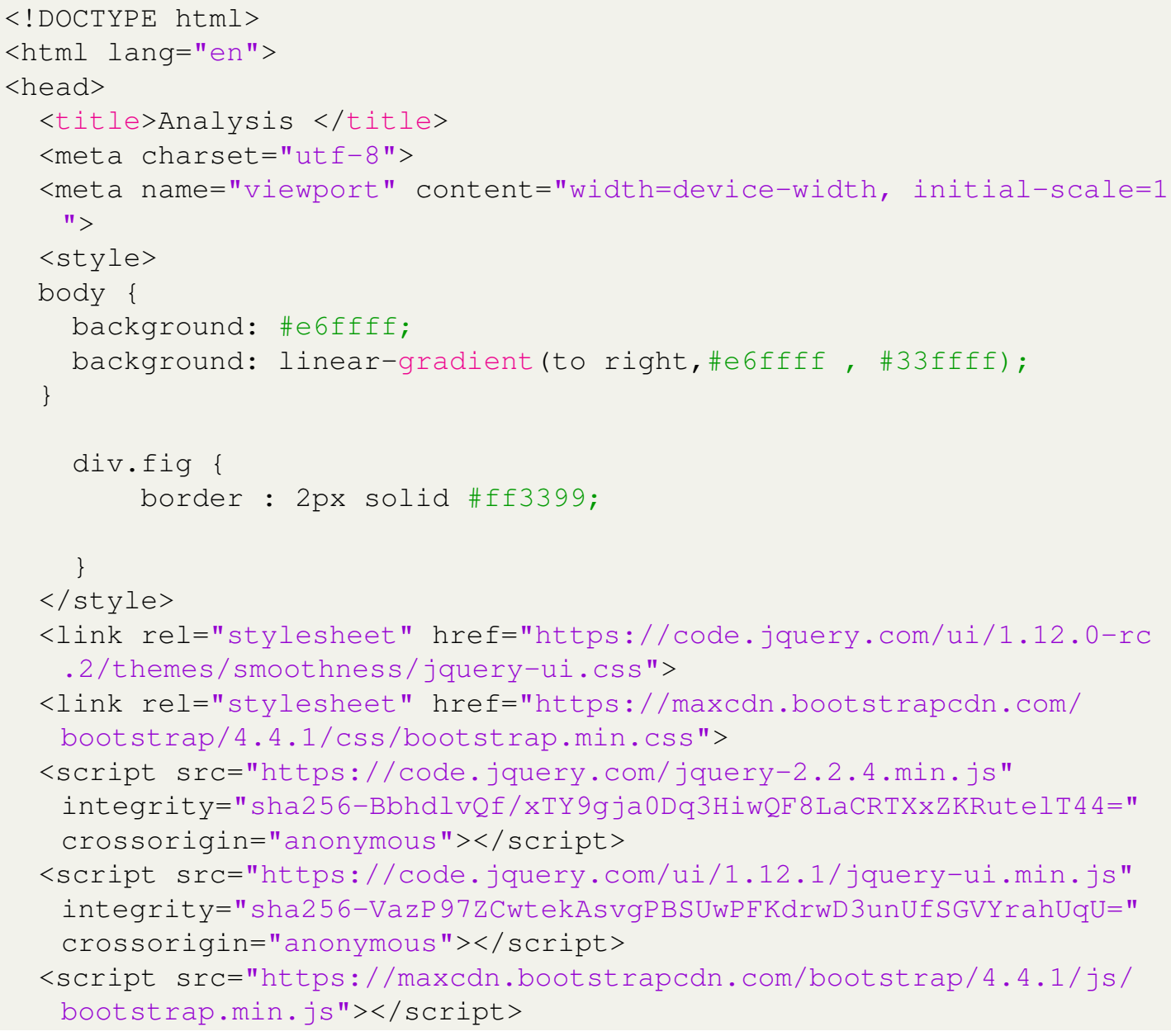




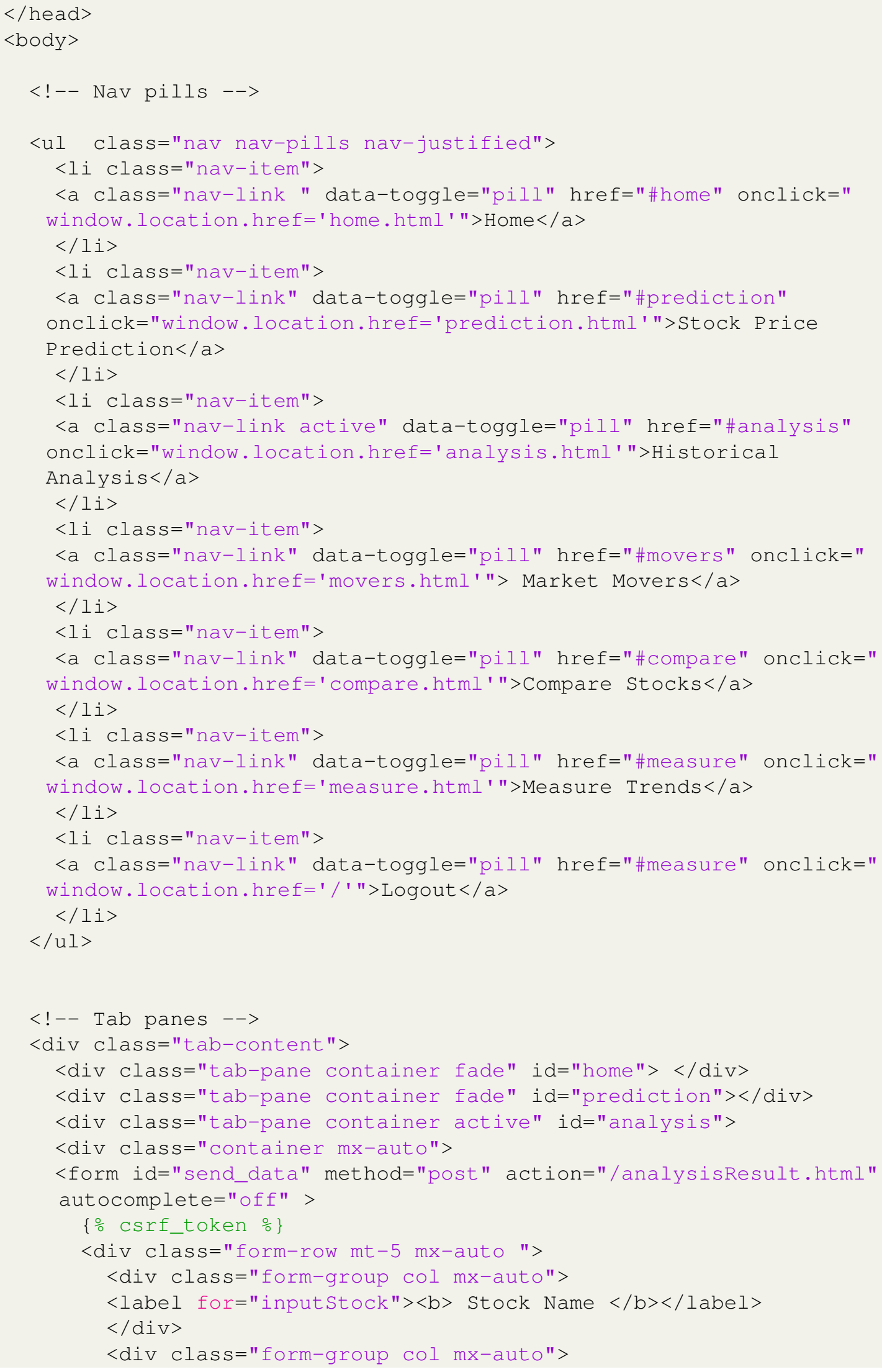




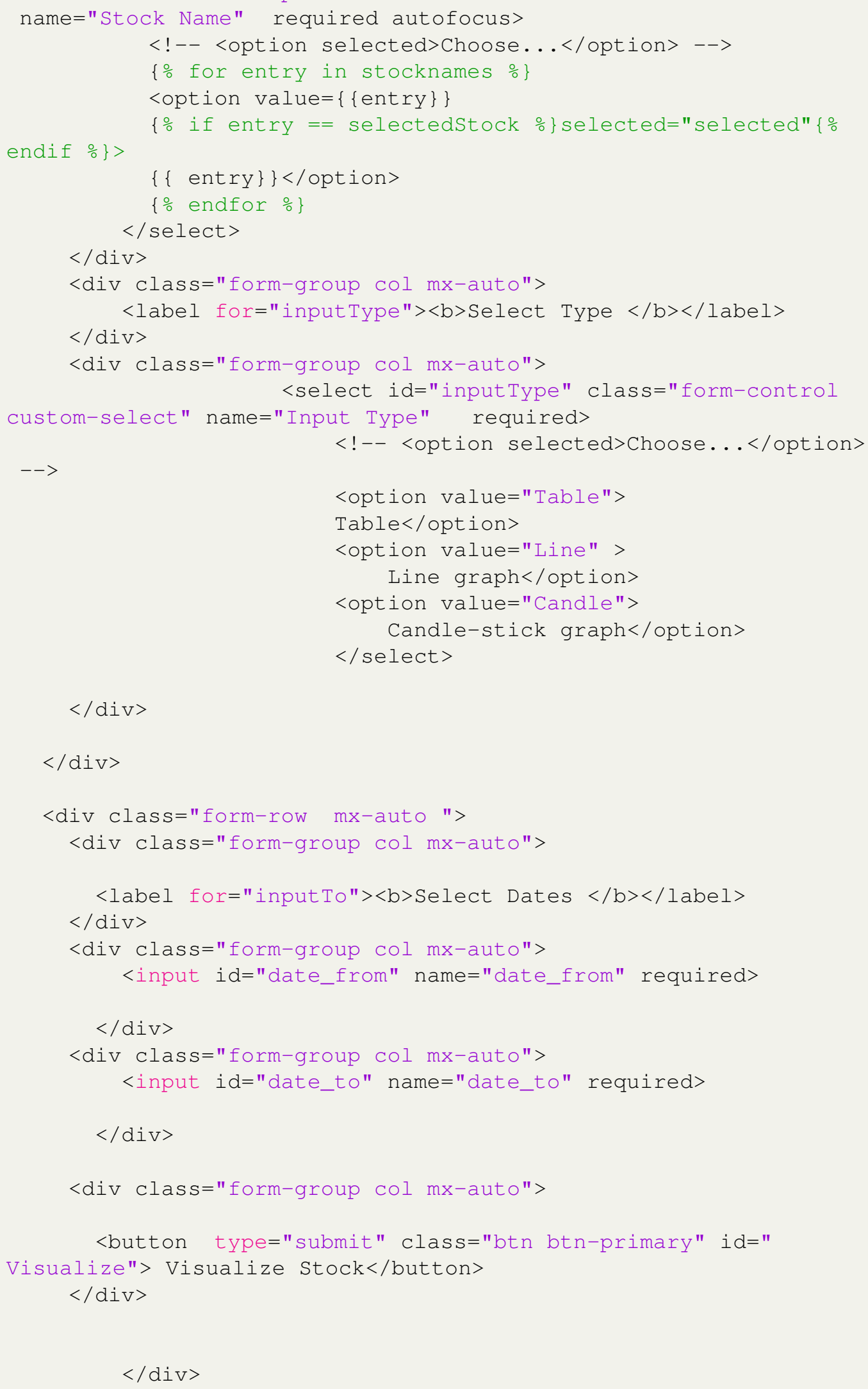




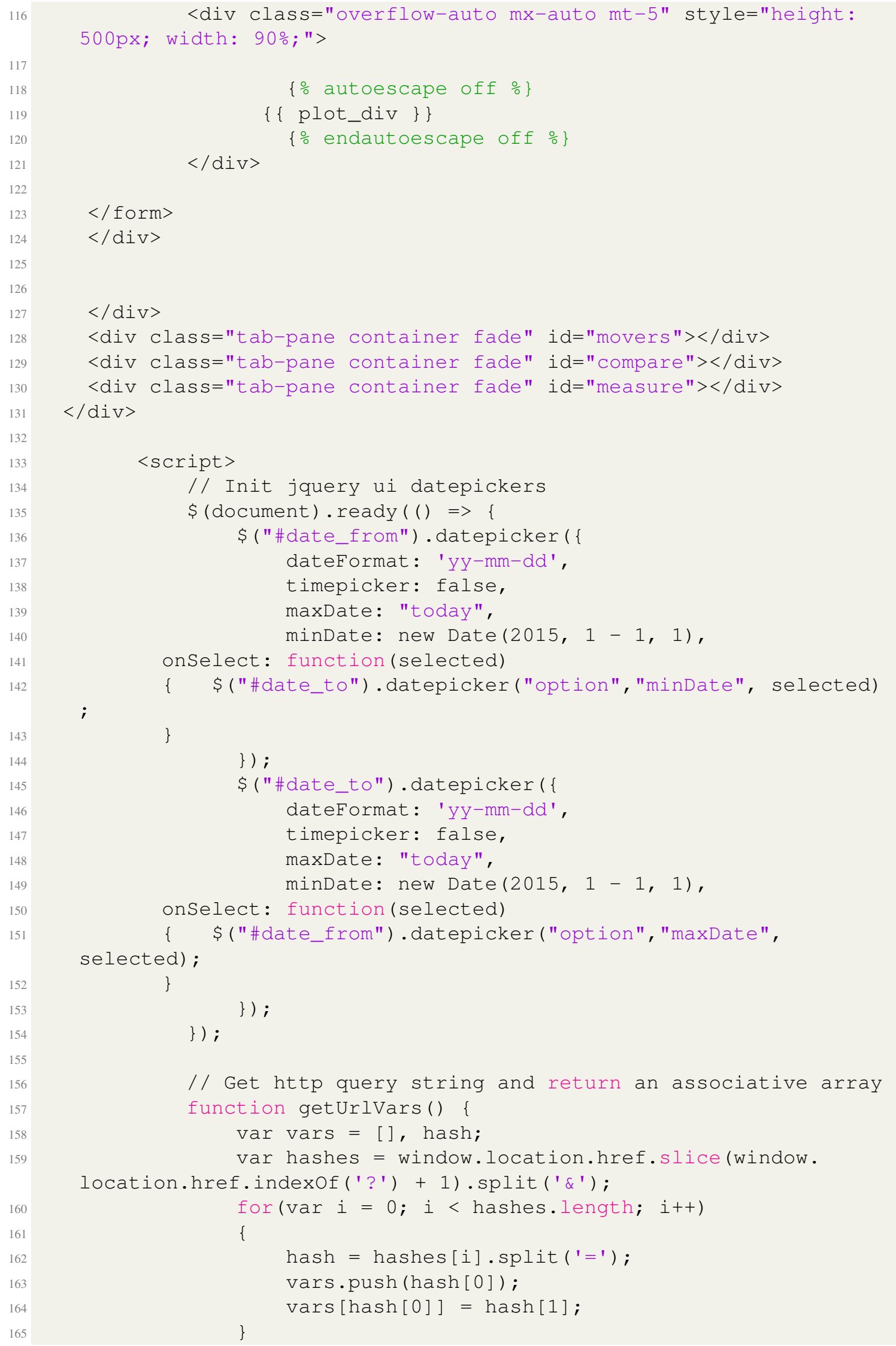




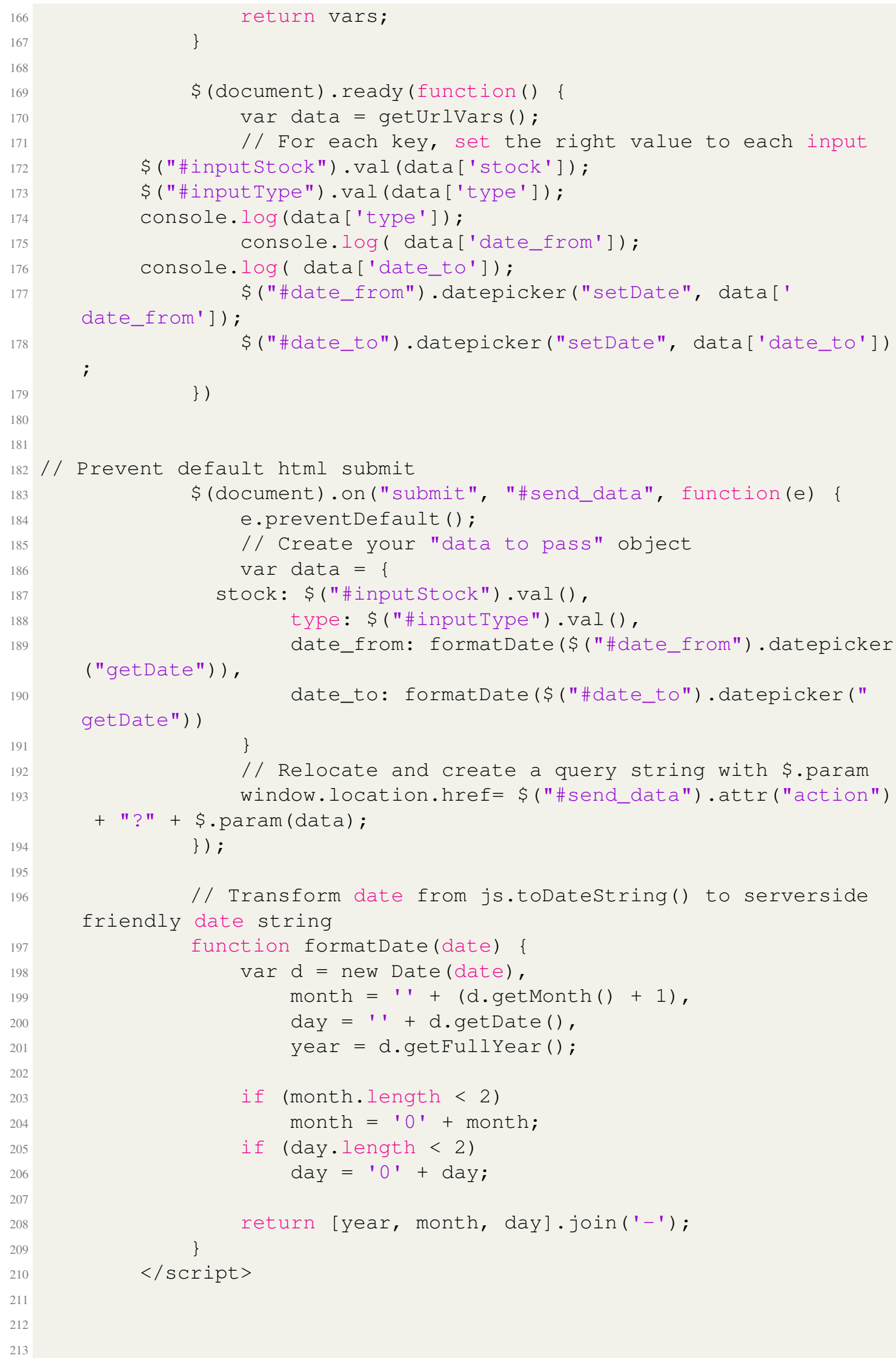


$</$ body $>$

$</$ html $>$

\section{Code A.2.3.9: analysisResultLine.html}

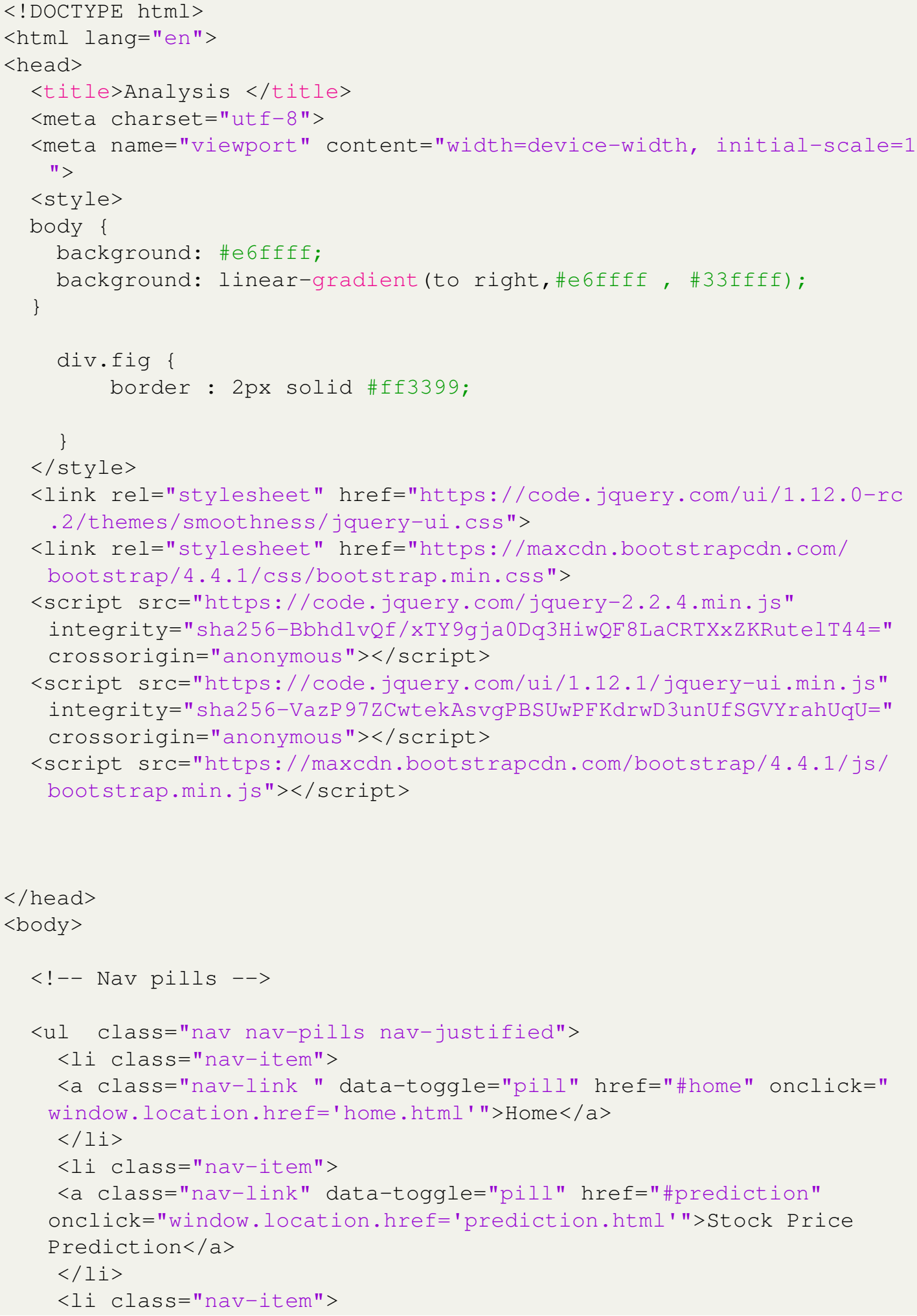


<a class="nav-link active" data-toggle="pill" href="\#analysis" onclick="window.location.href='analysis.html'" $>$ Historical Analysis $</ a>$

$</ 1 i>$

$<$ i class="nav-item" $>$

$<a$ class="nav-link" data-toggle="pill" href="\#movers" onclick=" window.location.href='movers.html'" $>$ Market Movers</a> 
$<!--<o p t i o n$ selected>Choose...</option>

<option value="Table"> Table</option>

<option value="Line">

Line graph</option>

<option value="Candle" $>$

Candlestick chart</option>

$</$ select $>$

$</$ div $>$

$</$ div $>$

<div class="form-row mx-auto ">

<div class="form-group col mx-auto">

$<$ label for="inputTo" $\rangle\langle$ b $\rangle$ Select Dates $\langle/$ b $\rangle\langle/$ label $\rangle$ $</$ div $>$

<div class="form-group col mx-auto">

<input id="date_from" name="date_from" required>

$</$ div $>$

<div class="form-group col mx-auto">

<input id="date_to" name="date_to" required>

$</$ div $>$

<div class="form-group col mx-auto">

<button type="submit" class="btn btn-primary" id=" Visualize" $>$ Visualize Stock</button $>$ $</$ div $>$

$</$ div $>$

<div class="overflow-auto mx-auto mt-5" style="height: 500px; width: $100 \% ; ">$

$\left\{\frac{\circ}{\circ}\right.$ autoescape off $\left.\div\right\}$

$\{\{$ datadf | safe\}\}

$\left\{\frac{\circ}{0}\right.$ endautoescape $\left.\frac{\circ}{\circ}\right\}$

$</$ div $>$

$</$ form $>$

$</$ div $>$

$</ \operatorname{div}\rangle$

<iv class="tab-pane container fade" id="movers" $></$ div $\rangle$

<div class="tab-pane container fade" id="compare" $></$ div $\rangle$

<div class="tab-pane container fade" id="measure" $></$ div $\rangle$ 


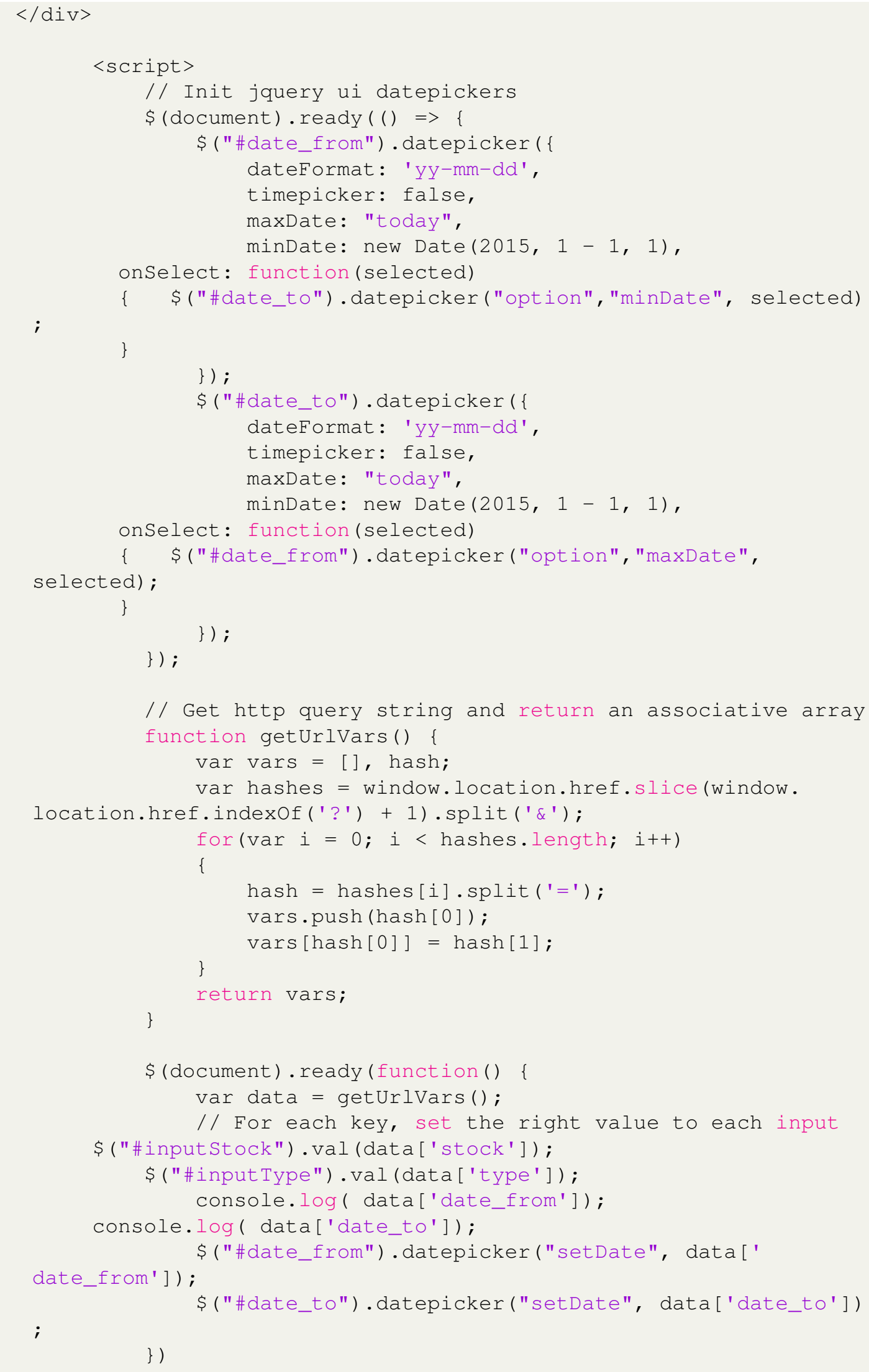




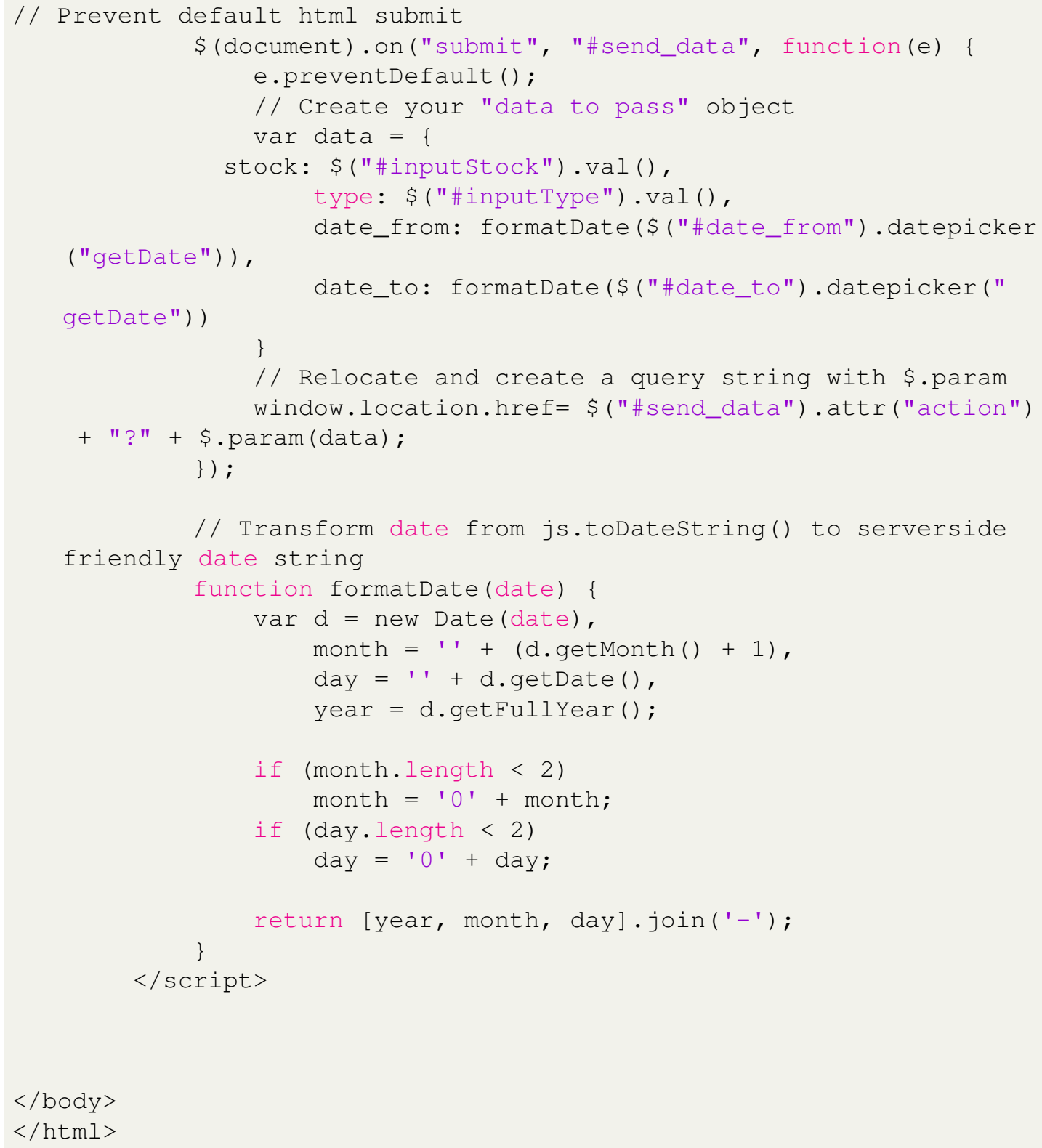

\section{Code A.2.3.10: analysisResultTable.html}

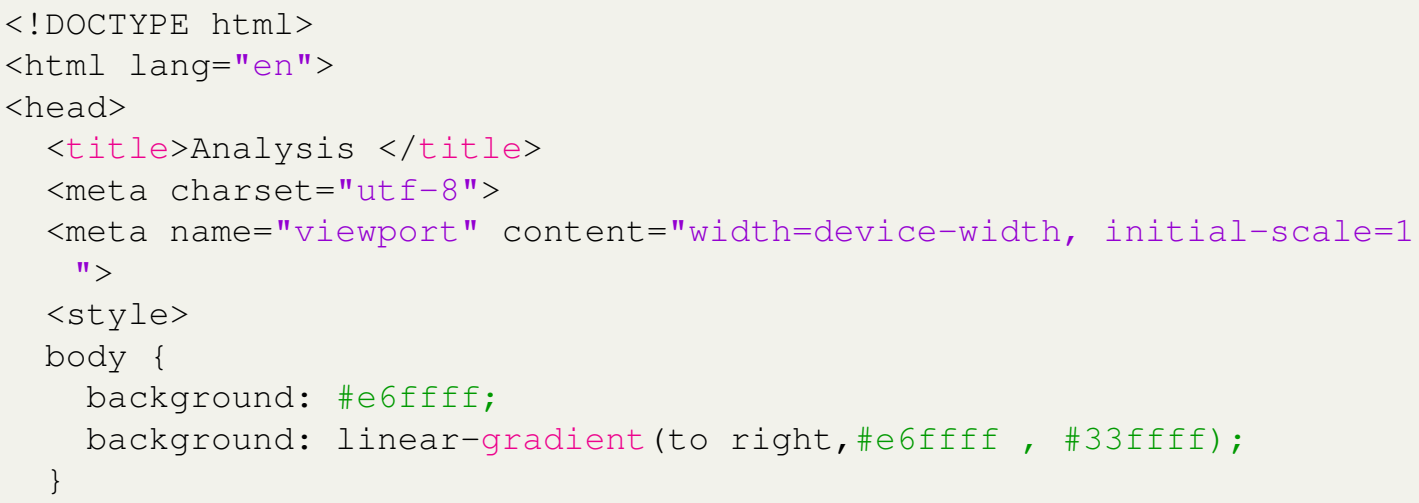




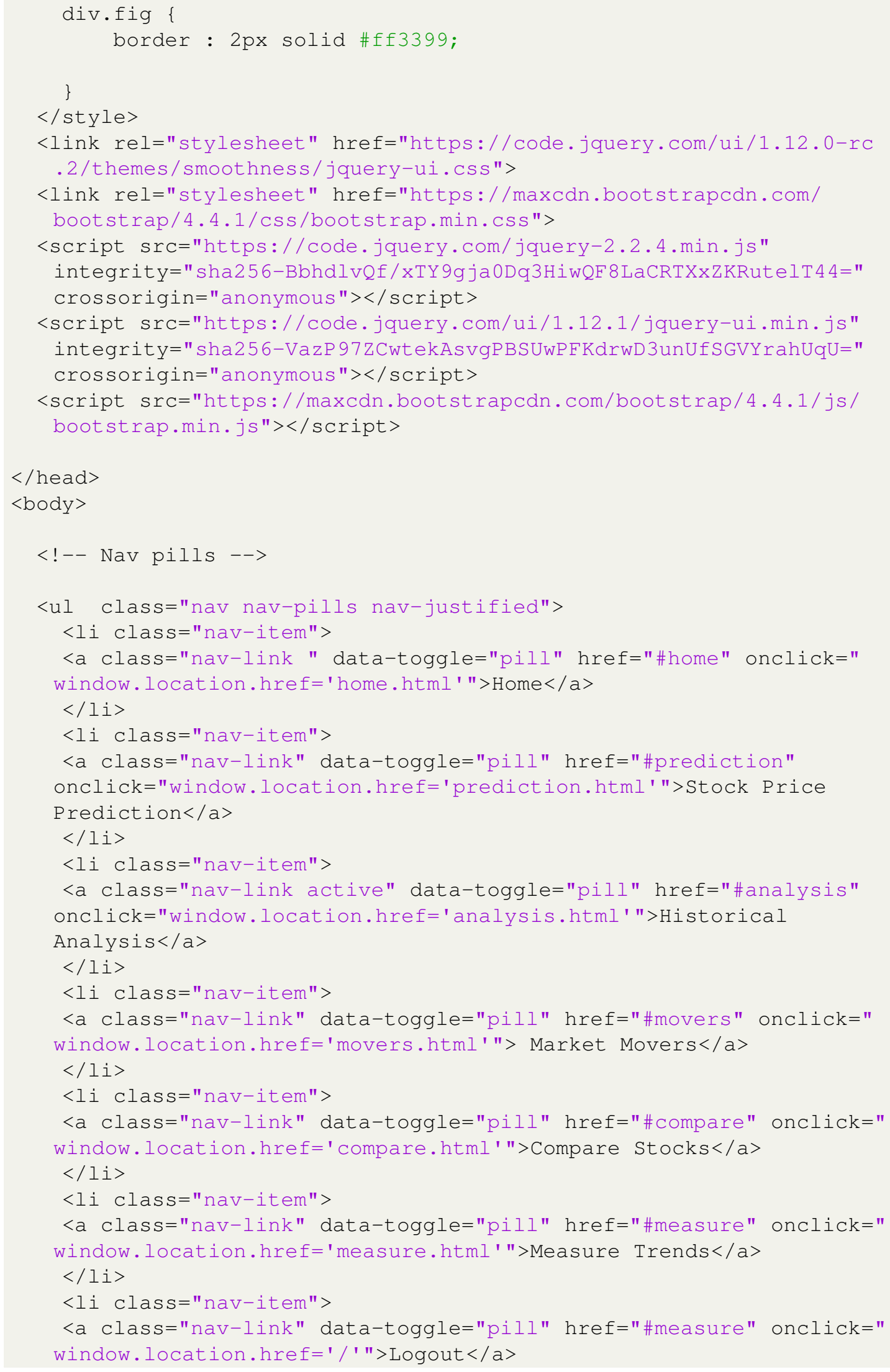




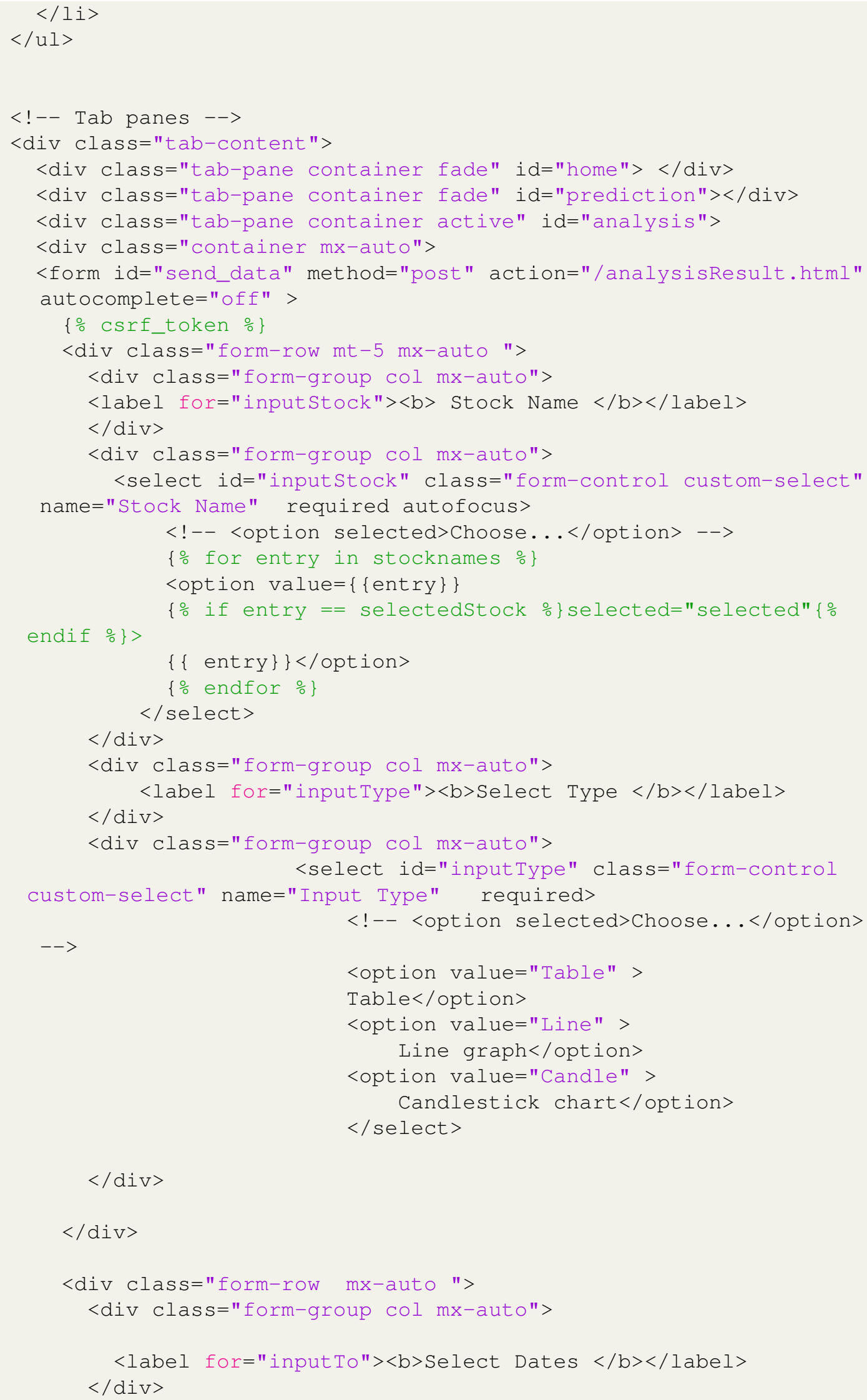




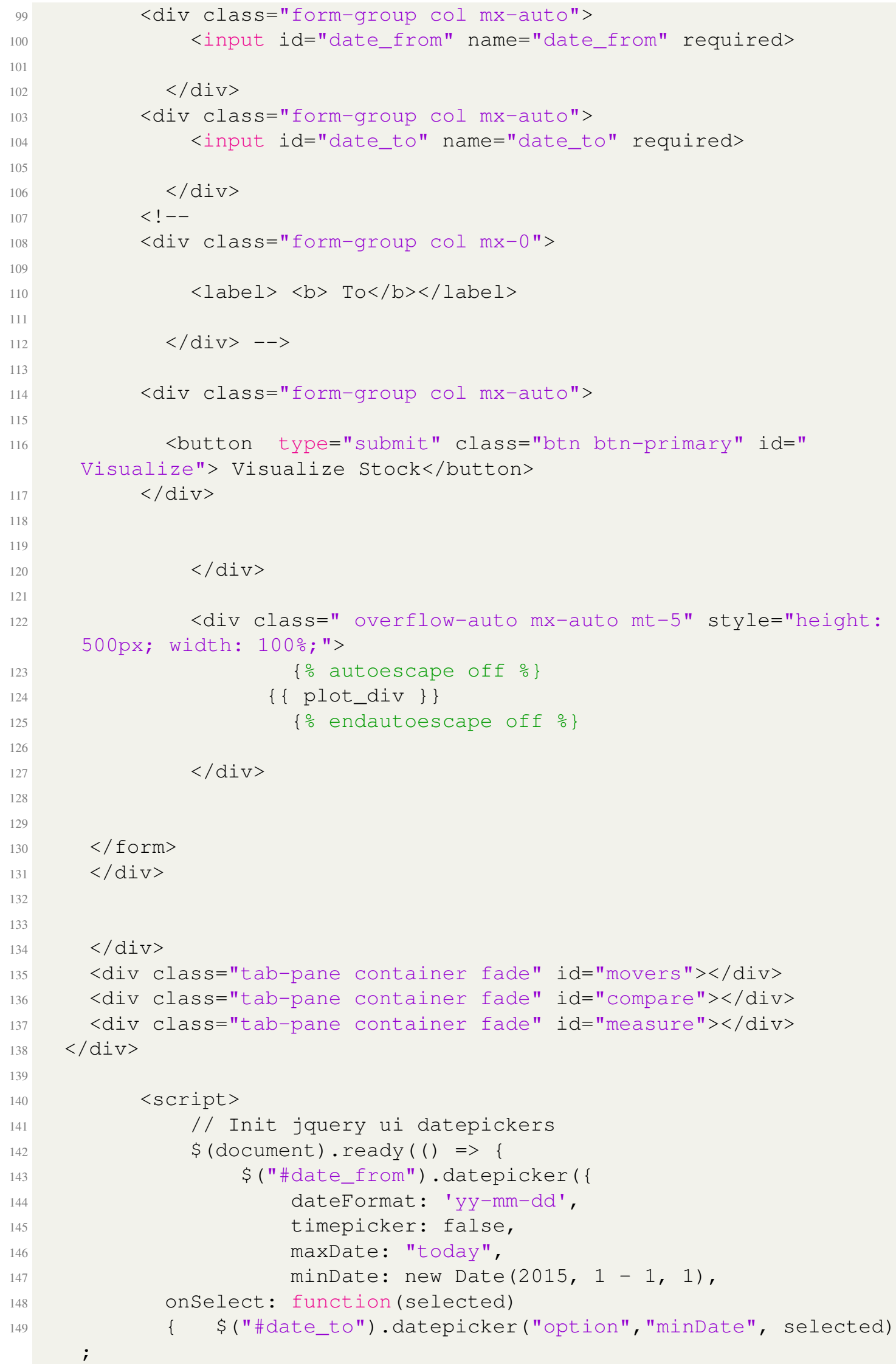




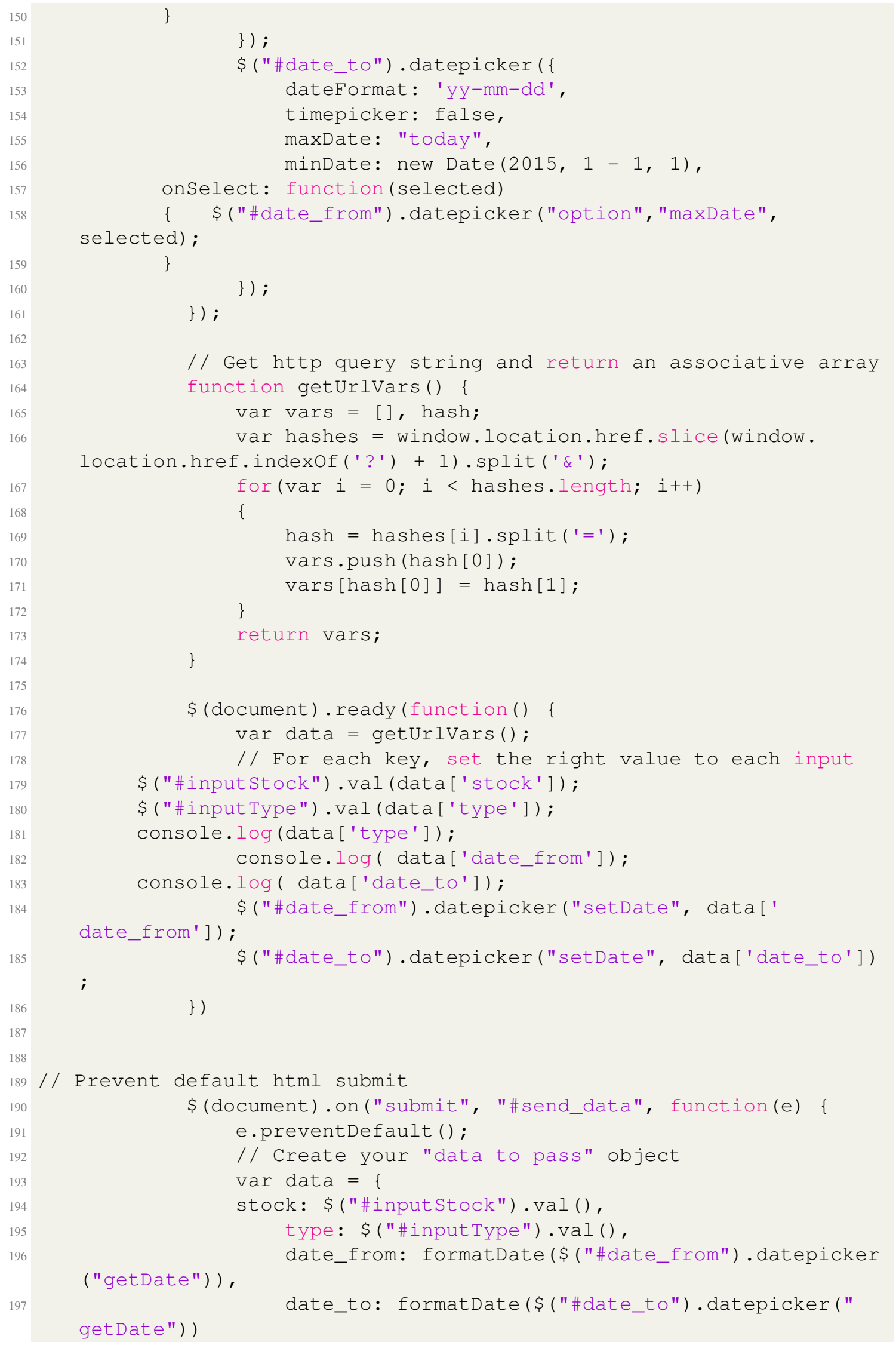




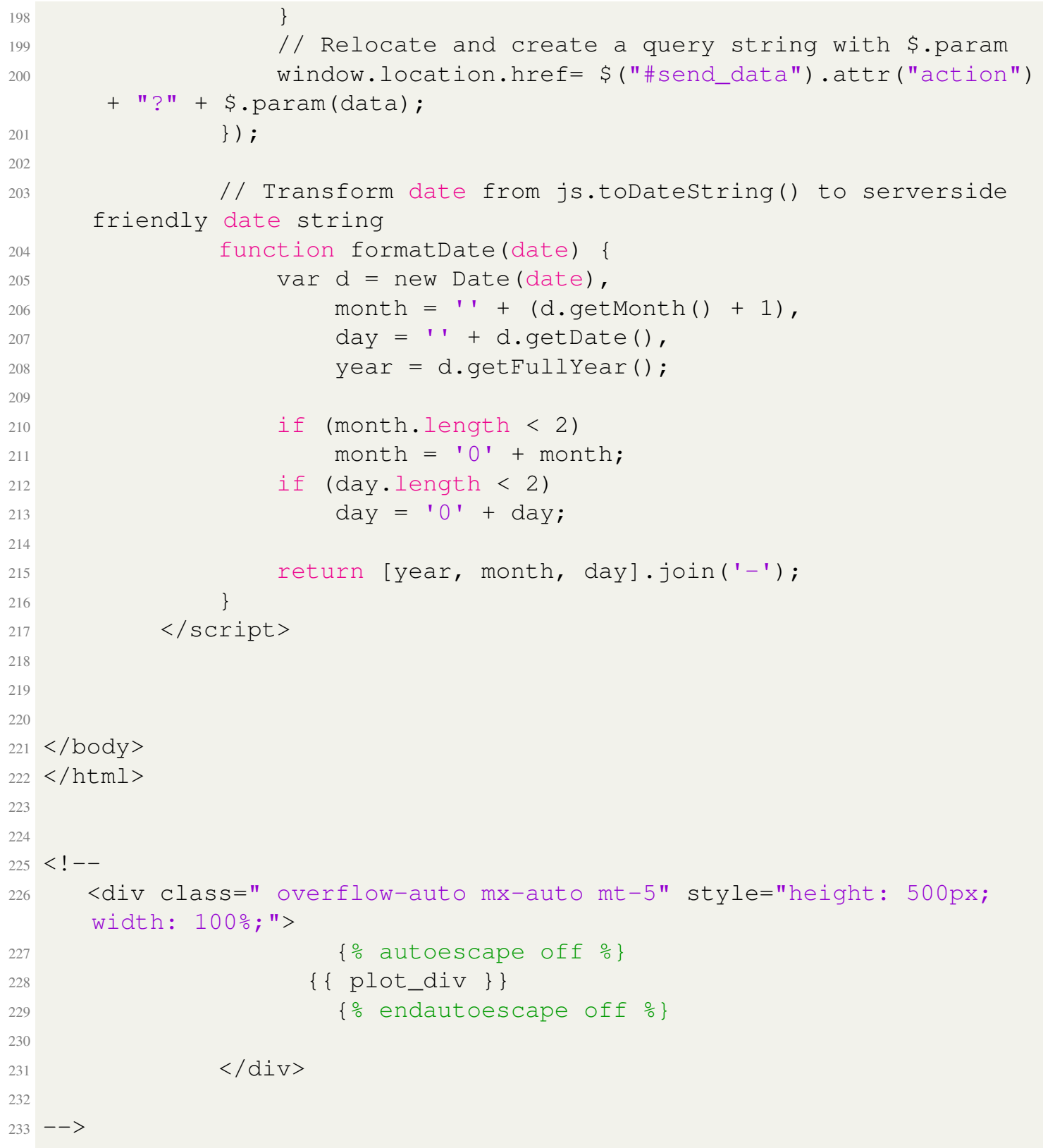

\section{Code A.2.3.11: analysisResultCandlestick.html}

\section{For comparing two stocks}

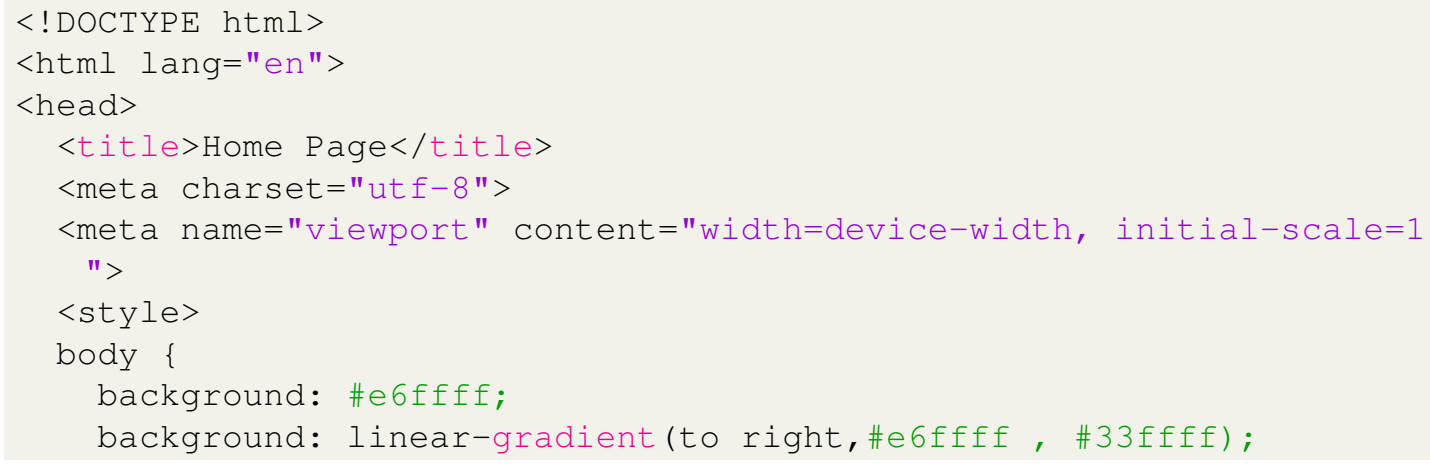




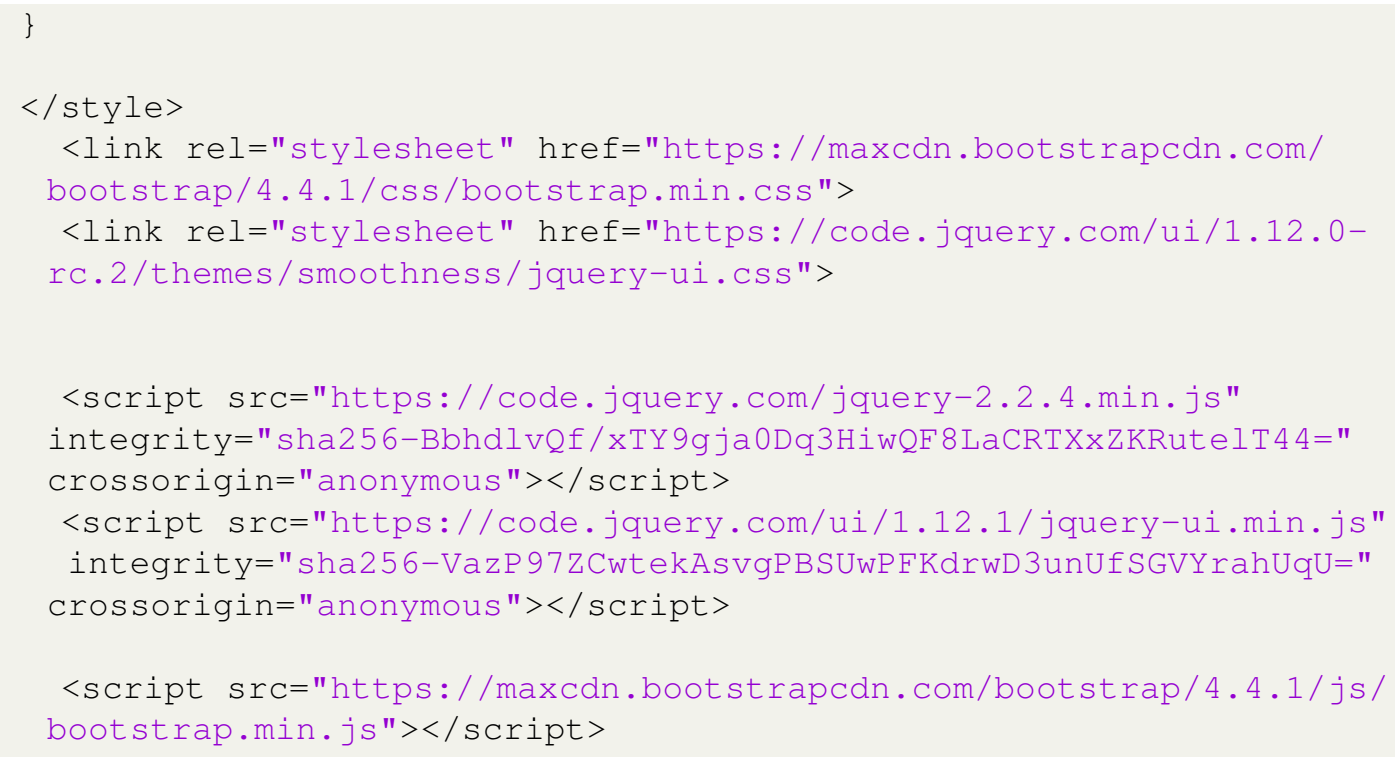




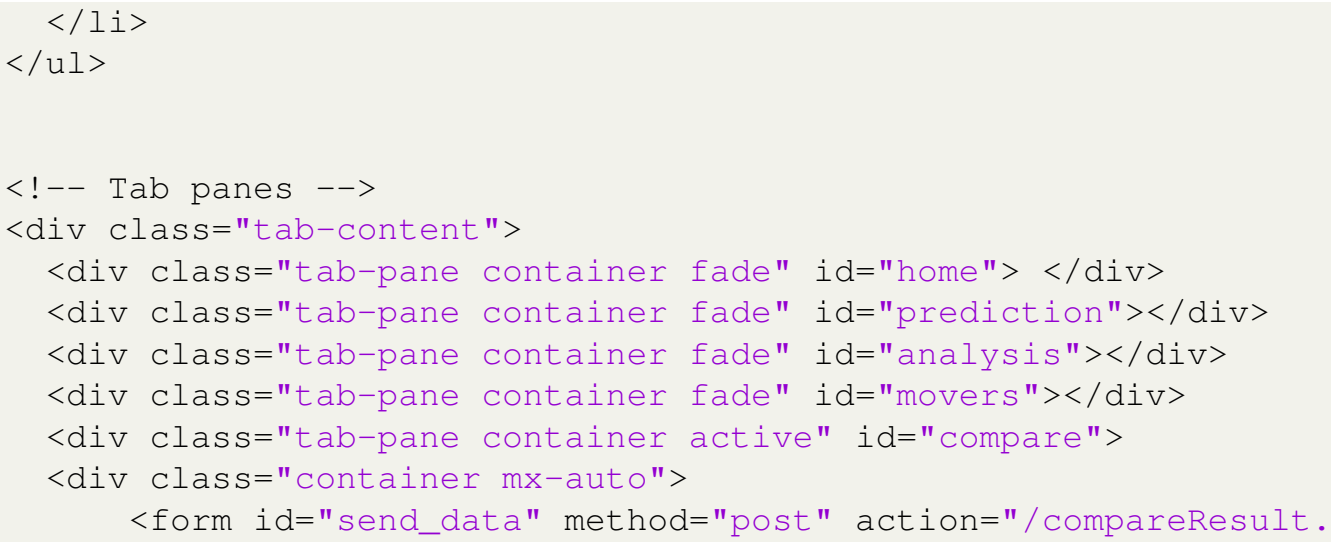




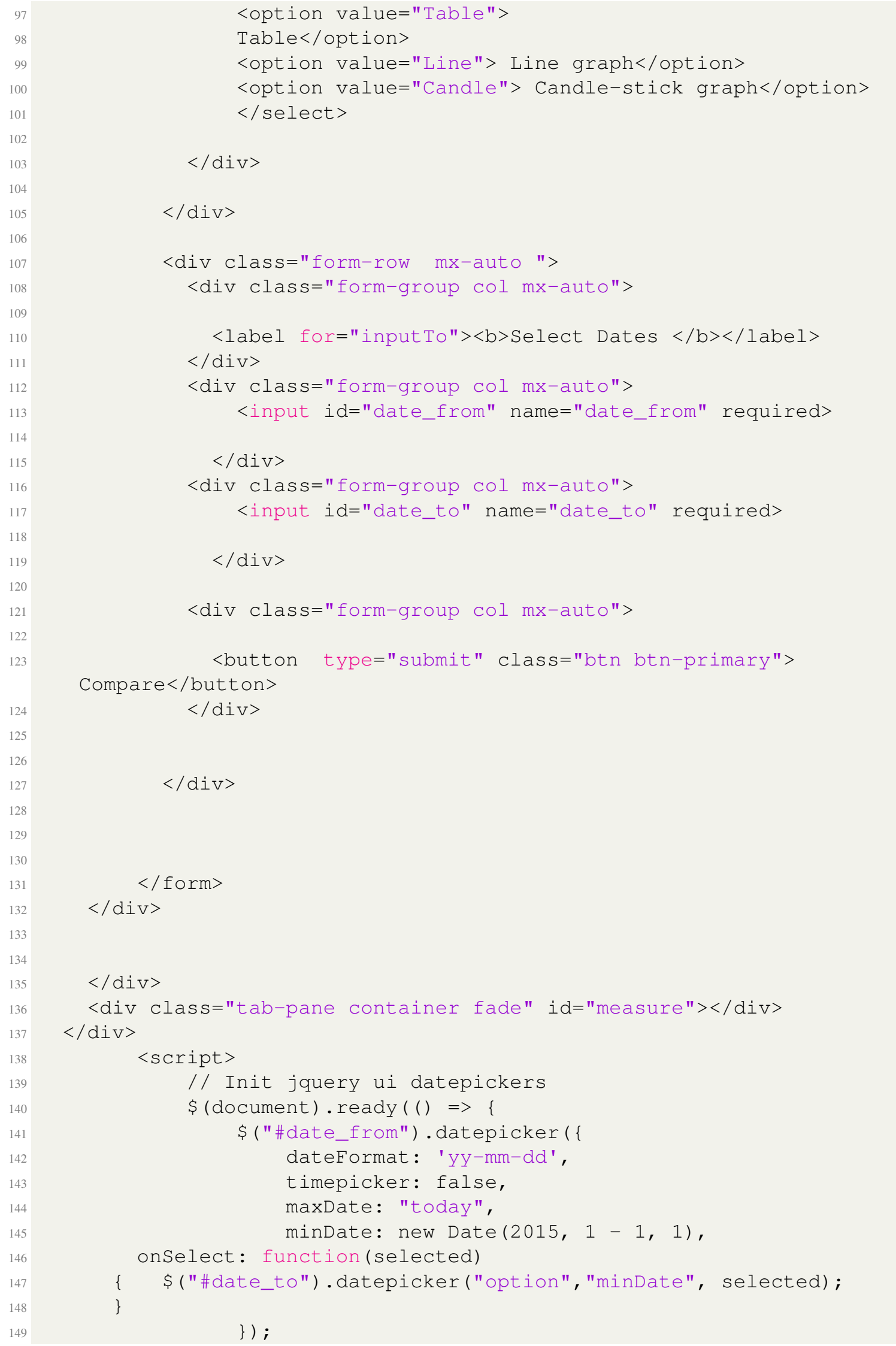




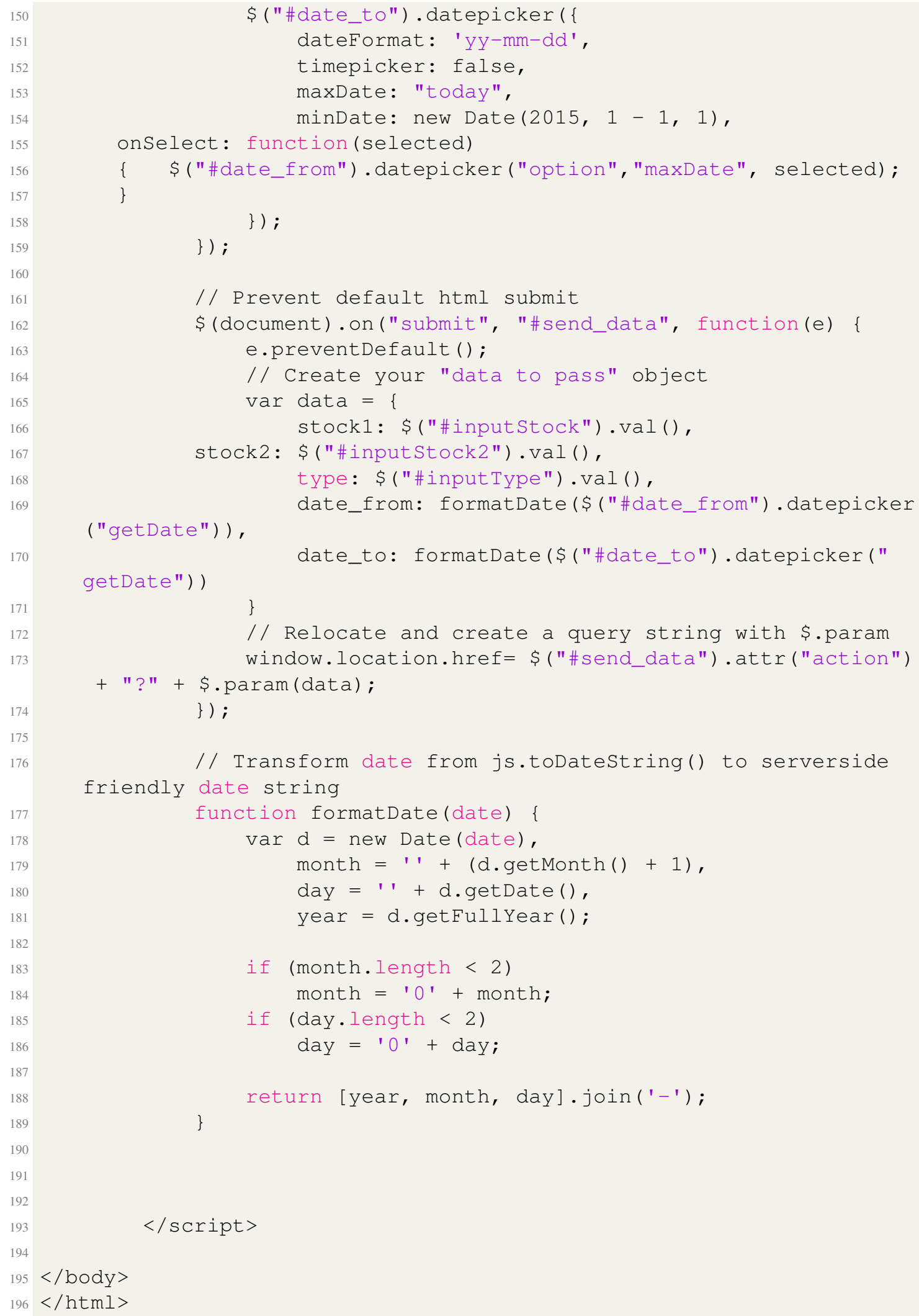

$196</$ html $>$

\$("\#date_to").datepicker ( \{ dateFormat: 'yy-mm-dd', timepicker: false, maxDate: "today", minDate: new Date(2015, $1-1,1)$, onselect: function (selected)

Code A.2.3.12: compare.html 


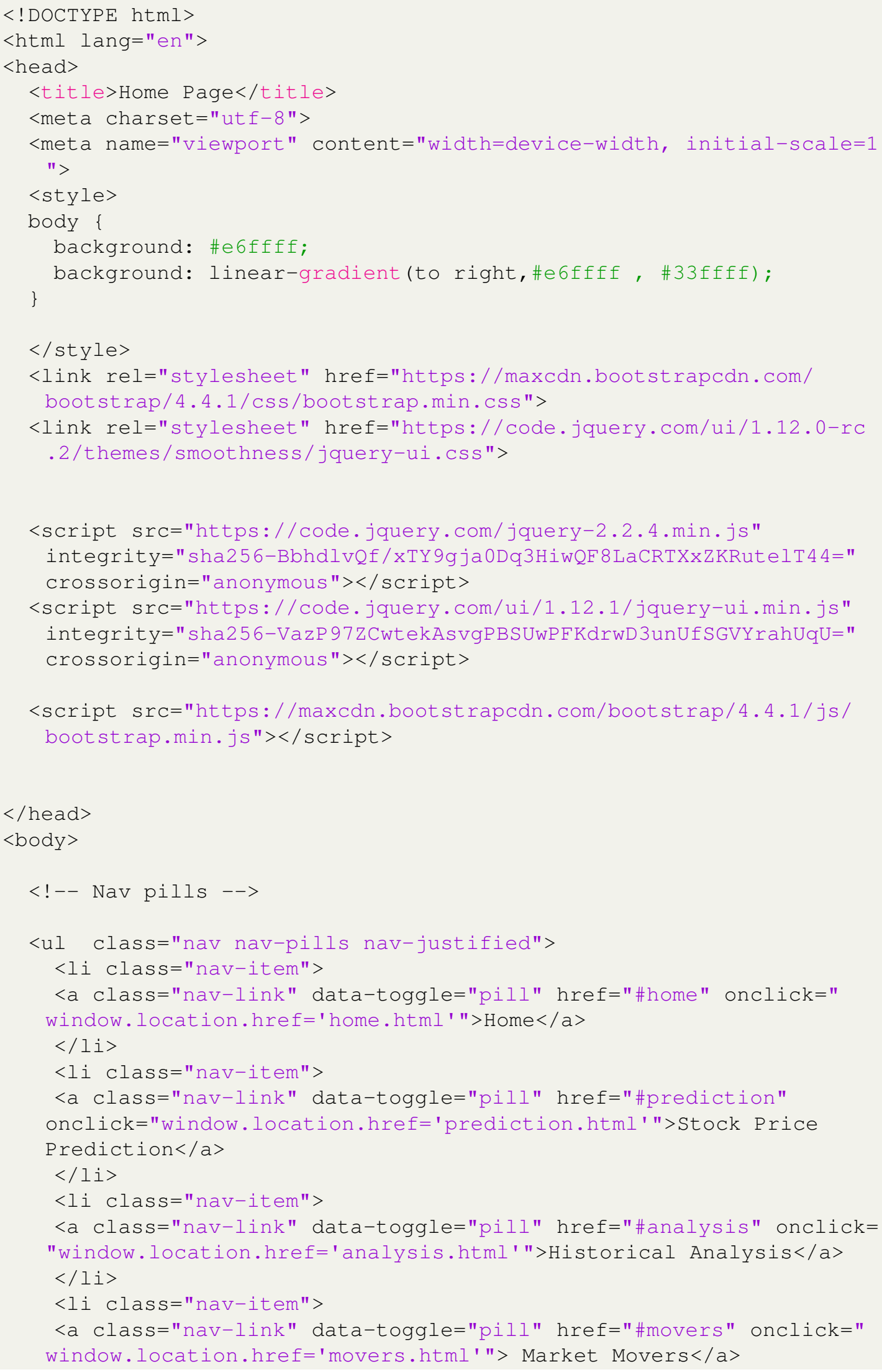




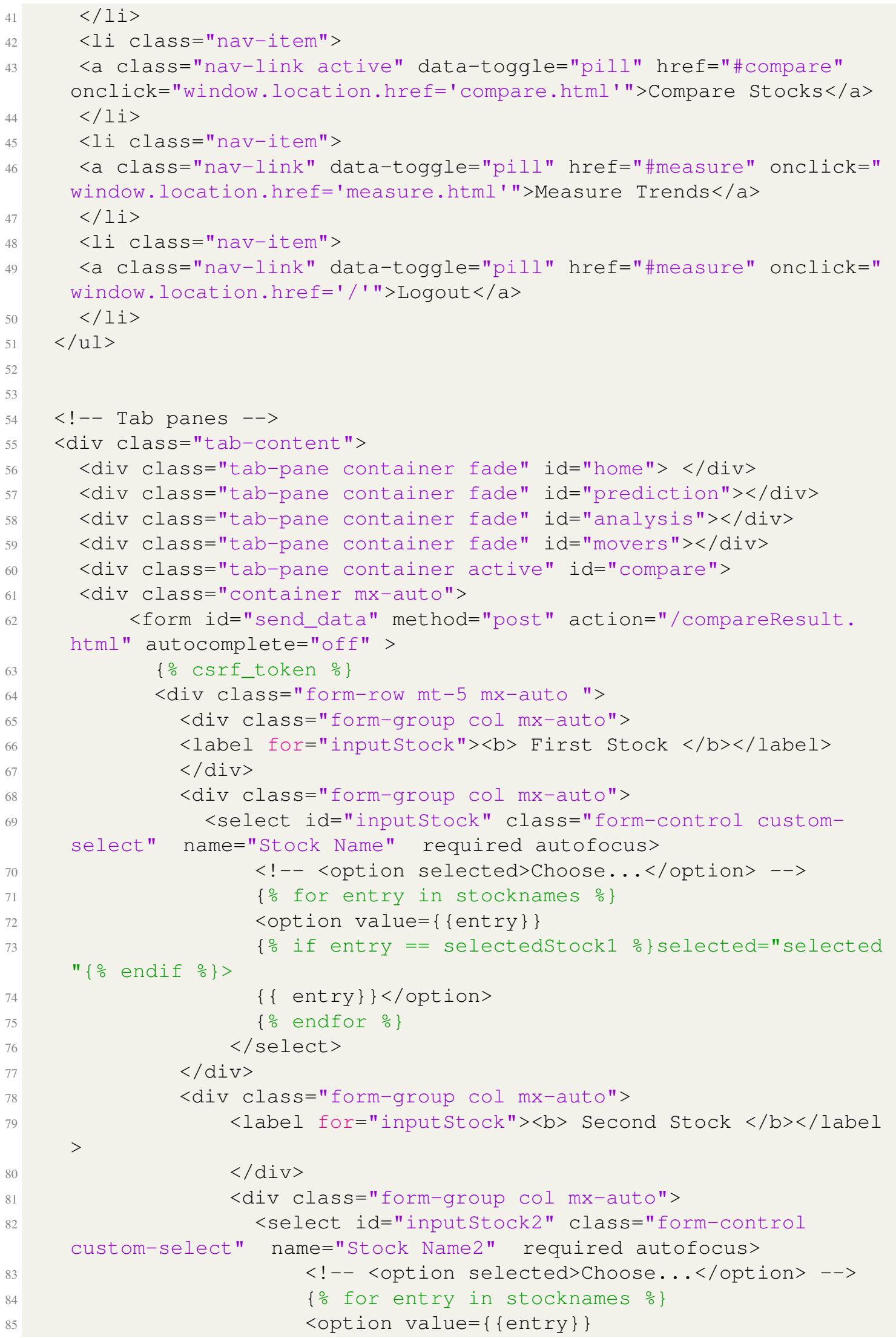




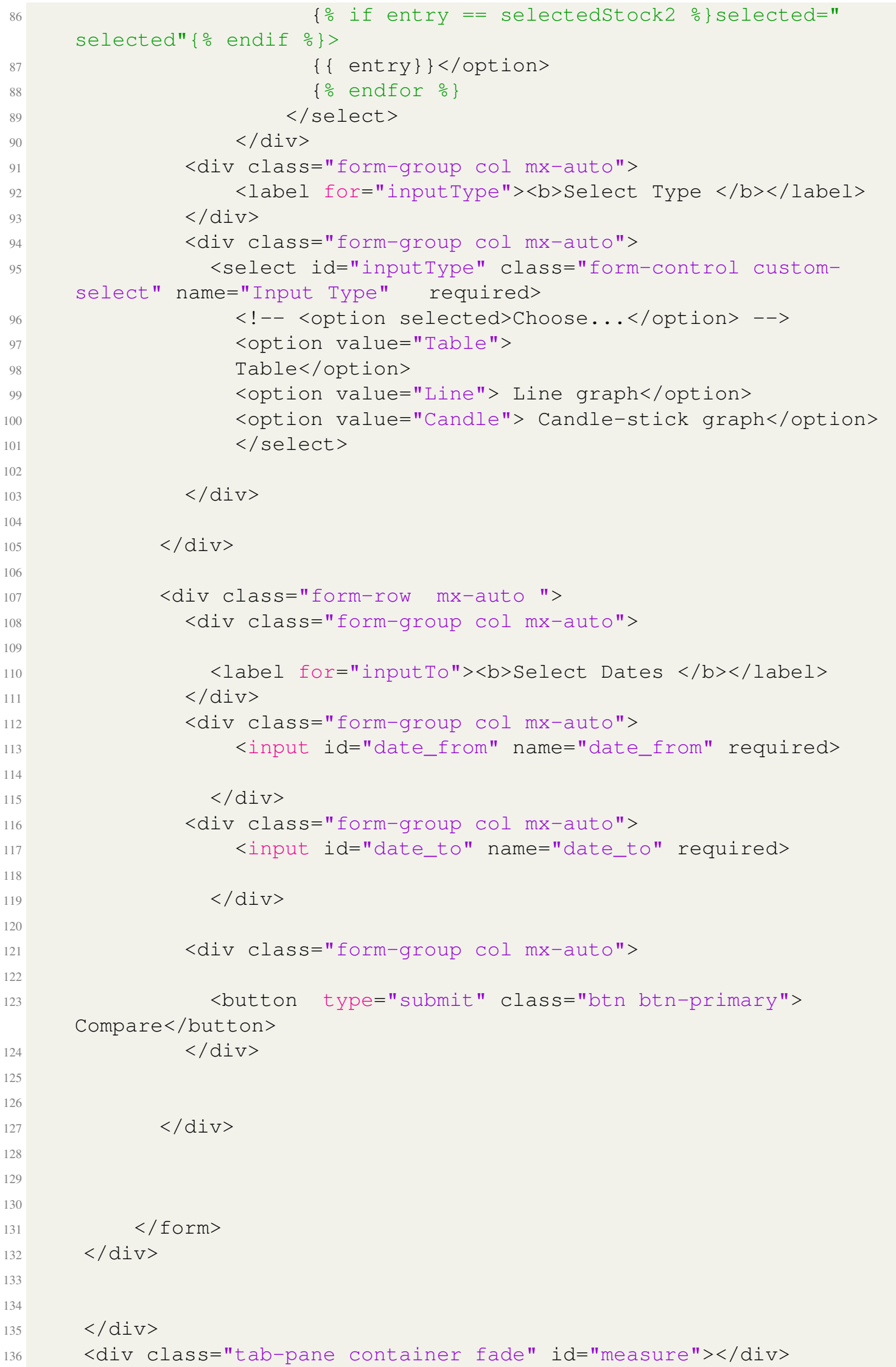




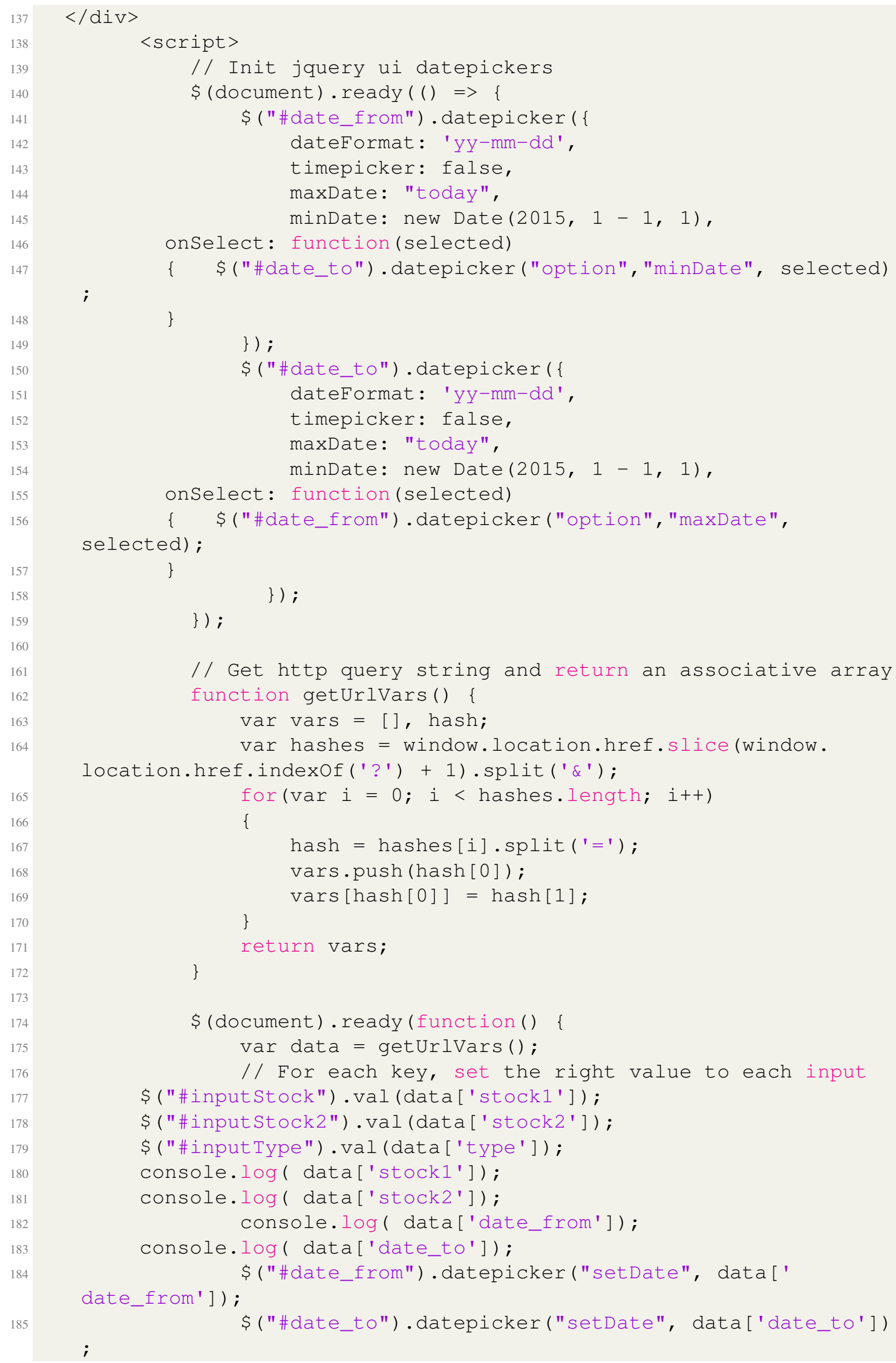




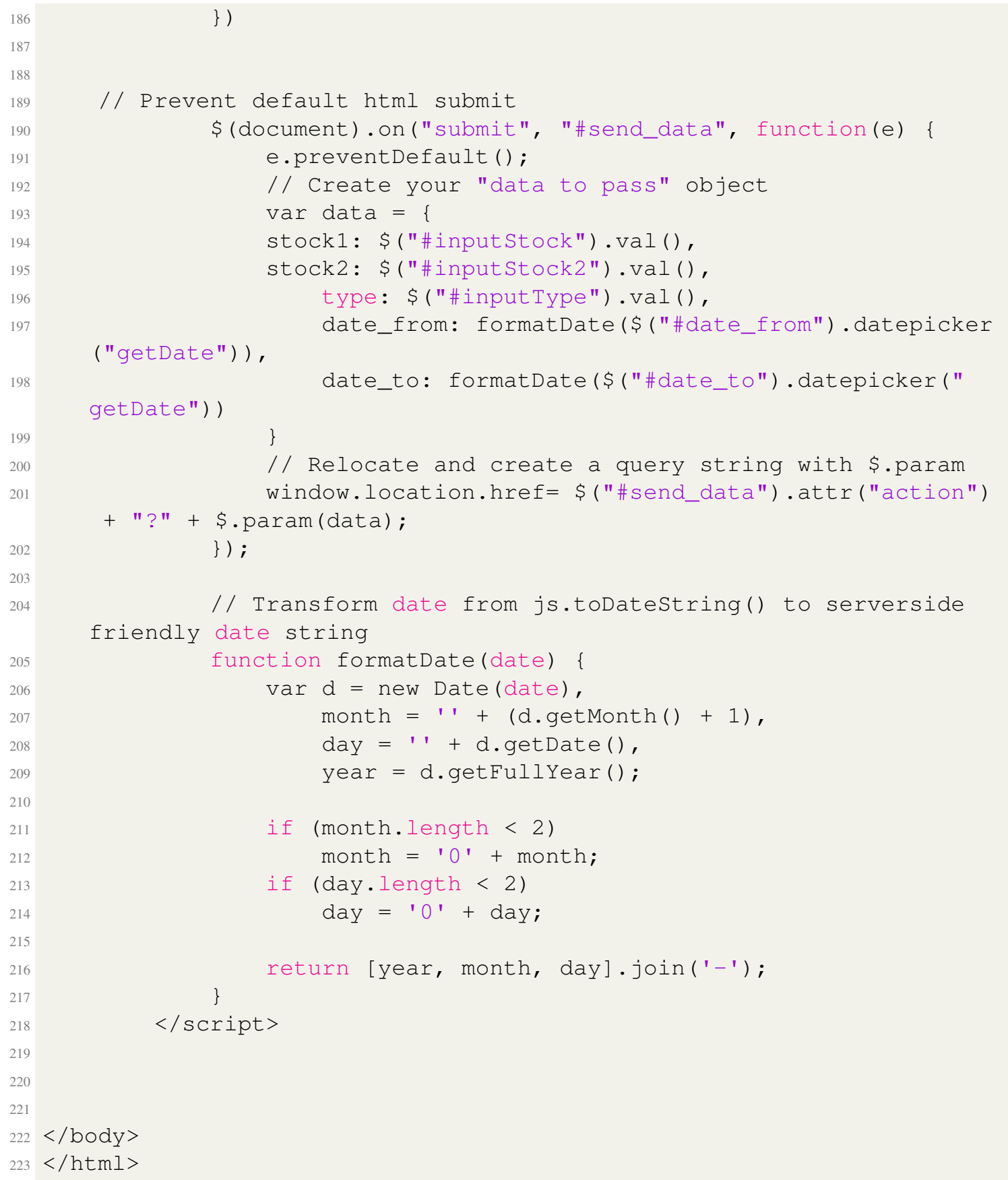

\section{Code A.2.3.13: compareResult.html}

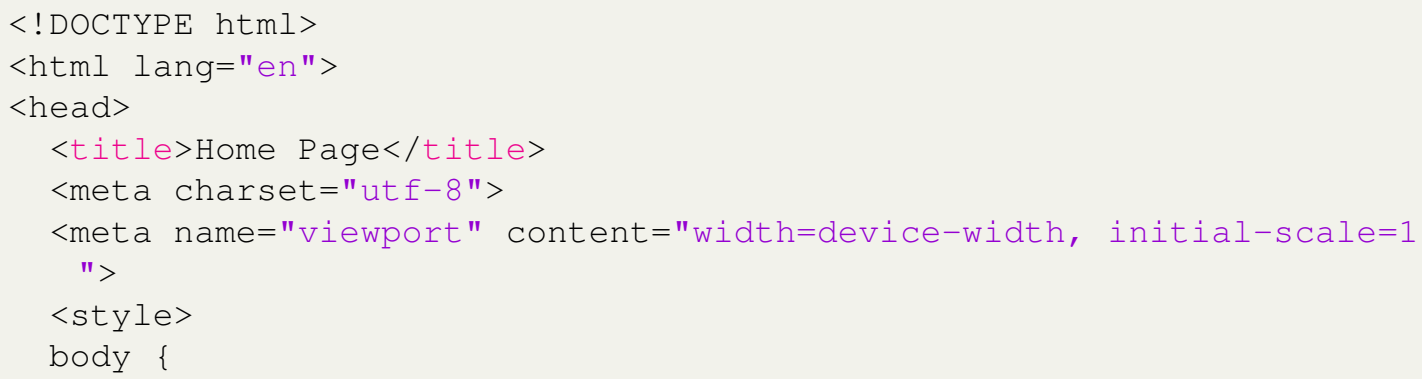




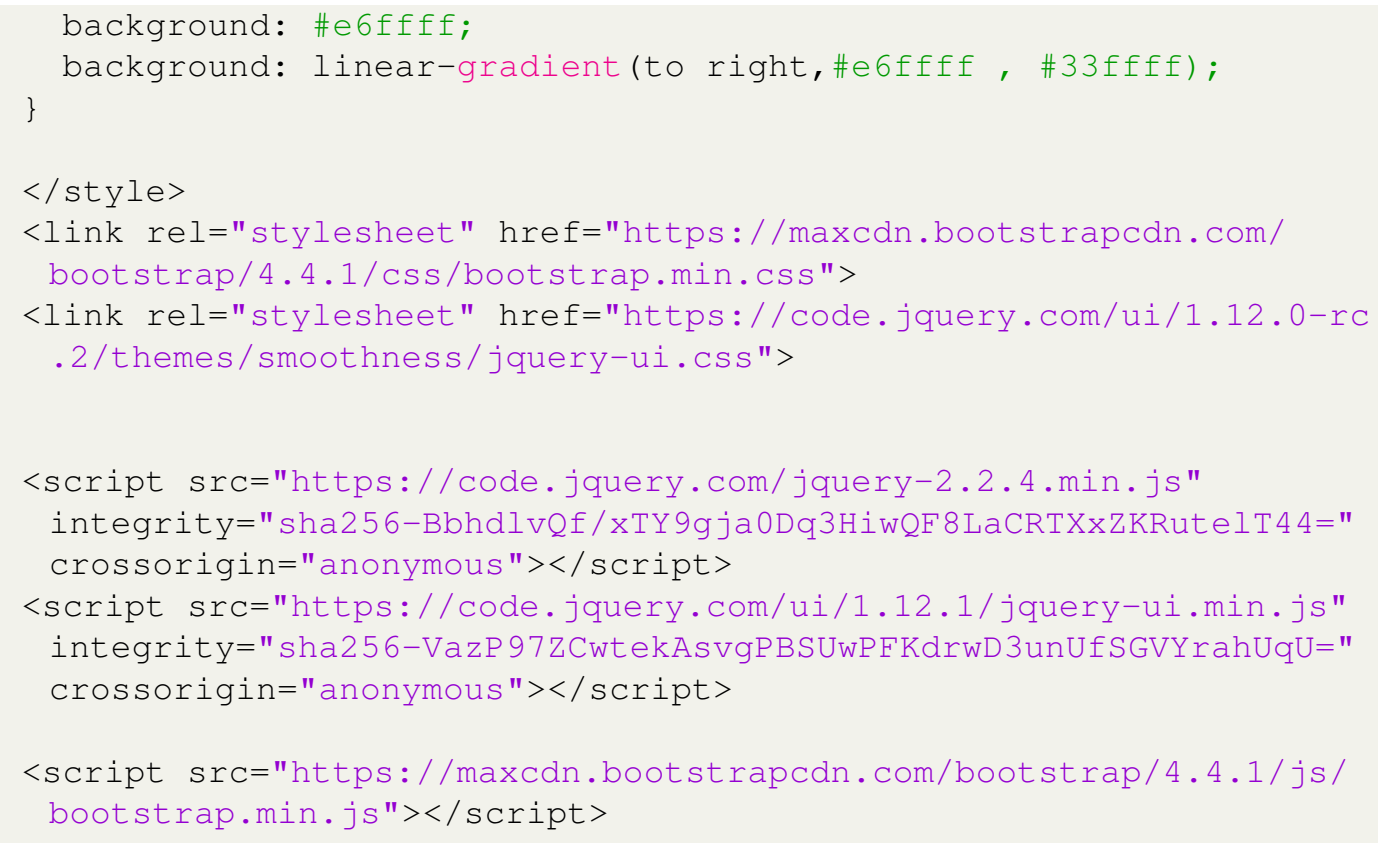




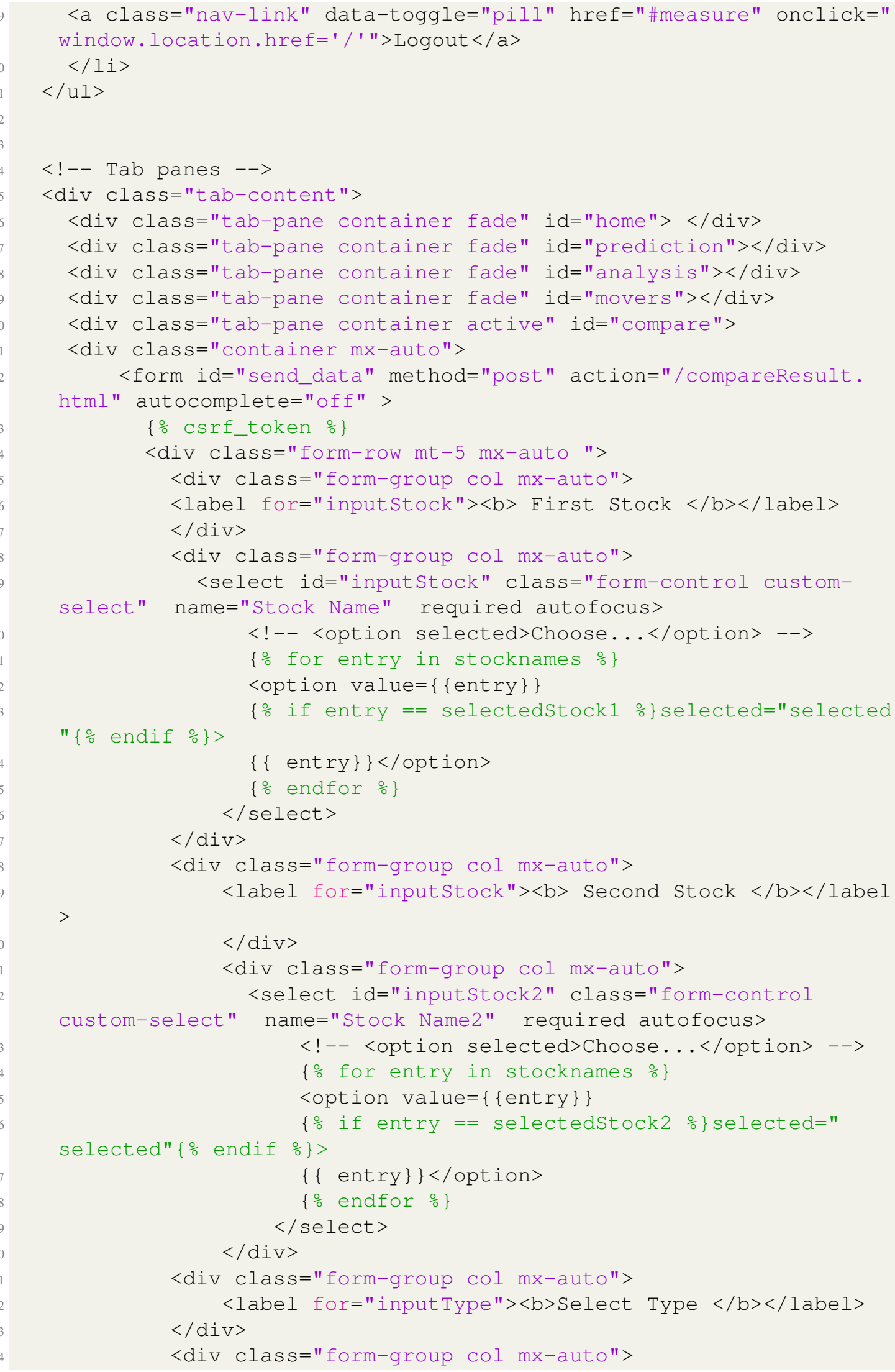




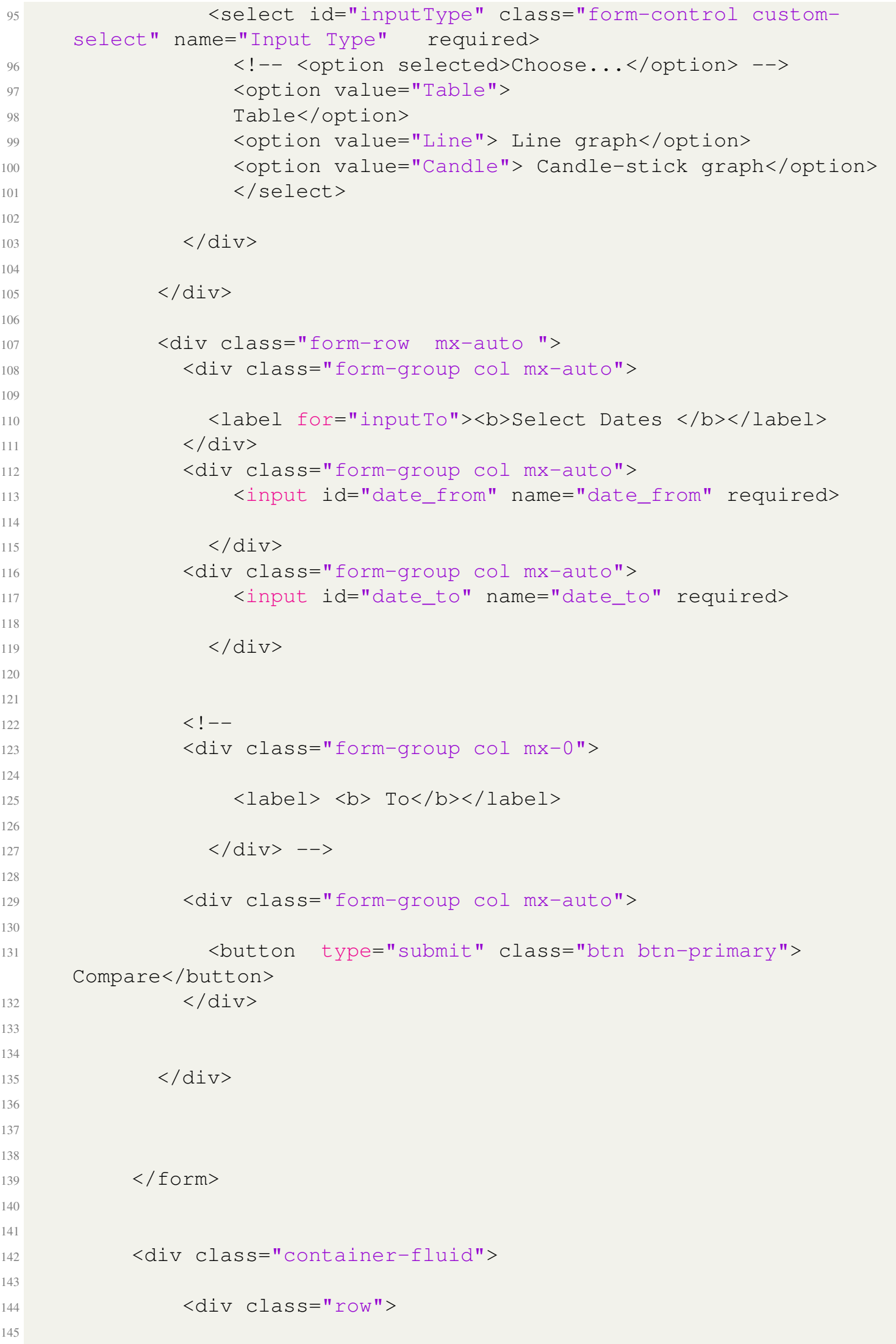




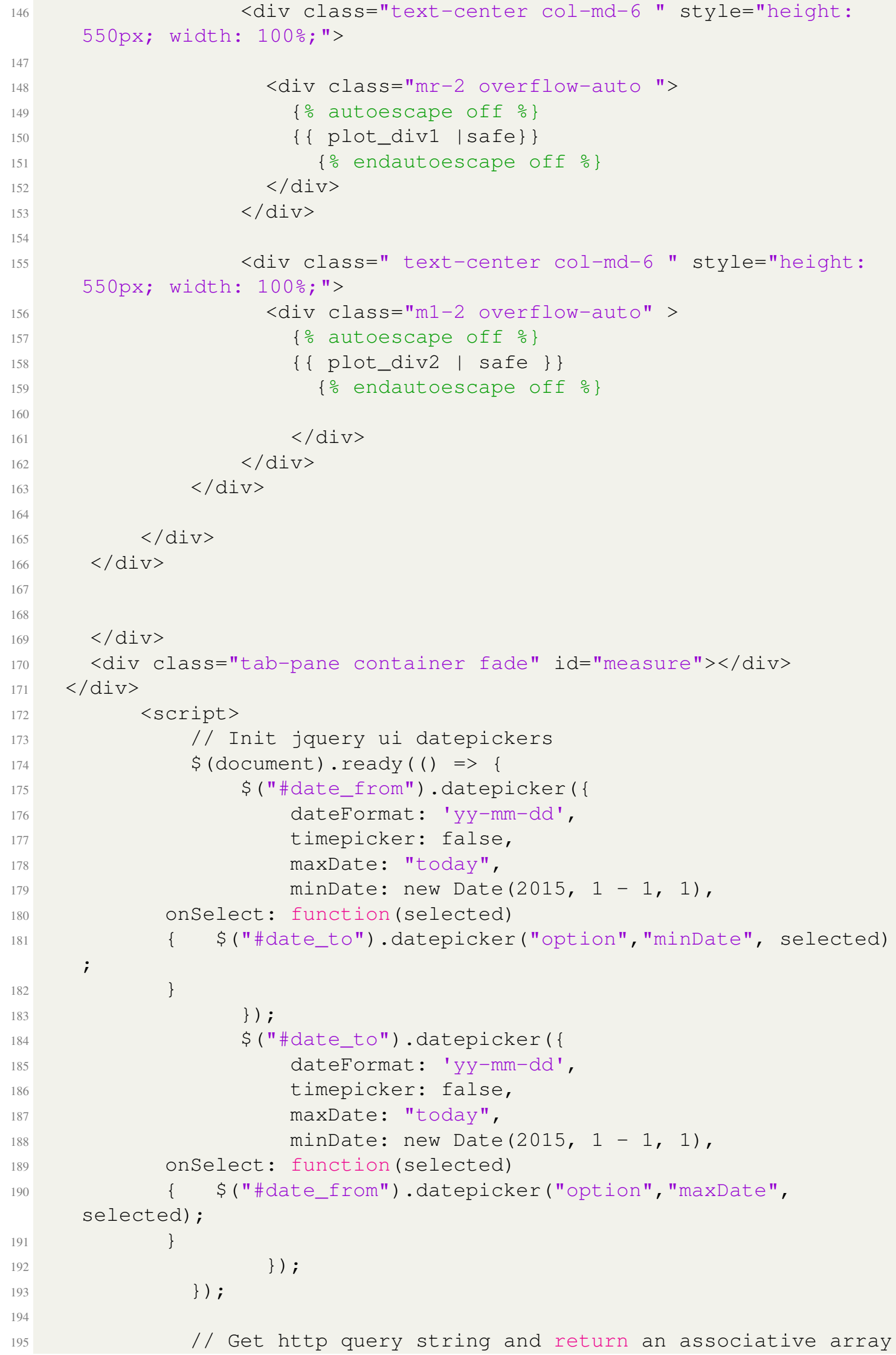




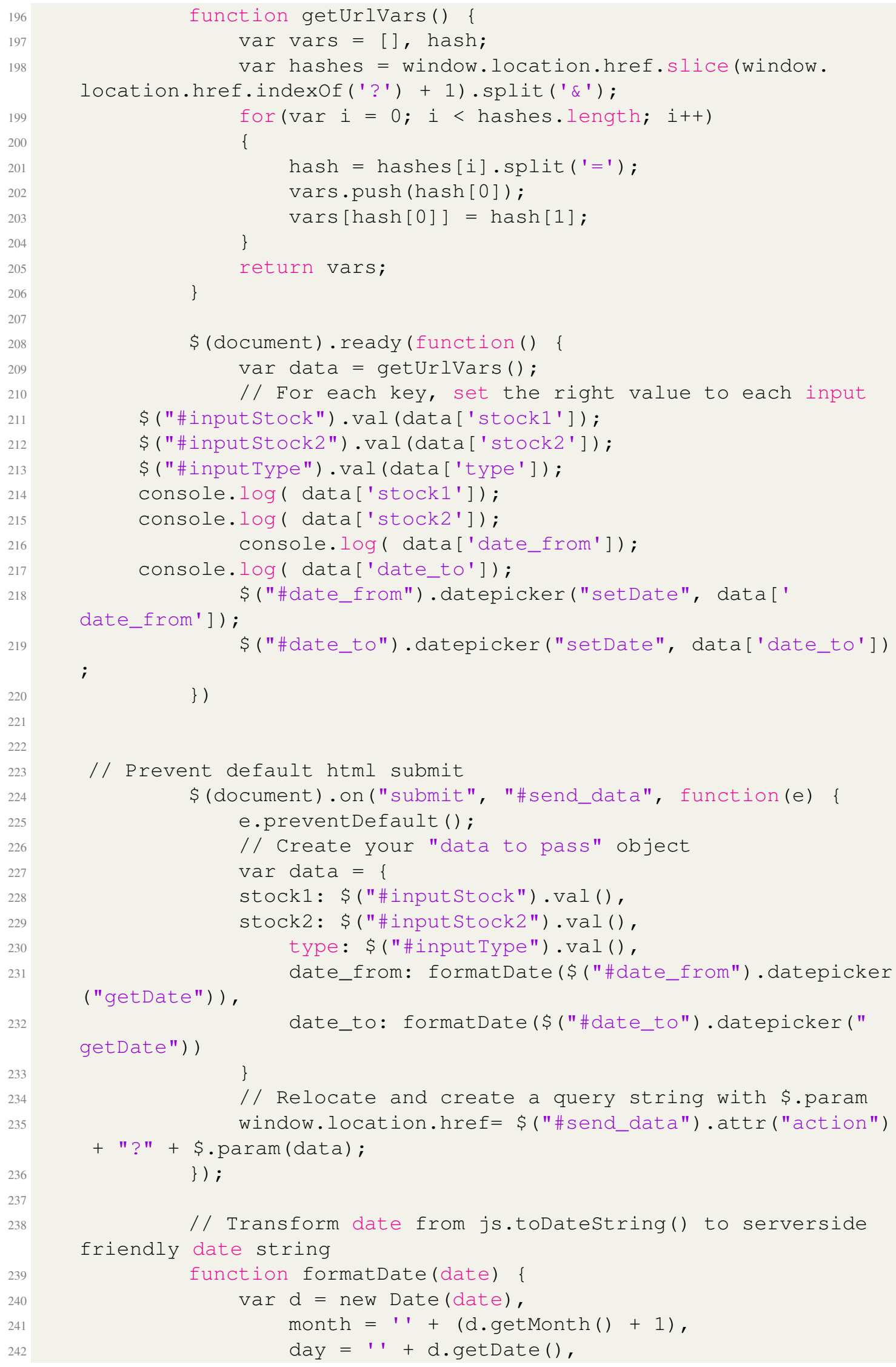




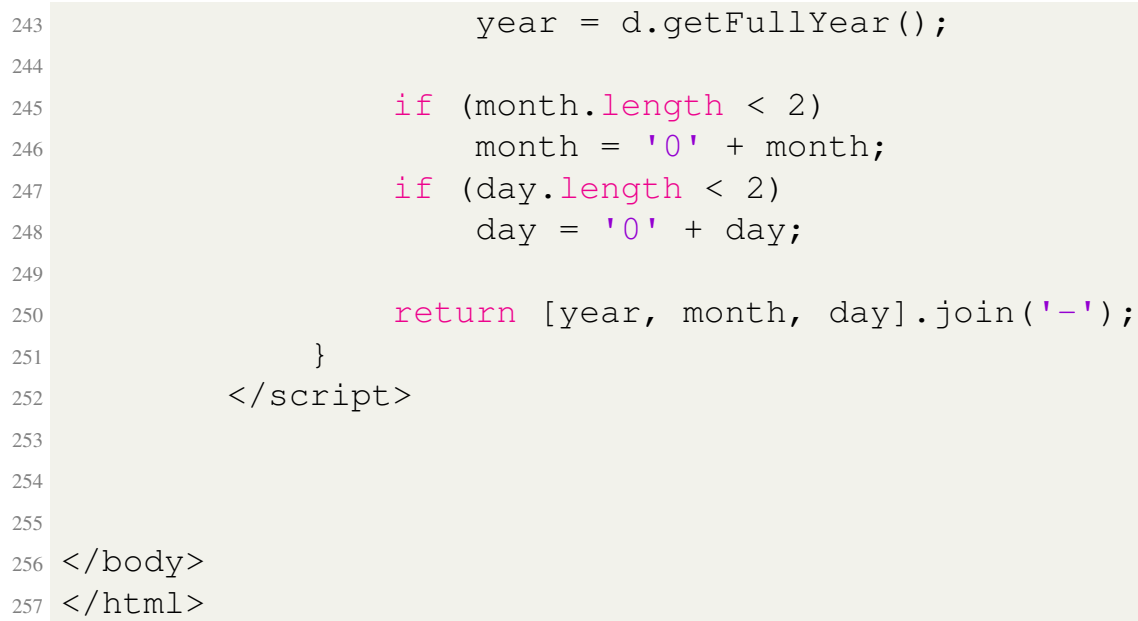

\section{Code A.2.3.14: compareCandleStick.html}

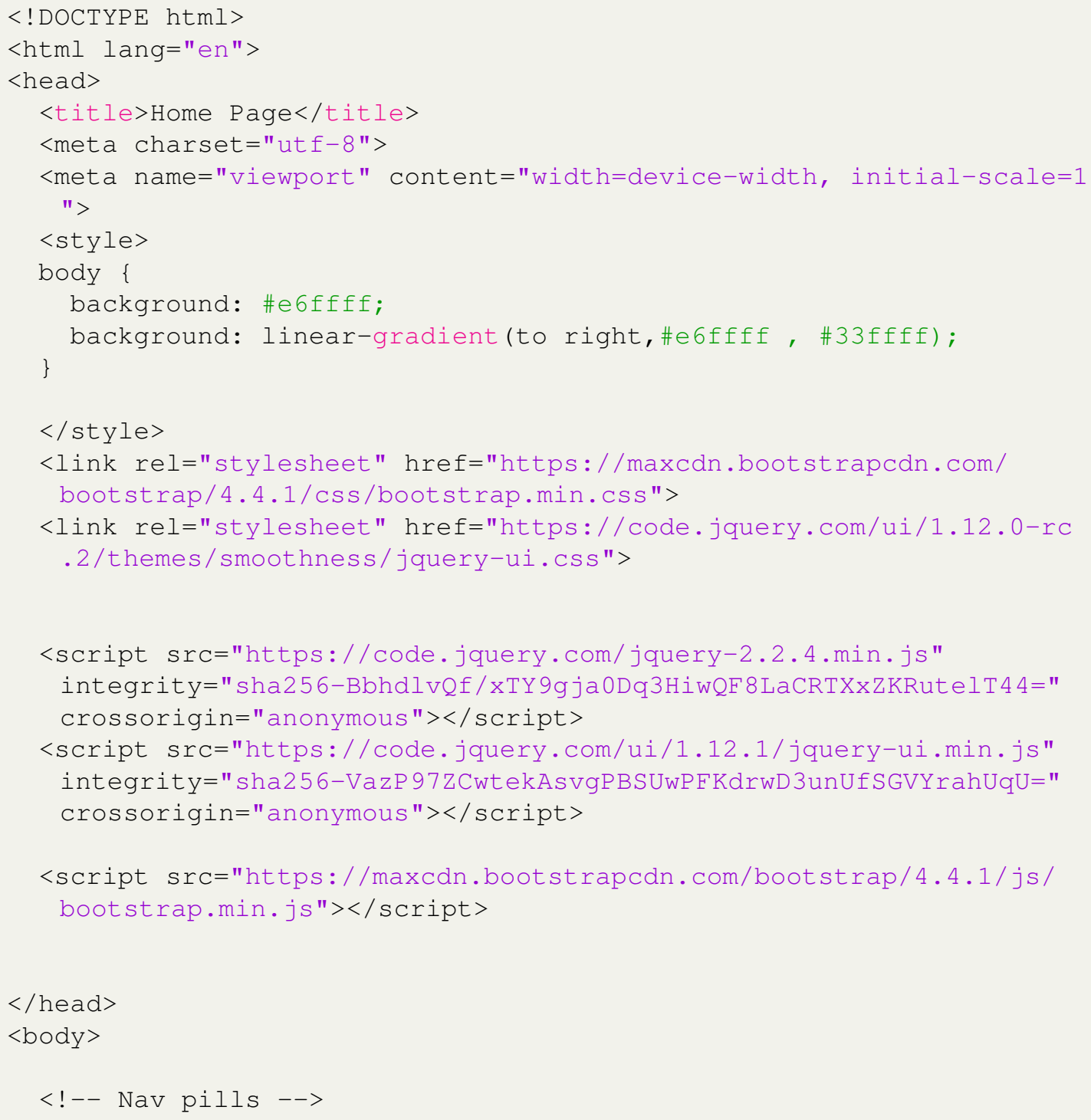




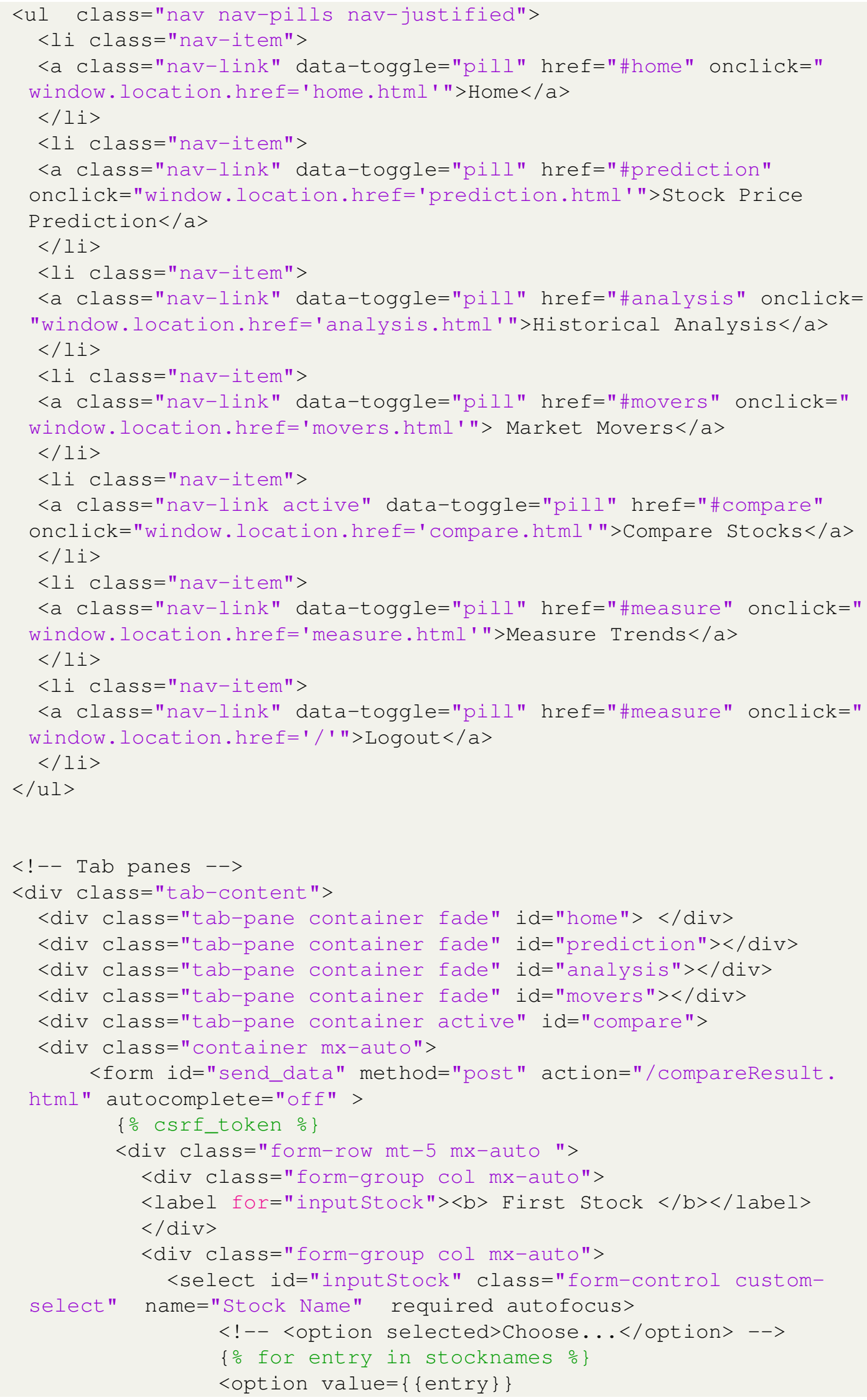




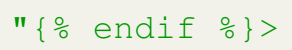




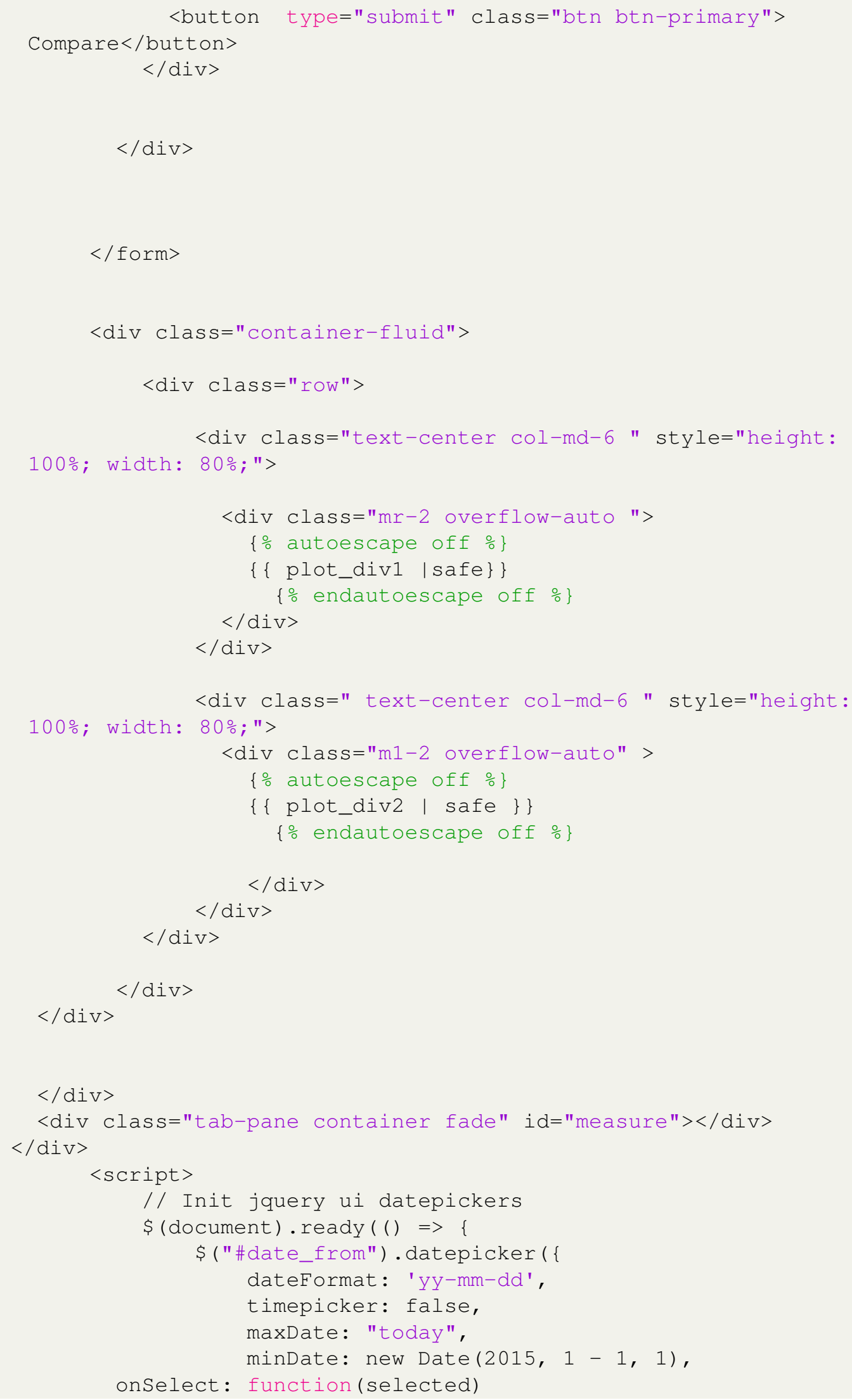




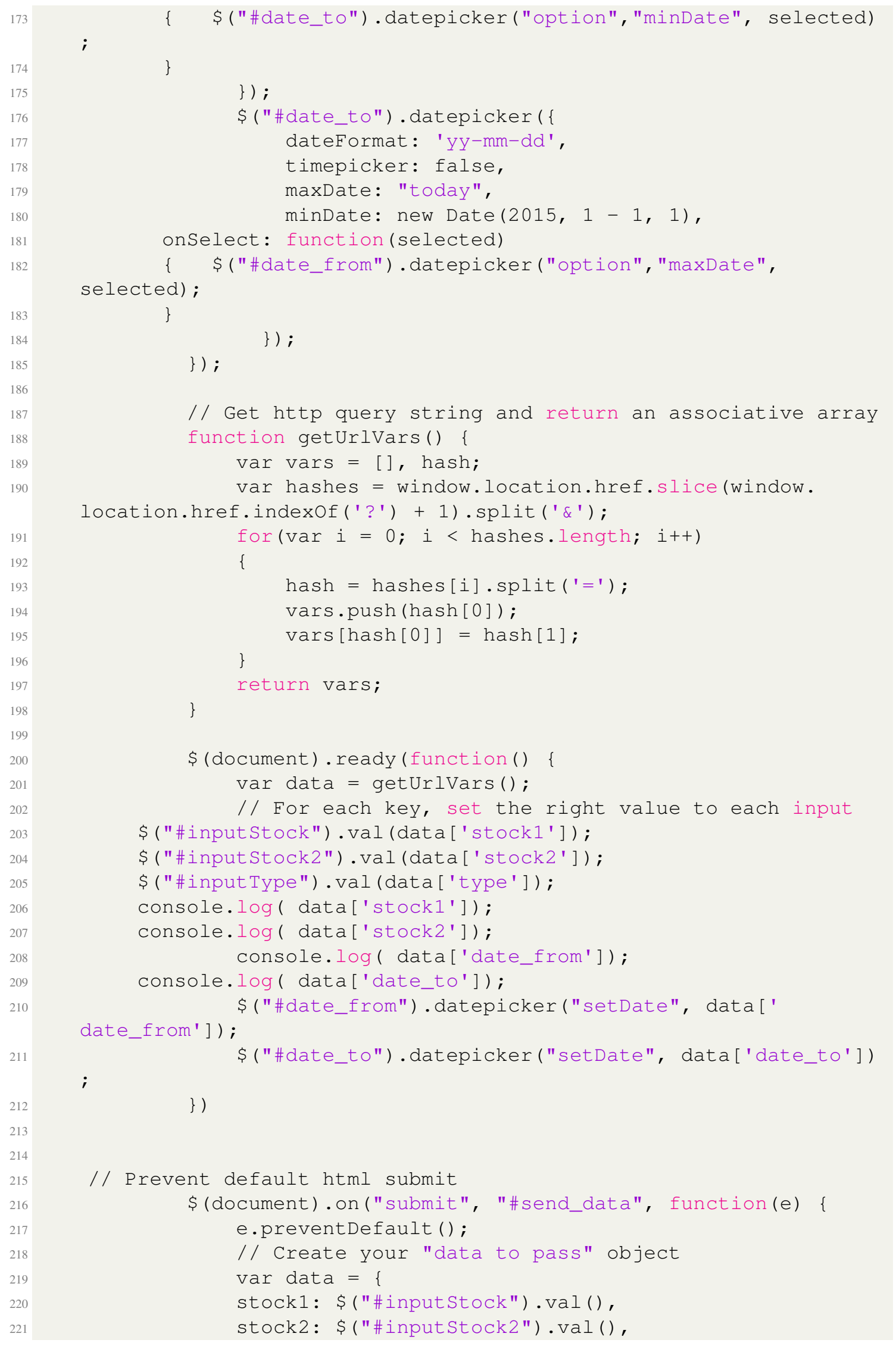




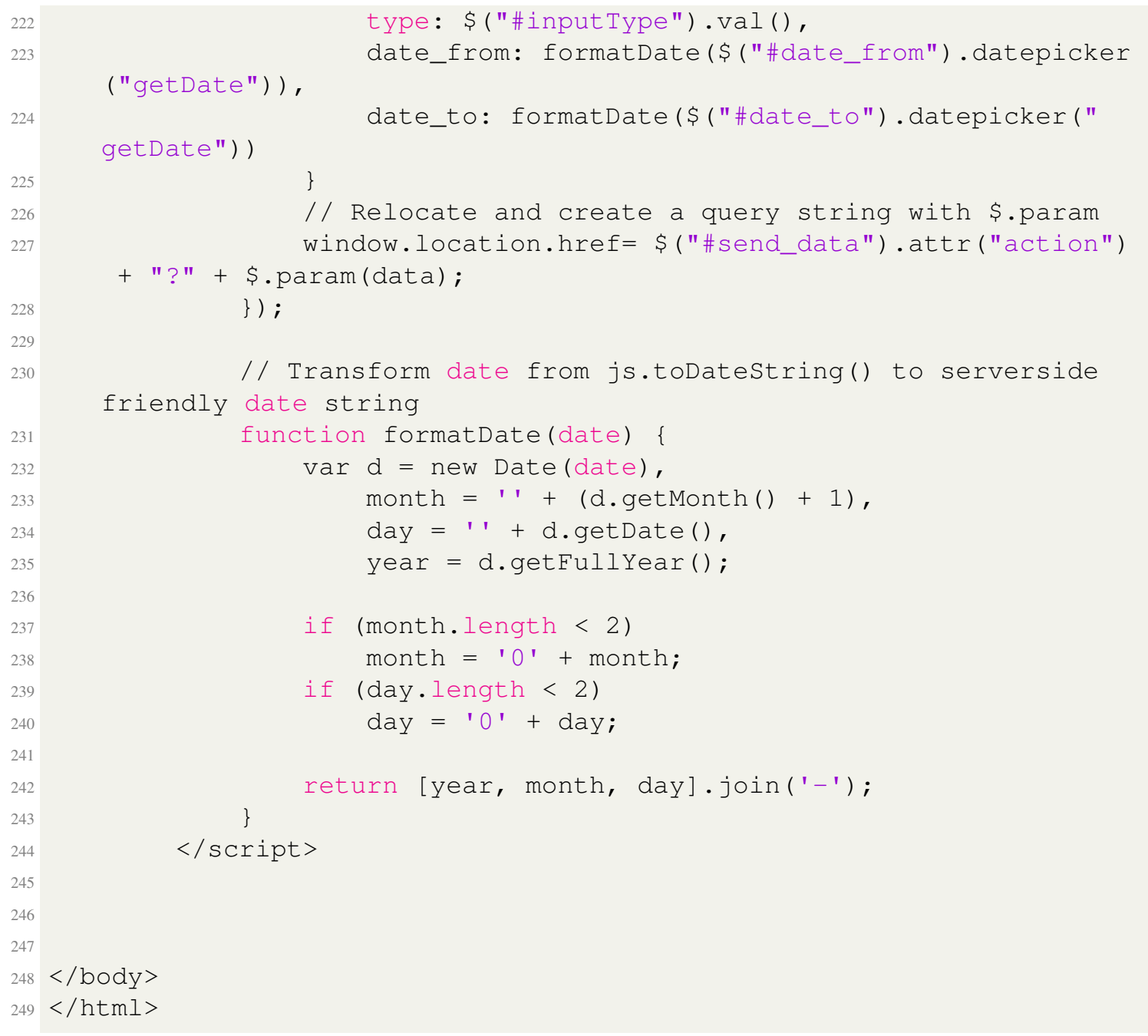

\section{Code A.2.3.15: compareResultLine.html}

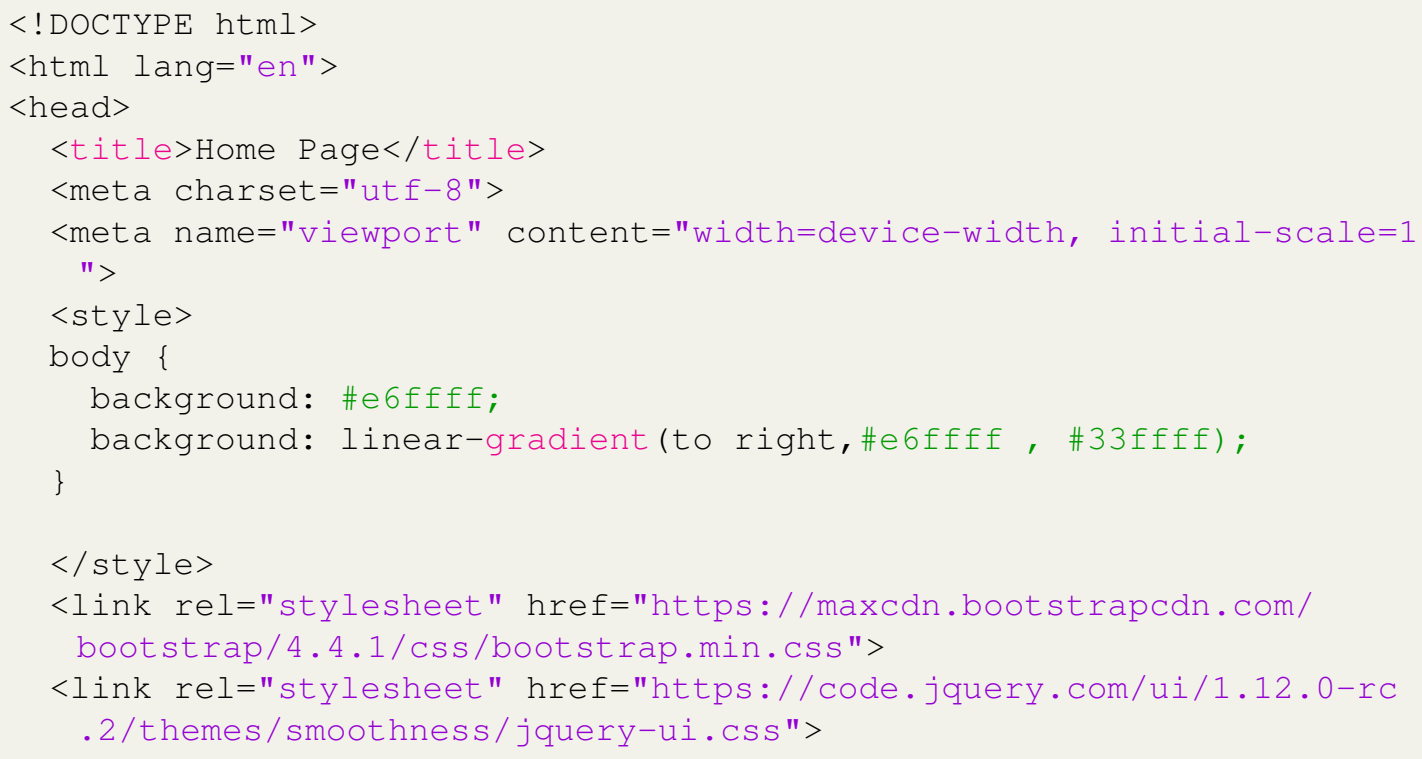


$$
\text { . }
$$

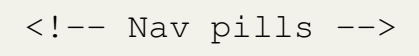




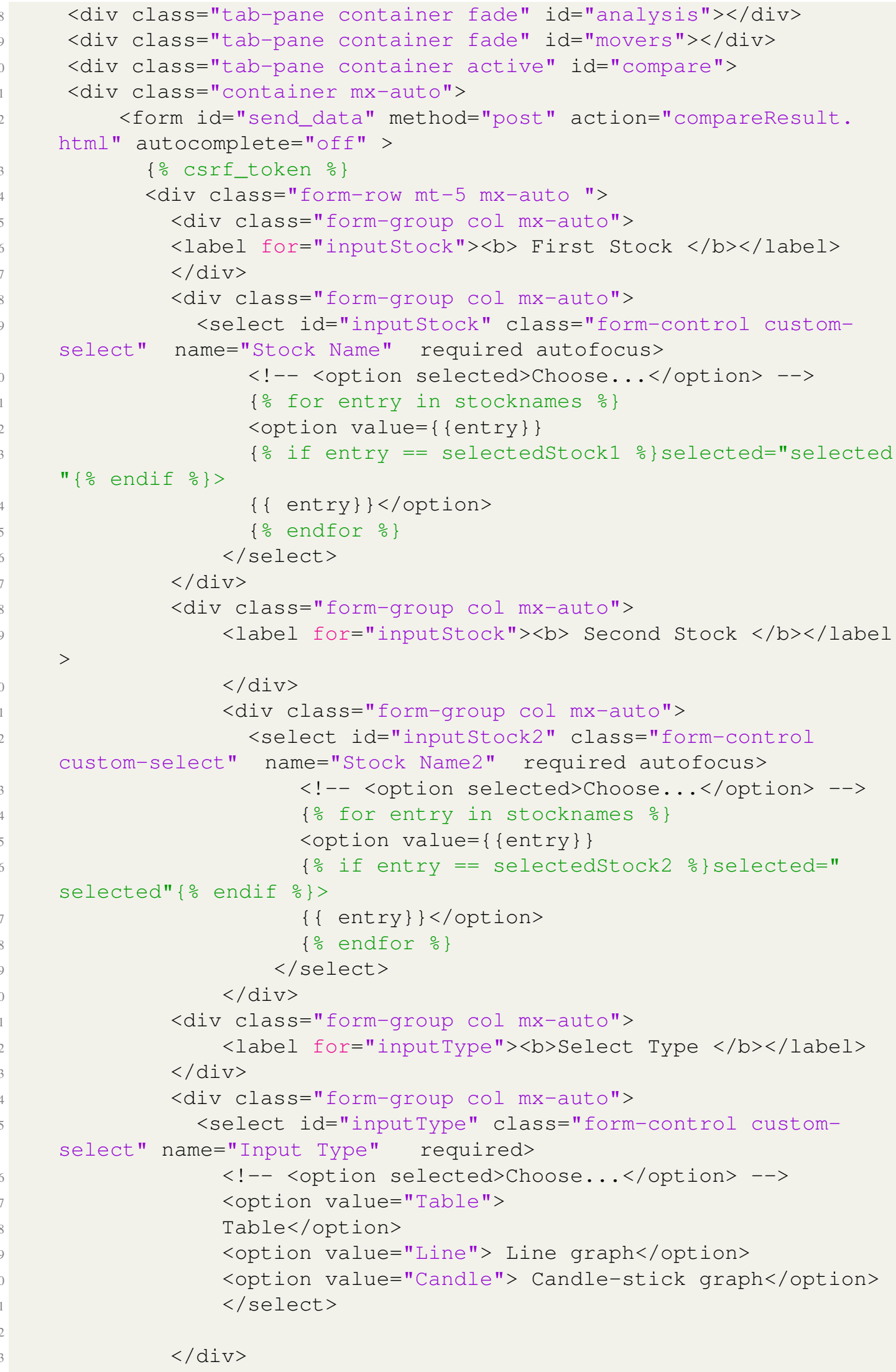




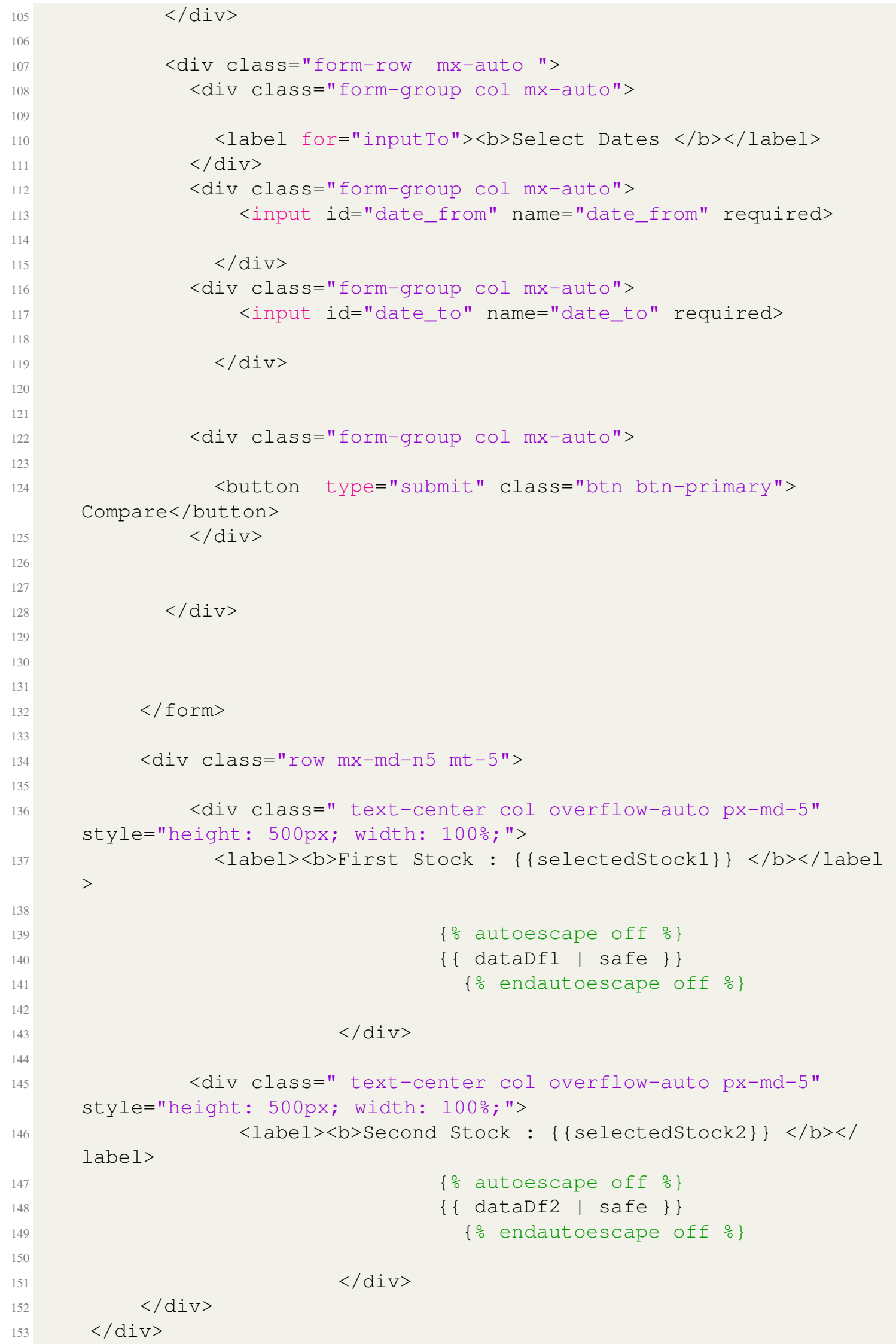




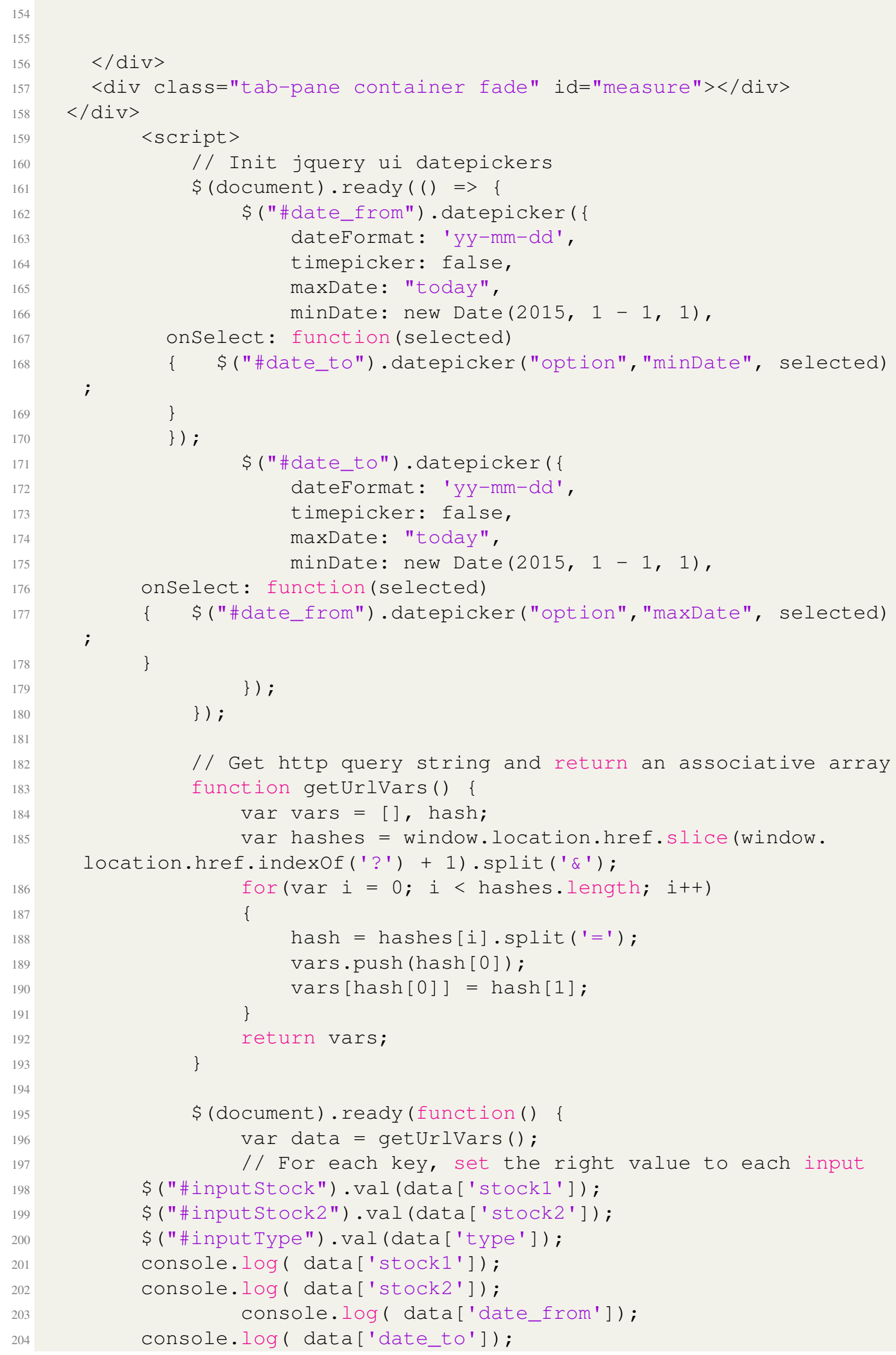

$</$ div $>$

$<$ div class="tab-pane container fade" id="measure" $></$ div $>$ $</ \operatorname{div}>$

$<$ script $>$

// Init jquery ui datepickers

$\$$ (document) .ready ( () $=>$ \{

\$("\#date_from") .datepicker ( \{

dateFormat: 'yy-mm-dd',

timepicker: false,

maxDate: "today",

minDate: new Date (2015, $1-1,1)$,

onselect: function (selected)

$\{\quad$ \$("\#date_to").datepicker("option", "minDate", selected)

;

\}

\});

\$("\#date_to").datepicker ( \{

dateFormat: 'yy-mm-dd',

timepicker: false,

maxDate: "today",

minDate: new Date(2015, 1 - 1, 1),

onselect: function(selected)

\{ \$("\#date_from").datepicker("option","maxDate", selected)

; \}

\});

\}) ;

// Get http query string and return an associative array function getUrlVars() \{

var $\operatorname{vars}=[]$, hash;

var hashes = window.location.href.slice(window.

location.href.indexOf('?') + 1).split('\&');

for (var $i=0 ; i<$ hashes.length; $i++)$

\{

hash = hashes[i].split ('=');

vars.push (hash [0]);

vars[hash[0]] = hash[1];

\}

\}

return vars;

\$ (document). ready (function() \{

var data $=$ geturlVars ();

// For each key, set the right value to each input

\$("\#inputStock").val (data ['stock1']);

\$("\#inputStock2") .val (data ['stock2']);

\$("\#input Type").val (data ['type']);

console.log ( data['stock1']);

console.log( data['stock2']);

console.log ( data['date_from']);

console.log ( data['date_to']); 


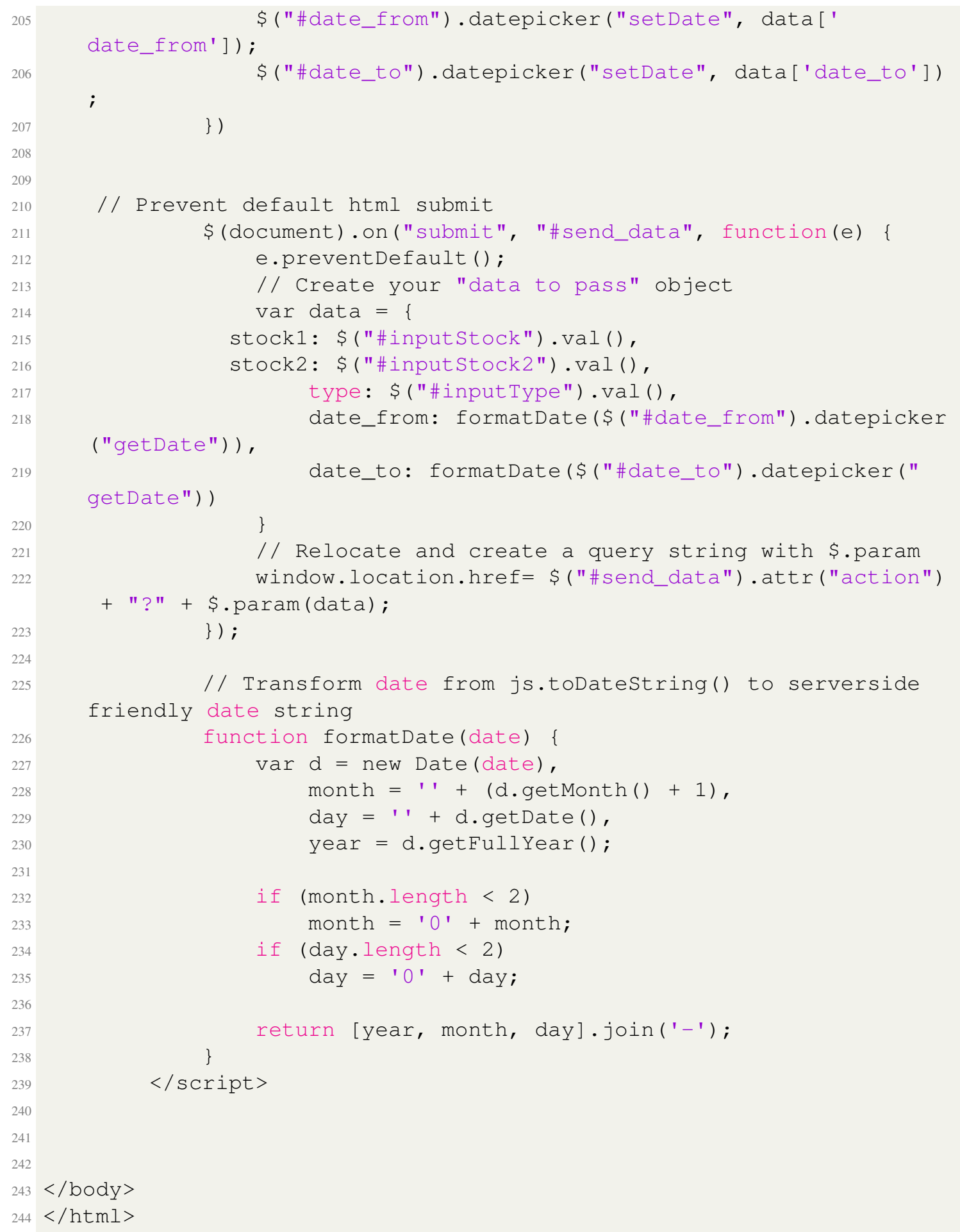

Code A.2.3.16: compareResultTable.html

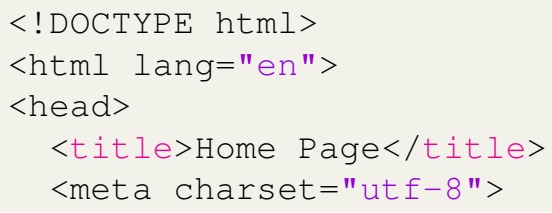




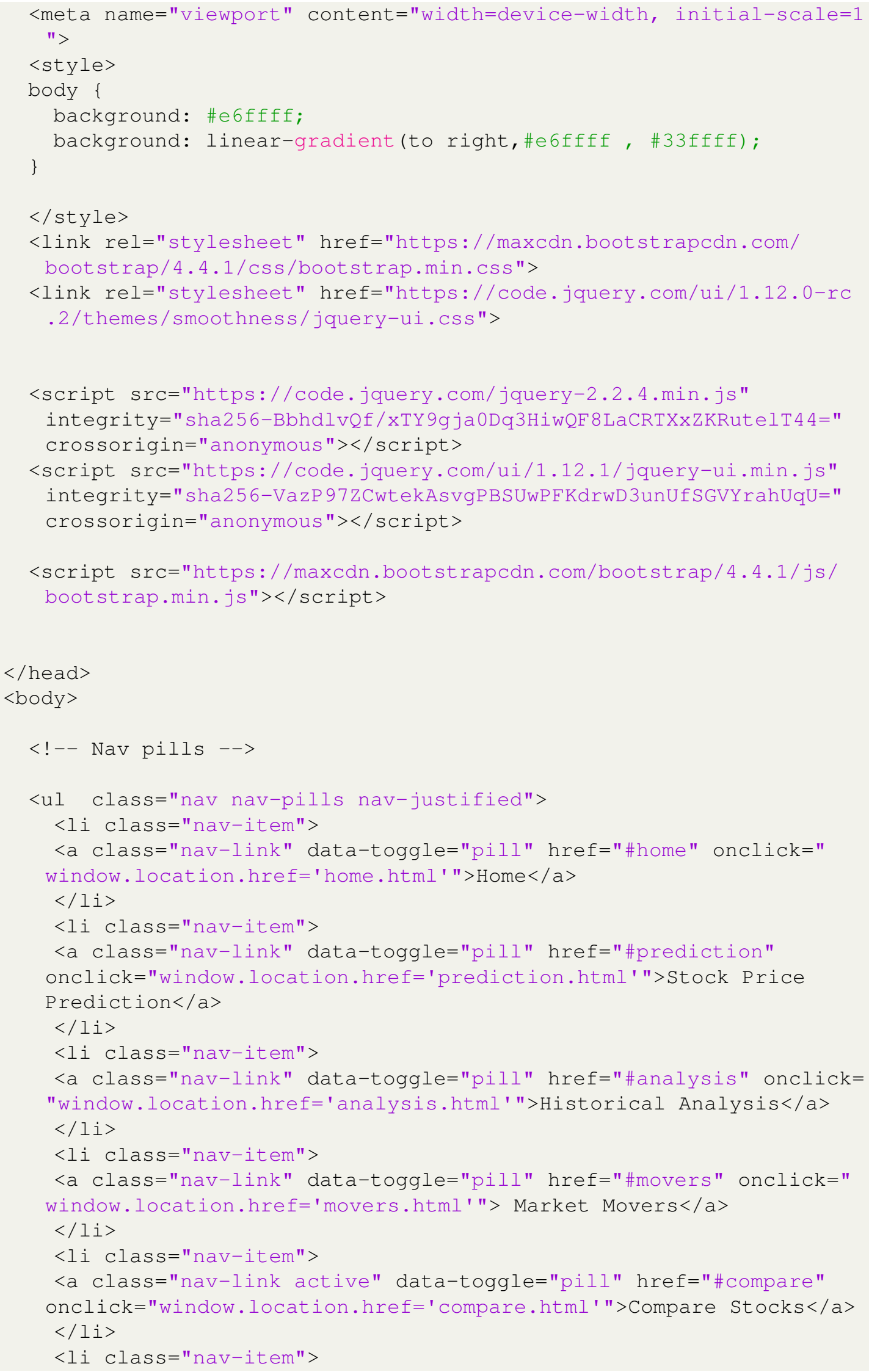




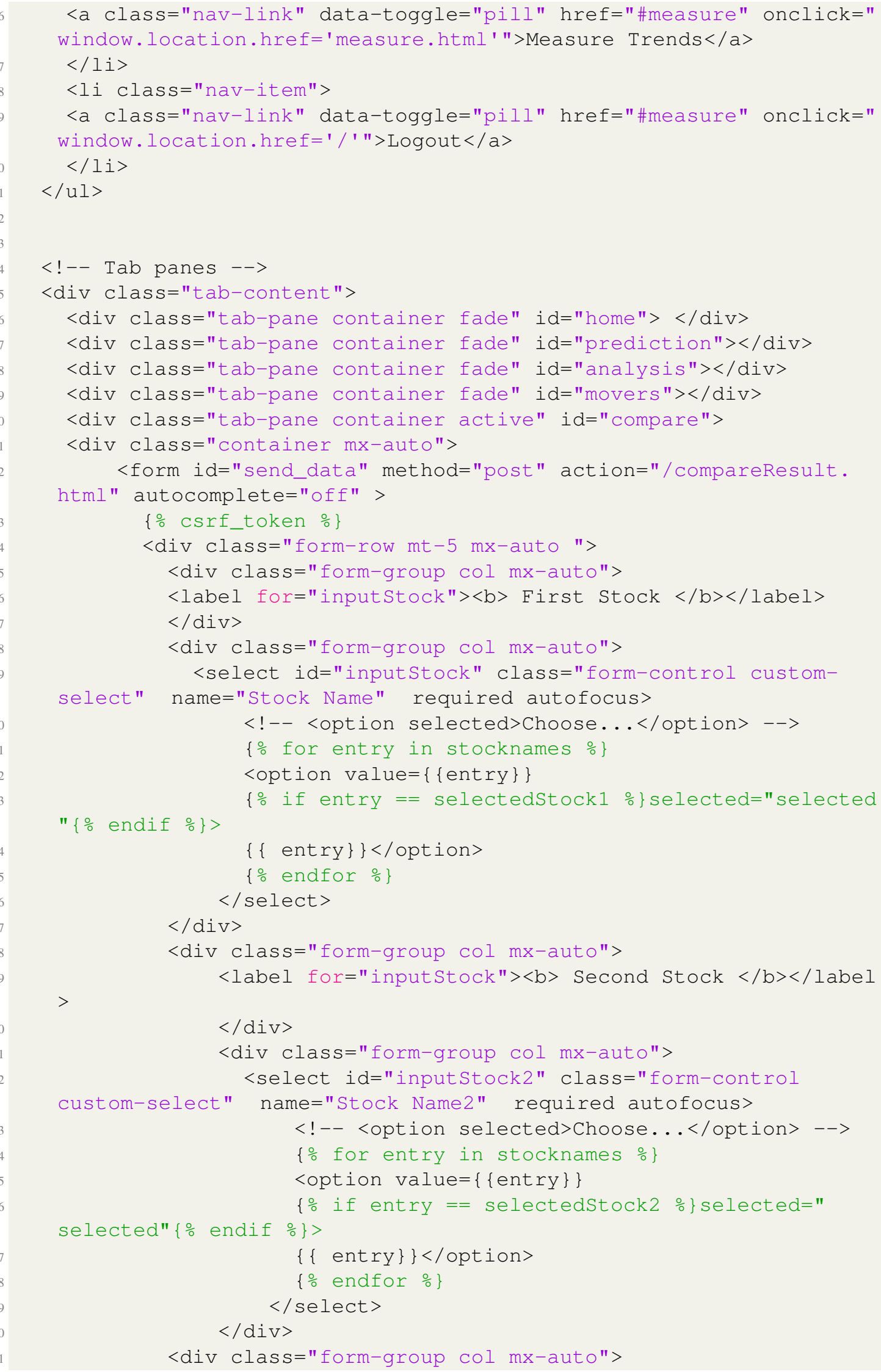




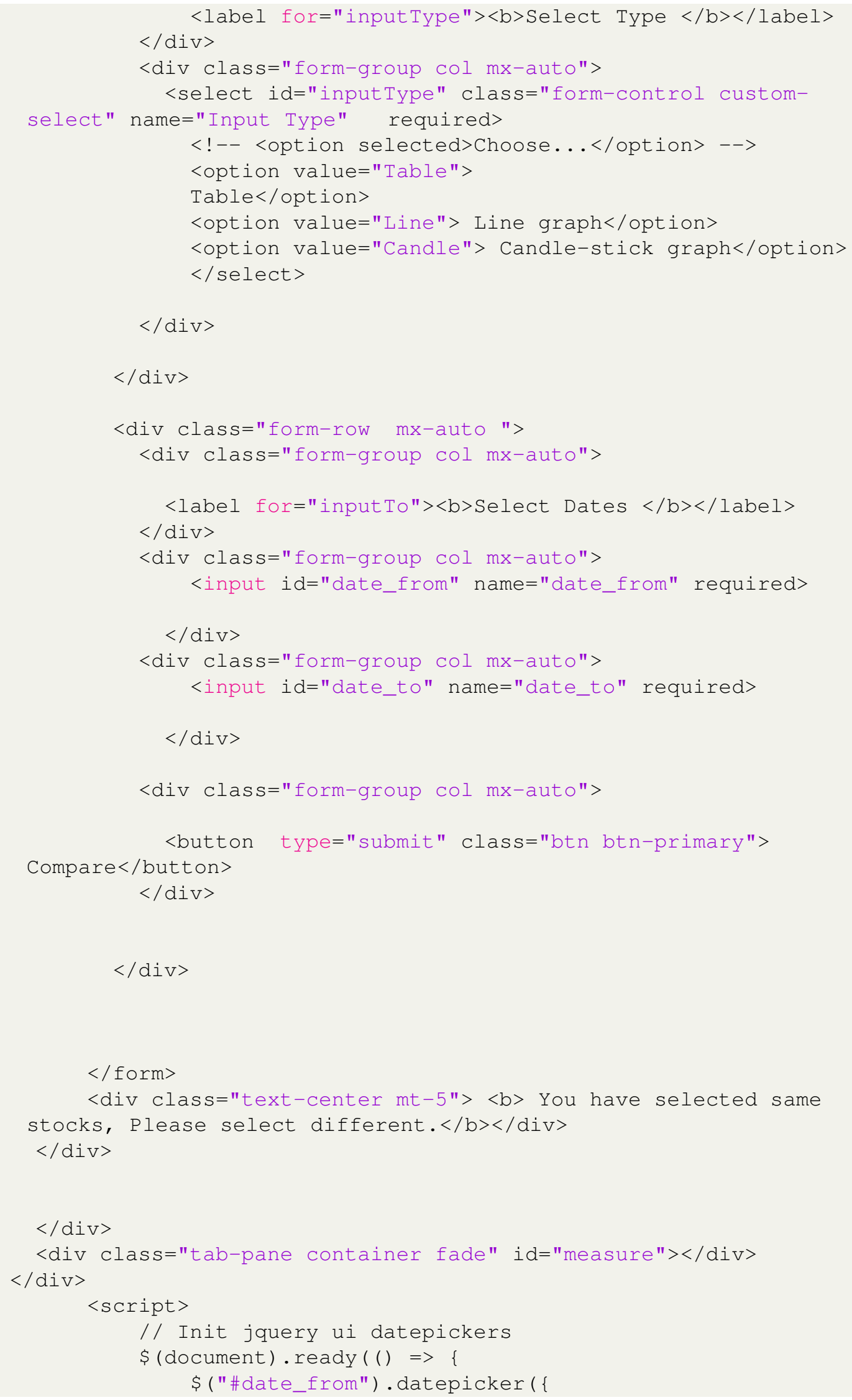




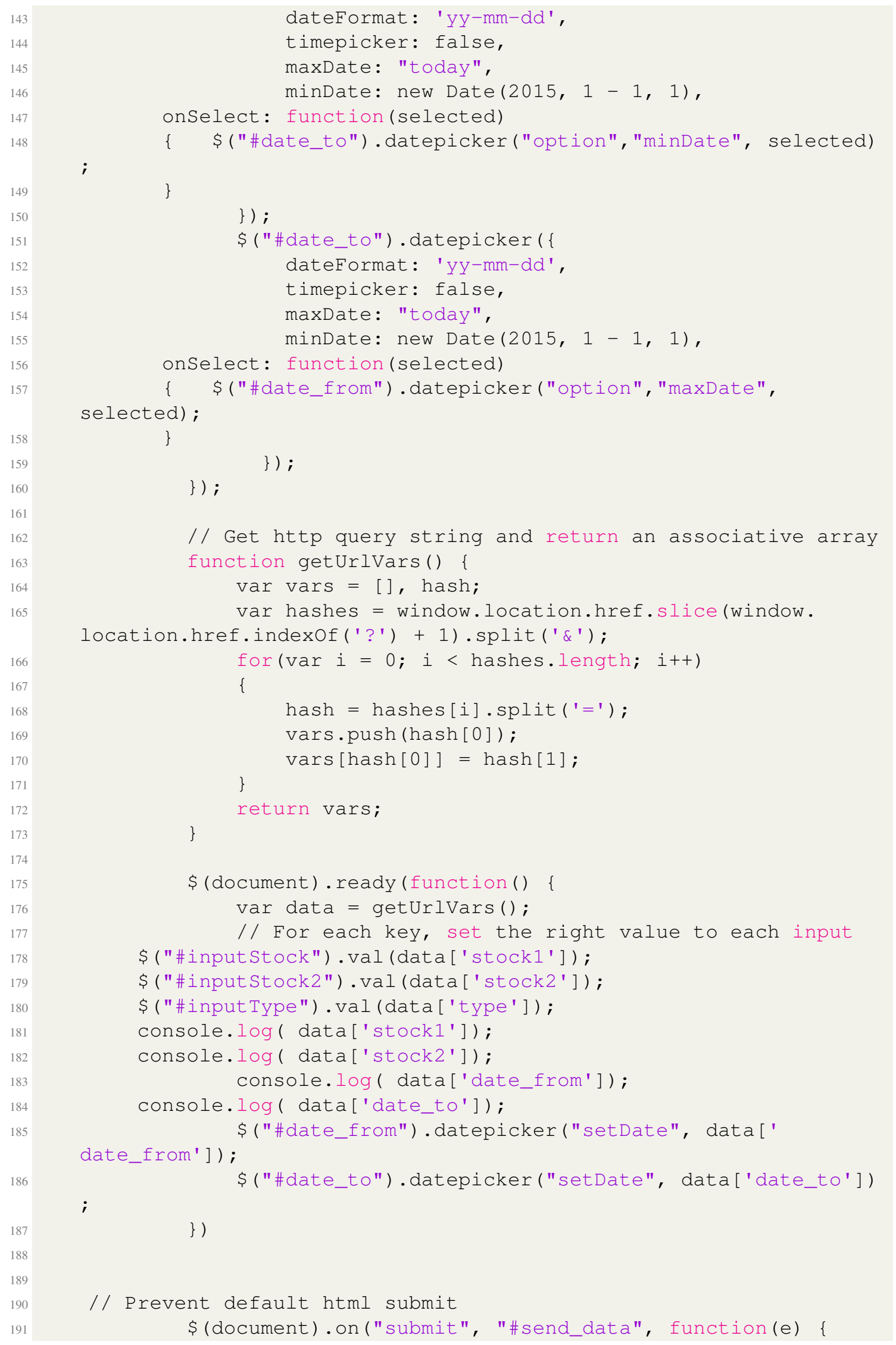




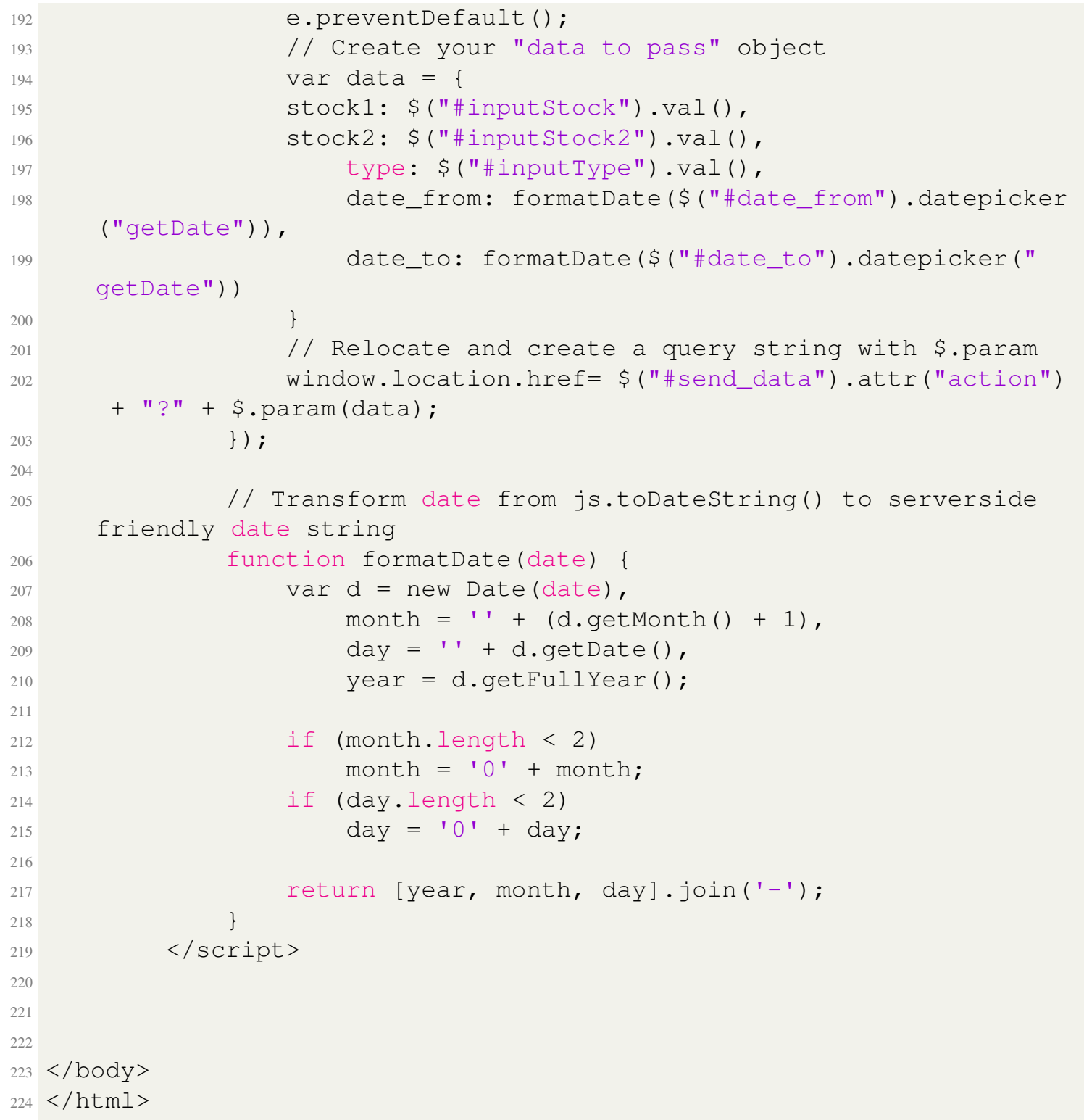

Code A.2.3.17: compareSameStock.html

\section{For finding trend in stock data}

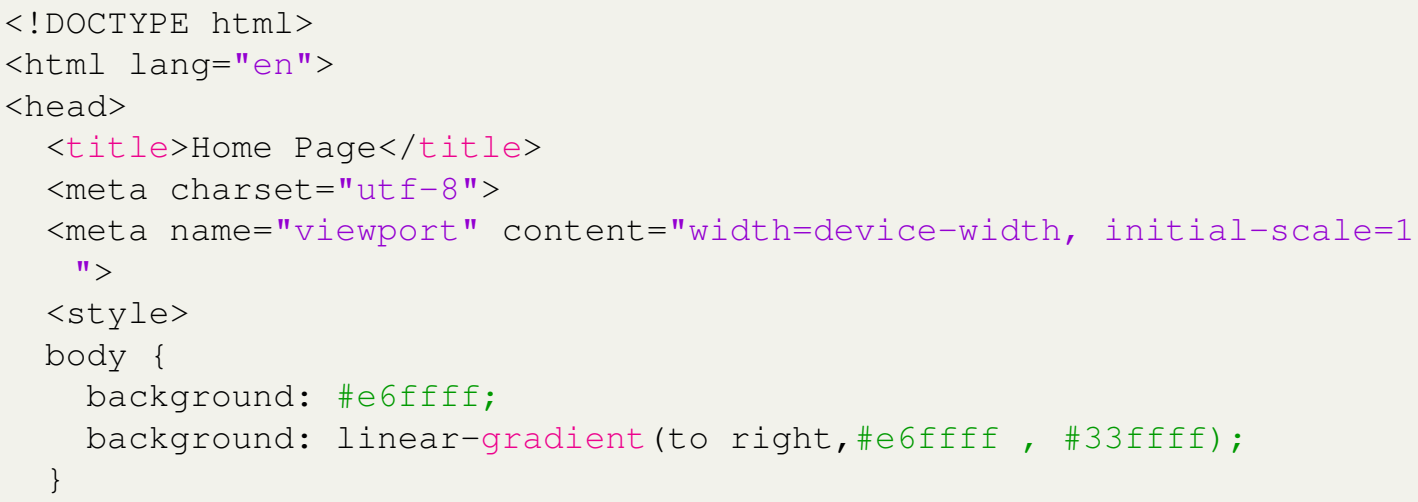




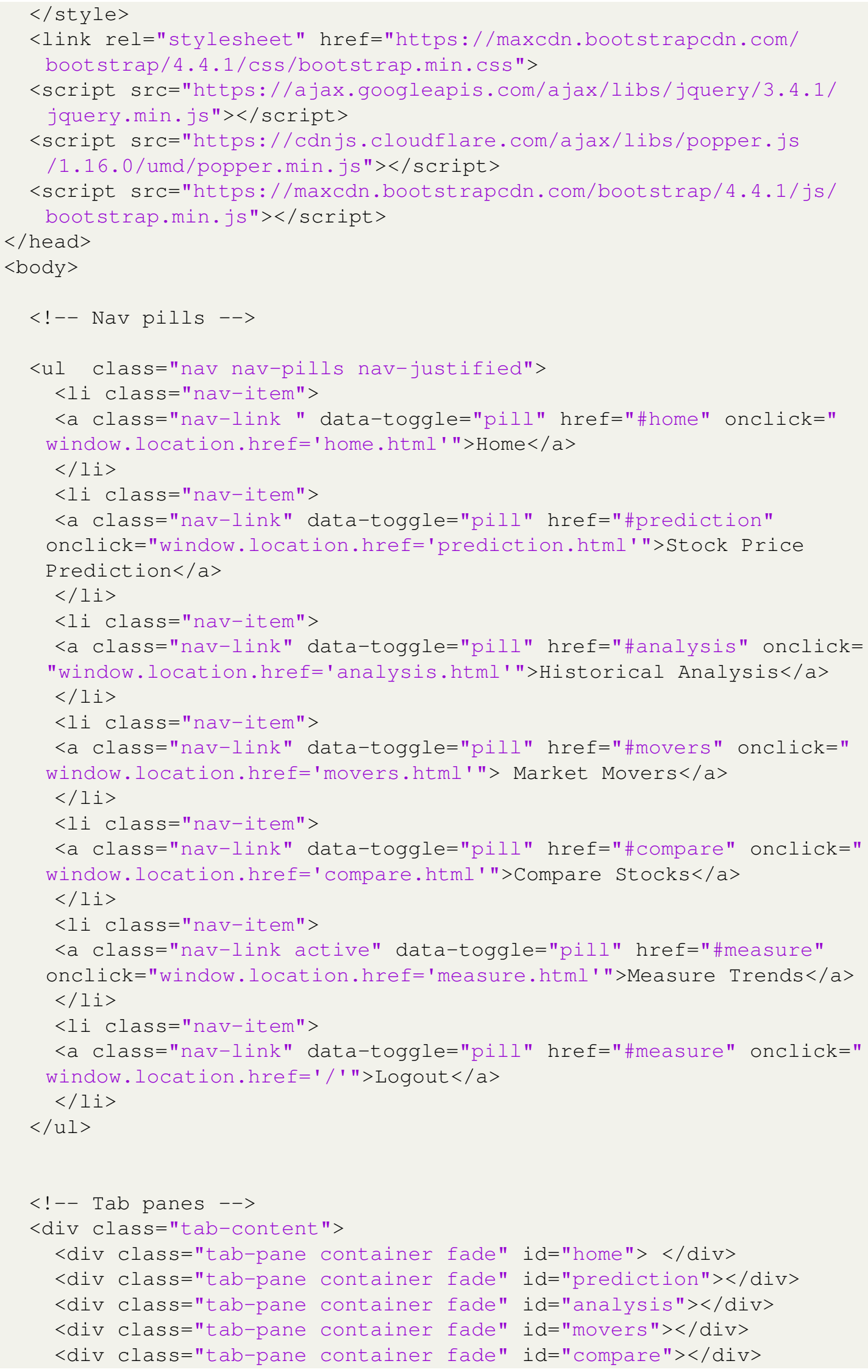




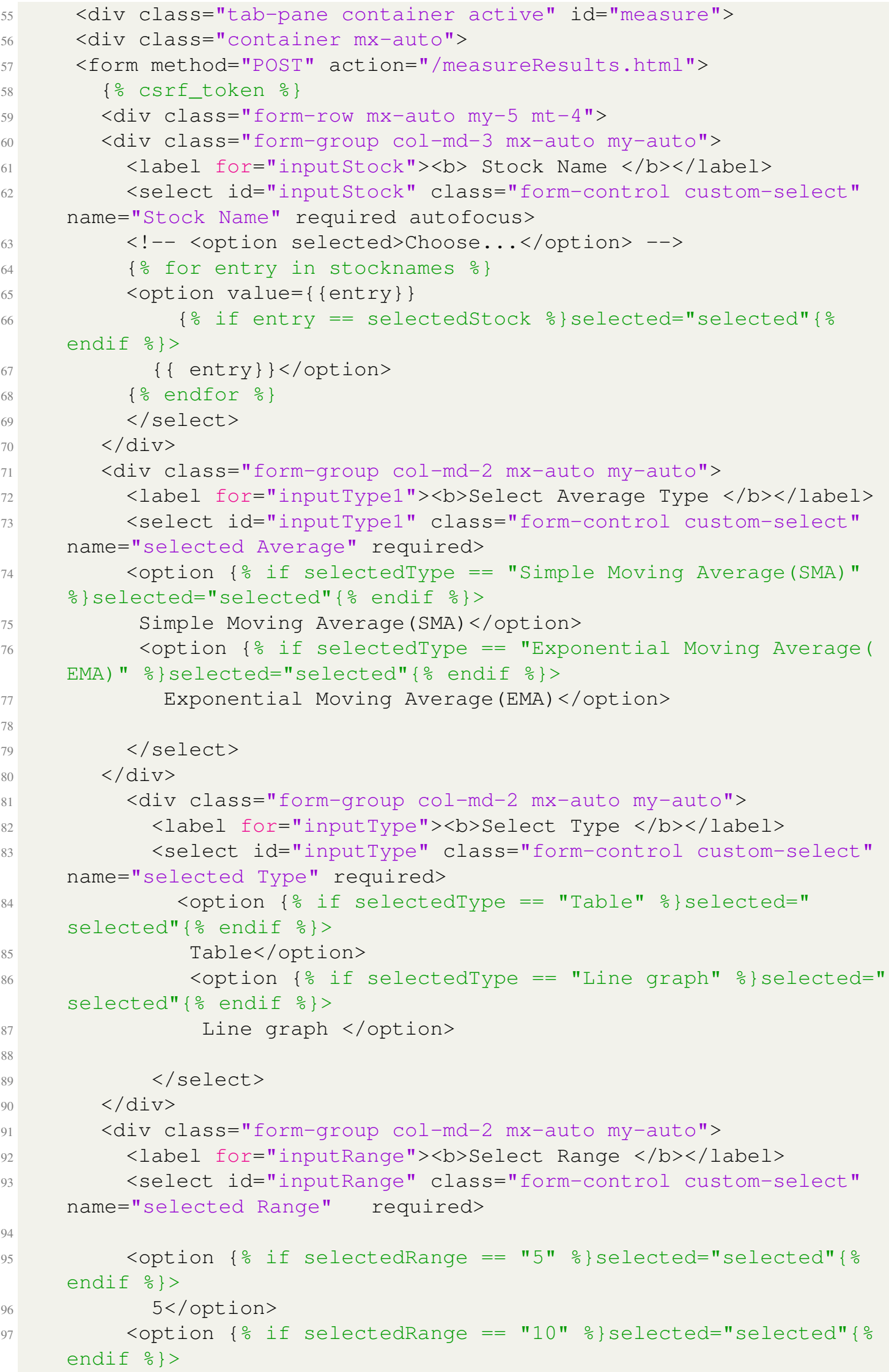




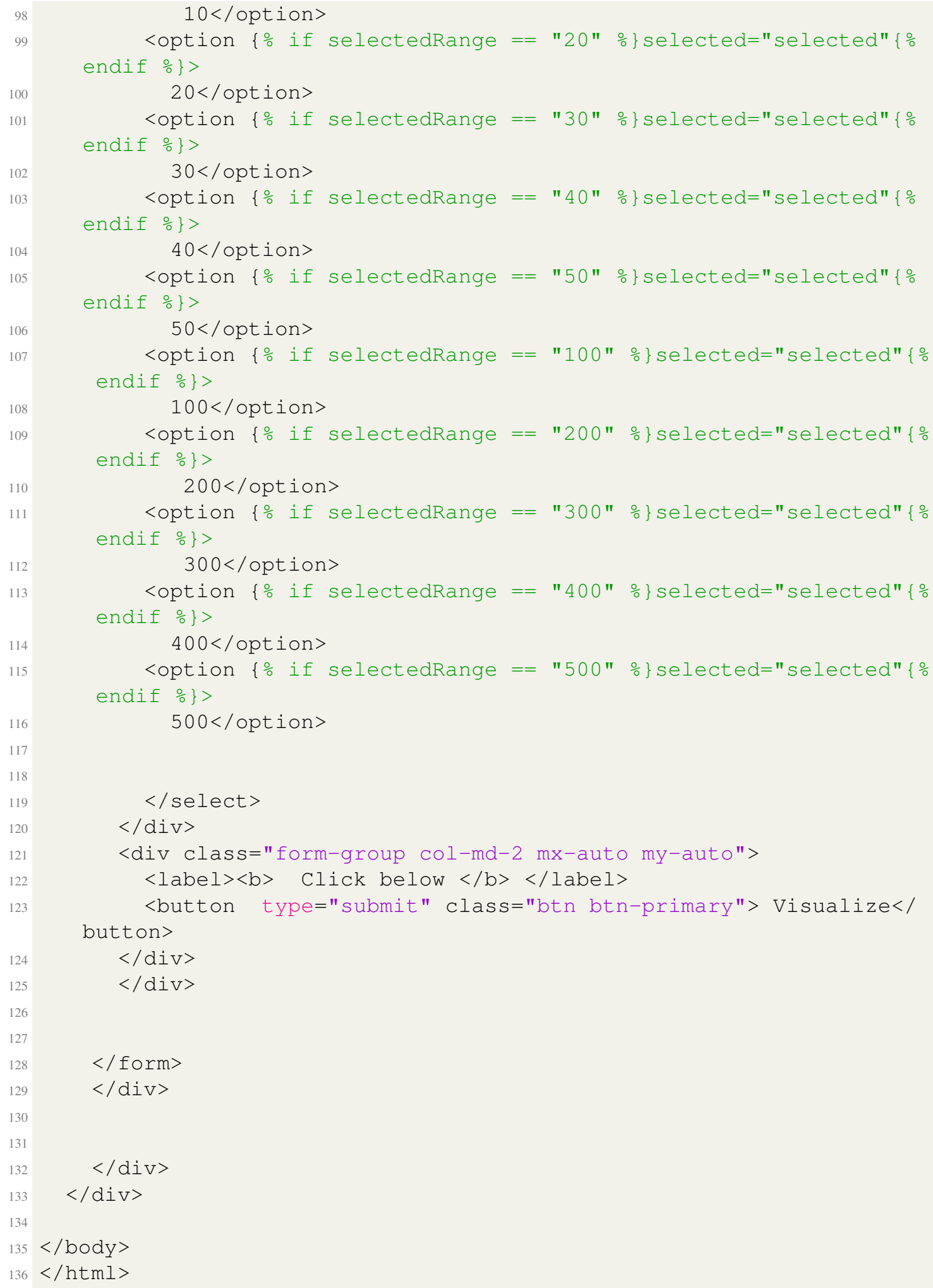

Code A.2.3.18: measure.html

$<$ !DOCTYPE html>

<html lang="en"> 


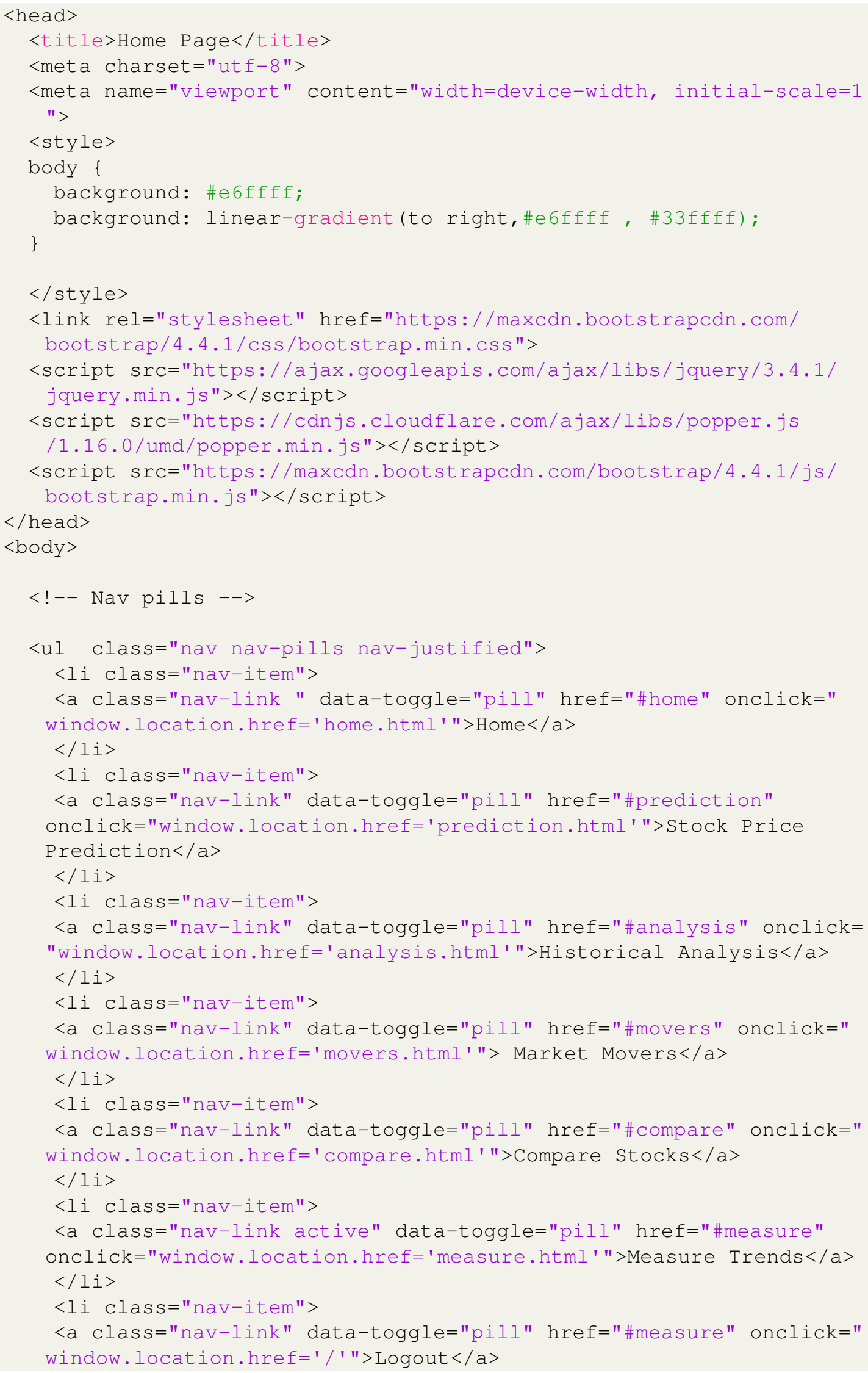




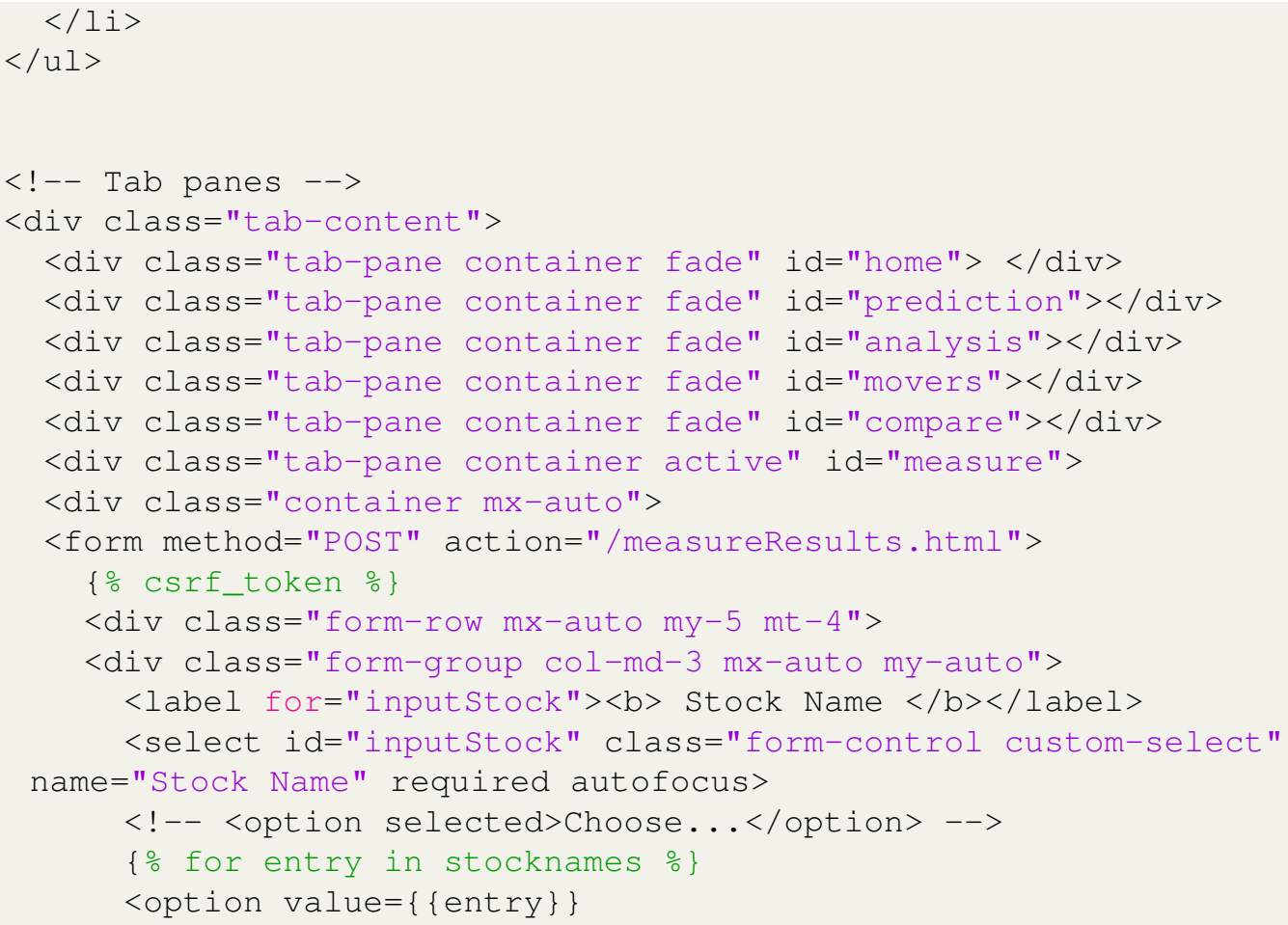




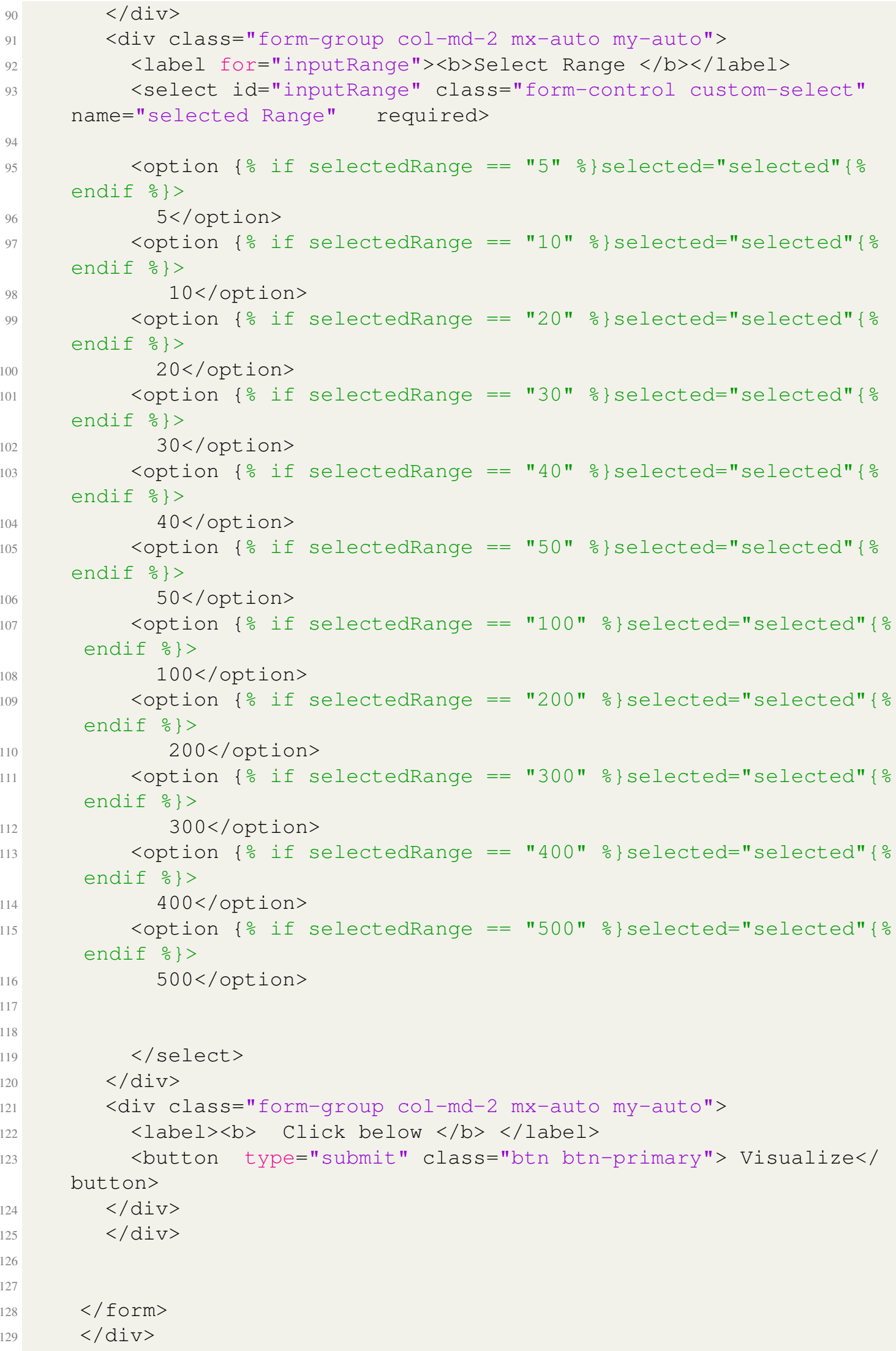




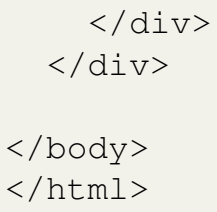

\section{Code A.2.3.19: measureResults.html}

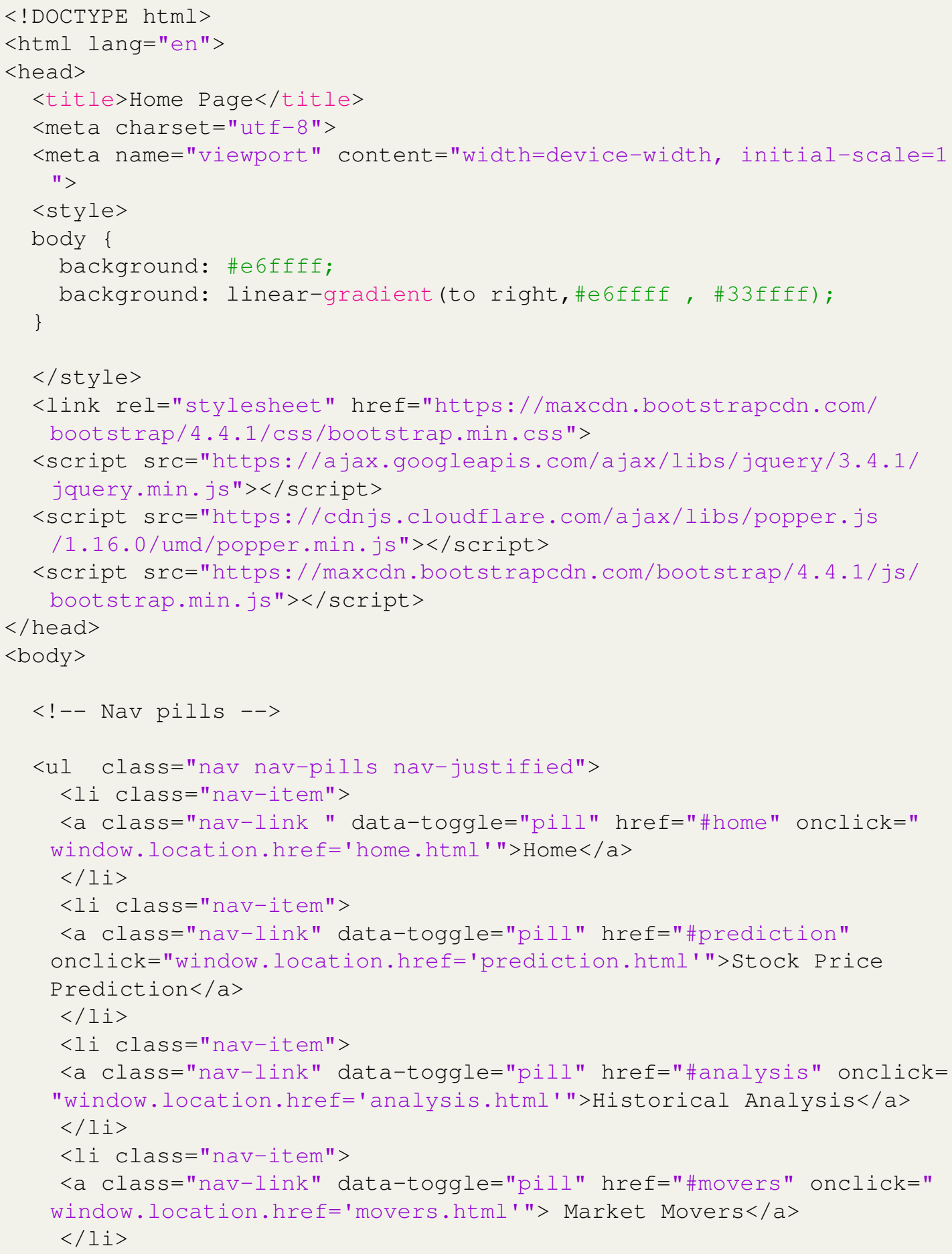




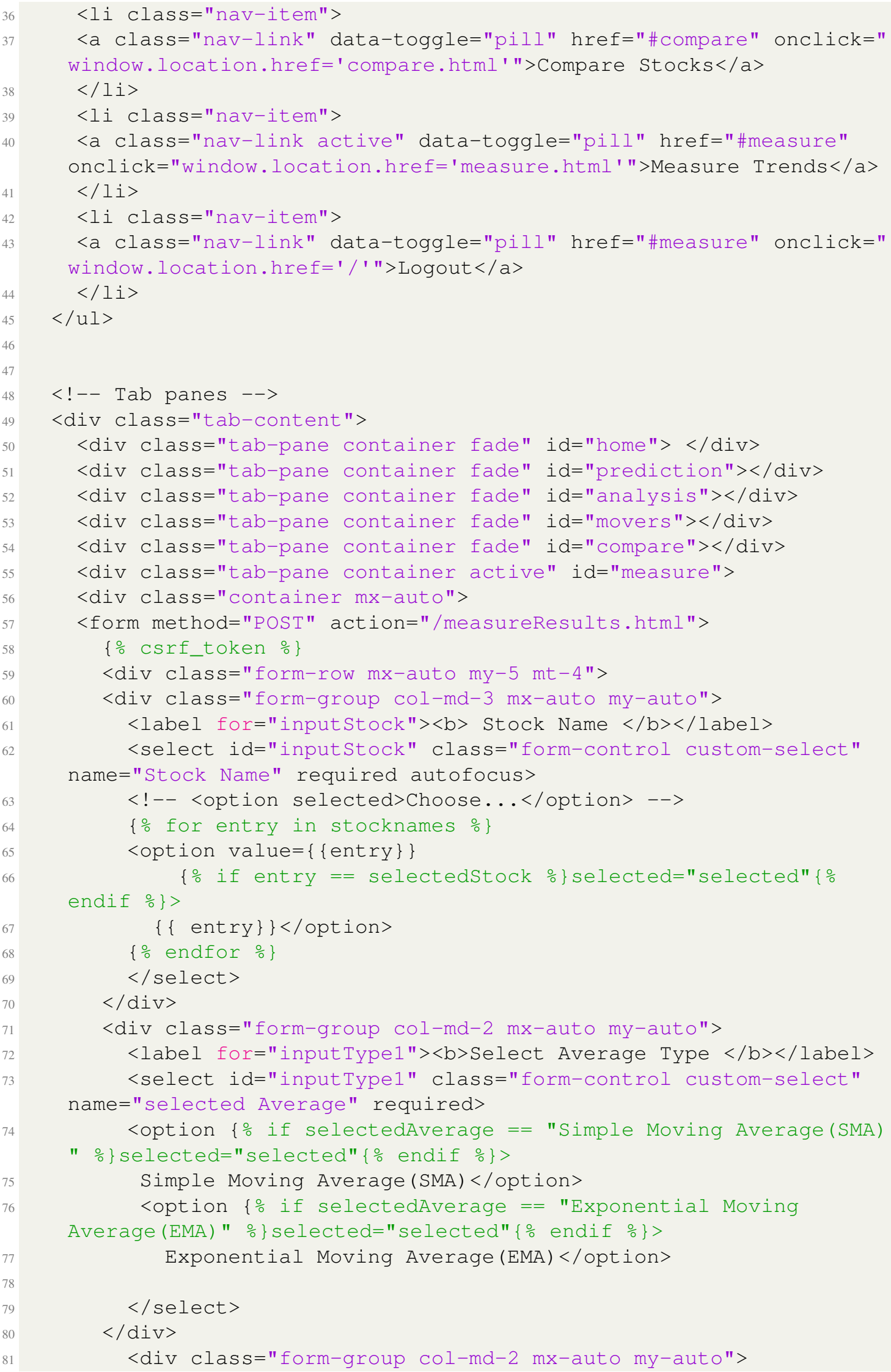




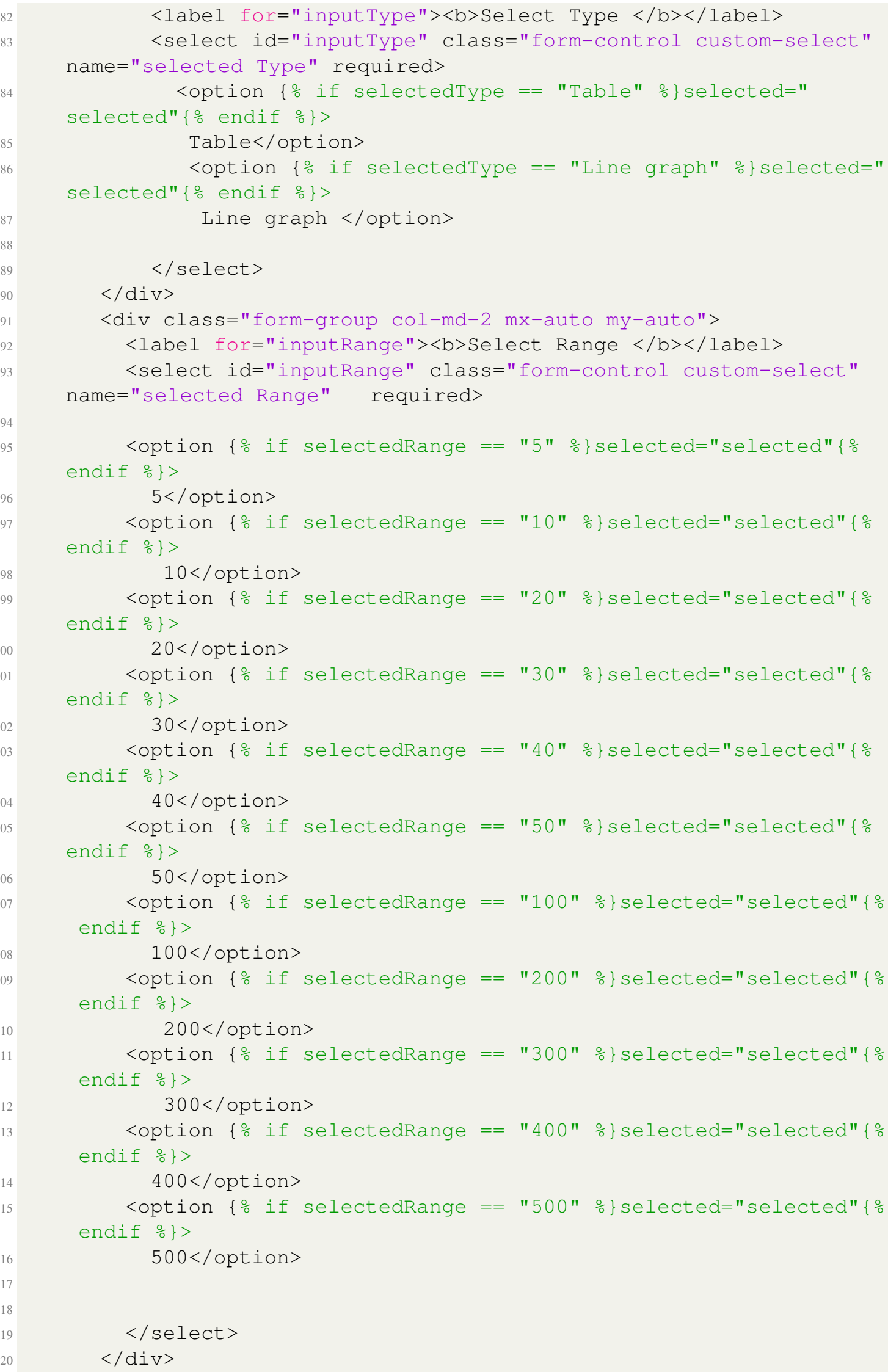




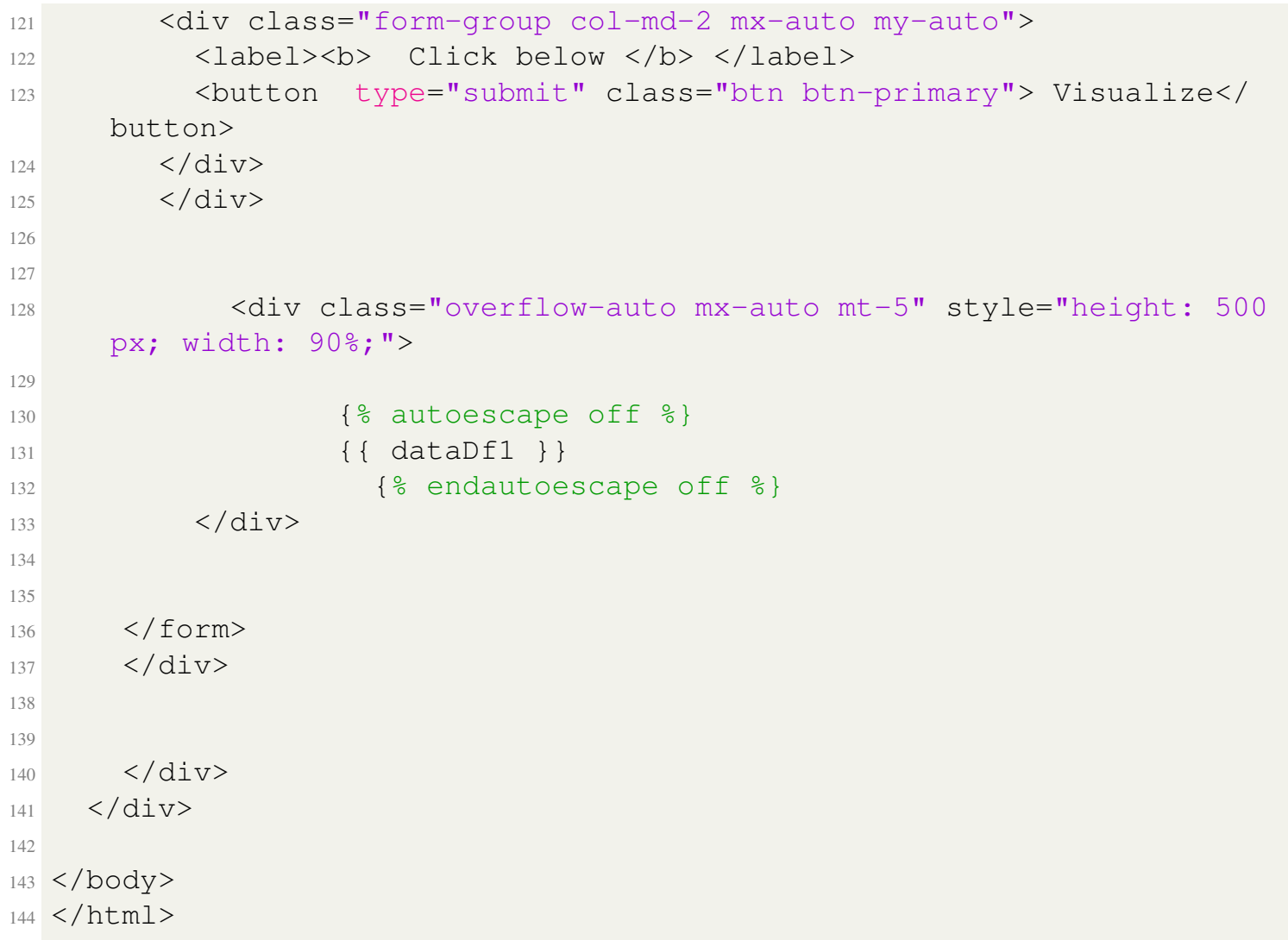

Code A.2.3.20: measureResultLine.html

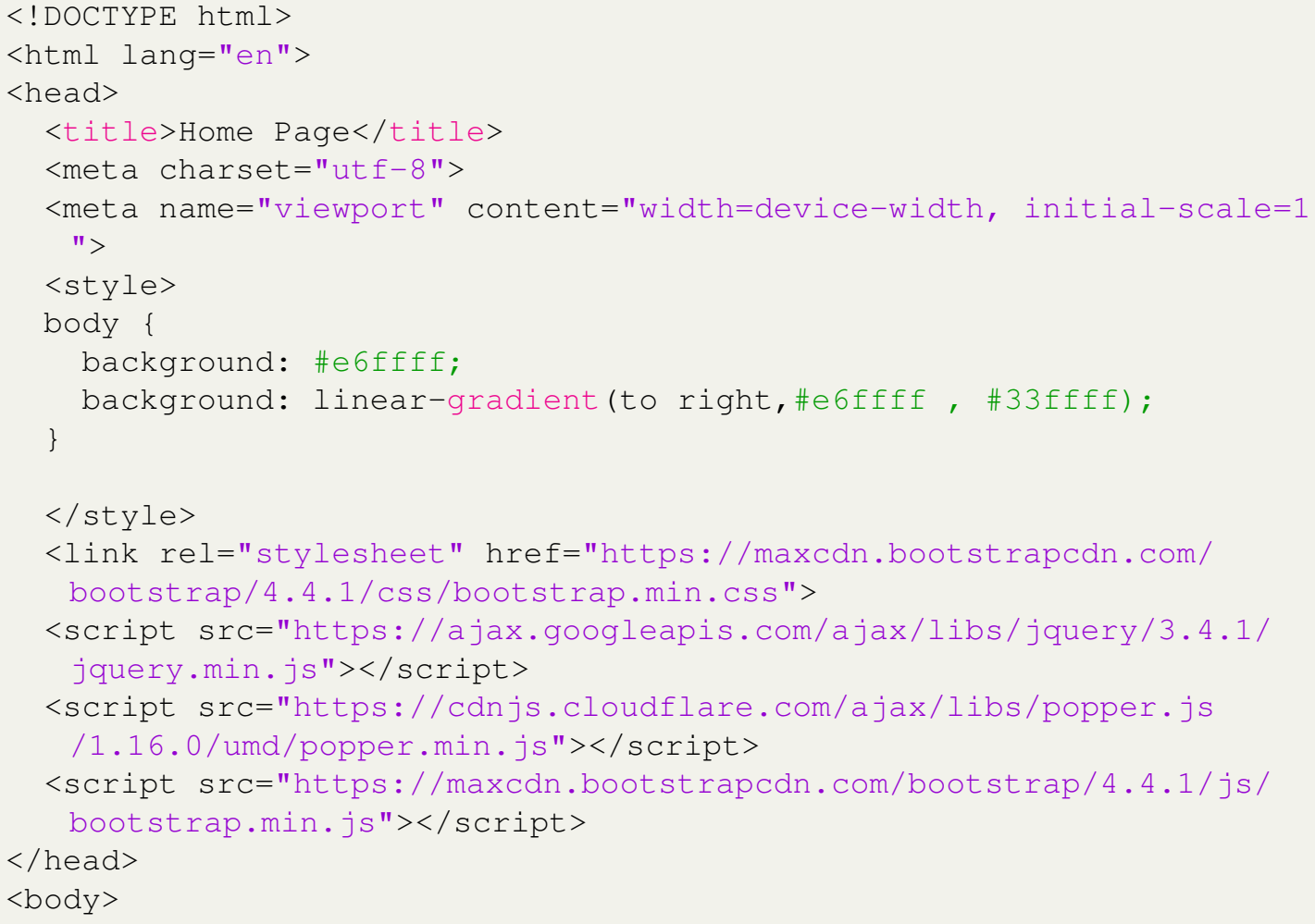




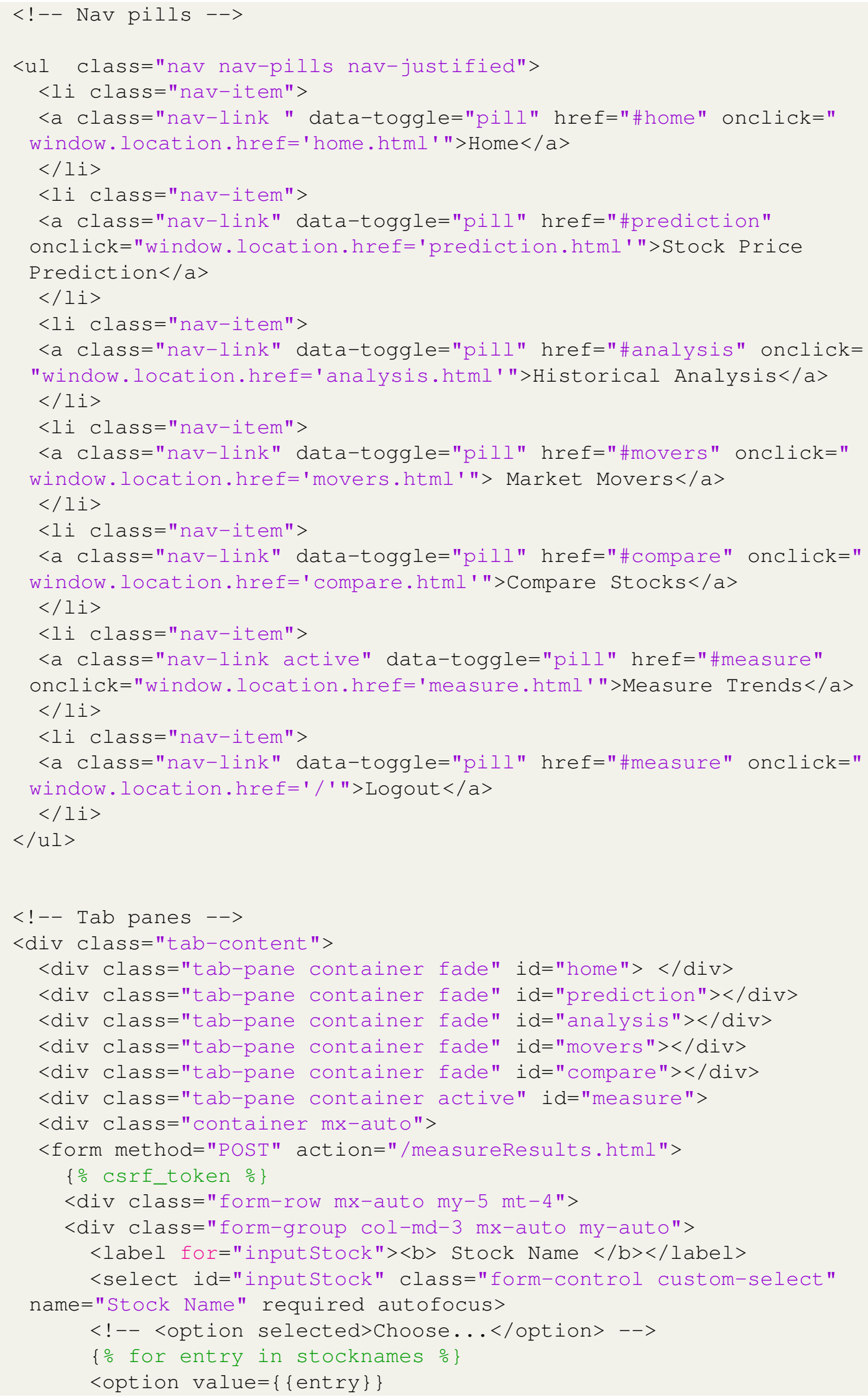




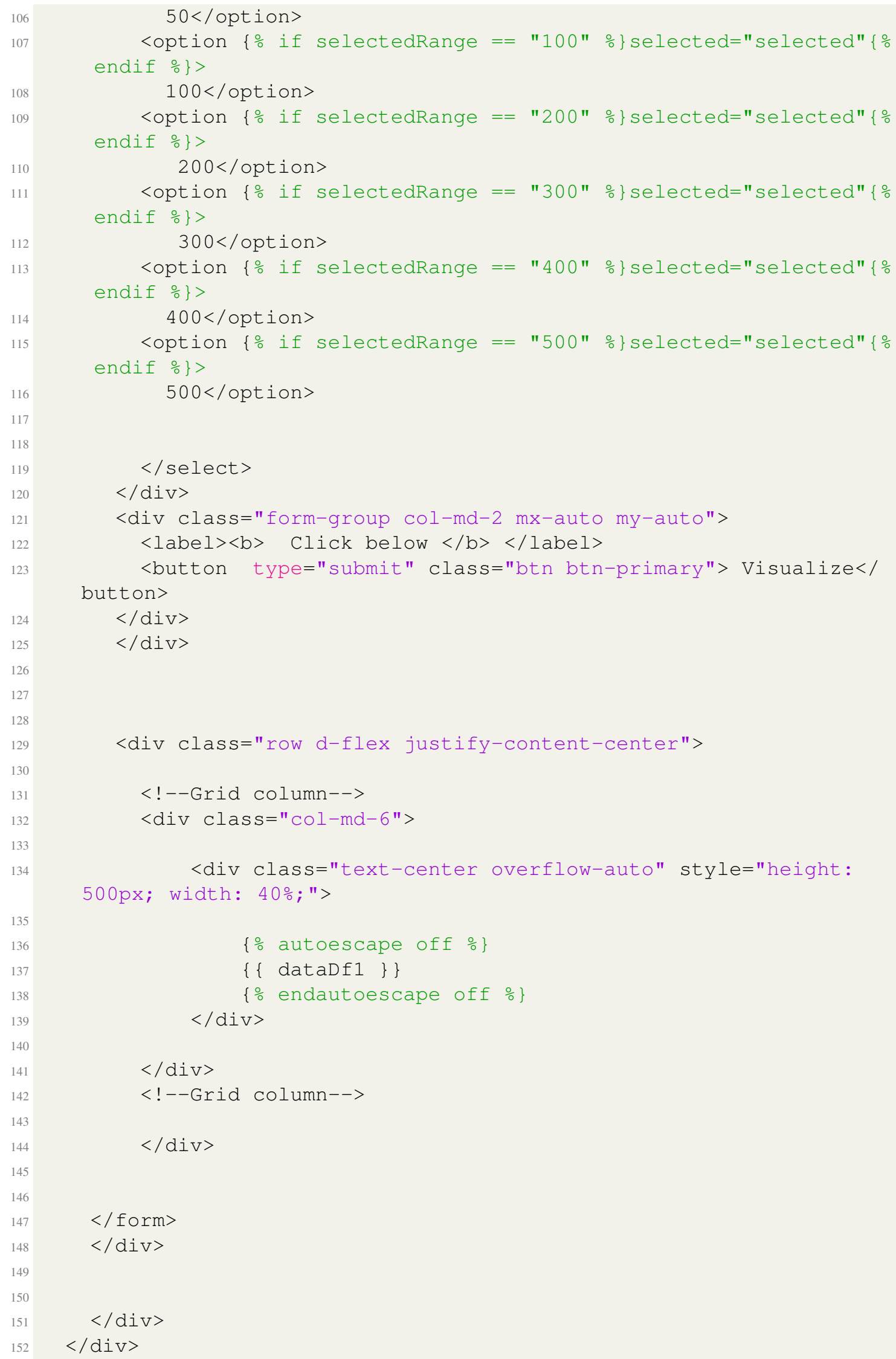




\section{Code A.2.3.21: measureResultTable.html}

\section{For market movers- gainers and losers}

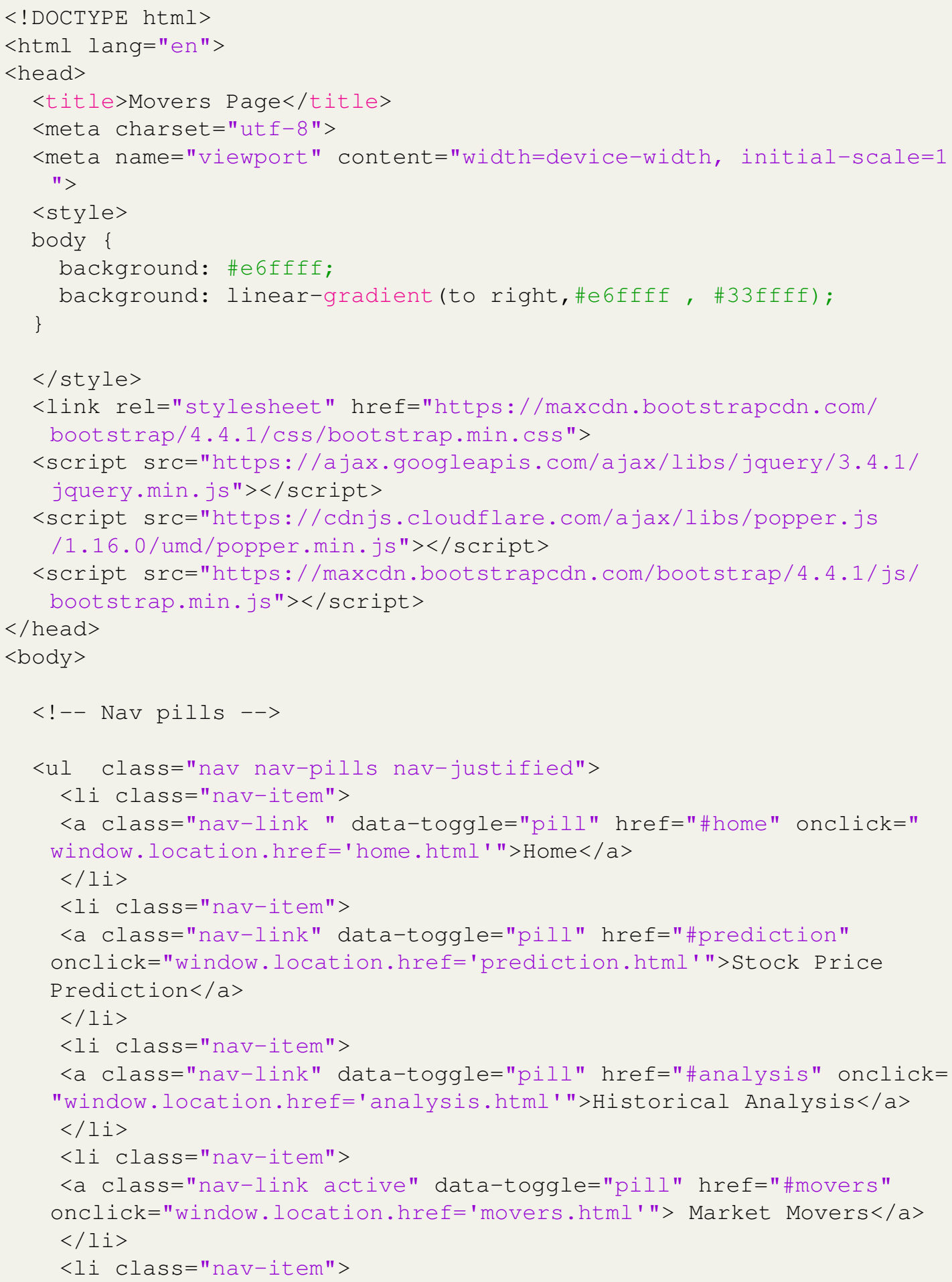




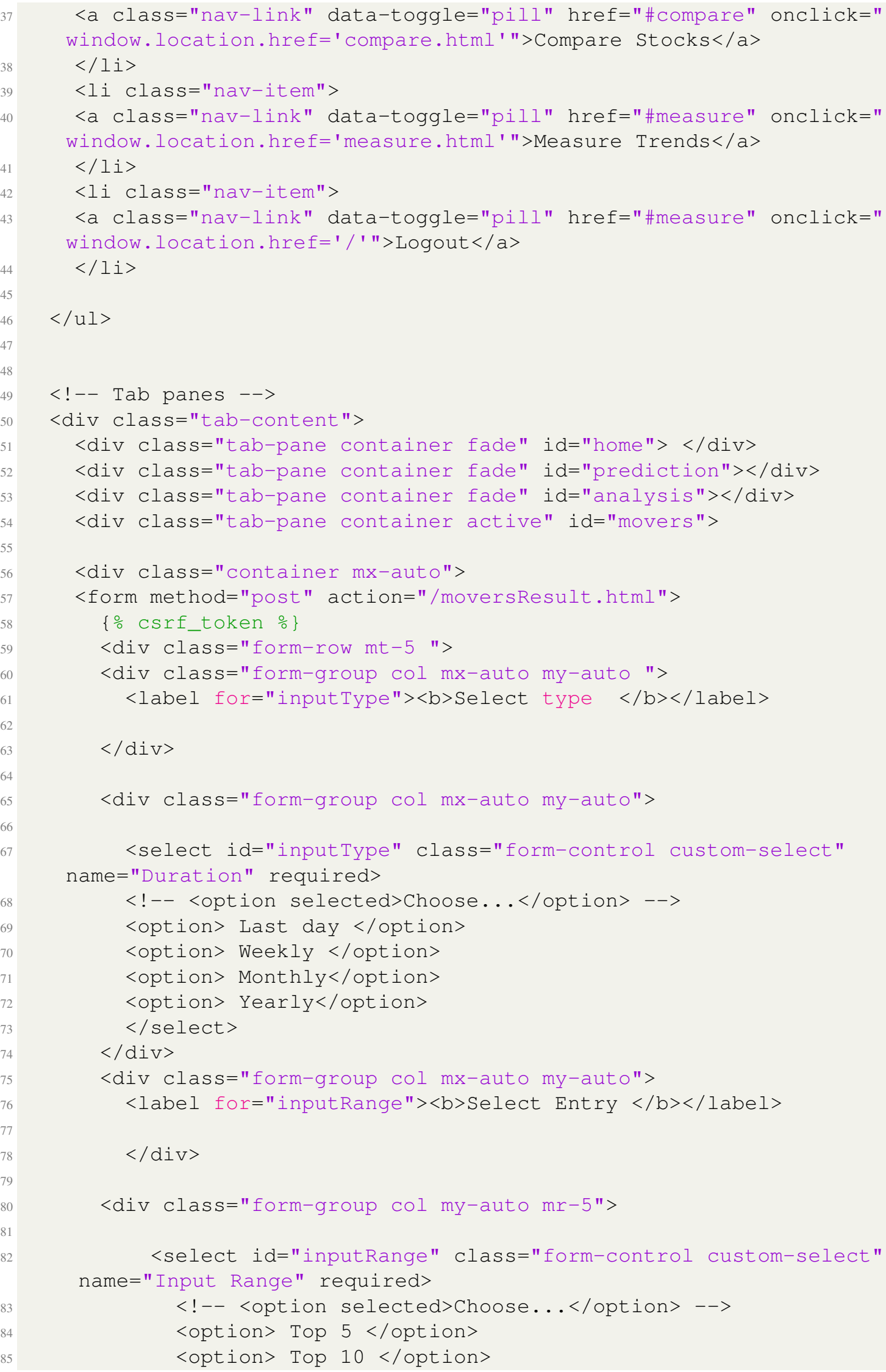




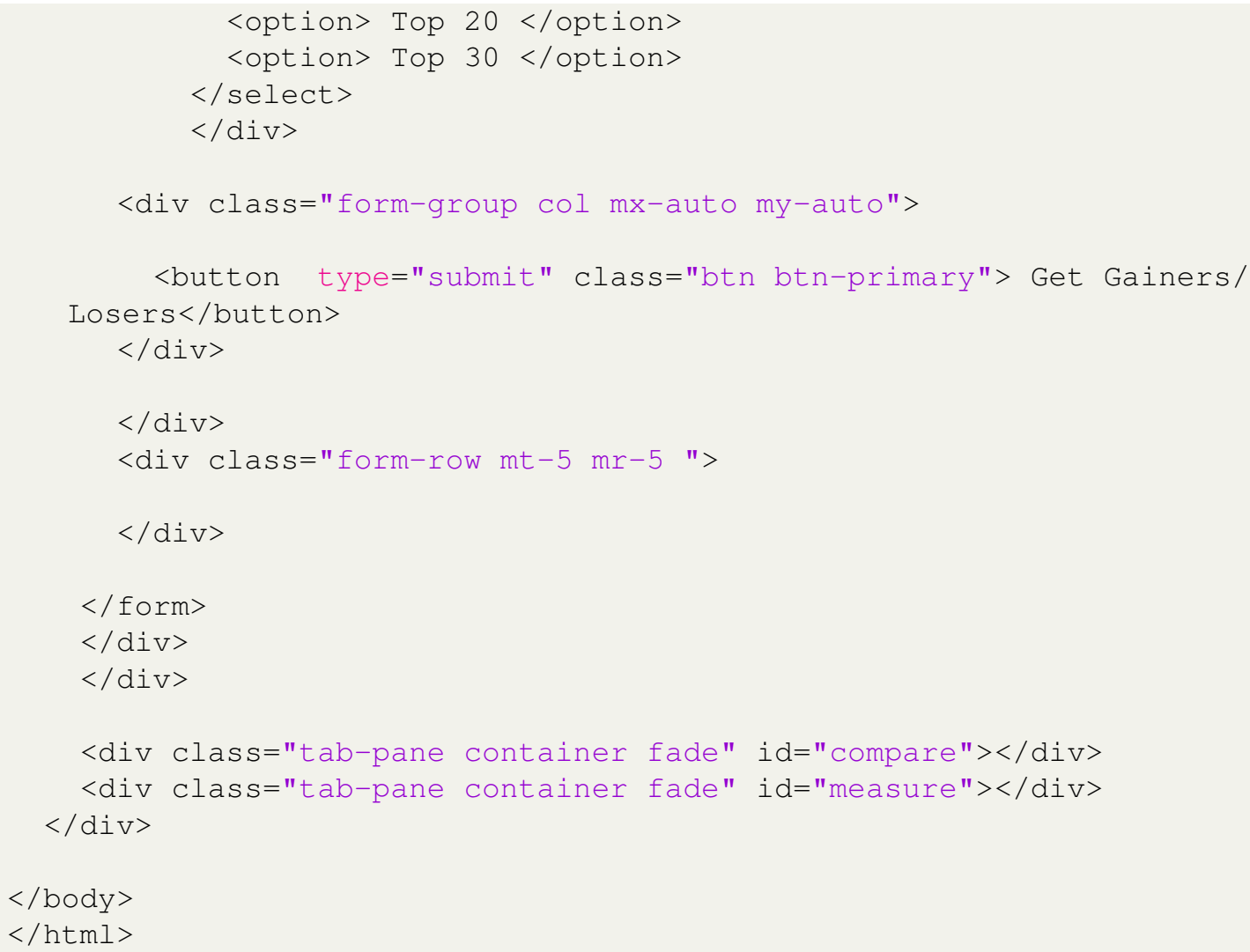

\section{Code A.2.3.22: movers.html}

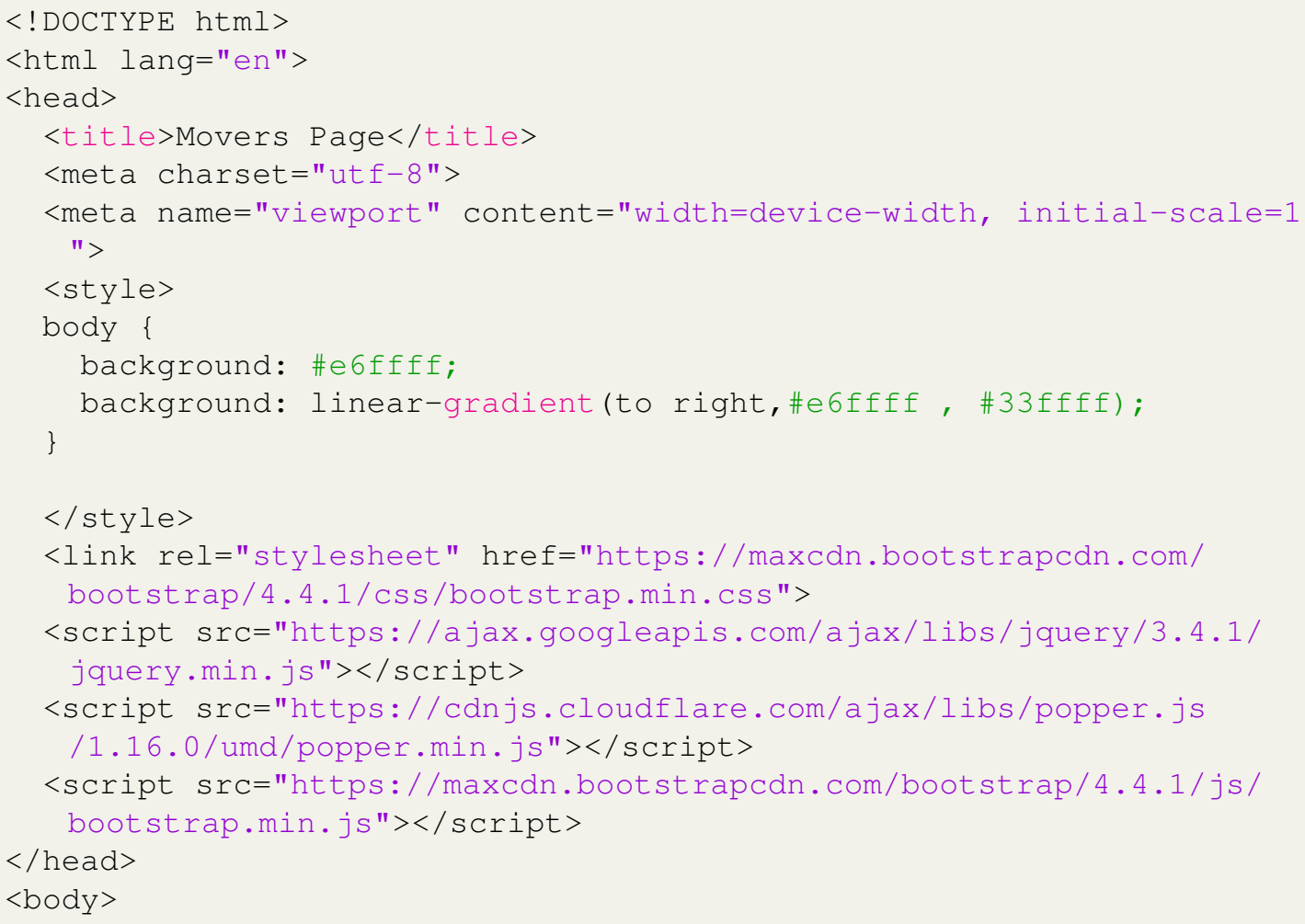




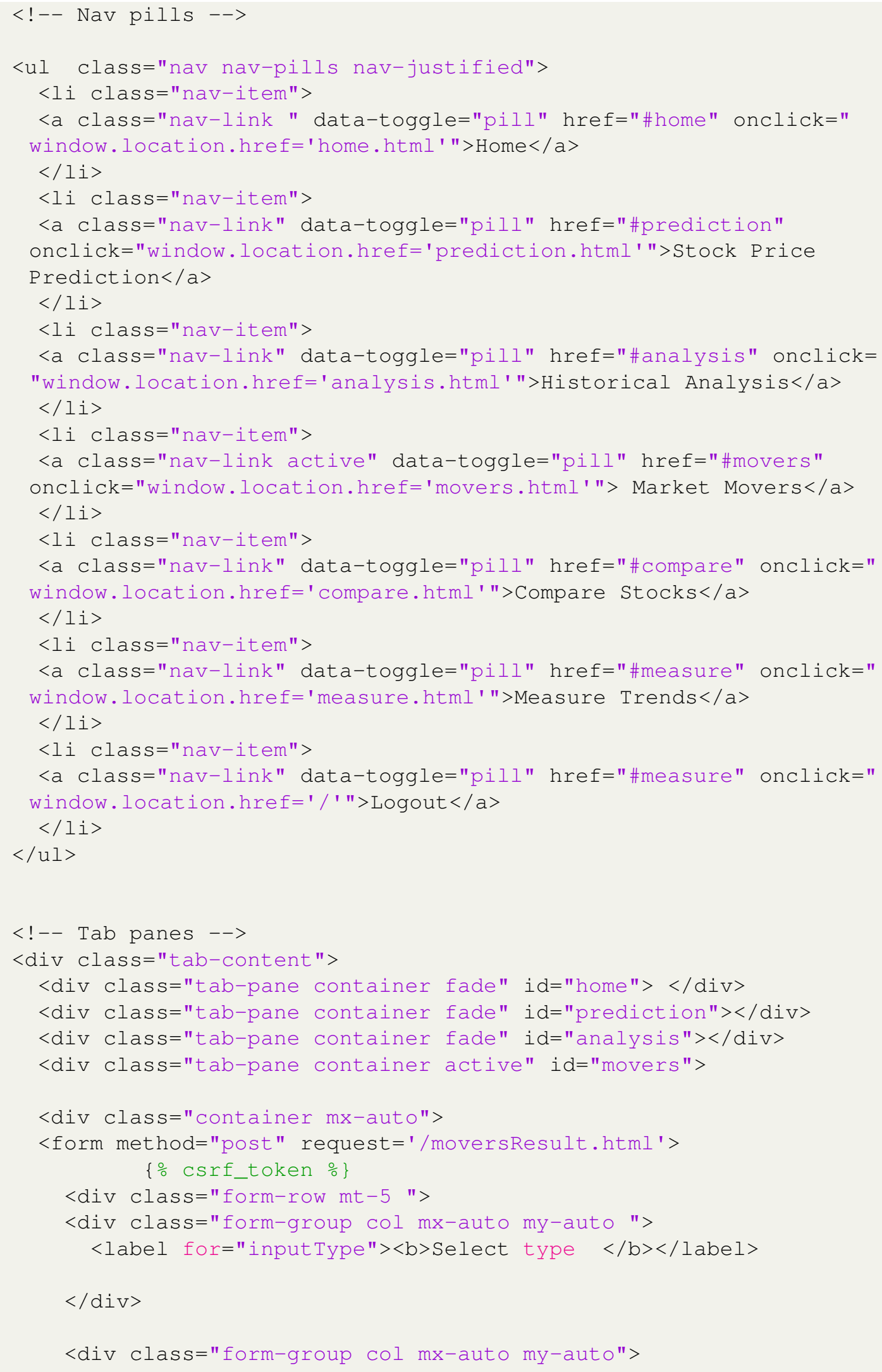




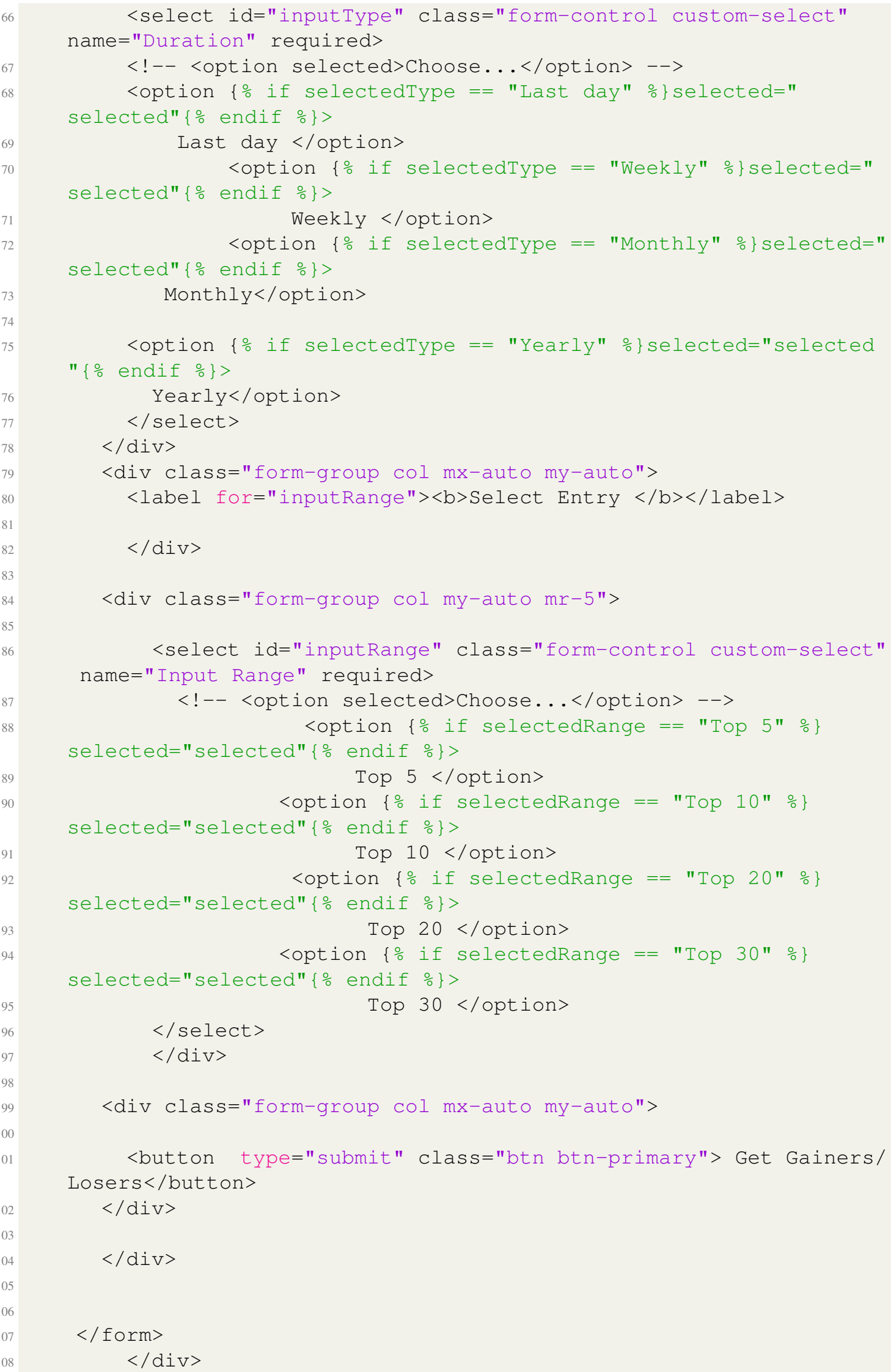




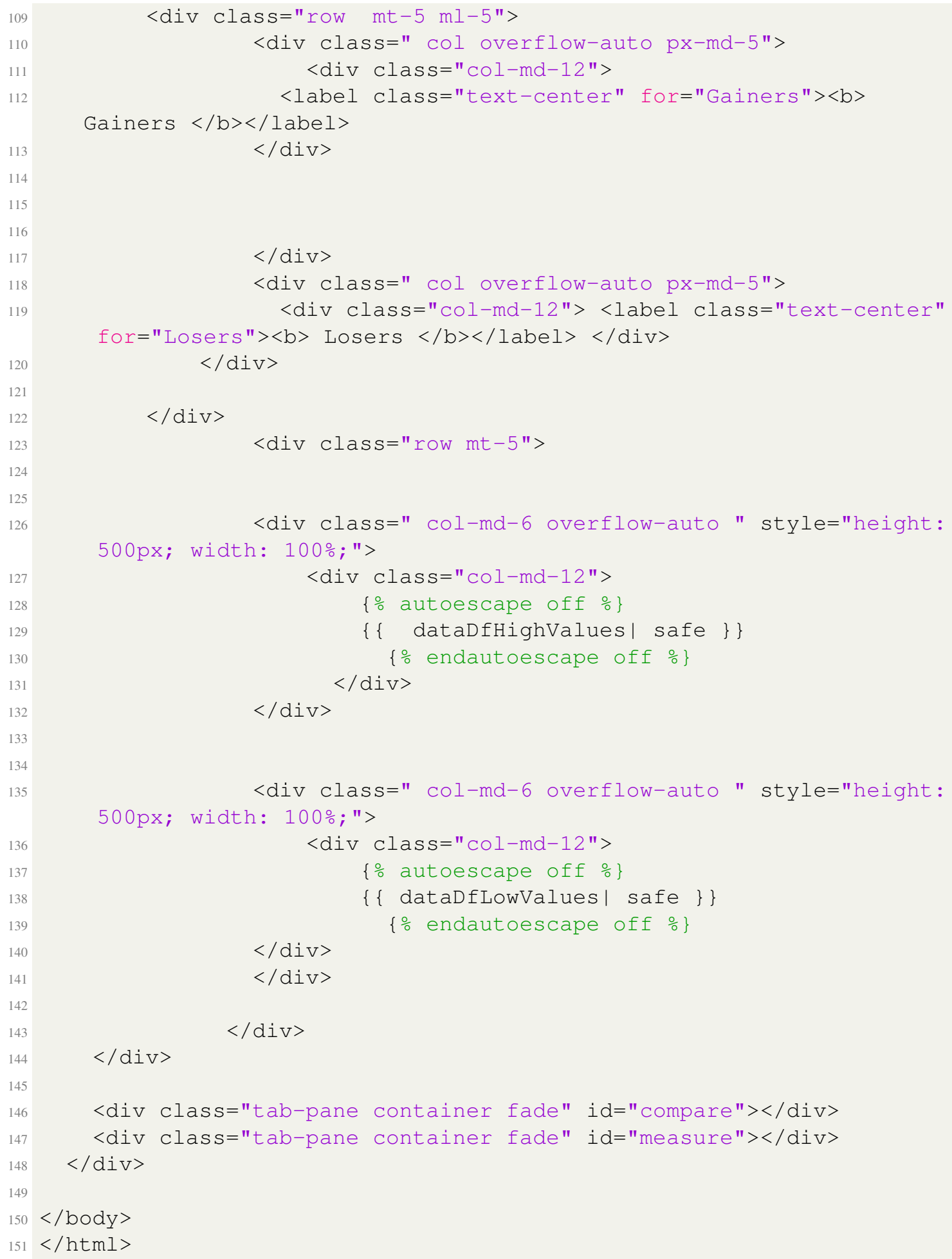

Code A.2.3.23: moversResult.html

\section{For predicting stock price}

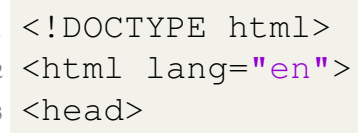




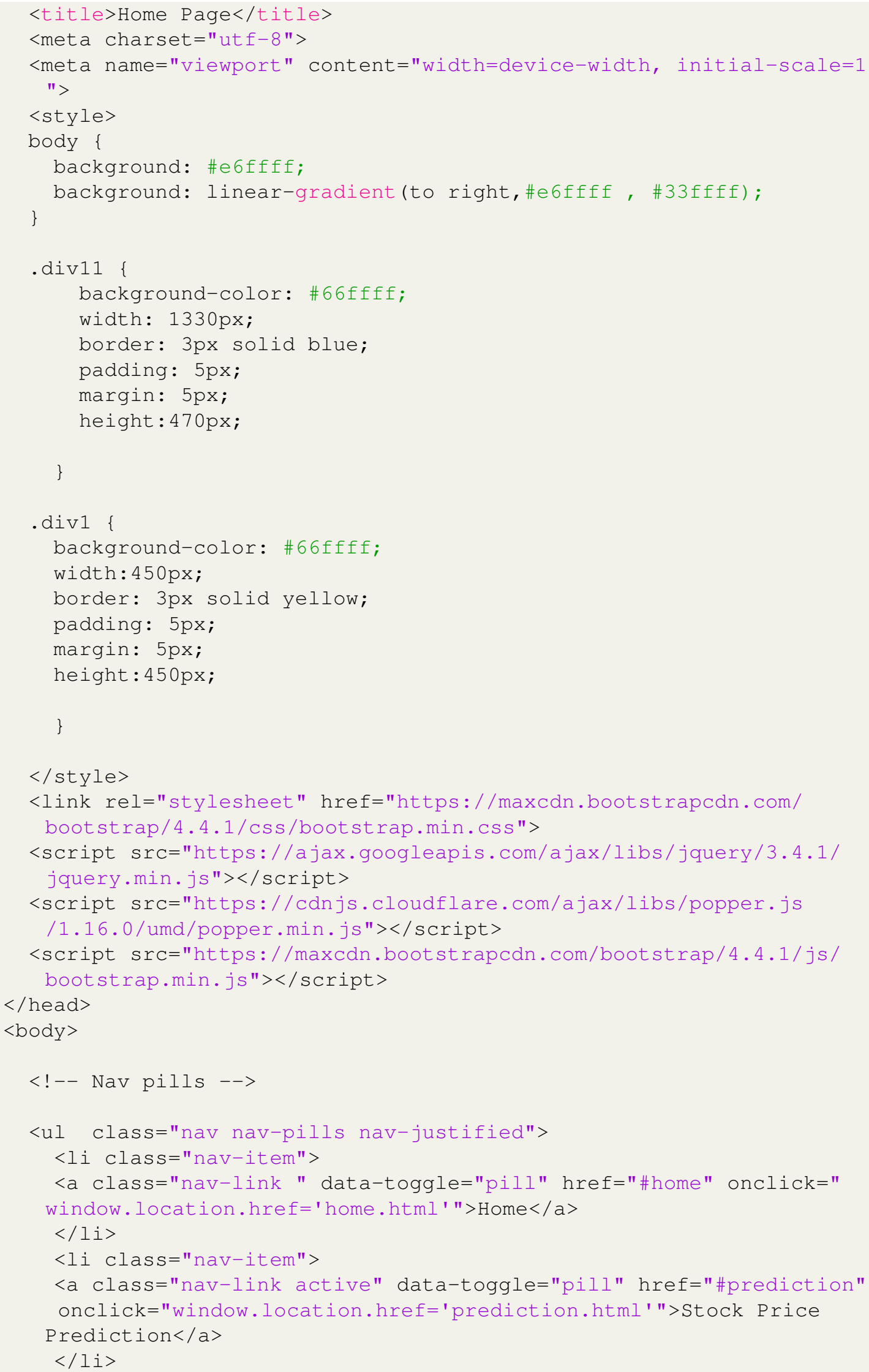




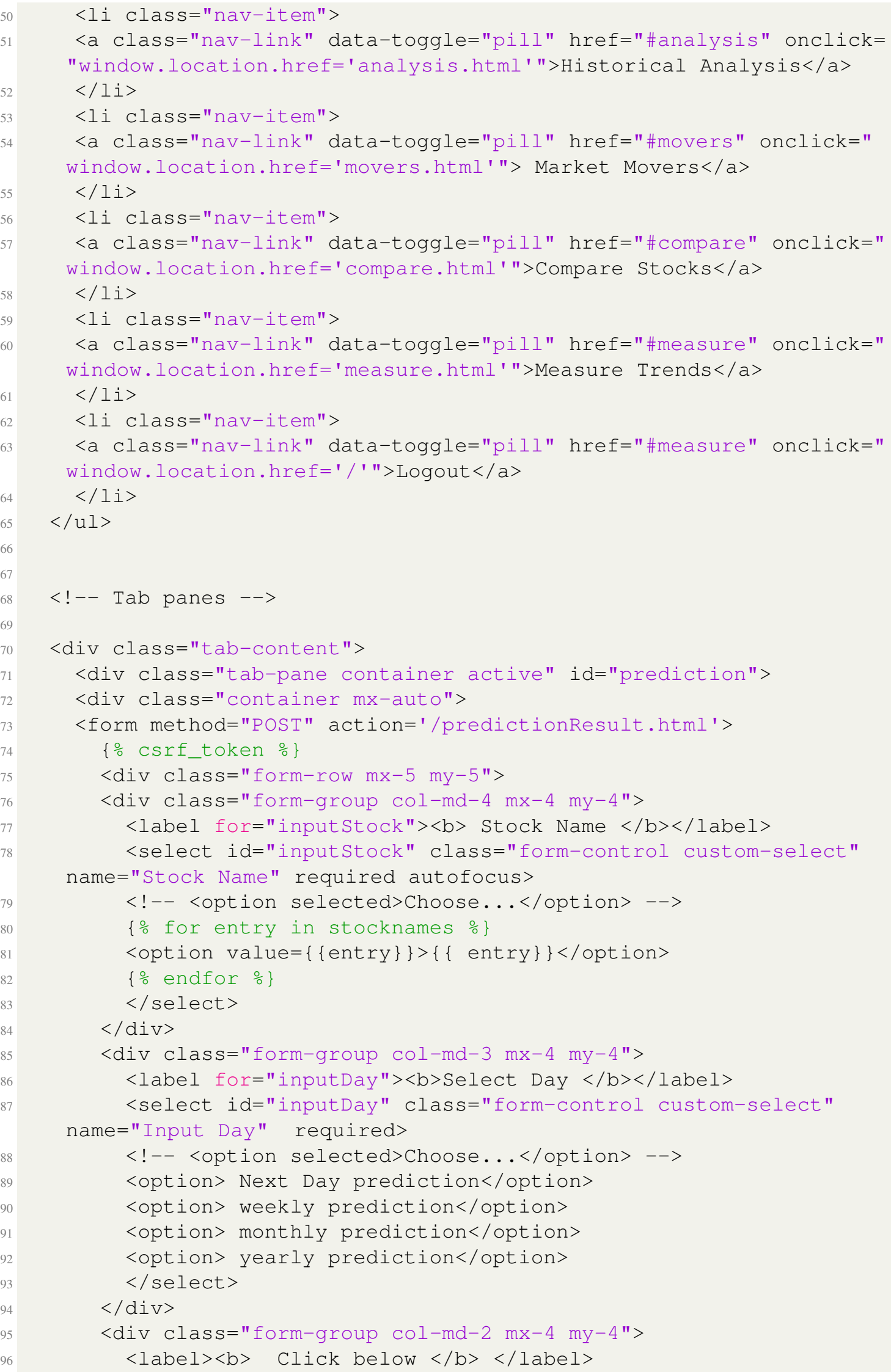




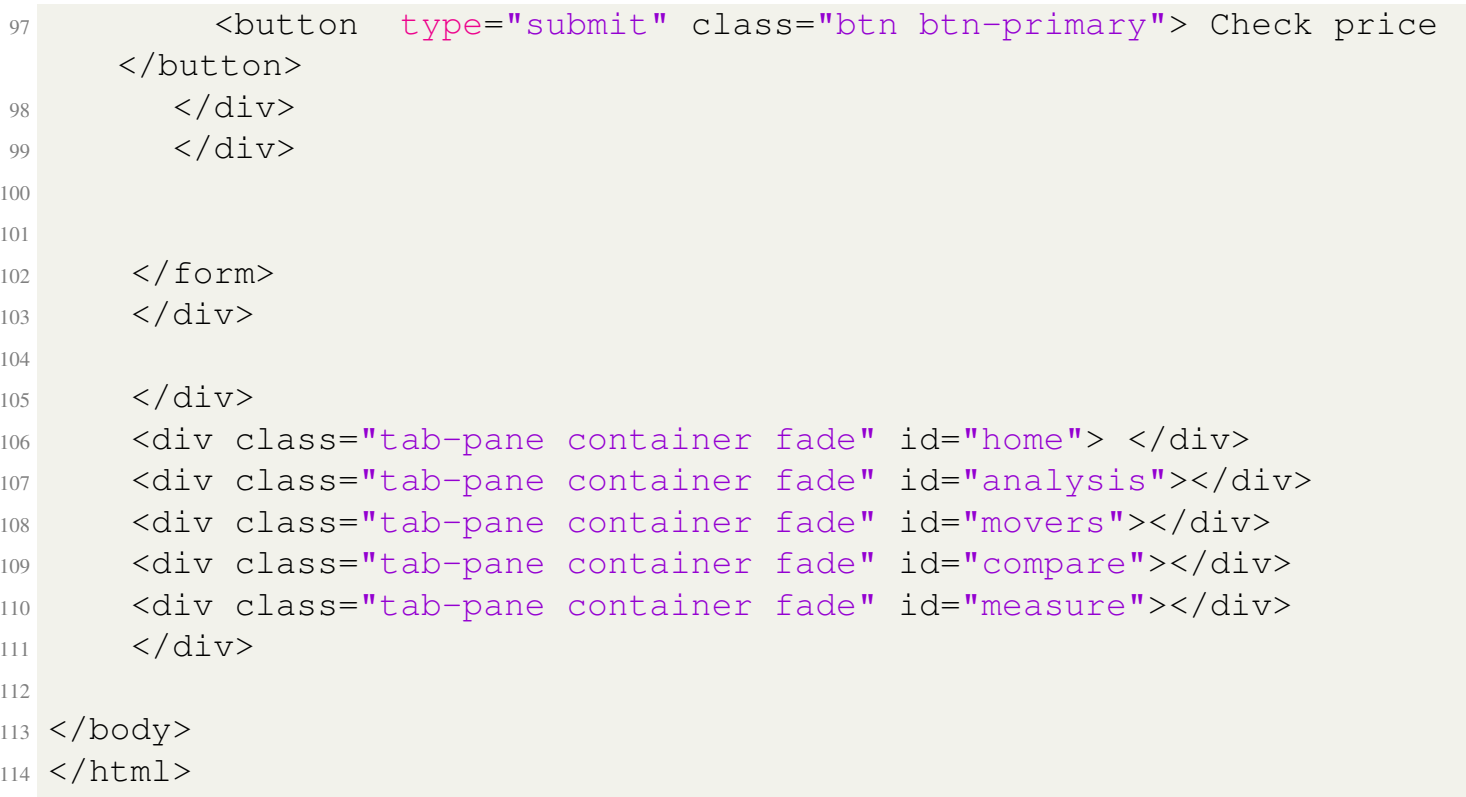

Code A.2.3.24: prediction.html

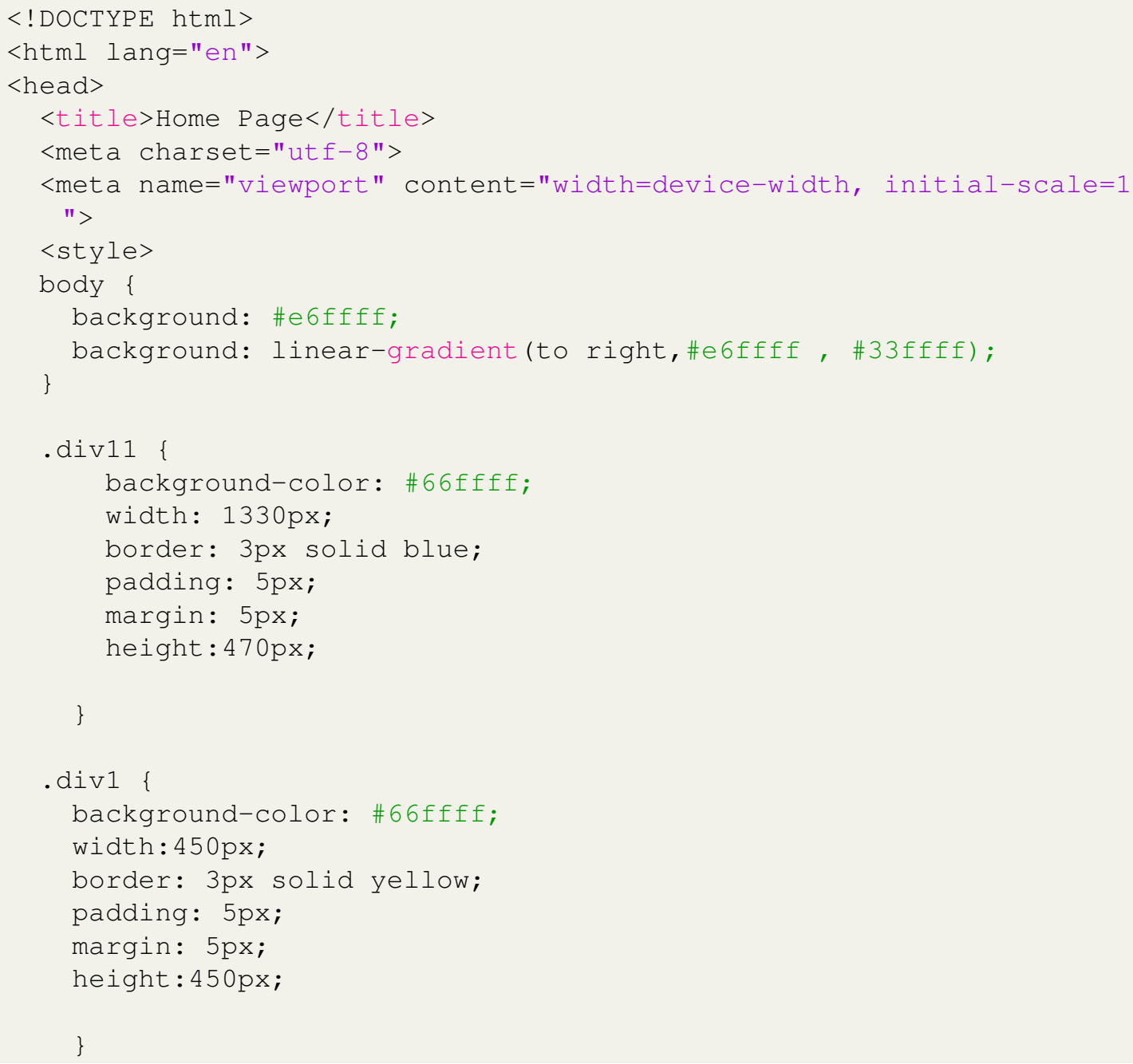




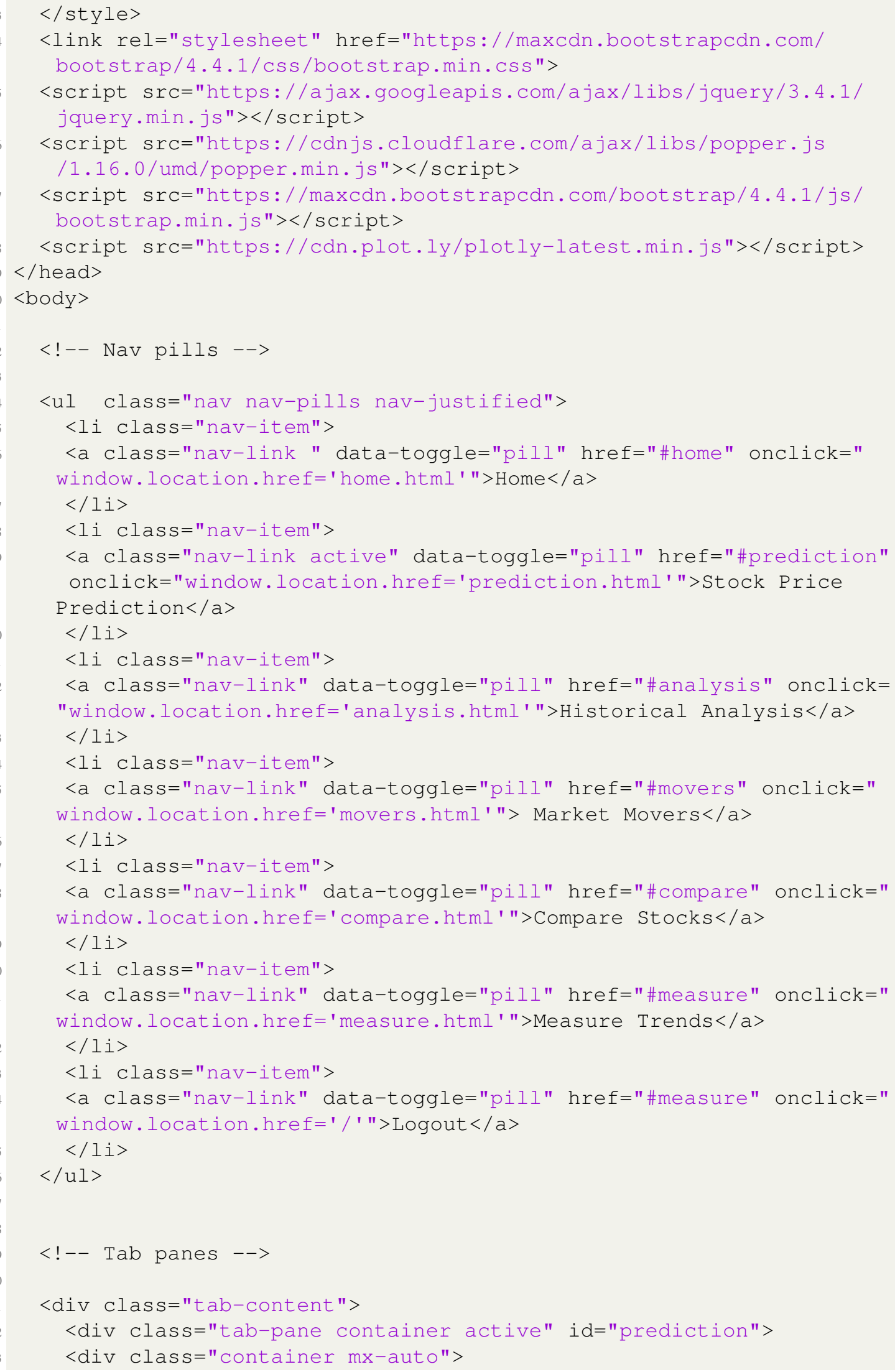




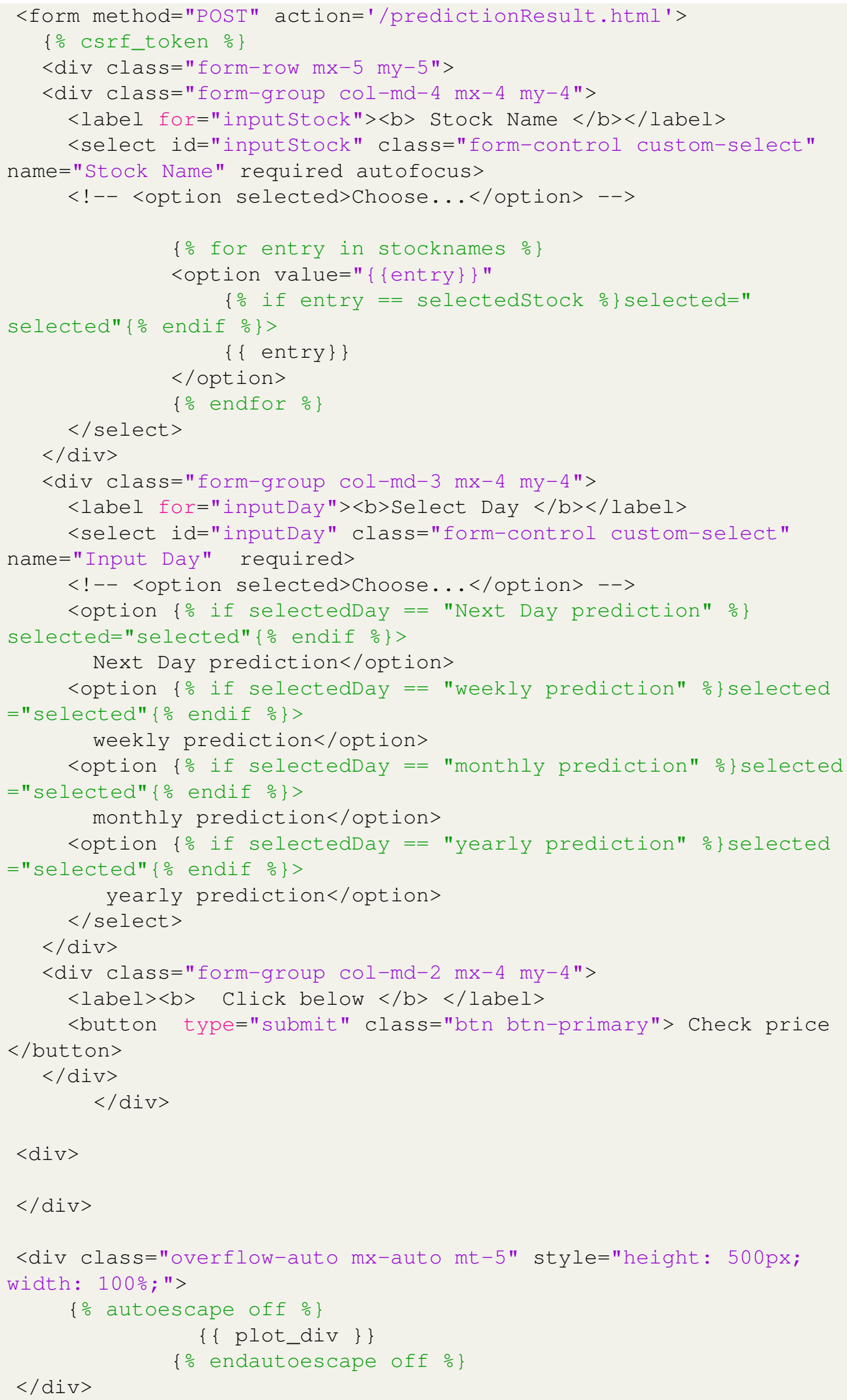




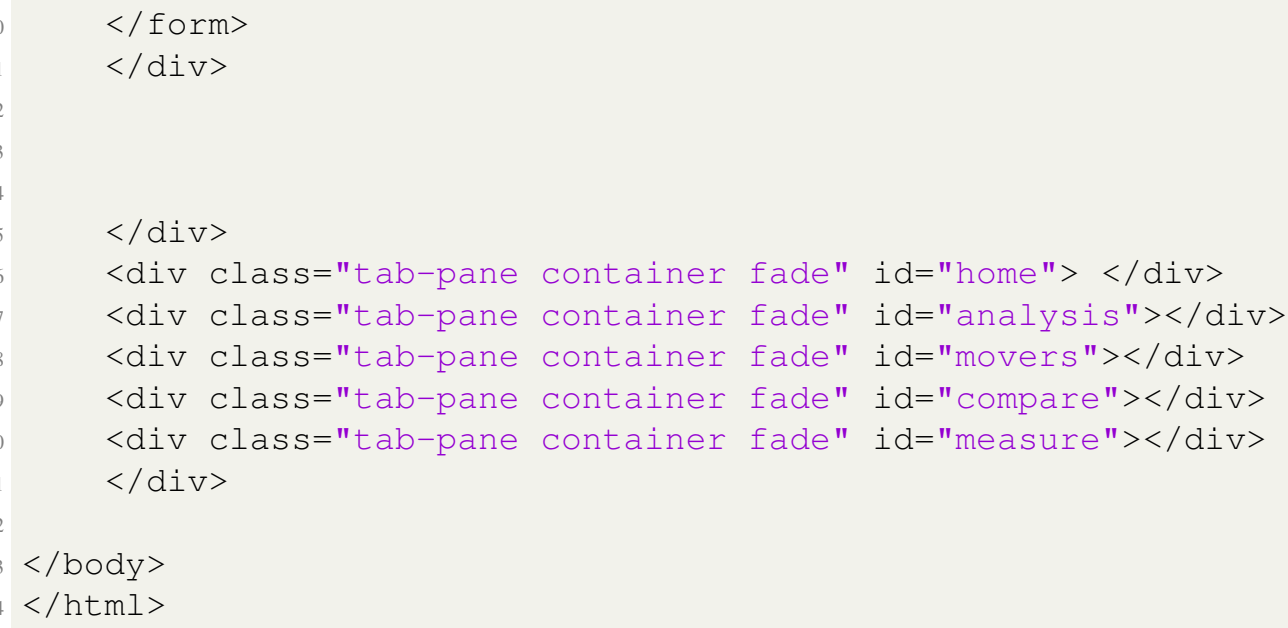

Code A.2.3.25: predictionResult.html

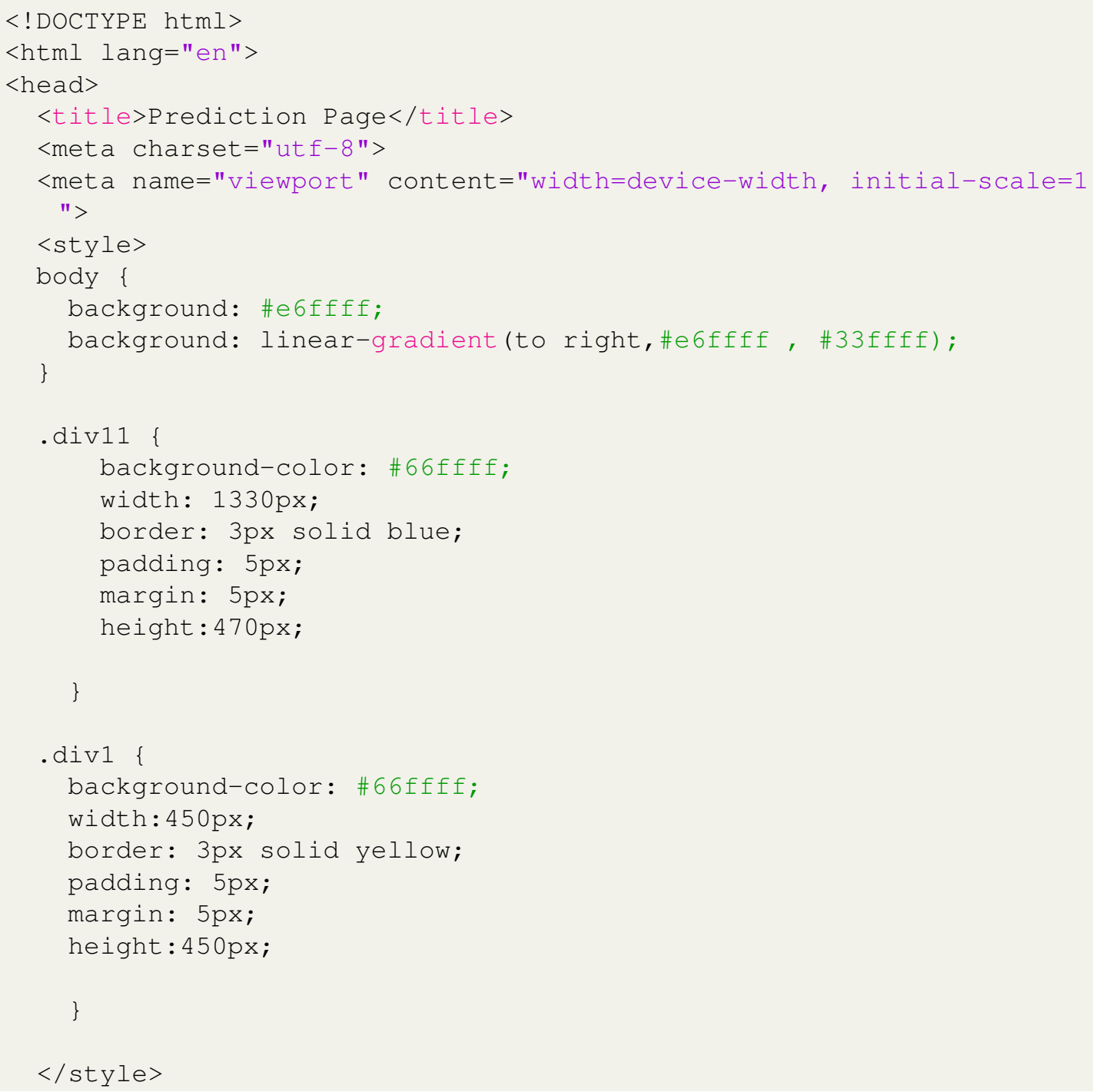


<link rel="stylesheet" href="https://maxcdn.bootstrapcdn.com/ bootstrap/4.4.1/css/bootstrap.min.css">

<script src="https://ajax.googleapis.com/ajax/libs/jquery/3.4.1/ jquery.min.js" $></$ script $>$

<script src="https://cdnjs.cloudflare.com/ajax/libs/popper.js /1.16.0/umd/popper.min.js" $></$ script $>$

<script src="https://maxcdn.bootstrapcdn.com/bootstrap/4.4.1/js/ bootstrap.min.js" $></$ script $>$

$</$ head $>$

$<$ body $>$

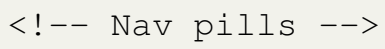




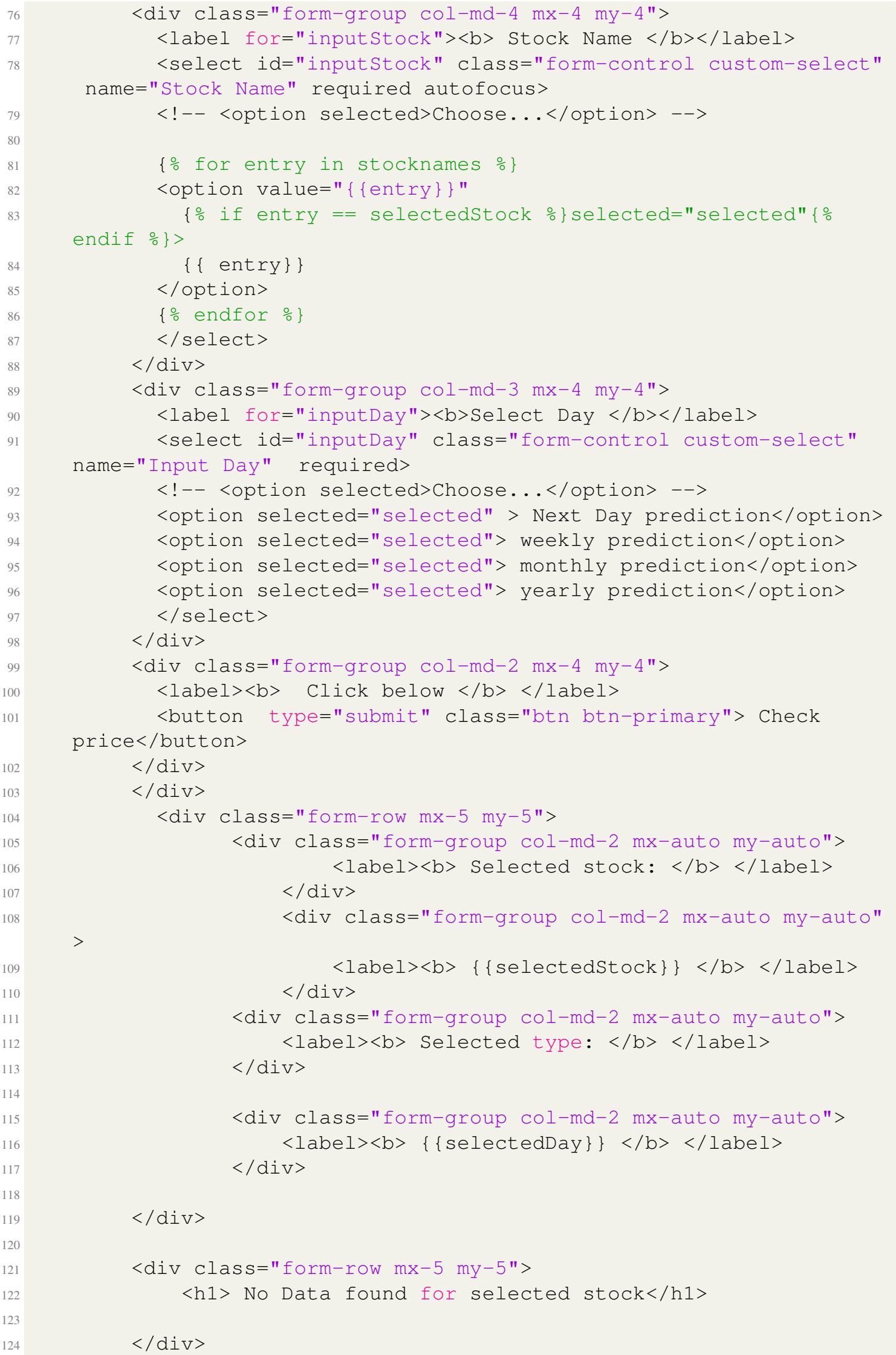




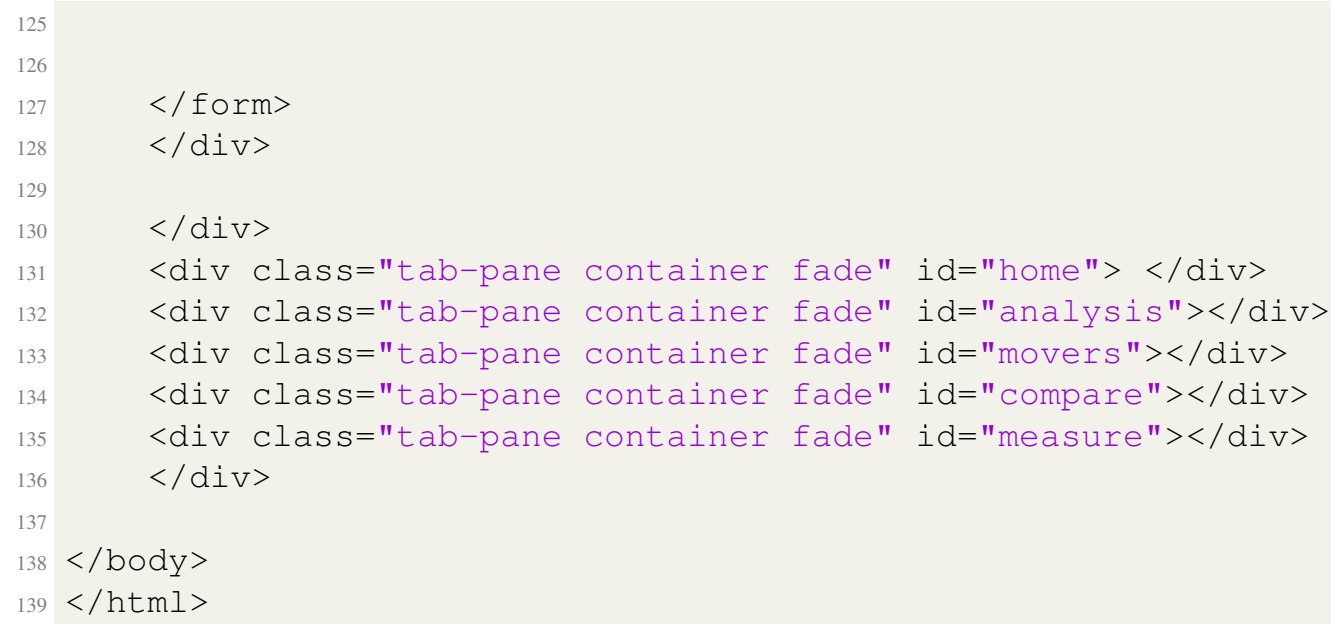

Code A.2.3.26: predictionResultNoData.html

\section{For login and signup page}

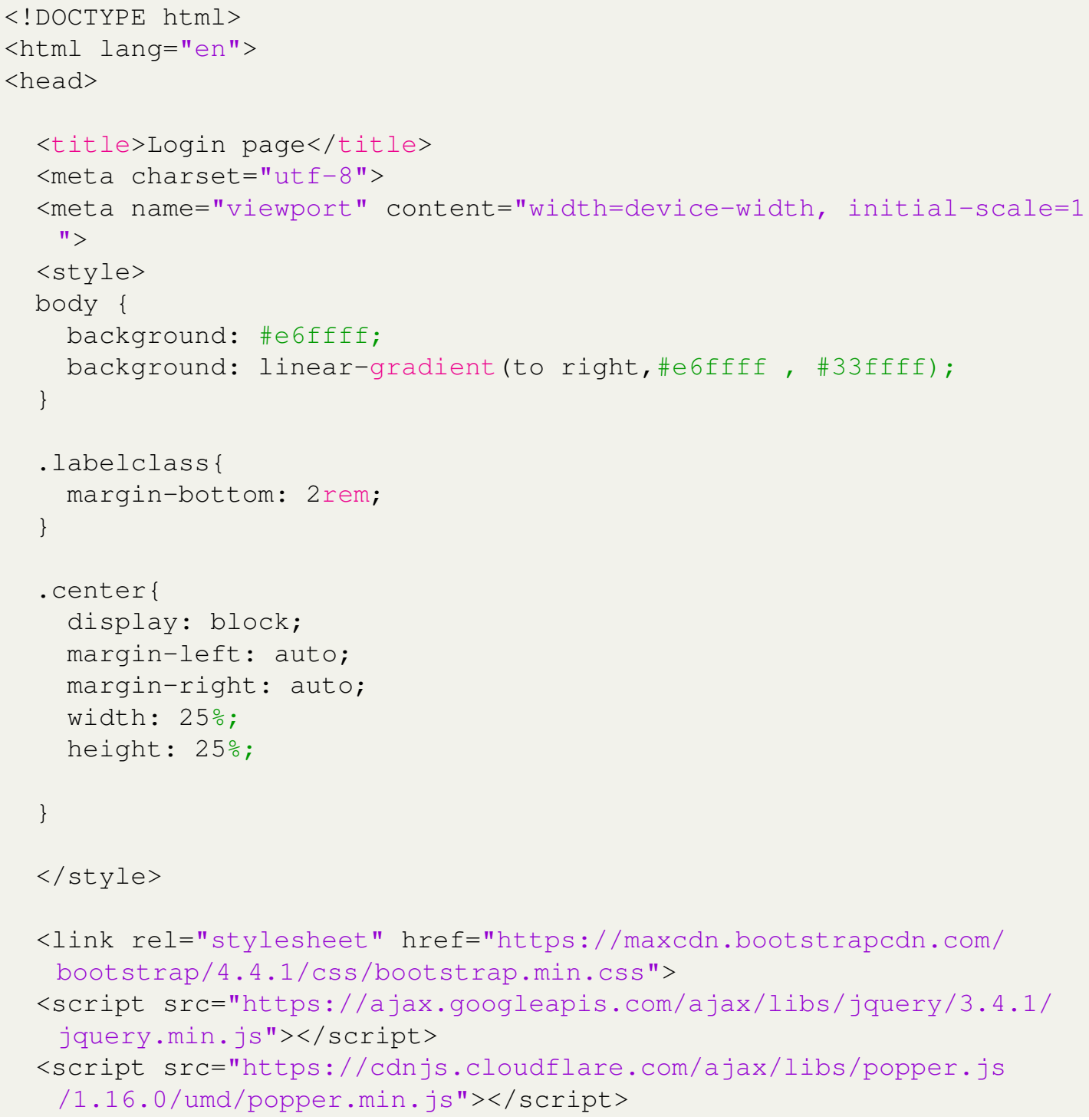




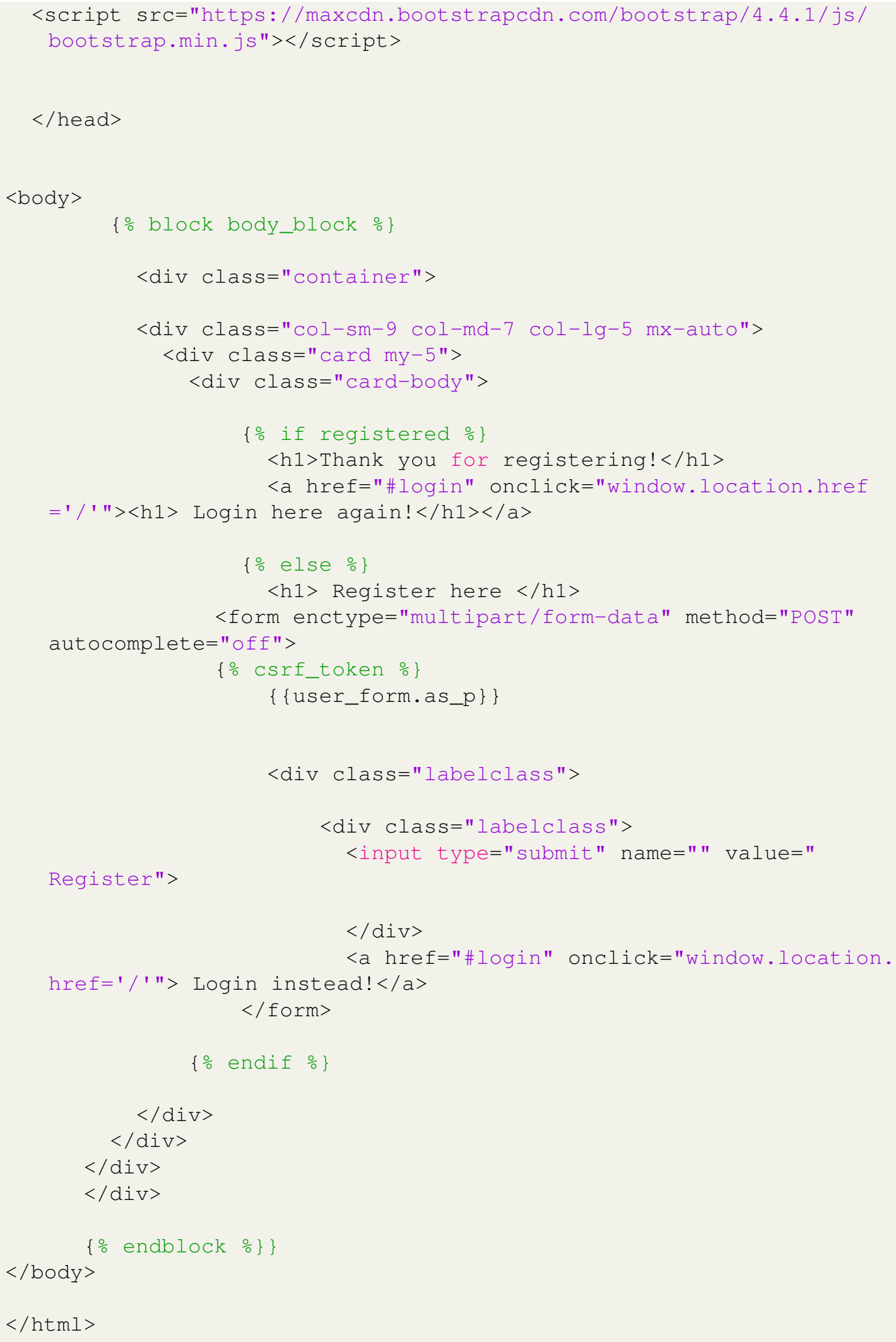

Code A.2.3.27: signup.html 


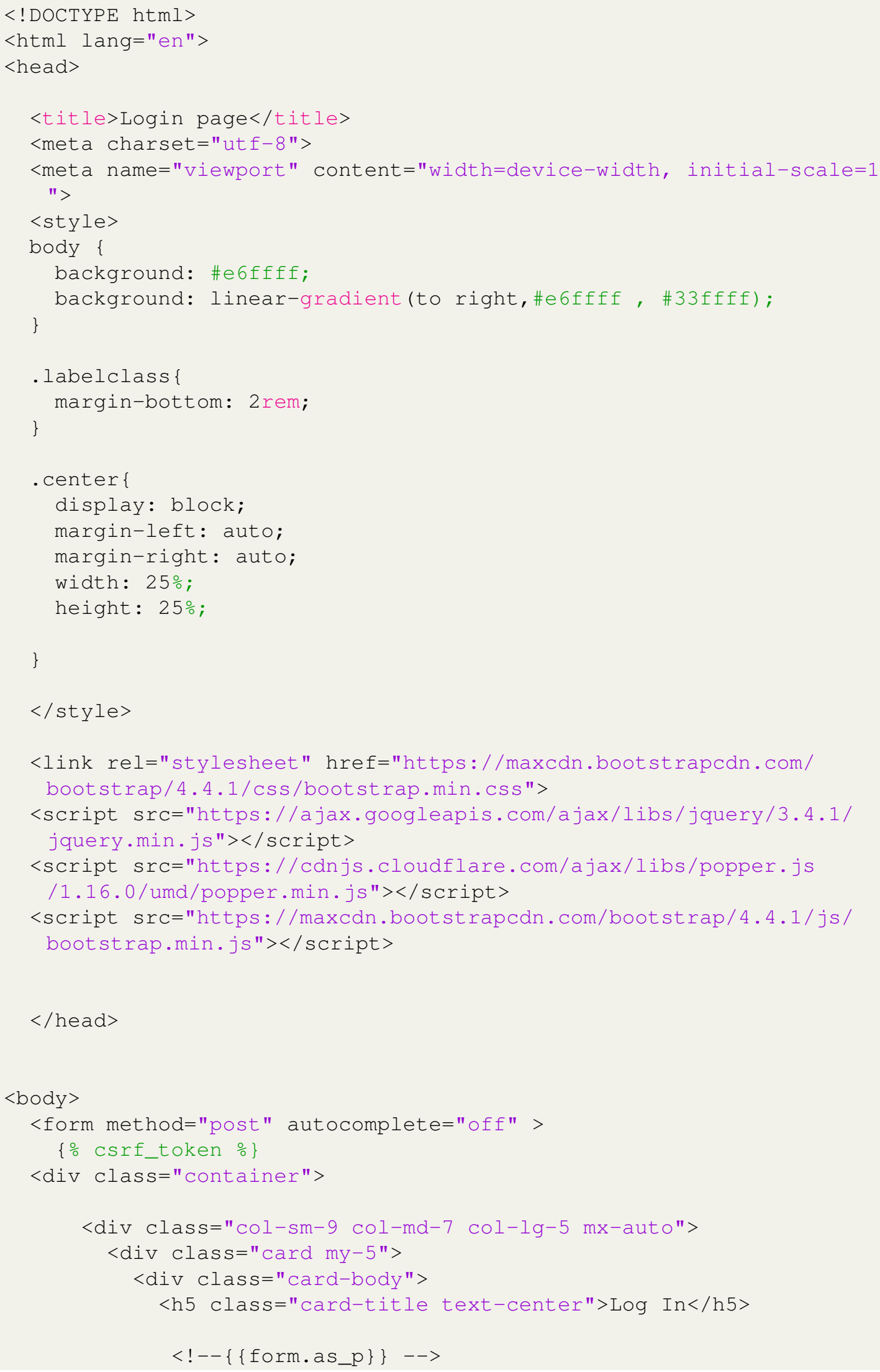




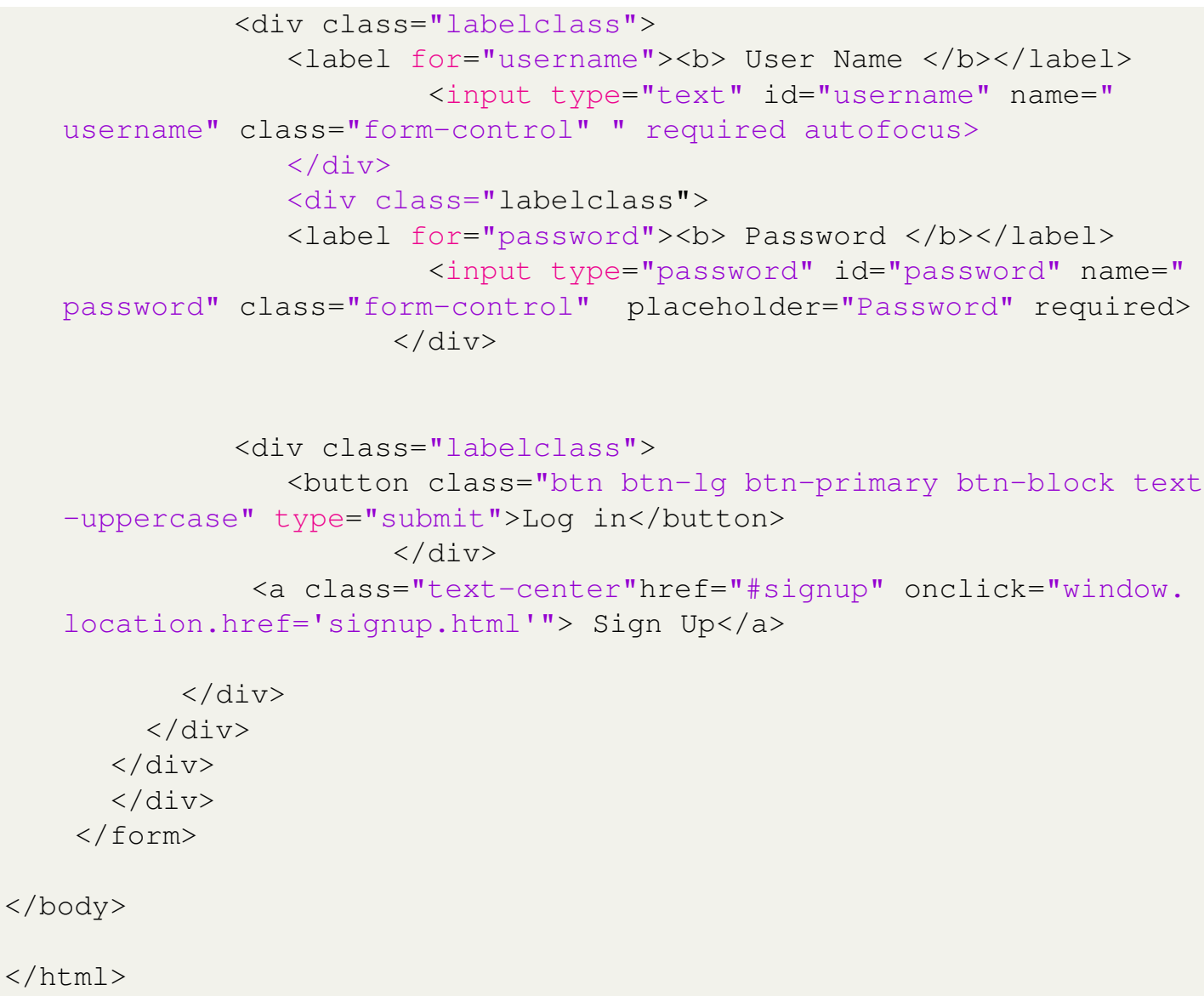

Code A.2.3.28: Loginpage.html

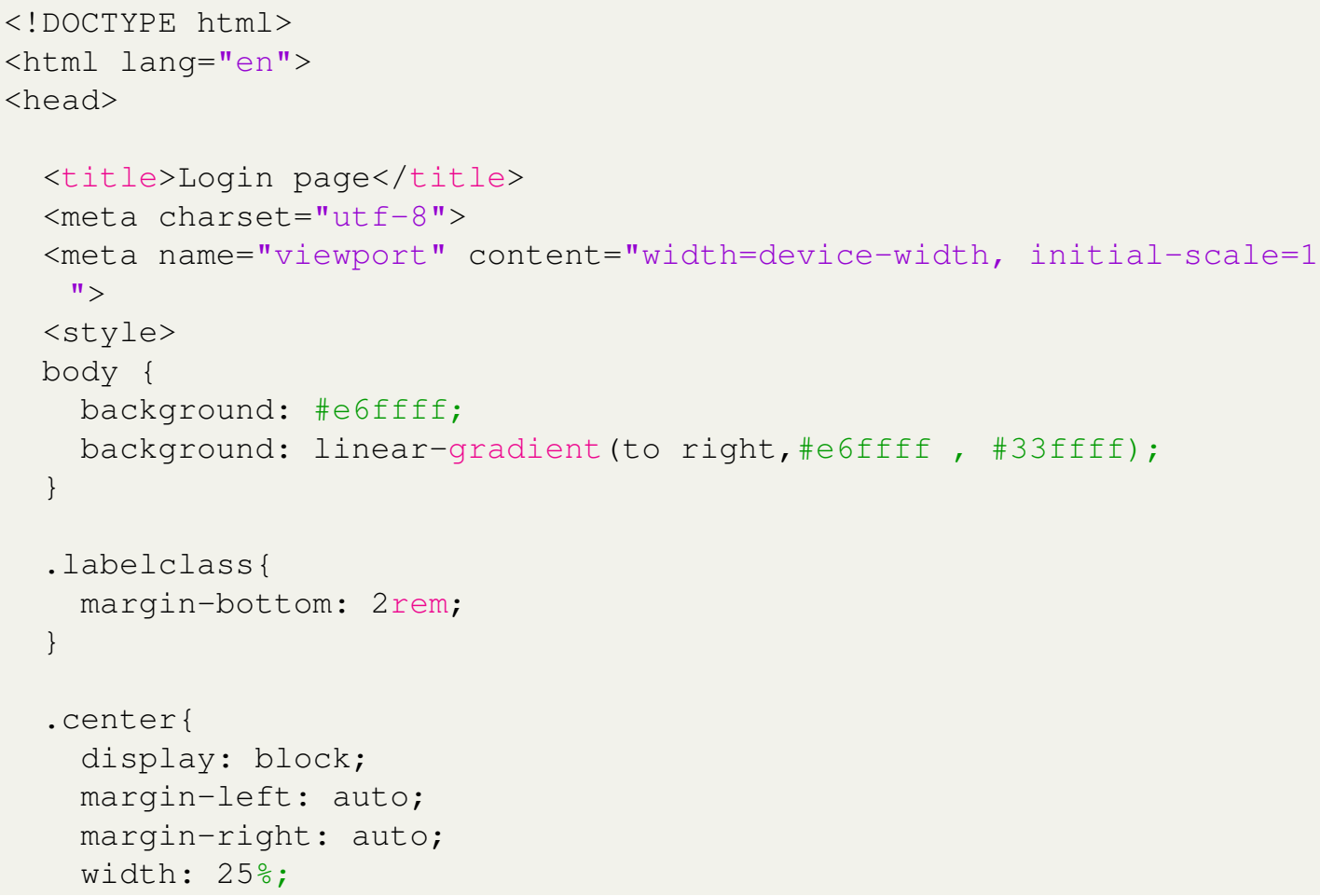




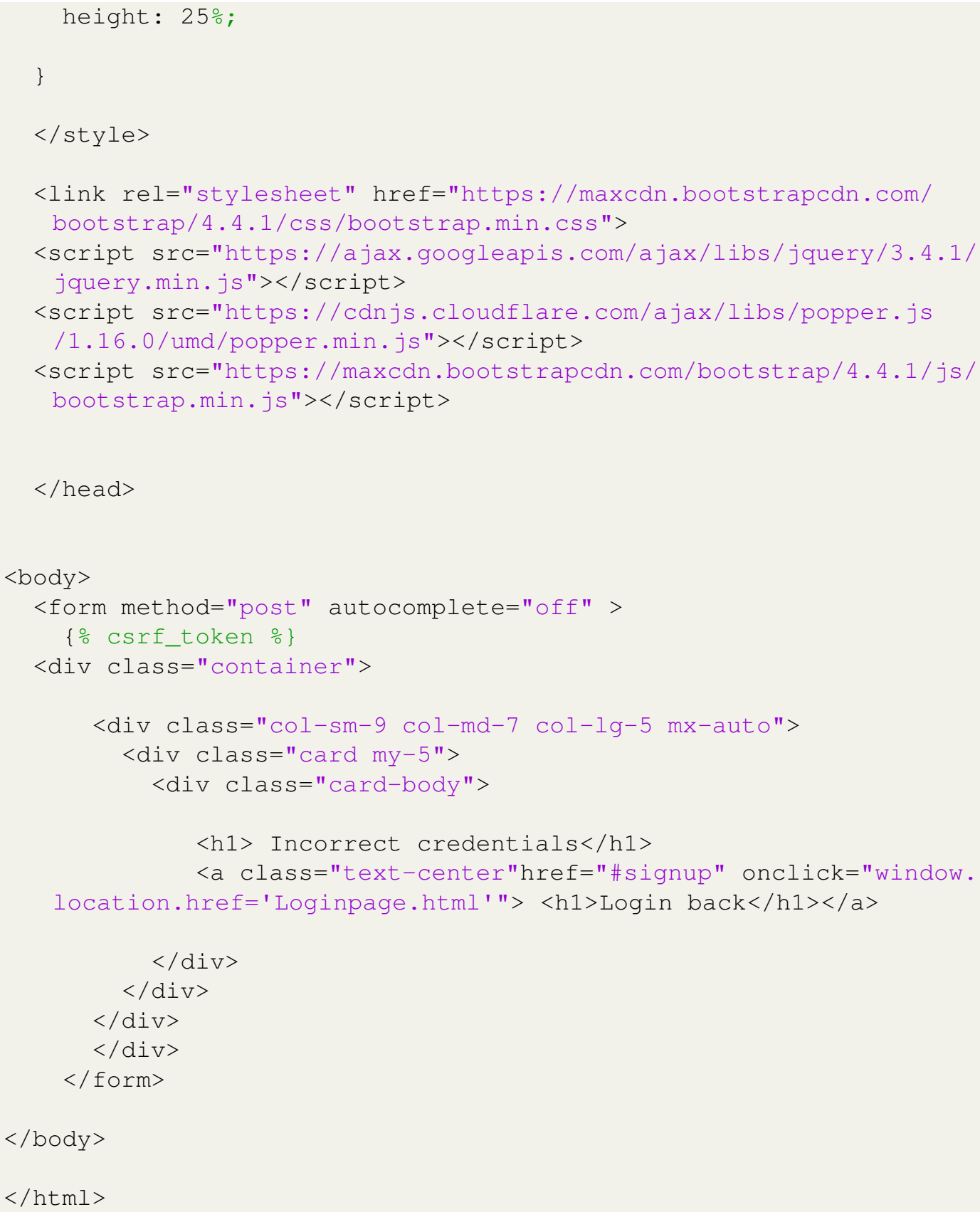

\section{Code A.2.3.29: invalidLogin.html}

\section{Home page}

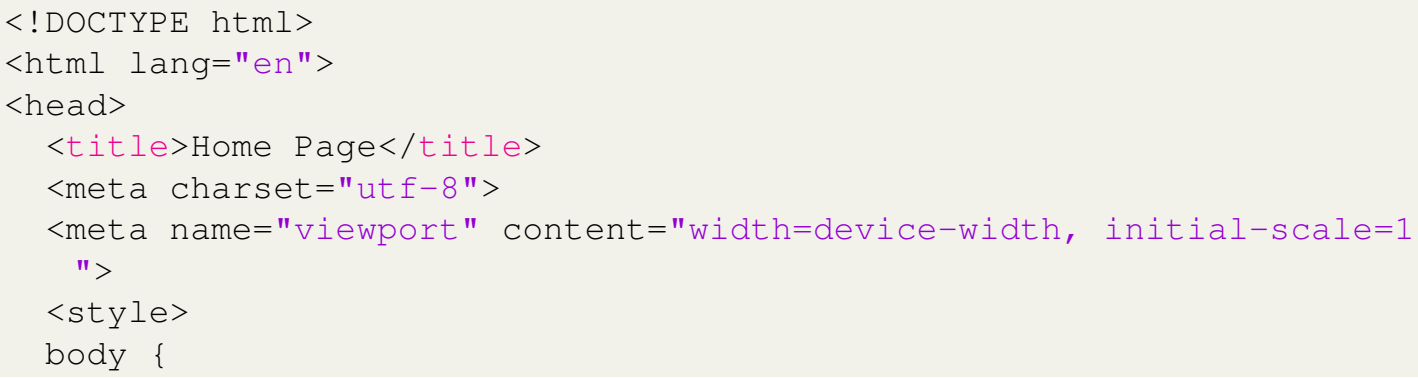




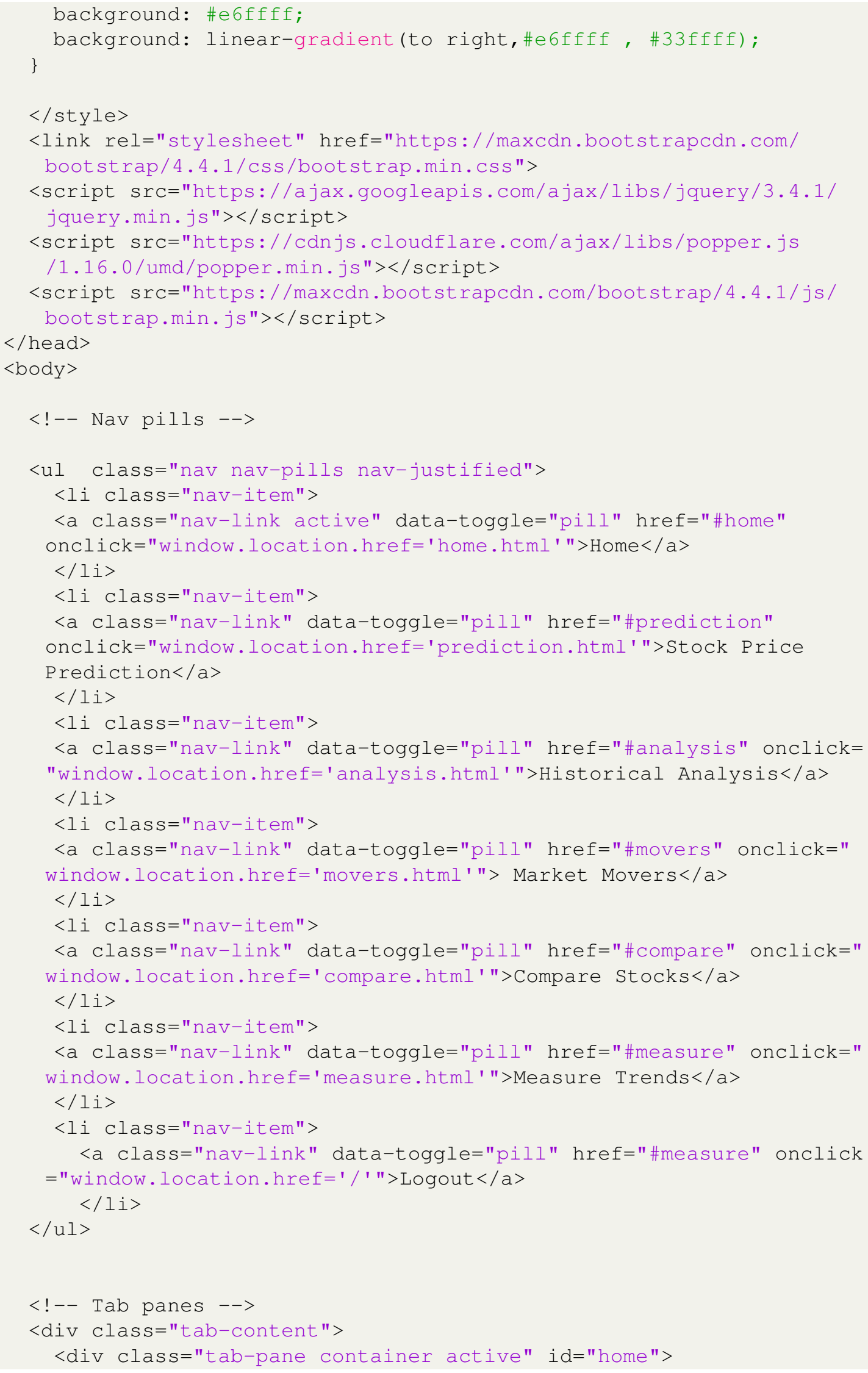




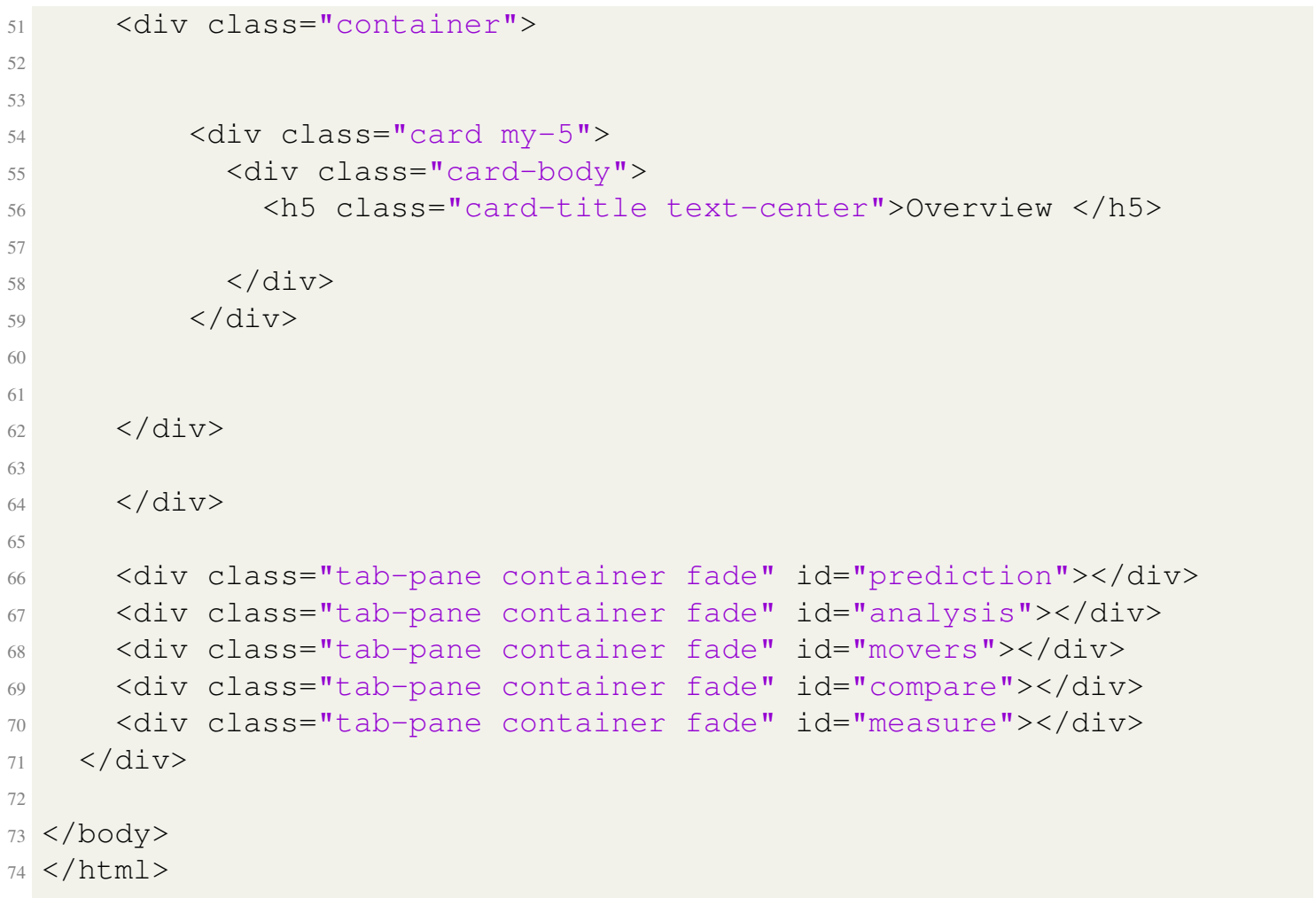

\section{Code A.2.3.30: home.html}

\section{A.2.4 Execution}

The execution of Django project is done with the help of manage.py file and command used is - python manage.py runserver

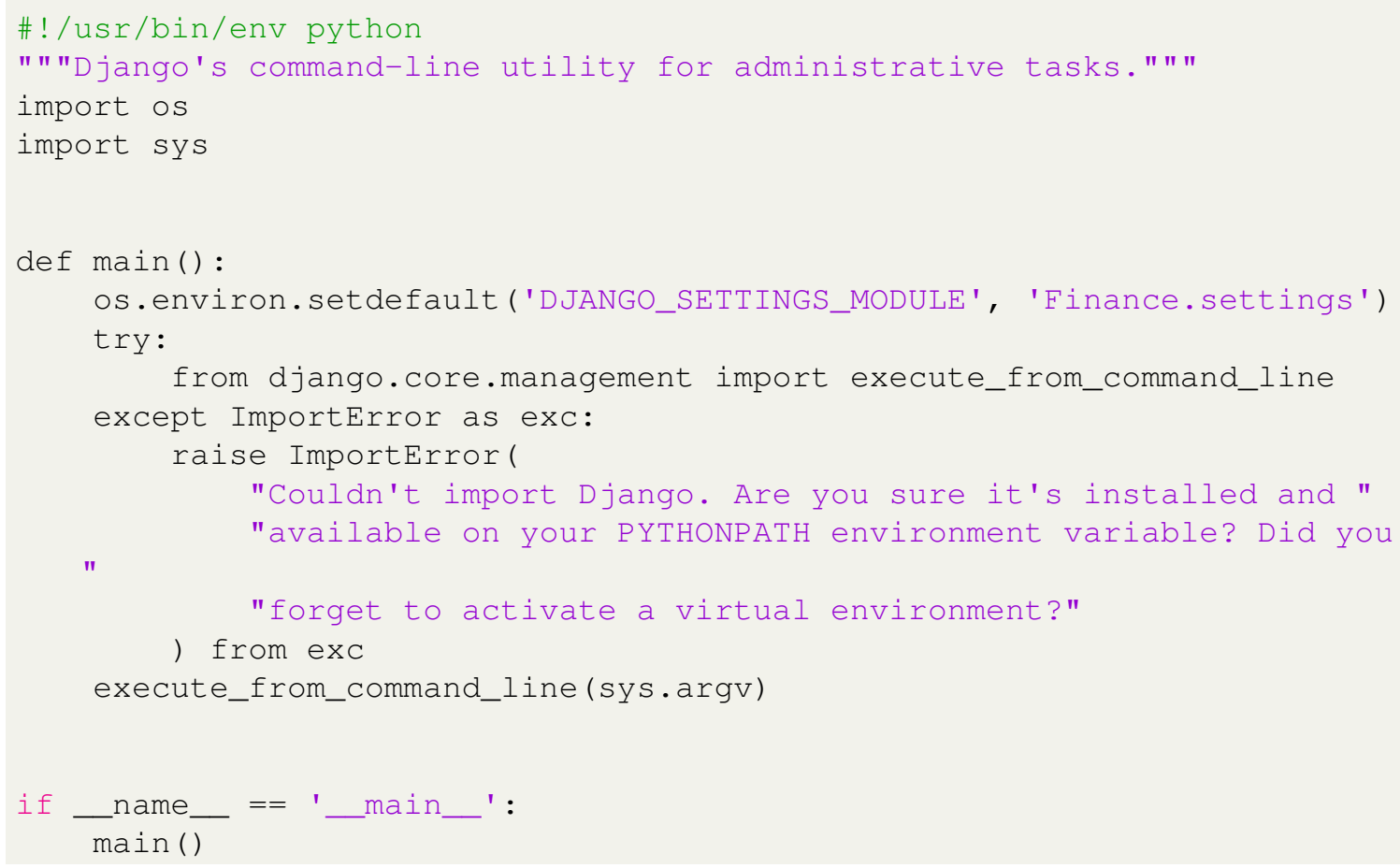




\section{Code A.2.4.1: manage.py}

\section{A.2.5 Initial testing of jobs using ab-benchmark tool}

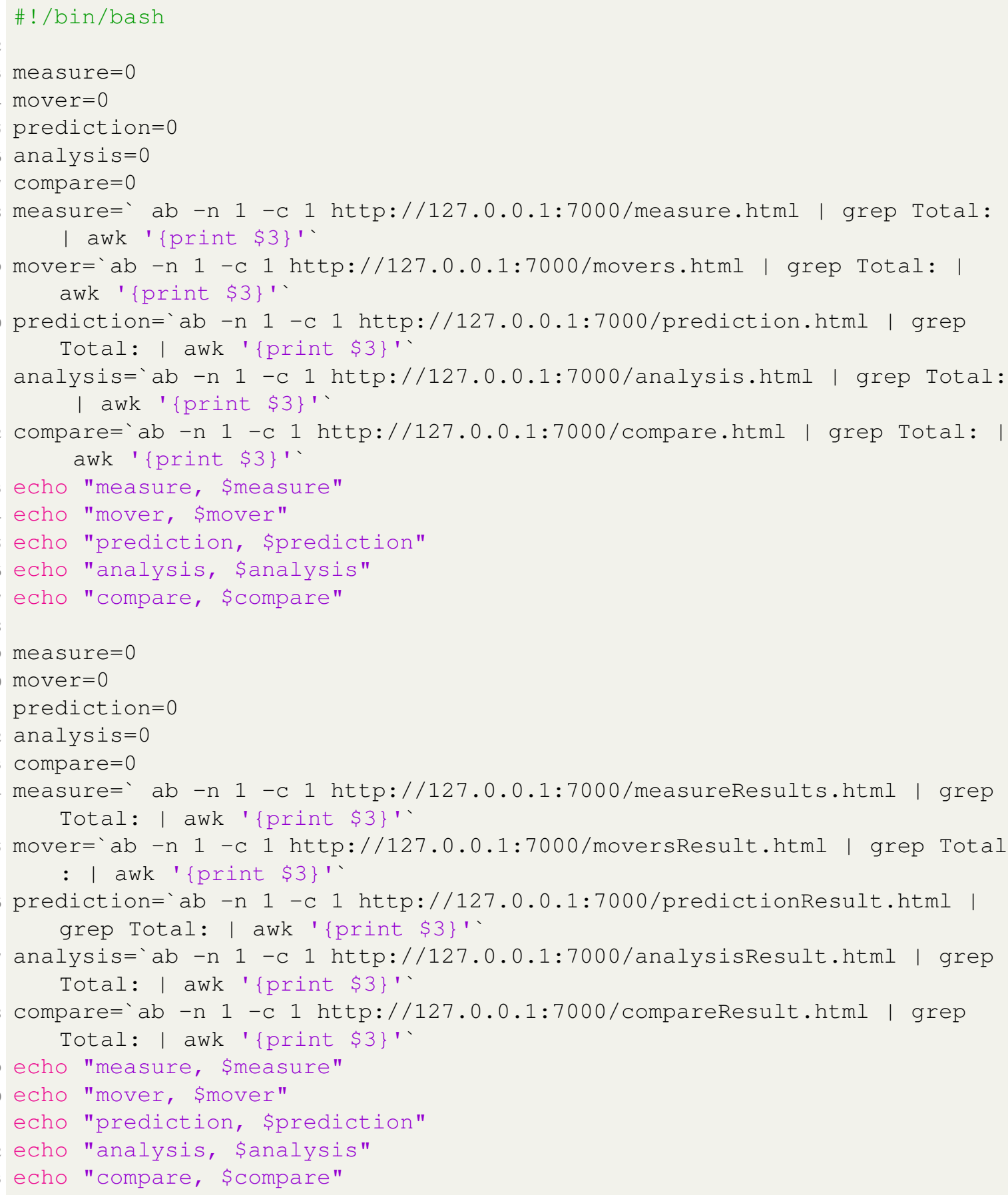

Code A.2.5.1: Execution time for all the types of job requests using ab-benchmark tool

Below is the output generated using above script - 


\begin{tabular}{|c|c|c|}
\hline Type of Job & GET Request(in ms) & POST Request(in ms) \\
\hline measure & 12 & 25 \\
\hline mover & 2 & 22 \\
\hline prediction & 7 & 21 \\
\hline analysis & 10 & 20 \\
\hline compare & 18 & 20 \\
\hline
\end{tabular}

Table A.1: Execution time for all the types of job requests using ab-benchmark tool

\section{A.3 Comparison of appilcation hosted on IBM and AWS Cloud}

\section{A.3.1 Web application on IBM Cloud}

A free tier account was created on IBM Cloud with basic services provided by the cloud to deploy web application. A Django application can be deployed by creating a cloud foundry app with the help of Django management script. Web application was successfully deployed on IBM Cloud and given below are images indicating the successful deployment of web application-

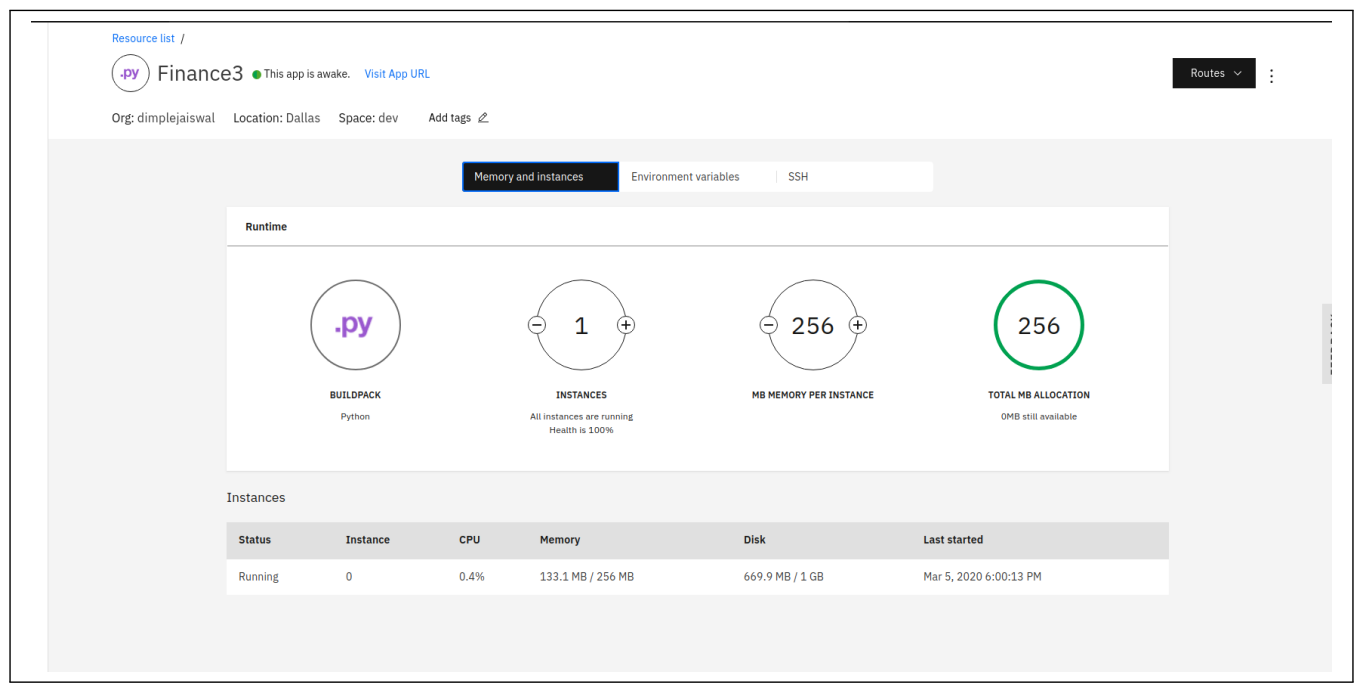

Figure A.4: Dashboard of web application hosted on IBM Cloud 


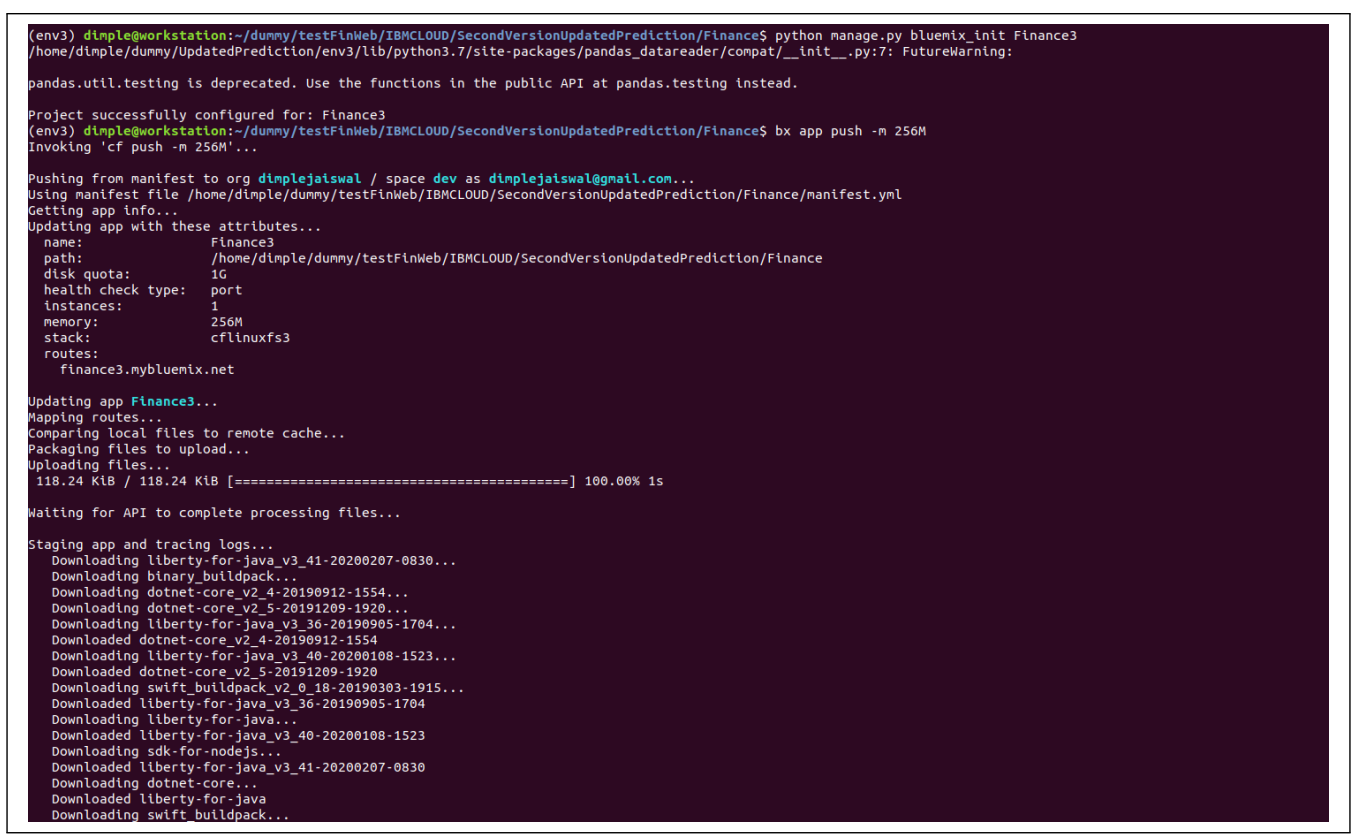

Figure A.5: Deployement of web application on IBM Cloud- part 1

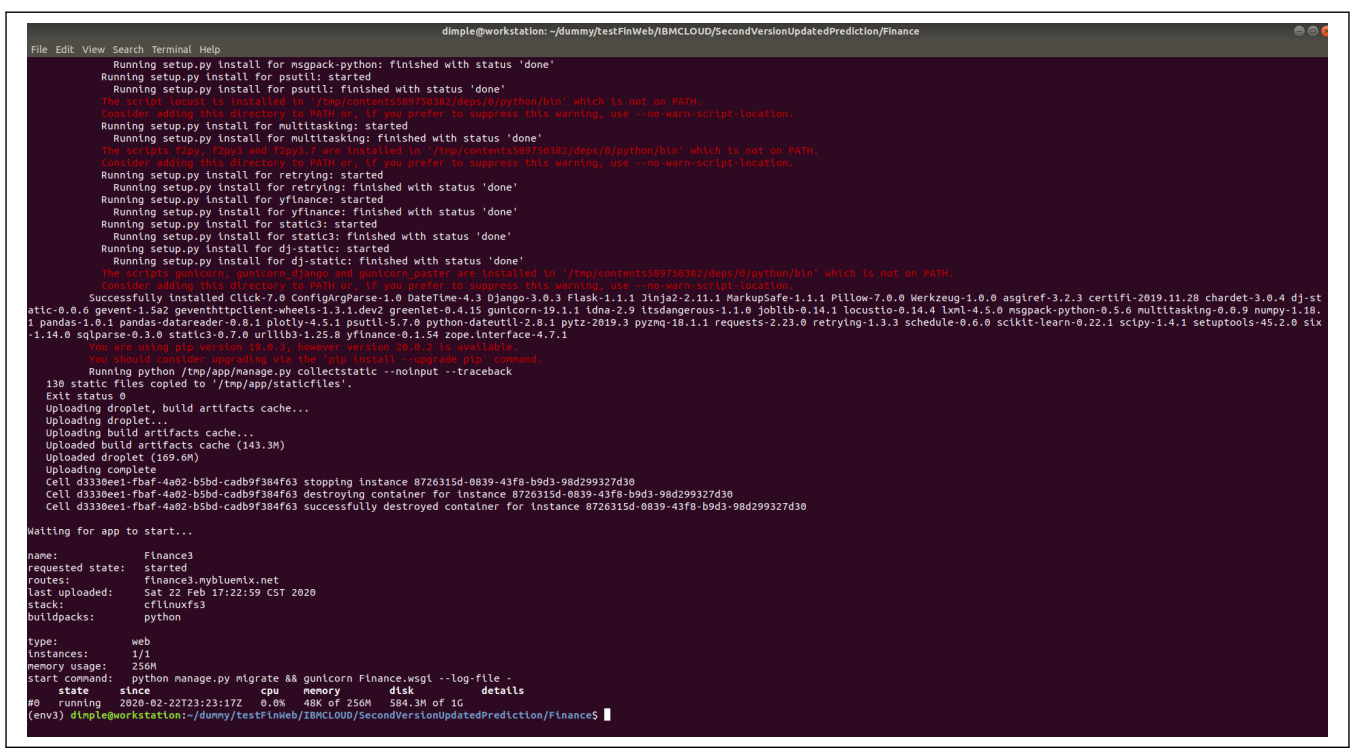

Figure A.6: Deployement of web application on IBM Cloud- part 2

\section{A.3.2 Web application on AWS Cloud}

A free tier account was created on AWS Cloud. Free tier account of AWS Cloud provides a variety of services and dashboard provide a lot of information for analysis. A Django application can be deployed with the help of AWS Elastic Beanstalk. Given below are images indicating the successful deployment of web application- 


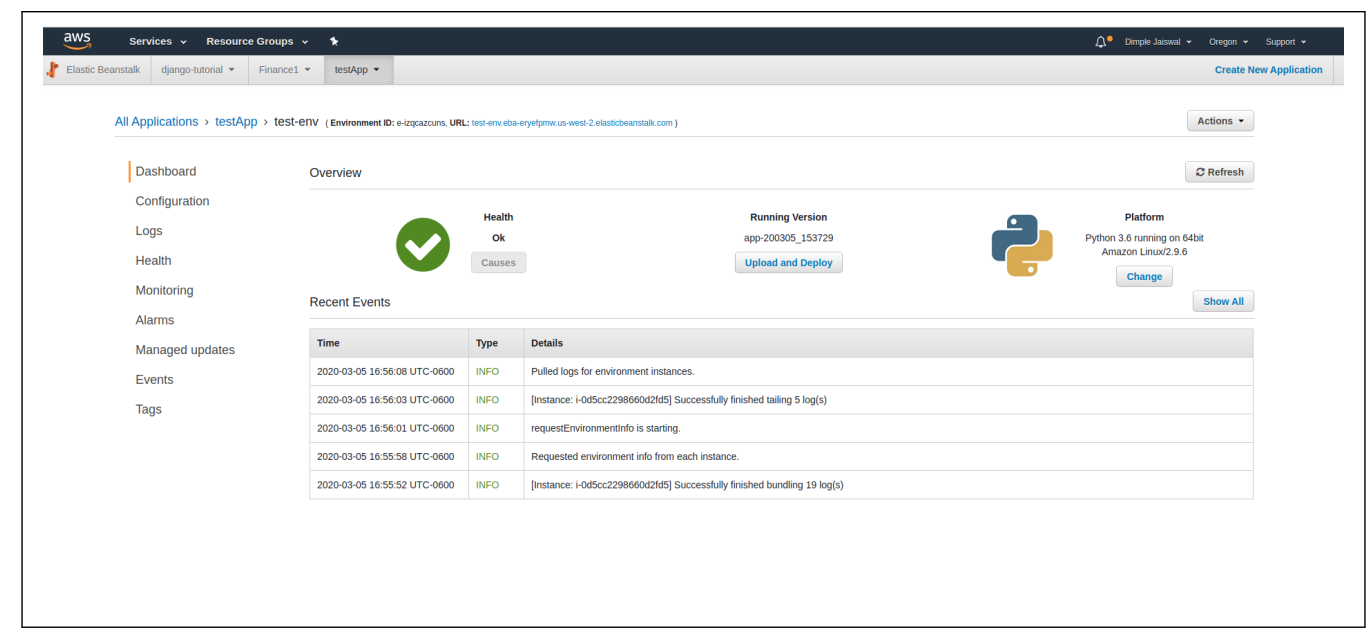

Figure A.7: Dashboard of web application hosted on AWS Cloud

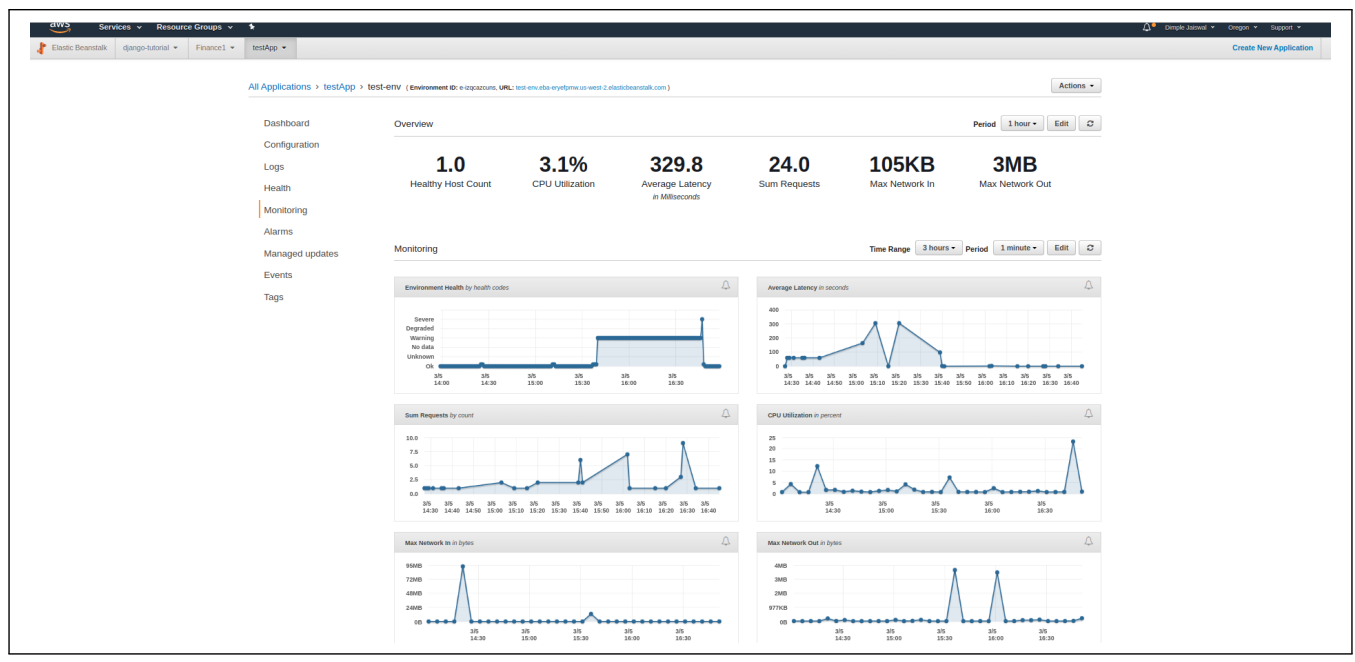

Figure A.8: Dashboard for monitoring performance of web application

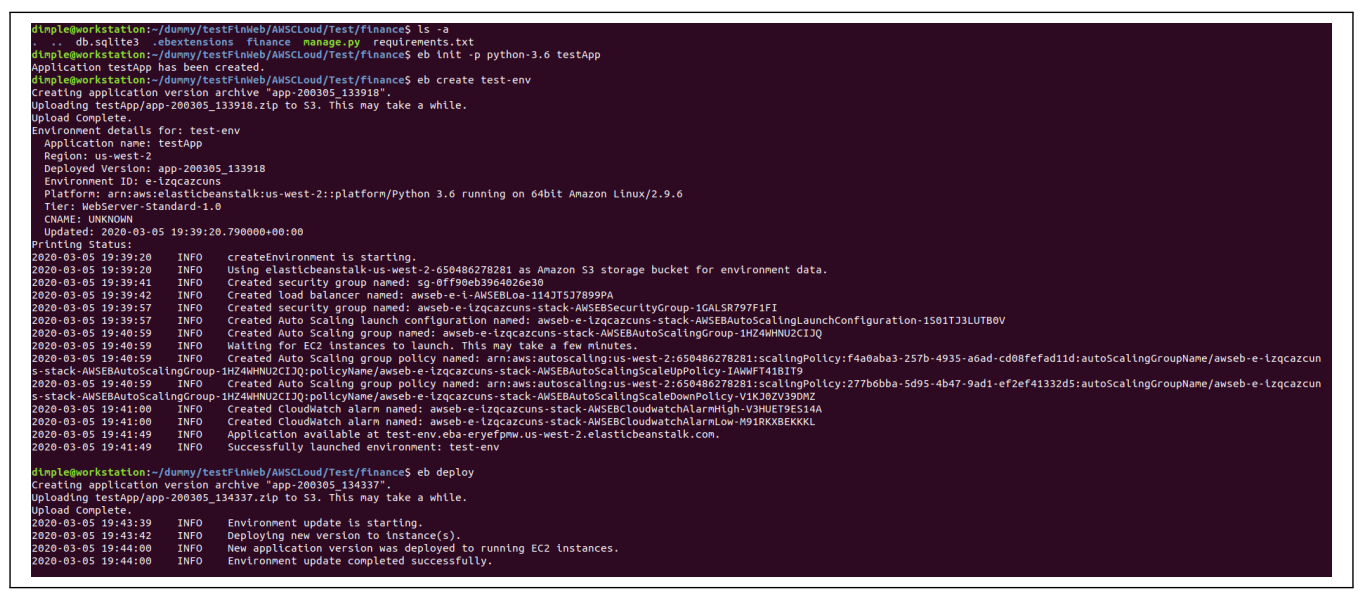

Figure A.9: Deployement of web application on AWS Cloud 


\section{A.3.3 Stress load testing using ab-benchmark tool \\ Stress load testing was conducted after hosting the web application on both- IBM and AWS Cloud using Apache bechmarking tool. Below given is the shell script and its output.}

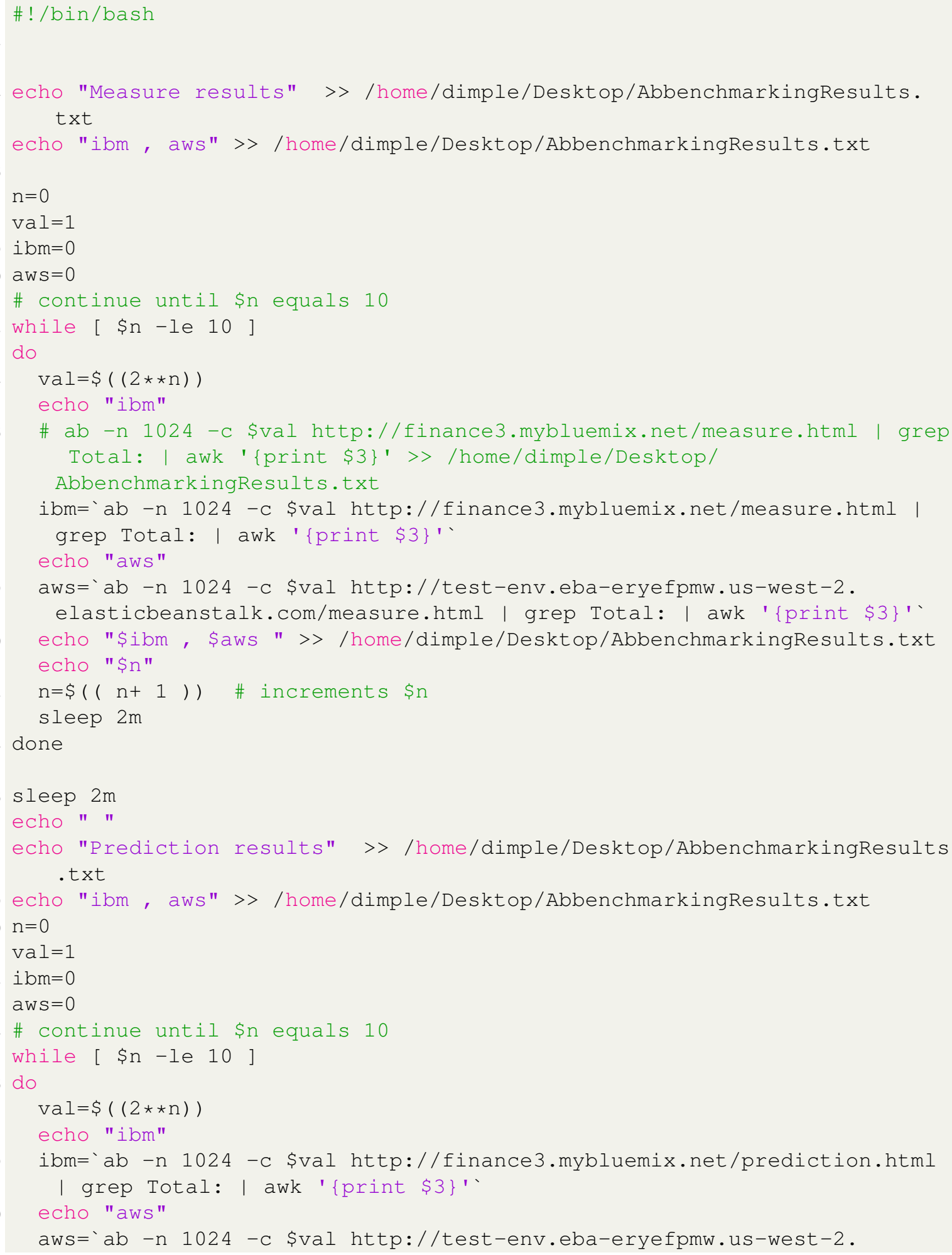




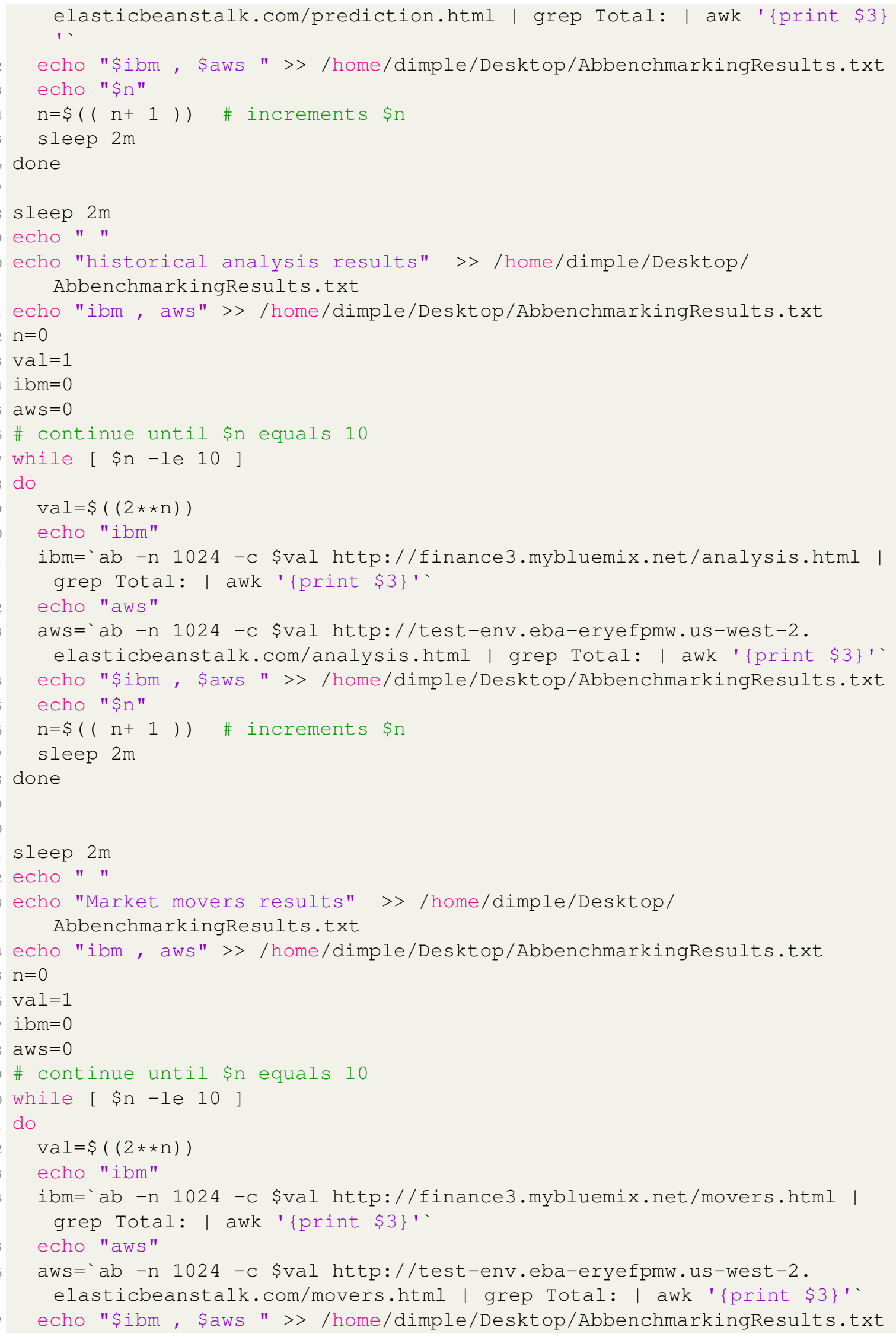




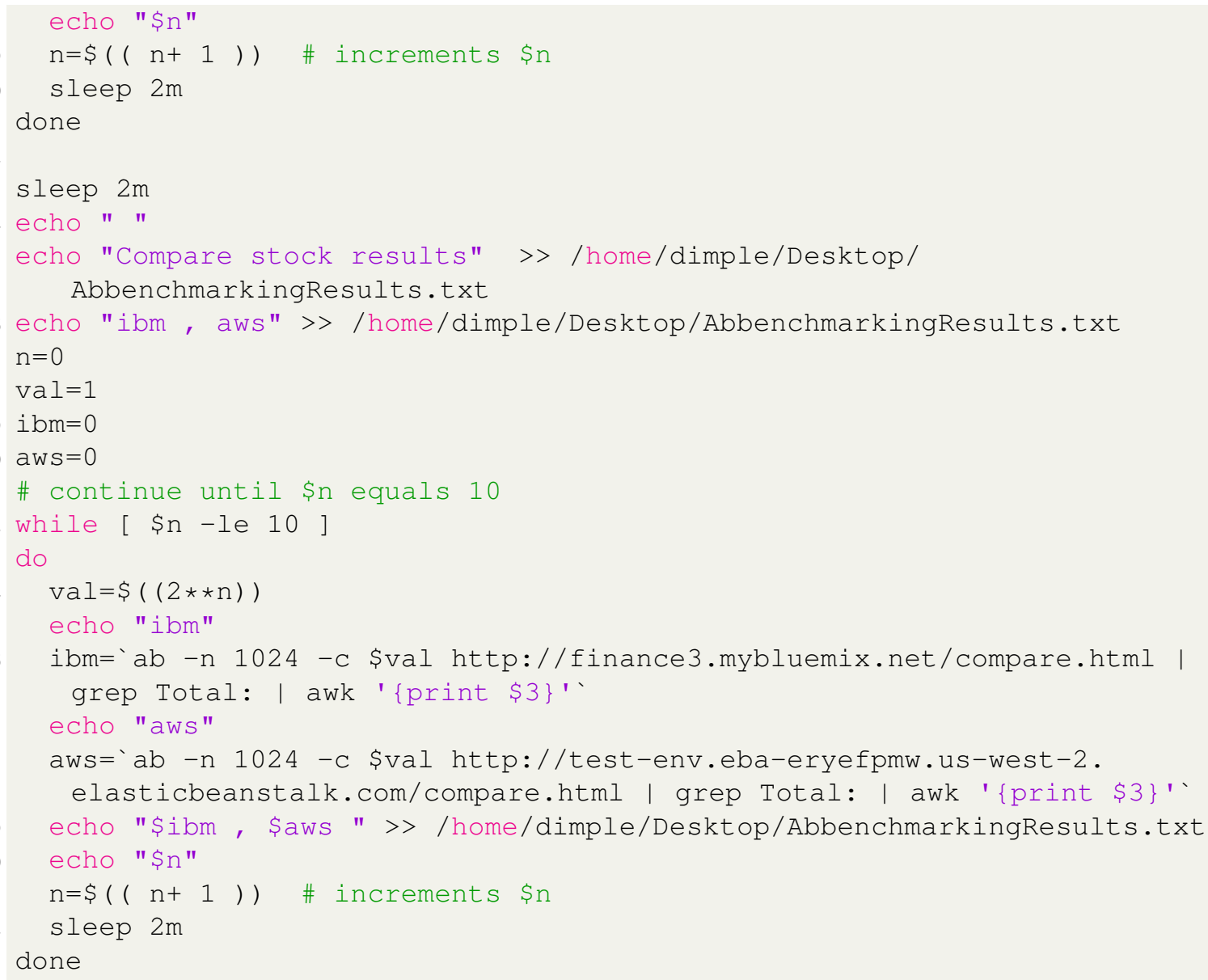

Code A.3.3.1: Shell script for load testing

Below is the output generated by shell script for load testing-

\begin{tabular}{|c|c|c|}
\hline Total Number of & Measure Trends & Results \\
\hline Concurrent Users & IBM(in ms) & AWS(in ms) \\
\hline 2 & 234 & 279 \\
\hline 4 & 238 & 266 \\
\hline 8 & 249 & 287 \\
\hline 16 & 368 & 328 \\
\hline 32 & 752 & 366 \\
\hline 64 & 1434 & 446 \\
\hline 128 & 2856 & 716 \\
\hline 252 & 5241 & 1565 \\
\hline 512 & 1280 & - \\
\hline 1024 & 1070 & - \\
\hline \multicolumn{2}{|c|}{} \\
\hline Total Number of & Prediction & Results \\
\hline Concurrent Users & IBM(in ms) & AWS(in ms) \\
\hline 2 & 178 & - \\
\hline
\end{tabular}




\begin{tabular}{|c|c|c|}
\hline 4 & 179 & - \\
\hline 8 & 191 & - \\
\hline 16 & 188 & 297 \\
\hline 32 & 20515 & 119 \\
\hline 64 & 6617 & 120 \\
\hline 128 & 1470 & 120 \\
\hline 252 & 4568 & 121 \\
\hline 512 & 695 & 126 \\
\hline 1024 & 868 & 170 \\
\hline Total Number of & Historical Analysis & Results \\
\hline Concurrent Users & IBM(in ms) & AWS(in ms) \\
\hline 2 & 228 & 119 \\
\hline 4 & 234 & 120 \\
\hline 8 & 240 & 119 \\
\hline 16 & 318 & 121 \\
\hline 32 & 564 & 121 \\
\hline 64 & 1194 & 121 \\
\hline 128 & 2589 & 121 \\
\hline 252 & 4960 & 124 \\
\hline 512 & 746 & 128 \\
\hline 1024 & 1157 & 168 \\
\hline Total Number of & Market Movers & Results \\
\hline Concurrent Users & IBM(in ms) & AWS(in ms) \\
\hline 2 & 106 & 119 \\
\hline 4 & 110 & 120 \\
\hline 8 & 2960 & 120 \\
\hline 16 & 126 & 119 \\
\hline 32 & 188 & 119 \\
\hline 64 & 186 & 120 \\
\hline 128 & 424 & 120 \\
\hline 252 & 548 & 122 \\
\hline 512 & 528 & 128 \\
\hline 1024 & 587 & 168 \\
\hline Total Number of & Compare Stock & Results \\
\hline Concurrent Users & IBM(in ms) & AWS(in ms) \\
\hline 2 & 336 & 119 \\
\hline 4 & 345 & 119 \\
\hline 8 & 419 & 119 \\
\hline 16 & 632 & 119 \\
\hline
\end{tabular}




\begin{tabular}{|c|c|c|}
\hline 32 & 1266 & 119 \\
\hline 64 & 2574 & 120 \\
\hline 128 & 4428 & 120 \\
\hline 252 & 14250 & 122 \\
\hline 512 & 1156 & 126 \\
\hline 1024 & 1201 & 157 \\
\hline
\end{tabular}

Table A.2: Shell script output for load testing

\section{A.4 Generation of training dataset}

To design and develop effective load balancing component using supervised machine learning technique, training dataset was required to train algorithm. Here, training dataset was generated using Locust, an open source load testing tool available to test how many concurrent users can be handled by a web-sites (or other systems). This is implemented by writing a locustfile. All the data generated using locust can be found here: https://github.com/dimplejaiswal95?tab=repositories. Below is the locustfile for designed financial web application -

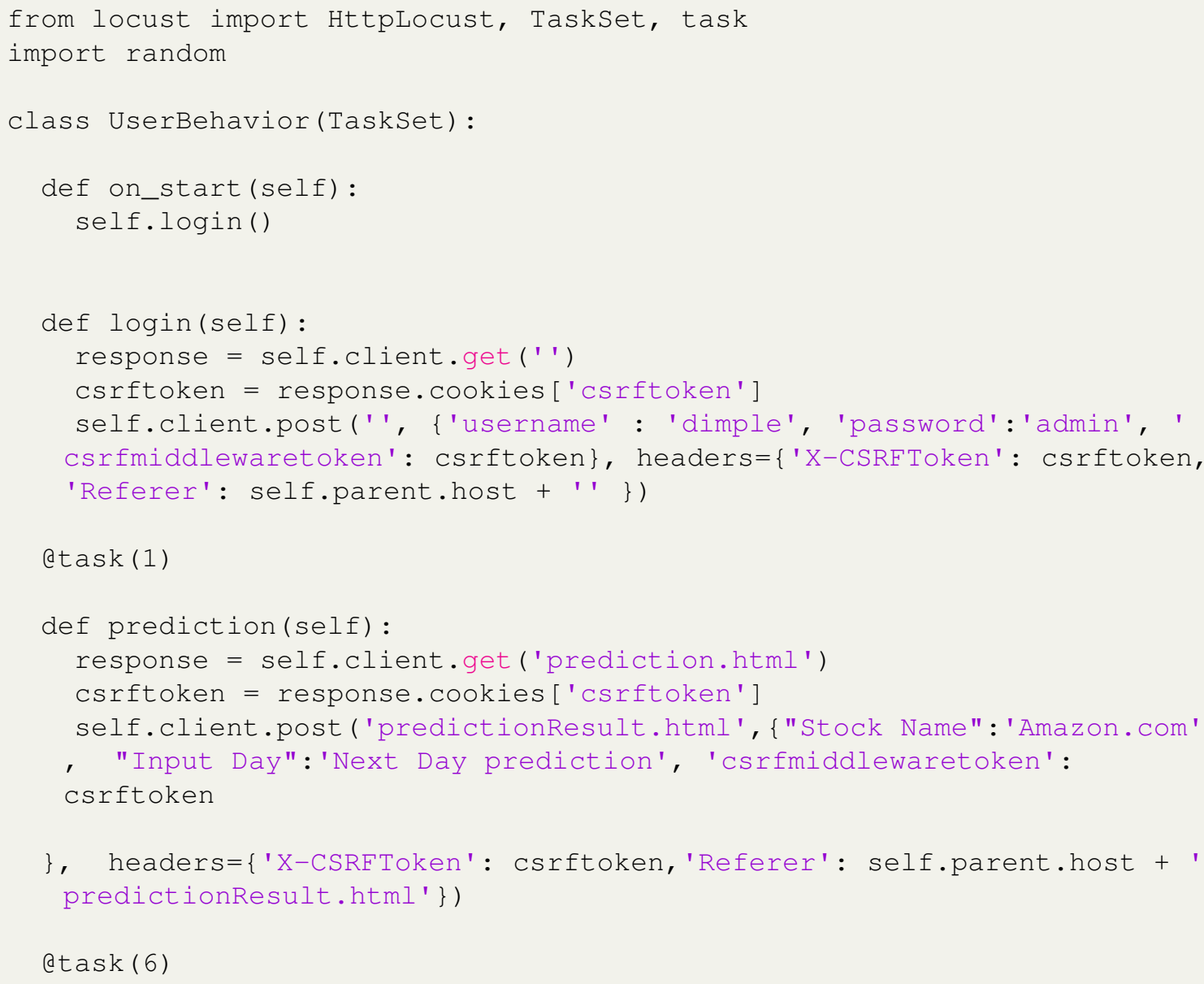




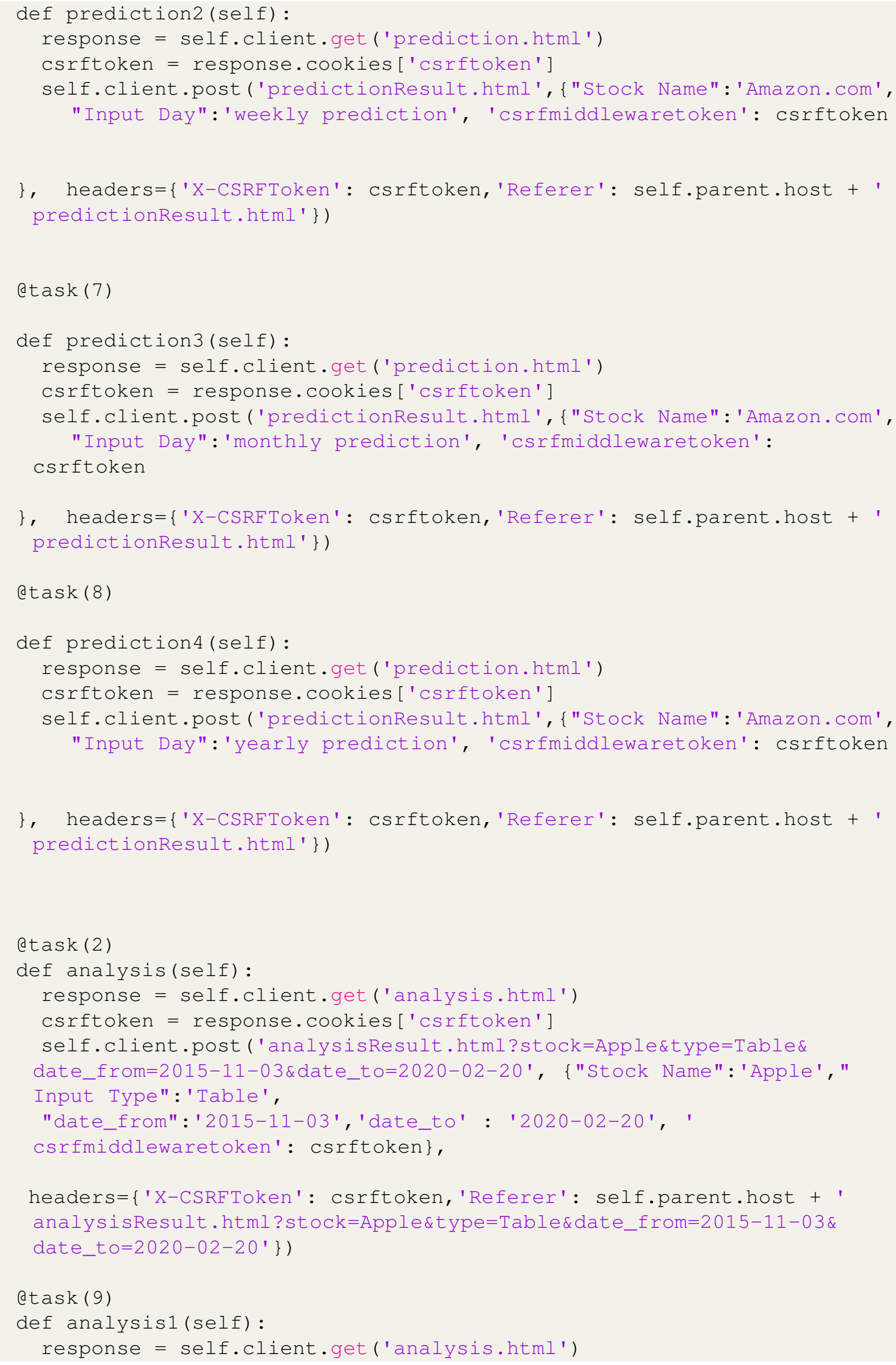




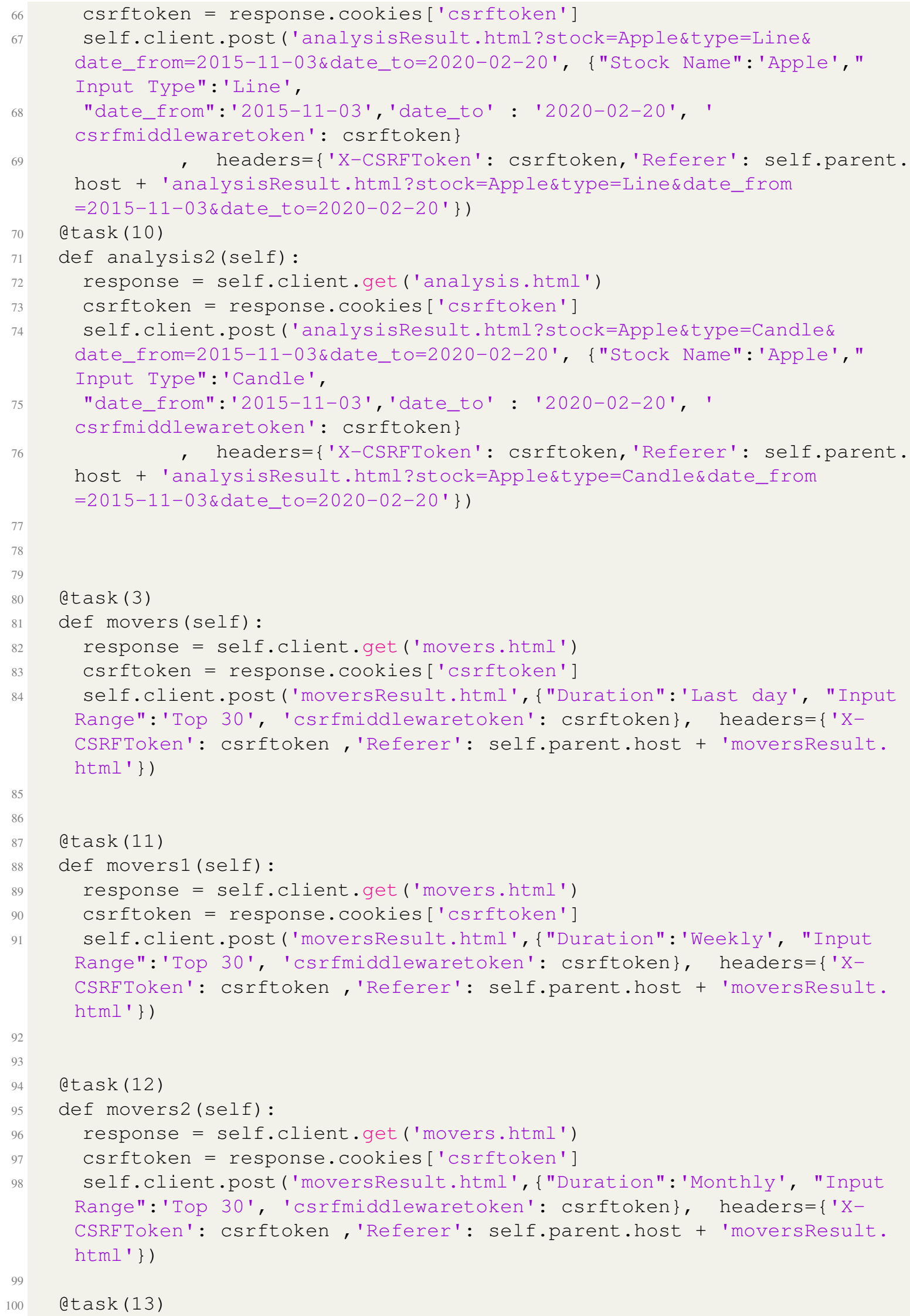




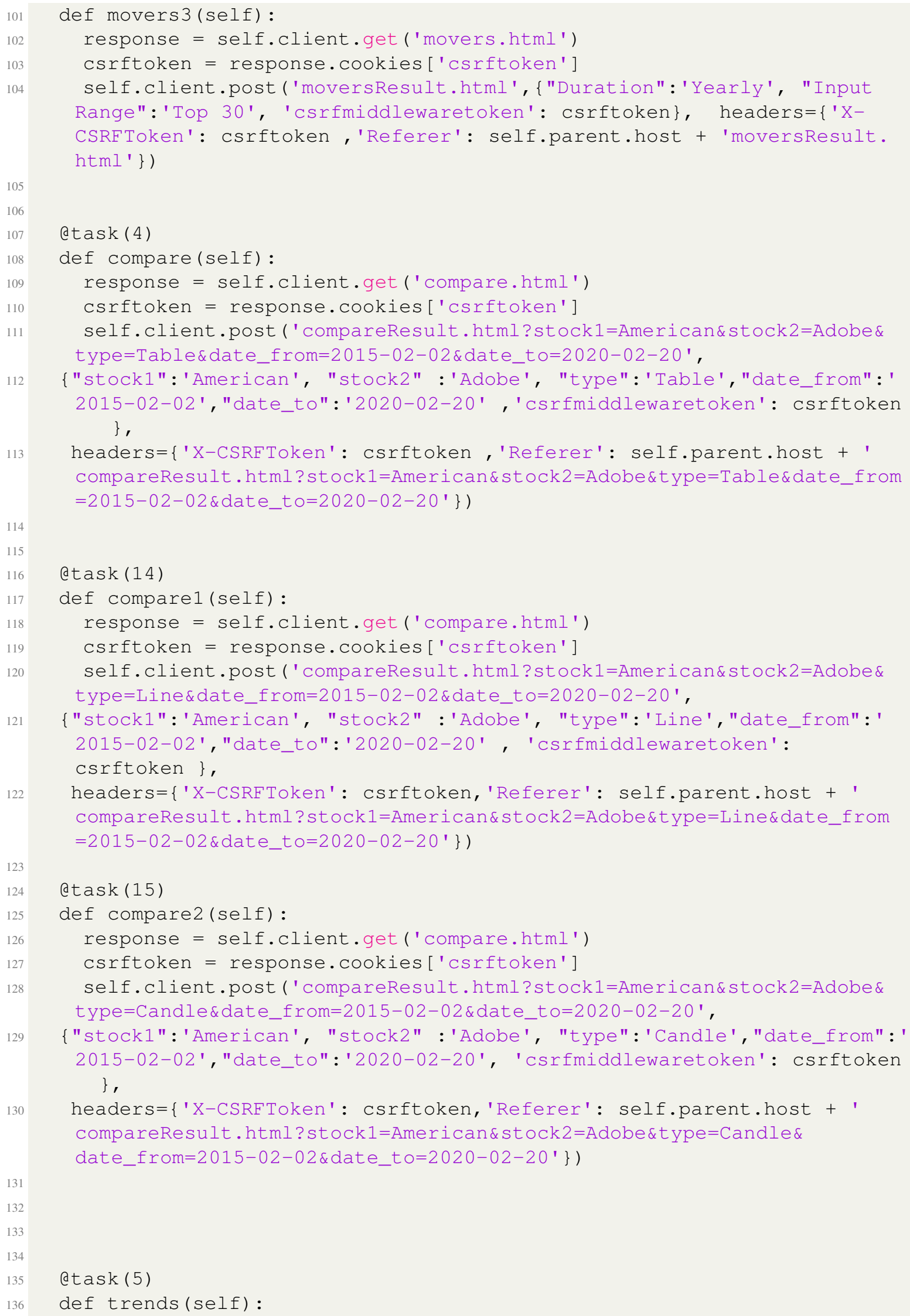

etask (4)

def compare(self): response = self.client.get ('compare.html') csrftoken = response.cookies ['csrftoken'] self.client.post (' compareResult.html?stock1=American\&stock2=Adobe\& type=Table\&date_from=2015-02-02\&date_to=2020-02-20',

\{"stock1":'American', "stock2" :'Adobe', "type":'Table', "date_from": ' 2015-02-02', "date_to":'2020-02-20', 'csrfmiddlewaretoken' : csrftoken , headers $=\{$ 'X-CSRFToken': csrftoken, 'Referer': self.parent.host + ' compareResult.html?stock1=American\&stock2=Adobe\&type=Table\&date_from $\left.=2015-02-02 \& d a t e \_t o=2020-02-200^{\prime}\right\}$ )

\section{@task (14)}

def comparel(self): response $=$ self.client.get ('compare.html') csrftoken = response.cookies['csrftoken'] self.client.post (' compareResult.html?stock1=American\&stock2=Adobe\& type=Line \&date_from=2015-02-02\&date_to=2020-02-20' ,

\{"stock1":'American', "stock2" :'Adobe', "type":'Line',"date_from": ' 2015-02-02', "date_to":'2020-02-20' , 'csrfmiddlewaretoken': csrftoken $\}$, headers $=\{$ 'X-CSRFToken': csrftoken, 'Referer' : self.parent.host + ' compareResult.html?stock1=American\&stock2=Adobe\&type=Line\&date_from $=2015-02-02 \& d a t e \_t o=2020-02-20$ ' $)$

\section{Qtask (15)}

def compare2(self): response $=$ self.client.get ('compare.html') csrftoken = response.cookies['csrftoken'] self.client.post ('compareResult.html?stock1=American\&stock2=Adobe\& type=Candle\&date_from=2015-02-02\&date_to=2020-02-20',

\{"stock1":'American', "stock2" :'Adobe', "type":'Candle',"date_from": ' 2015-02-02',"date_to":'2020-02-20', 'csrfmiddlewaretoken': csrftoken , headers $=\{$ 'X-CSRFToken': csrftoken, 'Referer': self.parent.host + ' compareResult.html? stock1=American\&stock2=Adobe\&type=Candle\& date_from $=2015-02-02 \&$ date_to $=2020-02-20$ ' \})

atask (5)

def trends (self): 


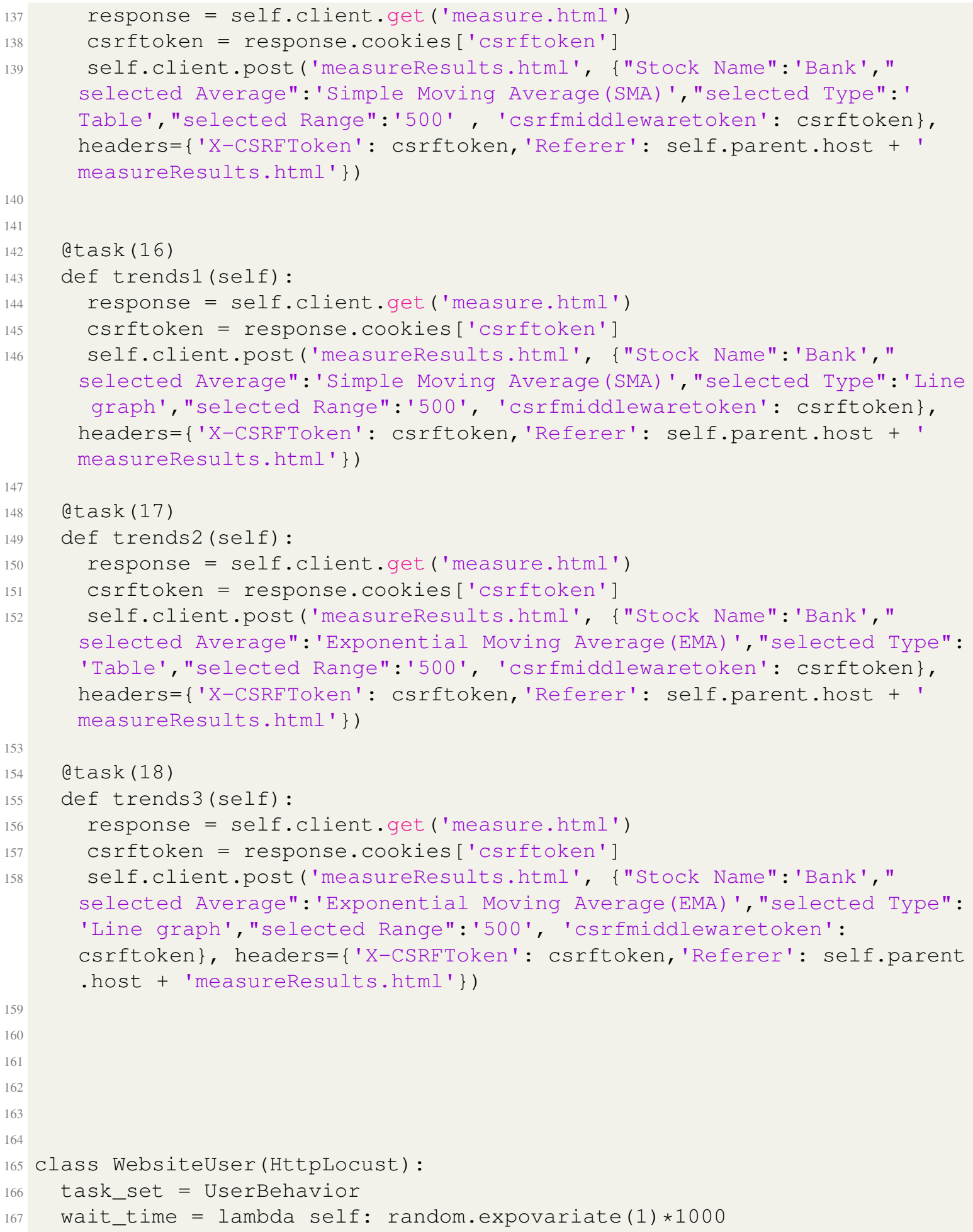

\section{Code A.4.0.1: locustfile.py}

The load testing using Locust is performed in an automated way for various number of users ranging from to 1 to 512 users, with varying number of concurrent users swarmed 
on web application. The testing result generated comprises of two files- distribution.csv and requests.csv for every case, i.e., for a given total number of users with number of concurrent users. The testing last for a long time. Below is the shell script to automate the testing and the figure displays an example of resulted directory created-

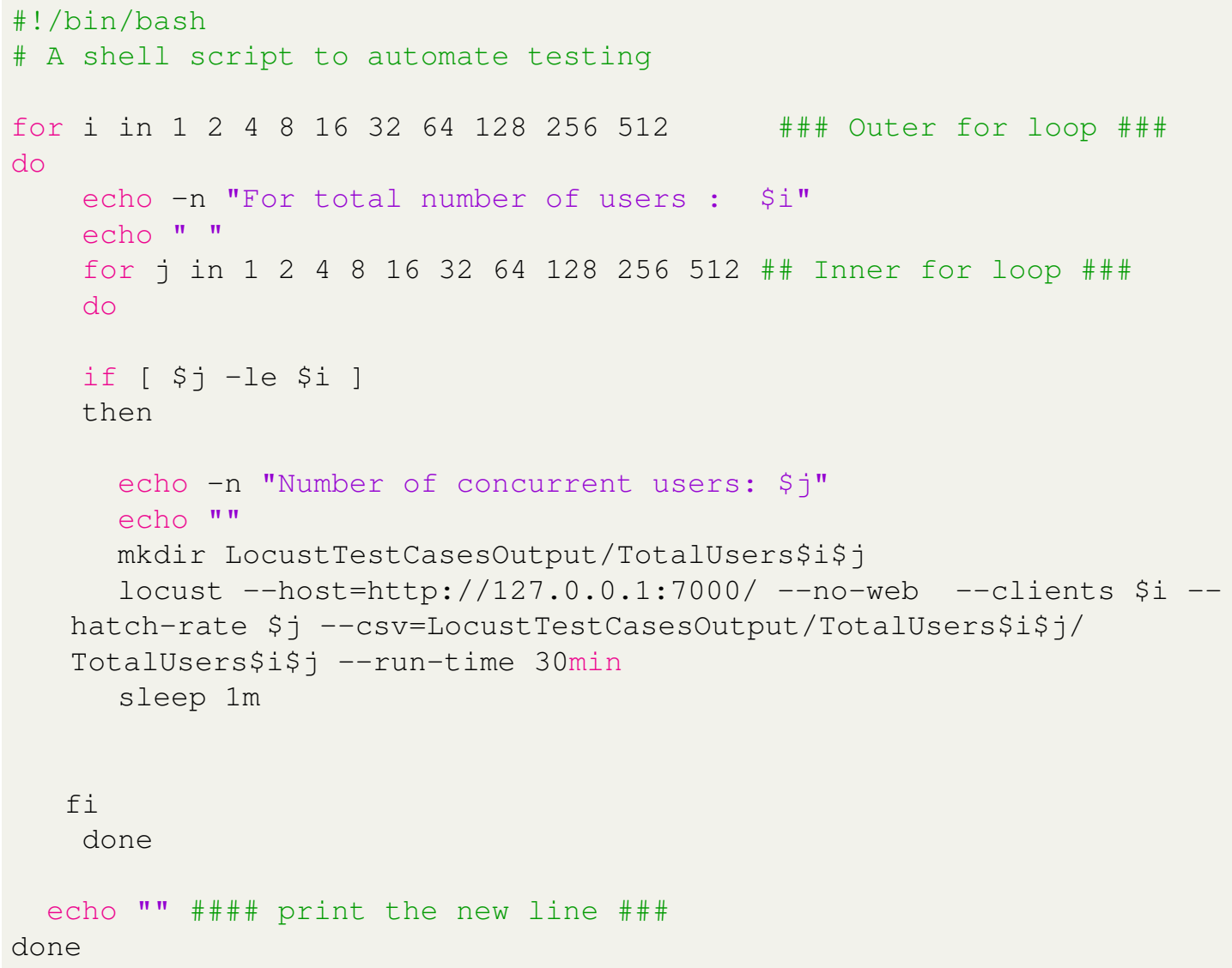

Code A.4.0.2: automateLocust.sh 


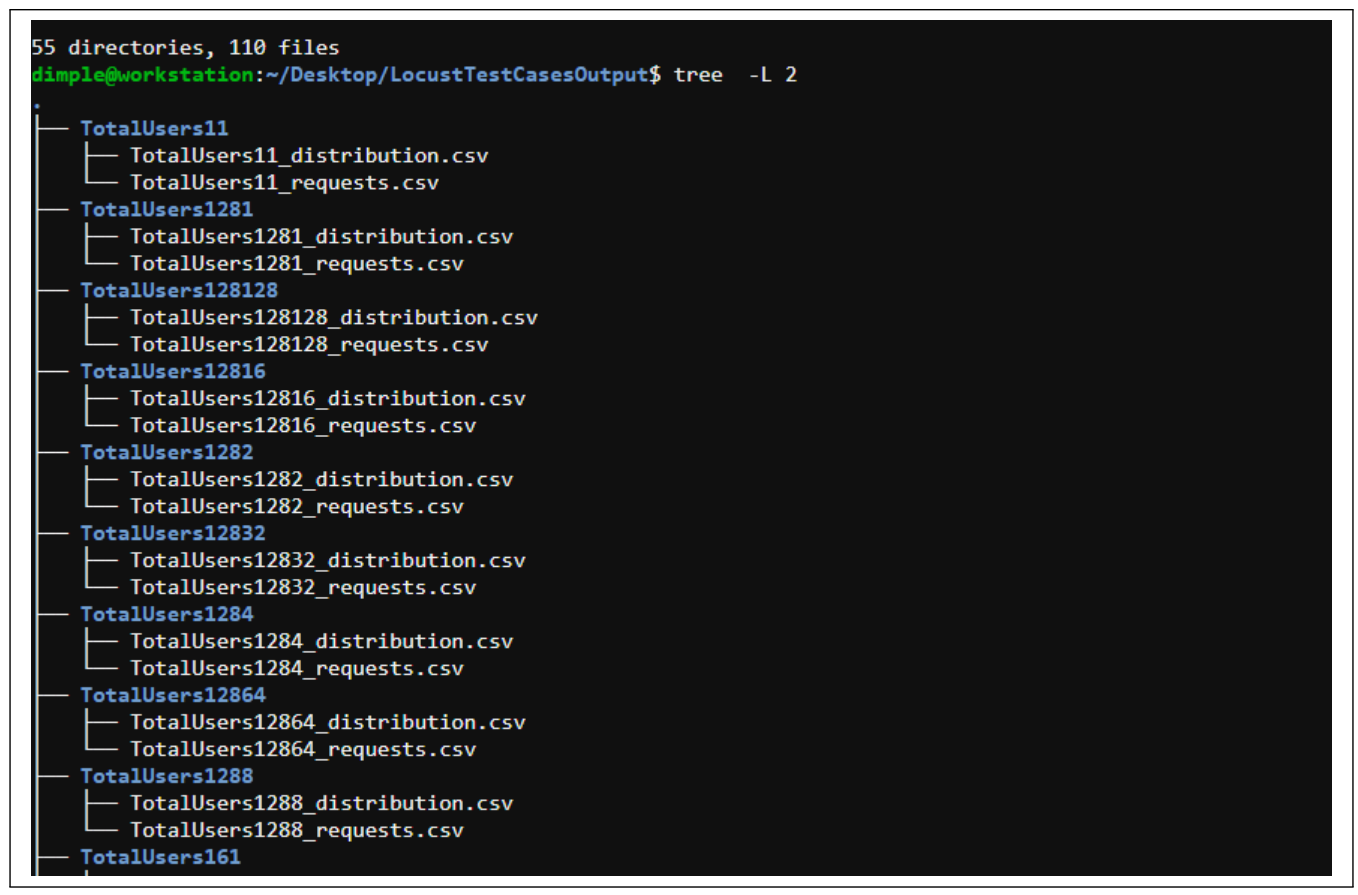

Figure A.10: Directory Tree Structure for output generated by automateLocush.sh

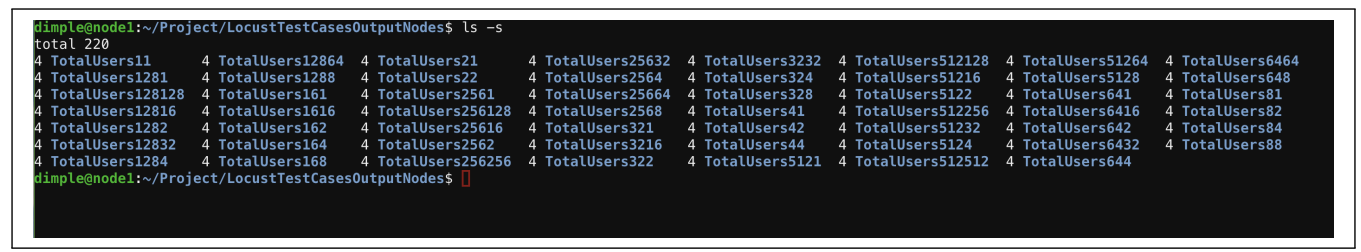

Figure A.11: Example of directory containing output generated by automateLocush.sh

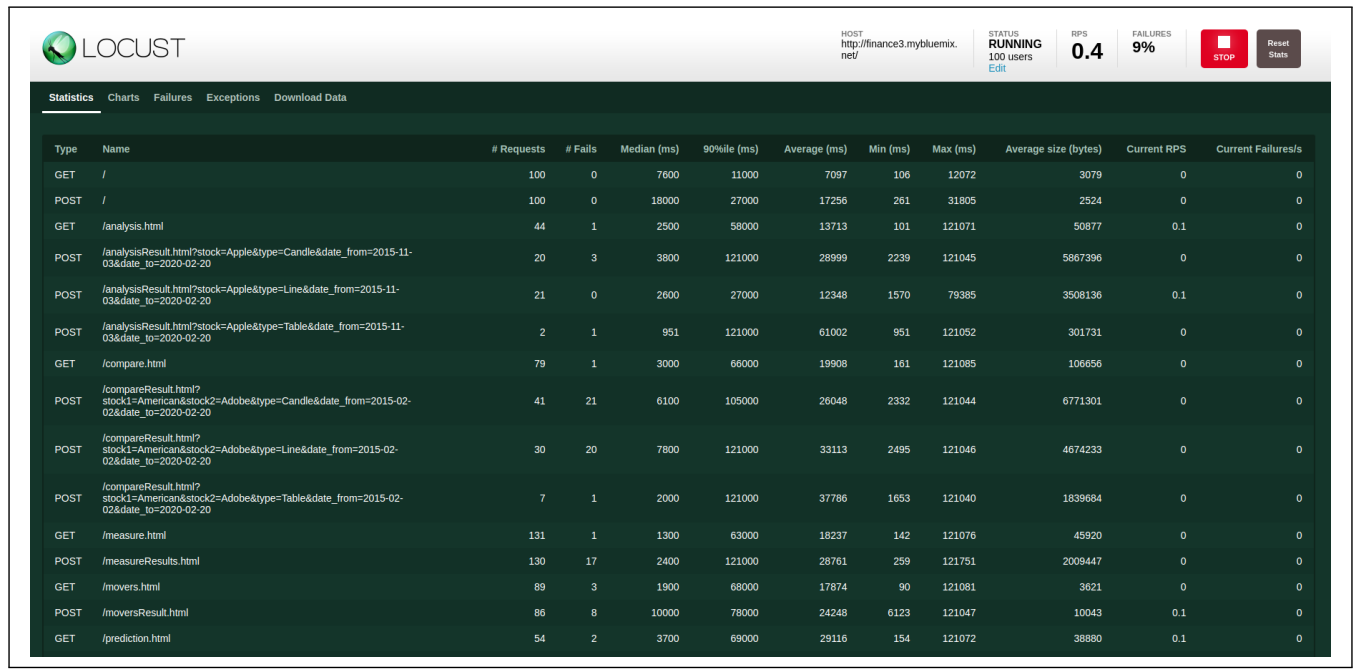

Figure A.12: Example of locust output on web interface

\section{A.5 Load Balancing Component}

A.5.1 Installation of NGINX and setup NGINX for cluster 


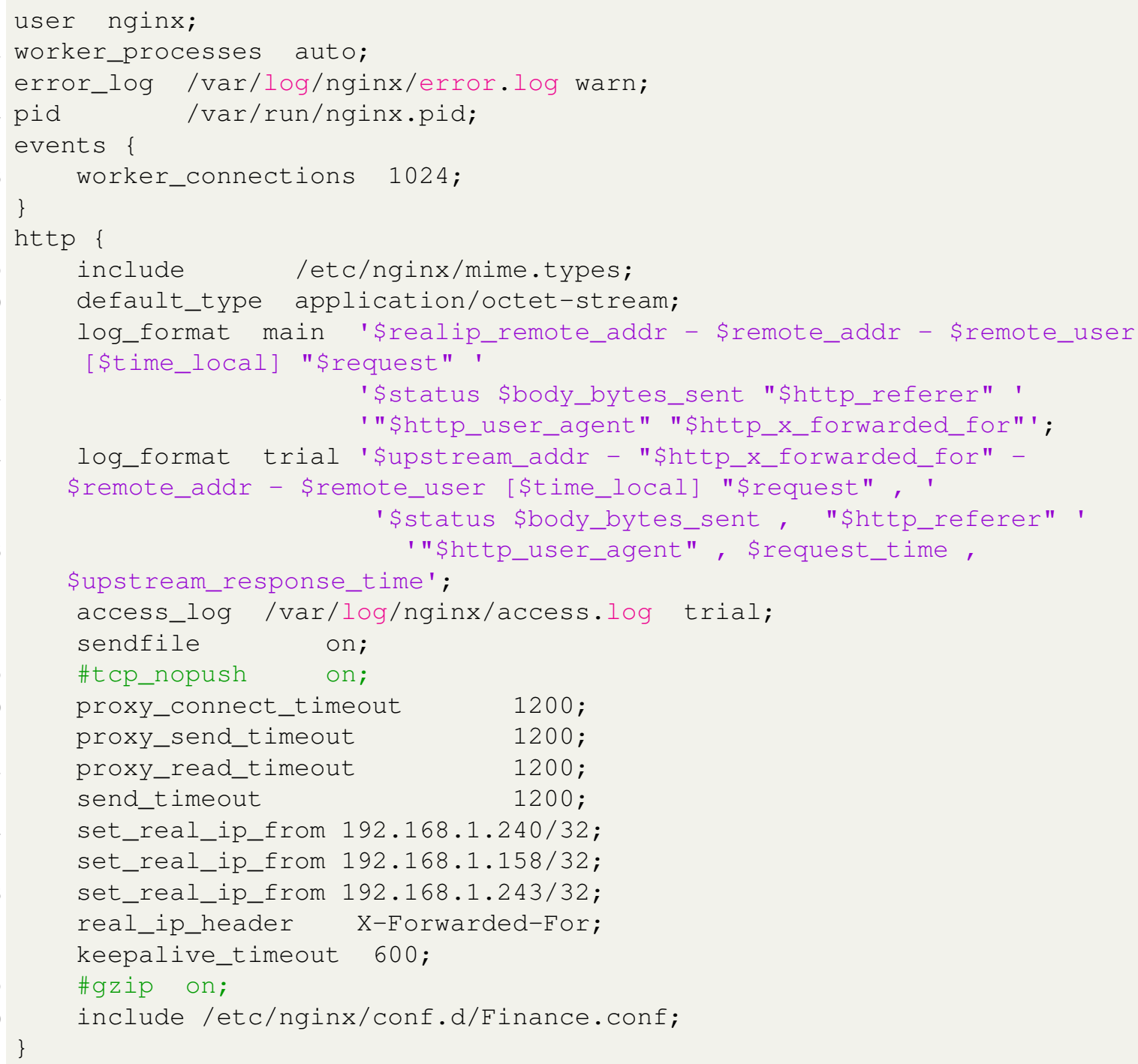

\section{Code A.5.1.1: Nginx Configuration file}

\section{A.5.2 Types of Load Balancing}

\section{A.5.2.1 Round Robin load balancing}

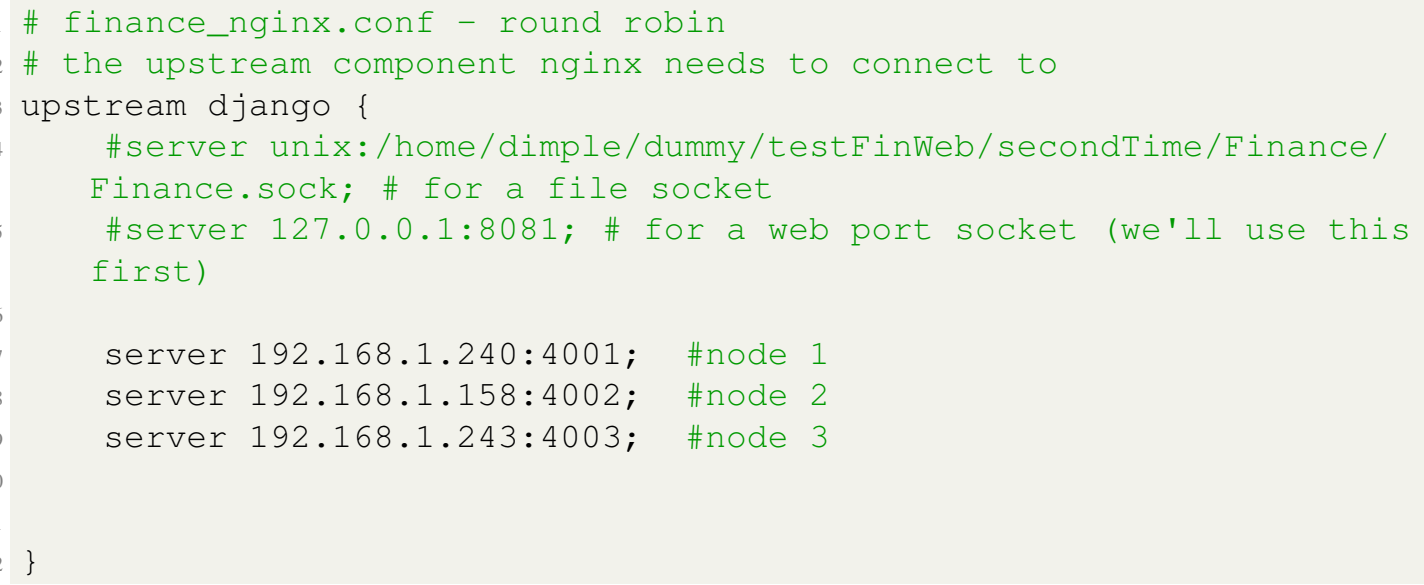




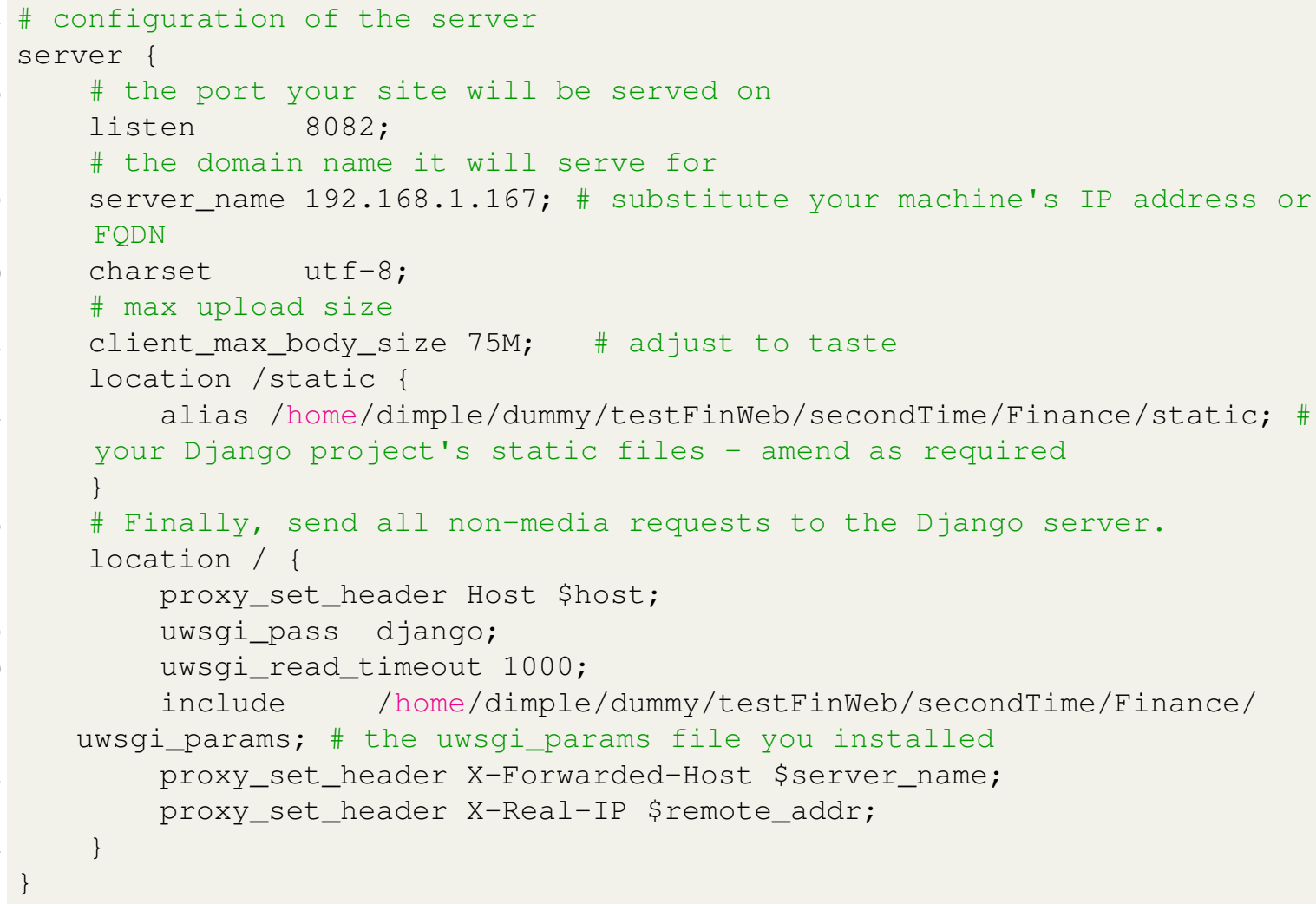

Code A.5.2.1: Round Robin load balancing

\section{A.5.2.2 Least connected load balancing}

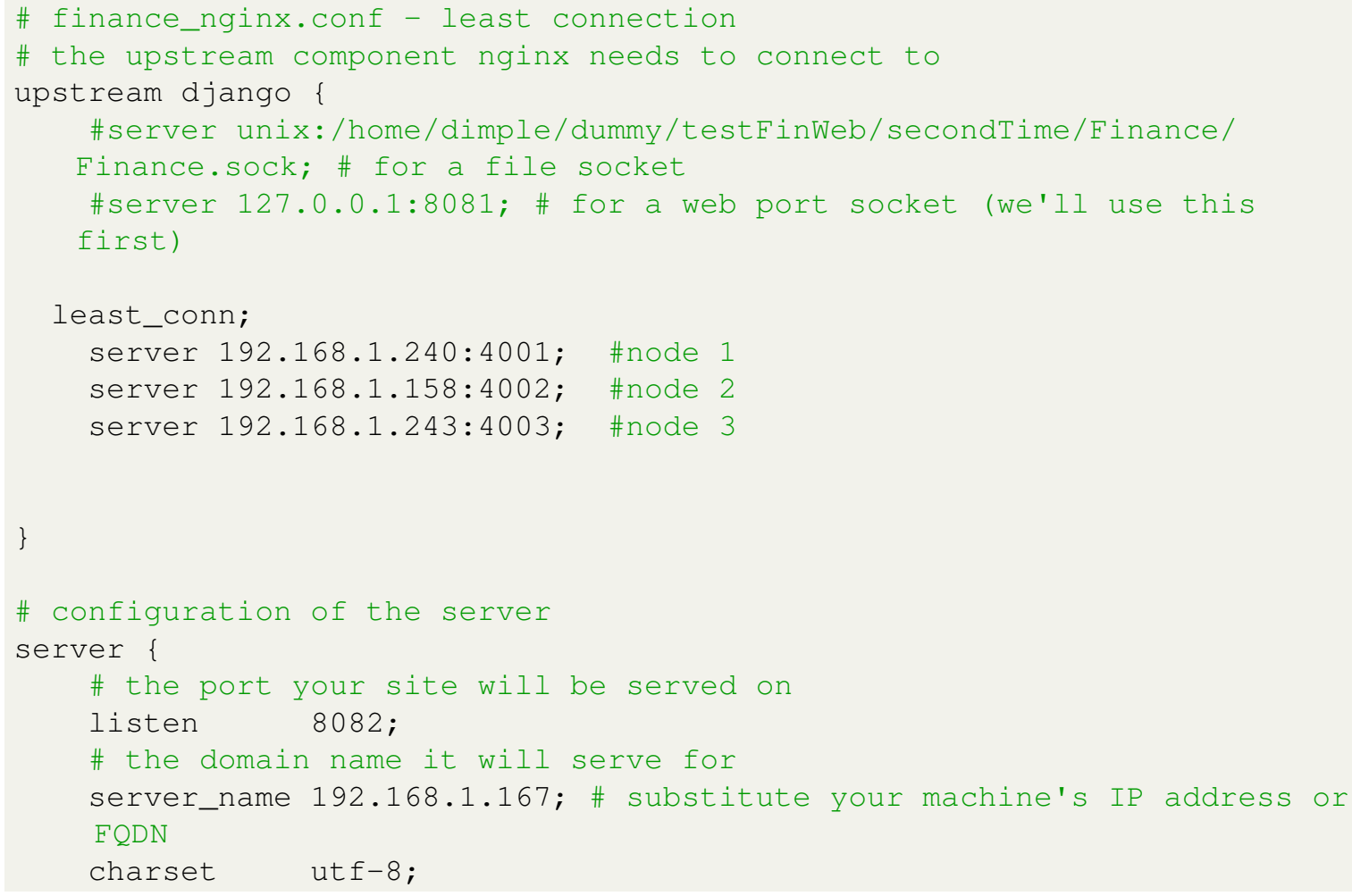




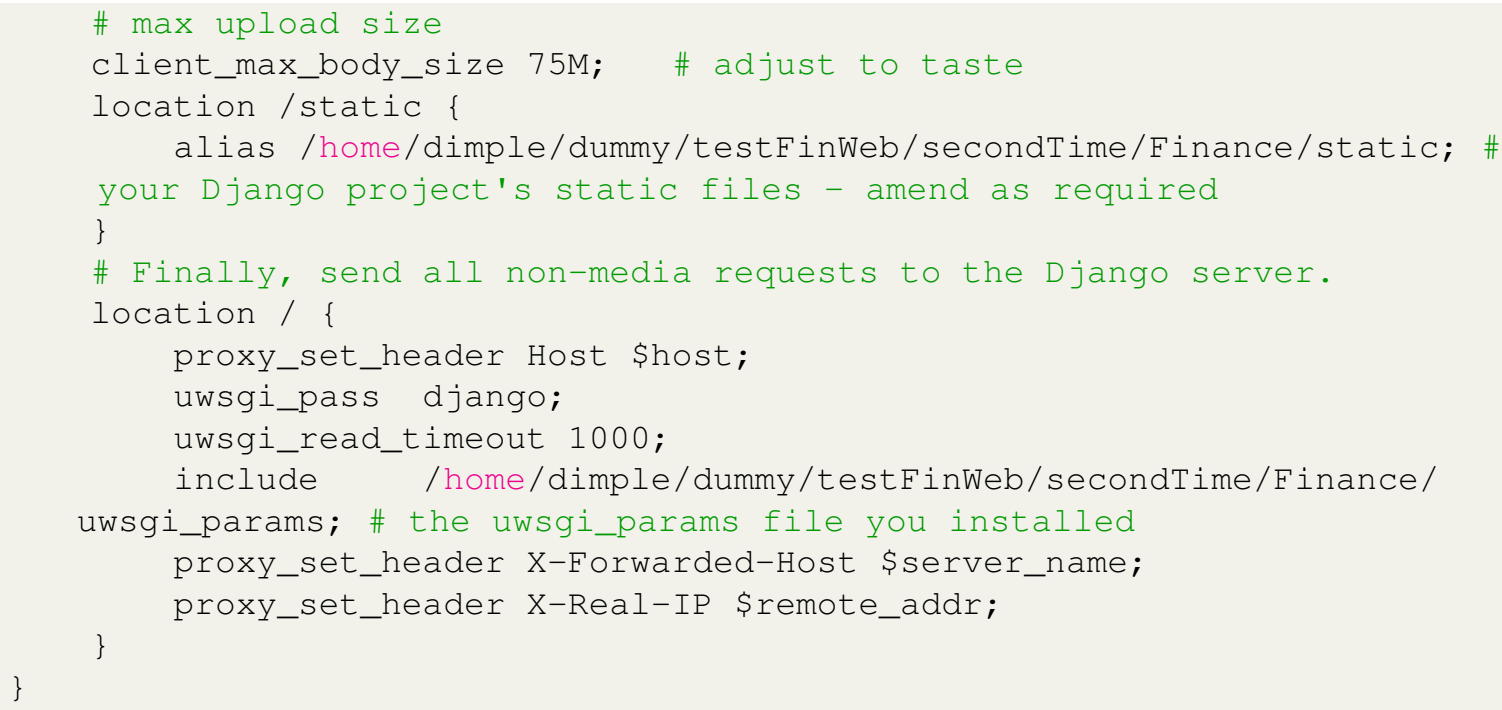

\section{Code A.5.2.2: Least connected load balancing}

\section{A.5.2.3 Session persistence load balancing}

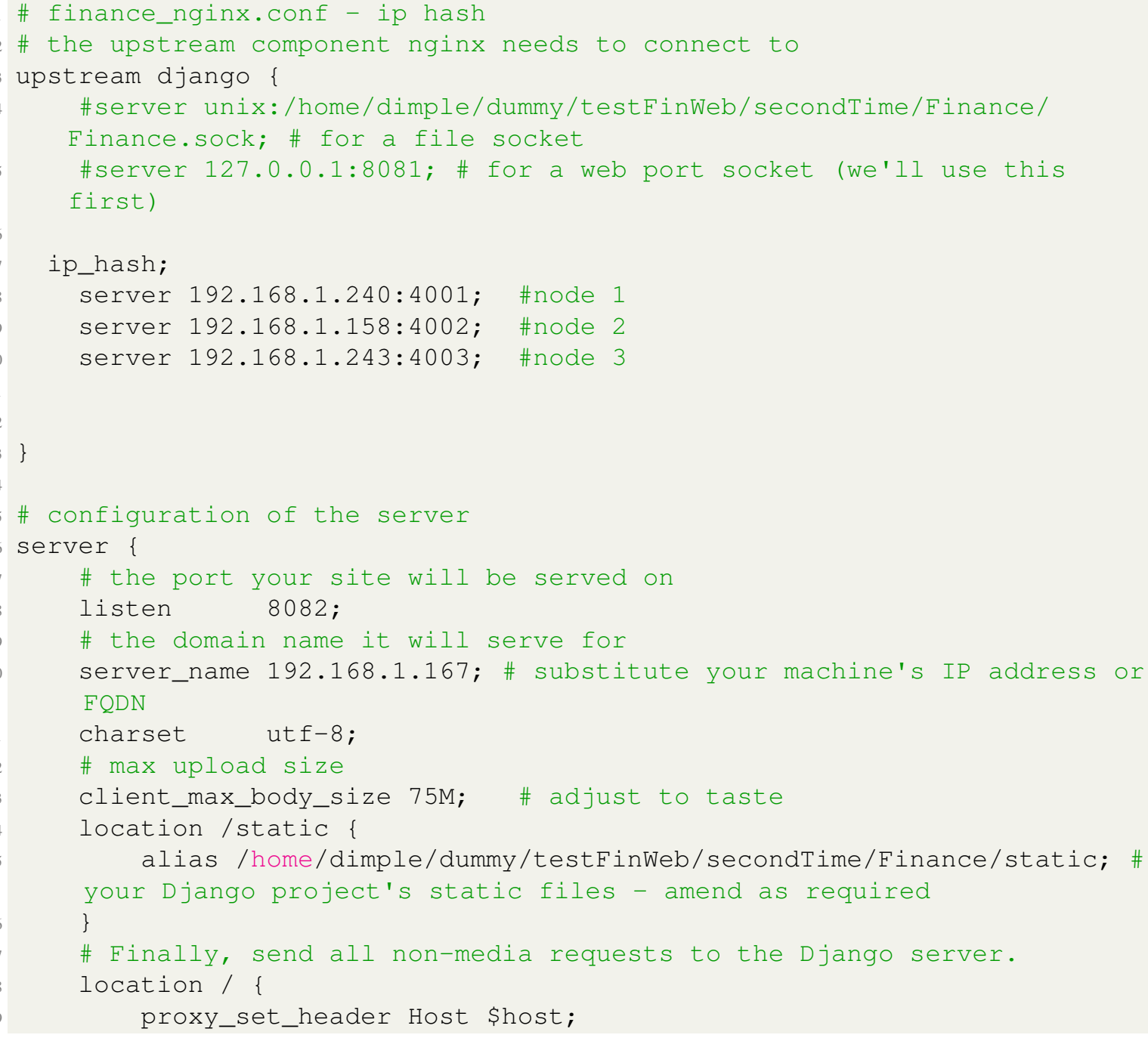




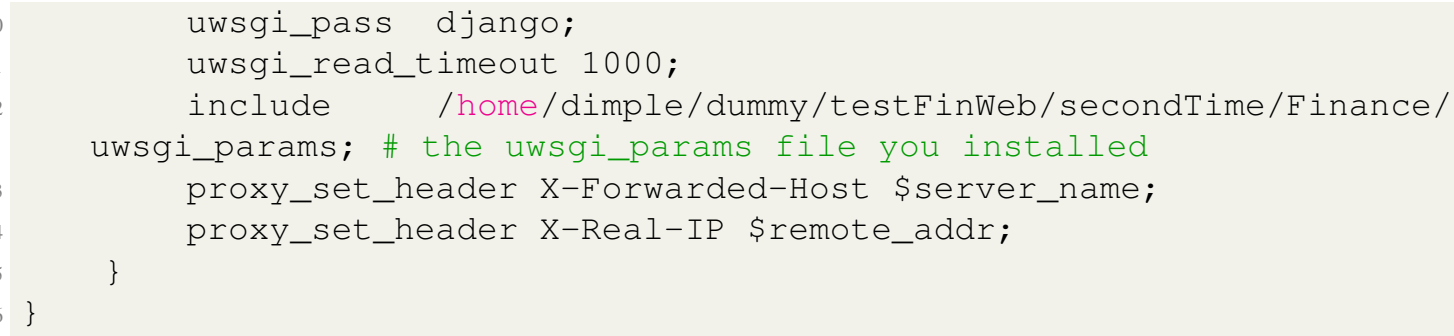

\section{Code A.5.2.3: Session persistence load balancing}

\section{A.5.2.4 Weighted load balancing}

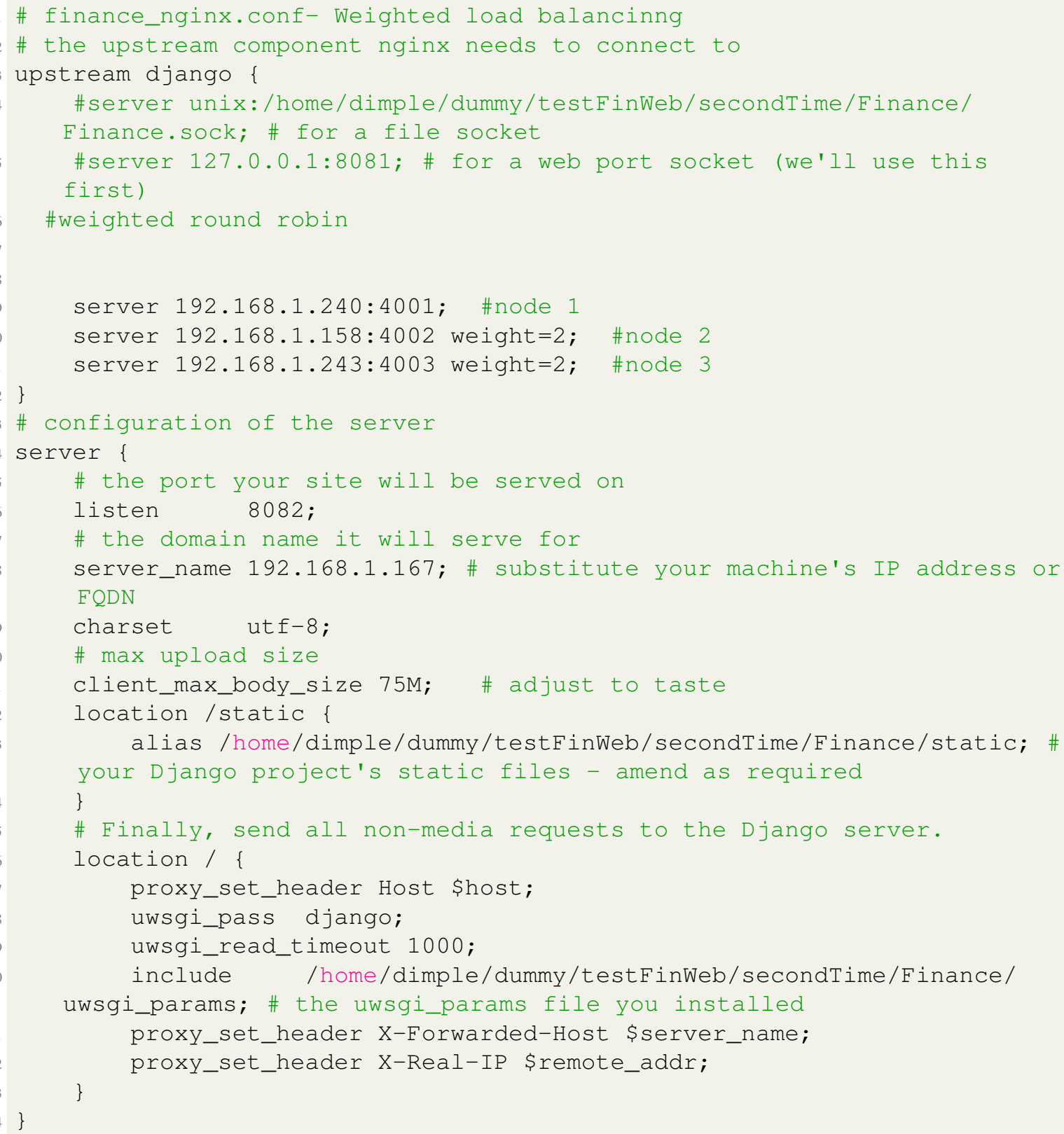

Code A.5.2.4: Weighted load balancing 


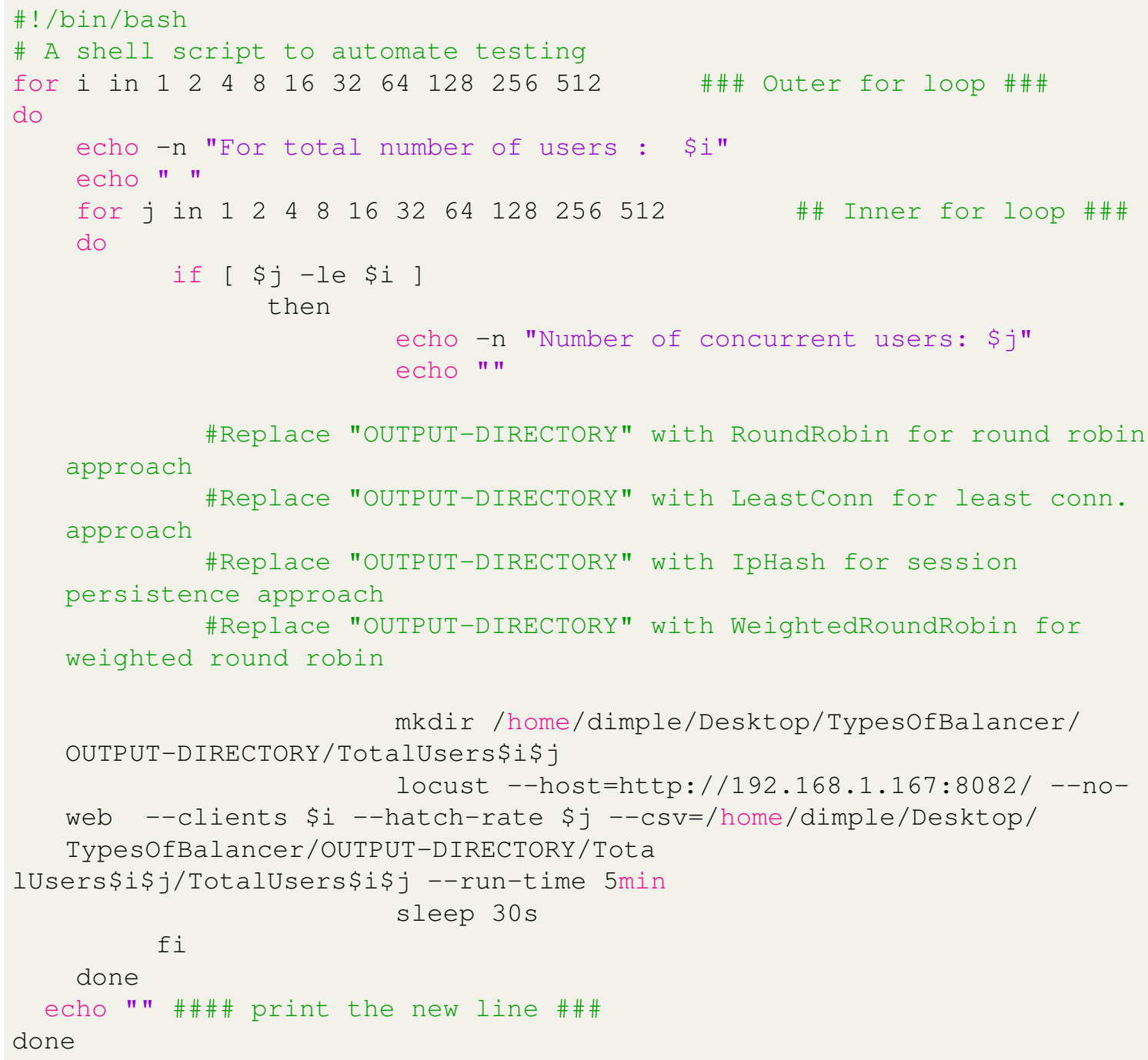

Code A.5.2.5: Locust stress testing for all types of load balancing approach

\section{A.6 Data Analysis}

A.6.1 Response time analysis for different types of job requests

Here is the example of response time analysis for different types of job requests when there are total 32 users accessing web application with 8 concurrent users at a time. The response time changes with the number of concurrent users accessing the application.

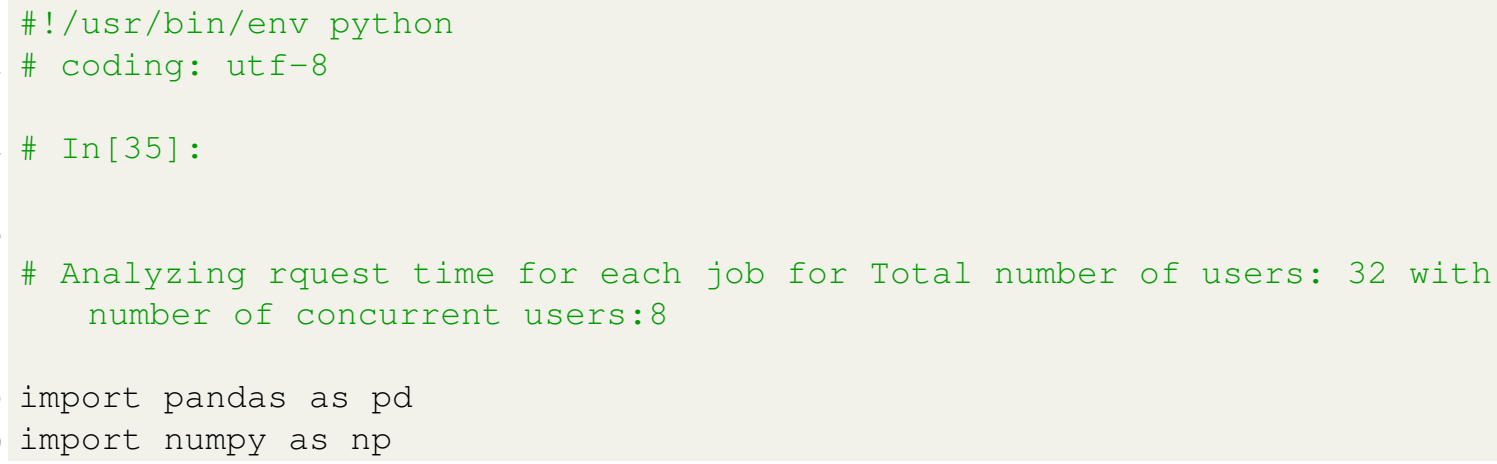




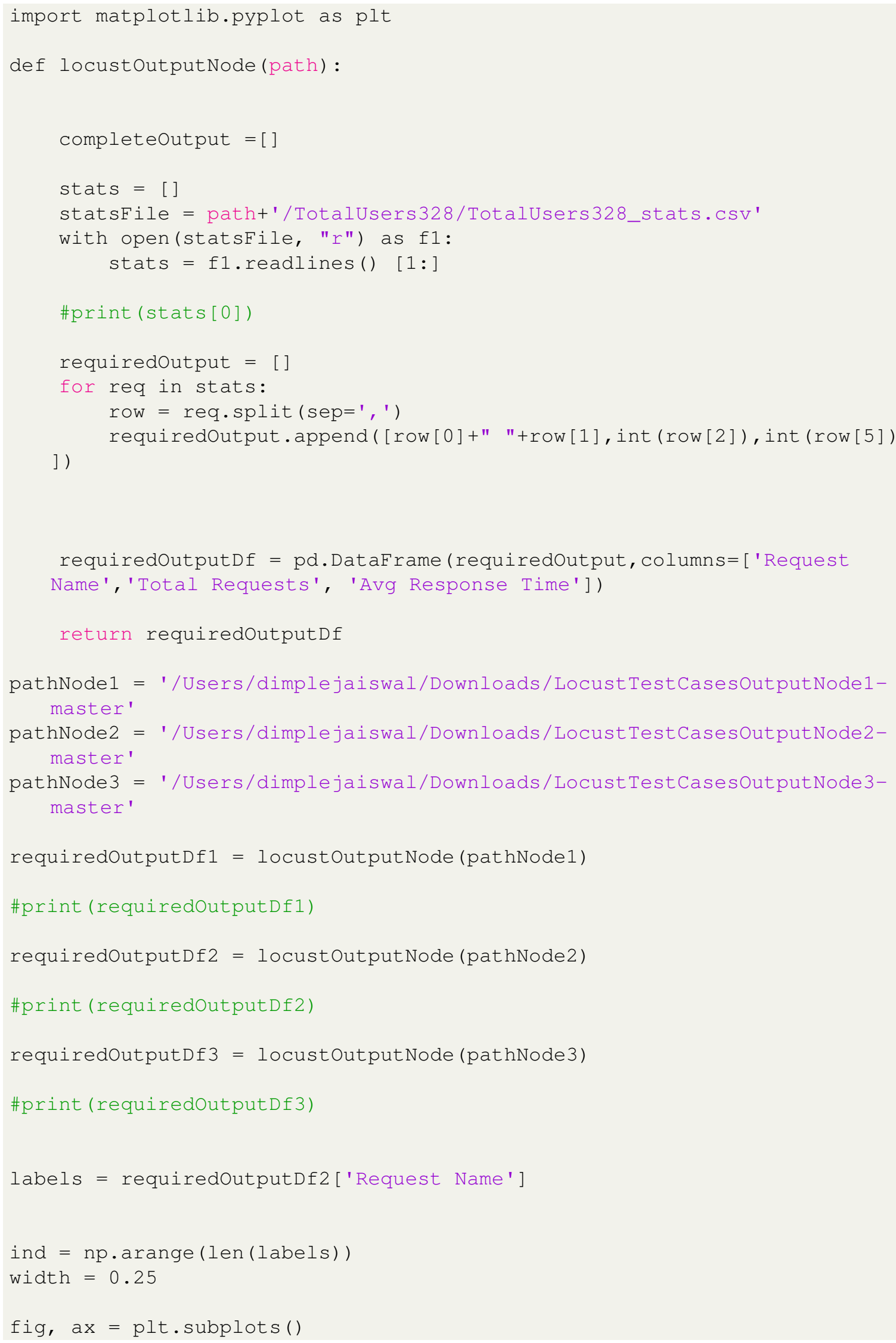




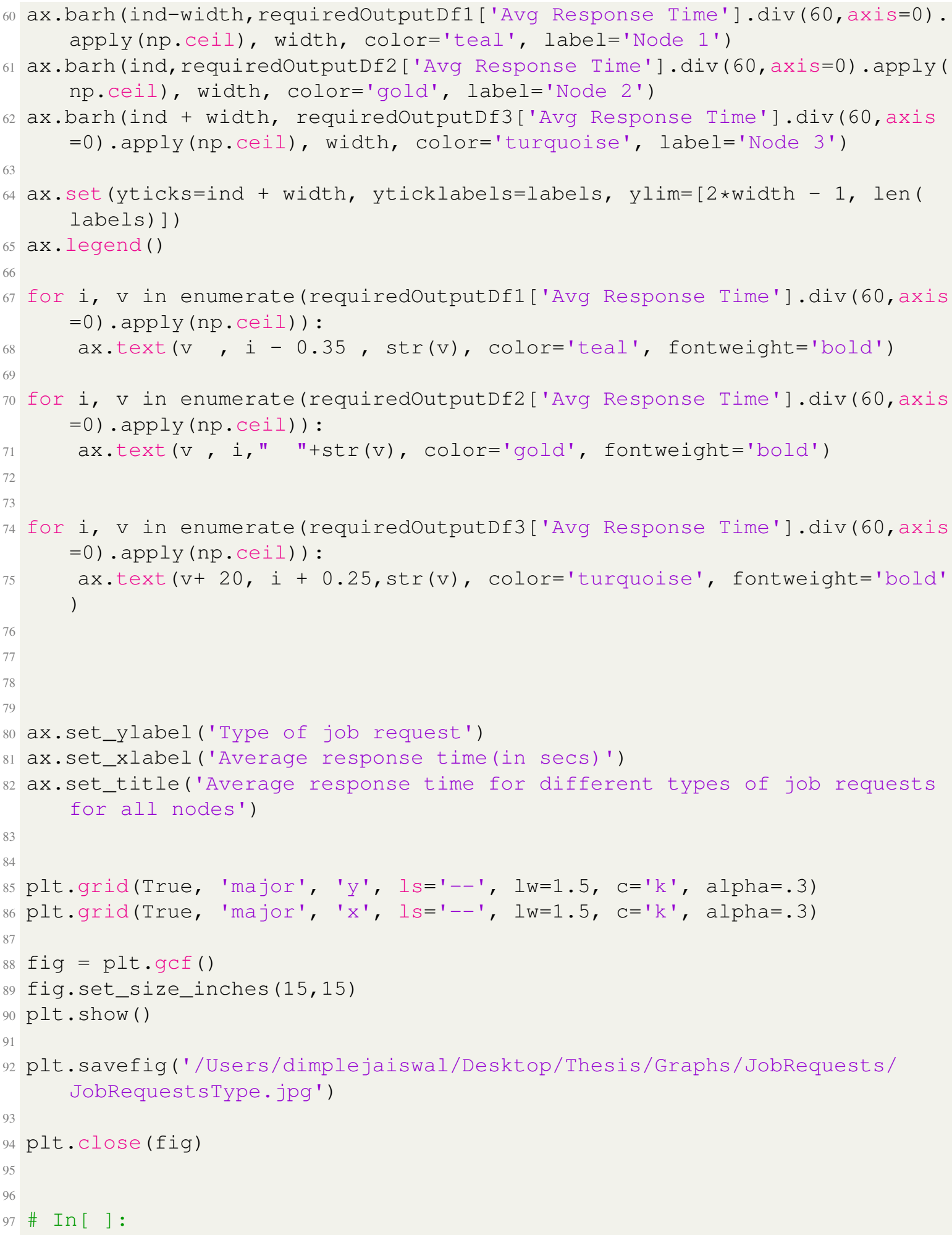

Code A.6.1.1: Response time analysis for different types of job requests

The above script creates the below given bar graph- 


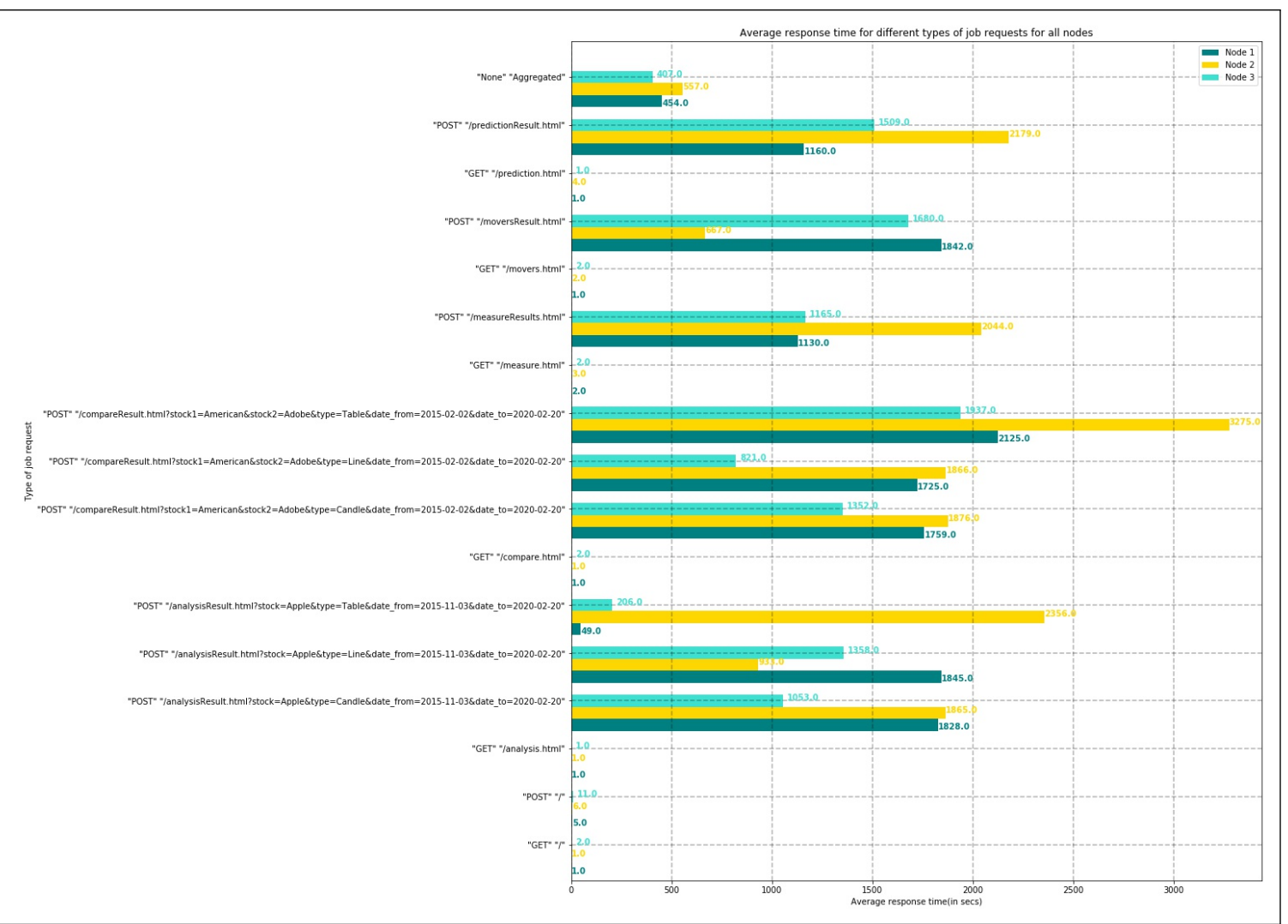

Figure A.13: Response time analysis for different types of job requests

\section{A.6.2 Data analysis of data generated by Locust testing at all the nodes}

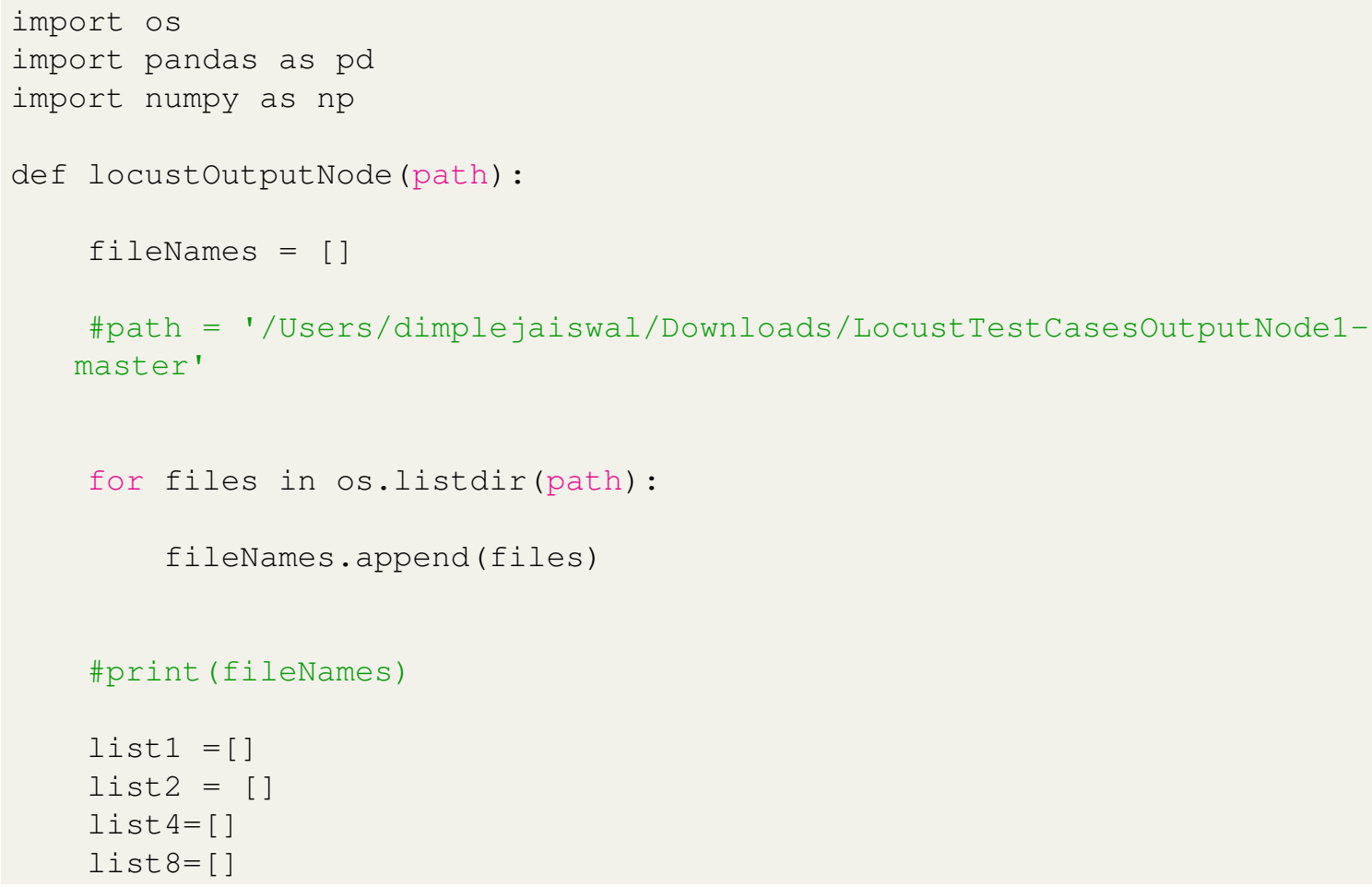




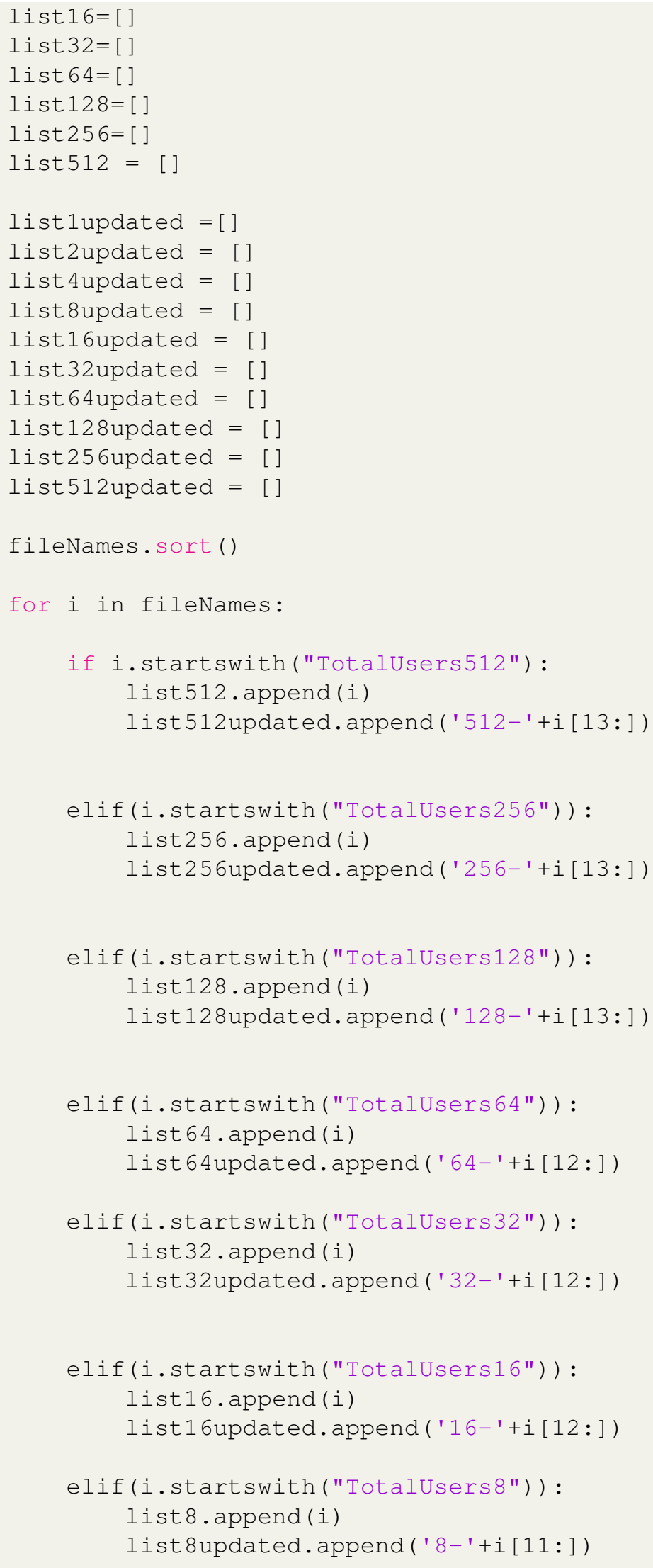




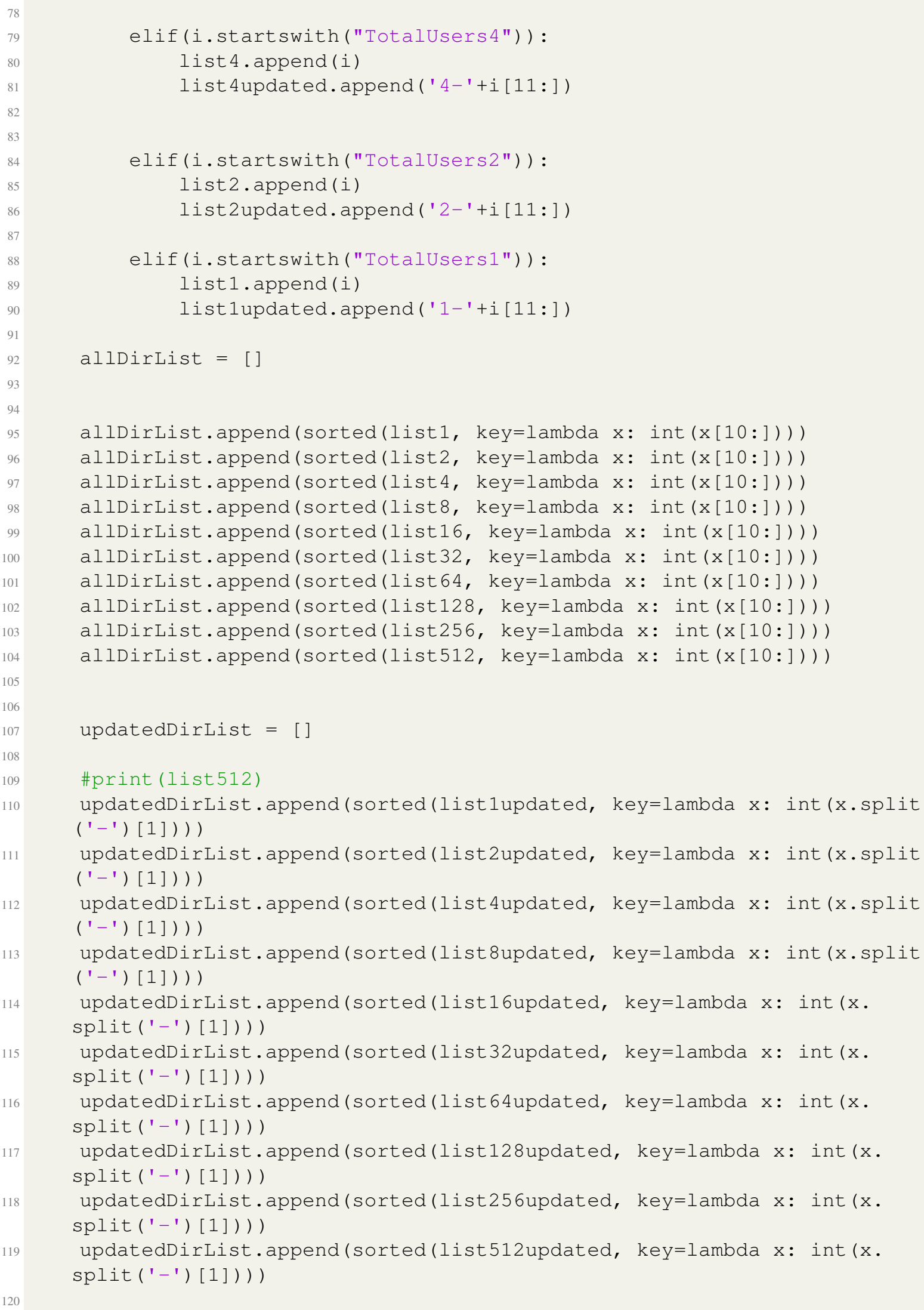

elif(i.startswith ("TotalUsers4")) : list 4 . append (i)

list 4 updated. append ('4- '+i [11: ])

elif(i.startswith ("Totalusers2")) :

list 2 . append (i)

list 2 updated.append ('2-'+i [11: ])

elif(i.startswith ("TotalUsers1")) : list 1 . append (i)

list 1 updated. append ('1- '+i [11: ])

allDirList $=[]$

allDirList. append (sorted(list1, $\operatorname{key}=1$ ambda $x$ : int (x[10:]))) allDirList. append(sorted(list2, $\operatorname{key}=1 \mathrm{ambda} x$ : int (x[10:]))) allDirList.append(sorted(list4, key=lambda $x$ : int (x[10:]))) allDirlist. append (sorted (list8, $\operatorname{key}=1$ ambda $x$ : int (x[10:]))) allDirList.append (sorted(list16, key=lambda $x$ : int(x[10:]))) allDirList.append (sorted(list32, key=lambda $x$ : int (x[10:]))) allDirList.append (sorted(list64, key=lambda $x$ : int (x[10:]))) allDirList.append (sorted(list128, key=lambda $x$ : int (x[10:]))) allDirList.append (sorted (list256, key=lambda $x$ : int (x[10:]))) allDirList. append (sorted(list512, key=lambda $x$ : int (x[10:])))

updatedDirList $=[]$

\#print (1ist512)

updatedDirList. append (sorted (listlupdated, key=lambda $x$ : int (x.split $\left.\left.\left({ }^{\prime}-{ }^{\prime}\right)[1]\right)\right)$ )

updatedDirList.append (sorted(list2updated, key=lambda $x$ : int (x.split $\left.\left.\left({ }^{\prime}-{ }^{\prime}\right)[1]\right)\right)$ )

updatedDirList. append (sorted (list4updated, key=lambda $x$ : int (x.split $\left.\left.\left({ }^{\prime}-{ }^{\prime}\right)[1]\right)\right)$ )

updatedDirList. append (sorted(list8updated, key=lambda $x$ : int (x.split $('-')[1]))$ )

updatedDirList. append (sorted (list16updated, key=lambda $x$ : int ( . split('-')[1])))

updatedDirList. append (sorted (list32updated, key=lambda $x$ : int ( . split('-')[1])))

updatedDirList. append (sorted (list64updated, key=lambda $x$ : int ( $x$. split('-')[1])))

updatedDirlist. append (sorted (list128updated, key=lambda $x$ : int ( $x$. split('-')[1])))

updatedDirList. append (sorted (list256updated, key=lambda $x$ : int ( $x$. split('-')[1])))

updatedDirList. append (sorted (list512updated, key=lambda $\mathrm{x}$ : int ( $\mathrm{x}$. split ('-')[1]))) 


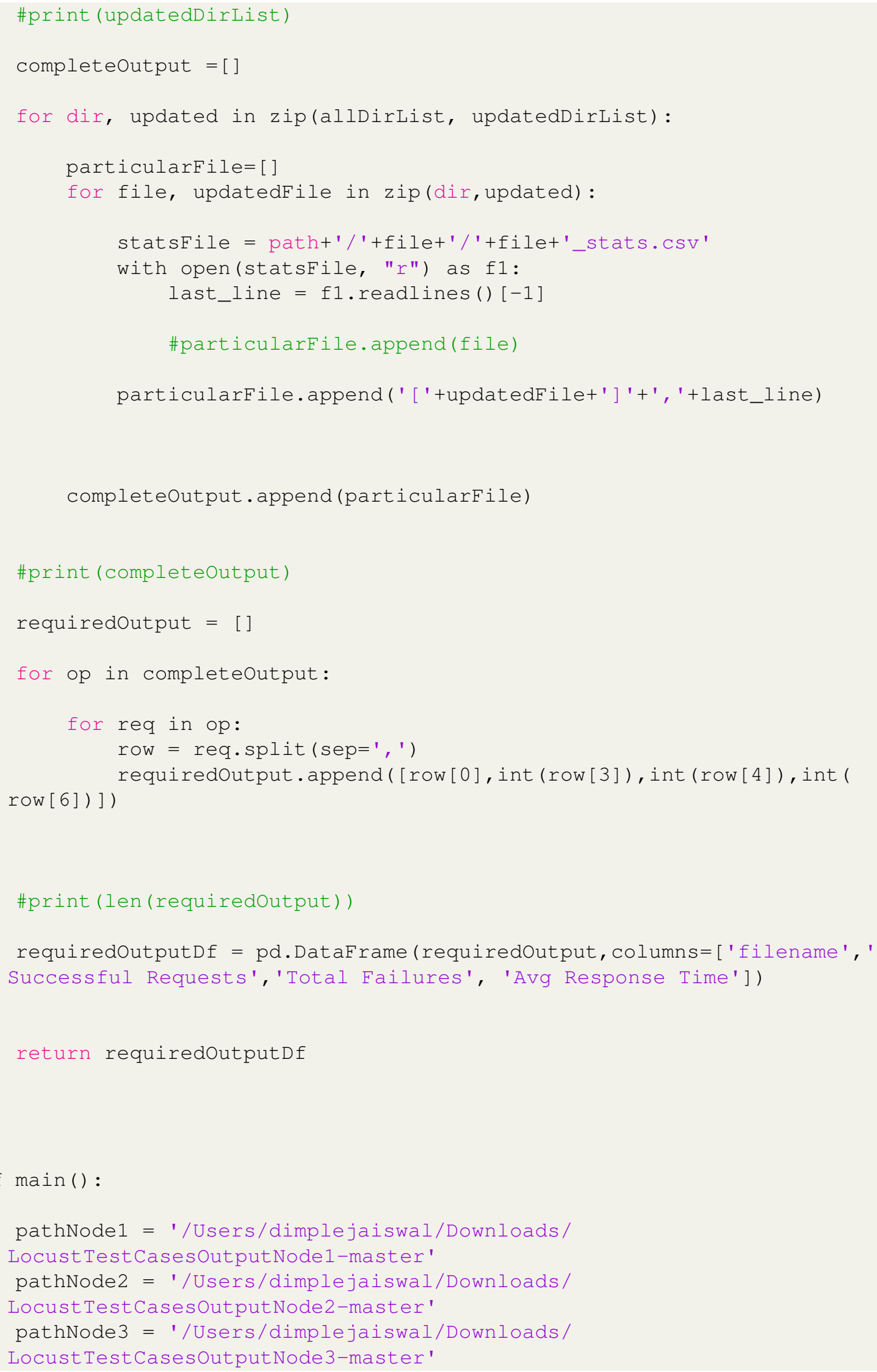




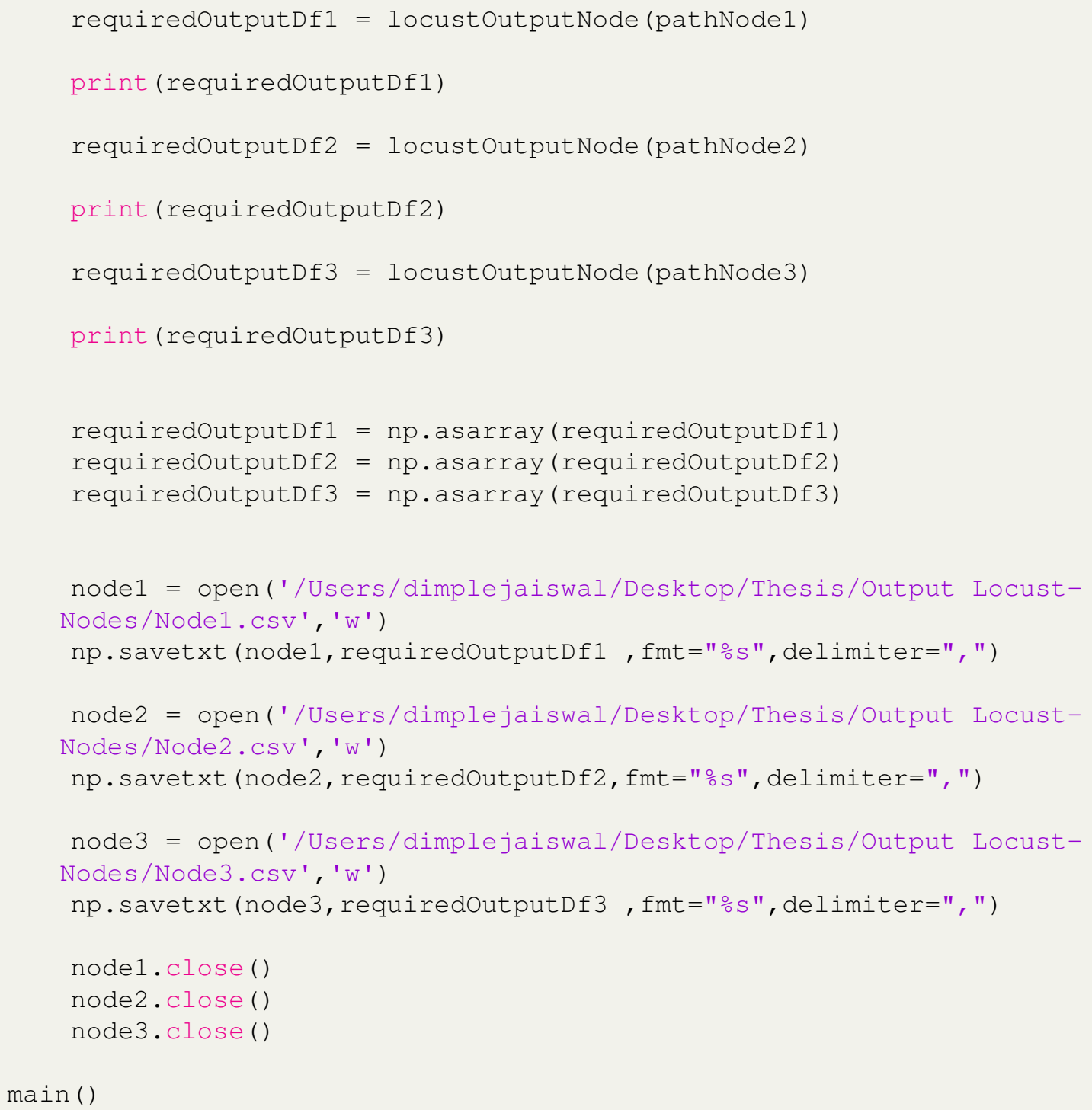

\section{Code A.6.2.1: Data preprocessing of data generated by Locust testing for all the nodes}

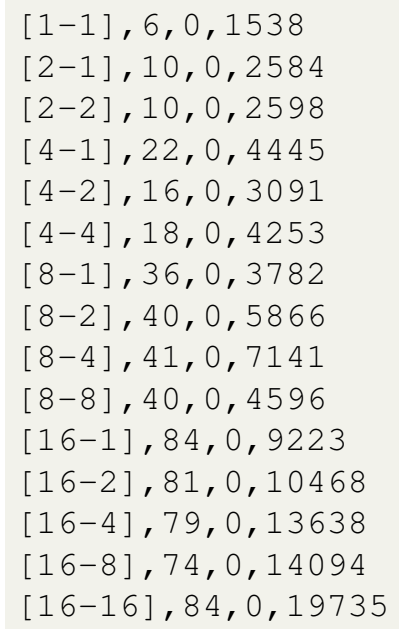




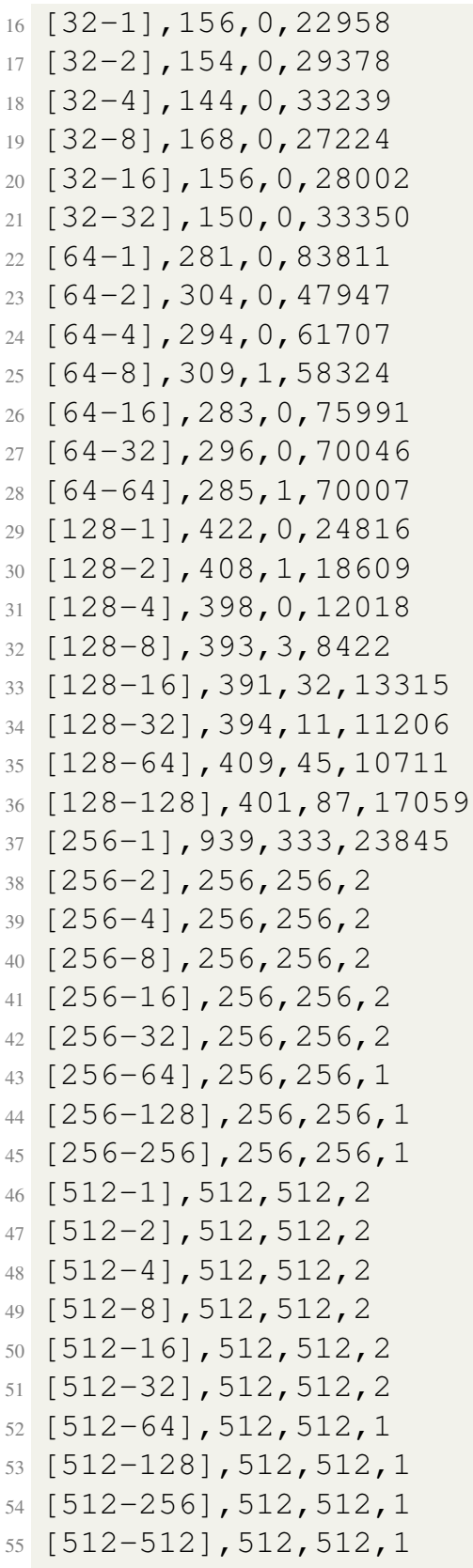

Code A.6.2.2: Output generated for node 1

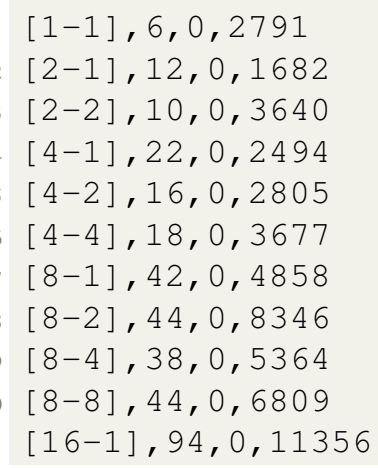




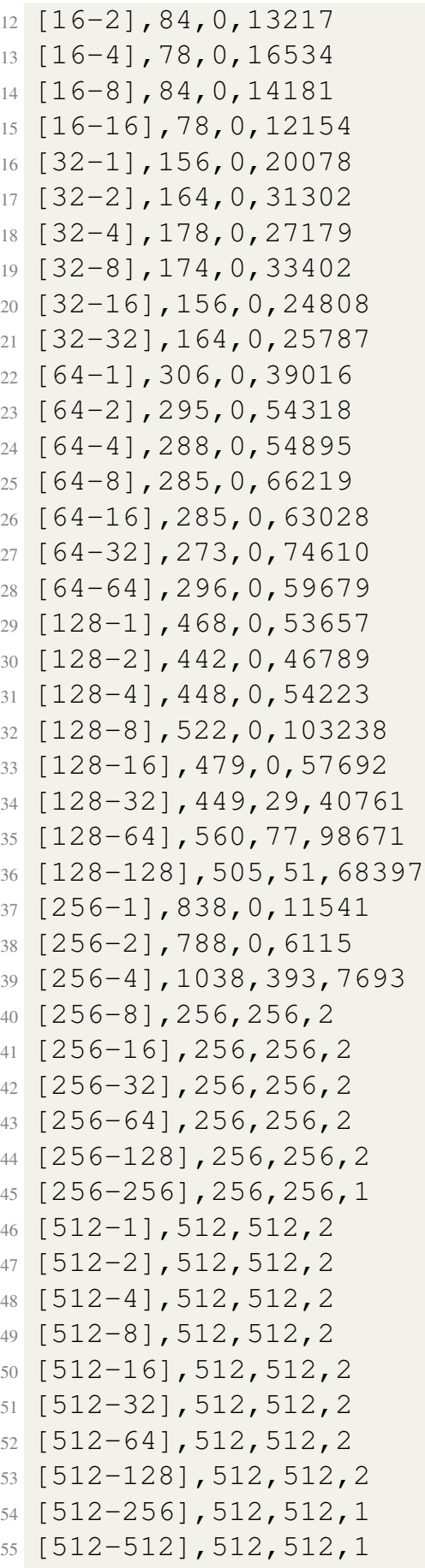

Code A.6.2.3: Output generated for node 2

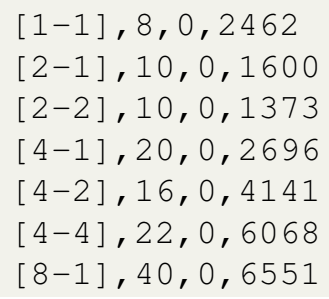




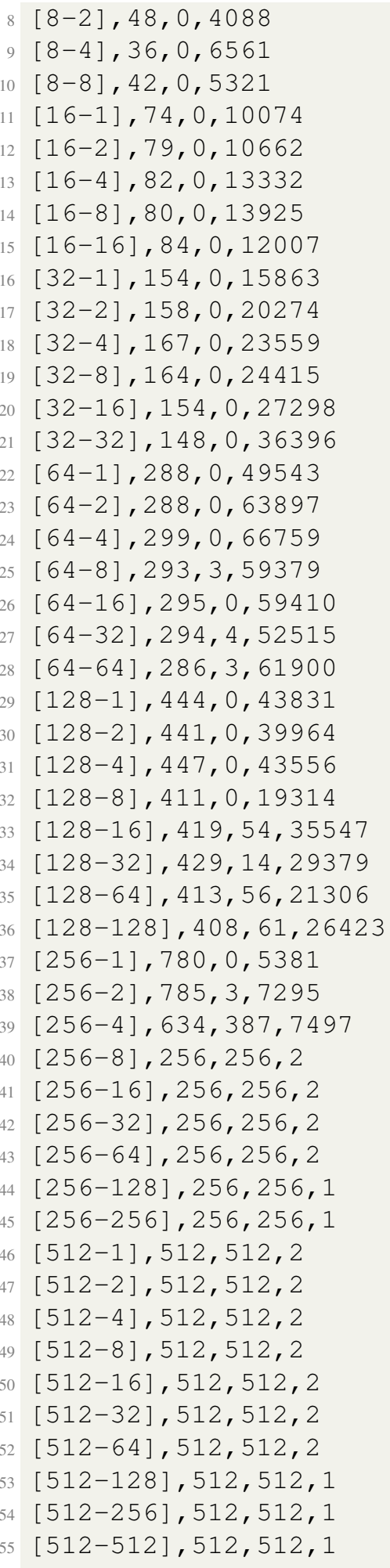

Code A.6.2.4: Output generated for node 3

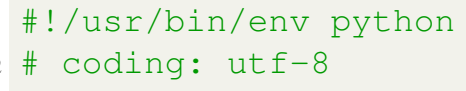




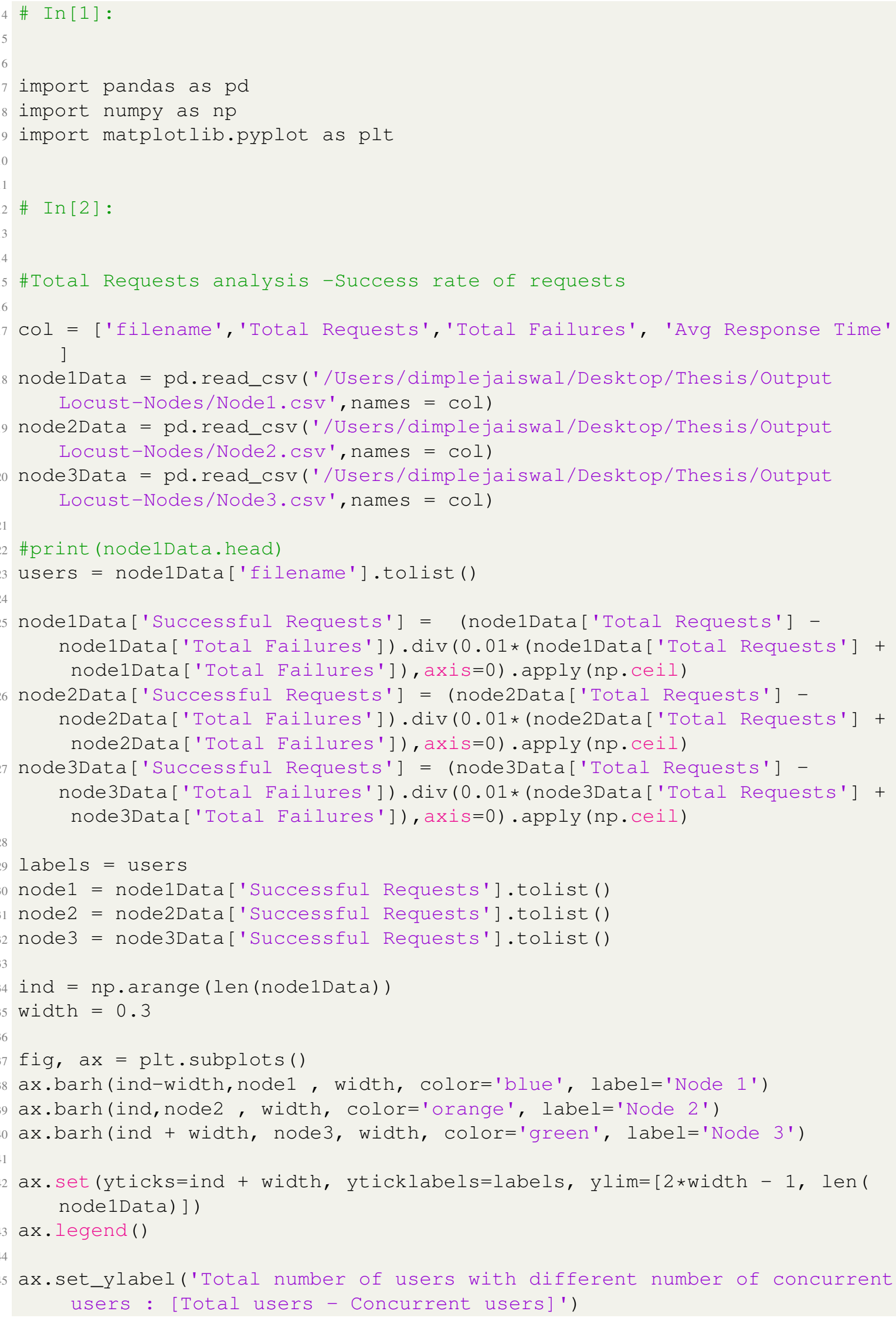




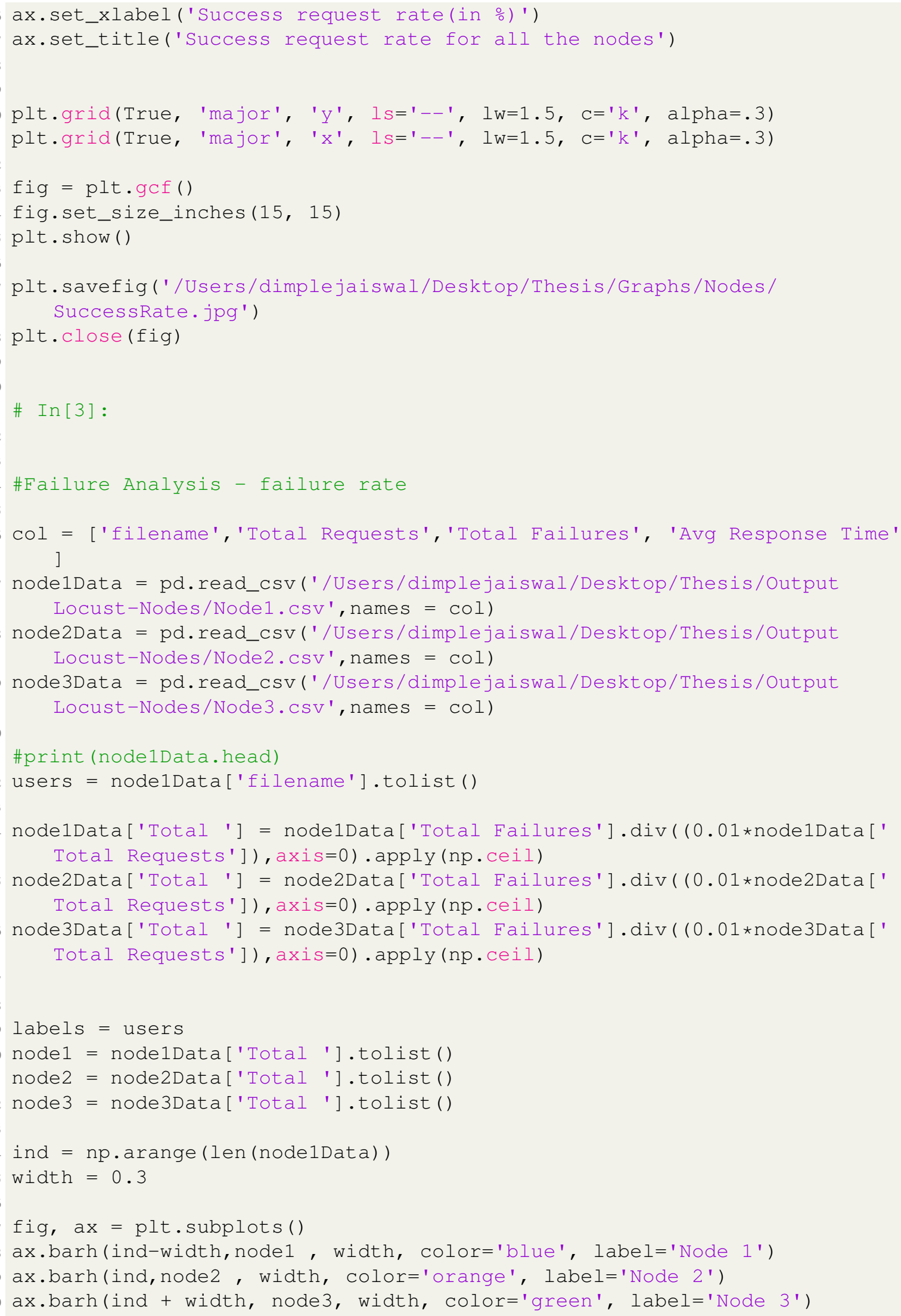




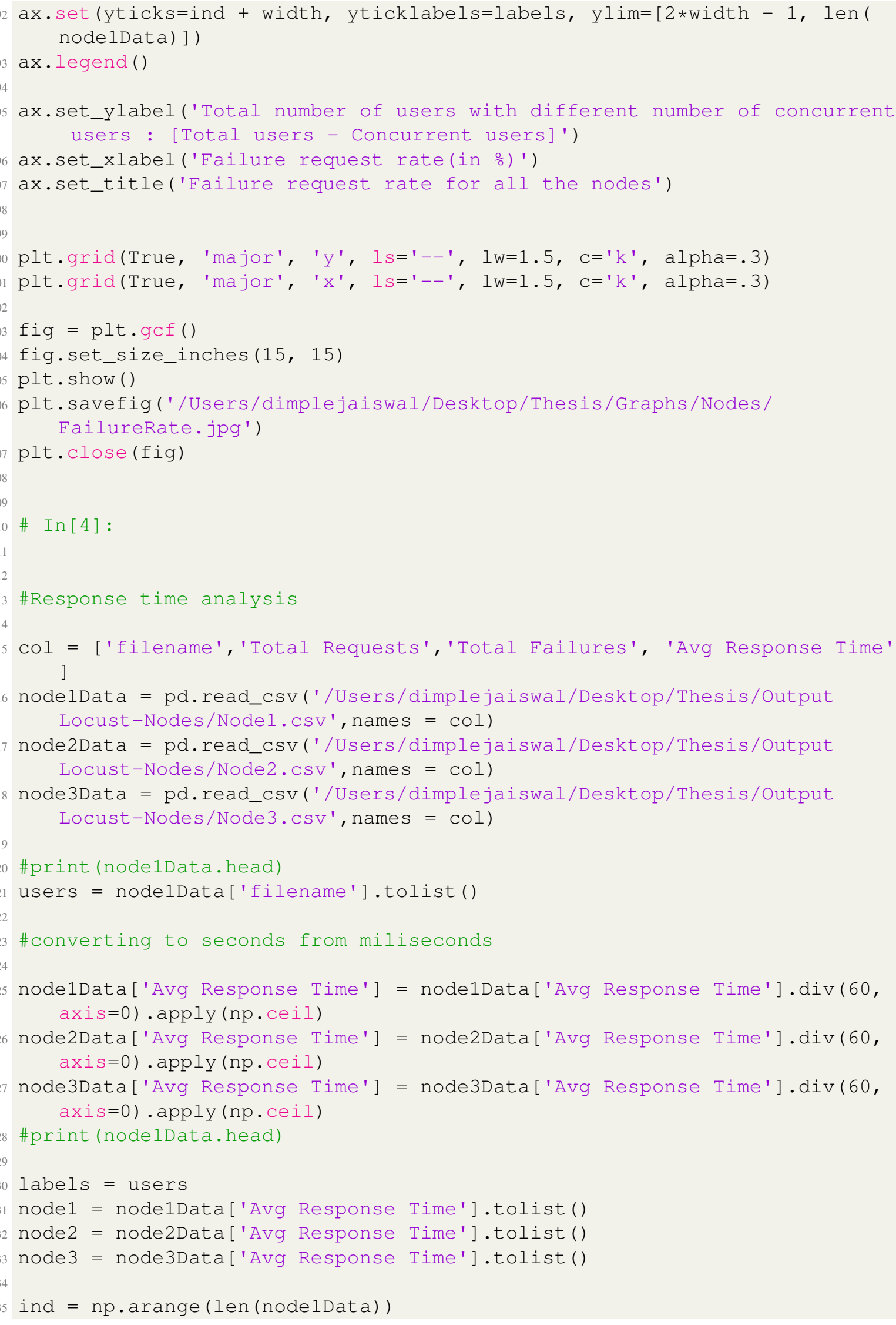




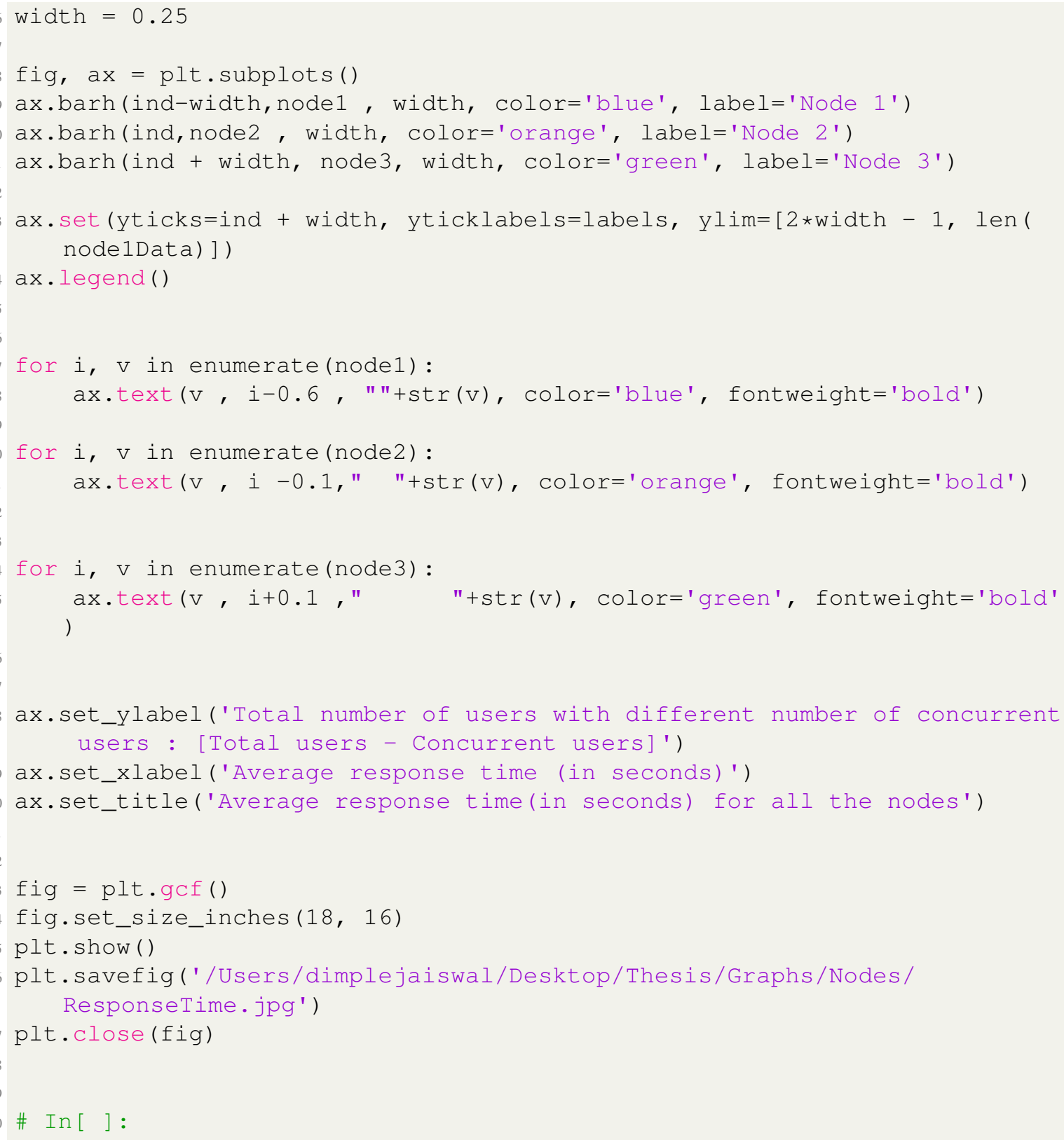

Code A.6.2.5: Analysis of data generated by locust for all nodes

Below are the figures generated using above script - 


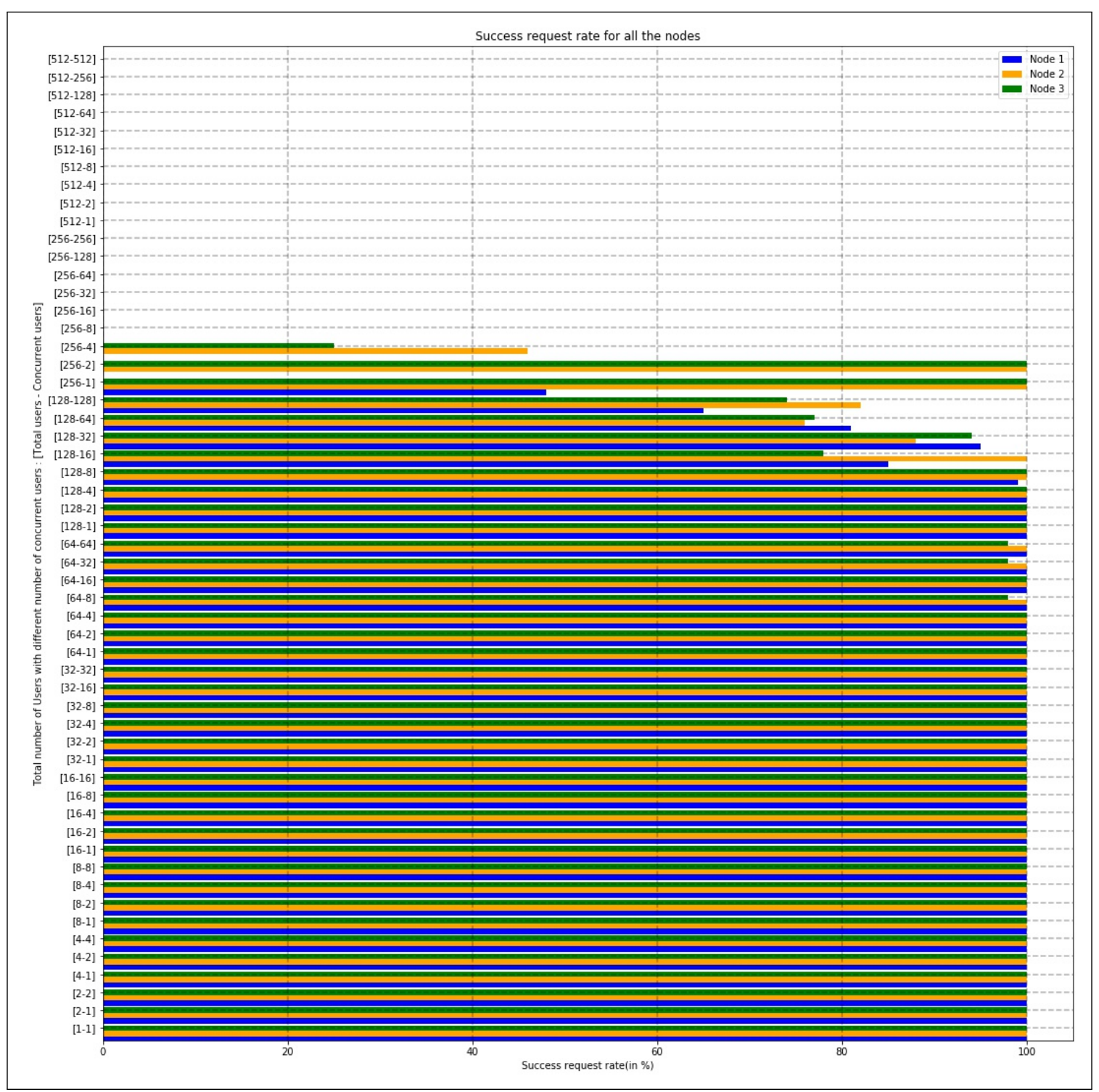

Figure A.14: Success request rate for all the nodes 


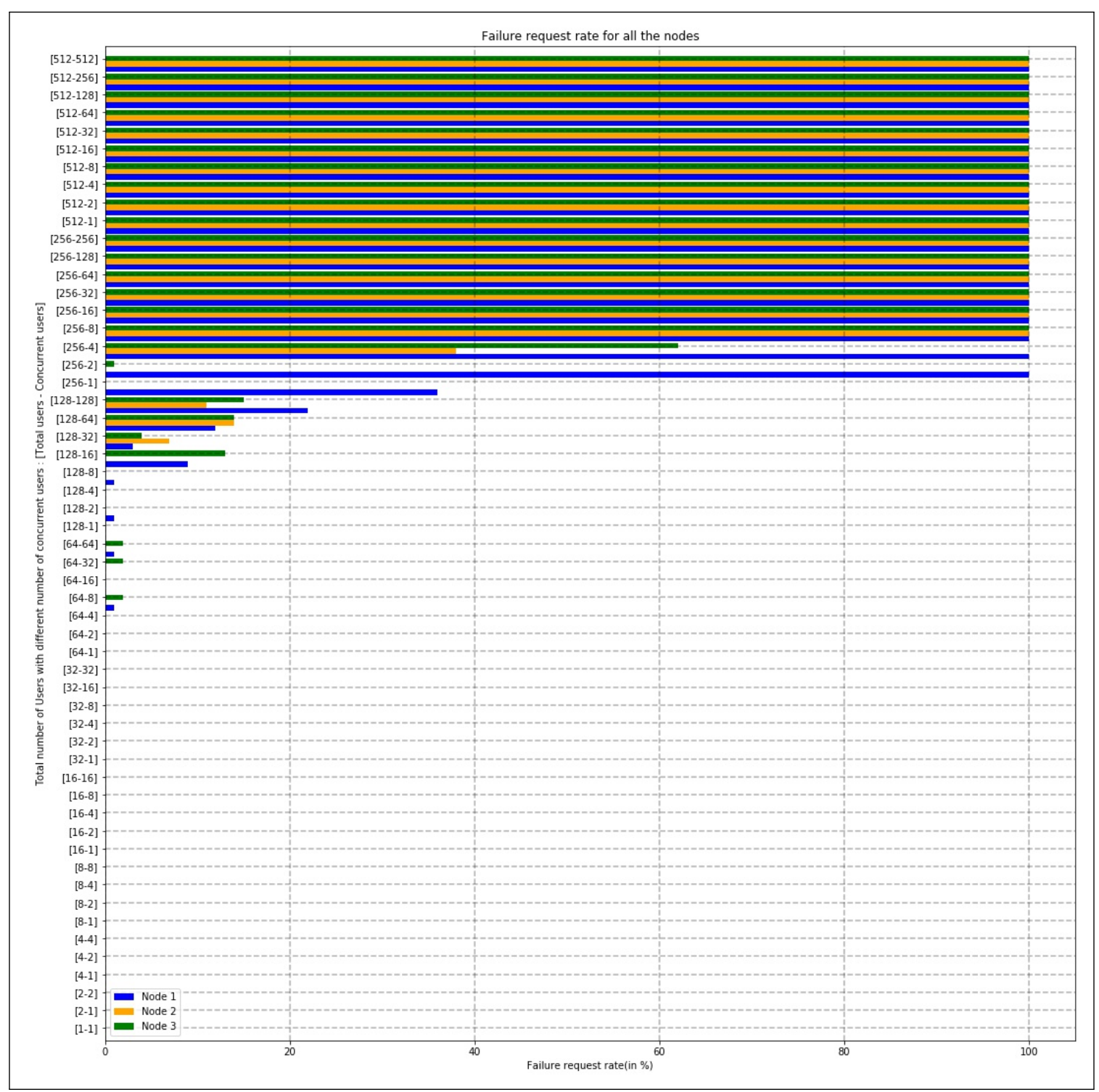

Figure A.15: Failure request rate for all the nodes 


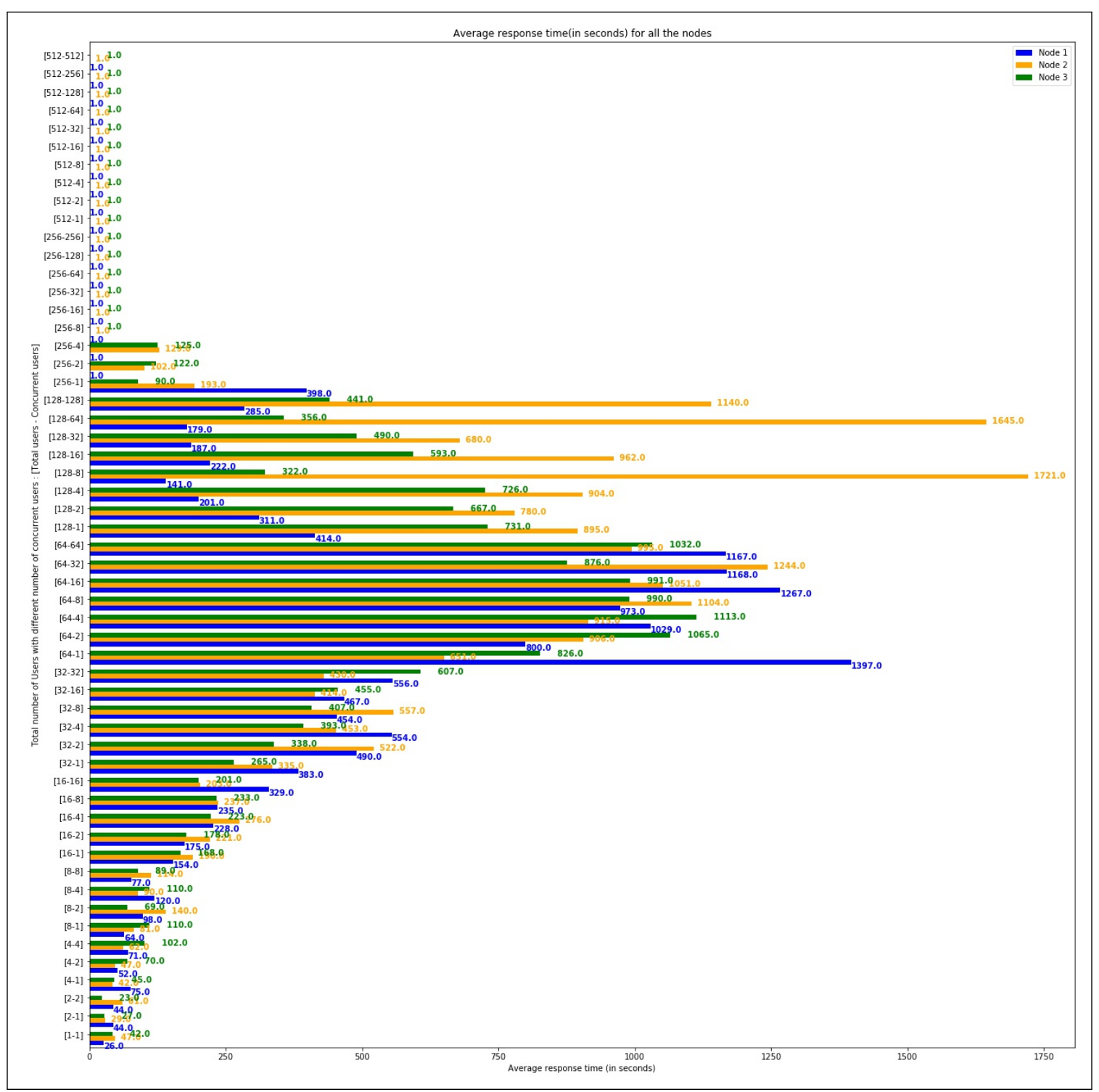

Figure A.16: Average response time(in seconds) for all the nodes

A.6.3 Data analysis of data generated by Locust testing using different types of load balancing approach

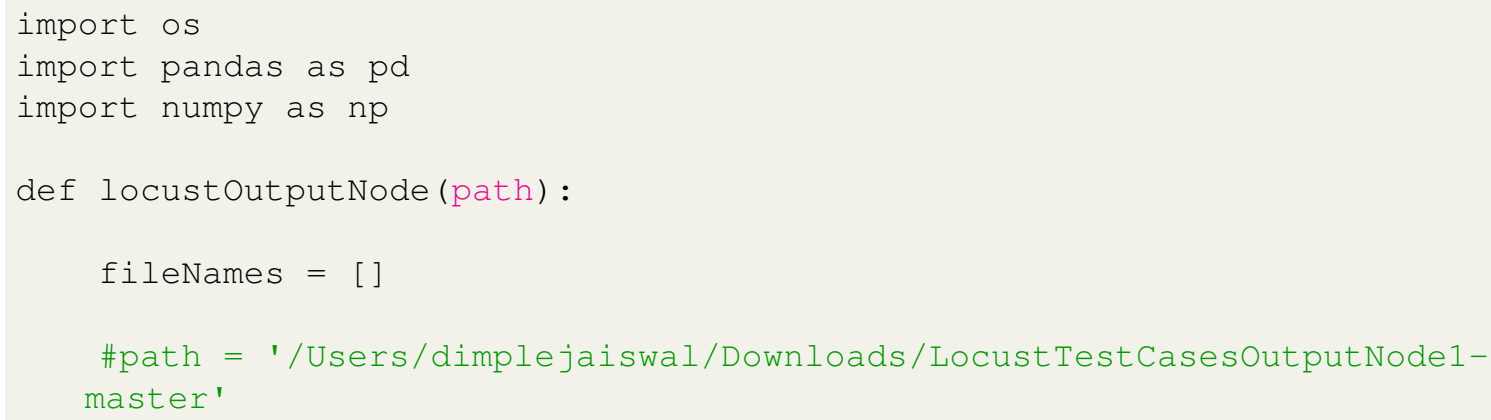




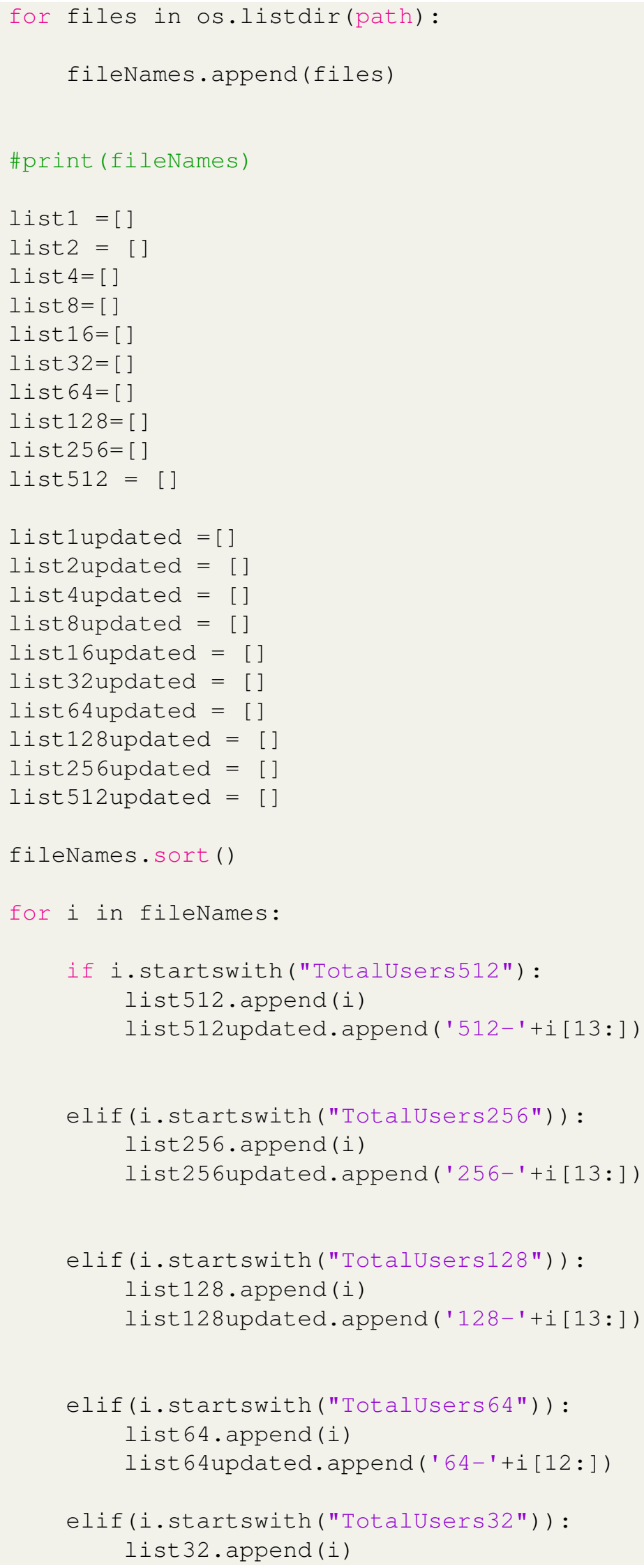




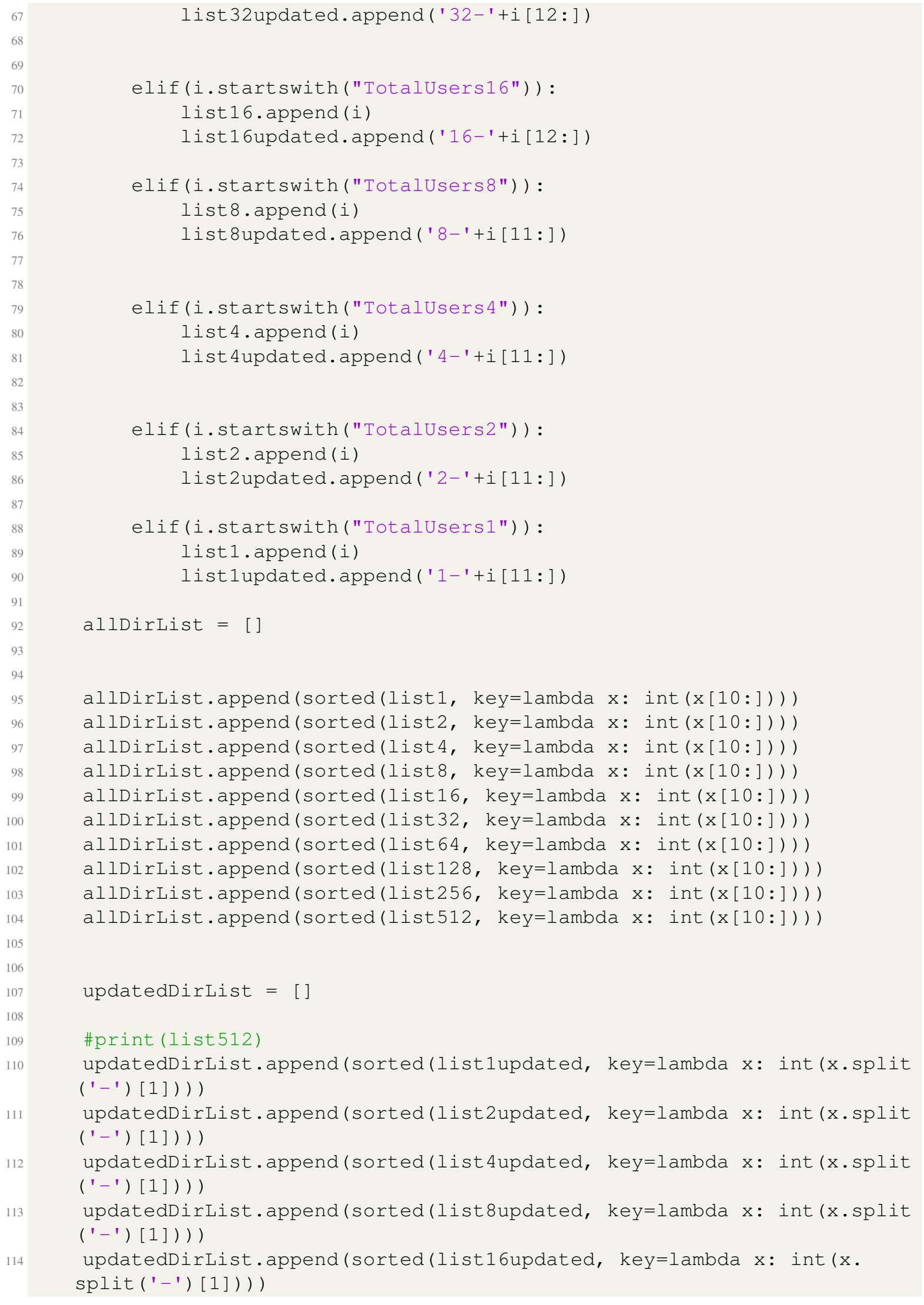




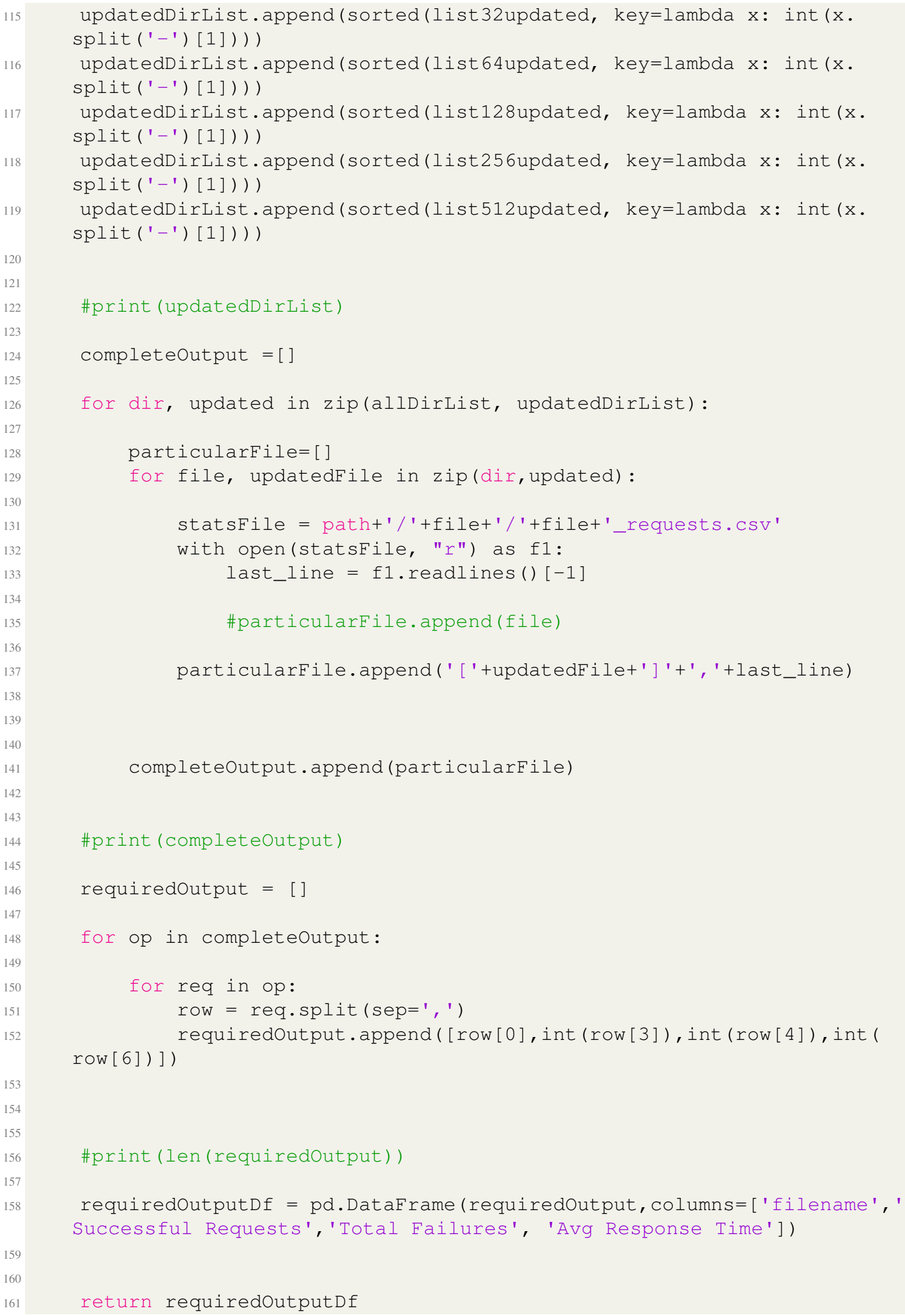




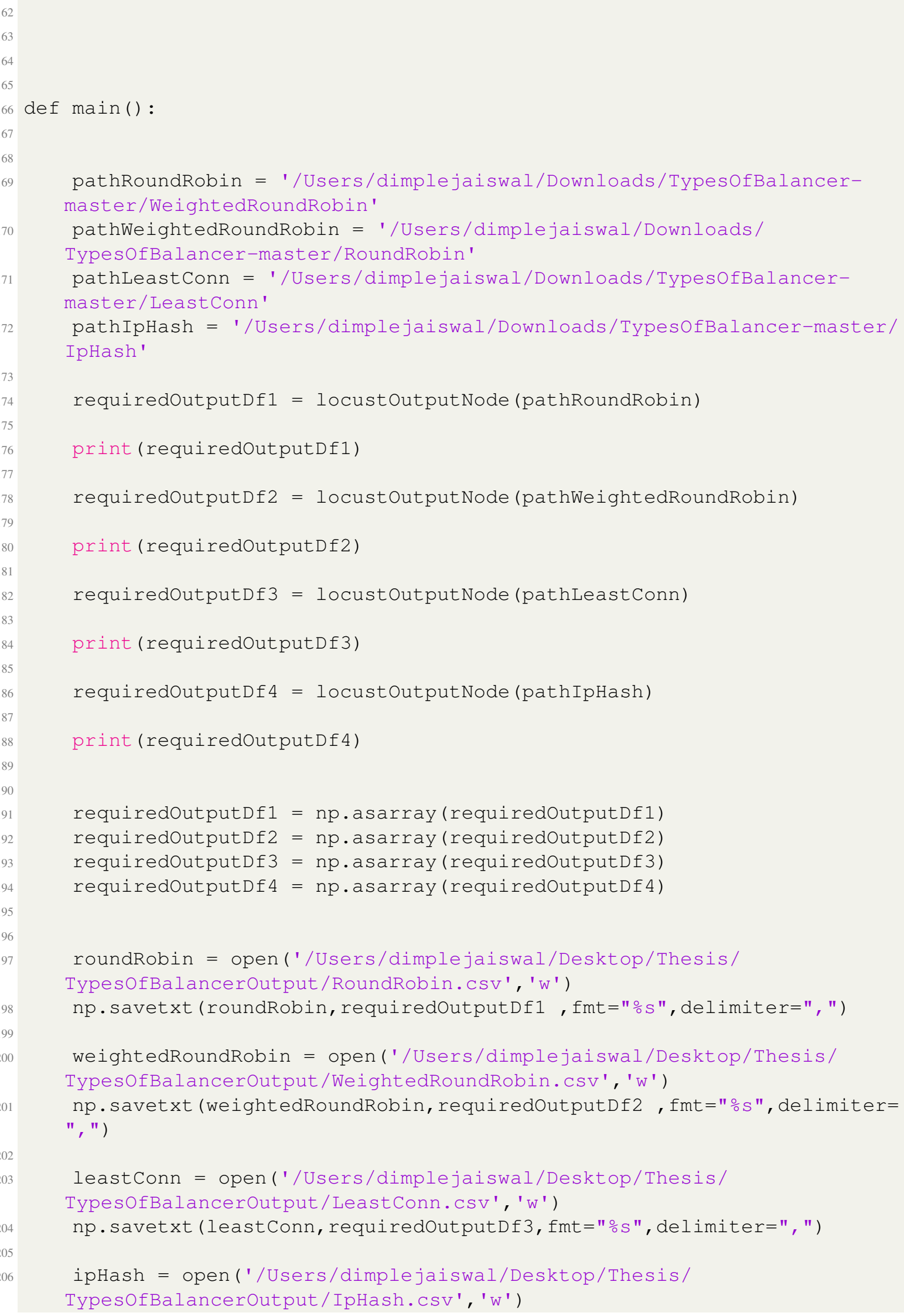




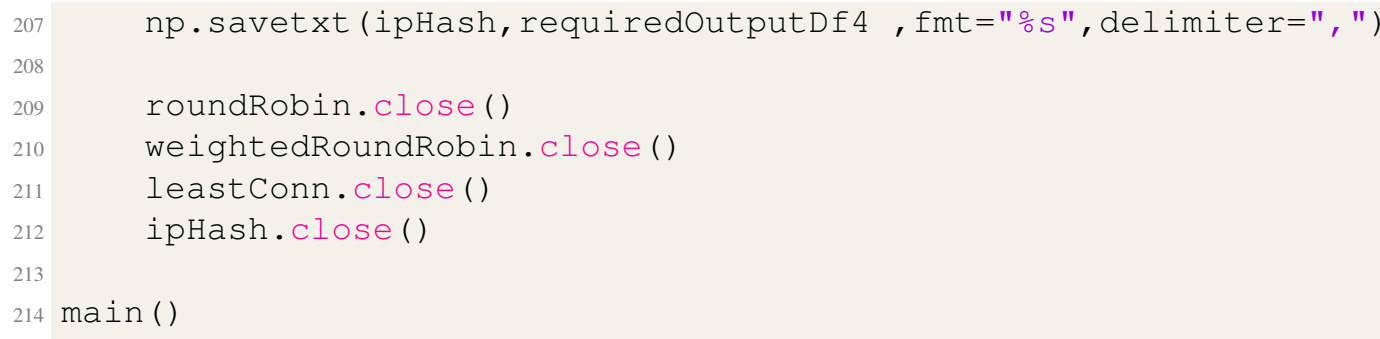

Code A.6.3.1: Data preprocessing of data generated by Locust testing using different types of load balancers

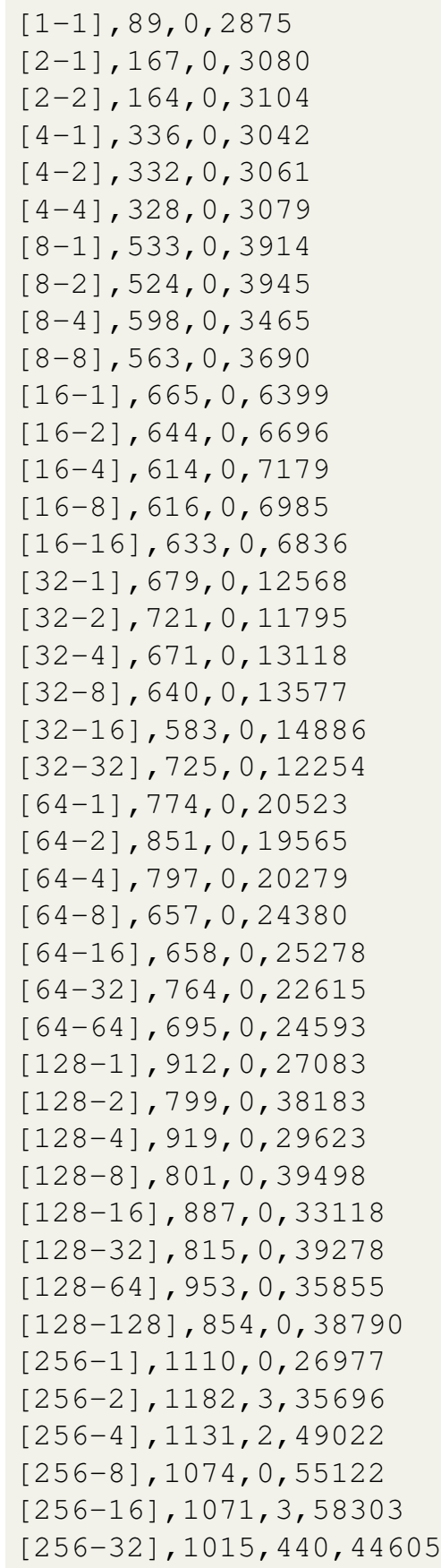




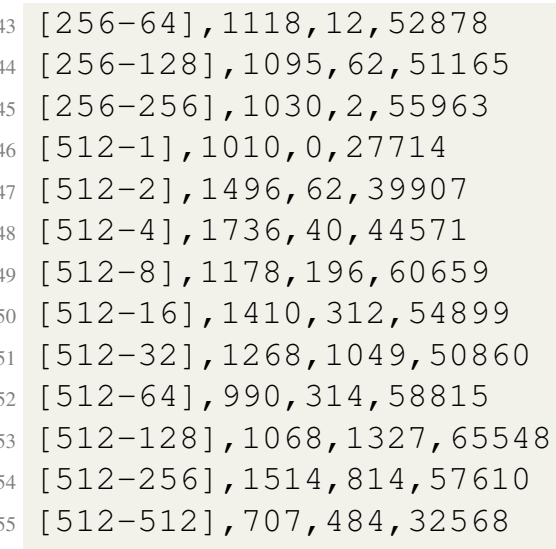

\section{Code A.6.3.2: Output generated using Round robin approach}

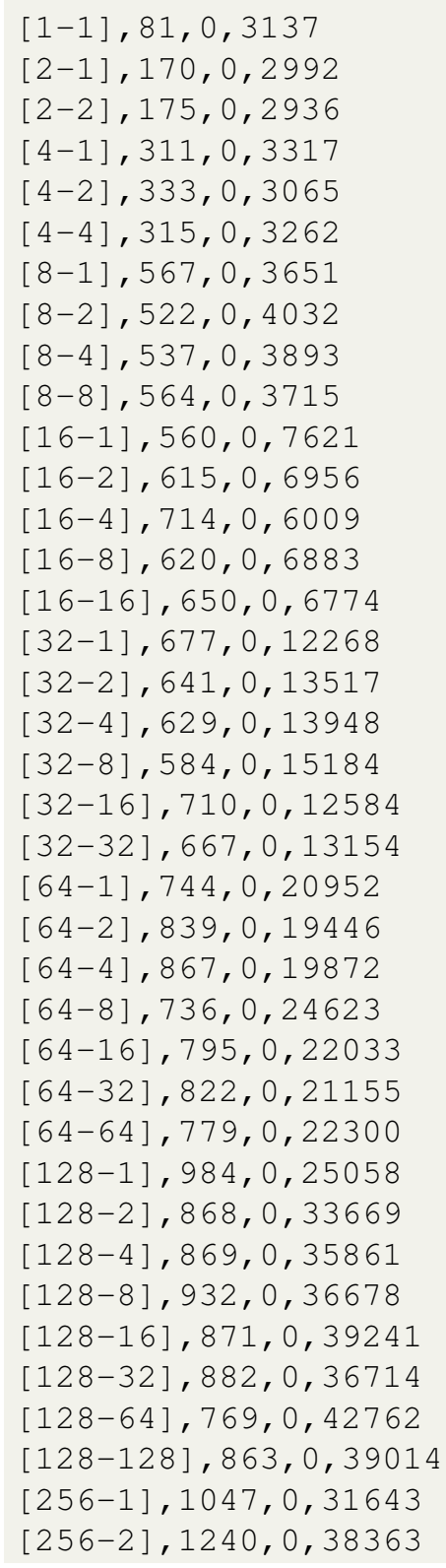




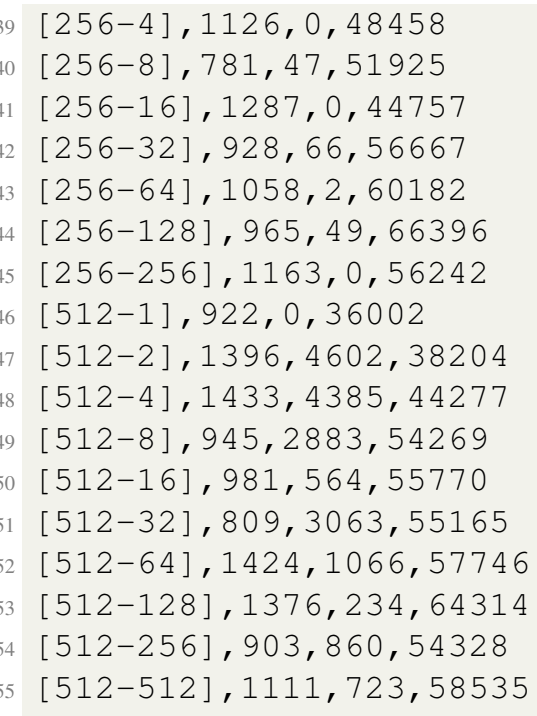

Code A.6.3.3: Output generated using Least connection approach

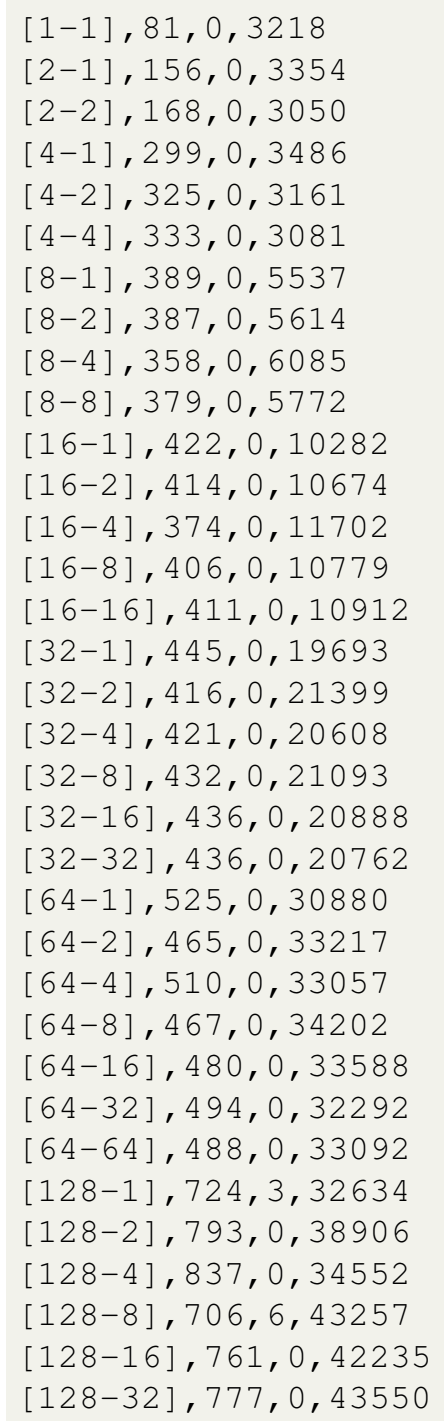




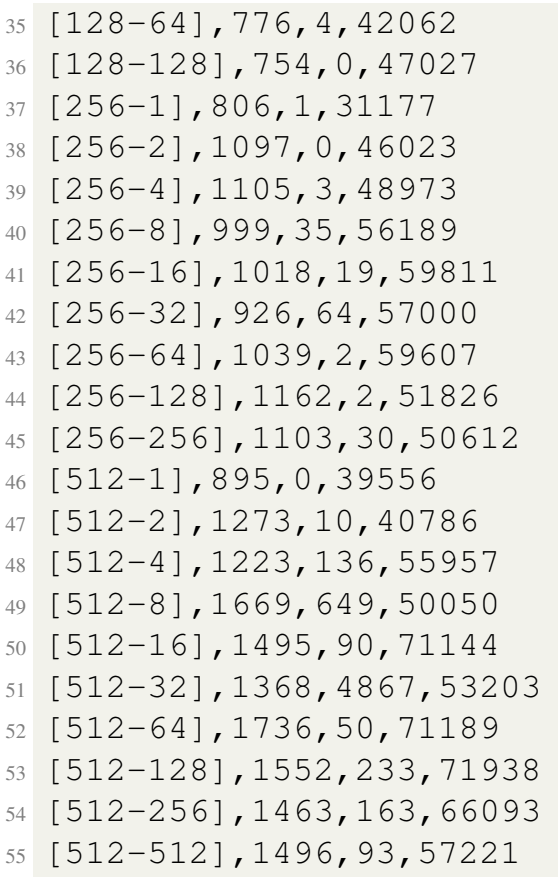

Code A.6.3.4: Output generated using Session persistance approach

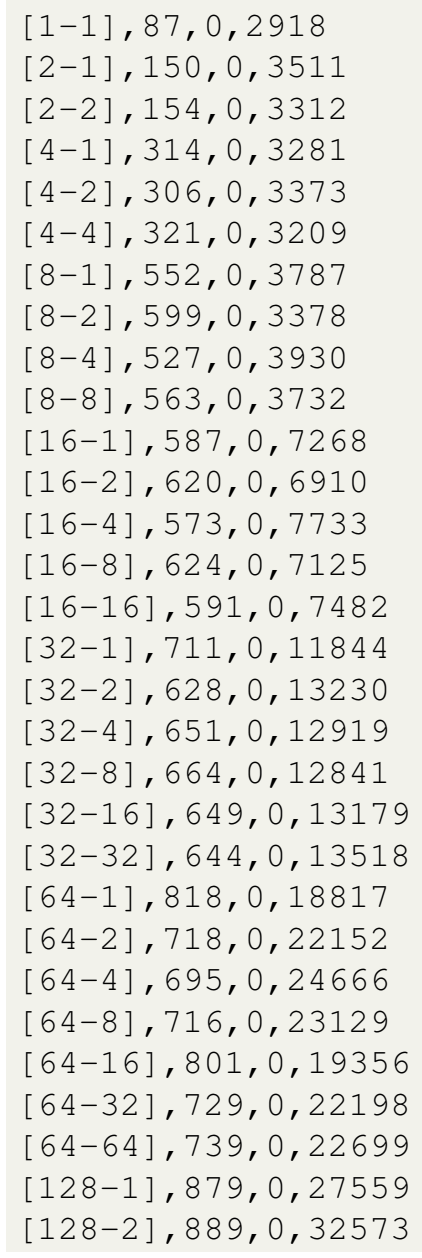




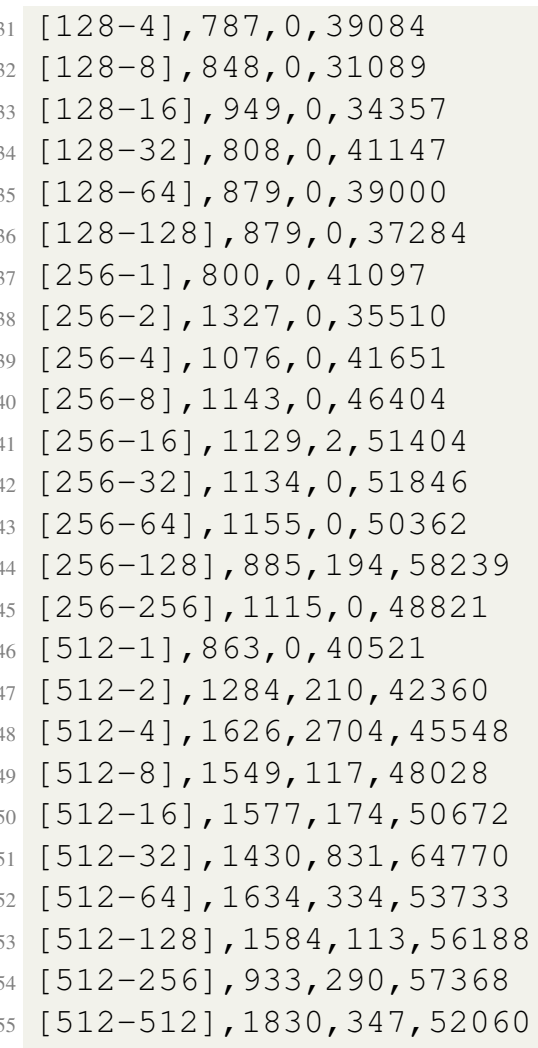

\section{Code A.6.3.5: Output generated using Weighted round robin approach}

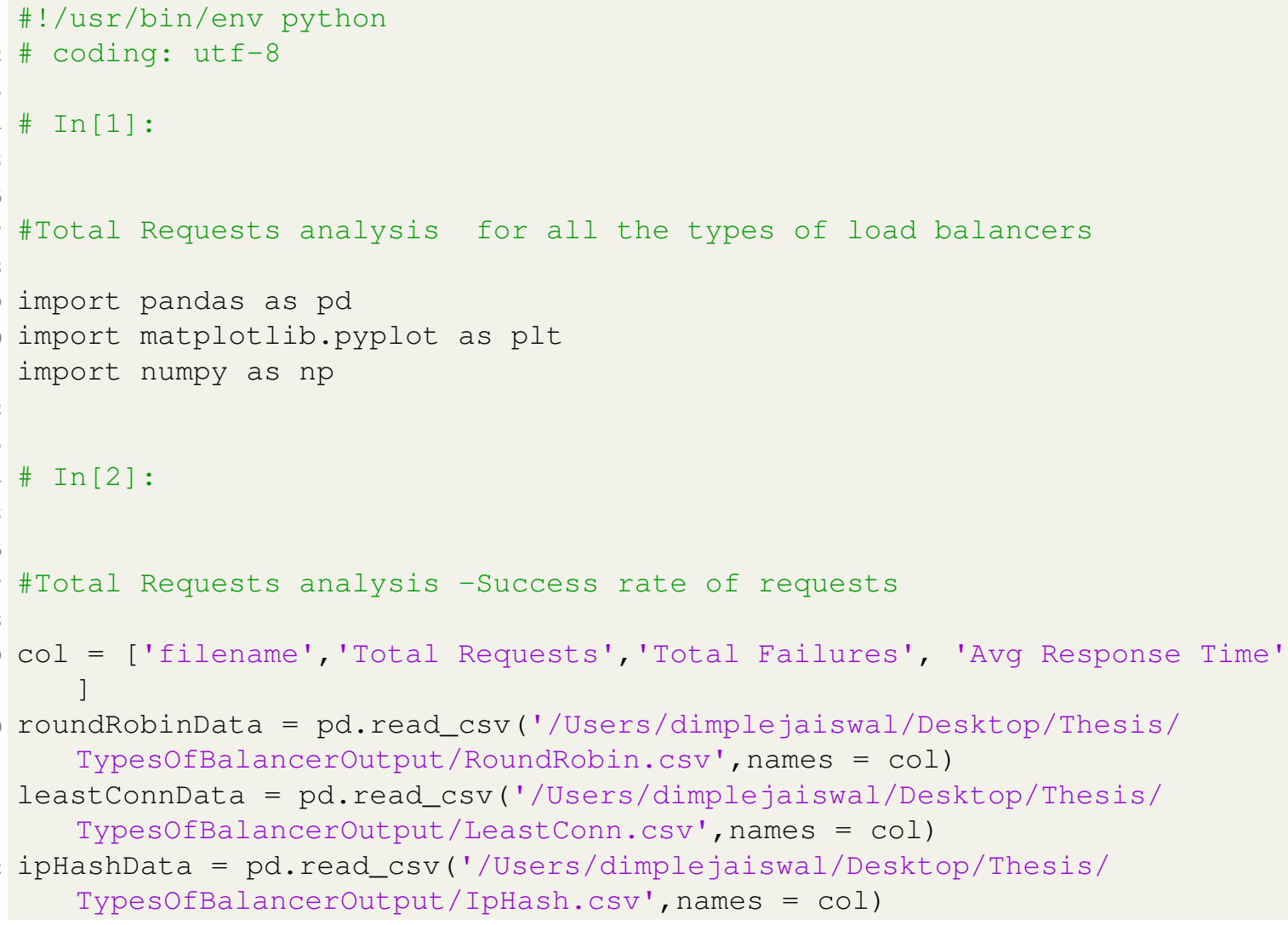




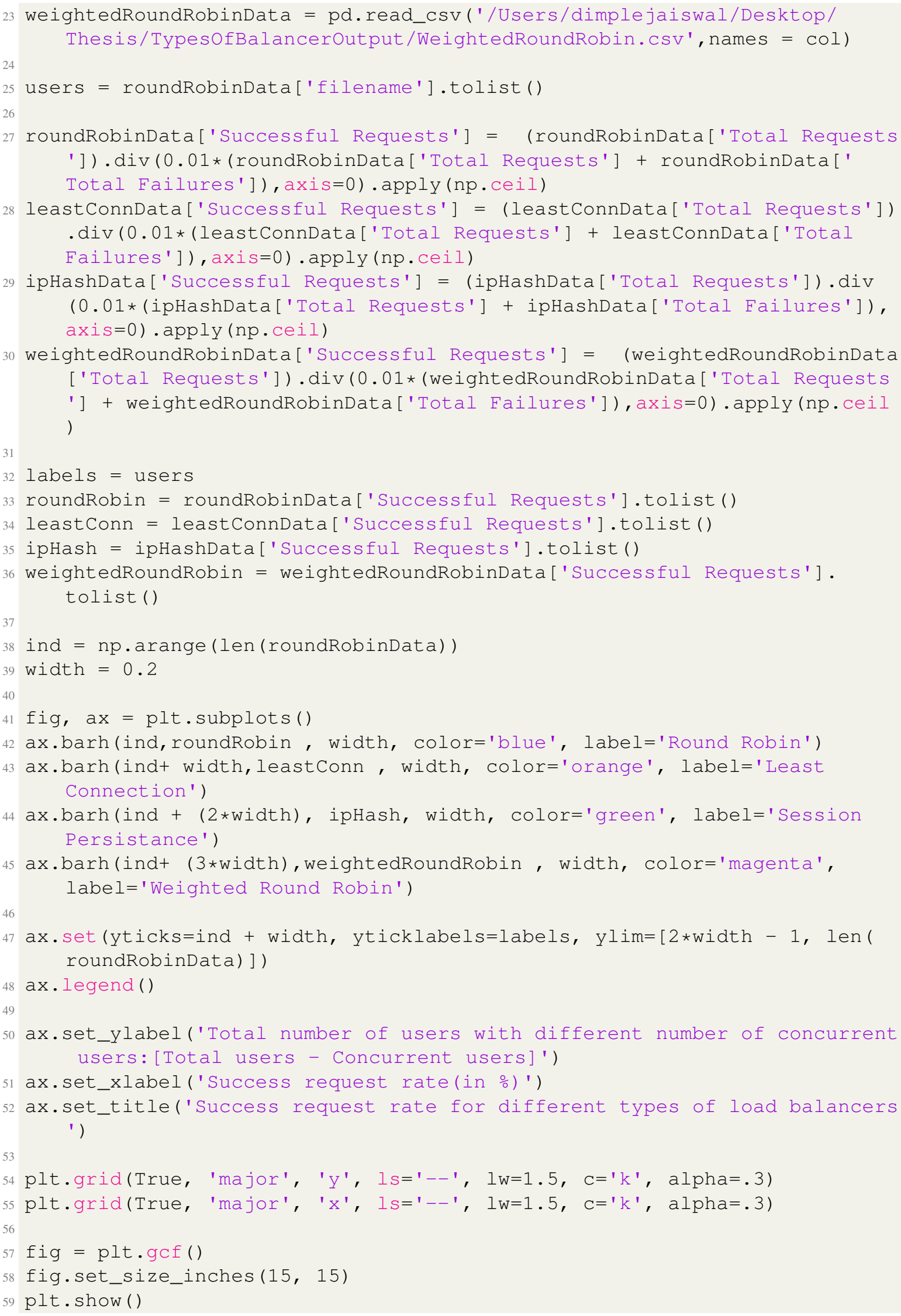




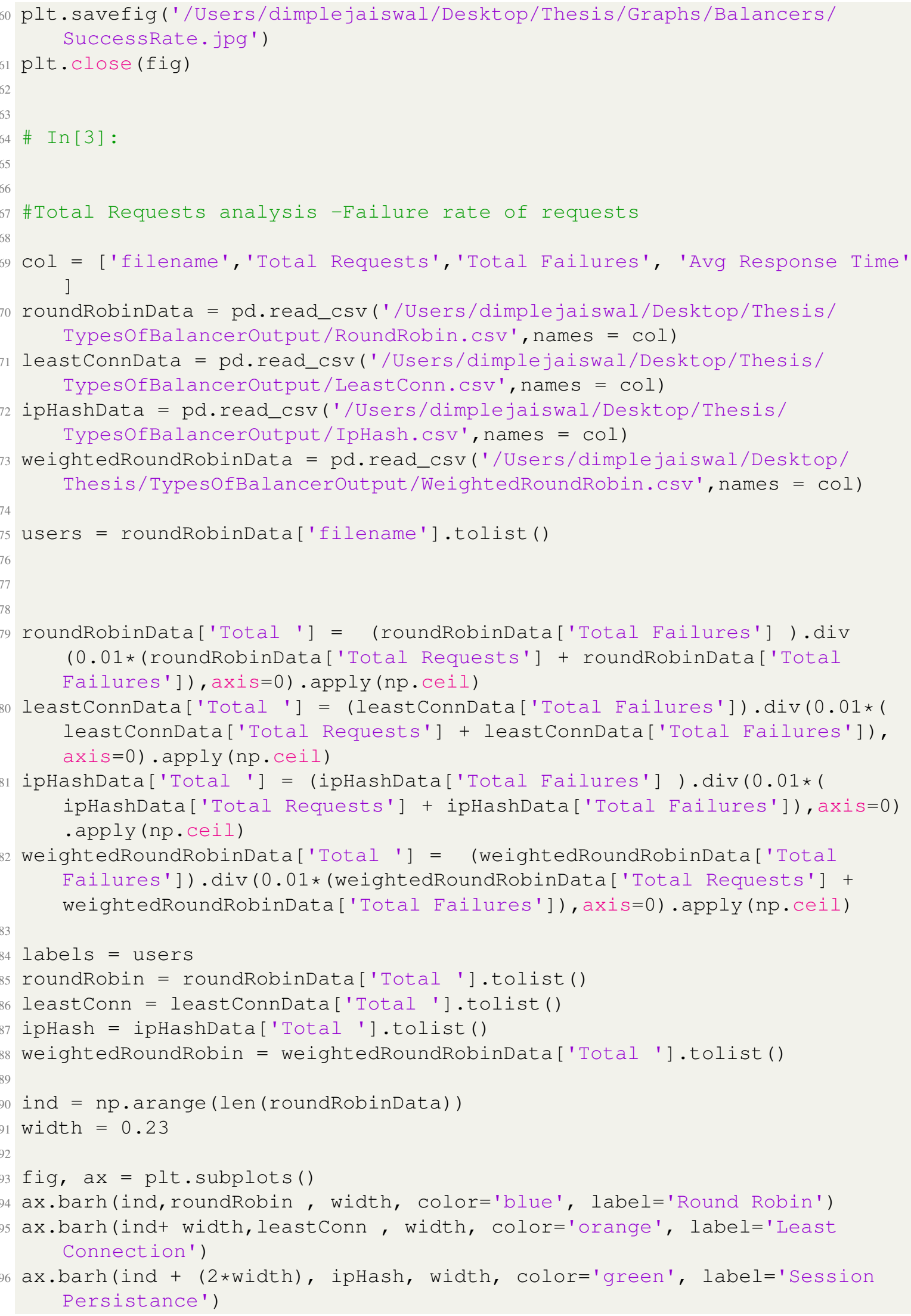




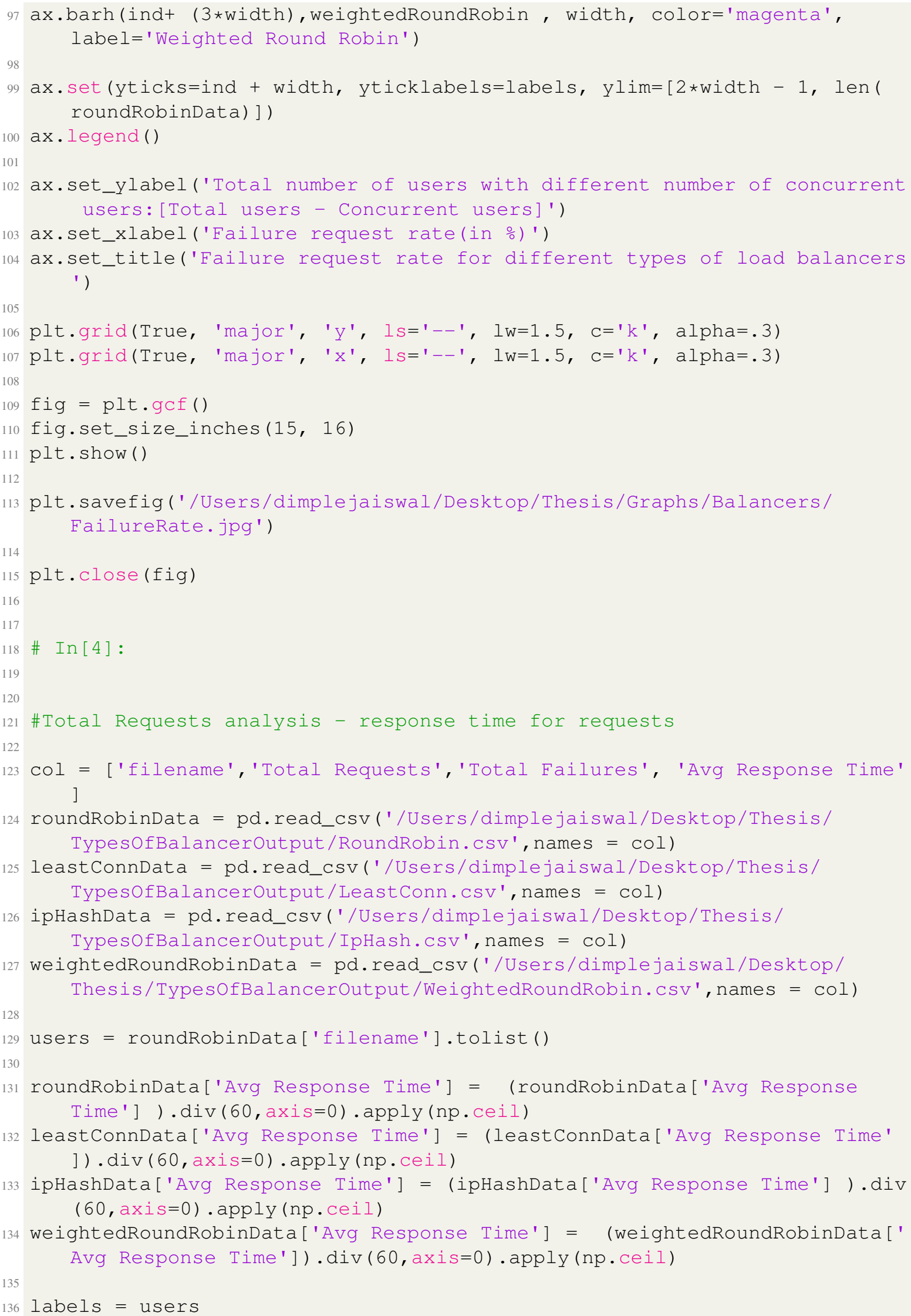




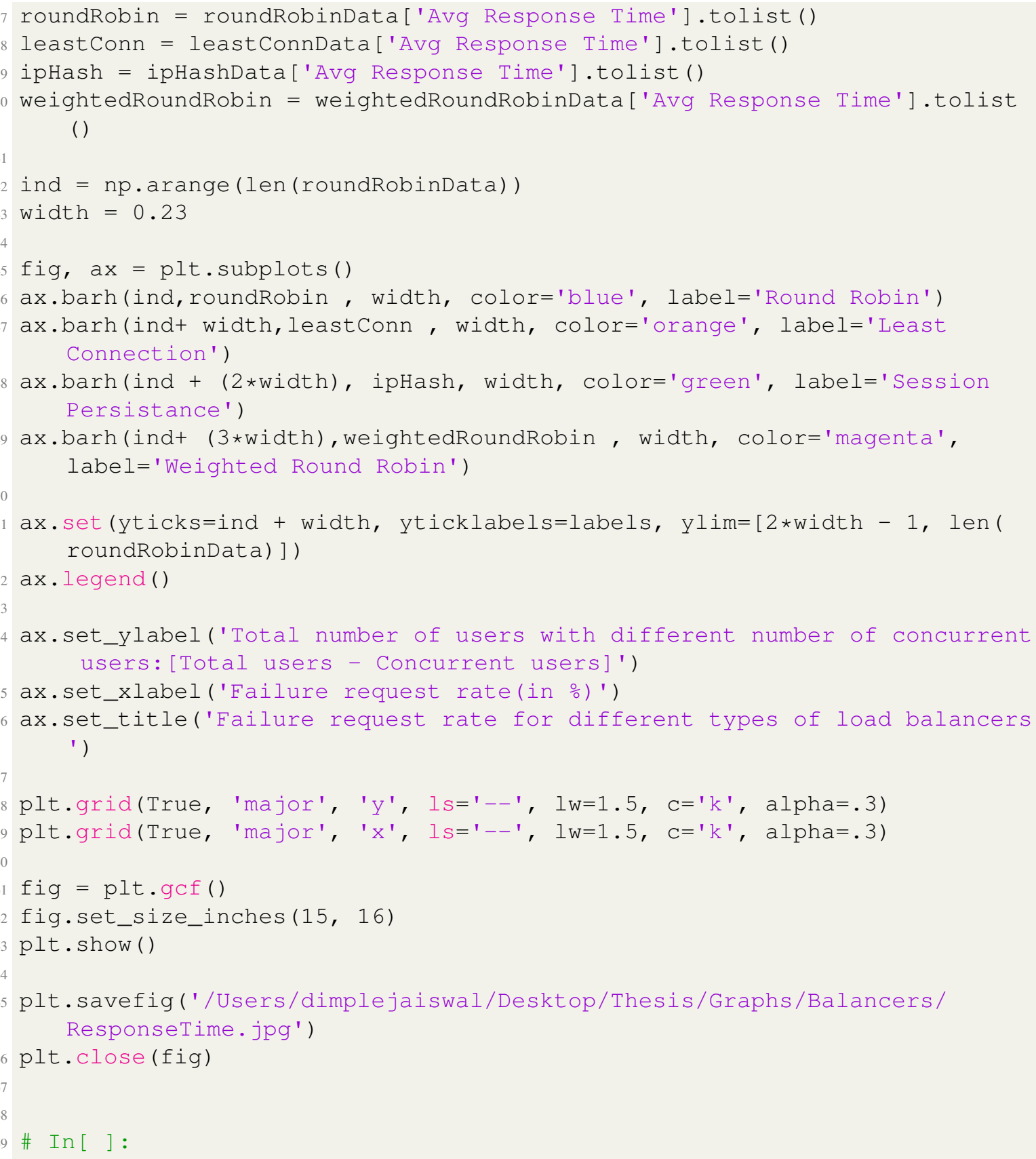

Code A.6.3.6: Analysis of data generated by locust using different types of load balancing technique

\section{Below are the figures generated using above script -}


Success request rate for different types of load balancers

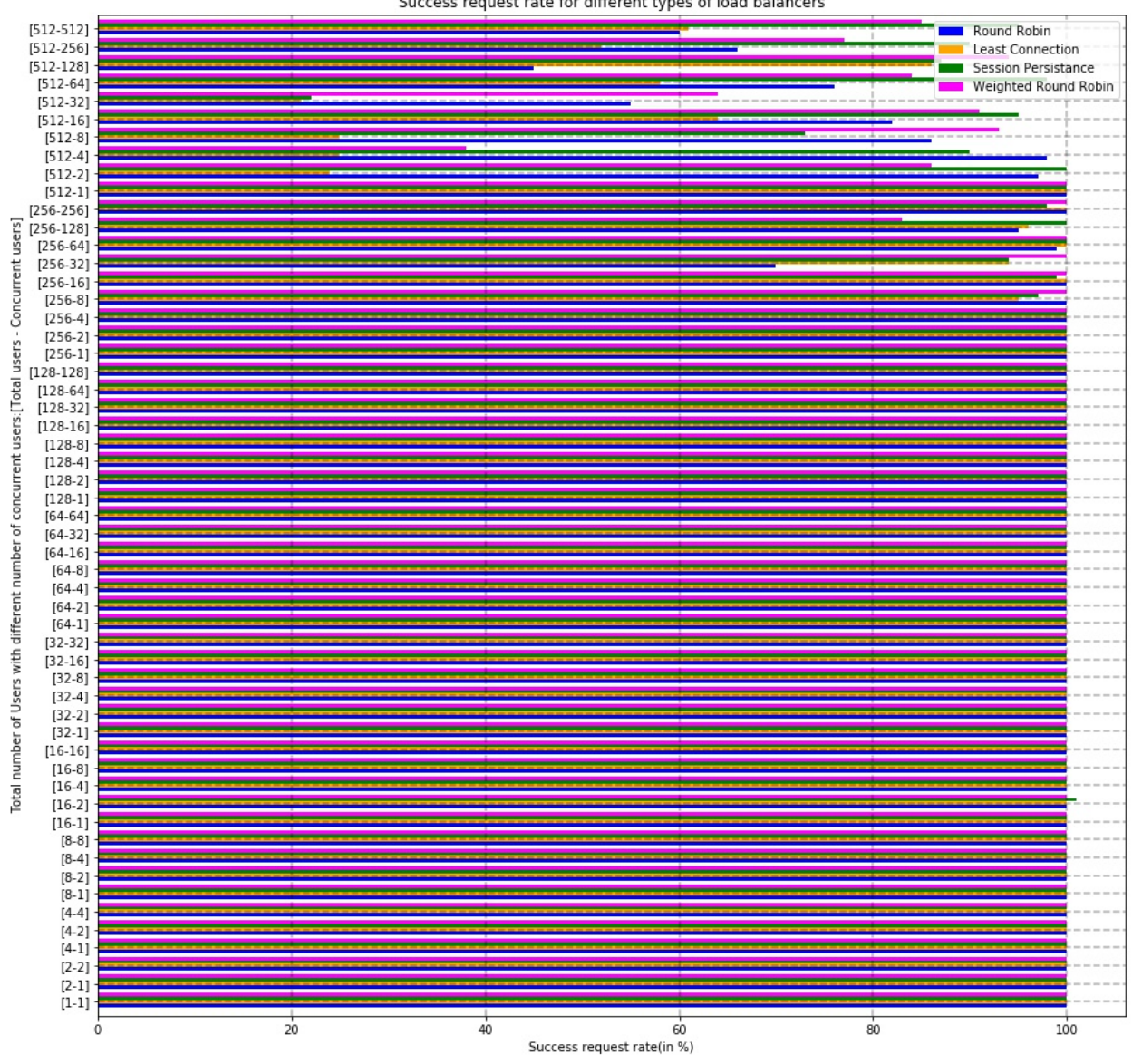

Figure A.17: Success request rate for different types of load balancers 


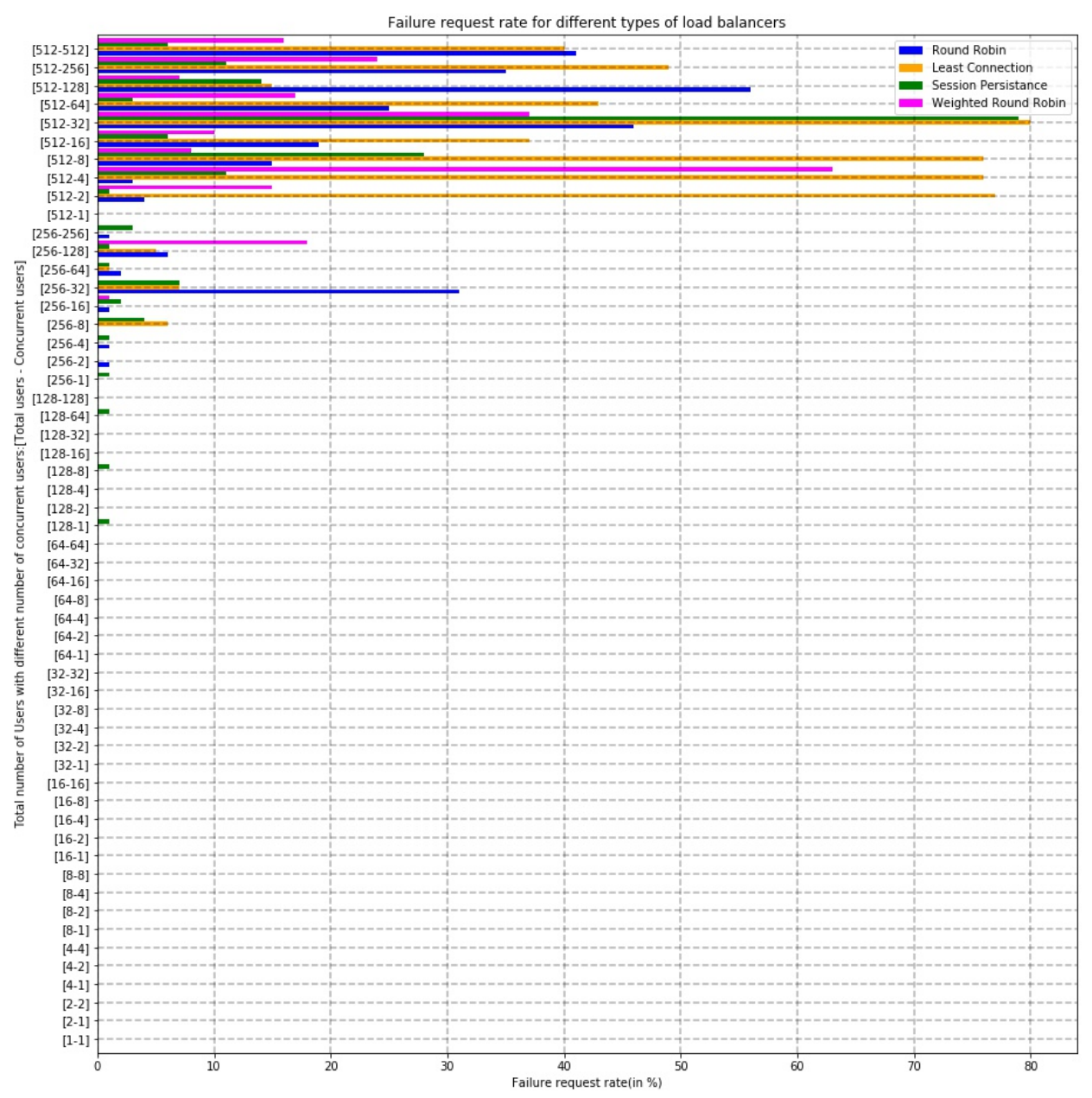

Figure A.18: Failure request rate for different types of load balancers 


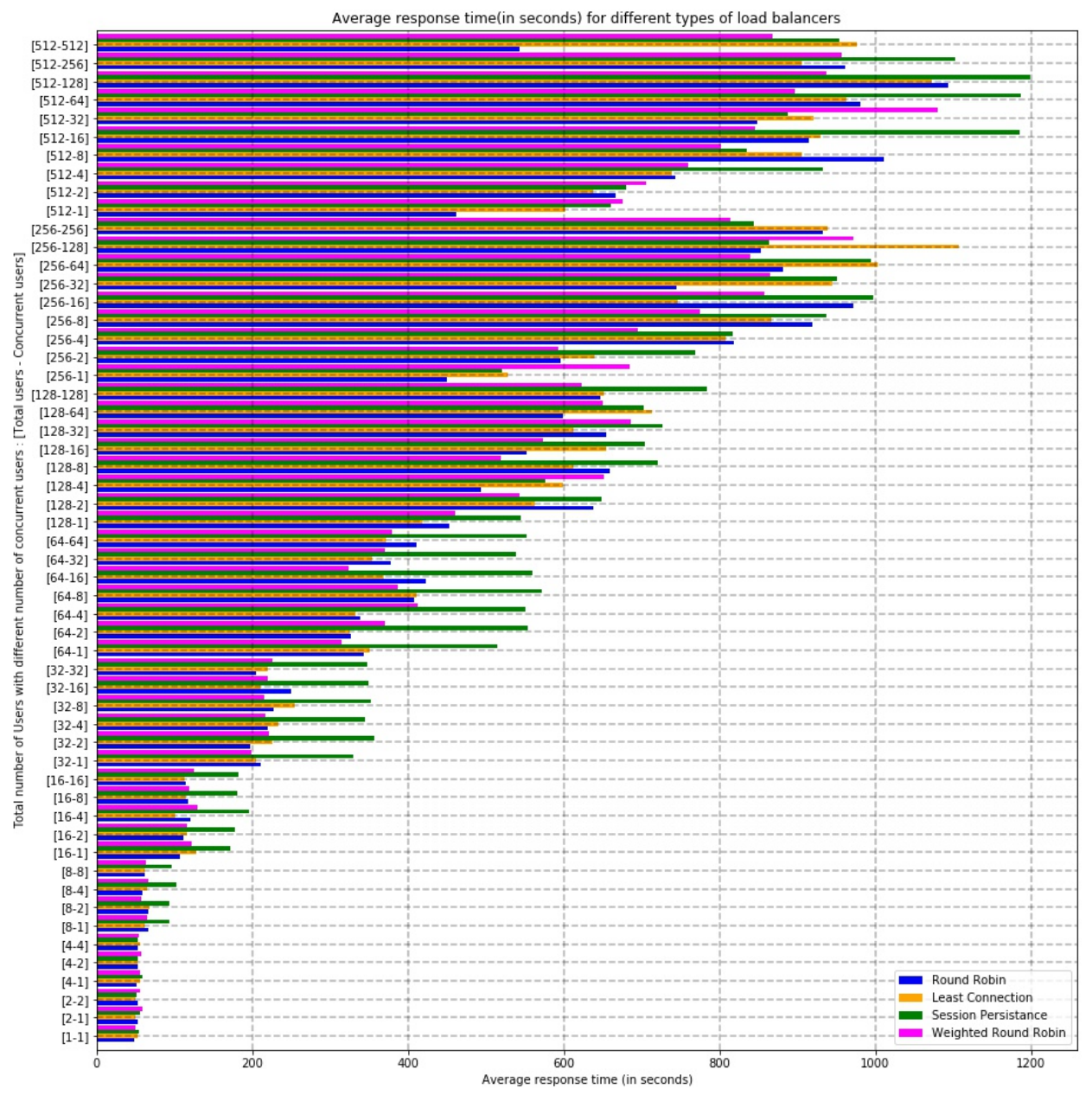

Figure A.19: Average response time(in seconds) for different types of load balancers

\section{A.6.4 Analysis of logs generated by nginx}

Nginx generates two types logs - access and error log. Given below script analyze logs generated while using different types of load balancing techniques.

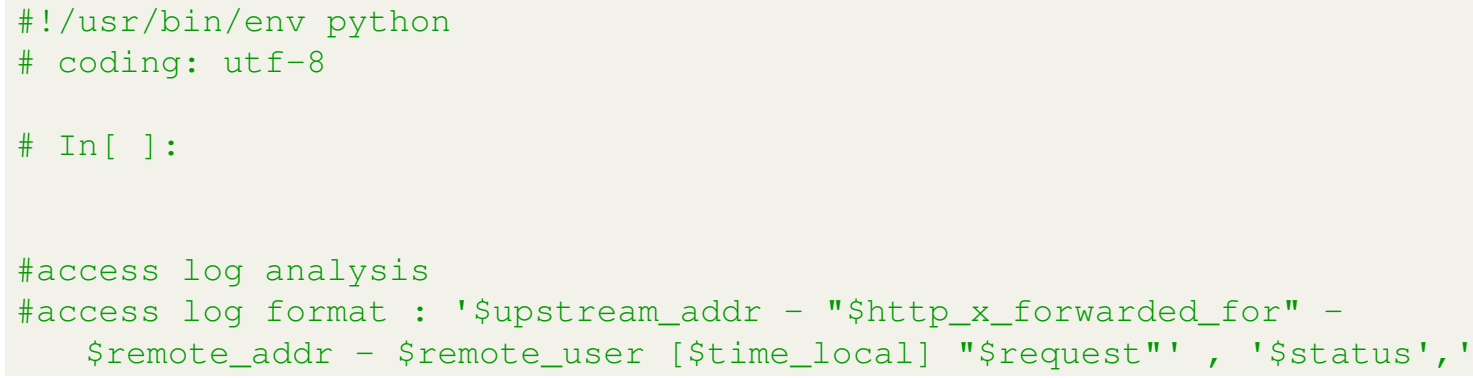




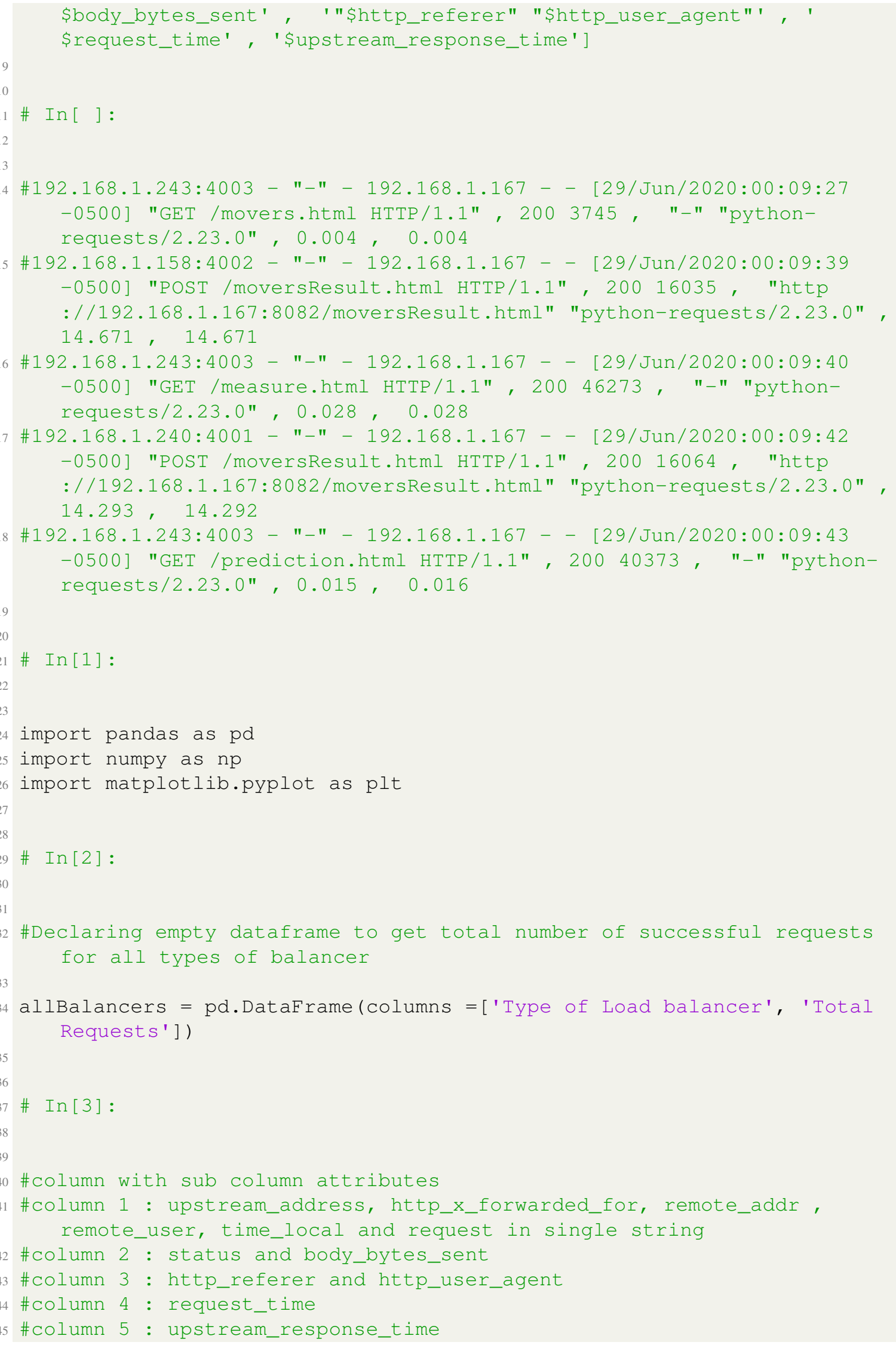




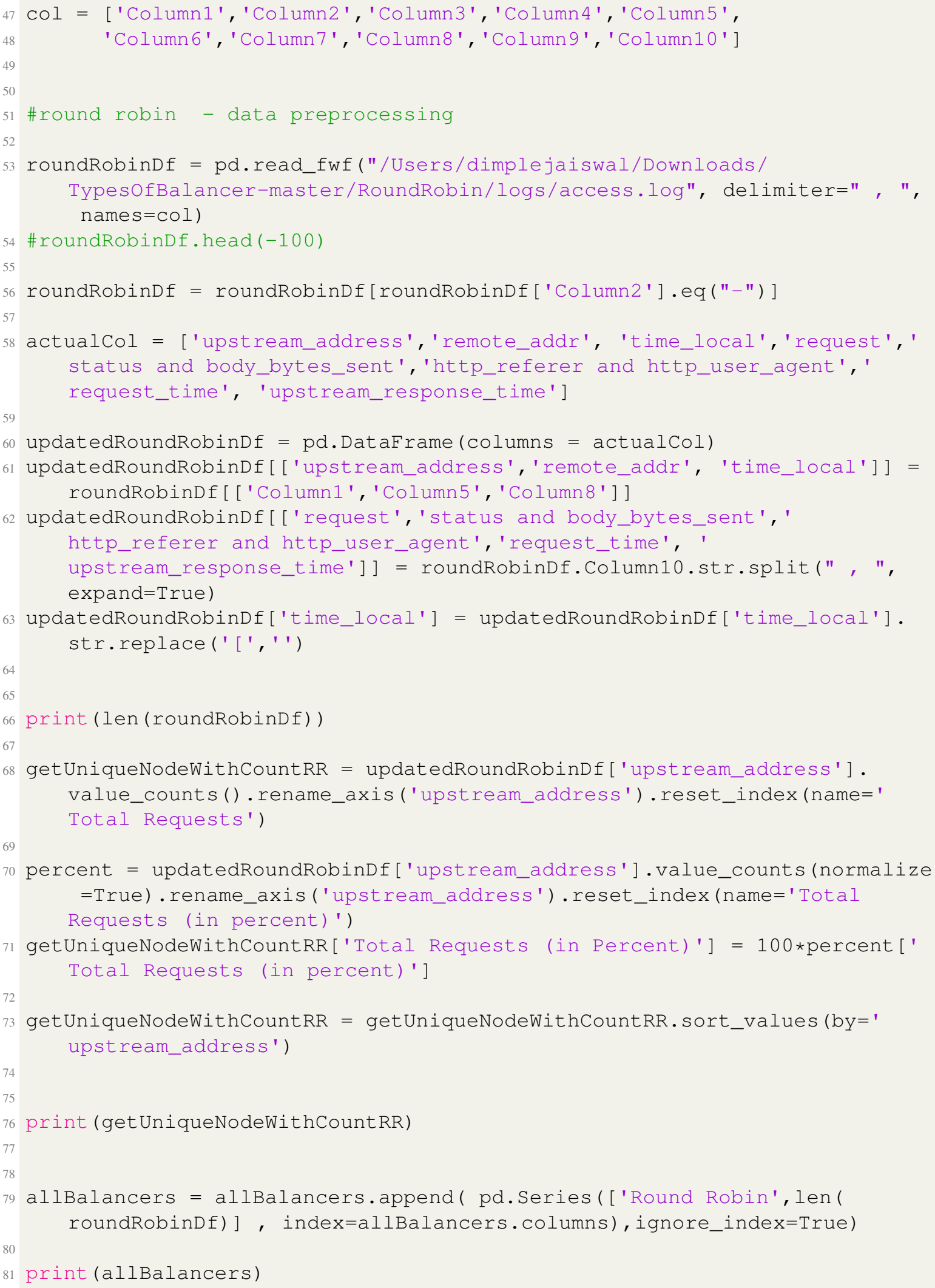




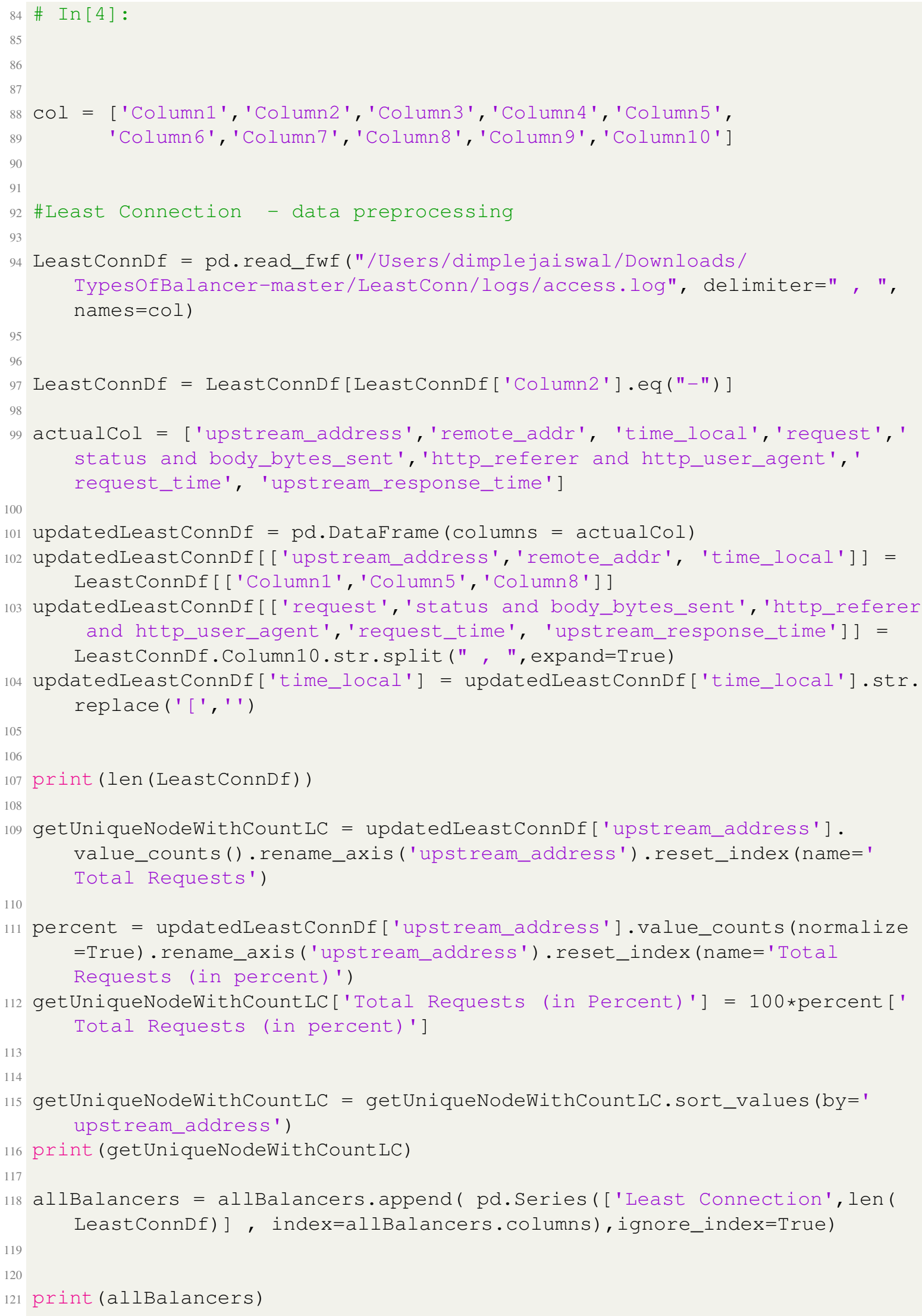




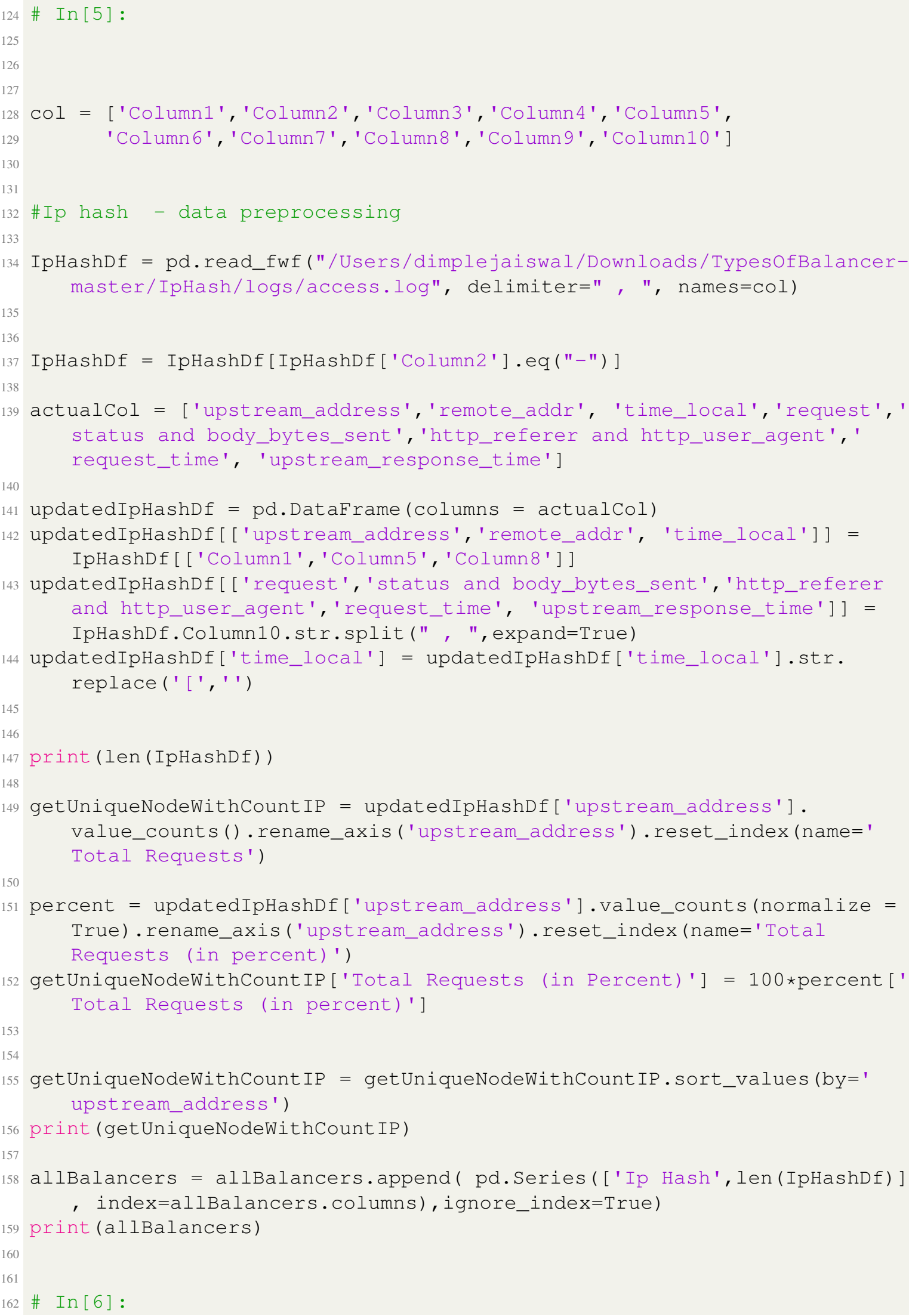




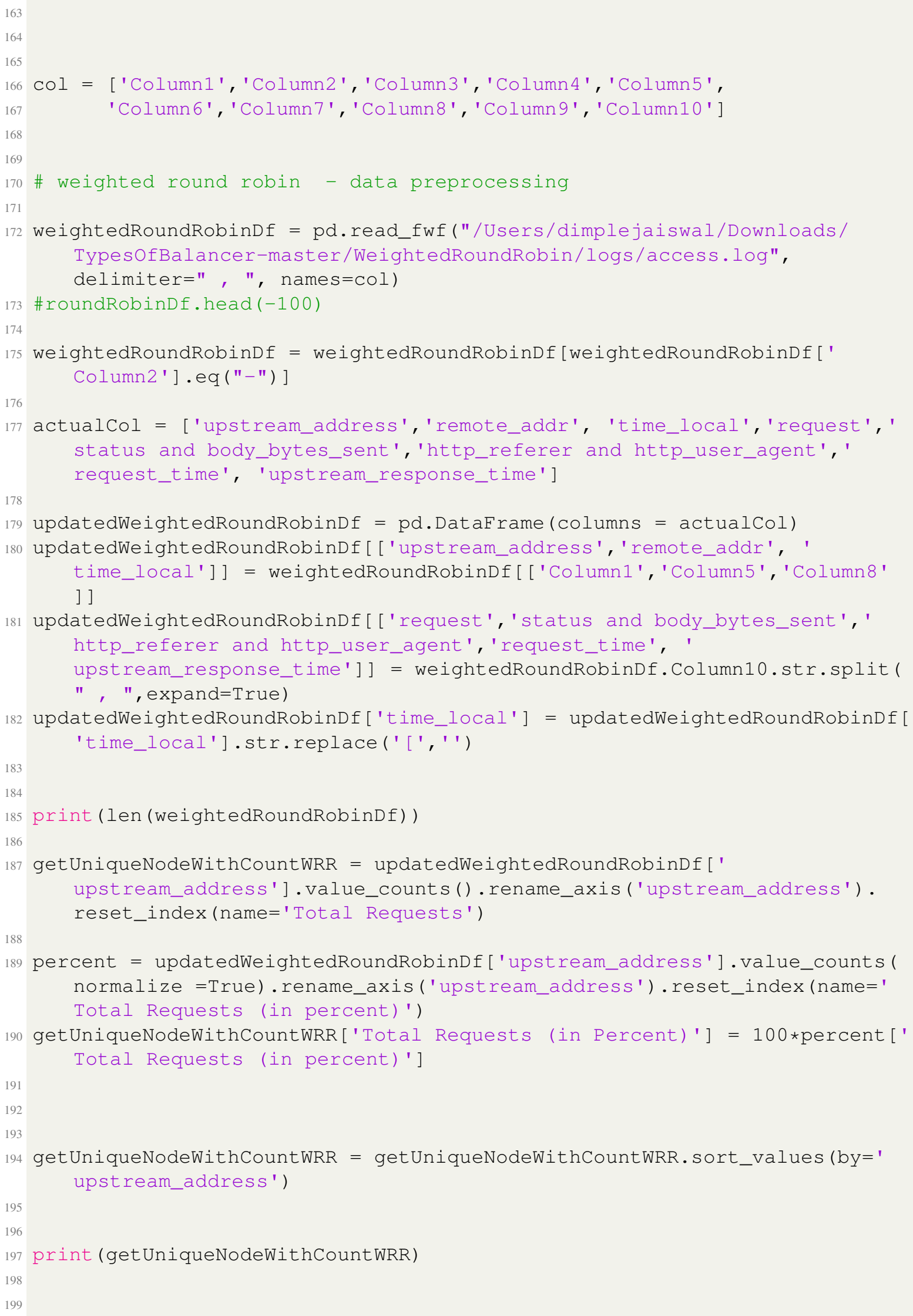


200 allBalancers = allBalancers.append( pd.Series(['Weighted Round Robin', len (weightedRoundRobinDf) ] , index=allBalancers.columns), ignore_index=True)

2 print (allBalancers)

203

\# In [8]:

206

207

208

$9 \mathrm{x}=$ allBalancers['Type of Load balancer']

$\mathrm{y}=$ allBalancers['Total Requests']

fig $=$ plt.figure()

ax = fig.add_subplot (111)

ax.set_ylim $(0,100000)$

plt.plot $\left(x, y, \operatorname{color}=\mathrm{g}^{\prime}\right)$

for $i, j$ in $\operatorname{zip}(x, y)$ :

ax. annotate $(\operatorname{str}(j), x y=(i, j)$, color='blue', fontweight='bold')

ax.set_ylabel ('Total number of successful requests')

ax.set_xlabel ('Type of load balancer')

ax.set_title('Total number of successful requests made by different types of load balancers')

fig.set_size_inches $(15,8)$

plt.show()

plt.savefig('/Users/dimplejaiswal/Desktop/Thesis/Graphs/LogAnalysis/ SuccessfulRequests.jpg')

plt.close (fig)

\# In [9]:

229

230

231 labels = ['Round Robin',' 'Least Connection','Ip Hash','Weighted Round Robin']

3 nodes $=[$ 'Node 2', 'Node 1', 'Node 3']

properArrangement $=$ pd.DataFrame $($ columns $=$ labels $)$

properArrangement['Round Robin'] = getUniqueNodeWithCountRR['Total Requests']

properArrangement ['Least Connection'] = getUniqueNodeWithCountLC['Total Requests']

237 properArrangement['Ip Hash'] = getUniqueNodeWithCountIP['Total Requests' ]

8 properArrangement ['Weighted Round Robin'] = getUniqueNodeWithCountWRR[' Total Requests']

properArrangement ['Type'] = nodes

properArrangement $=$ properArrangement.set_index (['Type'])

properArrangement = properArrangement.swapaxes ("index","columns")

242

243

$244 \mathrm{x}=\mathrm{np}$.arange(len(labels)) \# the label locations 


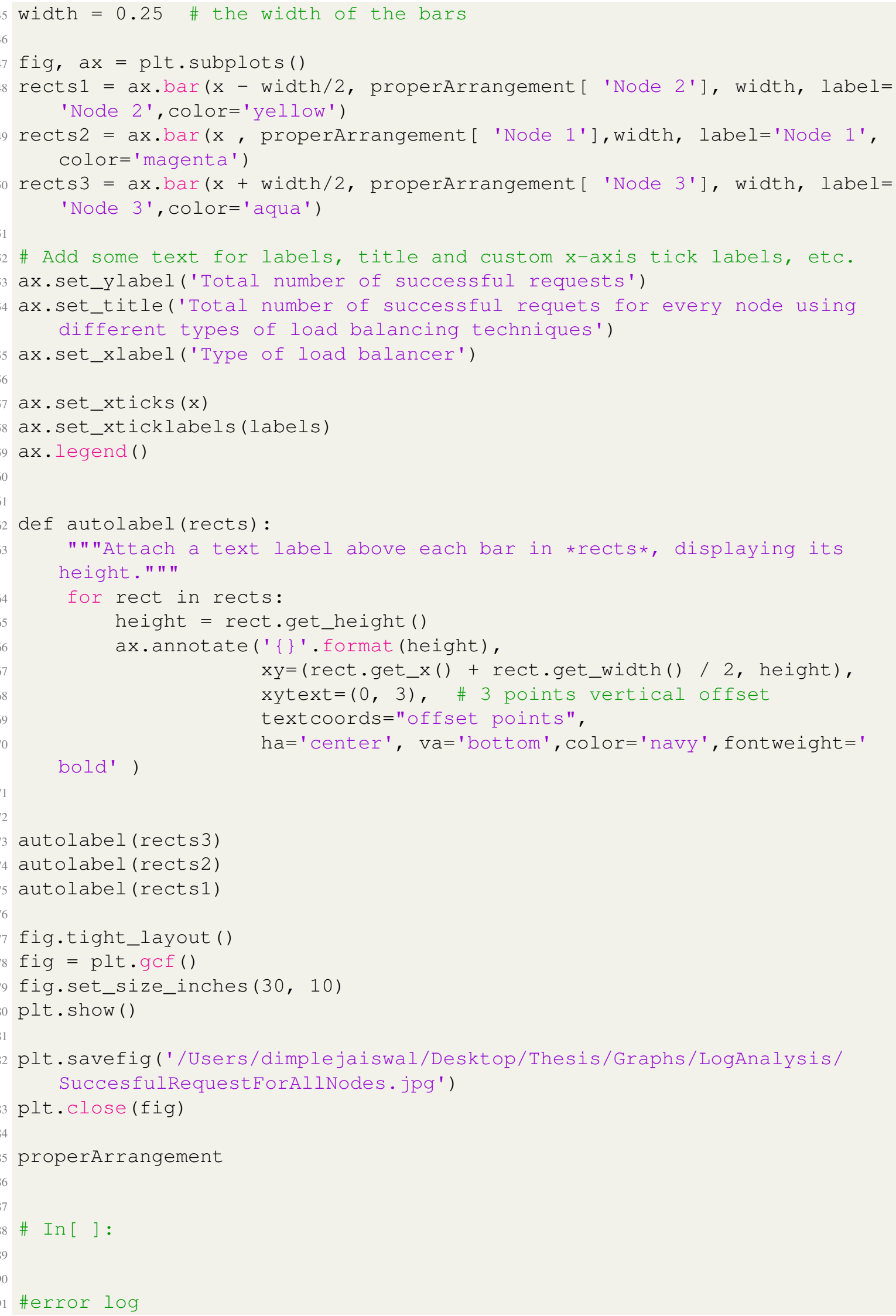


292

buffered to a temporary file/var/cache/nginx/uwsgi_temp /5/07/0000008075 while reading upstream, client: 192.168.1.167, server: 192.168.1.167, request: "POST /compareResult.html?stock1= American\&stock2=Adobe\&type $=$ Candle\&date_from=2015-02-02\&date_to =2020-02-20 HTTP/1.1", upstream: "uwsgi://192.168.1.158:4002", host: "192.168.1.167:8082", referrer: "http://192.168.1.167:8082/ compareResult.html?stock1=American\&stock2=Adobe\&type=Candle\& date_from $=2015-02-02 \&$ date_to $=2020-02-20$ "

\#2020/06/29 00:12:24 [warn] 14336\#14336: *363652 an upstream response is buffered to a temporary file/var/cache/nginx/uwsgi_temp /6/07/0000008076 while reading upstream, client: 192.168.1.167, server: 192.168.1.167, request: "POST/measureResults.html HTTP /1.1", upstream: "uwsgi://192.168.1.240:4001", host: "192.168.1.167:8082", referrer: "http://192.168.1.167:8082/ measureResults.html"

295

296

297 \# In [10]:

298

299

300 \# error dataframe for all types of balancers

301 errorBalancers $=$ pd.DataFrame(columns=['Type of load balancer', 'Total request failed'])

302

\# In [11]:

305

306

308 \#round robin error log

10 errorRR $=[]$

11 with open("/Users/dimplejaiswal/Downloads/TypesOfBalancer-master/ RoundRobin/logs/error.log",'r') as file:

312

13

14

316

18 countNode1 $=0$

19 countNode $2=0$

20 countNode $3=0$

countcluster $=0$

unreported $=[]$

for row in errorRR:

if 'upstream: "uwsgi://192.168.1.240:4001"' in row: count Node $1+=1$

elif 'upstream: "uwsgi://192.168.1.158:4002"' in row: count Node $2+=1$

elif 'upstream: "uwsgi://192.168.1.243:4003"' in row:

count Node $3+=1$ 


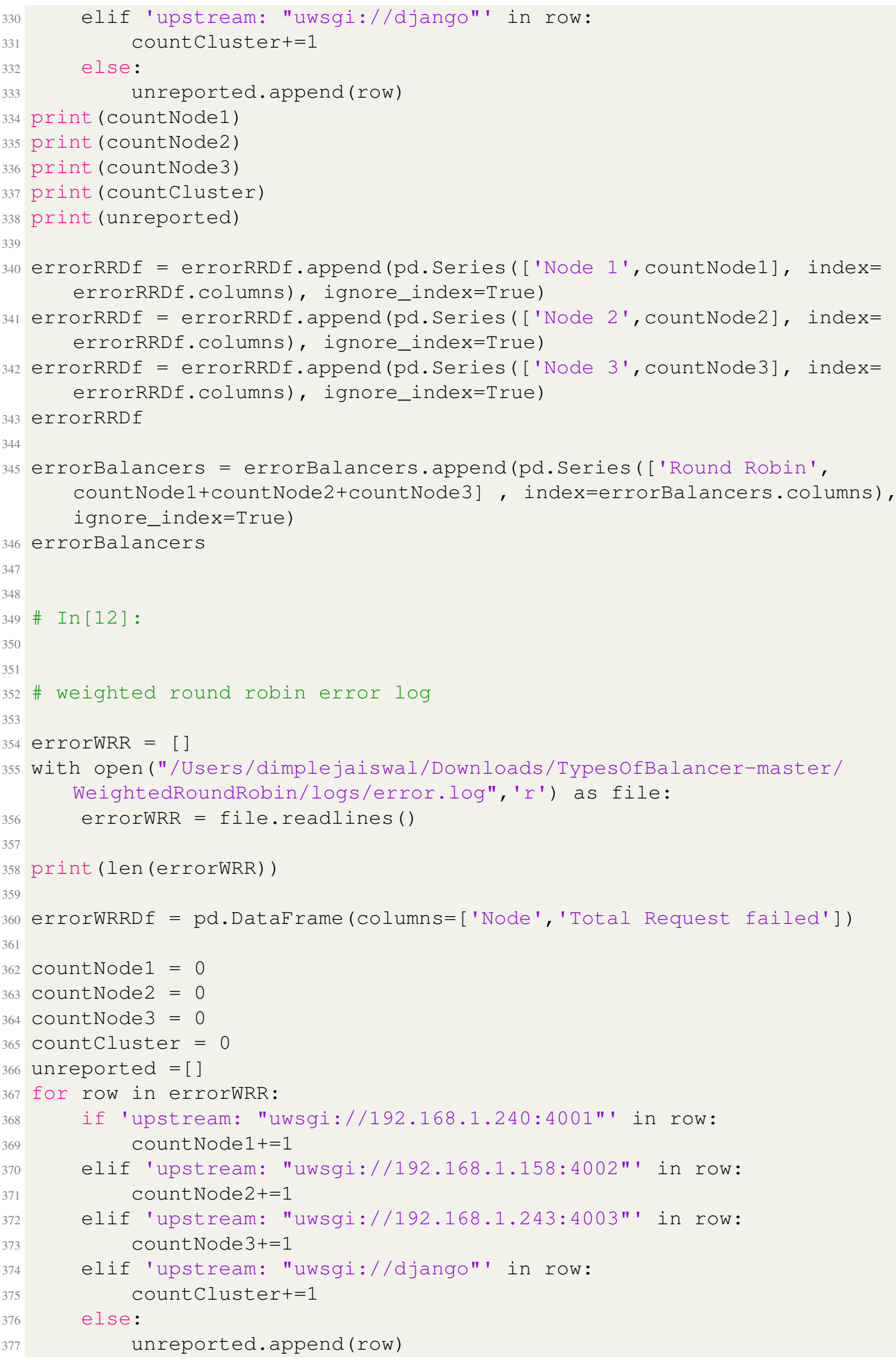




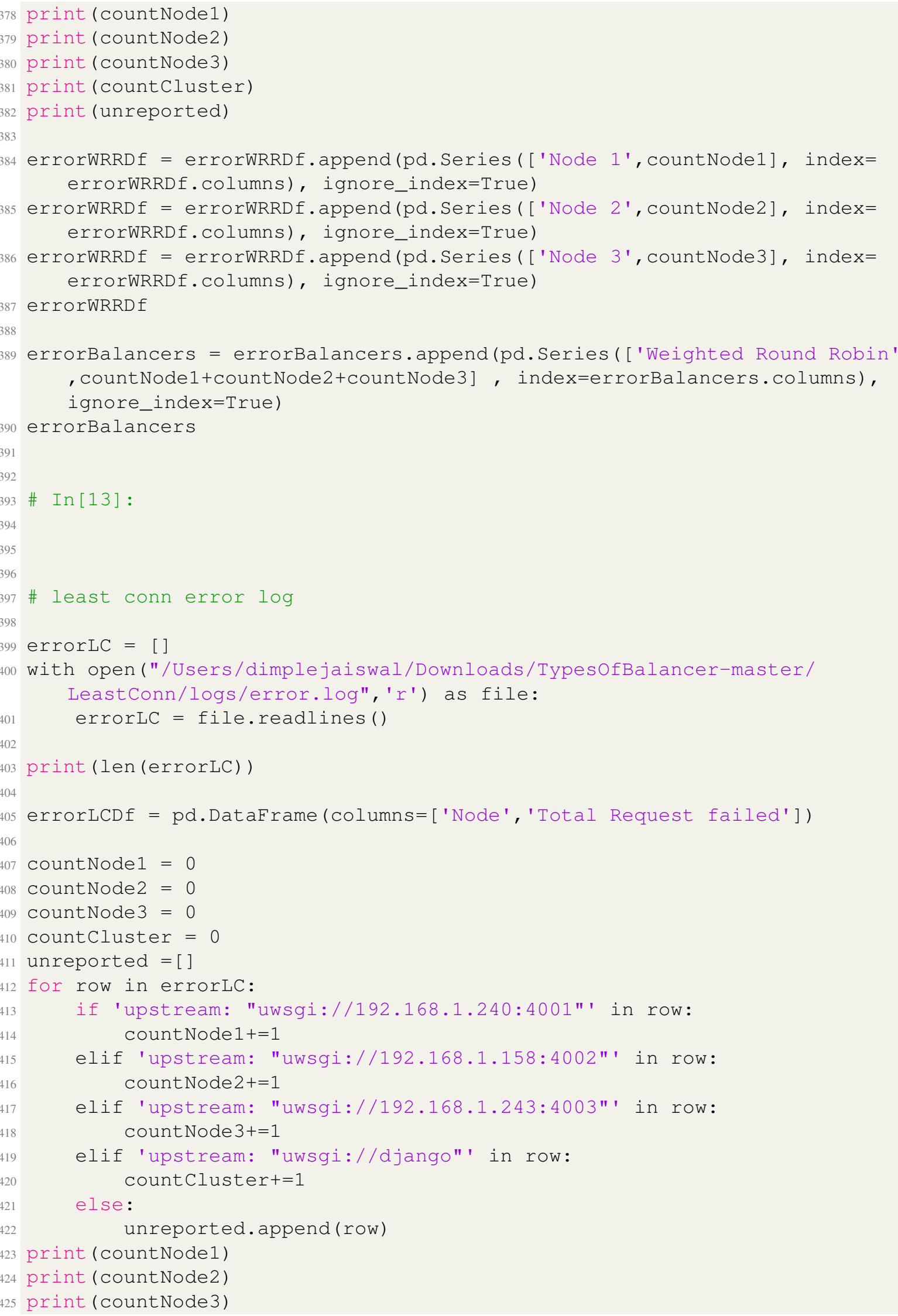




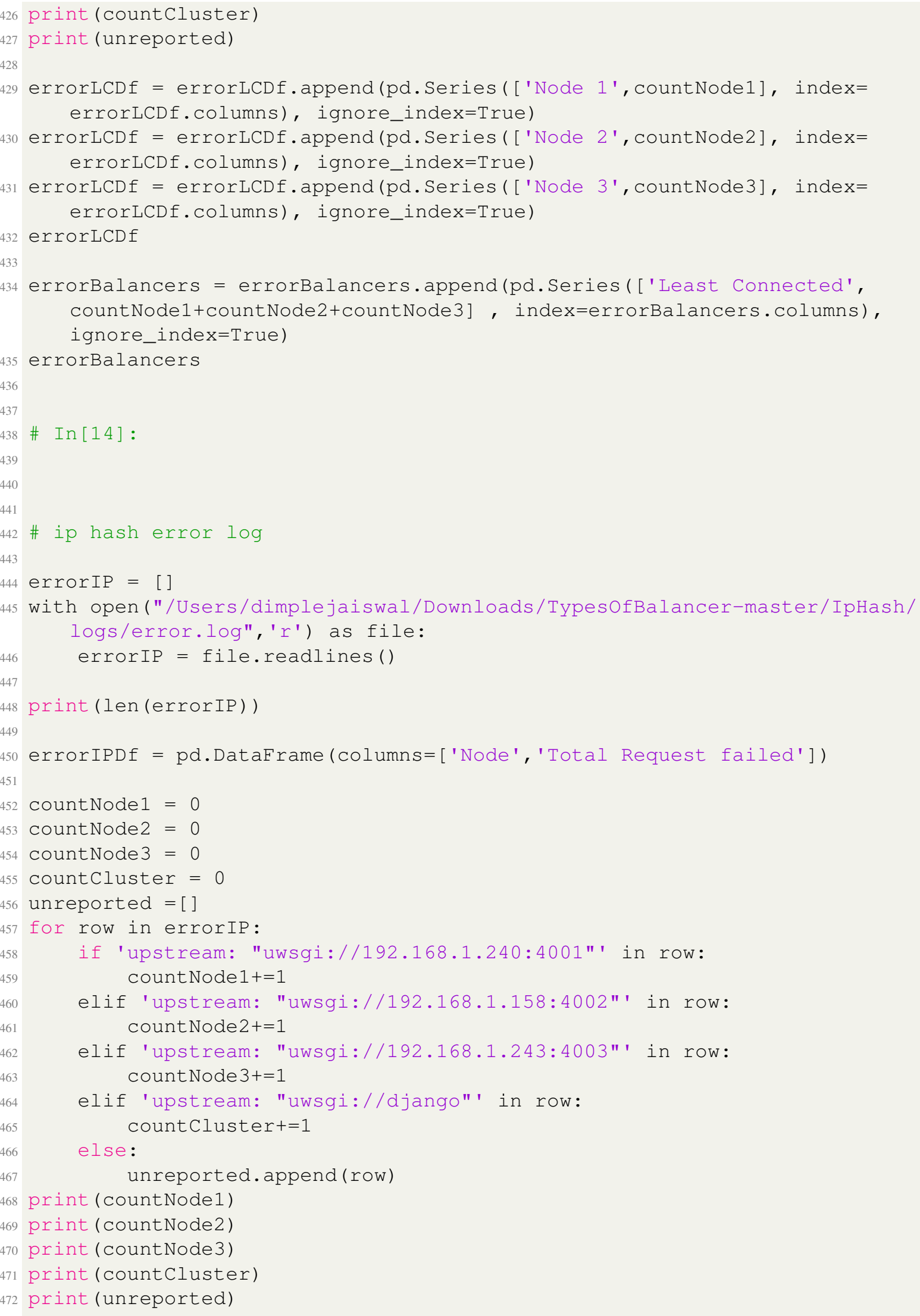




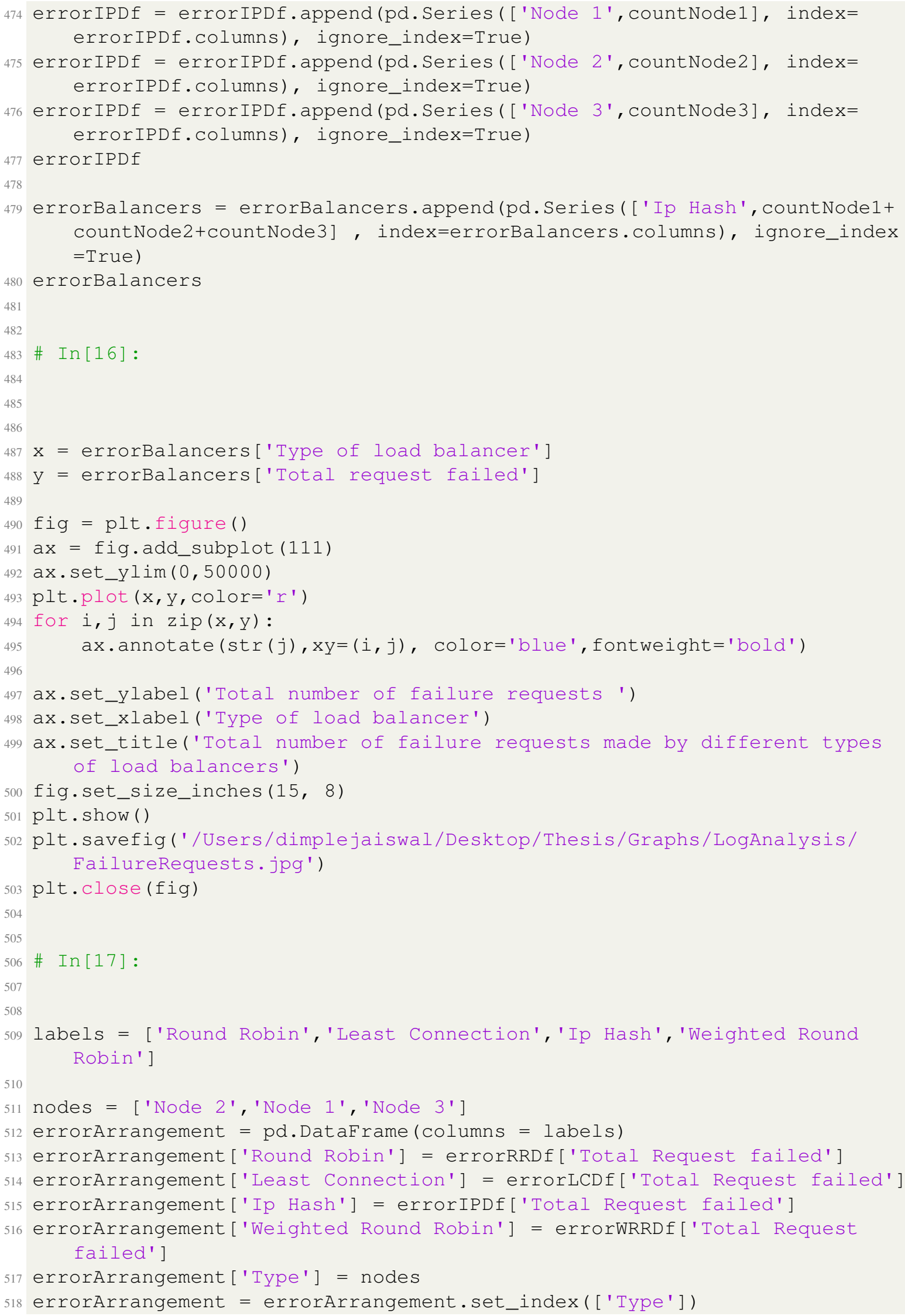




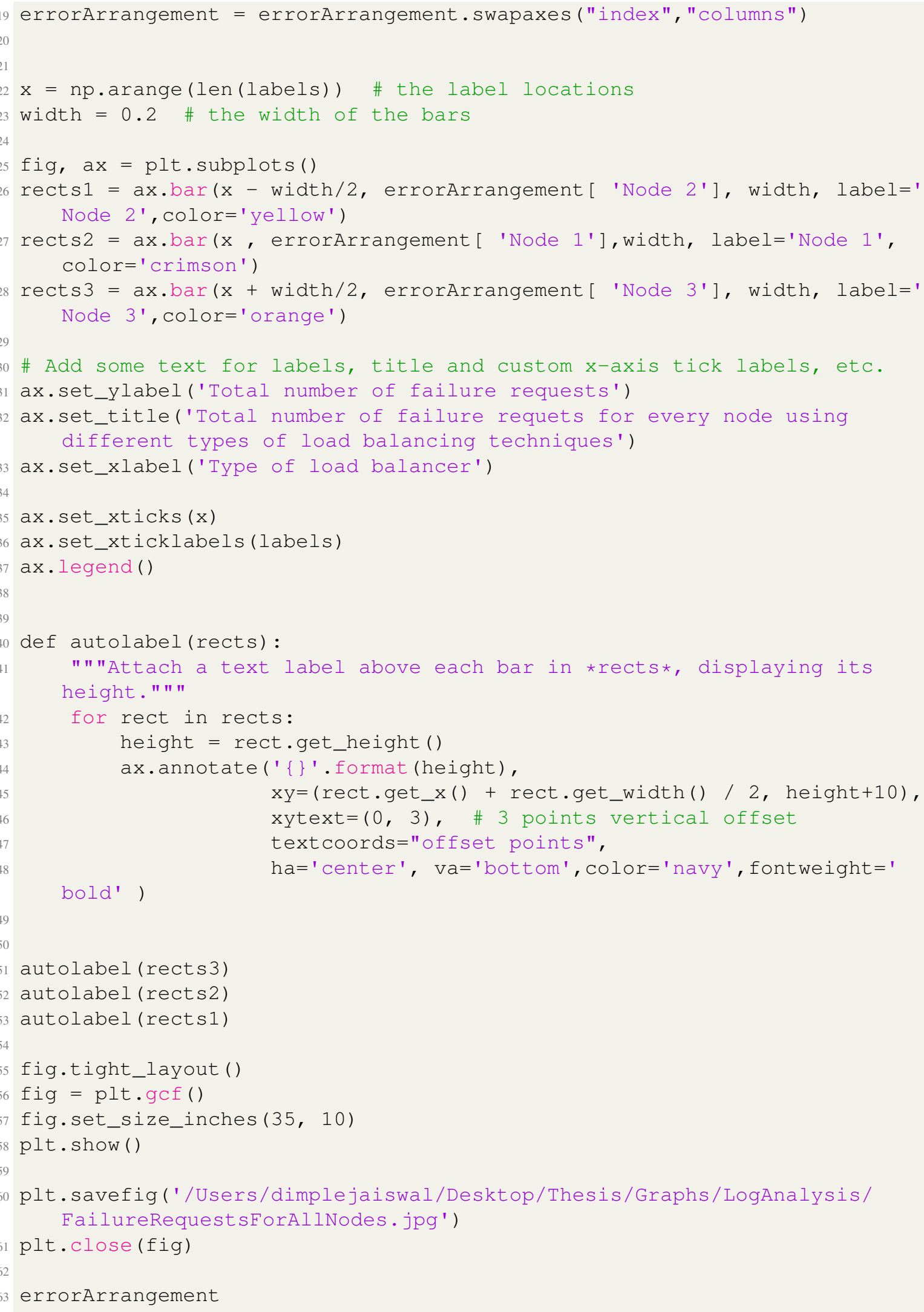


566 \# In [ ] :

Code A.6.4.1: Analysis of logs generated by nginx using different types of load balancing technique

Following are the figures generated by executing above script-

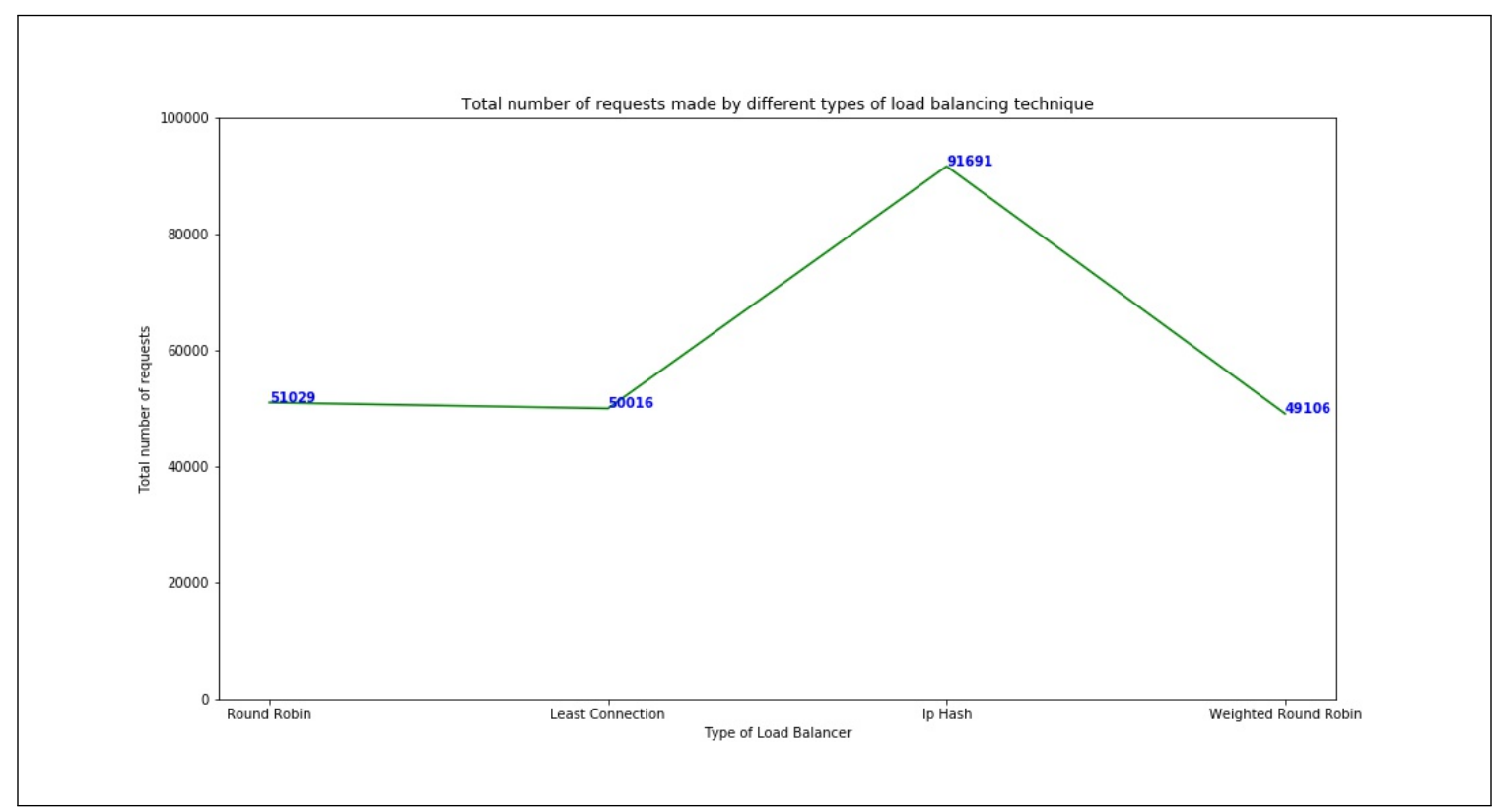

Figure A.20: Total number of requests made by different types of load balancing technique 


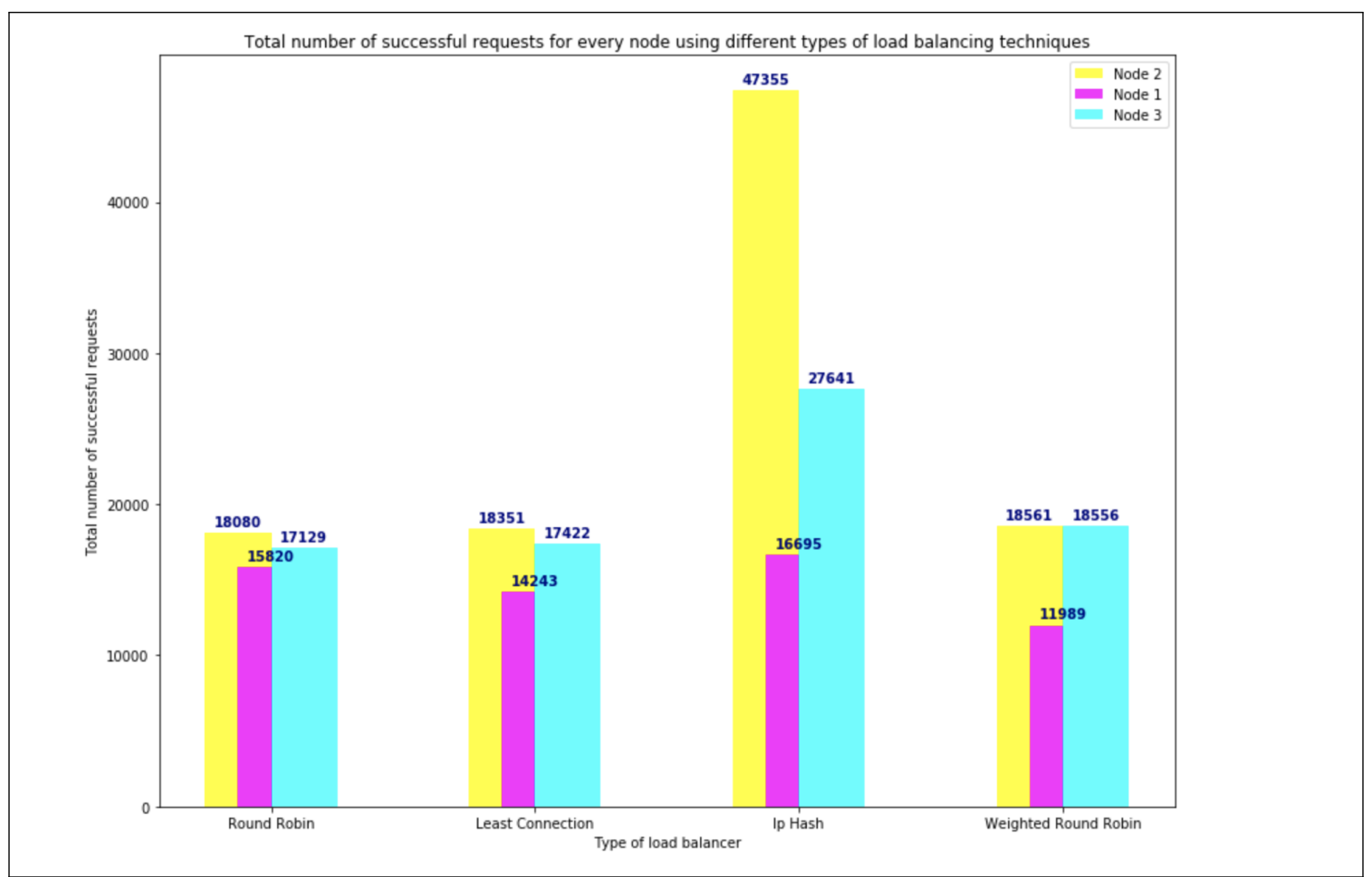

Figure A.21: Total number of successful requests for every node using different types of load balancing techniques

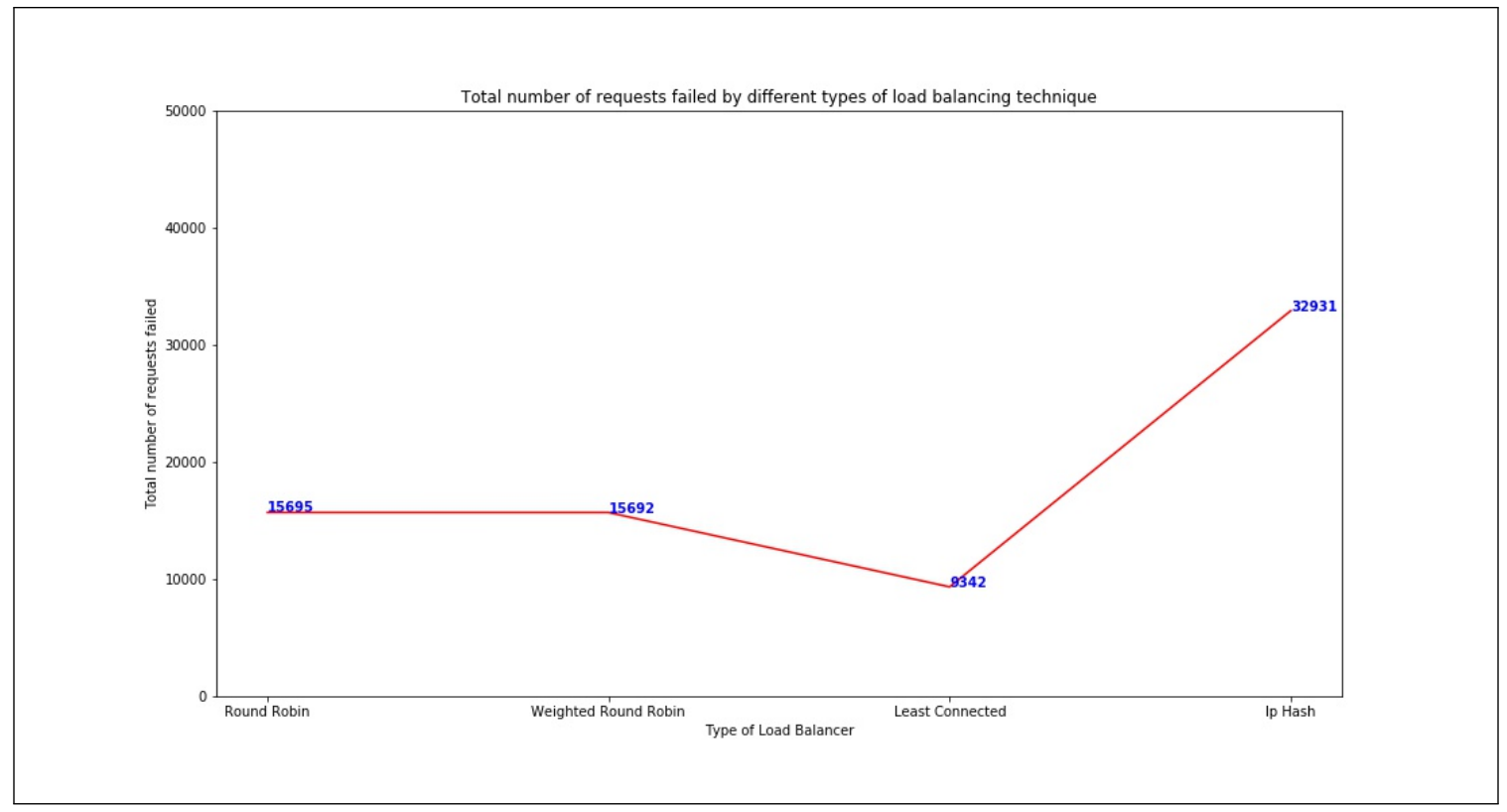

Figure A.22: Total number of requests failed by different types of load balancing technique 


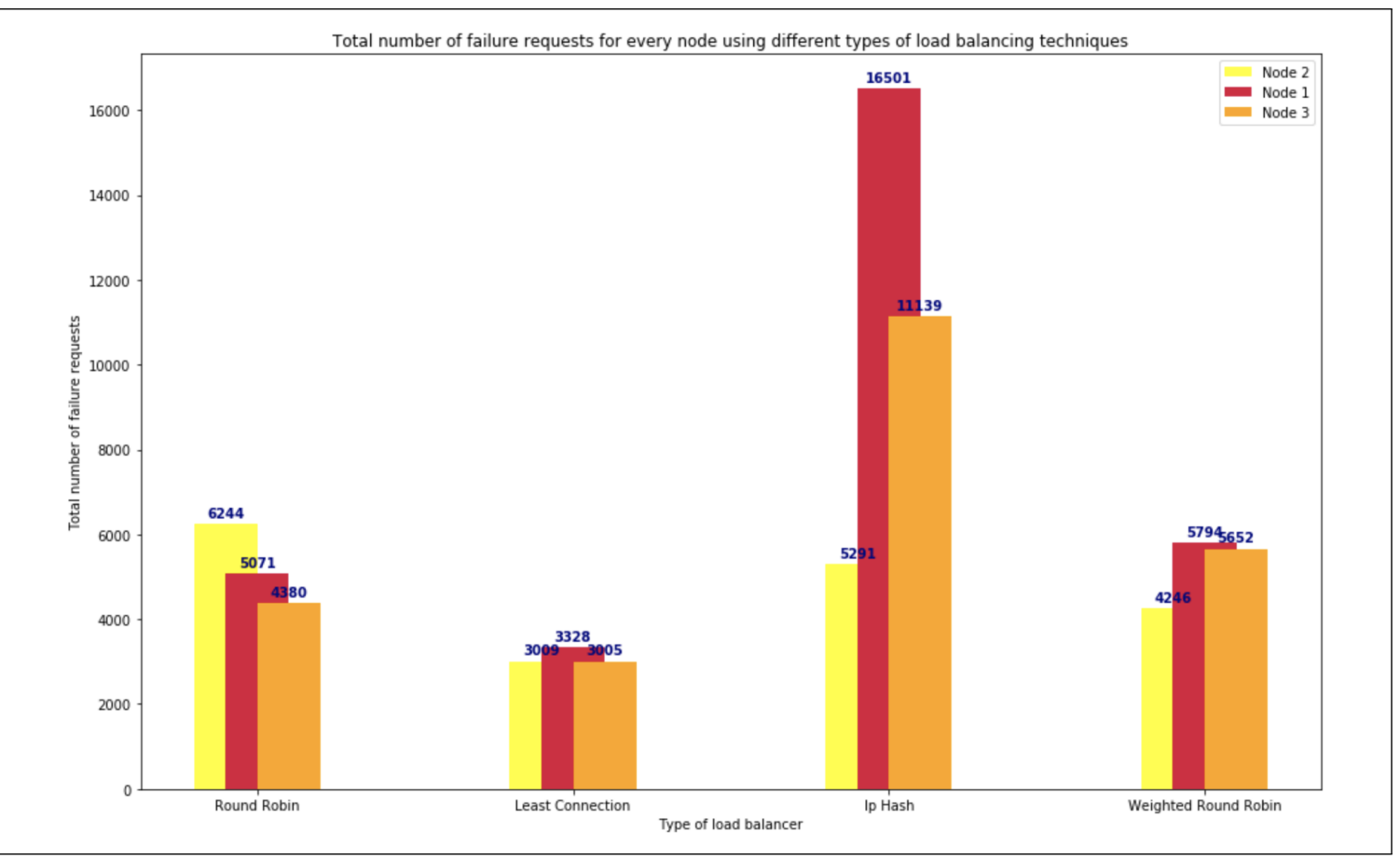

Figure A.23: Total number of failure requests for every node using different types of load balancing techniques

A.6.5 Data analysis of data generated by Locust testing for web application hosted on AWS cloud

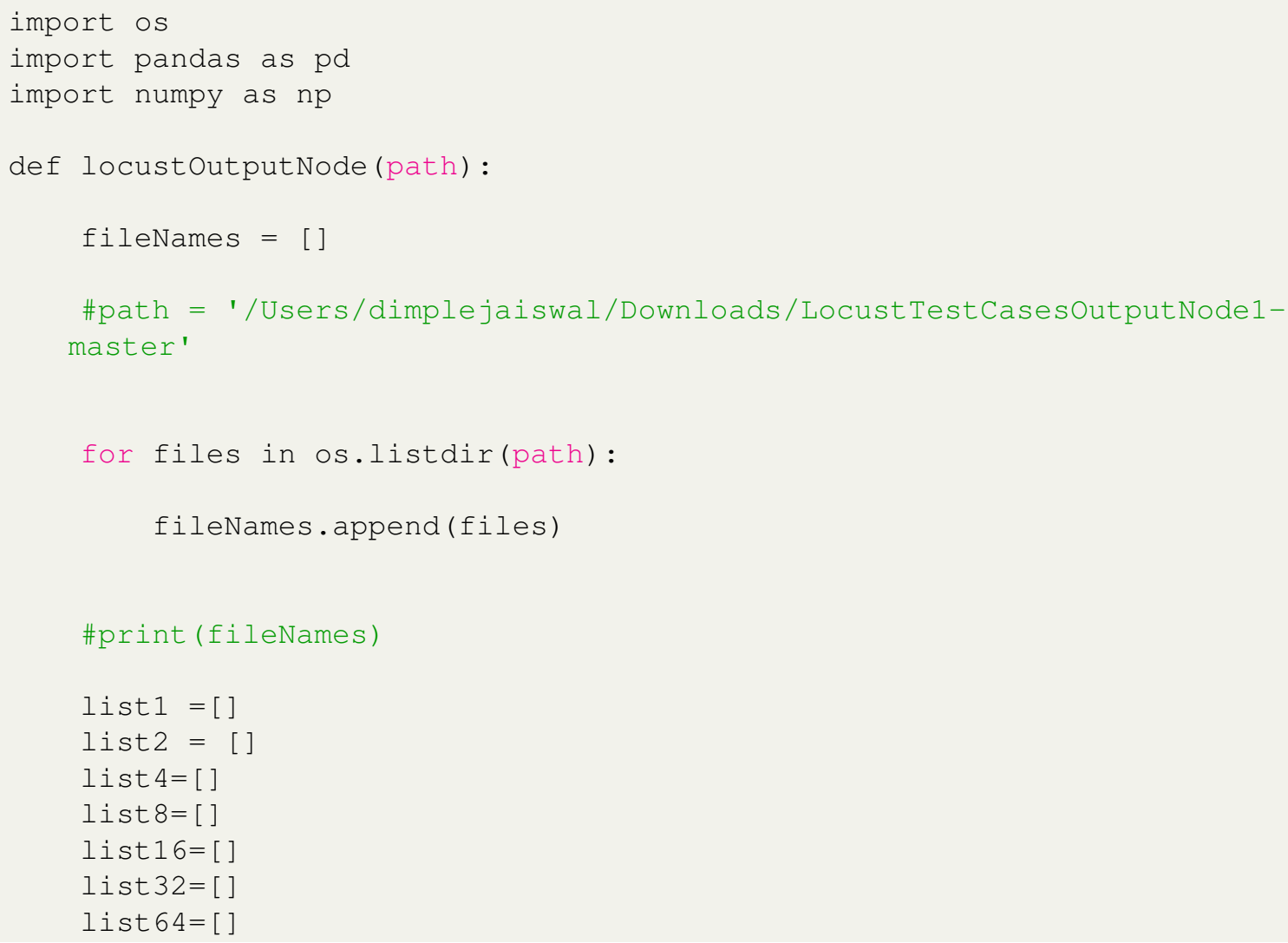




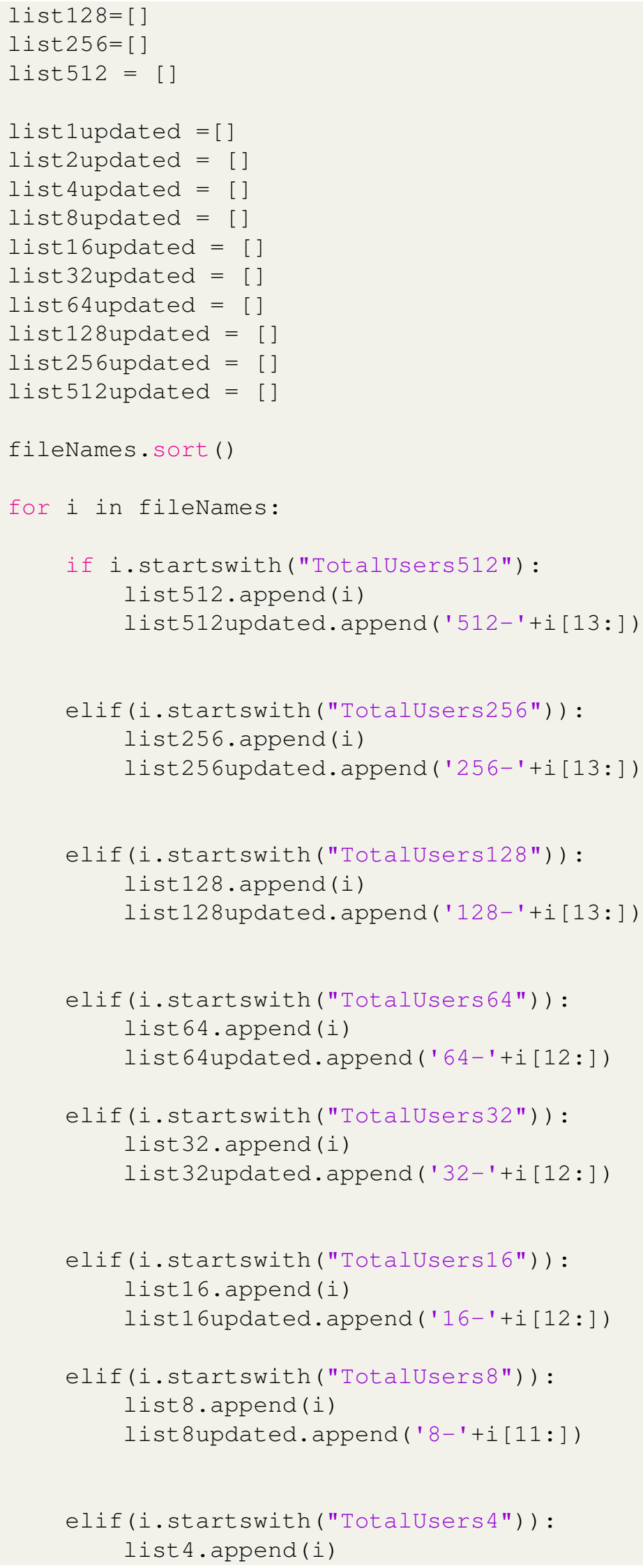




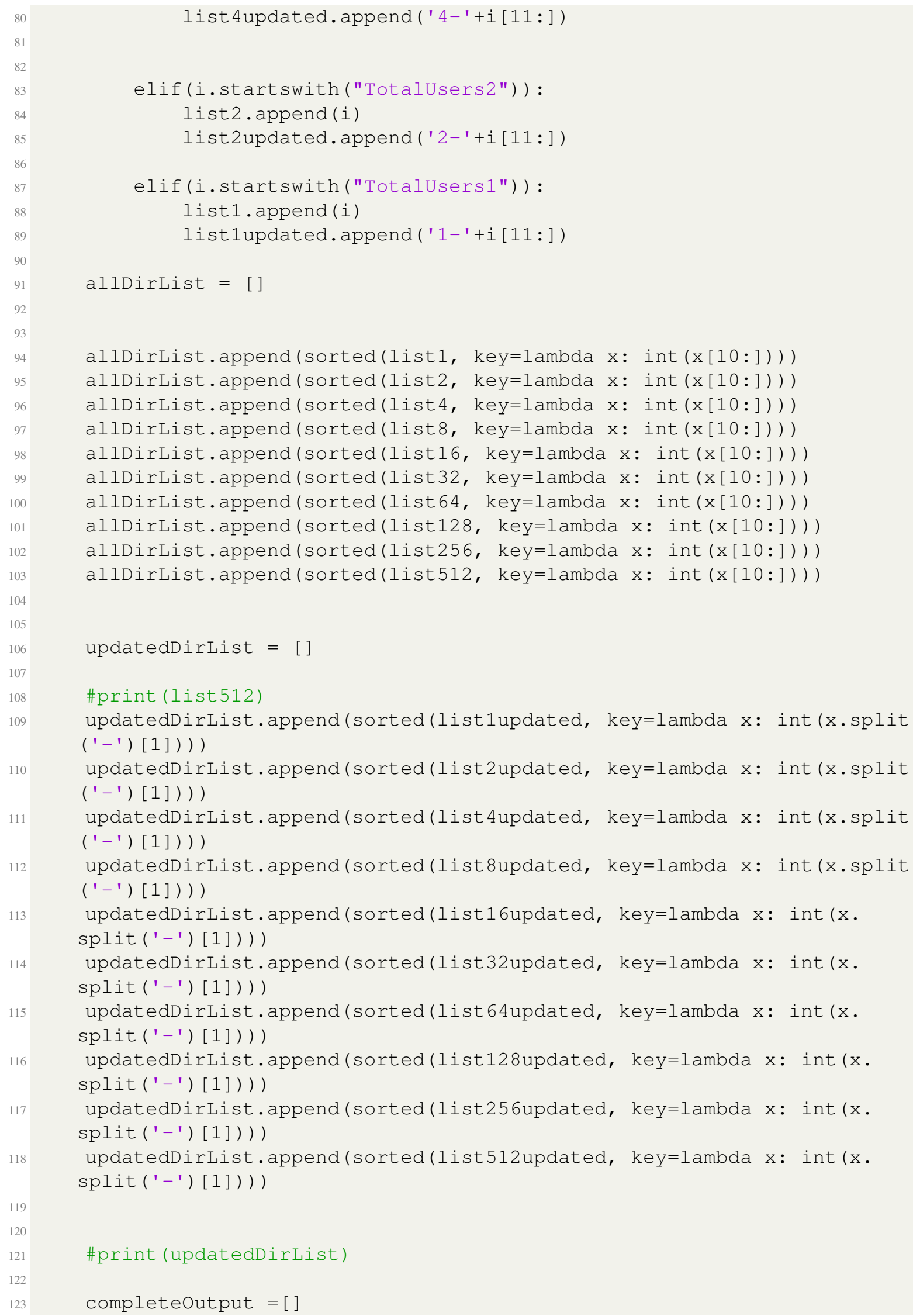




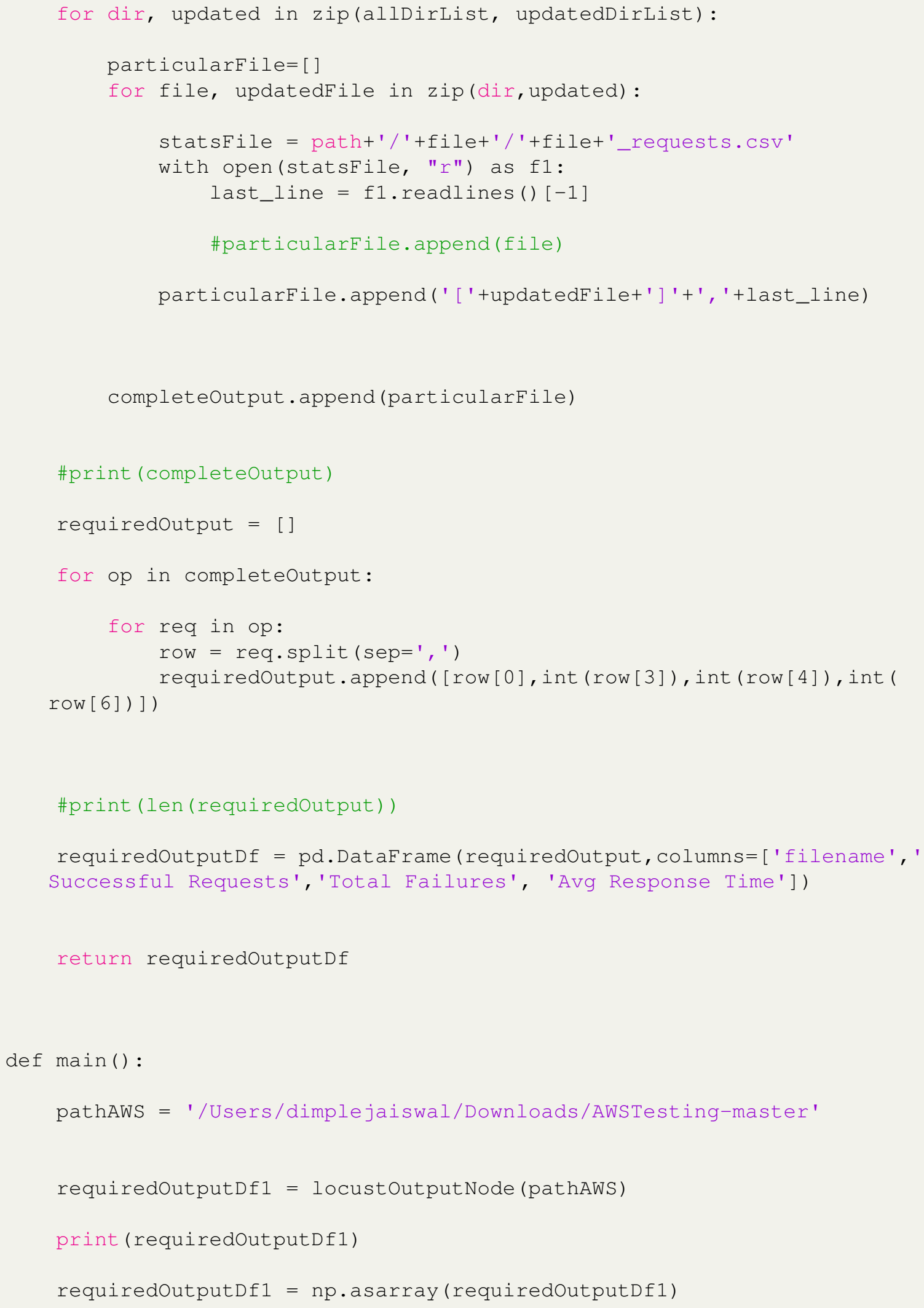




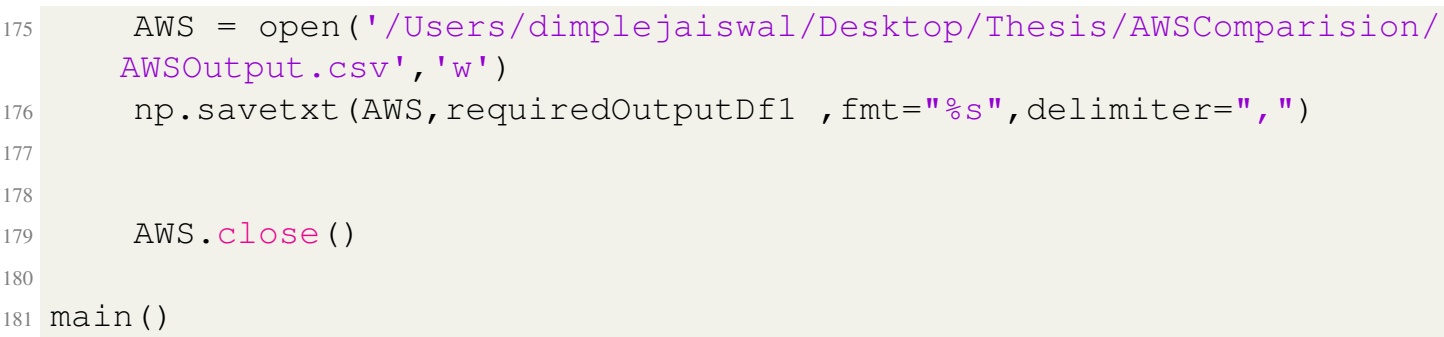

Code A.6.5.1: Data preprocessing of data generated by Locust testing for application on AWS cloud

Below is the output generated by above script-

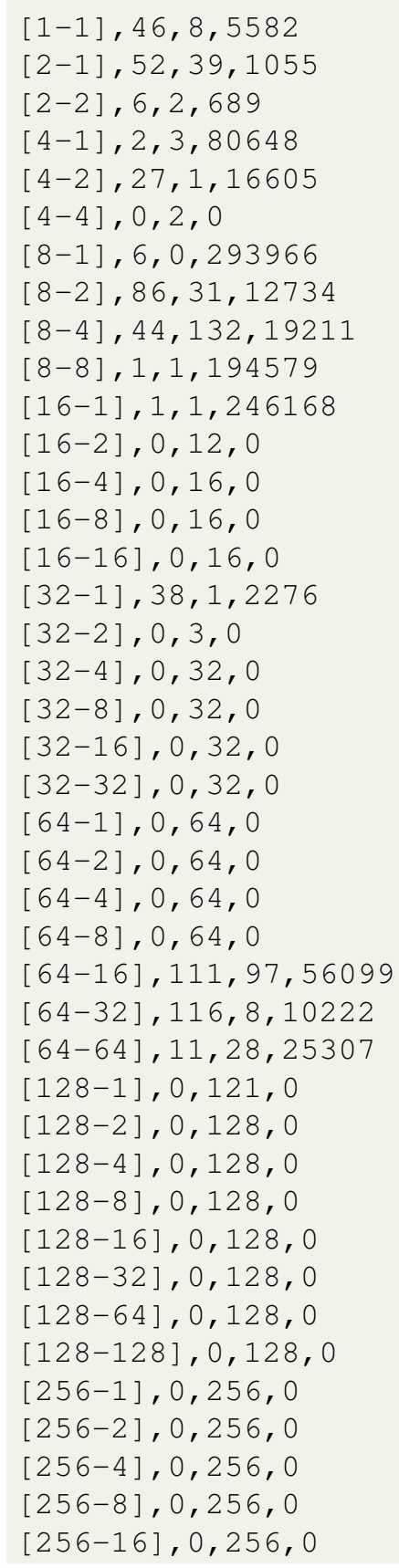




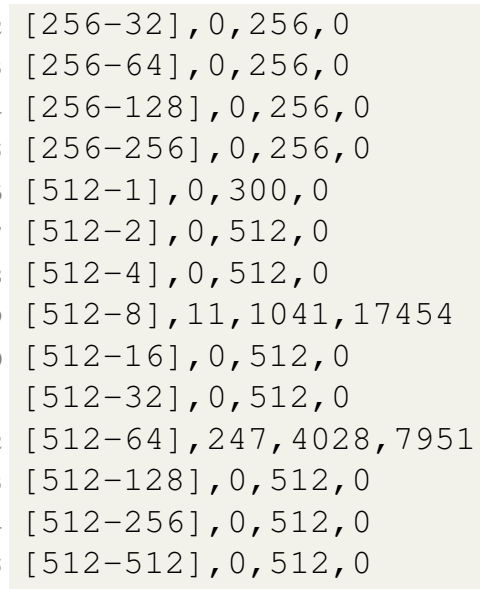

Code A.6.5.2: Output generated for node 1

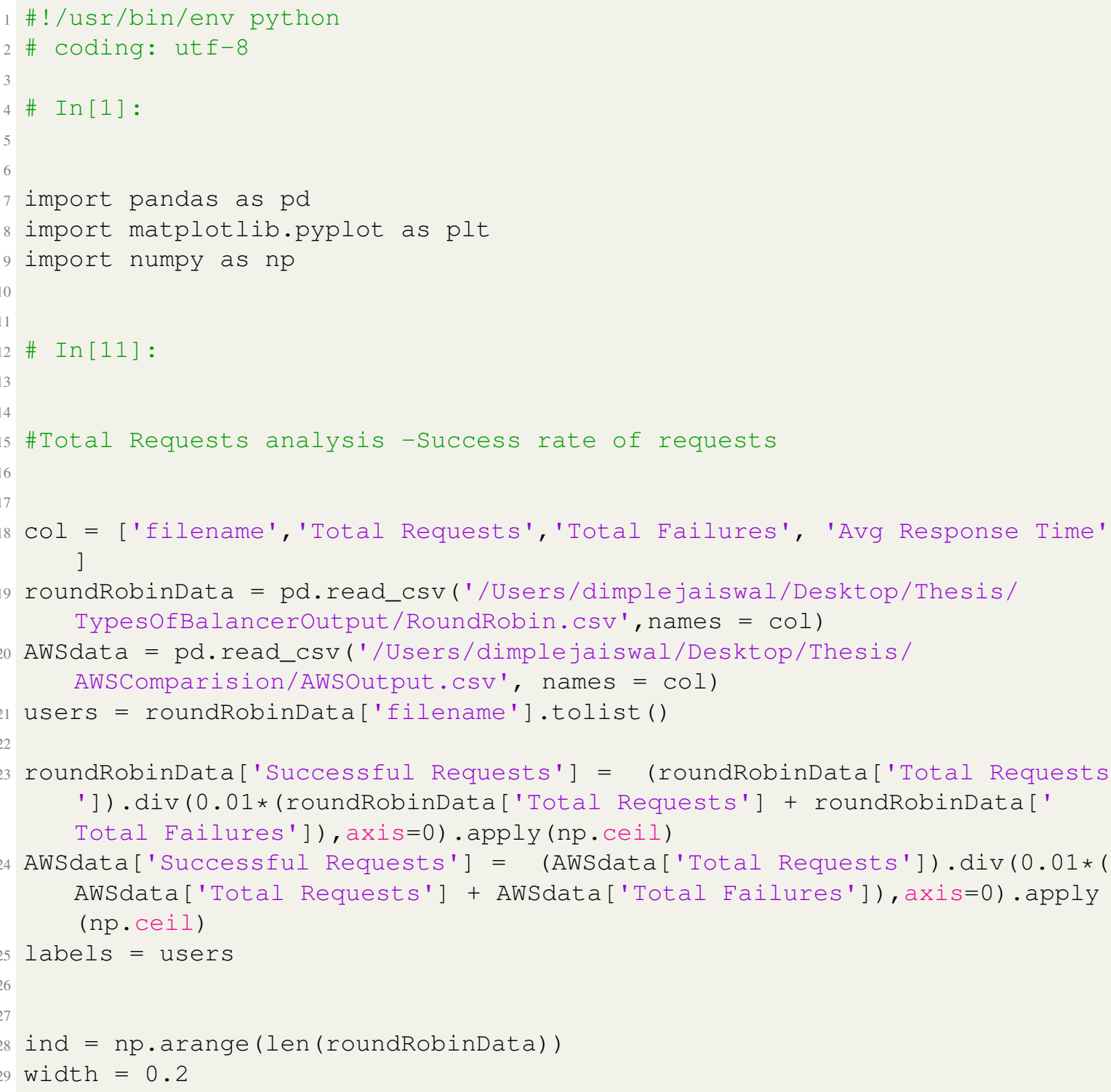




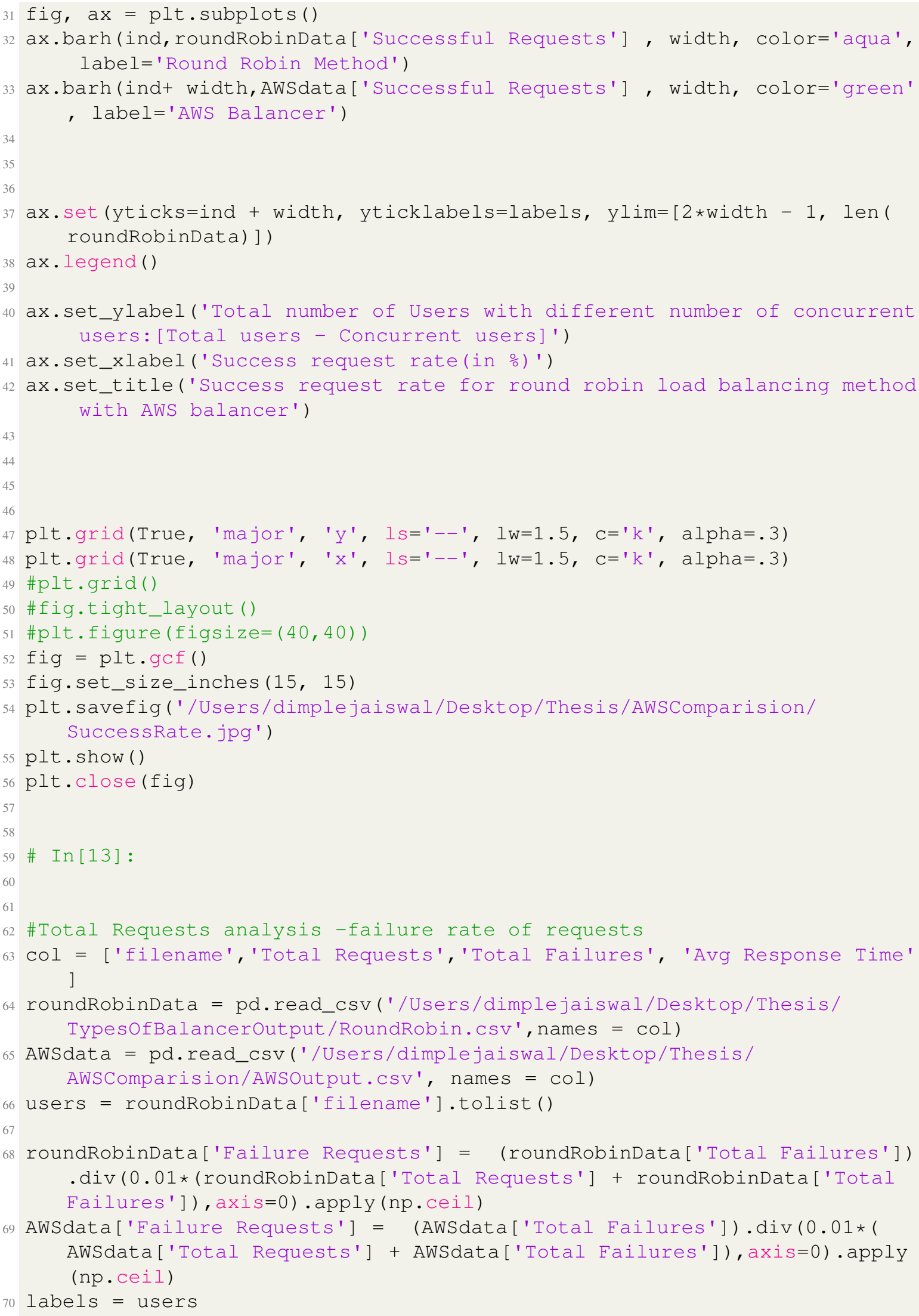




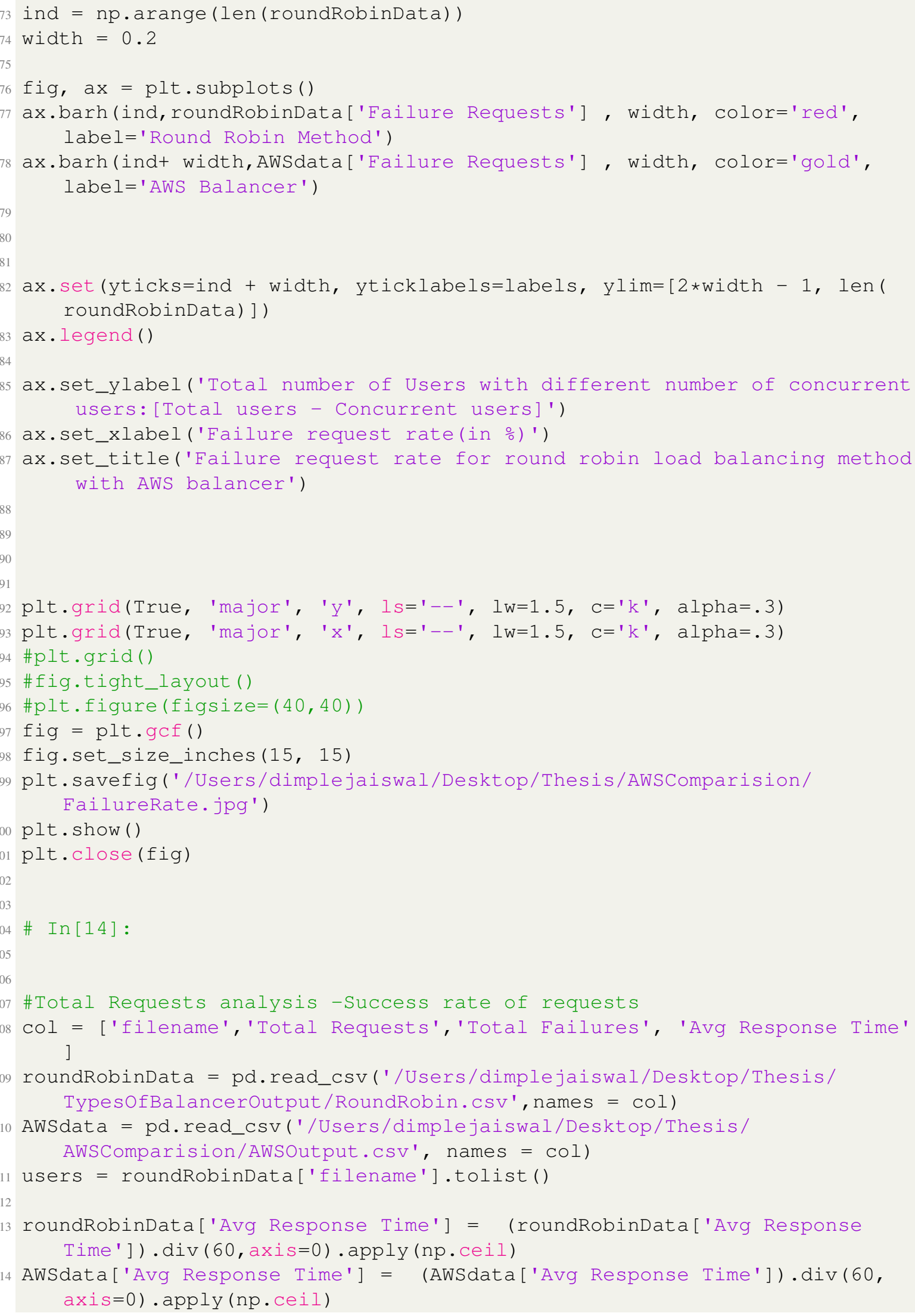




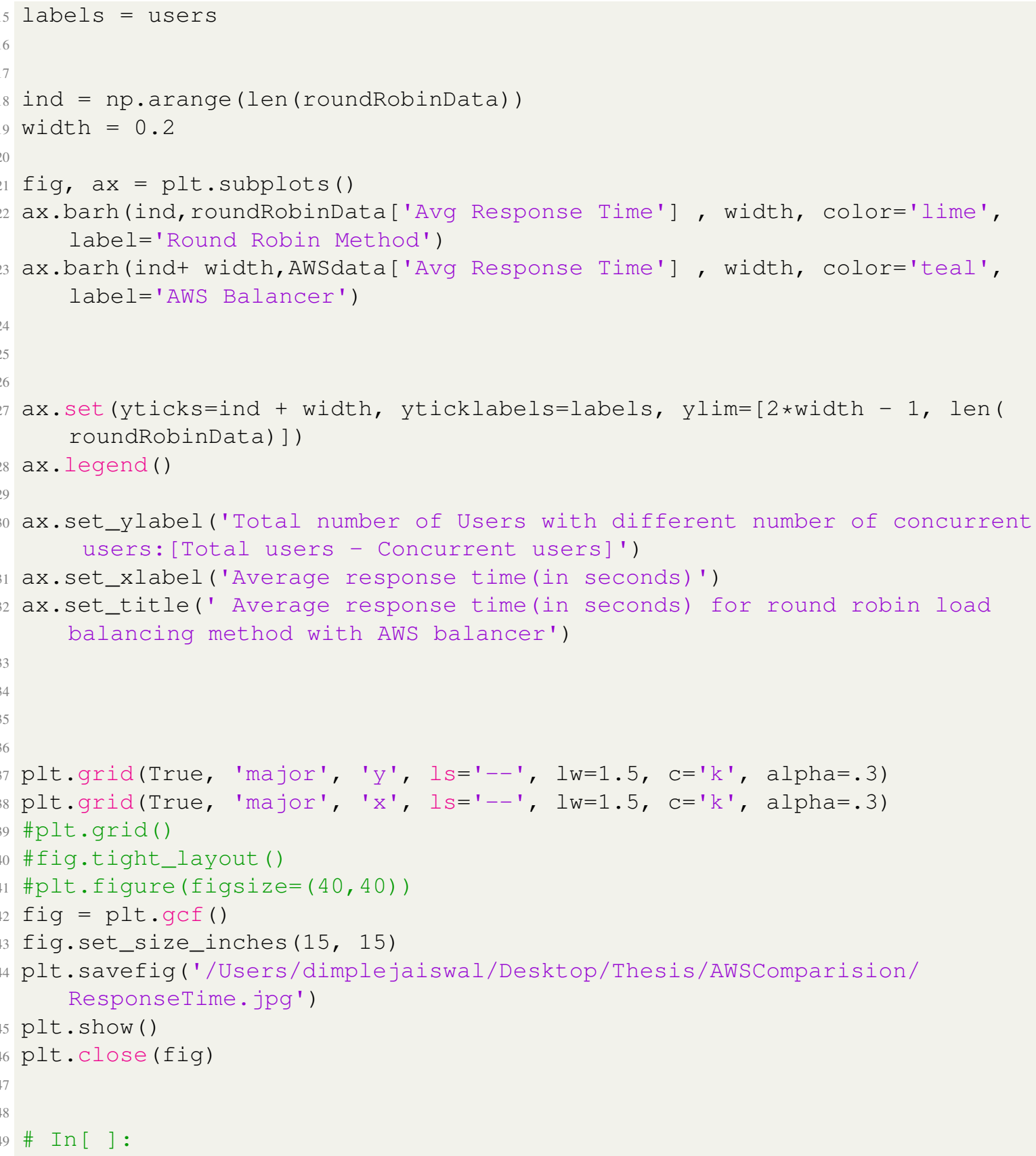

Code A.6.5.3: Analysis of data generated by Locust testing for application on AWS cloud with application using round robin approach on local cluster

Below are the figures generated using above script - 


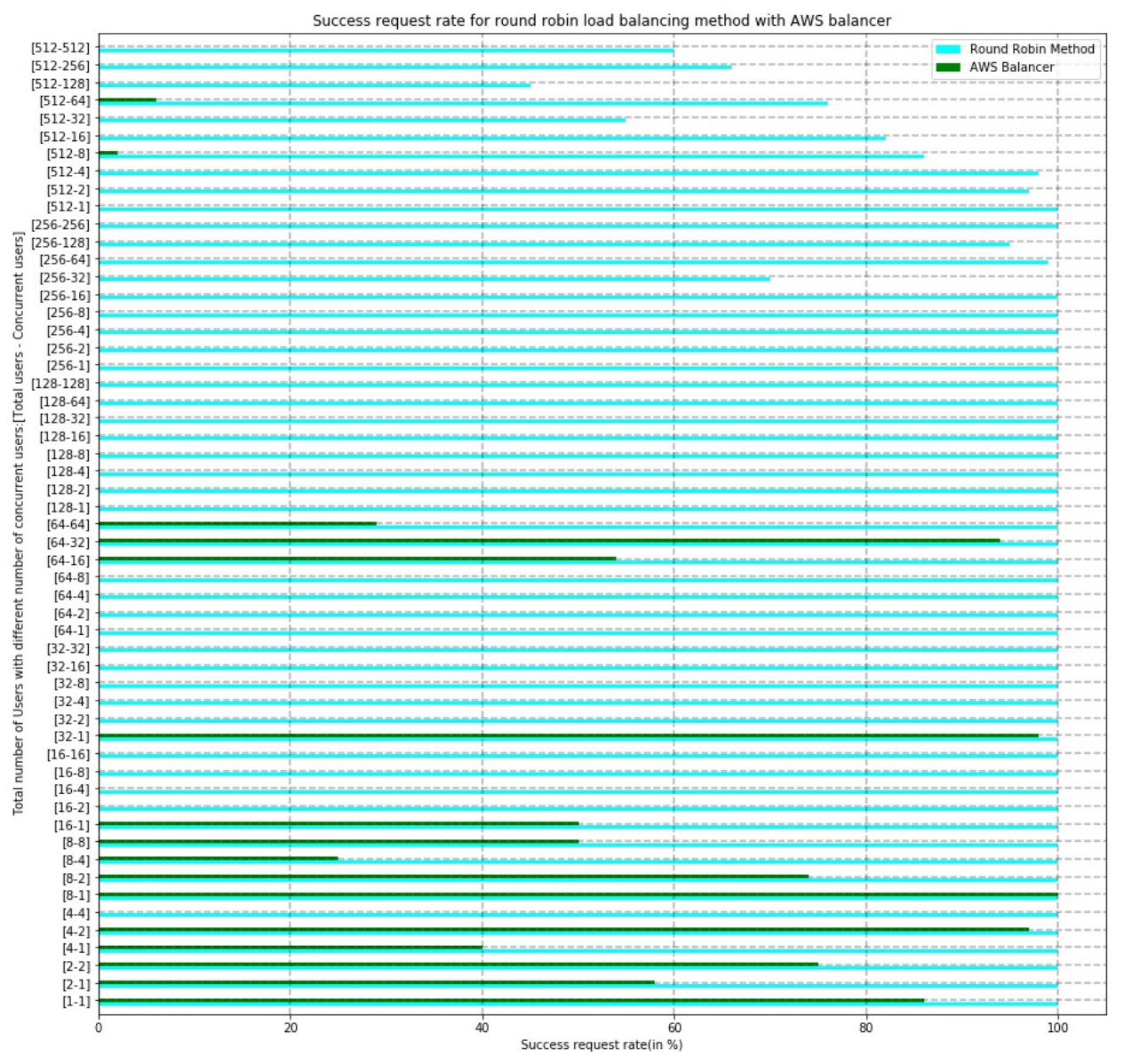

Figure A.24: Success request rate for round robin load balancing method with AWS balancer 


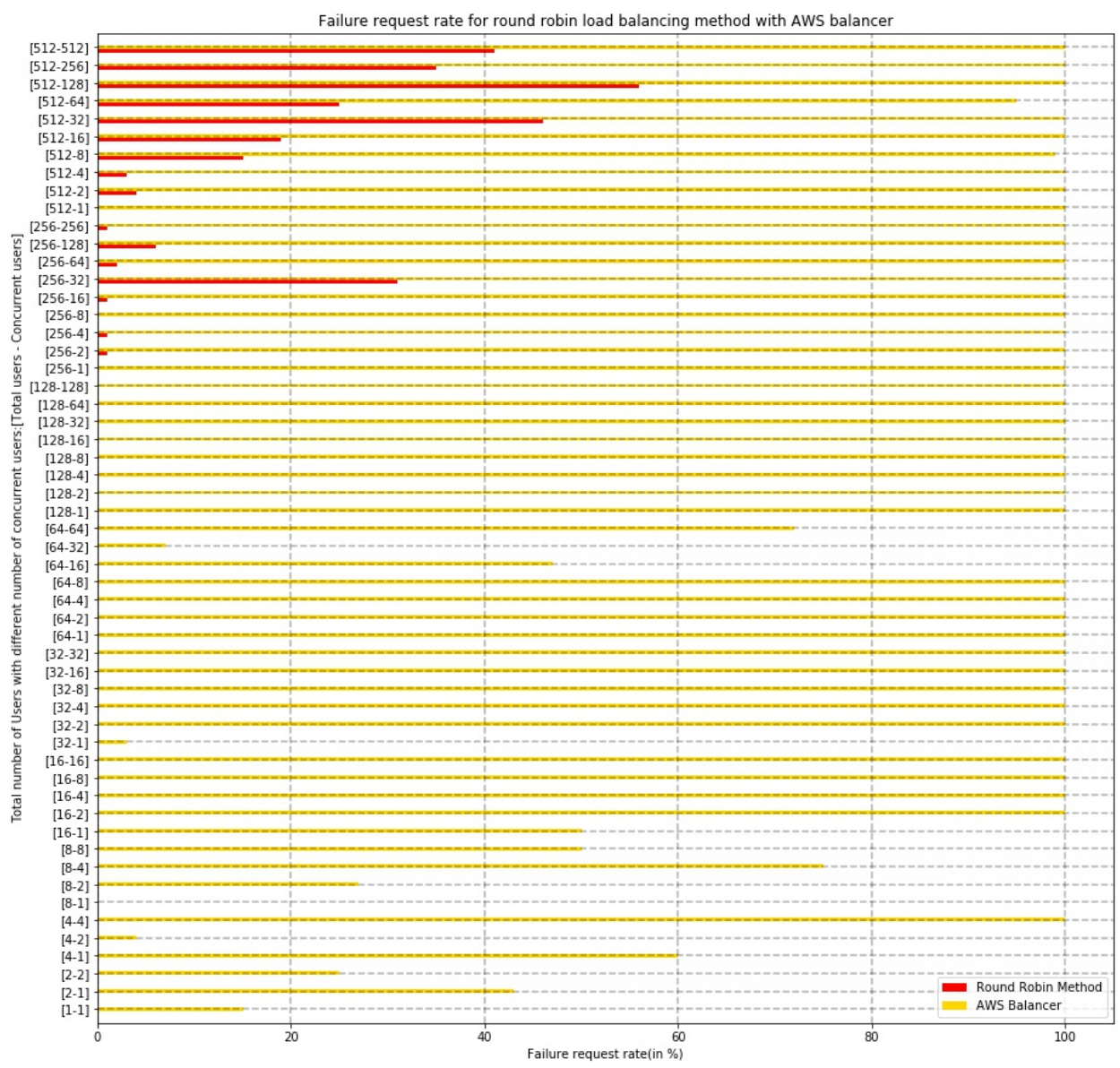

Figure A.25: Failure request rate for round robin load balancing method with AWS balancer 


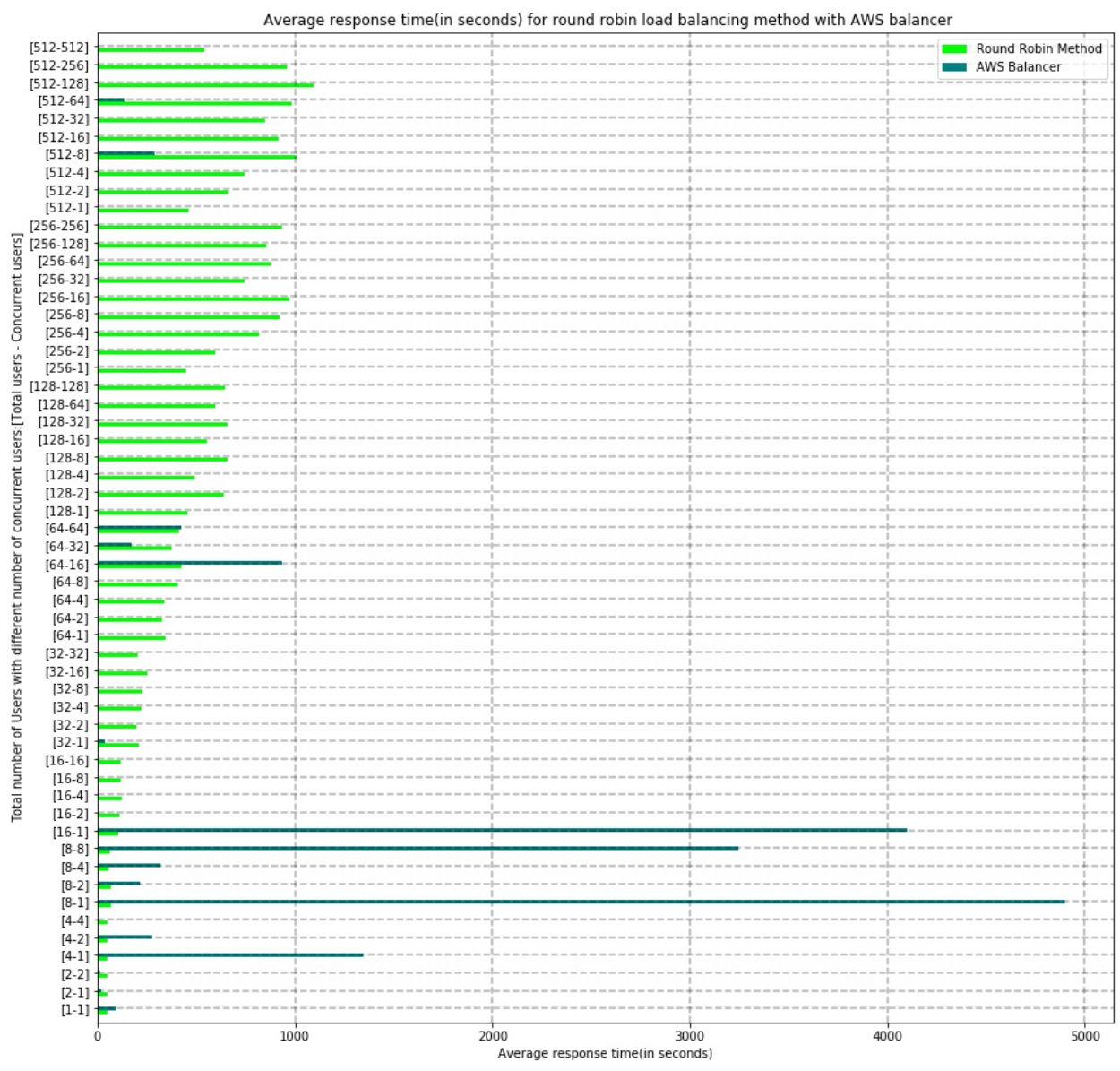

Figure A.26: Average response time(in seconds) for round robin load balancing method with AWS balancer 